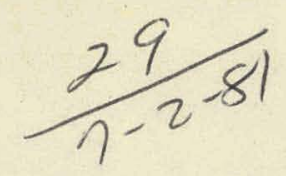

GEND-001

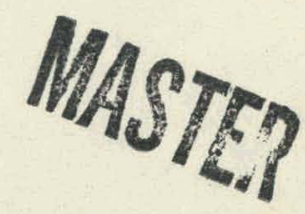

\title{
GEND PLANNING REPORT
}

\author{
Allied-General Nuclear Services \\ Argonne National Laboratory \\ EG\&G Idaho, Inc. \\ Electric Power Research Institute \\ Sandia National Laboratories
}

Published October 1980

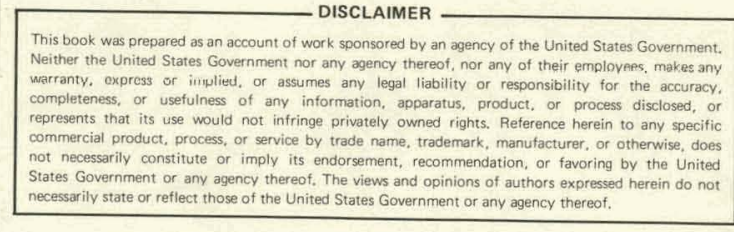

Prepared for the

U.S. Department of Energy

Three Mile Island Operations Office

Under DOE Contract No. DE-AC07-76ID01570 


\section{DISCLAIMER}

This report was prepared as an account of work sponsored by an agency of the United States Government. Neither the United States Government nor any agency Thereof, nor any of their employees, makes any warranty, express or implied, or assumes any legal liability or responsibility for the accuracy, completeness, or usefulness of any information, apparatus, product, or process disclosed, or represents that its use would not infringe privately owned rights. Reference herein to any specific commercial product, process, or service by trade name, trademark, manufacturer, or otherwise does not necessarily constitute or imply its endorsement, recommendation, or favoring by the United States Government or any agency thereof. The views and opinions of authors expressed herein do not necessarily state or reflect those of the United States Government or any agency thereof. 


\section{DISCLAIMER}

Portions of this document may be illegible in electronic image products. Images are produced from the best available original document. 
GEND REPORT

ISSUE RECORD

To facilitate maintenance of the Gend Report Document contents, the Gend Report will be controlled as a unit by use of "ISSUE NUMBERS".

Each transmittal of a Gend Report or Revision will be identified by an "ISSUE NUMBER".

Each release for inclusion in the Gend Report (or to indicate cancellation of an existing section) will be assigned the next "ISSUE NUMBER" not previously used.

As each new release is received by the Gend Report holder, the "ISSUE NUMBER" below that corresponds to the "ISSUE NUMBER" on the transmittal memoranda shall be crossed off. Thus, when an "ISSUE NUMBER" is crossed off and there. are numbers preceding this number that have not' been crossed off, it will be apparent that all released sections have either not been received or not filed in the Gend Report.

$\begin{array}{lllllllllll}001 & 011 & 021 & 031 & 041 & 051 & 061 & 071 & 081 & 091 \\ \because & 002 & 012 & 022 & 032 & 042 & 052 & 062 & 072 & 082 & 092 \\ 003 & 013 & 023 & 033 & 043 & 053 \cdots & 063 & 073 & 083 & 093 \\ 004 & 014 & 024 & 034 & 044 & 054 & 064 & 074 & 084 & 094 \\ 005 & 015 & 025 & 035 & 045 & 055 & 065 & 075 & 085 & 095 \\ 006 & 016 & 026 & 036 & 046 & 056 & 066 & 076 & 086 & 096 \\ 007 & 017 & 027 & 037 & 047 & 057 & 067 & 077 & 087 & 097 \\ 008 & 018 & 028 & 038 & 048 & 058 & 068 & 078 & 088 & 098 \\ 009 & 019 & 029 & 039 & 049 & 059 & 069 & 079 & 089 & 099 \\ 010 & 020 & 030 & 040 & 050 & 060 & 070 & 080 & 090 & 100\end{array}$


CONTENTS

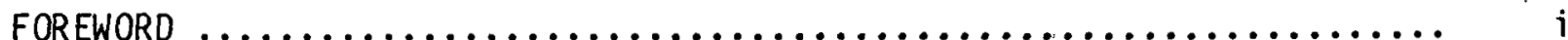

1.0 INSTRUMENTATION AND ELECTRICAL EQUIPMENT SURVIVABILITY $\ldots \ldots \ldots \ldots 1.0-1$

2.1 FISSION PRODUCT TRANSPORT AND DEPOSITION AND ENVIRONMENTS

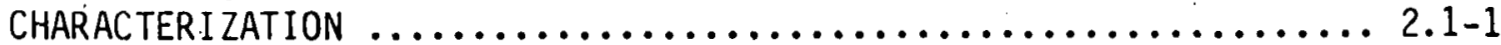

2.2 DECONTAMINATION AND PERSONNEL EXPOSURE CONTROL (Issued Under

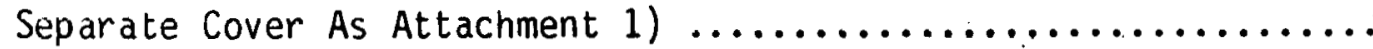

2.3 EARLY CONTAINMENT PENETRATION AND MONITORING $\ldots \ldots \ldots \ldots \ldots \ldots \ldots . . \ldots$

3.0 RADIOACTIVE WASTE HANDLING (Issued Under Separate Cover As

Attachment 2

4.0 (Combined with Section 2.0)

5.0 ARCHIVE SAMPLE REPOSITORY (TO Be Published Later) .............

6.1 PRIMARY SYSTEM PRESSURE BOUNDARY CHARACTERIZATION............ $6.1-1$

6.2 NUCLEAR STEAM SUPPLY SYSTEM MECHANICAL COMPONENTS RECOMMENDED FOR EXAMINATION DURING RECOVERY OF THE TMI-2 PLANT $\ldots \ldots \ldots \ldots \ldots . . \ldots .2-1$

7.1 CRITICALITY CONTROL STUDY (To Be Published Later).............

7.2 RECOMMENDATIONS ON IN-PLACE TMI-2 CORE DAMAGE EXAMINATIONS..... 7.2-1

7.3 SCOPING STUDIES OF THE ALTERNATIVE OPTIONS FOR DEFUELING, PACKAGING, SHIPPING, AND DISPOSING OF THE TMI-2 SPENT

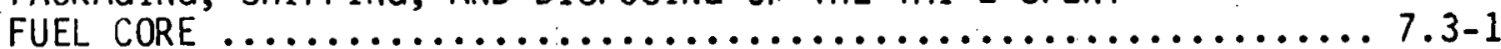

7.4 TMI-2 FUEL AND CORE COMPONENTS EXAMINATION.............. $7.4-1$ 
The Three Mile Island (TMI) Un it 2 accident on March 28, 1979 was and is of great concern to the nuclear industry; electric power generating companies and their customers, regulatory and other government agencies, the entire nuclear community, and to the country as a whole. While the accident resulted in only limited external plant radiation exposure, the plant itself suffered extensive damage with high radiation contamination within the reactor and auxiliary system facilities. TMI Unit 2 currently represents opportunities to provide information for the enhancement of the nuclear power industry safety and reliability of generic benefit to nuclear power technology. Having a common interest in assuring that this information is obtained during the TMI Unit 2 cleanup, four organizations have combined efforts to obtain this goal. They are: General Public Utilities (GPU), Electric Power Research Institute (EPRI), the Nuclear Regulatory Commission (NRC), and the Department of Energy (DOE). Hence the acronym GEND. Planning Groups have been formed to determine the scope of research activities to be performed to recover this valuable information. The "GEND" Planning Report covers the areas of: instrumentation and electrical equipment survivability; fission product transport; decontamination/radiation dose reduction technology; data bank organization and sample archive facility; characterization of primary system pressure boundary and mechanical components; core damage assessment; and fuel handling, removal, examination and disposal. Through the efforts of these groups, a list or "menu" of tasks which could be performed during the cleanup and recovery has been developed in each of the areas. Many of these tasks will form the bas is for the implementation phase of the program, the results of which will be used throughout the U.S. nuclear commun ity. 


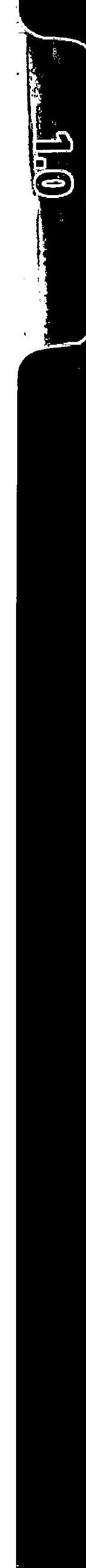




\section{Report of The \\ Instrumentation and Electrical Equipment \\ Survivability Planning Group \\ (Task 1.0)}

September 1980

1. 0-1 


\section{Planning Group Members}

Richard A. Damerow, Chairman, Sandia National Laboratories

John T. Bauer, General Atomic Company

Lloyd L. Bonzon, Sandia National Laboratories

Matthew Chiramal, U. S. Nuclear Regulatory Commission

Ronald A. Feit, U. S. Nuclear Regulatory Commission

Jack W. Malcomb, Bailey Controls Company

James Mock, EG\&G Technical Integration Office

Michael B. Murphy, Sandia National Laboratories

Lester C. Oakes, Oak Ridge National Laboratory

Gerald J. Sadauskas, Burns and Roe Company

Frank Silag, Bailey Controls Company

George Sliter, Electric Power Research Institute

Karl W. West, Oak Ridge National Laboratory 
Planning Group Members $. . \ldots \ldots \ldots \ldots \ldots \ldots \ldots \ldots \ldots \ldots \ldots \ldots \ldots \ldots . \ldots \ldots$

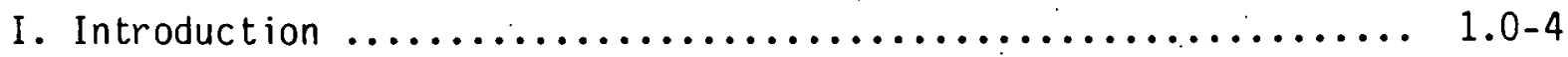

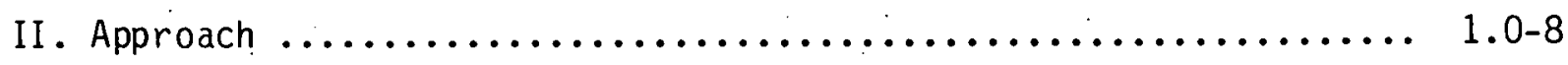

II . Preliminary Recovery List ........................ 1.0

IV. General Findings and Recommendations $\ldots \ldots \ldots \ldots \ldots \ldots \ldots \ldots \ldots . \ldots \ldots 2$

Appendix 1 -- Sumary of Reactor Building Instrumentation and

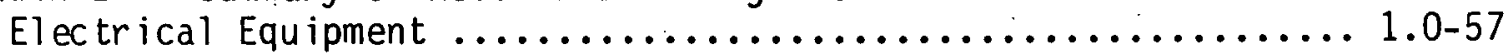

Appendix 2 -- Instrument Analys is Procedure ................. 1.0-62

Appendix 3 -- Selected Plant Drawings ...................... 1.0.65

Appendix 4 -. Piping and Instrumentation Diagram ............ 1.0-70

Appendix 5 -- Master List of TIM-2 In-Containment Instruments and Electrical Equipment ............................... 1.0.73 


\section{Introduction}

The Instrumentation and Electrical Equipment Survivability Planning Group (IEPG) was appointed to develop the planning guidance needed for the orderly recovery of data pertaining to the survivability of instrumentation and electrical equipment contained within the TMI Unit 2 reactor building.

During an accident, the safety of a nuclear reactor depends upon the proper functioning of certain items of instrumentation and electrical equipment; other items will be called on during post-accident recovery operations. Hence, a knowledge of the response of such equipment to accident conditions is crucial to an assessment of the safety of a nuclear plant under accident conditions. The TMI-2 accident subjected the in-containment electrical equipment and instrumentation to the effects of an accident environment which, while not one usually. treated in accident scenarios, is clearly possible. Thus, the data obtained from an analysis of the accident response of the TMI-2 equipment will be valuable in: 1) developing improved and more comprehensive standards for equipment qualification; 2) understanding how equipment designed under existing standards performed; 3) identifying equipment failure modes; and 4) assessing the safety of existing plants which use similar equipment. It should be noted that the TMI accident provides two time frames for equipment study in accomplishing these goals: a) the first few days of the accident 
in which much of the equipment was subjected to high heat, steam, chemical sprays, and explosion and high radiation levels; and b) the long period since the accident where long term degradation occurs.

One difficulty faced by the IEPG has been to devise a TMI-2 reentry program which strikes a reasonable balance between the needs of the nuclear industry and various government agencies on the one hand and the needs of the operating utility on the other. The industry/government group is concerned with the need to understand the effects on in-containment instrumentation and electrical equipment of prolonged exposure to significant levels of radiation and to various other hostile environments. The operating utility is concerned with the need to restore the plant to service with minimum delay.

A thorough analysis of all reactor building instrumentation and electrical equipment could be very costly in itself and is likely to be incompatible with the schedules one would employ in a. minimum time decontamination and reentry strategy. There has been considerable concern expressed that the decontamination procedures will be sufficiently damaging to the items within containment that much of the potential for assessing damage mechanisms will be lost. On the other hand, one must not lose sight of the fact that, without decontamination, "hands-on" examination and recovery of any equipment might not be practical. Again, these 
observations merely highlight the fact that the approach chosen necessarily involves balance and compromise.

Having made the point that consideration was given to striking a balance between the benefits of increased knowledge and the increased costs (monetary, personnel exposure, etc.) to be paid for these benefits, we will now turn to the purely technical goals of the IEPG.

Objectives - The objectives of this planning study were to:

1) Specify particular instrumentation and electrical equipment to be studied. The equipment might be. simply tested in place or it might be removed and thoroughly analyzed.

2) Define test objectives and methods for each component selected.

3) Develop a list of components and samples for archival storage.

Scope - The scope of this planning study includes all instrumentation and electrical equipment within the TMI-2 containment building. Certain items within this broad class are required for core recovery operations (for example, in-core reactor instruments) and may not be available for removal until the core is removed. However, the fact that their cables and connectors were exposed to the in-containment environment suggests that it would be unwise to eliminate them from our consideration.

This report lists some 228 pieces of equipment as candidates for further study and possible recovery and analysis. This list of candidates represents a broad sampling of equipment types 
generally with samples of a given type taken from several different locations in the containment building. Many of the items are presently under water. Some of the selections were chosen because they are representative of generic classes, while others were chosen because they have unique qualities or were known to behave in an unusual way during some stage of the accident. It is expected that as more information becomes available the study list will be further refined.

The planning group was divided on the questions of the depth of analysis required, the types of equipment to be analyzed, and the number of items required for analysis. The cost vs. benefit was the issue. The feeling of the group, however, was that as more thorough equipment status surveys, in situ tests and actual analyses of some representative instruments are made; answers to these questions will become more apparent. It was agreed that the longer the equipment remains in the containment building exposed to the degrading environments there the less useful the analysis information will be. 


\section{Approach}

The IEPG has done the following to accomplish the objectives previously given: First, information was gathered from the following persons and/or organizations:

1. Planning Group Members

2. Subcommittee Two (Qualification), Nuclear Power Engineering Committee, Institute of Electrical and Electronics Engineers (IEEE).

3. Industry Experts

4. National Laboratory Experts secondly, various reference documents were reviewed by the planning Group, including the following:

1. TMI-2 Lessons Learned Task Force Status Report and Short-Term Récommendations, NUREG-0578, July 1979.

2. rMI-2 Lessons Learned Task Force Final Report, NUREG-0585, October, 1979.

3. Investigation into the March 28, 1979, Three Mile Island Accident by Office of Inspection and Enforcement, NUREG-0600, August 1979 .

4. Analysis of Three Mile Island - Unit 2 Accident, NSAC-1, July 1979.

5. Supplement to Analysis of Three Mile Island-Unit 2 Accident, NSAC-1 Supplement, October 1979.

6. A list of in-containment instrumentation and electrical equipment dated June 19, 1979 (B\&R-GPU-R-008) and 
supplied to the planning group by GPU Service Corporation, October 2, 1979.

7. A list of Bailey instruments and associated drawings supplied by the Babcock \& Wilcox Company, October 15, 1979.

8. Relevant Architect and Engineer design drawings (i.e., P\&ID's, general arrangement drawings, etc.).

Finally, the selection of instruments and electrical equipment was made with the thought that the resulting data can serve several purposes:

1. A comparison of the actual performance of LOCA qualified equipment with its expected performance should lead to the development of improved qualification standards. These improvements can be expected to involve (a) what is required for proof of qualification, (b) changes in test type and level, and (c) testing against accident scenarios which are derived from the TMI-2 experience.

2. Related to the question of improved qualification standards is the assessment of the adequacy of existing standards. Operating data frnm TMI-2, when compared with the qualification standards in effect today, will allow evaluation of the extent to which present standards apply to this "real world" accident. That is, do the present qualification standards assure that key items of instrumentation and equipment can withstand this specific event. 
3. Analysis of equipment failure modes will provide important guidelines for design improvements. The failure of equipment that, by current understanding, should not have failed provides a fruitful area for study as does the survival of items predicted to fail. In general, failures that can be predicted on the basis of the environmental exposures are unlikely to advance our understanding although a few confirmation samples will be required.

4. An important outcome of the analysis of TMI-2 data will be an assessment of the vulnerability of the many existing plants which have equipment identical to that in TMI-2. Note that vulnerability has two important aspects: the first involves accident management, and the second involves pustodccident monitoring and recovery. As the lessons of this accident are assimilated, it is becoming clear that many other items beyond the normal (present standards) complement of $1 E$ equipment may be of crucial importance in an accident situation. TMI-2 thus provides an opportunity for assessing the response of typical equipment to an actual accident.

5. To the above Iist of general uses for the data, one must add a use which is specific to understanding the TMI-2 accident. This category includes calibration of instrument readings recorded during the accident and the 
determination of fission product transport and disposition. operator actions might also be more clearly evaluated. Certainly, the items which yield information specific to the accident (Item 5 above) should be recovered, as should class $1 E$ items that failed and other items which failed but should not have. Beyond this, it is difficult to argue that only recovery of items from the TMI-2 containment will answer the important general questions of nuclear safety. In this regard, at least two thoughts should be kept in mind. First, we cannot predict at this time what accuracies will be attained in the environmental maps which are being developed. Second, the radiation qualification requirements (approximately $2 \times 10^{4}$ rads total dose) for the TMI-2 instruments were not particularly severe by modern standards.

This document provides a list of items whose study will potentially serve one or more of the five purposes given above. The list can be reasonably decomposed into five different categories (discussed below), and a priority assessment has been provided for each item on the list.

IL is not expected that all of the items on this study list will actually be recovered from within the TMI-2 containment or that other items will not eventually be selected for recovery. Rather, this list can be adopted as a reasonable subset of all the items within the containment building. As data becomes available on these candidates for selection, choices can be made as to the need for recovery and analysis. Whether or not laboratory simulation 
is needed to provide additional information is a question that should be addressed on an item-by-item basis.

The study candidates may be associated with one or more of the following five categories. The letters $S, I, D, E$ and $A$ are abbreviations of the general categories used in the computer listing of candidate selections.

Safety Equipment (S)

This category includes both items currently designed as safety equipment and those items expected to receive this designation in future revisions of the appropriate regulatory guides. Incident-Related Equipment (I)

In this category we have included equipment which deserves particular attention as a result of its special relationship to the accident. For example, equipment whose output data was used in calculations of the accident development might well require recalibration to assess the likelihood that the conclusions drawn are correct. Also, equipment used by the operators as the accident developed should be particularly scrutinized to see if the data provided was correct or, at least, within the expected performance envelope given the accident conditions.

Equipment Yielding Equipment Design Data (D)

Analysis of equipment in this category can potentially contribute to improved equipment design. In particular, all failed equipment should be carefully considered. In addition, one must be 
alert to opportunities to assess operational margins and to explore actual performance versus expected performance.

Equipment Useful in the Determination of the Radiation Environment $(E)$

A variety of material properties undergo changes in response to radiation exposure. Particular materials whose radiation response make them possible "dosimeter" candidates include semiconductors, glass, plastics, elastomers, and plastomers. The equipment in this category has been chosen because it includes one or more of the items listed above. Naturally, most of the items chosen under the other categories will also include various samples of the "dosimeter" materials. In only a few cases do study candidates have only "dosimeter" utility.

Equipment Designated for Archive Storage (A)

Certain items have been selected for archive storage before any analysis has been attempted to preclude the loss of data which future investigations might prove to be useful. In addition, some items will be stored after analysis.

Having discussed the logic leading to the five cateyuries of equipment, there only remains the need to define the priority assignments and the description of the entire selection process will be complete.

Priority one is assigned to equipment of the following kinds:

1. IE or potential lE equipment.

2. Reactor control equipment. 
3. Equipment needed to understand the accident.

4. Equipment thought to be especially sensitive to the environments and therefore useful for establishing margins.

5. Equipment having properties especially useful in assessing damage or representative of important generic features.

Priority two includes duplicates of priority one items as well as items judged to have lower (relative to priority one) potential for information.

Priority three items are those whioh have some chance of adding to our store of knowledge but which are judged not to be of the same urgency as priority one or two.

Along with a discussion of our overall selection and priority assignment logic, it is worthwhile to state the principal impediment to proceeding to a firm selection list. This is the virtually complete unavailability of information pertaining to the performance of the equipment which is the object of nur study. The principd. lliter $1 a$ l available to the IEPG has been other investigatory reports and plant general arrangement drawings.

For example, all failed equipment should be the ulject of special attention. This would certainly include equipment which failed or had anomalous readings during the first few days of the accident and probably should include equipment failing later. To the best of our knowledge, no such list exists. Thus, in most instances, we were forced to make speoific instrument selections and general recommendations without the necessary material to justify in detail a particular selection. 


\section{Preliminary Recovery List}

Table I contains a listing of candidate items to be recovered. A brief statement of the rational and selection basis for the various items is given in Table II. The primary identification for each item is the tag number and the lists throughout this document are in tag number sequence. Additional information is provided as indicated by the various column headings. Table III provides an explanation of the various codes used; it is worthwhile, however, to supply a few additional comments about the computer listing. The information category column (INFO) shows the various information categories (see section II) to which the items belong. The status (ST) column will be used to indicate the present operating condition and readout capability of each instrument. This information is essential in establishing a pre-recovery test program and, as stated earlier, is unknown to us at this time. The work code column' will contain information regarding sample recovery plans. For example, the entry $A B X X$ means that in situ testing is required prior to recovery and that sample recovery cannot be performed until after core removal. The notation $A B$ means that in situ testing is required and that there are no restrictions on removal of the sample. Note that items coded A must have priority in the development of detailed testing procedures. The work code can be completed after the status of instruments is known. The special sealing program (SSP) column identifies those items which were subject to various additional sealing procedures to upgrade their resistance to water damage, etc. 
TMI UNIT 2 REACTOR BUILDING INSTRUMENT LISTING

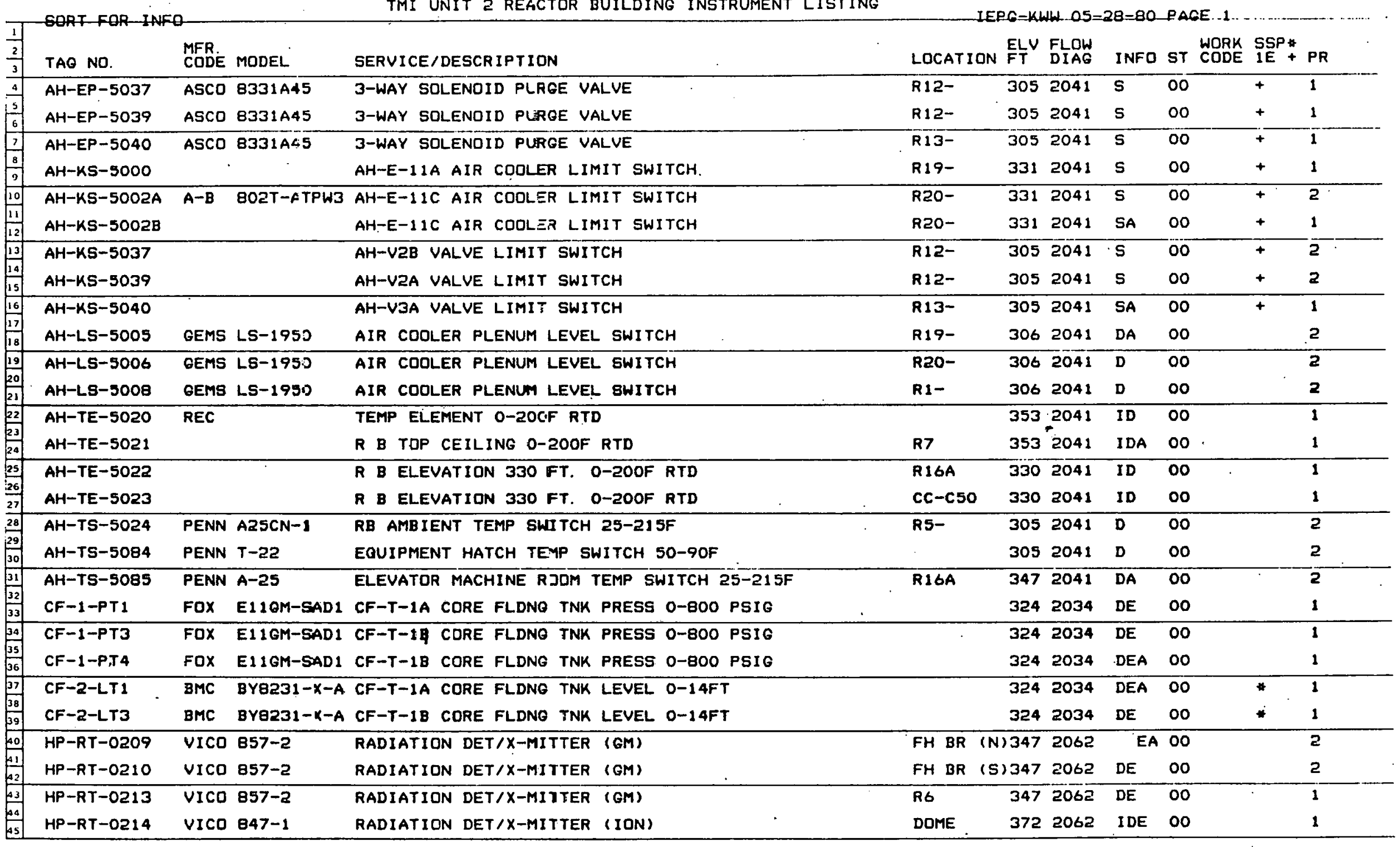


TABLE I (Cont'd)

TMI UNIT ב REACTOR BUILDING INSTRUMENT LISTING

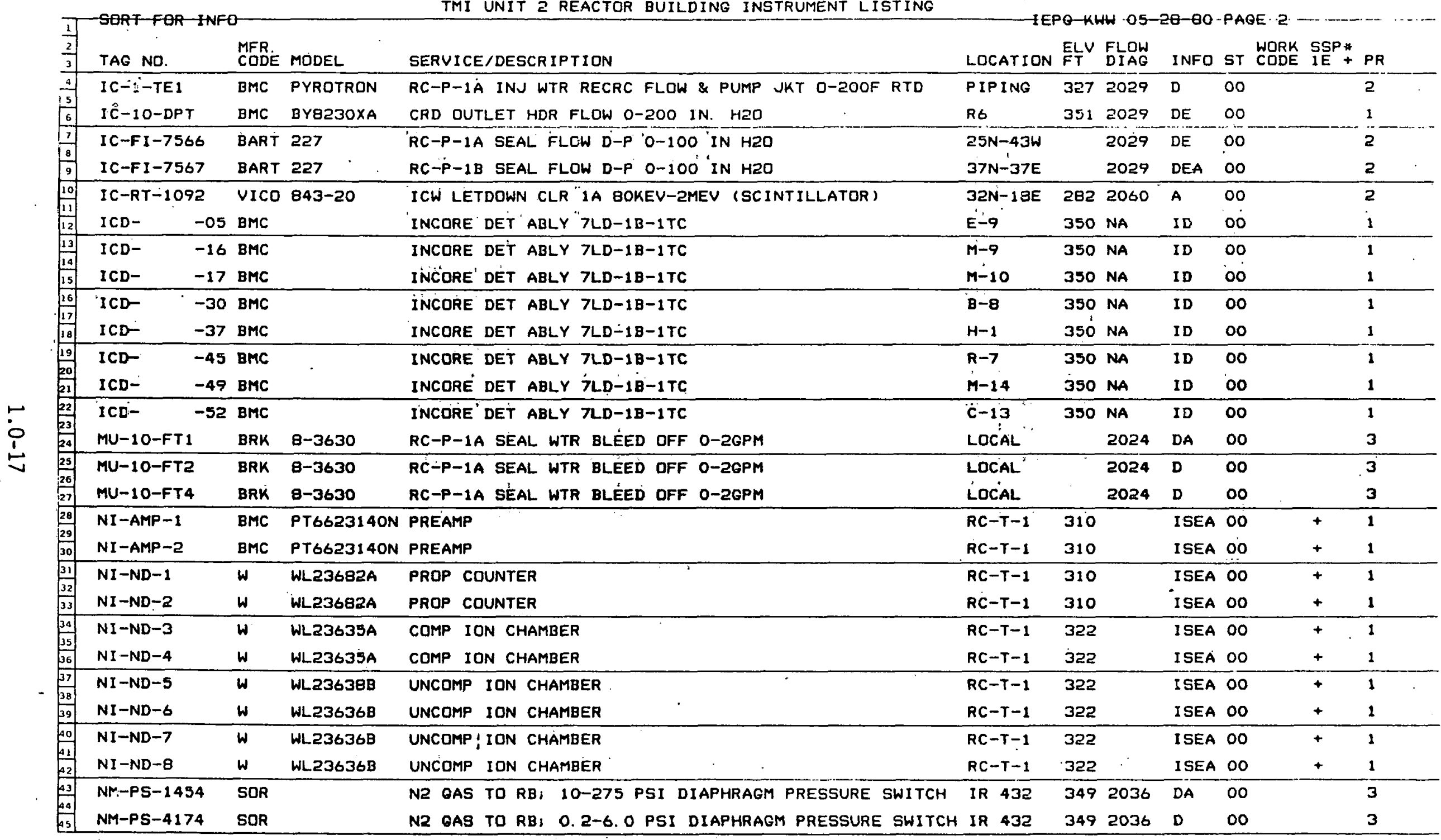


TMI UNIT 2 REACTOR BUILDING INSTRUMENT LISTING

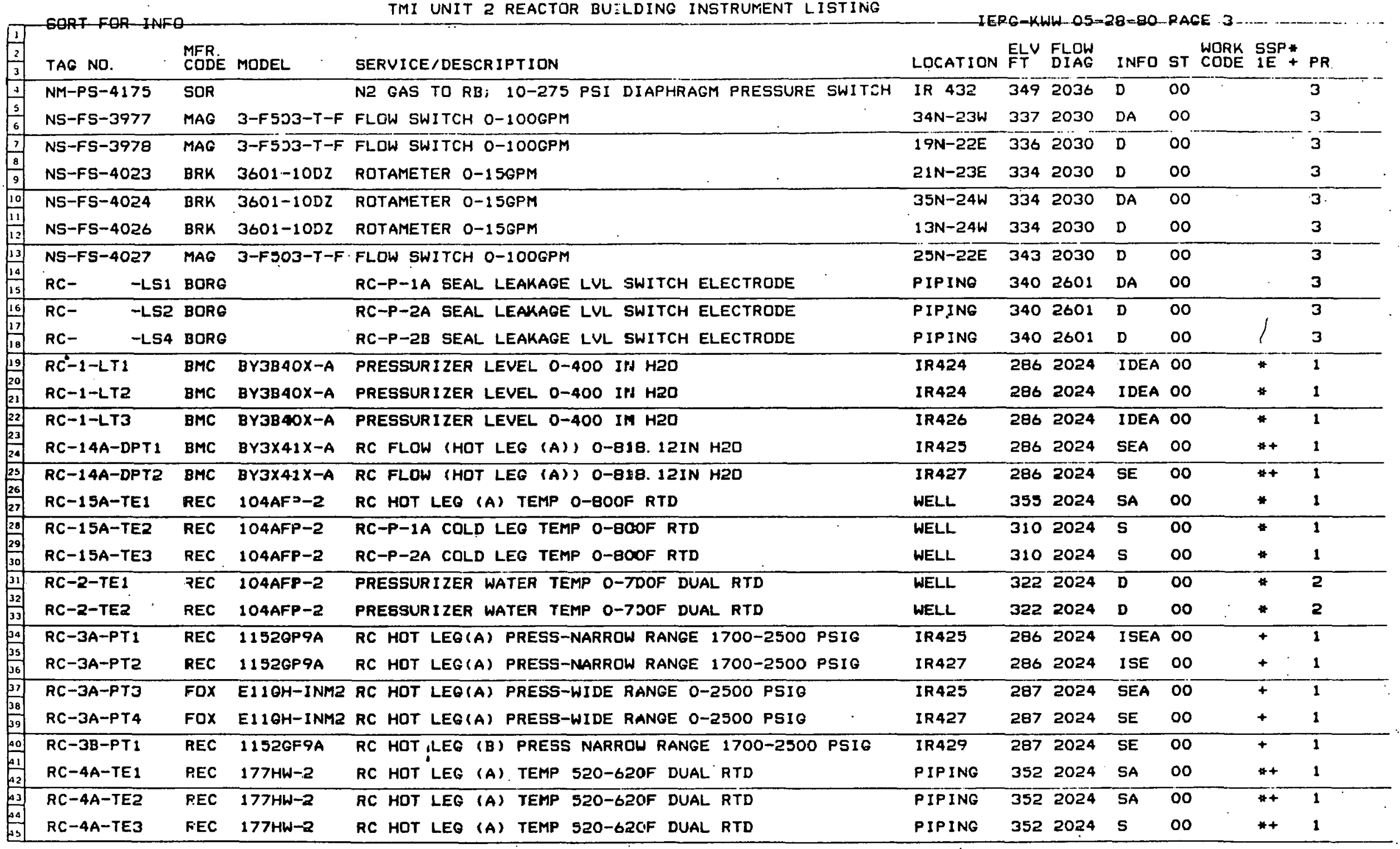


TABLE I (Cont'd)

TMI UNIT 2 REACTOR BUILDING INSTRUMENT LISTING

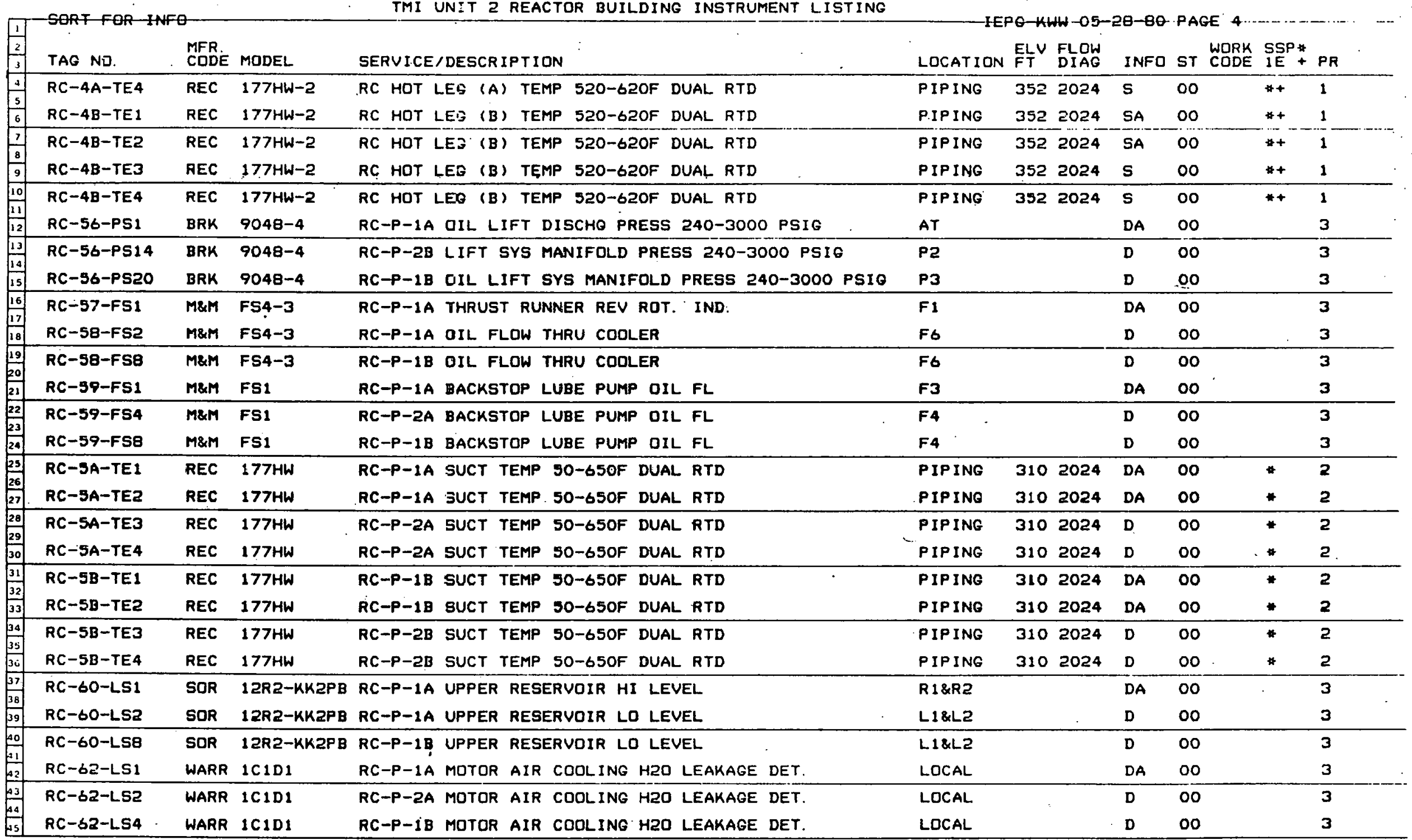


TMI UNIT 2 REACTOR BUILDING INSTRUMENT LISTING

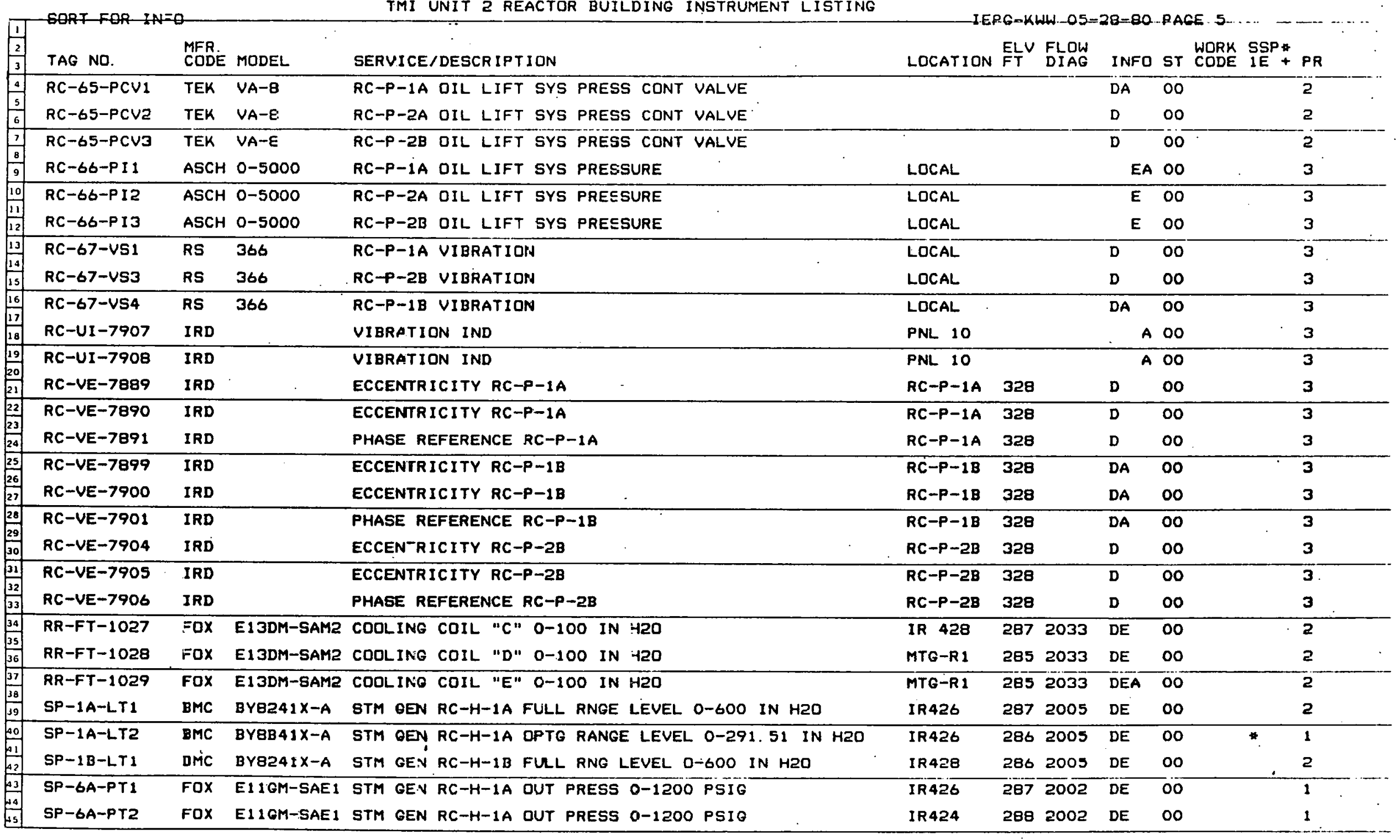


TMI UNIT 2 REACTOR BUILDING INSTRUMENT LISTING

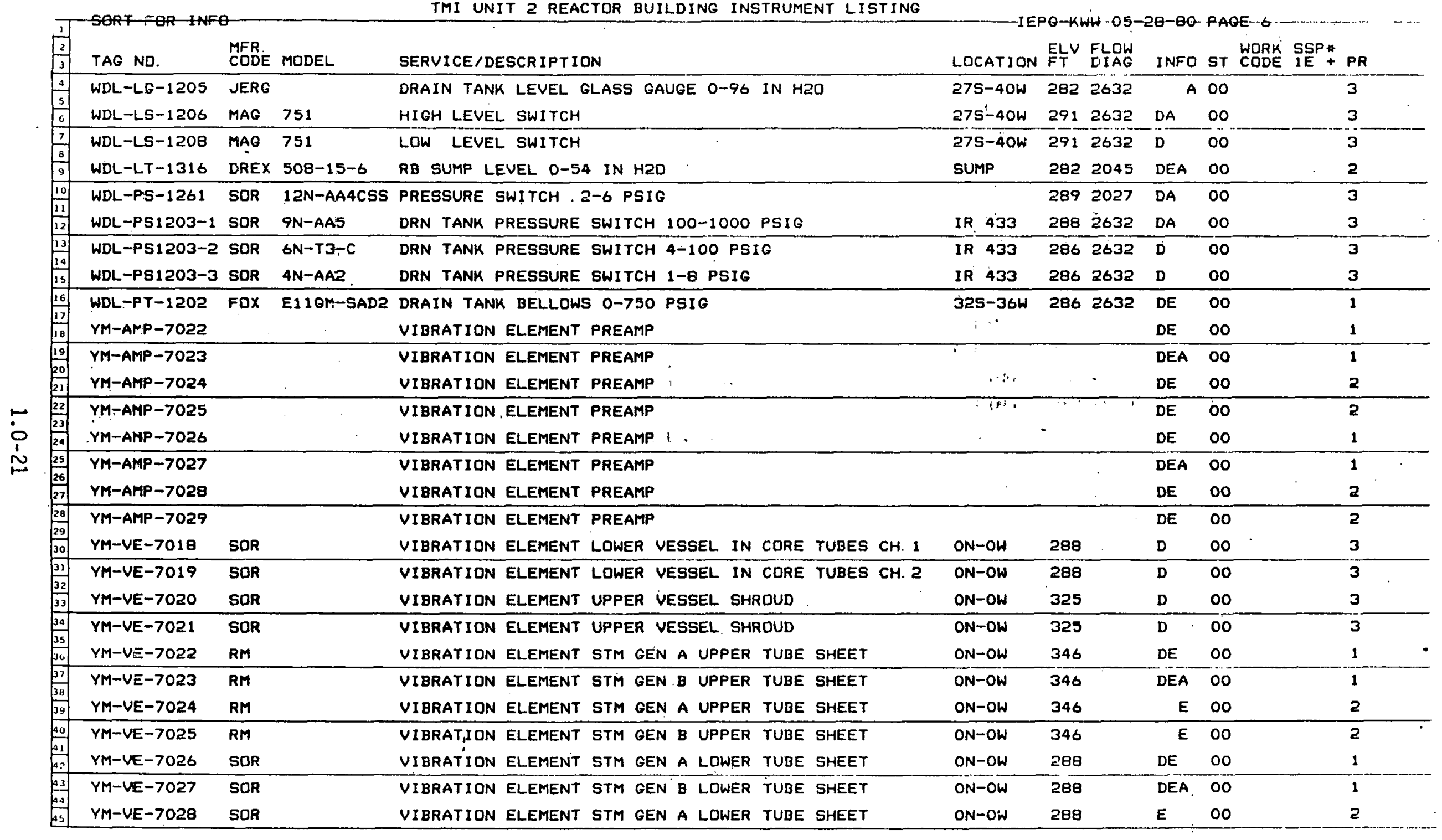


TABLE I (Cont'd)

TMI UNIT 2 REACTOR BUILDING INSTRUMENT LISTING

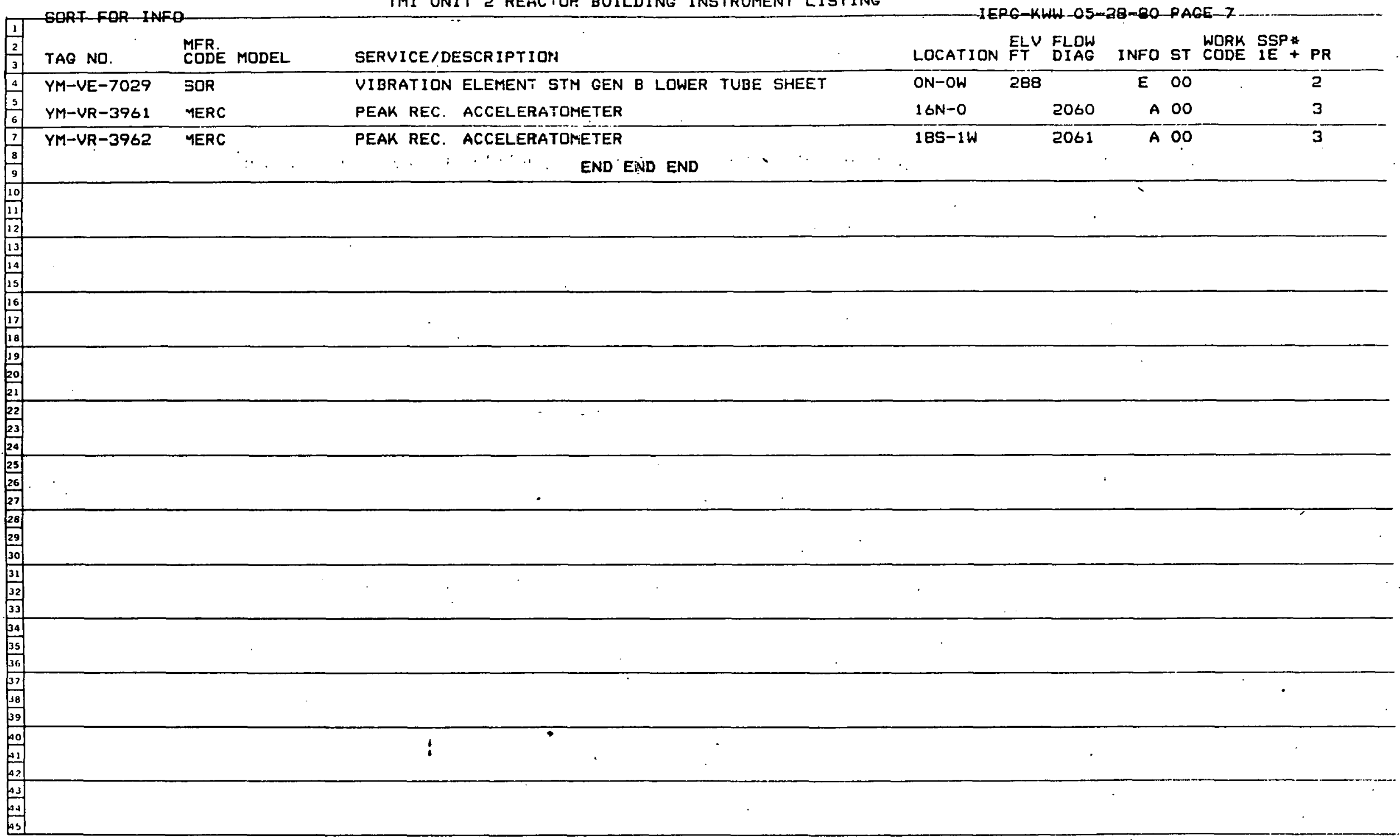


TMI UNIT 2 REACTOR BUILDING ELECTRICAL EQUIPMENT LISTING

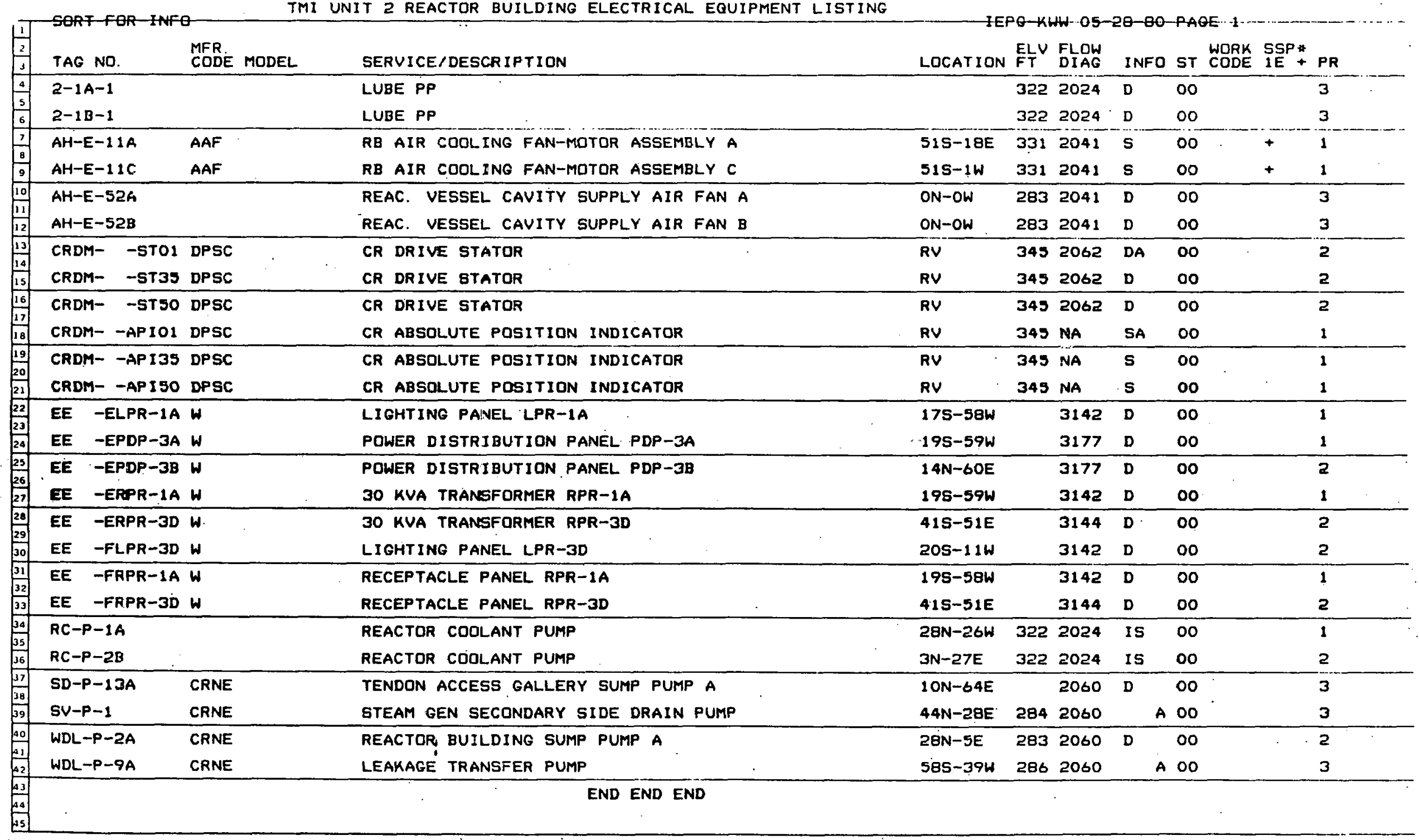




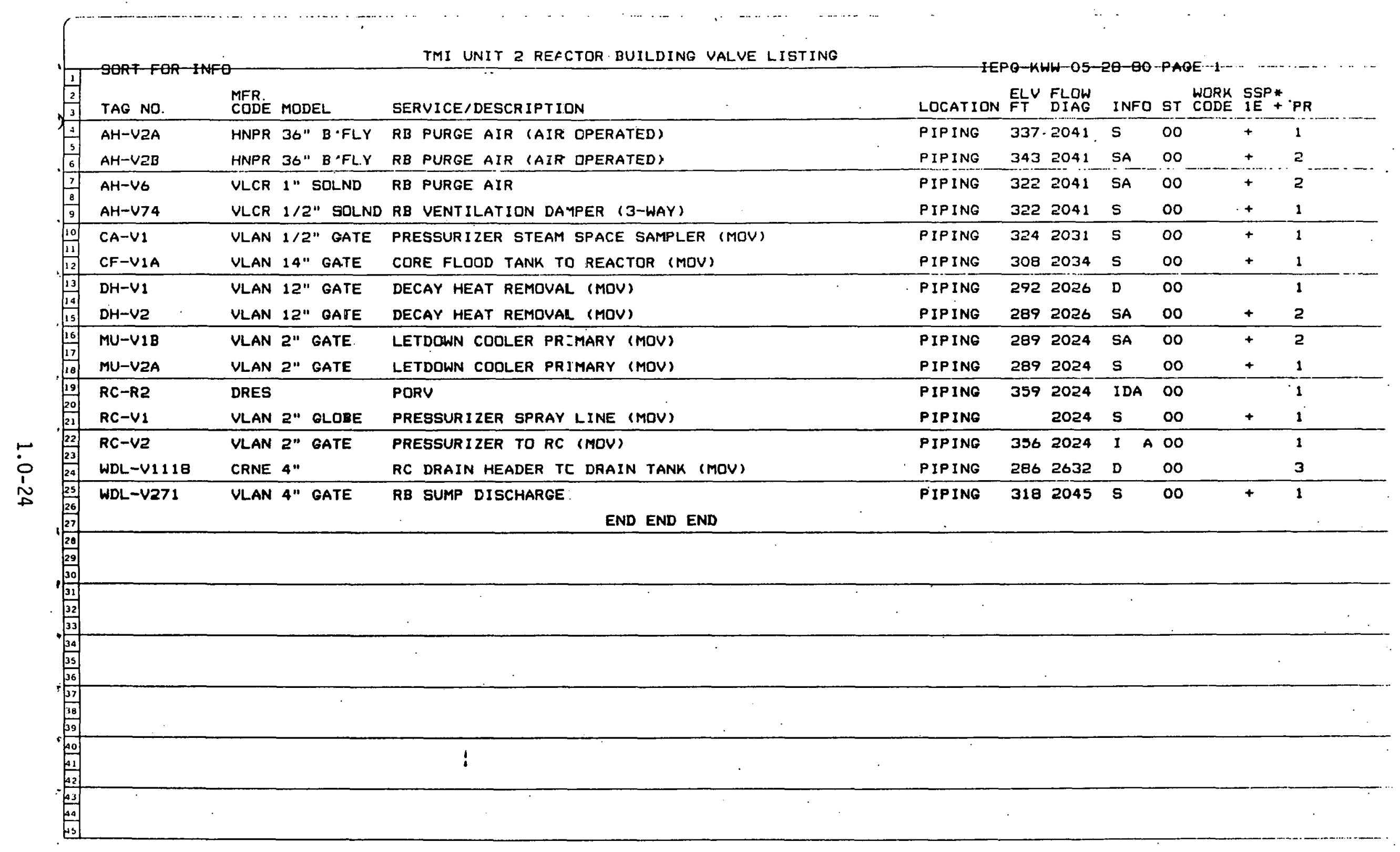


TMI UNIT $\supseteq$ REACTOR BUILDING PENETRATION LISTING

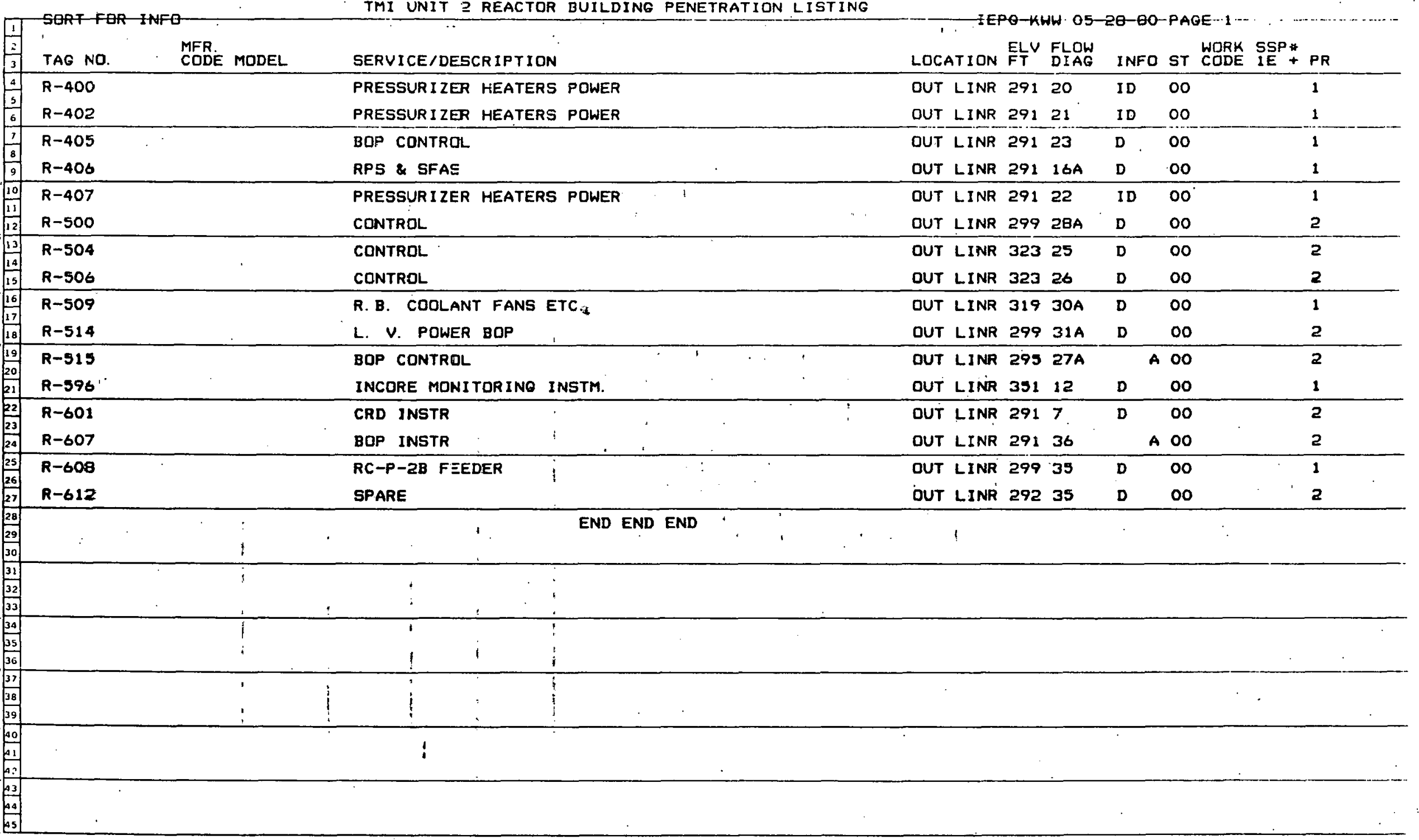



TABLE II (Cont'd)

TMI UNIT 2 CONTAINMENT BUILDING EQUIPMENT SELECTION BASIS

AND PRIORITY ASSIGNMENT

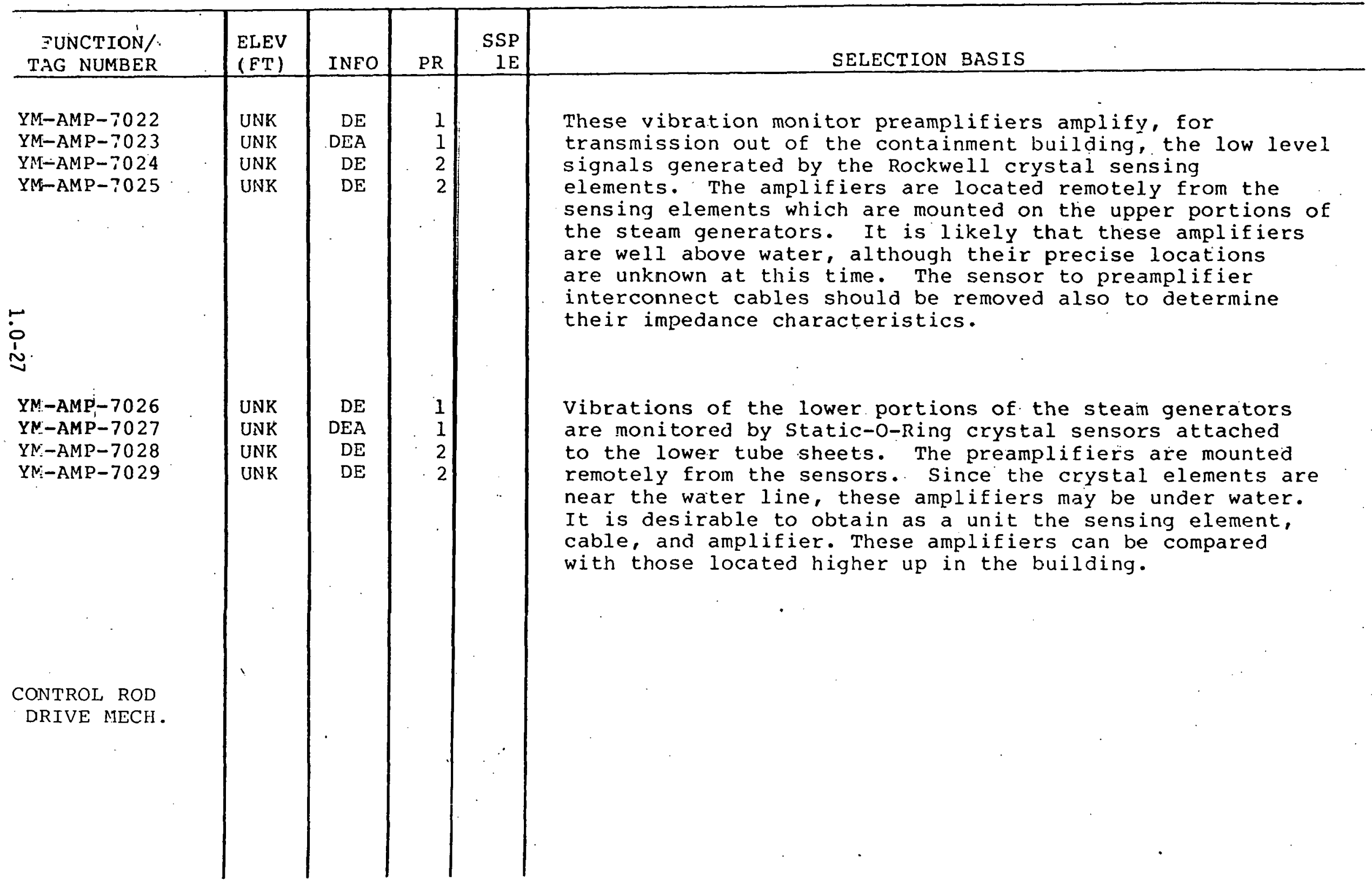


TABLE II (Cont'd)

TMI UNIT 2 CONTAINMENT BUILDING EQUIPMENT SELECTION BASIS

AND PRIORITY ASSIGNMENT

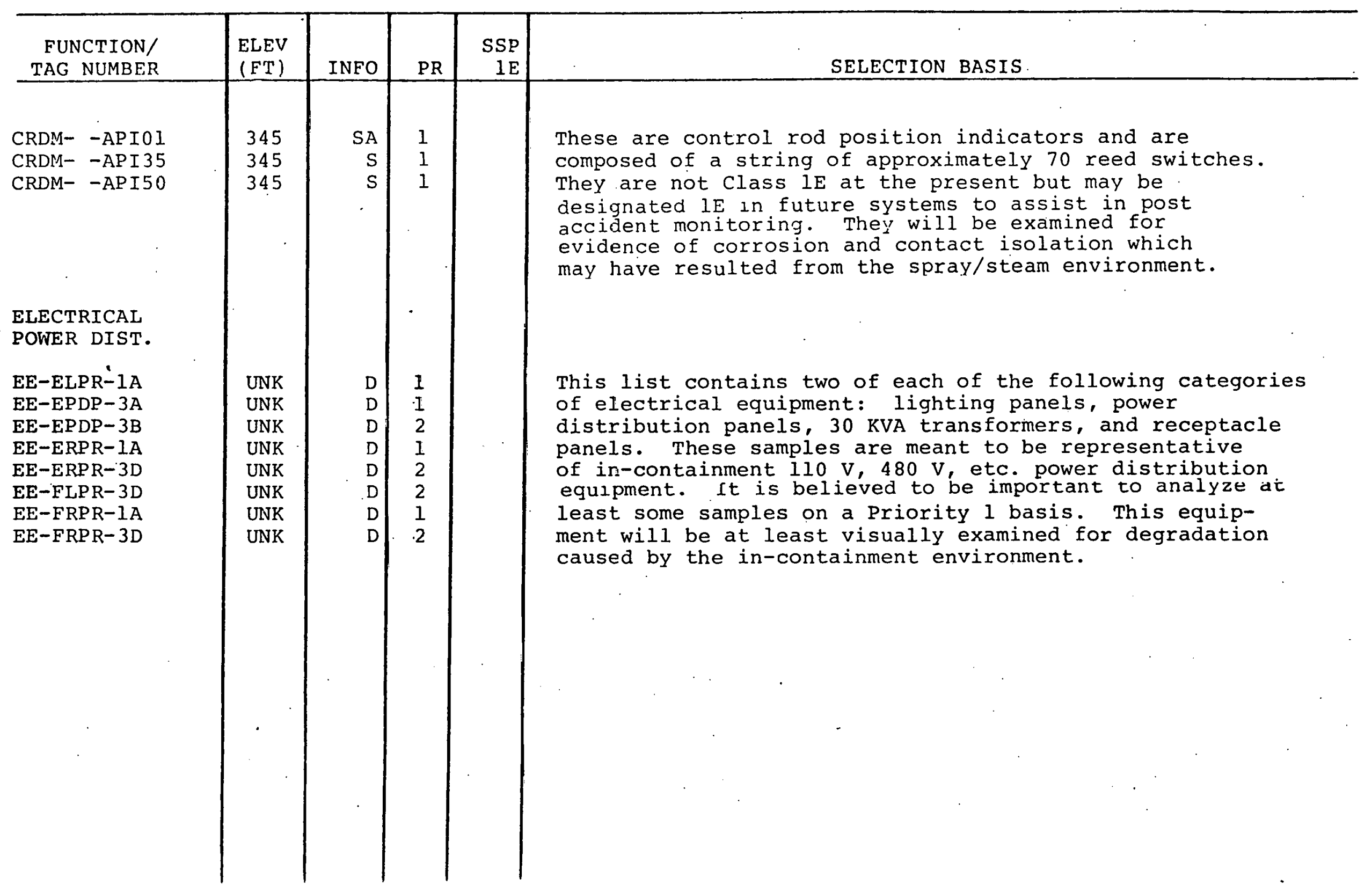


TABLE II (Cont'd)

TMI JNIT 2 CONTAINMENT BUILDING EQUIPMENT SELECTION BASIS

AND PRIORITY ASSIGNMENT

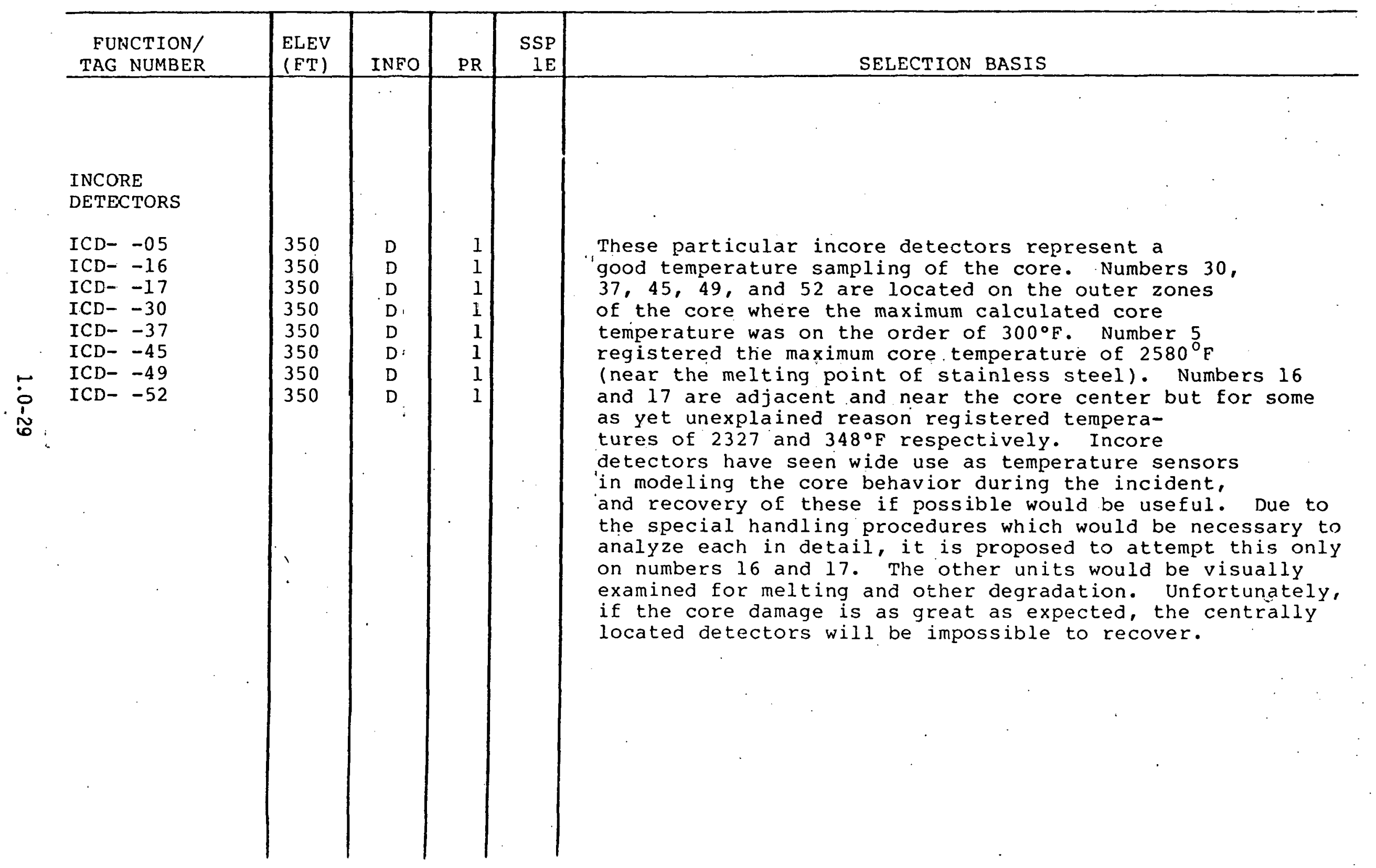


TABLE II (Cont'd)

TMI UNIT 2 CONTAINMENT BUILDING EQUIPMENT SELECTION BASIS

AND PRIORITY ASSIGNMENT

\begin{tabular}{|c|c|c|c|c|c|}
\hline $\begin{array}{l}\text { FUNCTION/ } \\
\text { TAG NUMBER }\end{array}$ & $\begin{array}{l}\text { ELEV } \\
(\mathrm{FT}) \\
\end{array}$ & INFO & PR & $\begin{array}{r}S S P \\
1 E \\
\end{array}$ & SELECTION BASIS \\
\hline $\begin{array}{l}\text { LEVEL AND } \\
\text { DIFFERENTIAI } \\
\text { PRESSURE } \\
\text { TRANSMITTERS }\end{array}$ & & & & & \\
\hline $\begin{array}{l}\text { CF-2-LTl } \\
\text { CF-2-LT3 } \\
\text { IC-10-DPT } \\
\text { RC-1-LT1 } \\
\text { RC-1-LT2 } \\
\text { RC-1-LT3 } \\
\text { RC-14A-DPT1 } \\
\text { RC-14A-DPT2 } \\
\text { SP-IA-LT1 } \\
\text { SP-1A-LT2 } \\
\text { SP-1B-LT1 }\end{array}$ & $\begin{array}{l}324 \\
324 \\
351 \\
286 \\
286 \\
286 \\
286 \\
286 \\
287 \\
286 \\
286\end{array}$ & $\begin{array}{r}\text { DEA } \\
D E \\
D E \\
\text { IDEA } \\
\text { IDEA } \\
\text { IDEA } \\
\text { SEA } \\
\text { SE } \\
\text { DE } \\
D E \\
\text { DE }\end{array}$ & $\begin{array}{l}1 \\
1 \\
1 \\
1 \\
1 \\
1 \\
1 \\
1 \\
2 \\
1 \\
2\end{array}$ & $\begin{array}{r}S \\
S \\
S \\
S \\
S \\
S E \\
S E\end{array}$ & $\begin{array}{l}\text { These level and differential pressure transmitters are } \\
\text { manufactured by Bailey Controls. Transmitters provide a } \\
\text { good representation of the various technologies used in } \\
\text { other instruments in that they contain gaskets and seals, } \\
\text { terminal blocks, printed circuit boards, conformal coating } \\
\text { and reasonably complex electronics. Analysis of environ- } \\
\text { mental effects on this class of instruments can provide } \\
\text { insight into effects on other instrument types. In } \\
\text { adition, electronic components in each could be used to } \\
\text { determine the total radiation dose seen at specific locations. } \\
\text { The Bailey transmitters are similar to Rosemont and Foxboro } \\
\text { transmizters. }\end{array}$ \\
\hline $\begin{array}{l}\text { - } \\
\dot{0} \\
\dot{o}\end{array}$ & & & & & $\begin{array}{l}\text { These particular Bailey transmitters are very similar in } \\
\text { both electrical, and mechanical design. The CF, IC, and } \\
\text { SP-1A-LFl and SP-1B-LTl transmitters have standard.NEMA } 4 \\
\text { housings which are not qualified for steam or spray environ- } \\
\text { ments, although they are specified for use in nuclear power } \\
\text { plants. The RC and SP-IA-LT2 transmitters have LocA } \\
\text { gualified housings. This qualification, although good for } \\
\text { a steam environment, does not apply to extended submersion. } \\
\text { A-1 these transmitters underwent the special sealing program } \\
\text { (SSP) except Sp-lA-LTl, SP-lB-LTl and IC-lo-DPT (note that } \\
\text { these units have the NEMA } 4 \text { housing also). Comparison of } \\
\text { trese units could provide insight into housing and } \\
\text { sealing designs. The CF and IC units located above water can }\end{array}$ \\
\hline
\end{tabular}


TABLE II'(Cont'd)

TMI UNIT 2 CONTAINMENT BUILDING EQUIPMENT SELECTION BASIS

AND PRIORITY ASSIGNMENT

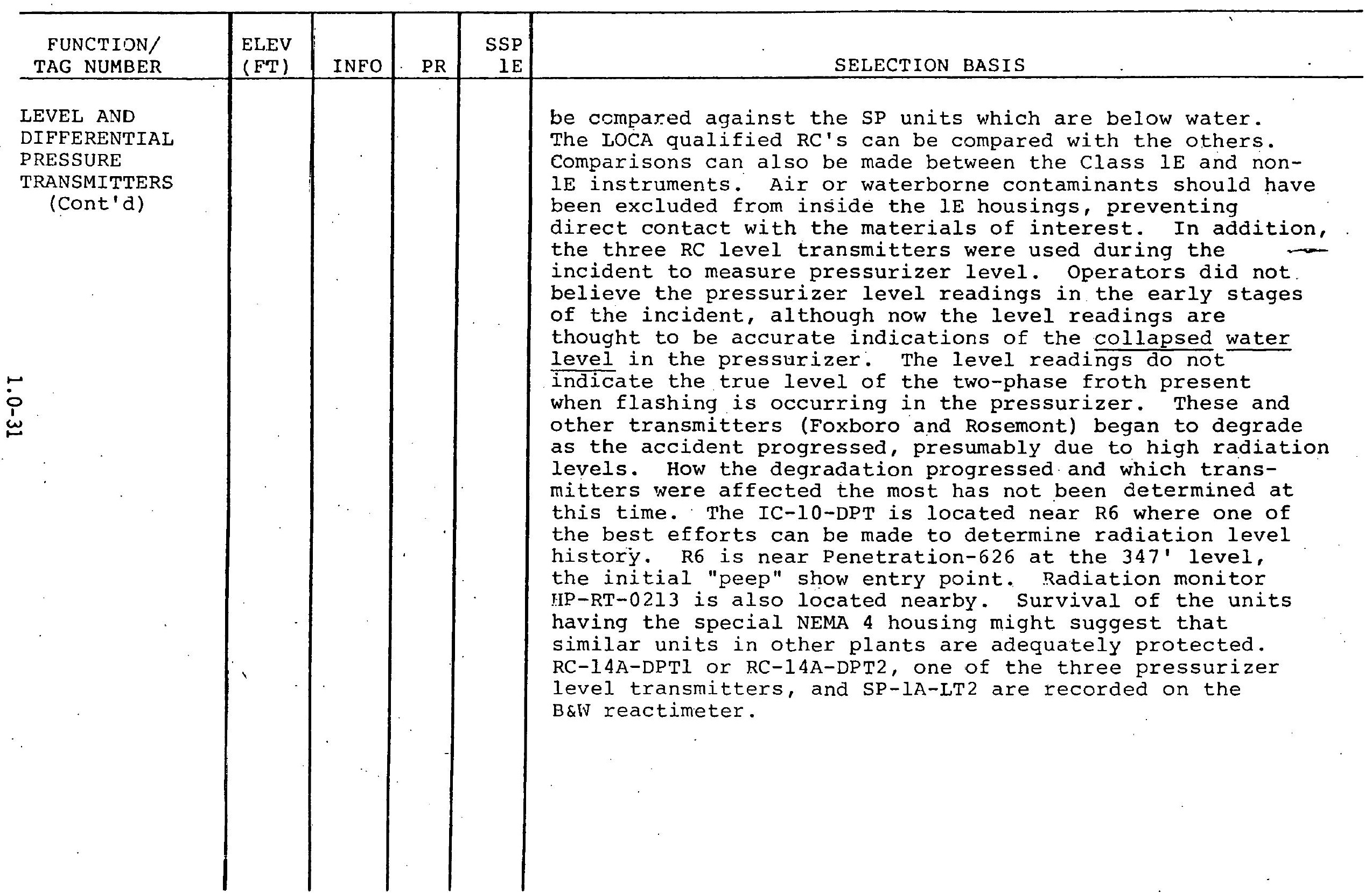




\section{AND PRIORITY ASSIGNMENT}

\begin{tabular}{|c|c|c|c|c|c|}
\hline $\begin{array}{l}\text { FUNCTION/ } \\
\text { TAG NUMBER } \\
\end{array}$ & $\begin{array}{l}\text { ELEV } \\
\text { (FT) }\end{array}$ & INFO & PR & $\begin{array}{r}\mathrm{SSP} \\
1 \mathrm{E} \\
\end{array}$ & SELECTION BASIS \\
\hline $\begin{array}{l}\text { PRESSURE AND } \\
\text { FLOW } \\
\text { TRANSMITTERS }\end{array}$ & & & & & $\begin{array}{l}\text { The following Foxboro and Rosemont Pressure and Flow } \\
\text { transmitters are similar to the Bailey level and } \\
\text { differential pressure transmitters discussed earlier } \\
\text { and, therefore, the measurement objectives stated for } \\
\text { the Eailey instruments apply also to these instruments. }\end{array}$ \\
\hline $\begin{array}{l}\text { CF-1-PTI } \\
\text { CF-1-PT3 }\end{array}$ & $\begin{array}{l}324 \\
324\end{array}$ & $\begin{array}{l}\mathrm{DE} \\
\mathrm{DE}\end{array}$ & $\begin{array}{l}1 \\
1\end{array}$ & & $\begin{array}{l}\text { Foxbaro pressure transmitters are used in most nuclear } \\
\text { plants and therefore any information obtained from analysis }\end{array}$ \\
\hline $\mathrm{CF}-1-\mathrm{PT} 4$ & 324 & DEA & 1 & & of these applies to other plants. Two of the selections \\
\hline $\mathrm{RC}-3 \mathrm{~A}-\mathrm{PT} 3$ & 287 & SEA & 1 & $E$ & are Class IE equipment. Both the Ell and El3 Foxboro \\
\hline $\mathrm{RC}-3 \mathrm{~A}-\mathrm{PT} 4$ & 287 & $\mathrm{SE}$ & 1 & $\mathrm{E}$ & instrument types are represented; however, the primary \\
\hline RR-FT-1027 & 287 & $\mathrm{DE}$ & 2 & & difference between the types is mechanical and not \\
\hline RR-FT-1028 & 285 & $\mathrm{DE}$ & 2 & & electrical. The CF transmitters are above containment \\
\hline RR-FT- 1029 & 285 & DEA & 2 & & flood level while the RC transmitters are below. CF units \\
\hline $\begin{array}{l}\text { SP-6A-PT1 } \\
\text { SP-6A-PT2 }\end{array}$ & $\begin{array}{l}287 \\
288\end{array}$ & $\begin{array}{l}\mathrm{DE} \\
\mathrm{DE}\end{array}$ & $\begin{array}{l}1 \\
1\end{array}$ & & $\begin{array}{l}3 \text { and } 4 \text { are on the same core flood tank, and one will be } \\
\text { place } 1 \text { in archive storage while the other two are analyzed. }\end{array}$ \\
\hline WDL-PT-1202 & 286 & $\mathrm{DE}$ & 1 & & $\begin{array}{l}\text { The below water units will be analyzed in the same manner } \\
\text { as those above water and the results compared. The WDL } \\
\text { transmitter and either SP-6A-PTl or SP-6A-PT } 2 \text { are } \\
\text { recorded on the B\&W reactimeter. All these Foxboro } \\
\text { instruments will be used to evaluate their radiation } \\
\text { hardness, ability to withstand immersion and very humid } \\
\text { condi=ions, and to compare to similar Bailey and Rosemont } \\
\text { designs. }\end{array}$ \\
\hline $\begin{array}{l}\mathrm{RC}-3 \mathrm{~A}-\mathrm{PTl} \\
\mathrm{RC}-3 \mathrm{~A}-\mathrm{PT} 2 \\
\mathrm{RC}-3 \mathrm{~B}-\mathrm{PTl}\end{array}$ & $\begin{array}{l}286 \\
286 \\
287\end{array}$ & $\begin{array}{r}\text { ISEA } \\
\text { ISE } \\
\text { SE }\end{array}$ & $\begin{array}{l}1 \\
1 \\
1\end{array}$ & $\begin{array}{l}E \\
E \\
E\end{array}$ & $\begin{array}{l}\text { Rosemont manufactures these pressure transmitters, all of } \\
\text { which are below water. They are all Class IE and of the } \\
\text { same model. The } 1152 \text { model is similar in function to the } \\
\text { Foxboro and Bailey transmitters except that this model is } \\
\text { of a rew design which has recently been qualified to more } \\
\text { stringent environmental levels. As a consequence, this }\end{array}$ \\
\hline
\end{tabular}


TMI UNIT 2 CONTAINMENT BUILDING EQUIPMENT SELECTION BASIS

AND PRIORITY ASSIGNMENT

\begin{tabular}{|c|c|c|c|c|c|}
\hline $\begin{array}{r}\text { FUNCTION/ } \\
\text { TAG NUMBER }\end{array}$ & $\begin{array}{l}\text { ELEV } \\
\text { (FT! }\end{array}$ & INFO & $\mathrm{PR}$ & $\begin{array}{r}\text { SSP } \\
1 \mathrm{E} \\
\end{array}$ & SELECTION BASIS \\
\hline 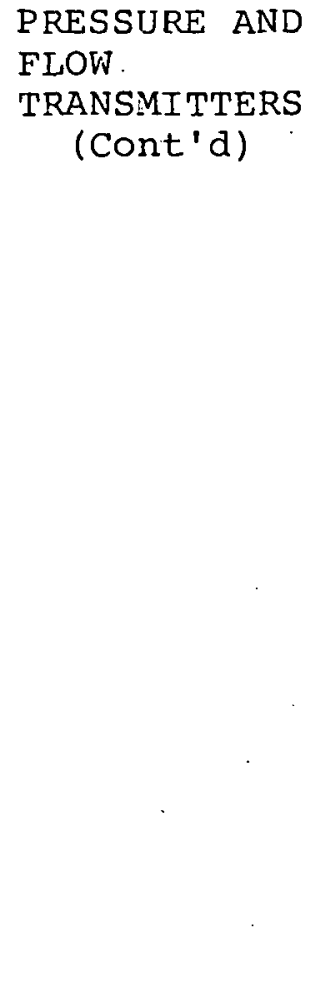 & & & $\therefore$ & & $\begin{array}{l}\text { instrument is widely called out. in new reactor designs. } \\
\text { All are of specific interest for possible calibration } \\
\text { because of their use during the incident to monitor steam/ } \\
\text { water pressures. Numbers } 3 A-P T 2 \text { and } 3 B-P T l \text { will be } \\
\text { analyzed first and } 3 A-P T 1 \text { will be held in reserve. } \\
\text { RC-3B-PTl is recorded on the } B \text { \&W reactimeter. } \\
\text { The } 1152 \text { transmitters are intended to meet the following } \\
\text { conditions: (1) qualified to IEEE } 323 / 344 \text { standards, } \\
\text { (2) gamma radiation integrated dose qualification to } \\
5 \times 10 \text { rads, ( } 3 \text { ) } 39 \text { seismic qualification, and steam- } \\
\text { pressure-chemical spray testing. It is possible to compare } \\
\text { the transmitter performance before, during, and after the } \\
\text { accident to published specifications and qualification } \\
\text { reports. In the comparison, it may be possible to deter- } \\
\text { mine if there are any performance distinctions between the } \\
\text { separate effects testing allowed by IEEE } 323 \text { and the } \\
\text { combined effects imposed on the transmitter during the } \\
\text { various accident phases. } \\
\text { operating experience data obtained on these transmitters } \\
\text { during the accident for such service conditions as } \\
\text { submersion or high radiation levels may be used to aid } \\
\text { in an extension of the qualification. }\end{array}$ \\
\hline $\begin{array}{l}\text { LIMIT } \\
\text { SWITCHES. }\end{array}$ & & & & & \\
\hline $\begin{array}{l}A H-K S-5000 \\
\lambda H-K S-5002 \lambda \\
A H-K S-5002 B \\
A H-K S-5037 \\
A H-K S-5039 \\
\Lambda H-K S-5040\end{array}$ & $\begin{array}{l}331 \\
331 \\
331 \\
315 \\
335 \\
305\end{array}$ & 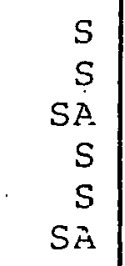 & $\begin{array}{l}1 \\
2 \\
1 \\
2 \\
2 \\
1\end{array}$ & \begin{tabular}{l|}
$E$ \\
$E$ \\
$E$ \\
$E$ \\
$E$ \\
$E$
\end{tabular} & $\begin{array}{l}\text { These switches were chosen as representations of Class } 1 \mathrm{E} \\
\text { limit switches. AH-5000, } 5002 \mathrm{~A} \text { and } 5002 \mathrm{~B} \text { will be removed } \\
\text { with fans } \mathrm{AH}-1 \mathrm{~A} \text { and } 1.1 \mathrm{C} \text {. A }-5037 \text { and } 5039 \text { will be } \\
\text { removed with } \mathrm{AH}-\mathrm{V} 2 \mathrm{~A} \text { and } \mathrm{V} 2 \mathrm{~B} \text {. Spares and archive samples } \\
\text { have been selected }\end{array}$ \\
\hline
\end{tabular}


TABLE II (Cont'd)

TMI UNIT 2 CONTAINMENT BUILDING EQUIFMENT SELECTION. BASIS

A.ND PRIORITY ASSIGNMENT

\begin{tabular}{|c|c|c|c|c|c|}
\hline $\begin{array}{r}\text { EUNCTION/ } \\
\text { TAG NUMBER }\end{array}$ & $\begin{array}{l}\text { ELEV } \\
\text { (FT) } \\
\end{array}$ & INFO & $\mathrm{PR}$ & $\begin{array}{r}S S P \\
1 E \\
\end{array}$ & SELECTION BASIS \\
\hline MOTORS, FAN & & & & & \\
\hline $\begin{array}{l}\mathrm{AH}-\mathrm{E}-11 \mathrm{~A} \\
\mathrm{AH}-\mathrm{E}-11 \mathrm{C} \\
\mathrm{AH}-\mathrm{E}-52 \mathrm{~A} \\
\mathrm{AH}-\mathrm{E}-52 \mathrm{~B}\end{array}$ & $\begin{array}{l}331 \\
331 \\
283 \\
283\end{array}$ & $\begin{array}{l}\text { S } \\
\text { S } \\
\text { D } \\
\text { D }\end{array}$ & $\begin{array}{l}1 \\
1 \\
3 \\
3\end{array}$ & $\begin{array}{l}\mathrm{E} \\
\mathrm{E}\end{array}$ & $\begin{array}{l}\text { These four large reactor building and vessel cavity air } \\
\text { cooling fans are representative samples of others in the } \\
\text { containment building. The two located at } 331 \text { feet are } \\
\text { class } 1 \text { equipment. The effects of radiation, humidity } \\
\text { and building spray can be evaluated on these motors. } \\
\text { The corresponding limit switches have been selected for } \\
\text { analysis also. The two units at } 283 \text { feet can be compared, } \\
\text { however probably little information can be obtained } \\
\text { since they are underwater. }\end{array}$ \\
\hline $\begin{array}{l}\text { MOTORS, REACTCR } \\
\text { COOLANT }\end{array}$ & & & & & \\
\hline $\begin{array}{l}R C-P-1 A \\
R C-P-1 B\end{array}$ & $\begin{array}{l}322 \\
322\end{array}$ & $\begin{array}{l}\text { IS } \\
\text { IS }\end{array}$ & $\frac{1}{2}$ & & $\begin{array}{l}\text { Juring the accident, all four reactor coolant pumps were } \\
\text { operated during abnormal coolant phase conditions. } \\
\text { Appraximately } 6 \text { minutes into the accident RCS conditions } \\
\text { were at saturation temperature and pressure. The four } \\
\text { pumps operated with a steam/water mixture until at } 73 \\
\text { and } 100 \text { minutes into the accident the two } B \text { loop and } \\
\text { two A loop pumps, respectively, were turned off. During } \\
\text { this operating time all experienced abnormal vibration } \\
\text { and probably cavitation. RC-P-2B was run for } 18 \text { minutes } \\
\text { later in the sequence and again vibrated badly. RC-P-IA } \\
\text { was finally restarted, and forced circulation was } \\
\text { achieved. RC-P-lA was used then to bring the plant to } \\
\text { a stabilized decay heat removal condition. These two } \\
\text { pumps should be examined for both electrical and } \\
\text { mechanical degradation resulting from the aforementioned } \\
\text { abnormal conditions as well as the high radiation levels } \\
\text { and building spray. The scope of this examination has } \\
\text { not been defined at this time. }\end{array}$ \\
\hline
\end{tabular}


TABLE II (Cont'd)

TMI UNIT 2 CONTAINMENT BUILDING EQUIPMENT SELECTION BASIS

AND PRIORITY ASSIGNMENT

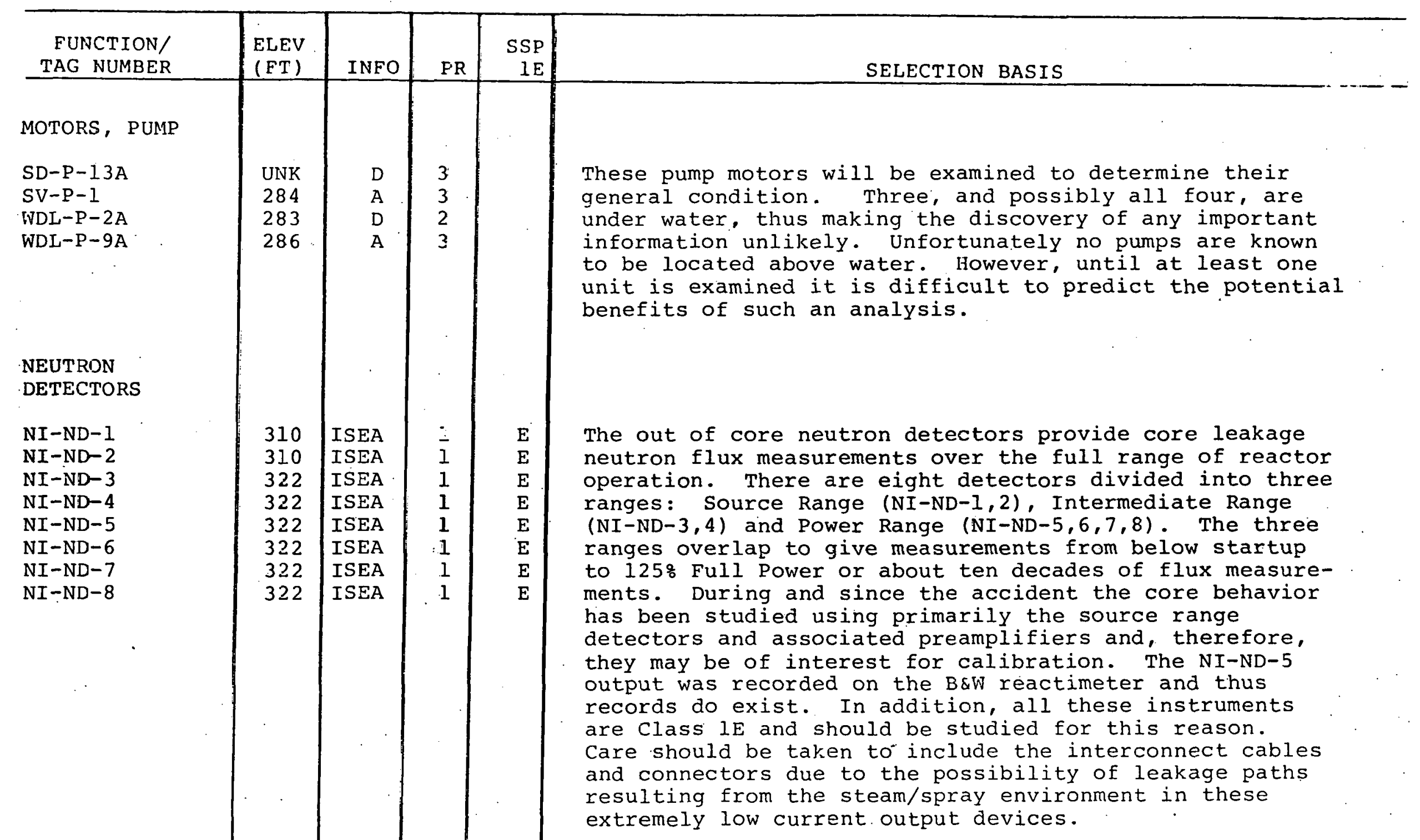


TABLE II (Cont'd)

TMI UNIT 2 CONTAINMENT BUILDING EQUIPMENT SELECTION BASIS

AND PRIORITY ASSIGNMENT

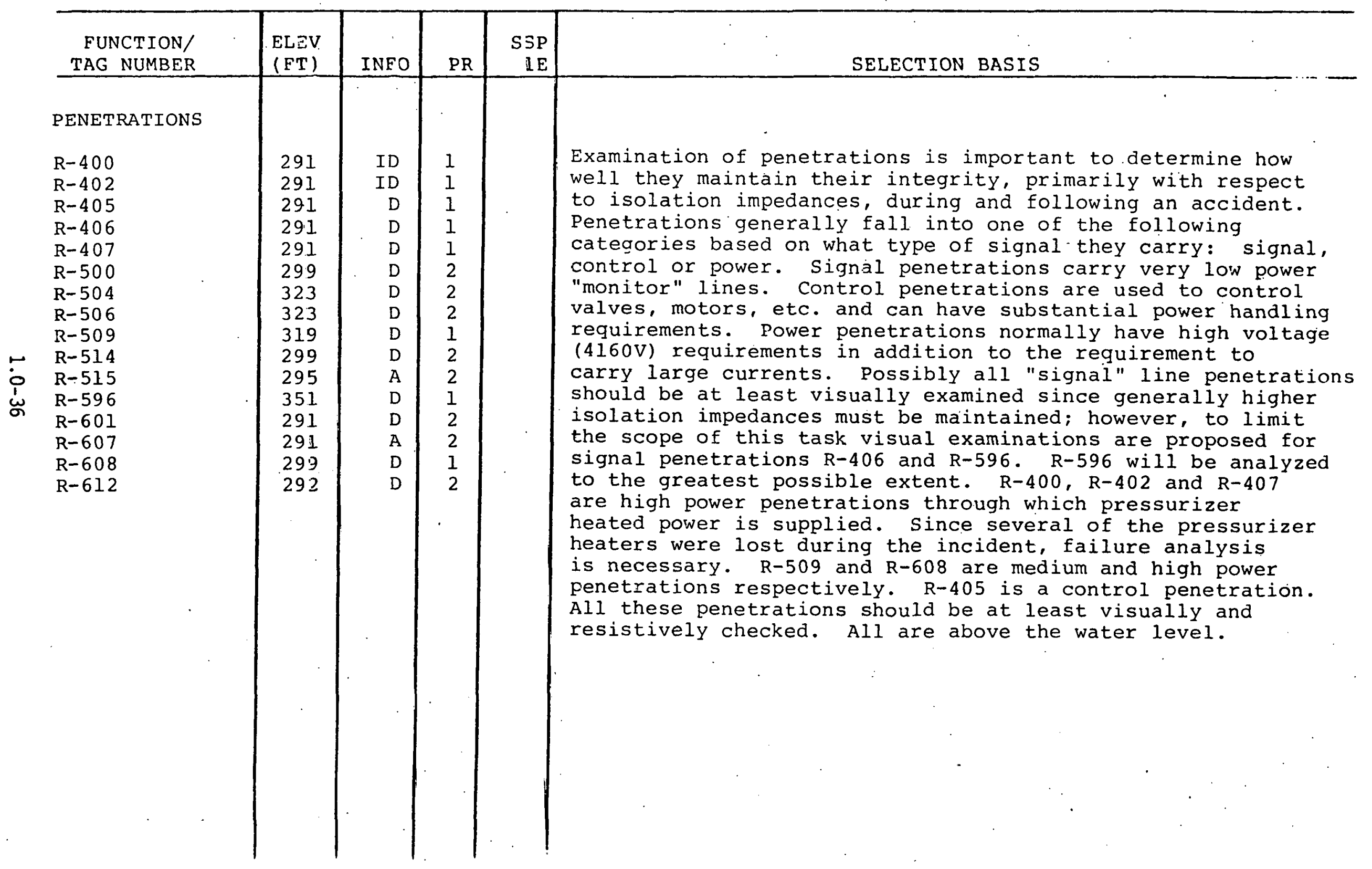


TABLE II (Cont'd)

TMI UNIT 2 CONTAINMENT BUILDING EQUIPMENT SELECTION BASIS

AND PRIORITY ASSIGNMENT

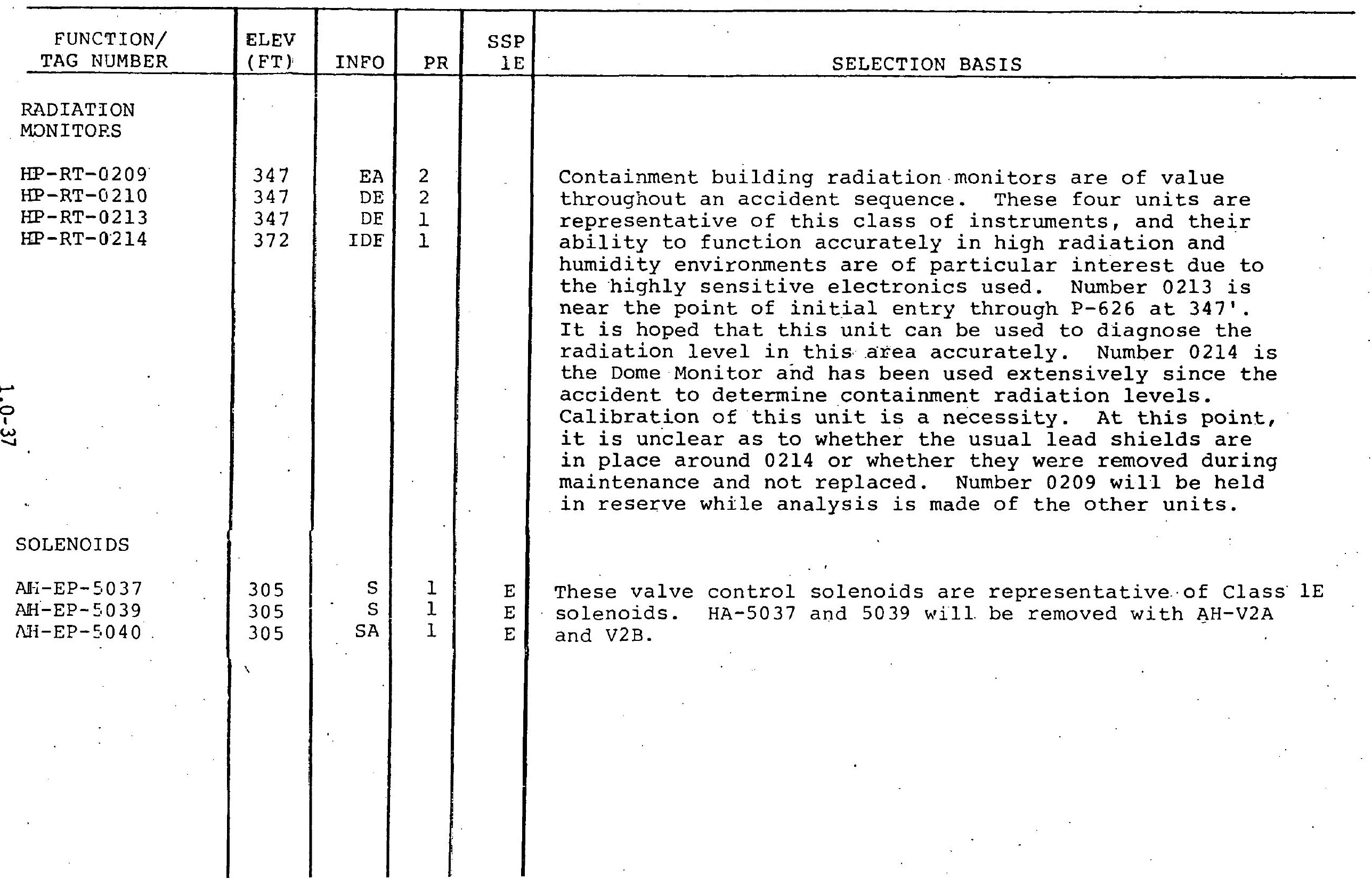


AND PRIORITY ASSIGNMENT

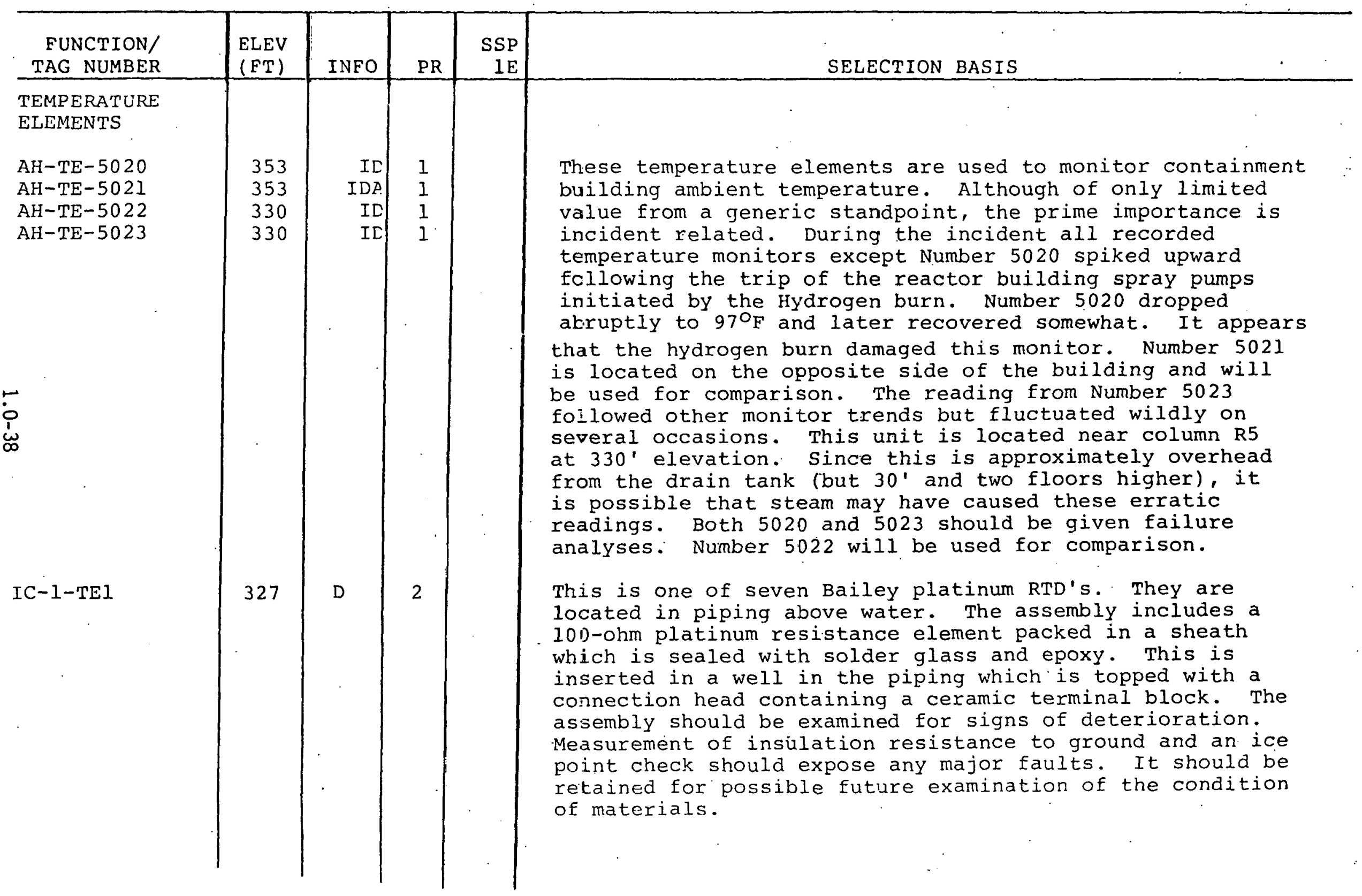


TABLE II (Cont'd)

TMI UNIT 2 CONTAINMENT BUILDING EQUIPMENT SELECTION BASIS

AND PRIORITY ASSIGNMENT

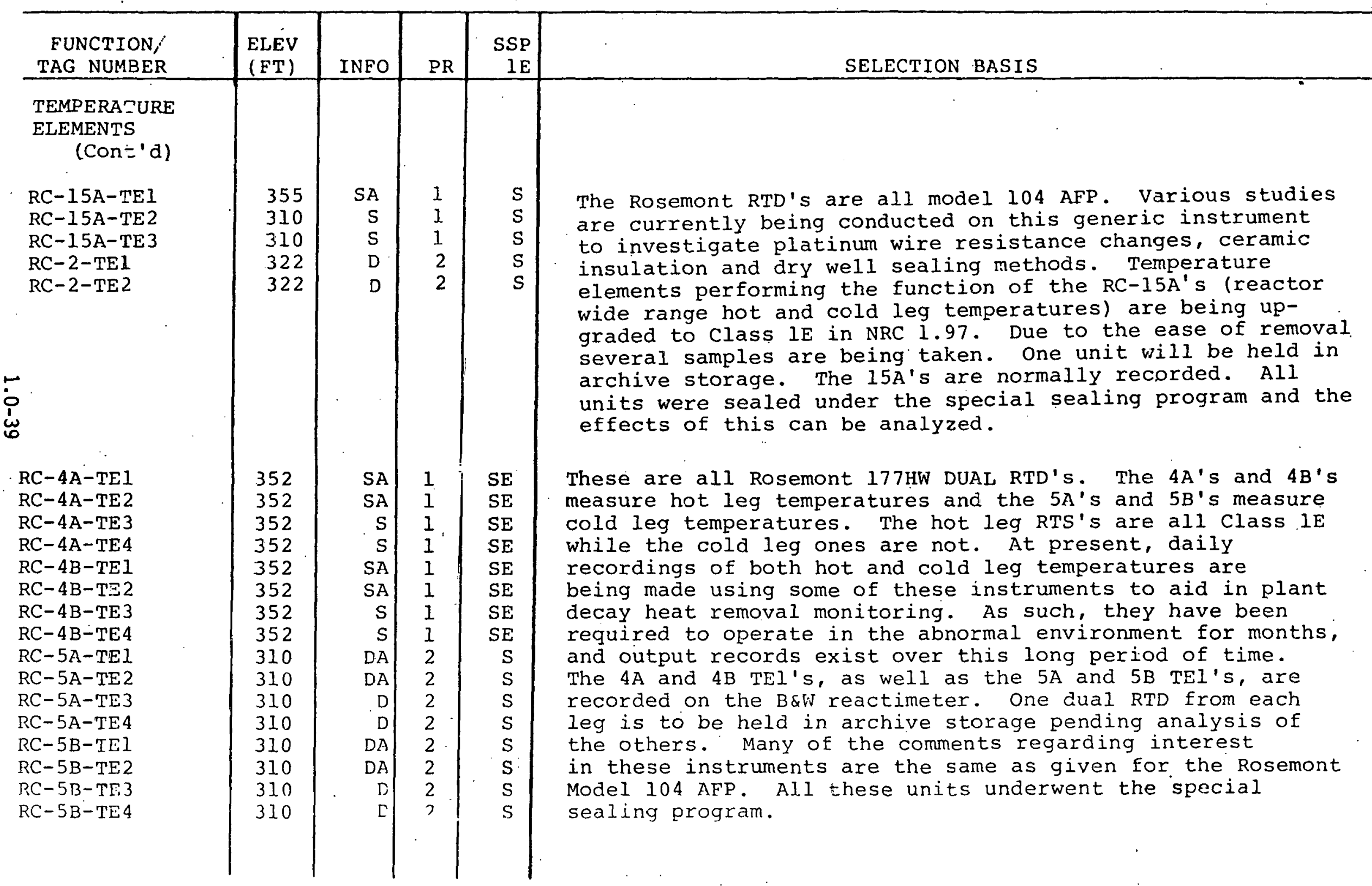


TABLE II (cont'd)

TMI UNIT 2 CONTAINMENT BUILDING EQUIPMENT SELECTION BASIS

AND PRIORITY ASSIGNMENT

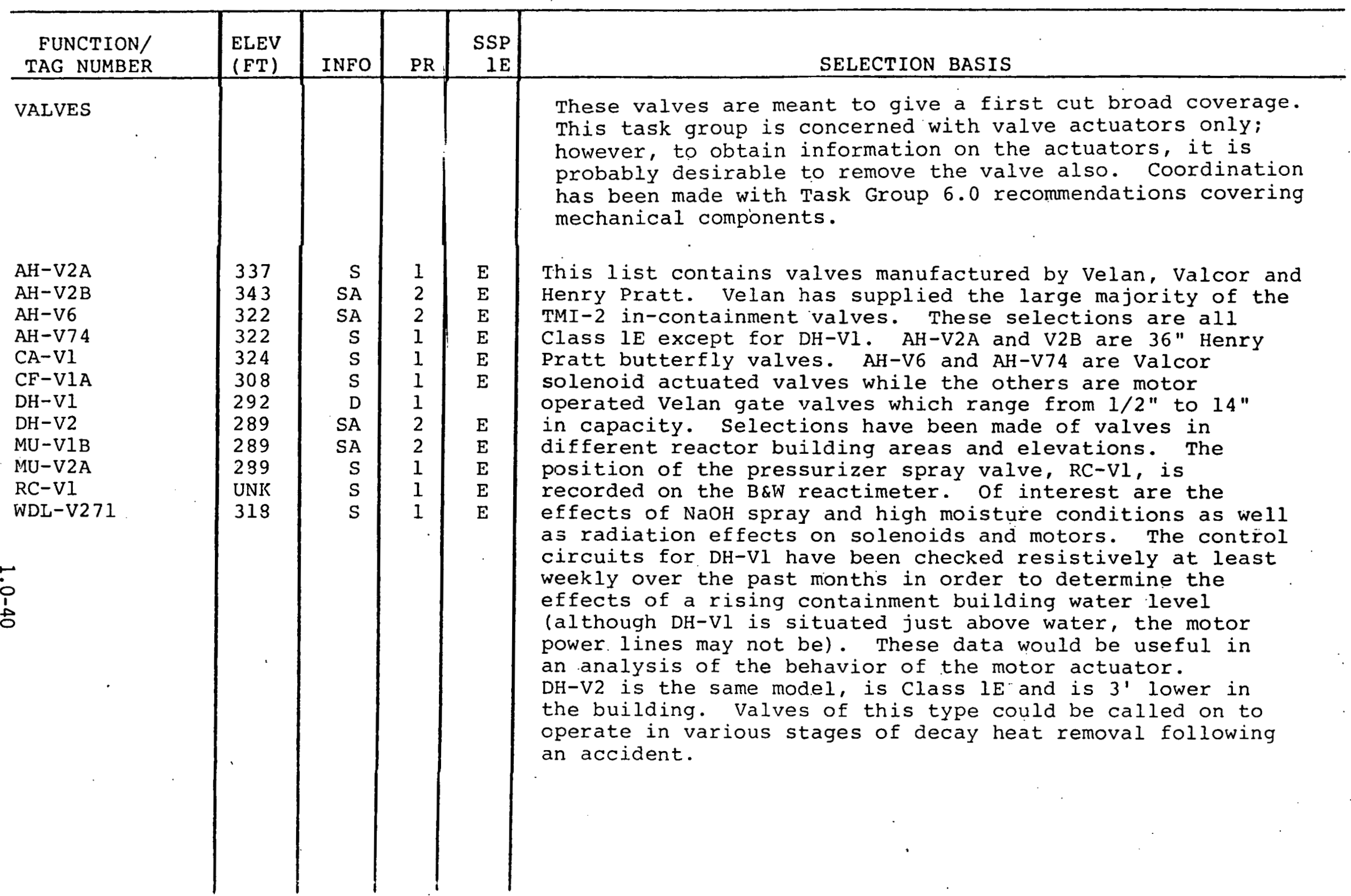


TABLE $\perp+$ (Cont'd)

TMI UNIT 2 CONTAINMENT BUILDING EQUIPMENT SELECTION BASIS

AND PRIORITY ASSIGNMENT

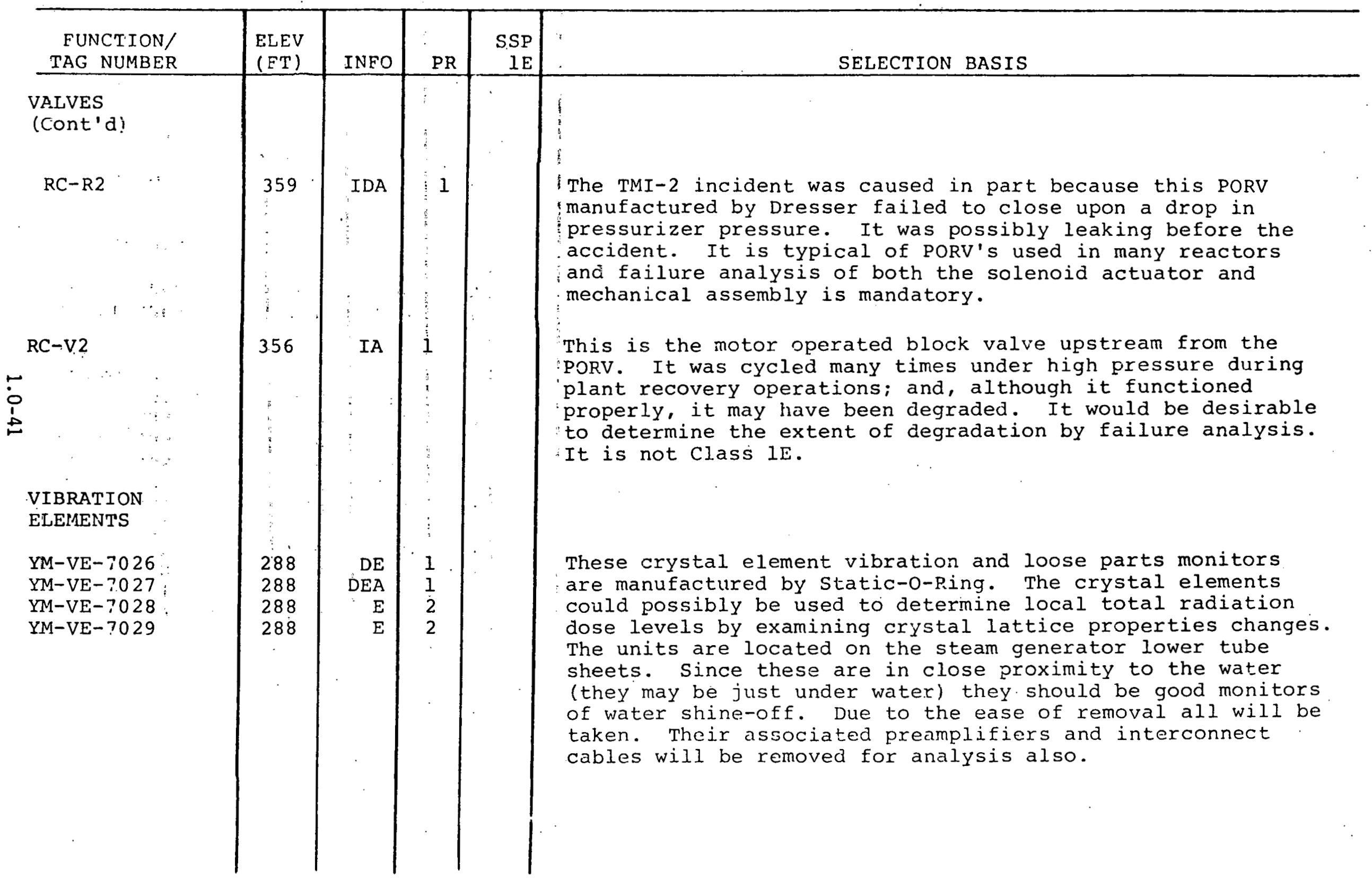


TABLE II (Cont'd)

TMI UNIT 2 CCNTAINMENT BUILDING EQUIPMENT SELECTION BASIS

AND PRIORITY ASSIGNMENT

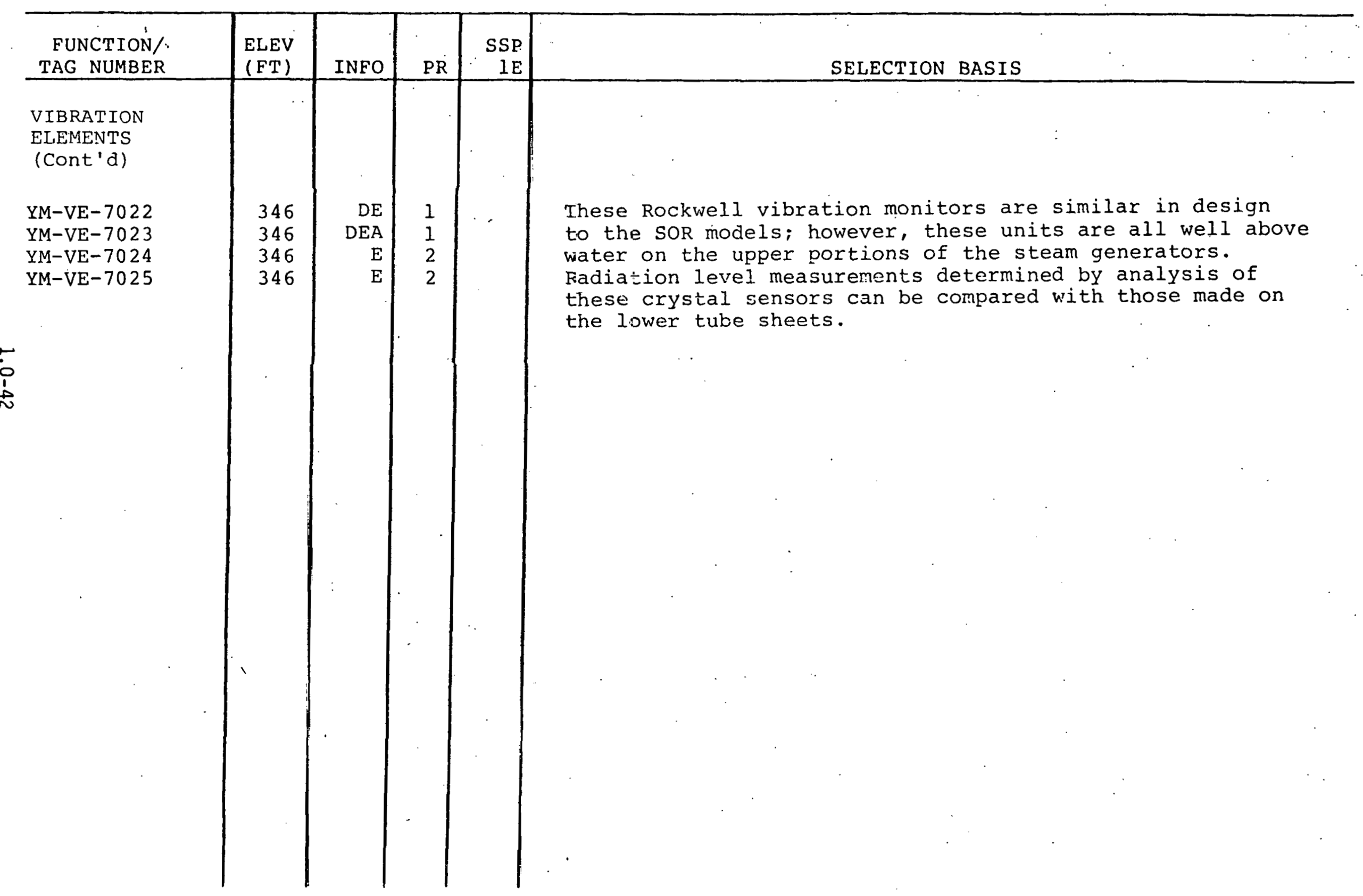


TABLE II (Cont'd)

TMI UNIT 2 CONTAINMENT BUILDING EQUIPMENT SELECTION BASIS

AND PRIORITY ASSIGNMENT

\begin{tabular}{|c|c|c|c|c|c|}
\hline $\begin{array}{l}\text { FUNCTION/ } \\
\text { TAG NUMBER }\end{array}$ & $\begin{array}{l}\text { ELEV } \\
\text { (FT) }\end{array}$ & INFO & PR & $\begin{array}{r}\text { SSP } \\
\text { lE }\end{array}$ & SELECTION BASIS \\
\hline$\frac{\text { MISCELLANEOUS }}{\text { GENERIC }}$ & & & & & Miscellaneous Generic Equipment \\
\hline
\end{tabular}

The TMI-2 incident provides an opportunity to acquire accident-tested equipment for systematic evaluation, at minimal cost.

The TMI-2 equipment is generically similar to equipment in all equivalent-aged plants (between 30 to 50 plants operating in the US). The TMI-2 equipment evaluation will supply information which is directly applicable to these US plants.

It is imperative to select a broad sampling of equipment to achieve maximum coverage and to discover any "surprises" in equipment degradation. Also, the NRC is particularly interested in the analysis of samples of all Class $1 \mathrm{E}$ equipment in order to verify the adequacy of existing environmental qualification methodology. Analysis of Class $I E$ and Non-Class $I E$ equipment required for post accident monitoring and control in operating nuclear plants is of interest. The TMI-2 data could be used in the near term to determine whether certain instrumentation and electrical. equipment would be capable of surviving an accident environment for the length of time its function is required. Based on these considerations, the following additional equipment selections have been made. Generally, three instruments of a given type have been chosen. Two for analysis and one for archive storage pending the analysis of the other two. 
TABLE II (Cont'd)

TMI UNIT 2 CONTAINMENT BUILDING EQUIPMENT SELECTION BASIS

AND PRIORITY ASSIGNMENT

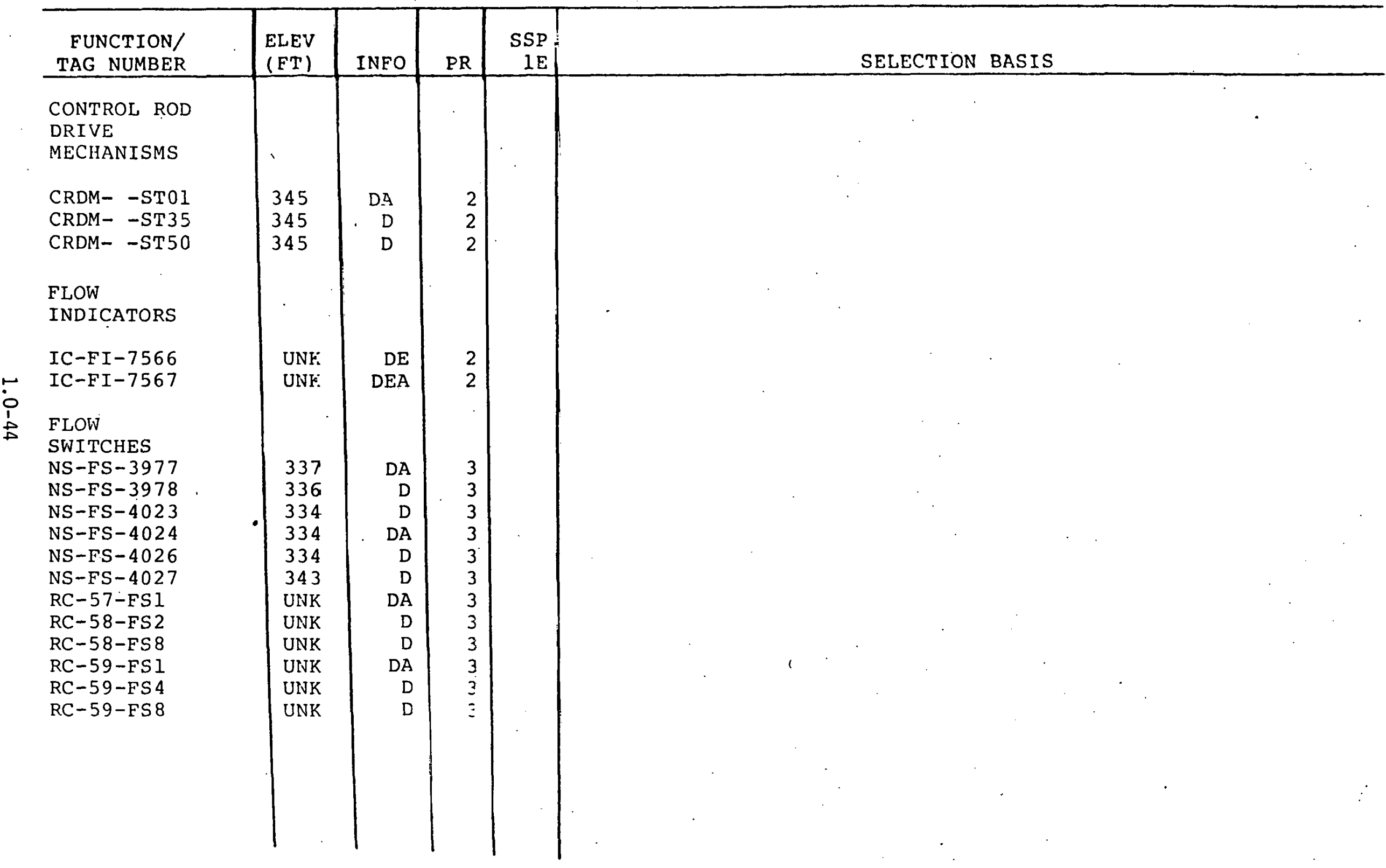


TABLE II (cont'd)

TMI UNIT 2 CONTAINMENT BUILDING EQUIPMENT SELECTION BASIS

AND PRIORITY ASSIGNMENT

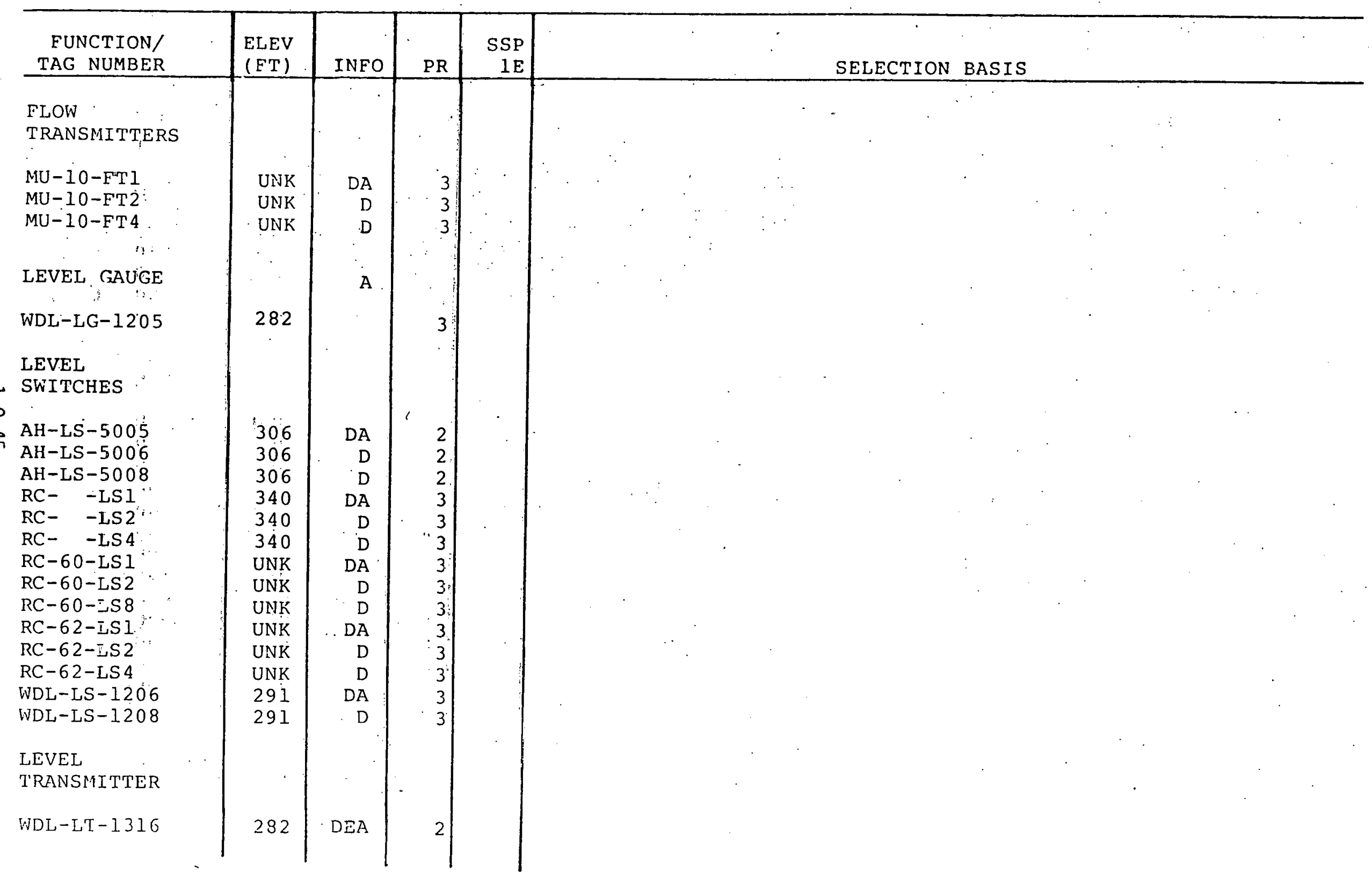


TABLE II (cont'd)

TMI UNIT 2 CONTAINMENT BUILDING EQUIPMENT SELECTION BASIS

AND PRIORITY ASSIGNMENT

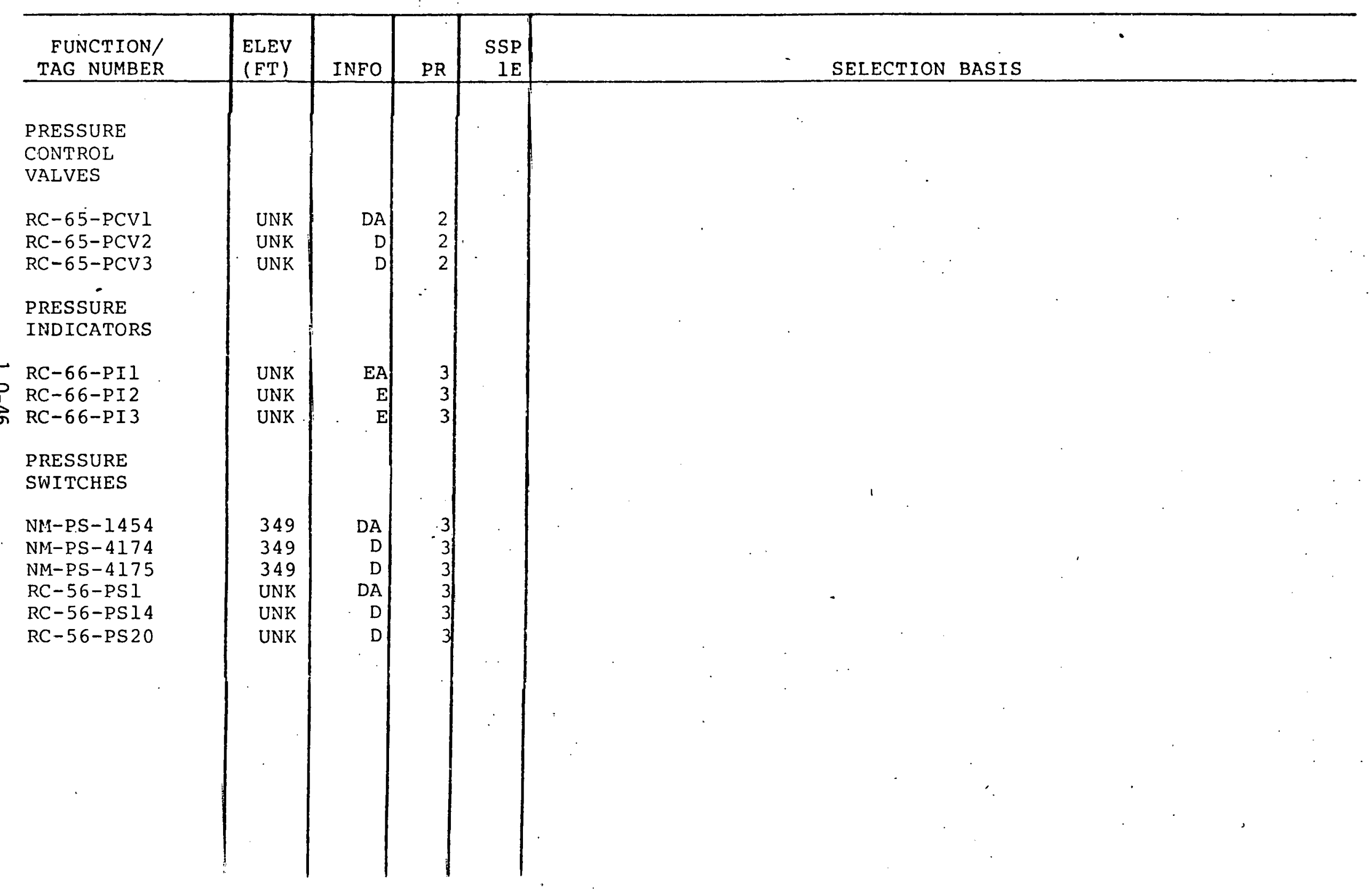


TABLE II (Cont'd)

TMI UNIT 2 CONTAINMENT BUILDIUG EQUIPISENT SELECTION BASIS AND PRIORITY ASSIGMMENT

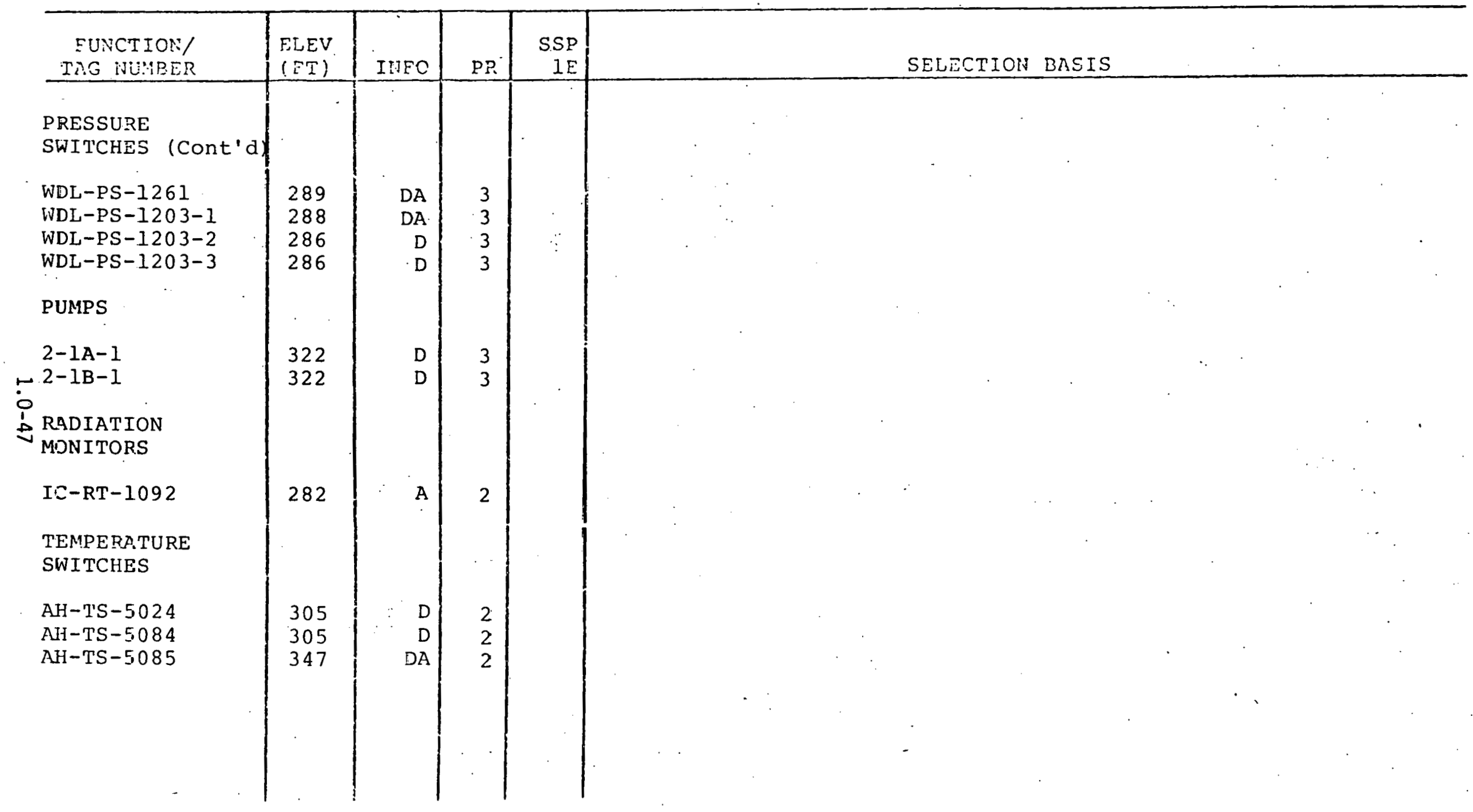


TABLE II (Cont'd)

TMI UNIT 2 CONTAINMENT BUILDING EQUIPMENT SELECTION BASIS

\section{- ANE PRIORITY ASSIGNMENT}

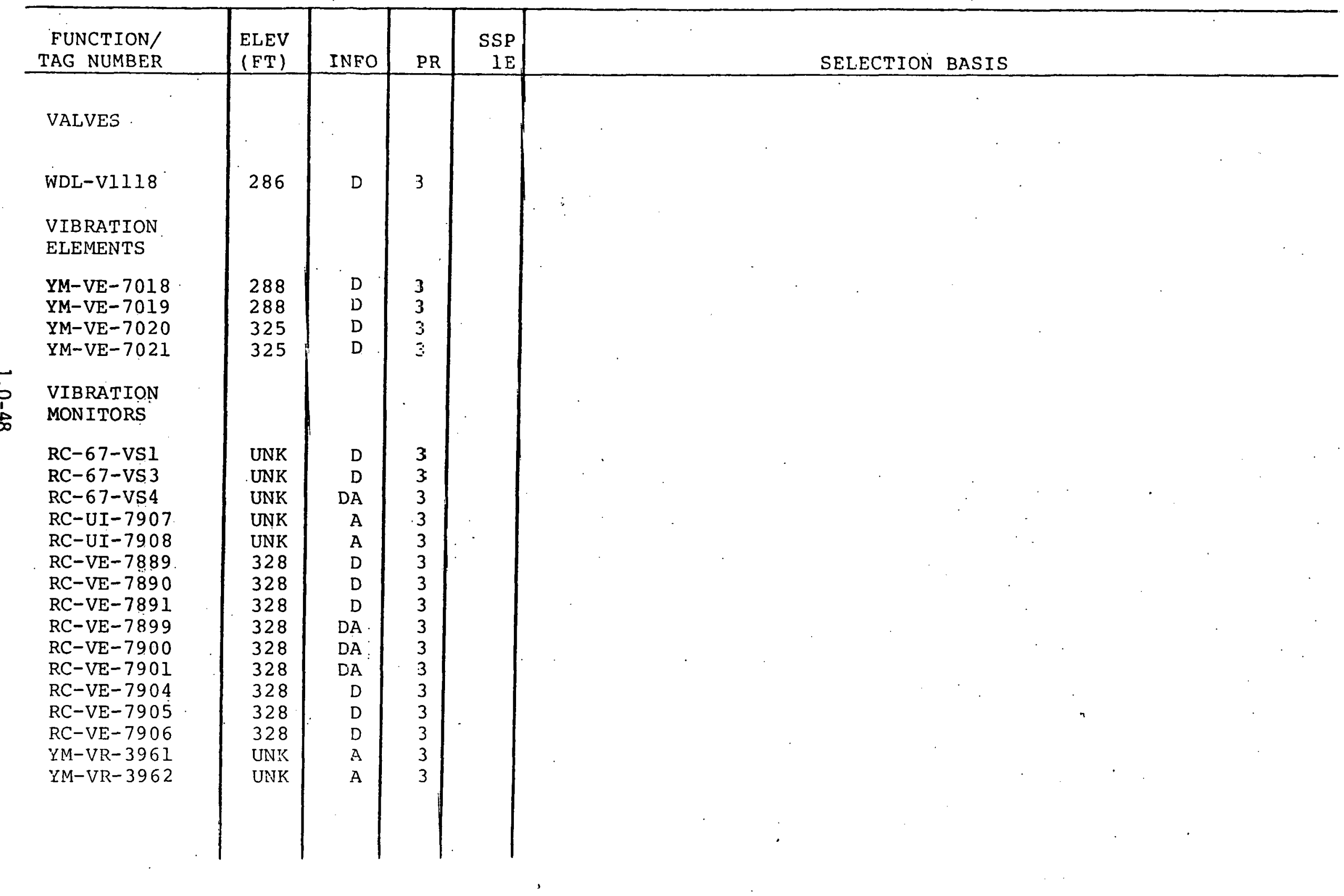




\section{TMI UNIT 2 REACTOR BUILDING INSTRUMENT IISTING CODES}

Tag Number

First Two Letters in Tag No:

Symbol

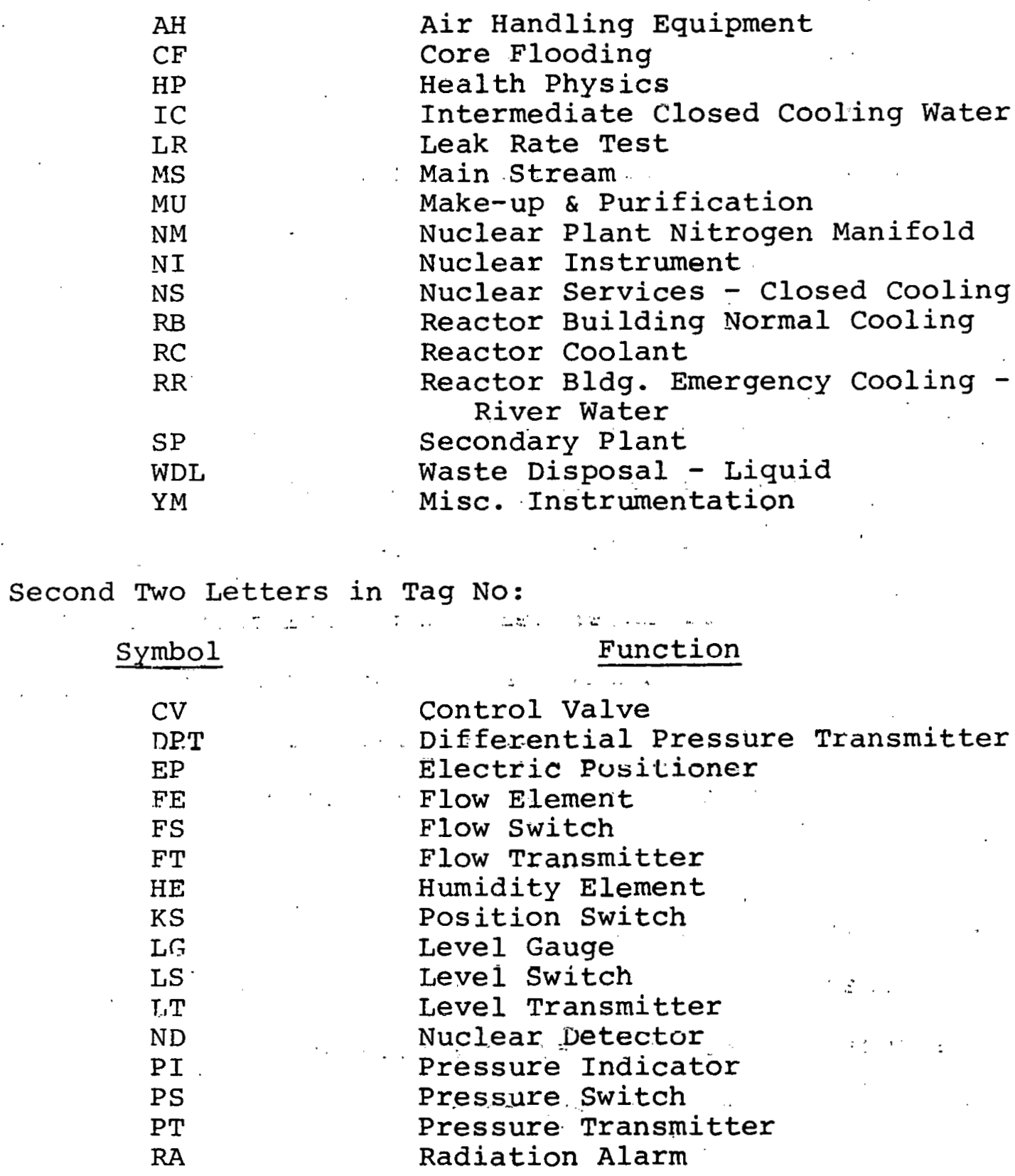


TABLE III (Cont'd)

Second Two Letters in Tag No: (Cont'd)

Symbol

RI

RT

SS

ST

$\mathrm{TE}$

TI

TS

UI

$\mathrm{VE}$

VS

Function

Radiation Indicator

Radiation Transmitter

Speed Switch

speed Transmitter

Temperature Element

Temperature Indicator

Temperature Switch

Multivariable Indicator

Vibration Element

Vibration Switch

Manufacturer code

Symbol

\section{Manufacturer}

$\mathrm{A}-\mathrm{B}$

AMI

ASCO

BDV

BMC

BING

RRK.

$B \& W$

DREX

ELPR

FOX

DRES

GEMS

IRD

JERG

$M \& M$

MNCO

MAG

PENN

REC

$\mathrm{RM}$

SOR

TEC

TEK

VICO

VLAN

VLCR

WARR

W
Allen-Bradley

Aminco

Automatic Switch Co.

Barksdale

Bailey

Bingham Pump

Broolks

Babcock \& Wilcox

Drexel Brooks

Electro Products

Foxboro

Dresser Valves

GEMS Sensor Div. De Laval Turbine

IRD

Jerguson Gage \& Valve

McDonnell \& Miller

Minco Products

Magnatrol

Penn Controls

Rosemont Engineering Co.

Rockwell

Static-0-Ring

Thermo Electric

Taktro (Applied Power Ind.)

Victoreen Instrument Div.

Velan

Valcor

Warrick

Westinghouse 


\section{Information (INFO) Category code}

Each instrument selected for information retrieval or storage will have a code containing one or more of the five information categories shown below.

$\begin{array}{cll}\text { S } & \text { Safety } \\ \text { I } & \text { Incident } \\ \text { D } & \text { Design } \\ \text { E } & \text { Environment } \\ \text { A } & \text { Archive }\end{array}$

Example: SIEA

Status (ST) Code

Operating

$\begin{array}{cc}\text { Yes } & \text { Y } \\ \text { No } & \mathrm{N} \\ \text { Unknown } & ?\end{array}$

Example: YC

\section{Readout}

C Continuous

$D$ On Demand

$\mathrm{N}$ Neither

Work Code

A

Insitu Testing Removal

A

B
B $\mathrm{X} \quad \mathrm{X}$

XX Needed for Core Removal

Example: $\quad A B X X$ 
IV. General Findings and Recommendations

Findings

The facts, observations, etc. given below have had varying degrees of impact on our work and are presented with the idea of providing the reader with some additional perspective on the task at hand. No particular significance is attached to the order in which the items are presented.

1. A coordinated list of instrumentation and electrical equipment within the reactor building was not readily available. We have compiled what we believe to be the most comprehensive list available. It is included in Appendix 5.

2. An up-to-date account of the operational status of the instrumentation and electrical equipment within the reactor building does not exist.

3. Ag of Auyusl 1980, the water level in the reactor building had reached roughly the $290.7 \mathrm{ft}$. elevation; therefore, much of the equipment of interest is under water (for reference, the elevation of the lower level of the reactor building is 282.5 feet).

4. A particularly important activity is to obtain a dooumented history of selected instruments. This includes manufacturing details and qualification procedures, as well as performance during the accident. 
5. The value of the information obtained as a result of analysis of the recovered samples is strongly dependent on the accuracy of the environmental history (including radiation exposure) which results from TWG Task 2 and upon the timely recovery of the equipment.

6. It is possible that "a materials analysis of selected components (i.e., cables, glass seals, semiconductors) can provide another source of data for estimating the radiation environment (this clearly interfaces with the efforts of the Task 2 planning group).

7. A definitive working arrangement between the various study groups and GPU/Met Ed must be developed.

8. The decontamination plan adopted could have a severe impact on the usefulness of this equipment study.

Recommendations

The general recommendations resulting from this study are given below. General guidance for sample analysis is contained in Appendix 2 .

1. As soon as possible, a remote test program (i.e., testing from the cable or control room) for instruments, electrical equipment, etc., should be established. 
2. The operational status for all instruments and electrical equipment within the reactor building should be determined. A diagnosis of out-of-spec equipment should be attempted. This work should be done prior to decontamination and without disturbing the equipment.

3. The predicted behavior of class IE electrical equipment should be correlated with actual performance. If significant discrepancies exist, further analysis would be warranted. A similar correlation should be attempted for selected items of non-lE electrical equipment.

4. An instrumentation and electrical equipment data book should be compiled to document, in readily available form, all pertinent pre and post accident information.

5. Items 1 through 4 should be accomplished as soon as possible. Items 1 through 3 are obviously time urgent; the urgency of item 4 is simply that much of the data needed for accurate planning would be contained in such a compilation.

6. The IEPG, or a similar group, should be tasked to continue in the role of technical monitor and interface between the community of users and the various groups under contract to implement the instrument and electrical equipment recovery plan. In addition, the 
IEPG should be tasked to continue the development and refinement of this recovery plan as new information becomes available.

7. Obviously, budgets need to be established and funding managers appointed for the various tasks required to execute this plan.

8. Written and approved procedures must be developed for the removal and testing of all items. If possible, the recovery procedure for each type of equipment should be reviewed by the equipment manufacturer, or an equivalent expert, prior to any physical contact (including decontamination) with the equipment.

9. Plans need to be developed for temporary and permanent storage sites for recovered material.

10. Photographs and/or TV videotape recordings of equipment should be used (a) to assist in preparing for equipment retrieval, and (b) to provide a part of the historical record for removed equipment (this effort may interface with the work of Task Group 4.0).

11. An attempt should be made to identify and store those i.tems of equipment not initially selected to be part of the survivability study. Final disposal of any item should be done only after approval by the TWG.

12. A representative of the IEPG should be present at TMI at least during the early phase of the recovery operation. 
13. A plan for the disposition of the electrical equipment which is removed for study needs to be developed. The plan would specify a procedure for designating a company or agency to perform the physical analysis and summarize the results.

14. Immediate coordination needs to begin with the plant decontamination team to arrive at an acceptable plan. 
Appendix 1

\title{
Summary of Reactor Building Instrumentation and Electrical Equipment
}

\begin{abstract}
The summary data given in this Appendix is based on the master list given in Appendix 5. The purpose of this list is to show the different kinds of equipment in the containment building and their elevations.
\end{abstract}


- TMI UNIT 2 CONTAINMENT BUILDING EQUIPMENT SUMMARY

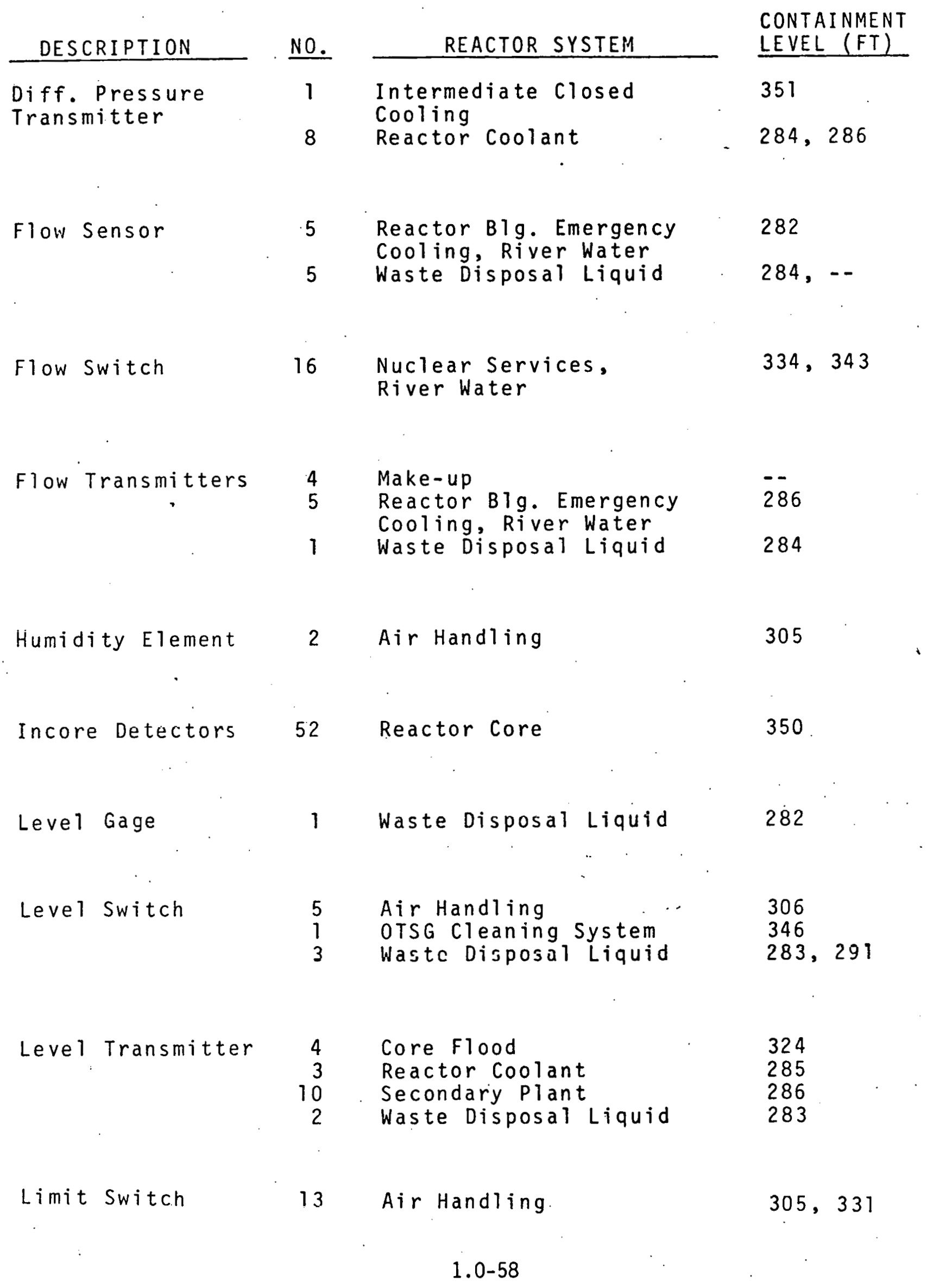


TMI UNIT 2 CONTAINMENT BUILDING EQUIPMENT SUMMARY (CONT:)

\begin{tabular}{|c|c|c|c|}
\hline DESCRIPTION & NO. & REACTOR SYSTEM & $\begin{array}{l}\text { CONTAINMENT } \\
\text { LEVEL (FT) }\end{array}$ \\
\hline $\begin{array}{l}\text { Miscellaneous } \\
\text { Equipment }\end{array}$ & -- & Not Listed & -- \\
\hline $\begin{array}{l}\text { Movable Incore } \\
\text { Detector Drives }\end{array}$ & 2 & Reactor Core & 350 \\
\hline $\begin{array}{l}\text { Nuclear Detectors } \\
\text { Out-of-core }\end{array}$ & 8 & Nuclear Instrumentation & 310,322 \\
\hline $\begin{array}{l}\text { Penetrations } \\
\therefore \\
\ddots\end{array}$ & $\begin{array}{r}10 \\
3 \\
5 \\
4 \\
3 \\
1 \\
2 \\
2 \\
2\end{array}$ & Not Applicable & $\begin{array}{l}291 \\
295 \\
299 \\
319 \\
323 \\
348 \\
351 \\
355 \\
359\end{array}$ \\
\hline $\begin{array}{l}\text { Pressure Switch } \\
\because\end{array}$ & $\begin{array}{l}3 \\
2 \\
6\end{array}$ & $\begin{array}{l}\text { Nuclear Plant Nitrogen } \\
\text { Reactor Coolant } \\
\text { Waste Disposal Liquid }\end{array}$ & $\begin{array}{l}349 \\
\overline{282}, 286,289\end{array}$ \\
\hline $\begin{array}{l}\text { Pressure } \\
\text { Transmitter }\end{array}$ & $\begin{array}{r}4 \\
16 \\
4 \\
5\end{array}$ & $\begin{array}{l}\text { Core Flood } \\
\text { Reactor Coolant } \\
\text { Secondary Plant } \\
\text { Waste Disposal Liquid }\end{array}$ & $\begin{array}{l}324 \\
287 \\
284,288 \\
285\end{array}$ \\
\hline$:$ & & & \\
\hline Pumps/Fan Motors & 35 & "Several systems" & "Several Levels" \\
\hline Radiation Monitors & $\begin{array}{r}12 \\
2\end{array}$ & $\begin{array}{l}\text { Health Physics } \\
\text { Intermediate Closed } \\
\text { Cooling. }\end{array}$ & $282,347,372$ \\
\hline
\end{tabular}


TMI UNIT 2 CONTAINMENT BUILDING EQUIPMENT SUMMARY (CONT.)

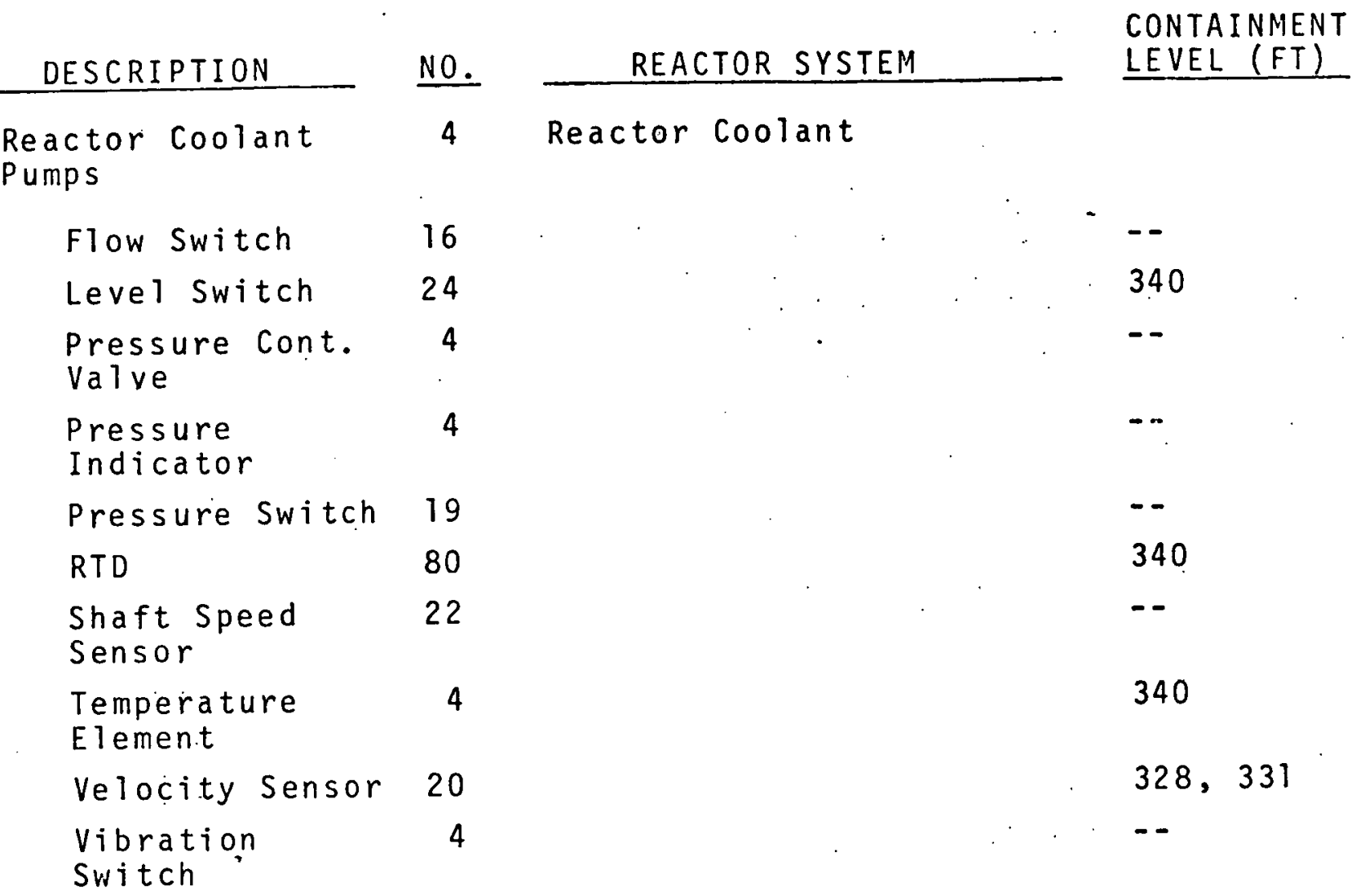

Solenoid

3 Air Handling

305

Temperature Sensors

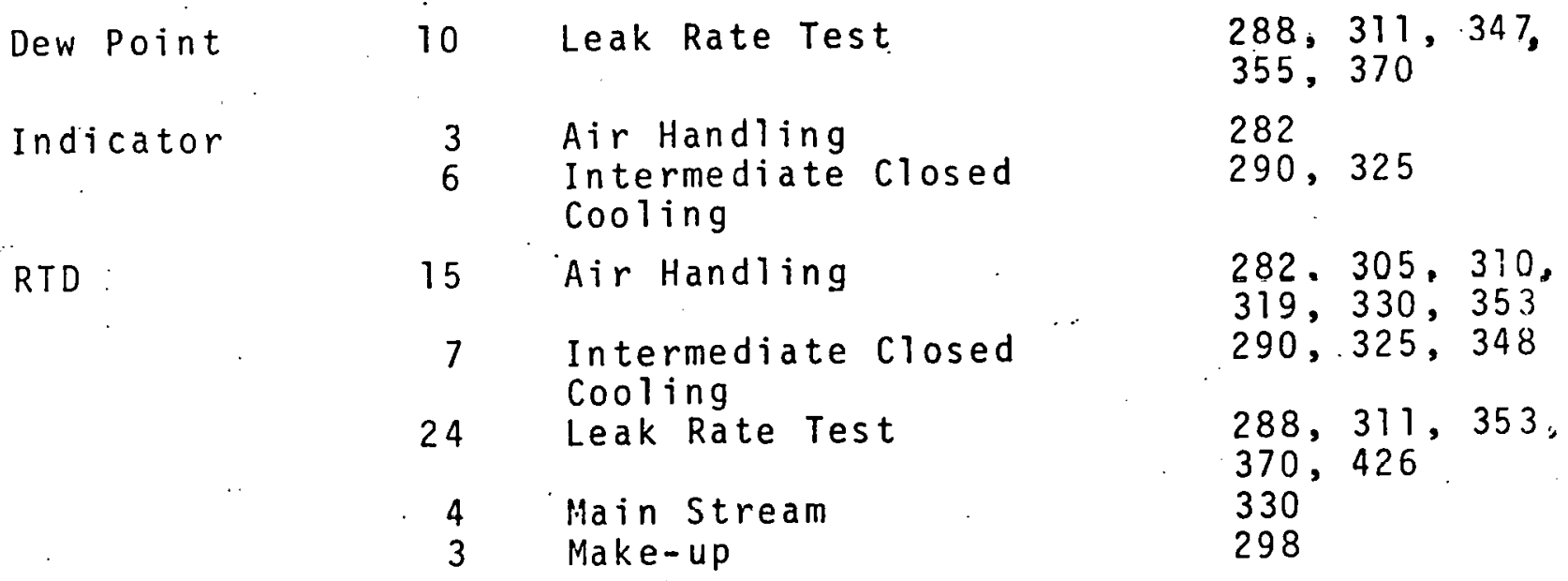


TMI UNIT 2 CONTAINMENT BUILDING EQUIPMENT SUMMARY (CONT.)

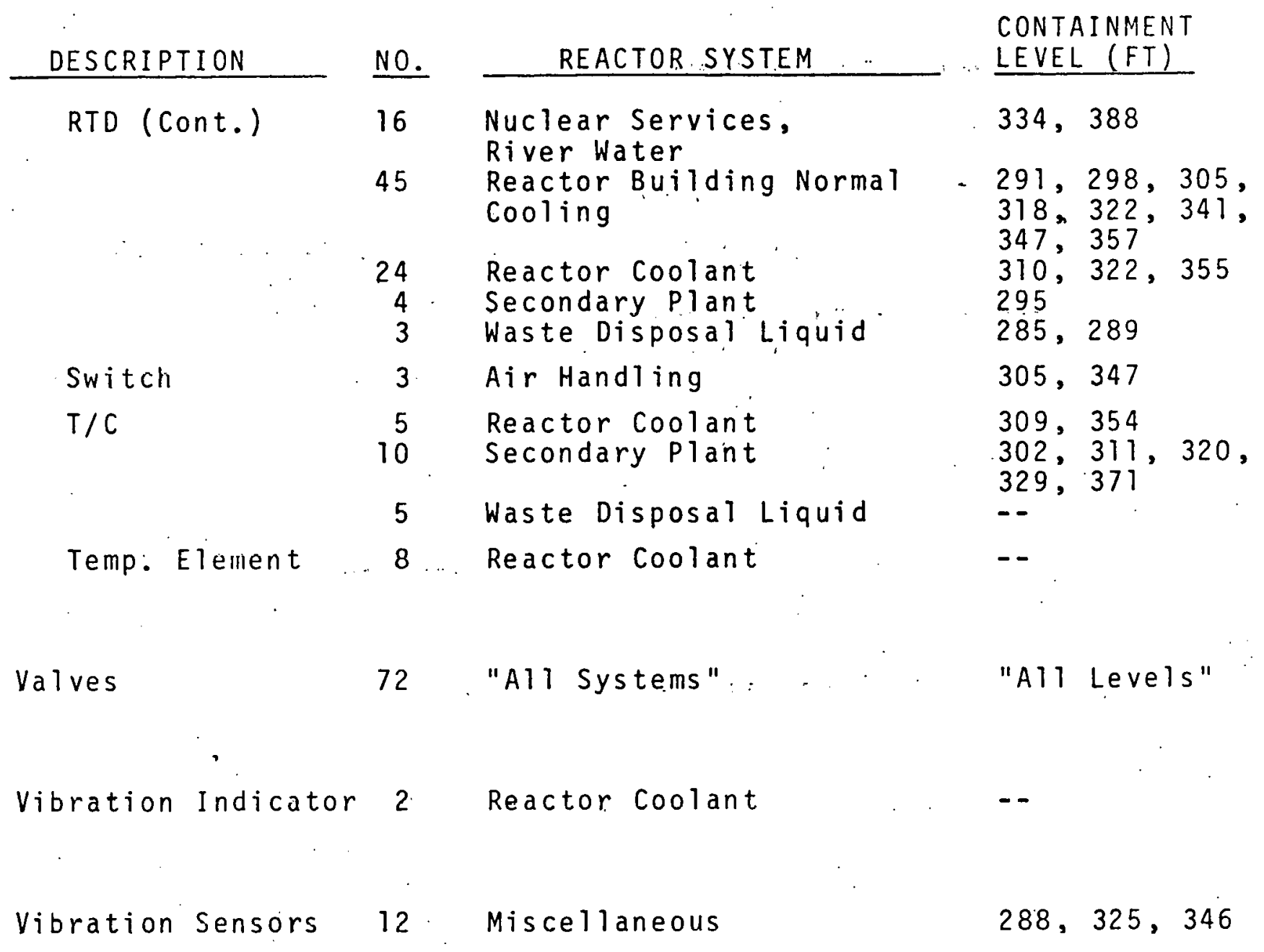


Appendix 2

Instrument Analysis Procedure

\section{Introduction}

All samples which are removed from the containment building, except perhaps some of those destined for archive storage, will eventually undergo an analysis to determine their functionality and degradation, if any. Consequently, a specific handling and analysis procedure must be generated for each sample type. While these detailed procedures may be quite involved for some, for others the procedure might be reasonably simple. In addition, background data packages must be assembled which may include (a) data traces during and after the event, (b) equipment histories, and (c) qualification data. It is recognized that only a portion of these data may exist for a given sample since not all are class $1 \mathrm{E}$ and data traces either may not have been recorded during the incident or such data may have been lost.

\section{Typical Instrument Analysis Procedure}

The tasks shown below outline what might be necessary in the analysis of an instrument such as pressure transmitter. Many pressure transmitters are safety related $1 \mathrm{E}$ instruments and possess electrical, electronic, mechanical and materials properties characteristic of many of the in-containment instruments. Information obtained from such a device might be of incident related, generic and/or environment analysis interest.

1. Records Retrieval (Data Package)
A. Manufacturer's specifications
B. Manufacturer's test data
C. Plant data traces
1. Control room, alarm computer and reactimeter sources
D. Babcock and Wilcox "noise team" data
E. Detailed information on where the instrument was installed
F. Determine environmental history and levels
1. From environment study group 
2. Test in Place

A. Electrical measurements

1. DC resistance, output current or voltage, capacitance, time domain reflectometry, power spectral density, calibration characteristics

B. Operate in Place

i. Is it possible?

2. Will degradation occur?

3. Record data

C. Photograph before removal

D. Expert examination before disturbance

3. Remove and Ship

A. Cable and piping disconnects and mounting procedures and removal

B. Handling procedures and packaging

C. Shipment

4. Decontaminate

A. Determine proper procedure for external and internal components

B. Is water dry-out necessary?

5. Bench Tests Before Disassembly

A. Record measurable parameters and compare to manufacturer specifications

6. Disassemble

7. Test After Disassembly

A. Functionality of electronic circuits

1. Failure analysis of electronic components

B. Examination and analysis of gaskets and seals

C. Insulation resistance measurements of interconnect wires, lugs and terminal blocks

D. Mechanical examination of functionality and to detect corrosion and stress points

E. Analyze external cables 
8. Determine Disposition of Components

A. For in-containment environment determination

B. Archive storage

C. Disposal

9. Simulate Environments on "New" Devices and "New" Subcomponents

A. Determine environment exposure type and levels

B. Compare degradation differences after exposures

10. Report Results

A. Should contain information relating to:

1. Operability

2. Causes of degradation or failure if applicable

3. Adequacy of qualification testing

4. Calibration of incident recorded data 


\section{Appendix 3}

\section{Selected Plant Drawings}

This Appendix contains plan views of the 282.5, 305, and $347.5 \mathrm{ft}$. levels and, also, a side view of the reactor and containment structure. These are the main plant drawings used in the sample selection process. 


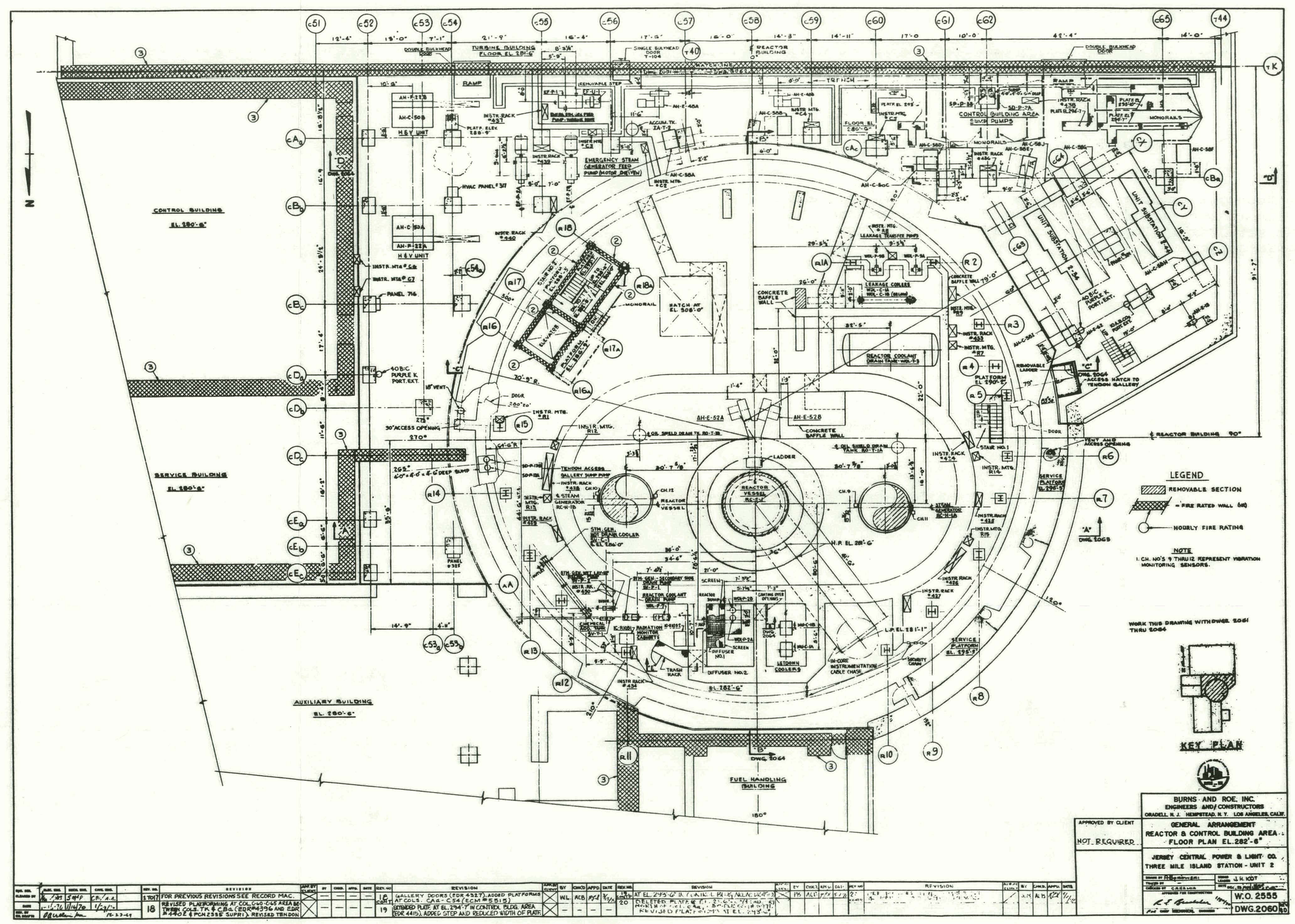




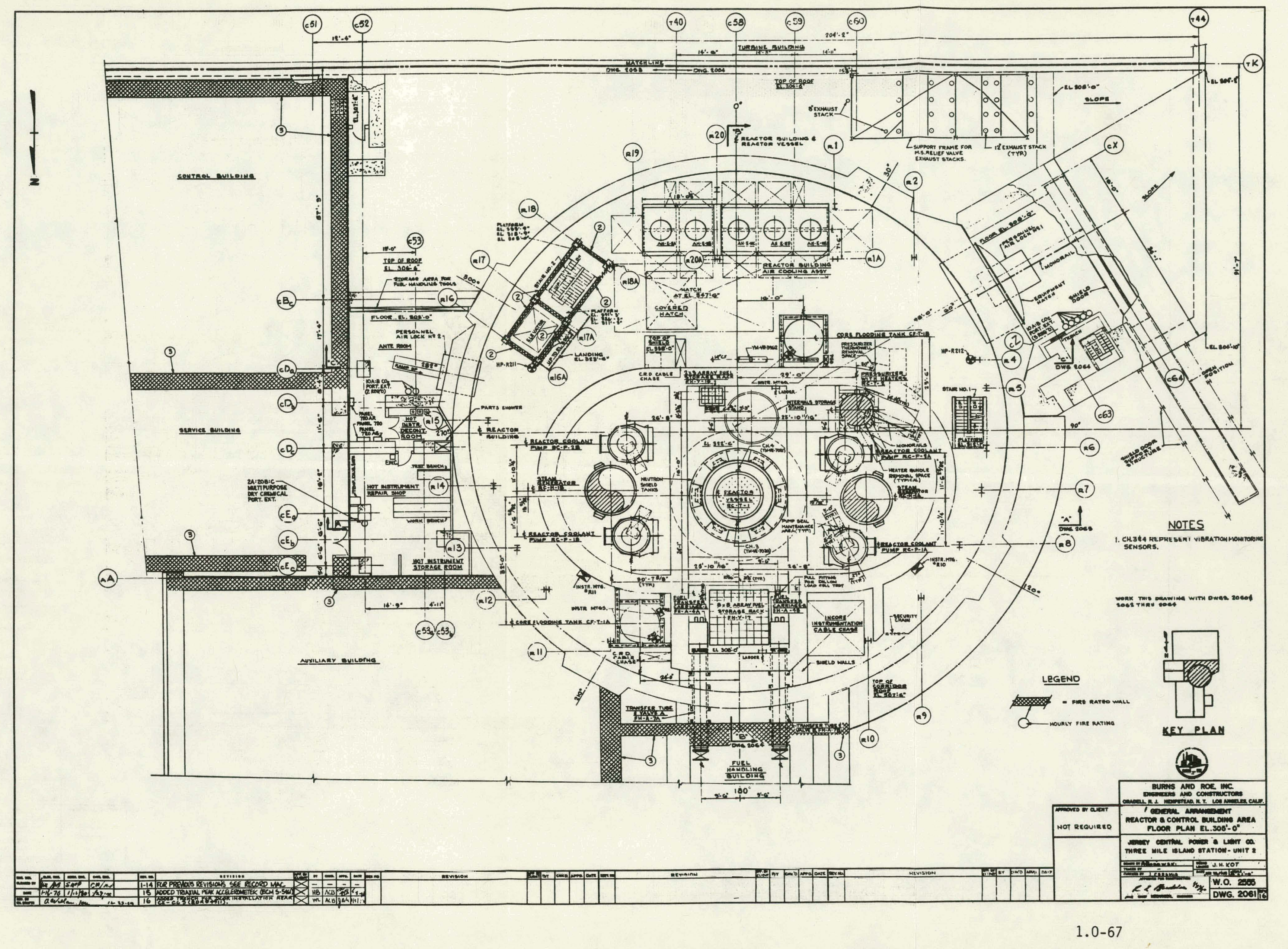




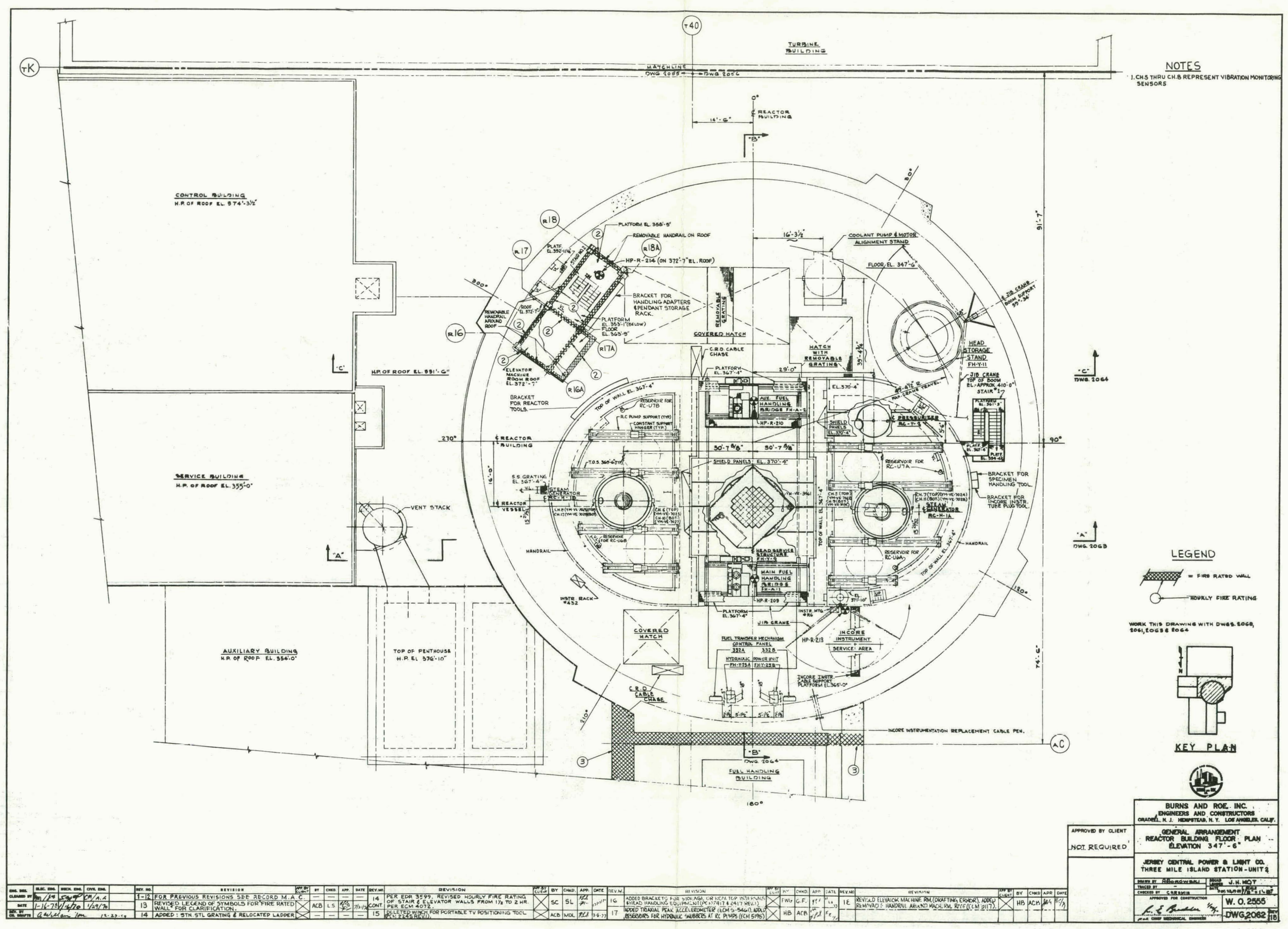




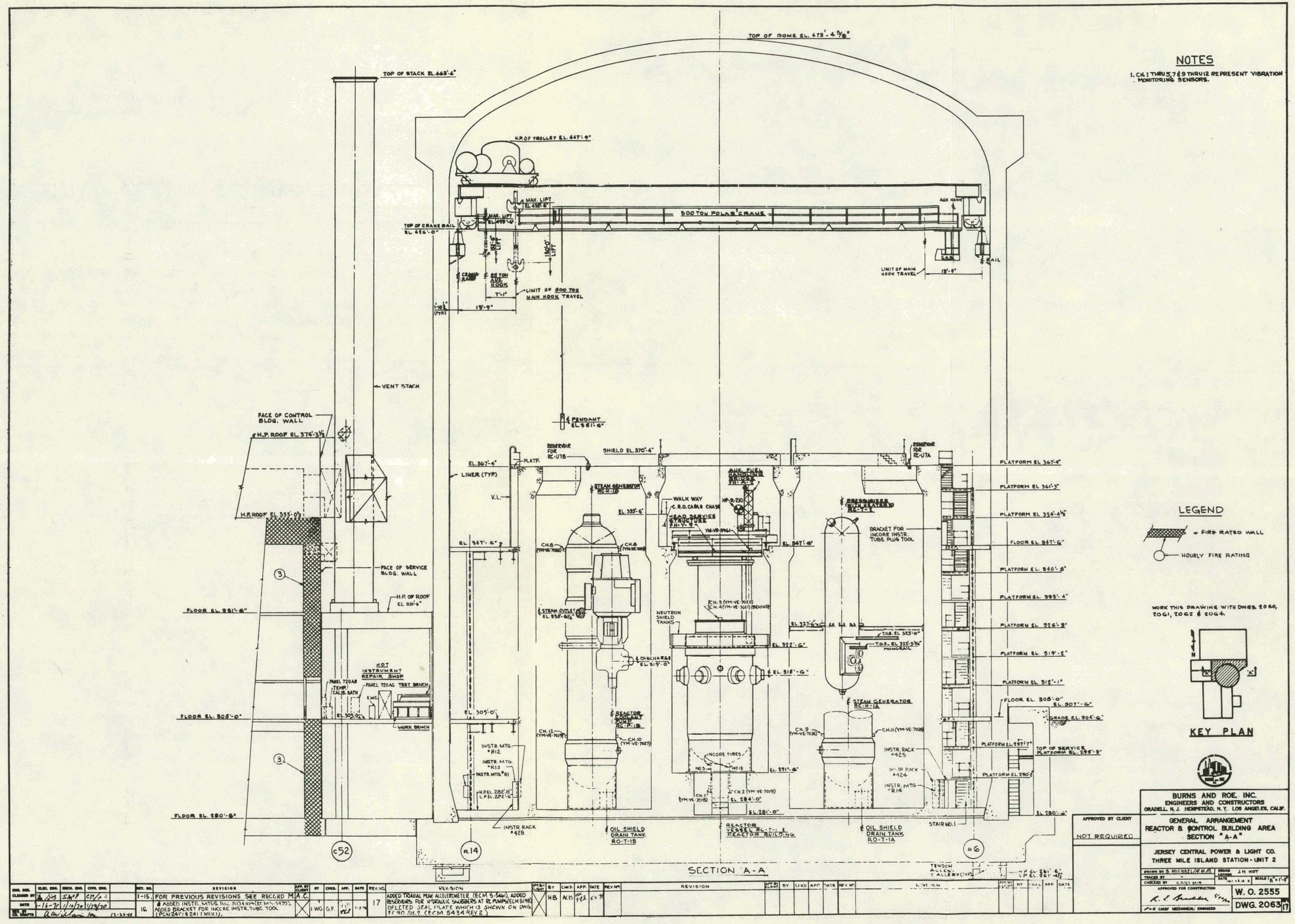




\section{Appendix 4}

Piping and Instrumentation Diagram

This Appendix provides a schematic of the TMI-2 plant which shows the locations of many of the samples selected. In some cases the notation will apply only to the function since the location of the transmitter is often separate from the sample point. Instruments are indicated by circles or squares. Those items selected as analysis candidates are designated by partially blackening the appropriate circle or square. Valves which have been selected also have partially blackened circles or squares appended. Partially blackened triangles have another meaning (see symbol sheet below). 


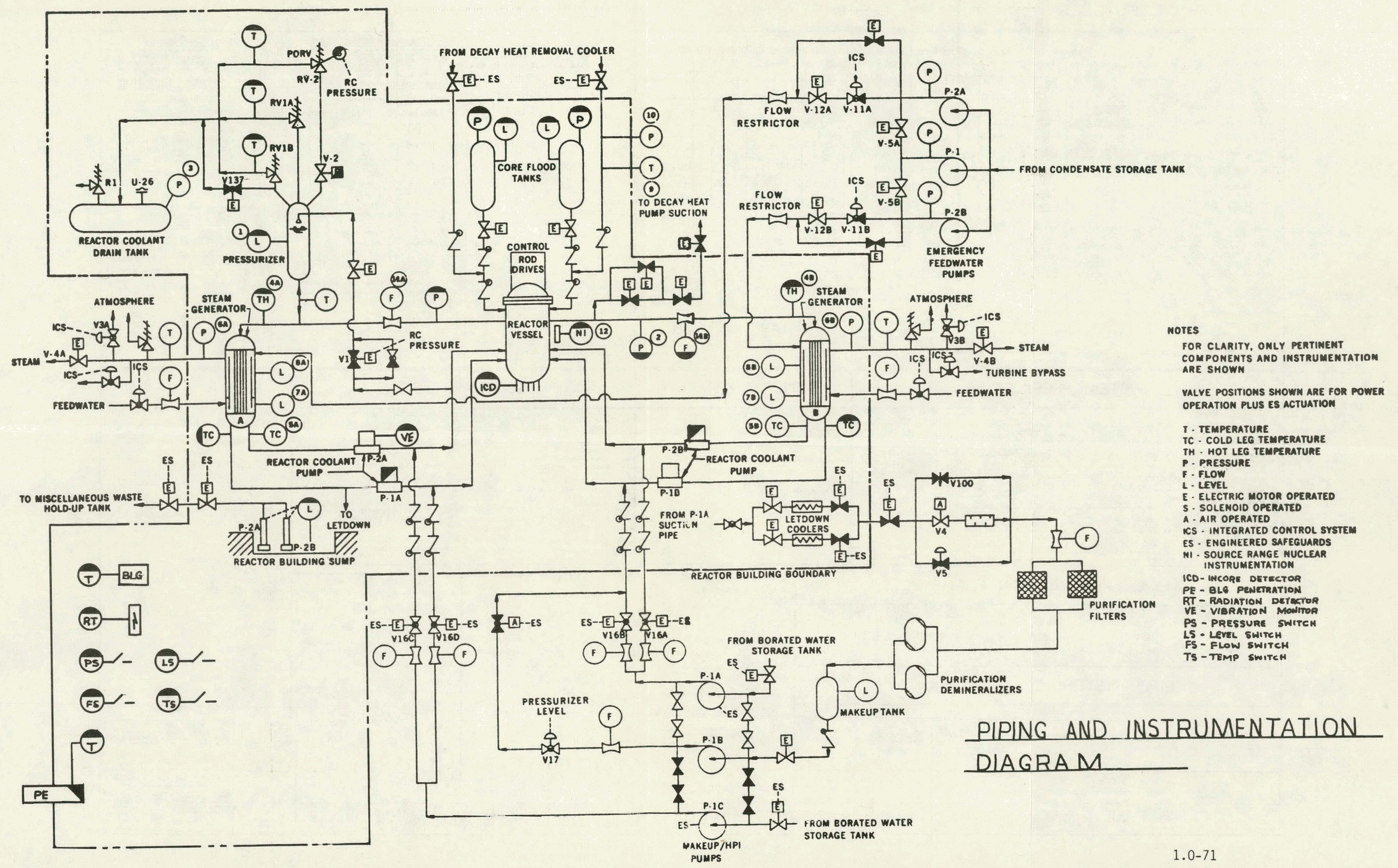




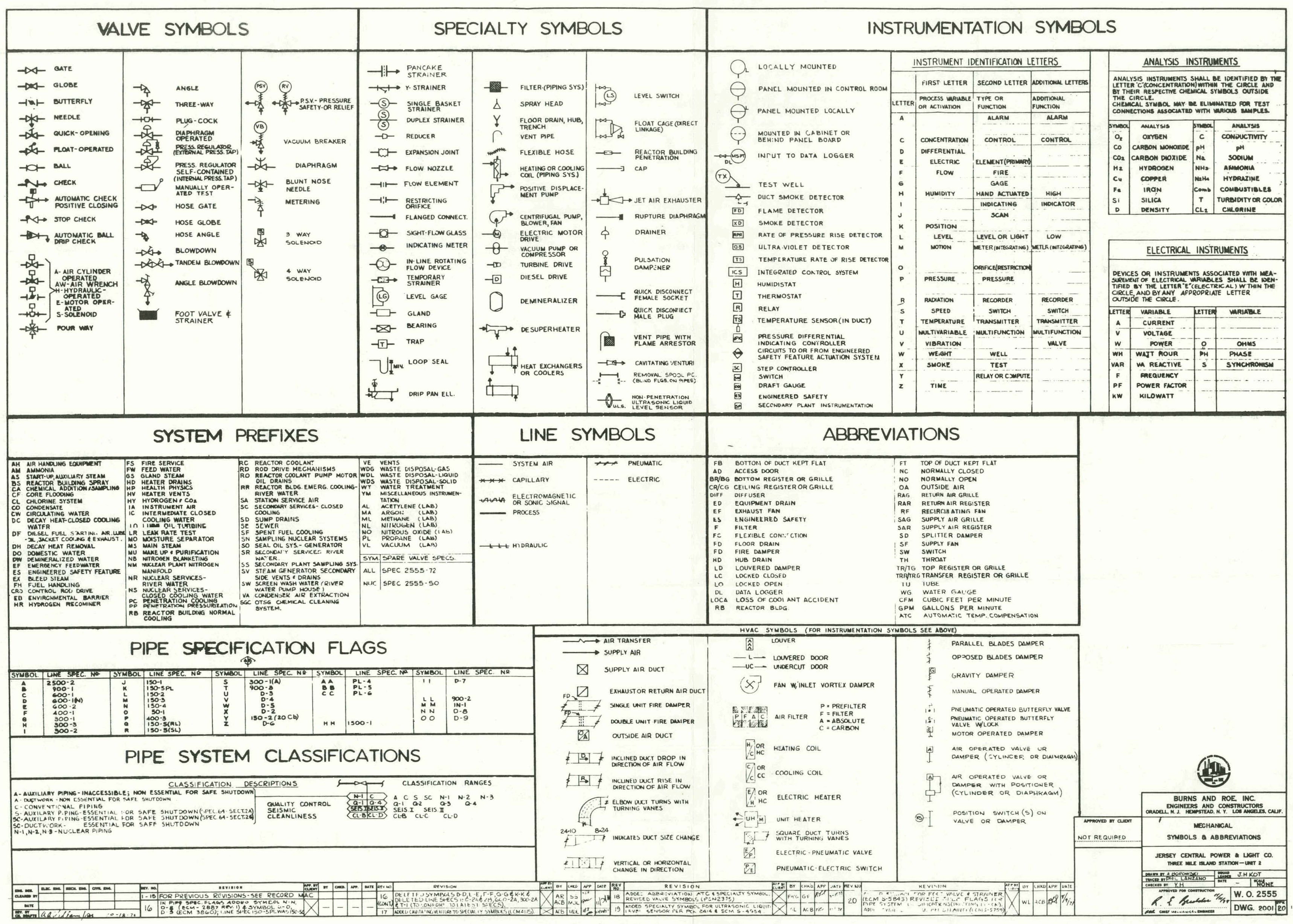




\section{Appendix 5 \\ Master List of TMI-2 In-Containment Instruments and Electrical Equipment}

This Appendix contains the complete list of. Instruments and Electrical Equipment contained within the TMI-2 containment building. It is complete in the sense that a considerable effort has been expended to create a data base which incorporates the various other lists which exist. It is certainly complete in the sense that all items which have been. considered for inclusion in the sample recovery plan are given here whether or not they were ultimately selected. It is unlikely to be complete if judged against the standard that no errors exist or that no omissions have been made. 


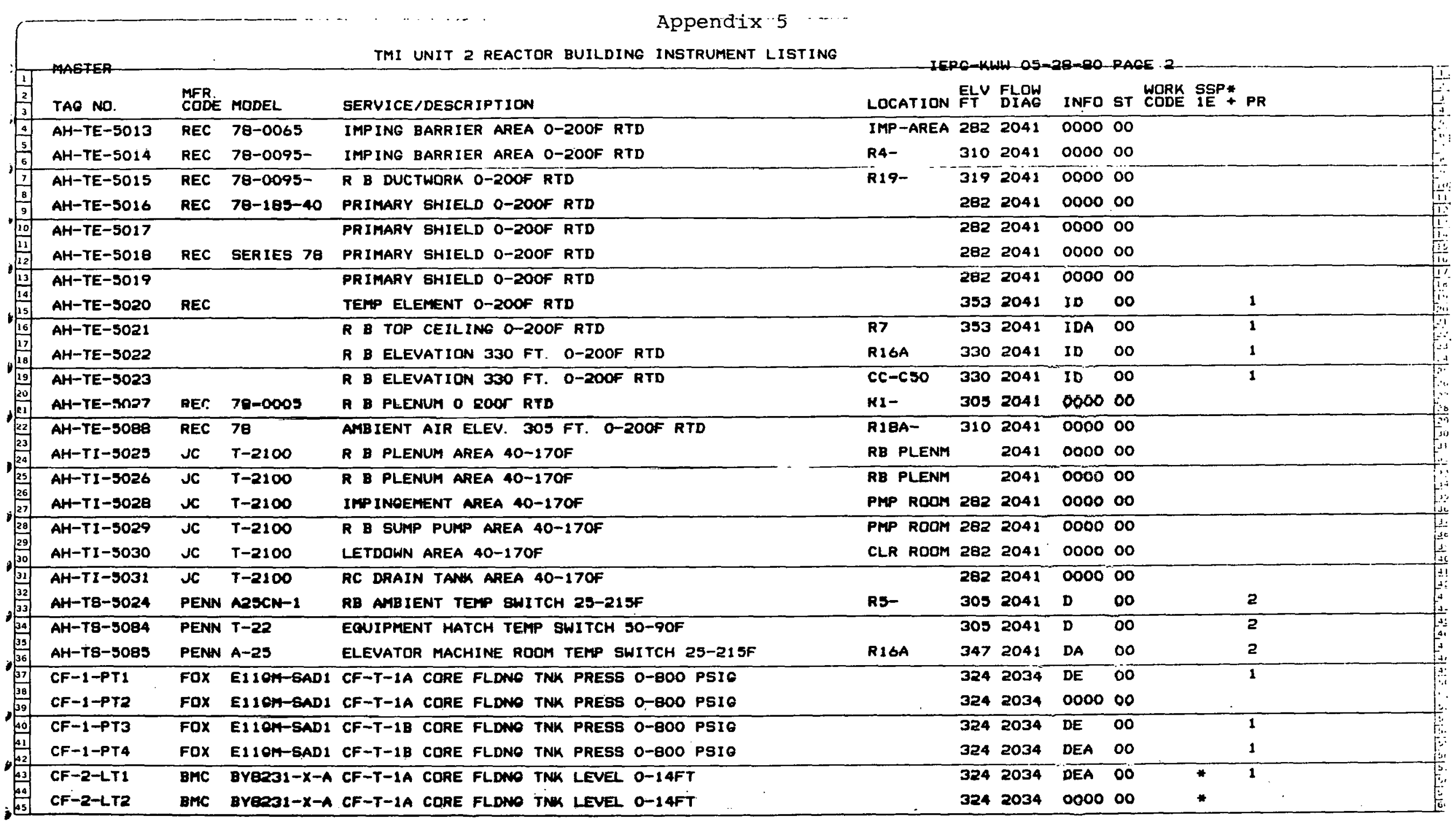


TMI UNIT 2 REACTOR BUILDING INSTRUMENT LISTING

\begin{tabular}{|c|c|c|c|c|c|c|c|c|c|c|c|c|}
\hline 1 & -MASTER & & & & & & & & & & & . \\
\hline 2 & TAQ ND. & MFR. & MODEL & SERVICE/DESCRIPTION & LOCATION F & $\begin{array}{l}\text { ELV } \\
\text { FT }\end{array}$ & $\begin{array}{l}\text { FLOW } \\
\text { DIAG }\end{array}$ & INFO & ST & $\begin{array}{l}\text { WORK SSP* } \\
\text { CODE IE + }\end{array}$ & PR & 童 \\
\hline$\frac{4}{5}$ & $C F-2-L T 3$ & BMC & BYB231-X-A & CF-T-1B CORE FLDNG TNK LEVEL O-14FT & & 324 & 2034 & DE & 00 & * & 1 & $\frac{1}{3}$ \\
\hline$\frac{5}{6}$ & $C F-2-L T 4$ & BmC & BYB231-X-A & CF-T-1B CORE FLDNG TNK $0-14 F T$ & & 324 & 2034 & 0000 & $\infty$ & * & & -1 \\
\hline$\frac{7}{8}$ & DC-TX-2011 & MERC & & LEAKAGE COOLER WDL-C-1A INLET TEMPERATURE & $505-26 w$ & & 2196 & 0000 & 00 & & & 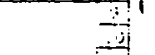 \\
\hline 9 & DC-TX-2012 & MERC & & LEAKAOE COQLER WDL-C-1A OUTLET TEMPERATURE & $505-264$ & & 2196 & 0000 & oo & & & $\because$ \\
\hline$\frac{10}{11}$ & $D C-T X-2013$ & MERC & & LEAKAQE COOLER WDL-C-1B INLET TEMPERATURE & $505-26 \mathrm{~W}$ & & 2196 & 0000 & 00 & & & $\frac{1}{1}$ \\
\hline 12 & $D C-T X-2014$ & MERC & & LEAKAGE COOLER HDL-C-1B OUTLET TEMPERATURE & $505-26 \mathrm{~W}$ & & 2196 & 0000 & 00 & & & (1) \\
\hline$\frac{13}{14}$ & HP-RA-0209 & VICO & & RADIATION ALARM & FH BR & & & 0000 & 00 & & & $\frac{11}{31}$ \\
\hline 15 & HP-RA-75BS-1 & & & RB EVACUATION HORN & RB & 281 & & 0000 & $\mathbf{0 0}$ & & & 。1 \\
\hline$\frac{16}{17}$ & HP-RA-7586-2 & & & RB EVACUATION HORN & RB & 281 & & 0000 & 00 & & & 2 \\
\hline 10 & HP-RA-7586-3 & & & RB EVACUATION HORN & $\mathbf{R B}$ & 281 & & 0000 & 00 & & & 翼 \\
\hline$\frac{19}{20}$ & HP-RI-0209 & VICO & $856-2$ & RADIATION IND/W-BIBTABLEB & $F H$ BR (N) & & & 0000 & 00 & & & 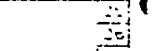 \\
\hline 21 & HP-RI-0210 & VICO & $856-9$ & RADIATION IND/W-BIBTABLES & FH BR (B) & & & 0000 & $\mathbf{0 0}$ & & & 1 \\
\hline 22 & HP-RI-A0211 & VICO & 958-3 & RADIATION ALARM/METER UNIT & PER HTCH & & & 0000 & 00 & & & is \\
\hline$\frac{24}{24}$ & HP-RI-A0212 & VICO & $858-3$ & RADIATION ALARM/METER UNIT & EQP HTCH & & & 0000 & 00 & & & 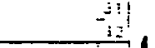 \\
\hline$\frac{25}{26}$ & $H P-A I-A 0213$ & VIC. & 858-3 & RADIATION ALARM/METER UNIT & INST PNL & & & 0000 & 00 & & & 年 \\
\hline 27 & MP-RT-0209 & VICO & $857-2$ & RADIATIDN DET/X-MITTER (OM) & FH BK (N): & 347 & 2062 & EA & $\infty 0$ & & 2 & $\stackrel{10}{\pi}$ \\
\hline 29 & HP-RT-0210 & VICO & $857-2$ & RADIATION DET/X-MITTER (OM) & FH BR (S): & 347 & 2062 & $\overline{D E}$ & 00 & & 2 & $\frac{11}{a}$ \\
\hline$\frac{29}{30}$ & HP-RT-0211 & VIco & $857-2$ & RADIATION DET/X-MITTER (OM) & & 309 & & 0000 & 00 & & & $\frac{3 y}{20}$ \\
\hline$\frac{31}{32}$ & HP-RT-0212 & VICO & $857-2$ & RADIATION DET/X-MITTER (OM) & & 309 & & 0000 & 00 & & & tit \\
\hline ;33 & HP-RT-0213 & VICO & $857-2$ & RADIATION DET/X-MITTER (OM) & R6 & 347 & 2062 & DE & 00 & & 1 & : \\
\hline 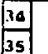 & Hr RT-0211 & VICO & $847-1$ & RADIATION DET/X-MITTER (ION) & DOME & 372 & 2062 & IDE & 00 & & 1 & $\frac{25}{3+1}$ \\
\hline 36 . & IC-1-TEI & вмс & PYROTRON & RC-P-IA INU WTR RECRC FLOW \& PUMP JKT O-ZOOF RTD & PIPINO & 327 & 2029 & D & 00 & & 2 & 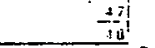 \\
\hline 37 & IC-1-TE2 & BMC & PYROTRON & RC-P-IA INJ WTR RECRC FLOW \& PUMP JKT D-200F RTD & PIPINO & 327 & 2029 & 0000 & 00 & & & 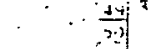 \\
\hline 39 & IC-I-TE3 & BMC & PYROTRON & RC-P-1A INJ WTR RECRC FLOW PUMP JKT D-200F RTD & PIP INO & 327 & 2029 & 0000 & 00 & & & 28 \\
\hline 40 & IC-1-TE4 & BMC & PYROTRON & RC-P-IA INJ WTR RECRC FLOW \& PUMP JKT 0-2OOF RTD & PIPINO & 325 & 2029 & 0000 & 00 & & & 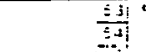 \\
\hline 整 & IC-10-DPT & BMC & BY8230XA & CRD OUTLET HDR FLOW 0-200 IN. H2O & R6 & 351 & 2029 & DE & $\mathbf{0 0}$ & & 1 & 3 \\
\hline 43 & IC-7-TEI & BMC & PYROTRON & LETDOWN CLR MU-C-1B OUT IC FLOW TEMP 0-2SOF RTD. & PIPINO & 290 & 2029 & 0000 & 00 & & & $\because$ \\
\hline 0 & IC-7-TE2 & Bme & PYROTRON & LETDOWN CLR MU-C-1A OUT IC FLOW TEMP O-25OF RTD & PIPINO & 290 & 2029 & 0000 & 00 & & & 6 \\
\hline
\end{tabular}




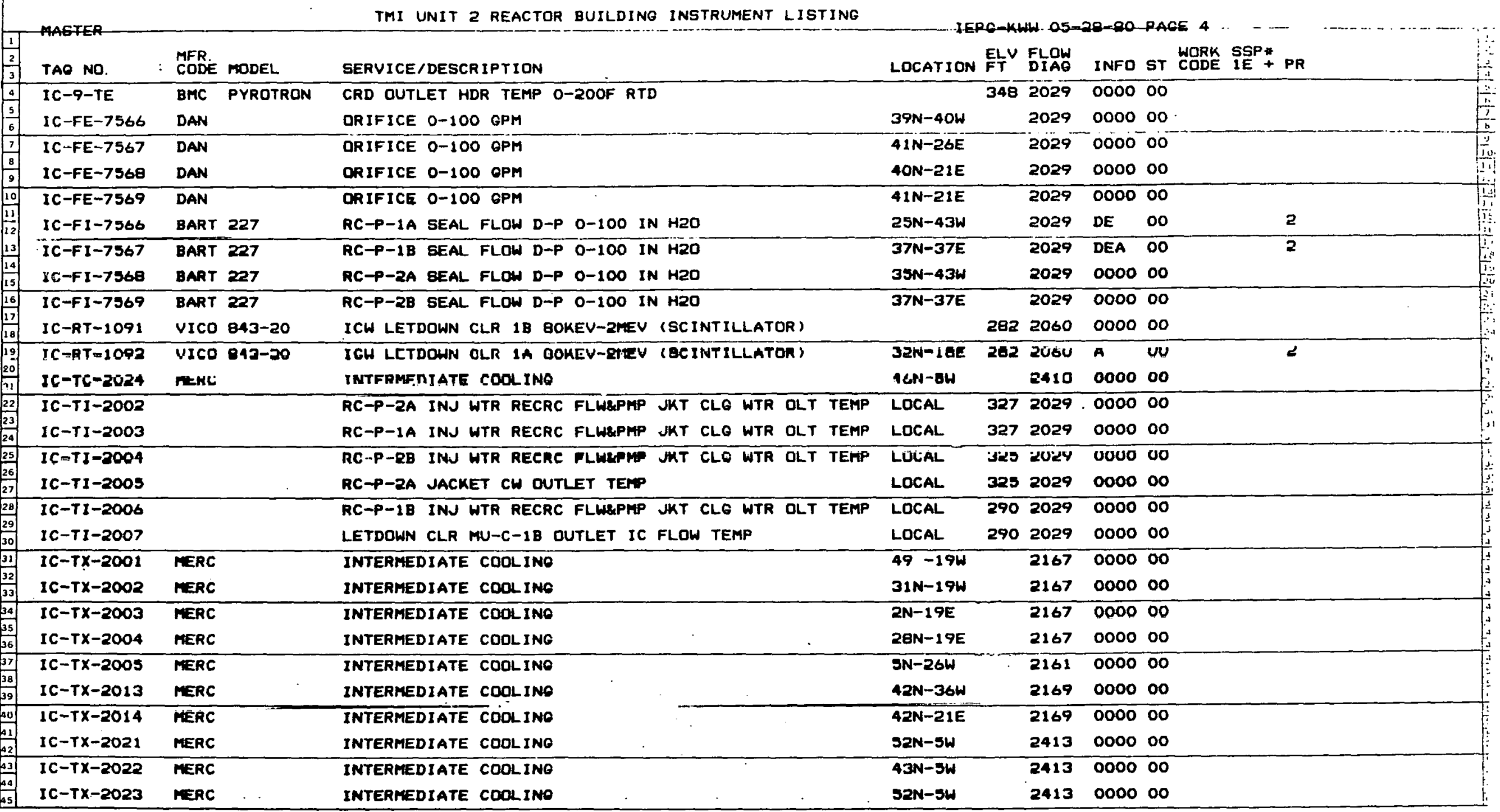


Appendix 5 (Cont'd.)

TMI UNIT 2 REACTOR BUILDING INSTRUMENT LISTING

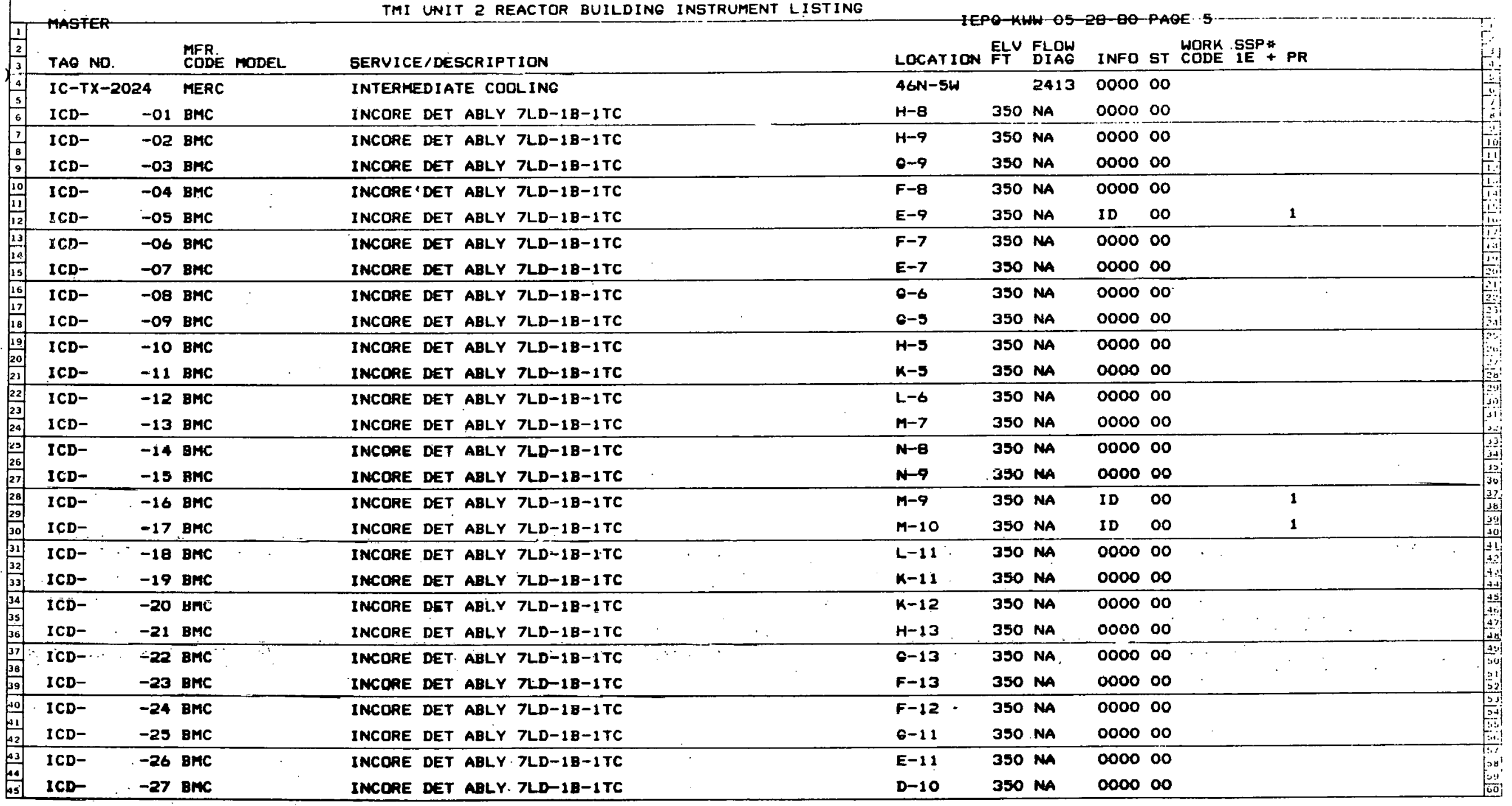




\section{Appendix 5 (Cont'd)}

TMI UNIT 2 REACTOR BUILDING INSTRUMENT LISTING

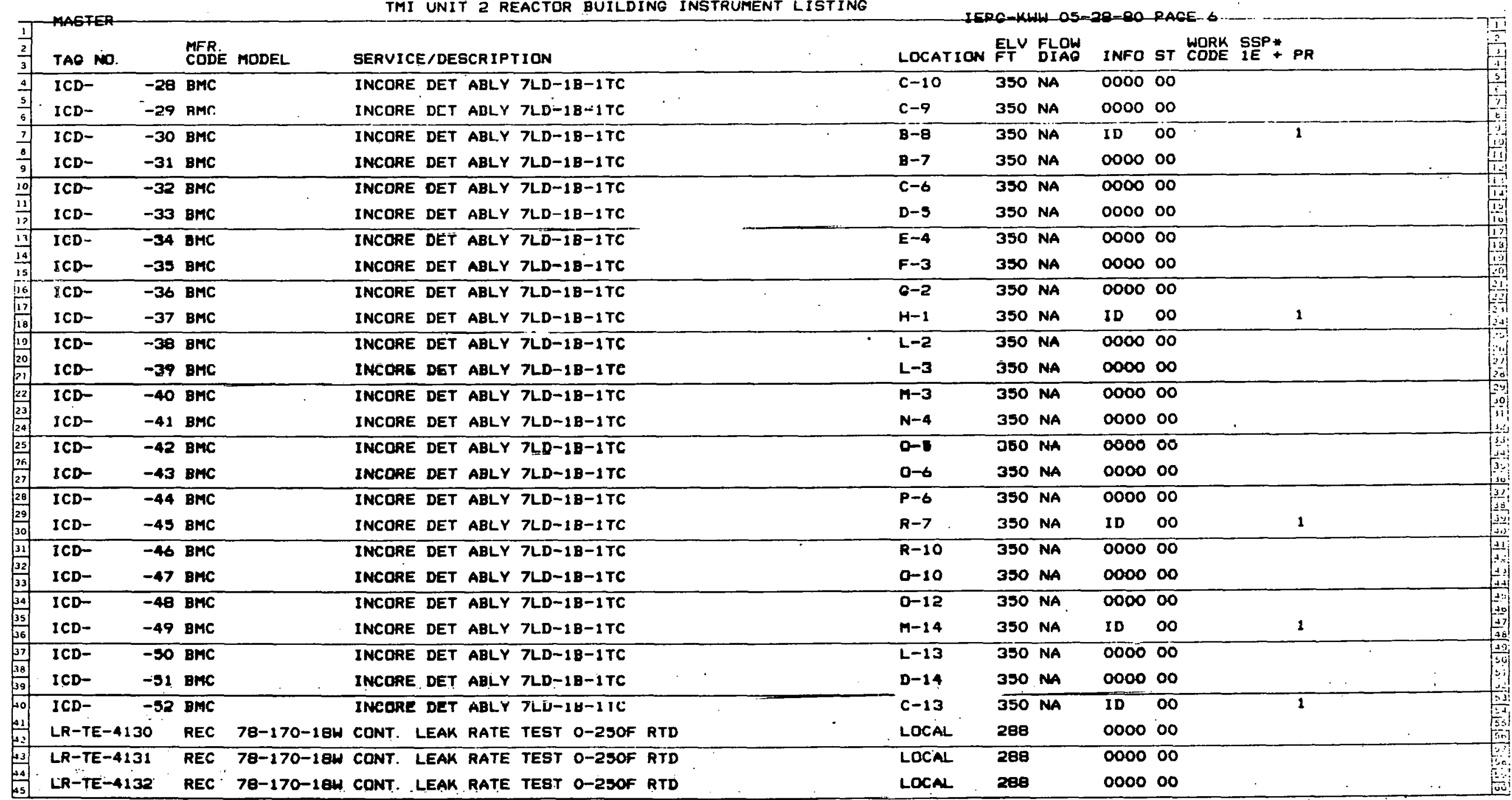




\section{Appendix 5 (Cont'd)}

TMI UNIT 2 REACTOR BUILDING INSTRUMENT LISTING

I MASTER TAC NO. LR -TE-4133

LR-TE-4134

LR

LR-TE-4135

LR-TE-4136

L.R-TE-4137

LR-TE-4138

LR-TE-4139

LR-TE-4140

LR-TE-4141

LR-TE-4142

LR-TE-4143

LR-TE-4144

LR-TE-4145

LR-TE-4146

LR-TE-4147

LR-TE-4148

LR-TE-4149

LR-TE-4150

LR-TE-4151

LR-TE-4152

LH-TE-4153

LR-TE-4164

LR-TE-4165

LR-TE-4166

LR-TE-4167

LR-TE-4168

LR-TE-4169

LR-TE-4170

LR-TE-4170

IEPG-KWH -05-28-80-PAGE-7

MFR. MODEL SERVICE/DESCRIPTION

REC 78-170-18W CONT. LEAK RATE TEST O-25OF RTD

REC 78-170-18 CONT. LEAK RATE TEST 0-25OF RTD

REC 78-170-18 CONT. LEAK RATE TEST O-25OF RTD

REC 78-170-18 CONT. LEAK RATE TEST 0-25OF RTD

REC 78-170-18 CONT. EEAK RATE TEST 0-25OF RTD

REC 78-170-18W CONT. LEAK RATE TEST O-25OF RTO

REC 78-170-18W CONT. LEAK RATE TEST 0-25OF RTD

REC 78-170-18W CONT. LEAK RATE TEST 0-ZSOF RTD

REC 78-170-18W CONT. LEAK RATE TEST 0-25OF RTD

REC 79-170-18W CONT. LEAK RATE TEST 0-25OF RTD

REC 79-170-18W CONT. LEAK RATE TEST 0-25OF RTD

REC 79-170-18W CONT. LEAK RATE TEST 0-250F RTD

REC 79-170-18W CONT. LEAK RATE TEST 0-250F RTD

REC 78-170-18W CONT. LEAK RATE TEST 0-25OF RTD

REC 78-170-18W CONT. LEAK RATE TEST 0-25OF RTD

REC 78-170-18W CONT. LEAK RATE TEST 0-25OF RTD

REC 78-170-18W CONT. LEAK RATE TEST O-25OF RTD

REC 78-170-18W CONT. LEAK RATE TEST 0-25OF RTD

REC 78-170-18W CONT. LEAK RATE TEST O-250F RTD

REC 78-170-18W CONT. LEAK RATE TEST O-ZSOF RTD

REC 70 170-18W COMT. LEAK RATE TEST Q-ZSOF RTD

FOX 2711-AG

CONT. L.R. TEST DEW POINT O-1OOF

FOX 2711-AO CONT. L.R. TEST DEW POINT 0-100F CONT. L. R. TEBT DEW POINT 0-10OF

CDNT. L. R. TEST DEW POINT O-10OF

CONT. L. R. TEST DEW POINT O-1OOF

OX $2711 \mathrm{AO}$

FoX. 2711 AO

CONT. L.R. TEST DEW POINT O-10OF

CONT. L. R. TEBT DEM POINT 0-10OF.

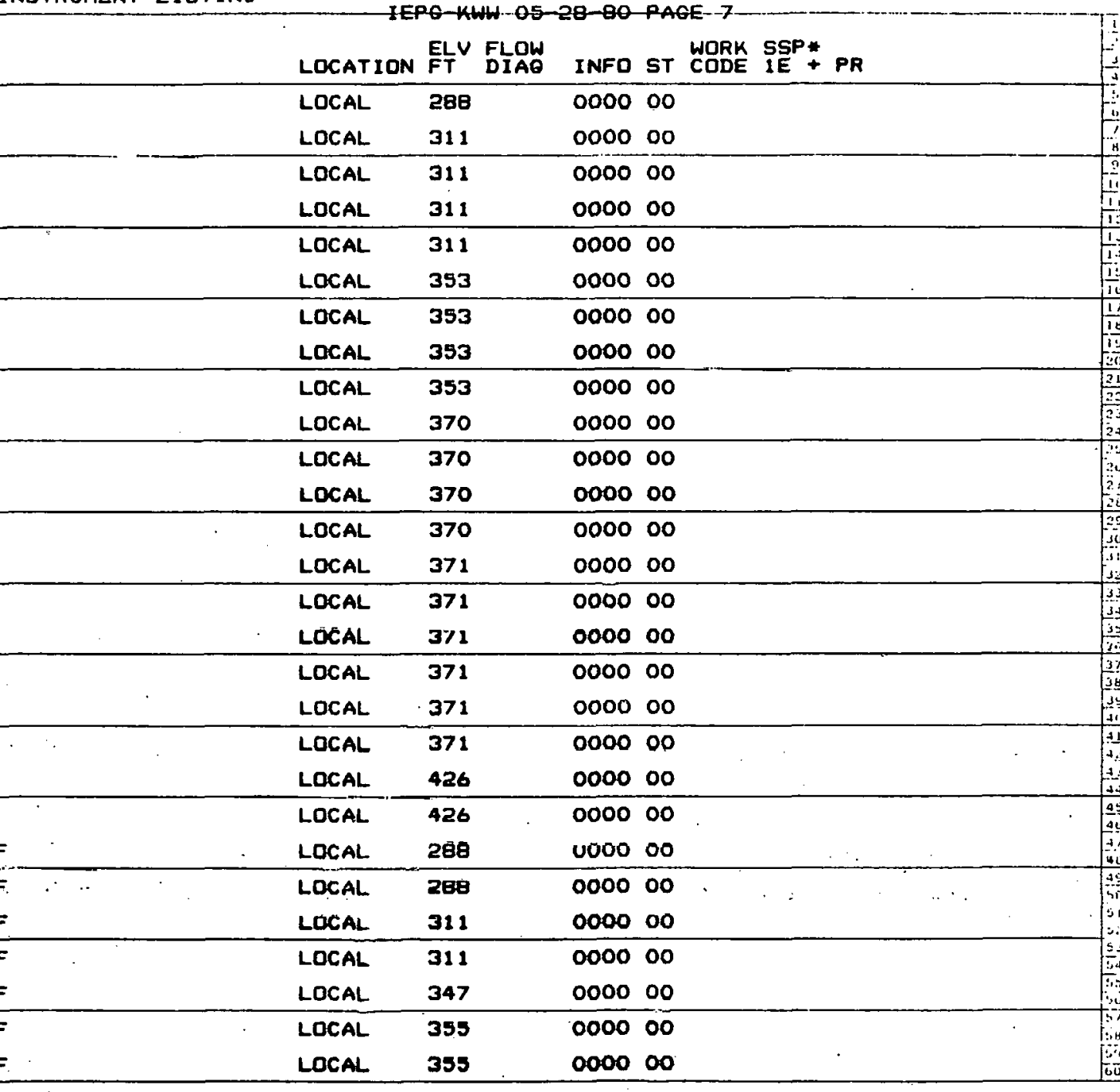




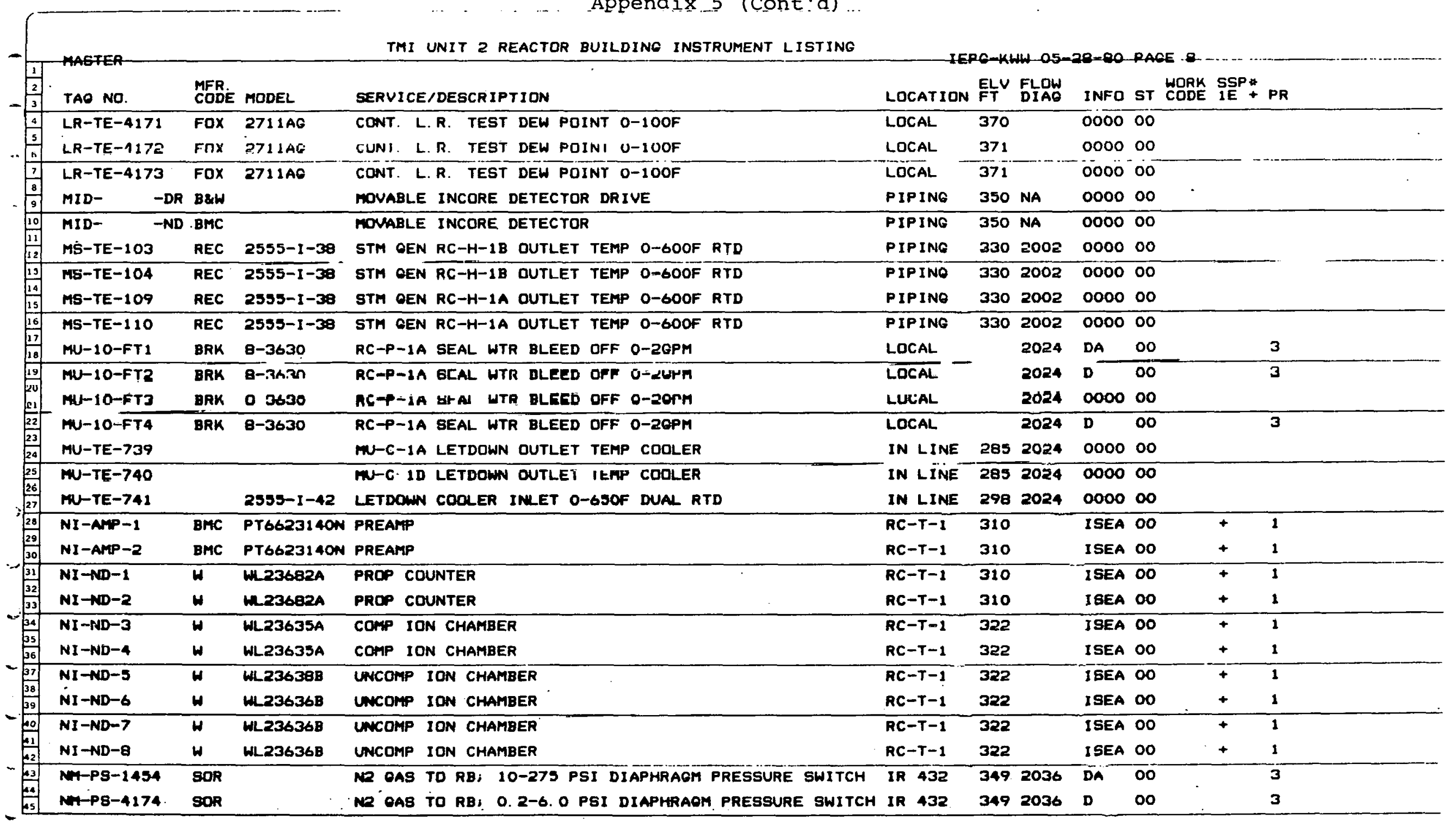




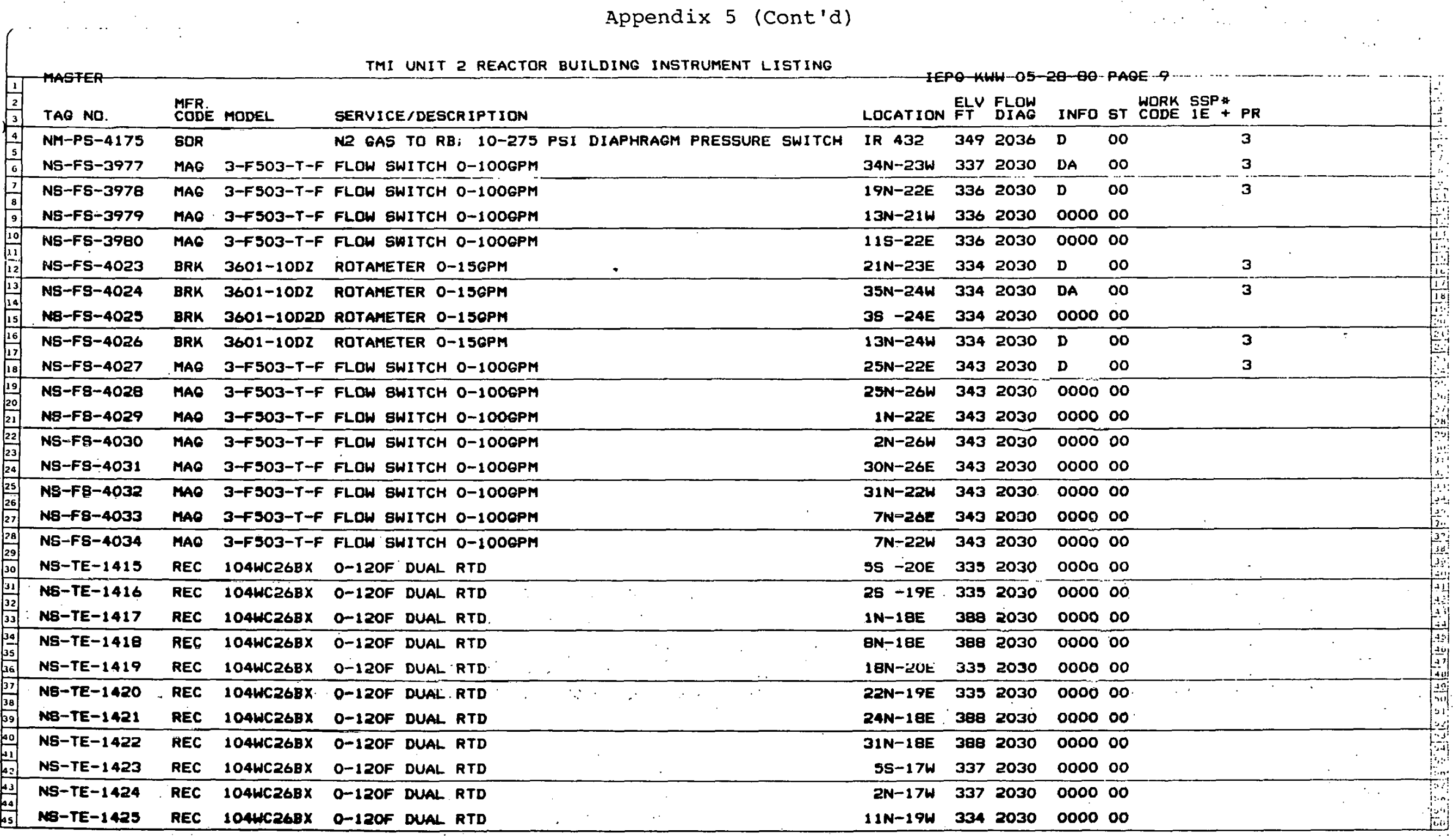


Appendix 5 (Cont'd)

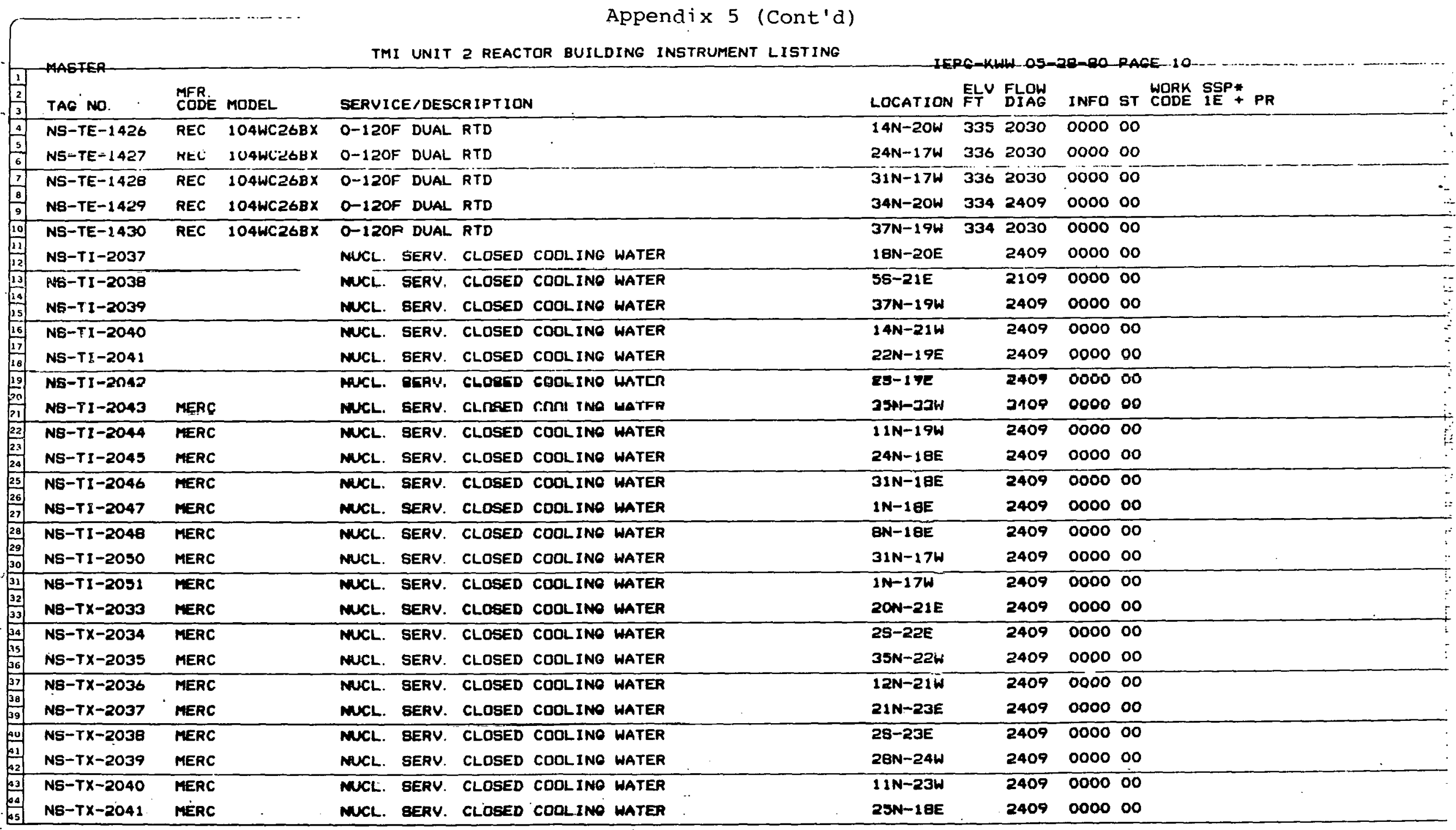

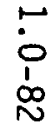


TMI. UNIT 2 REACTOR BUILDING INSTRUMENT LISTING

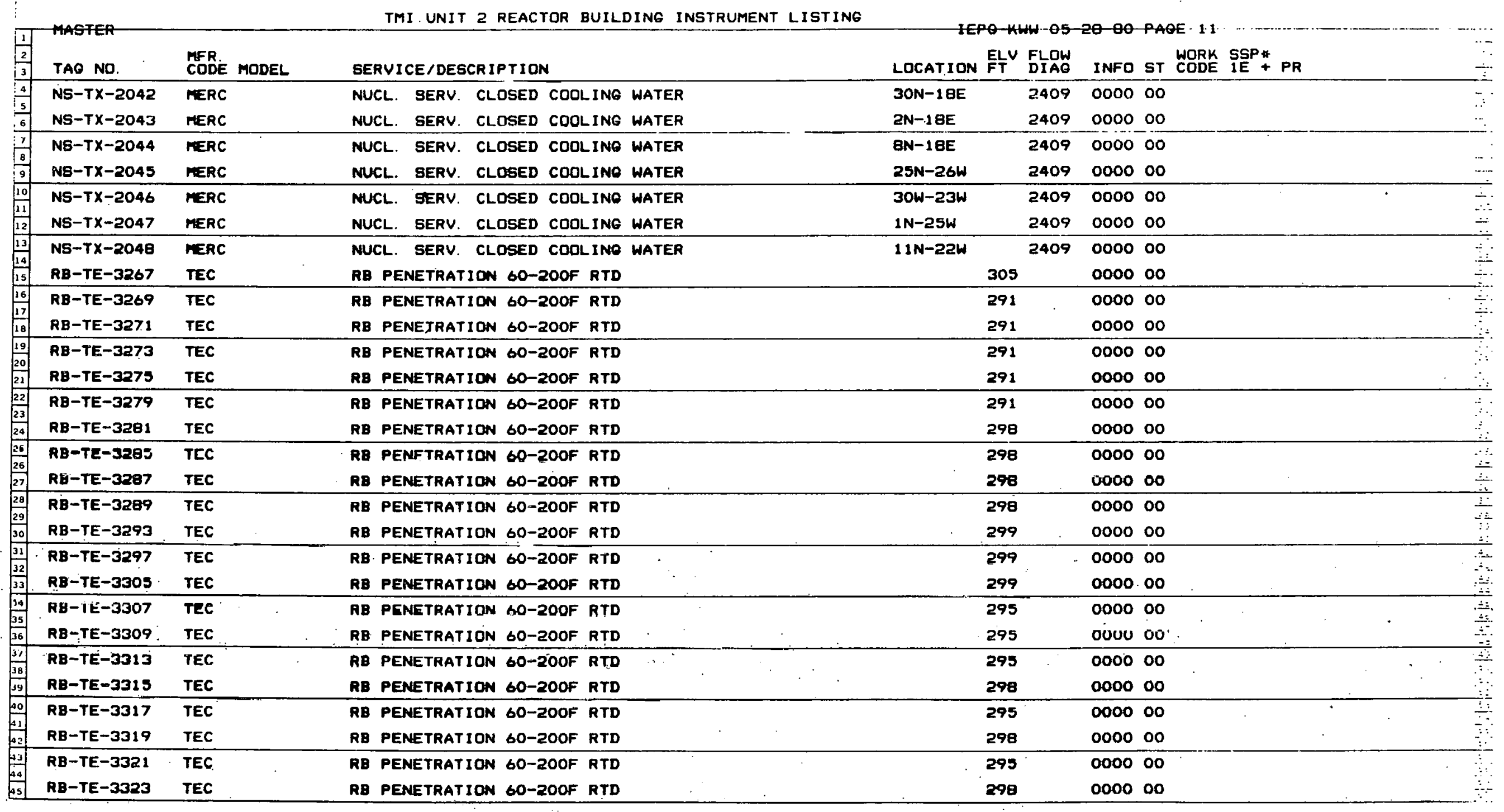


TMI UNIT 2 REACTOR BUILDING INSTRUMENT LISTING

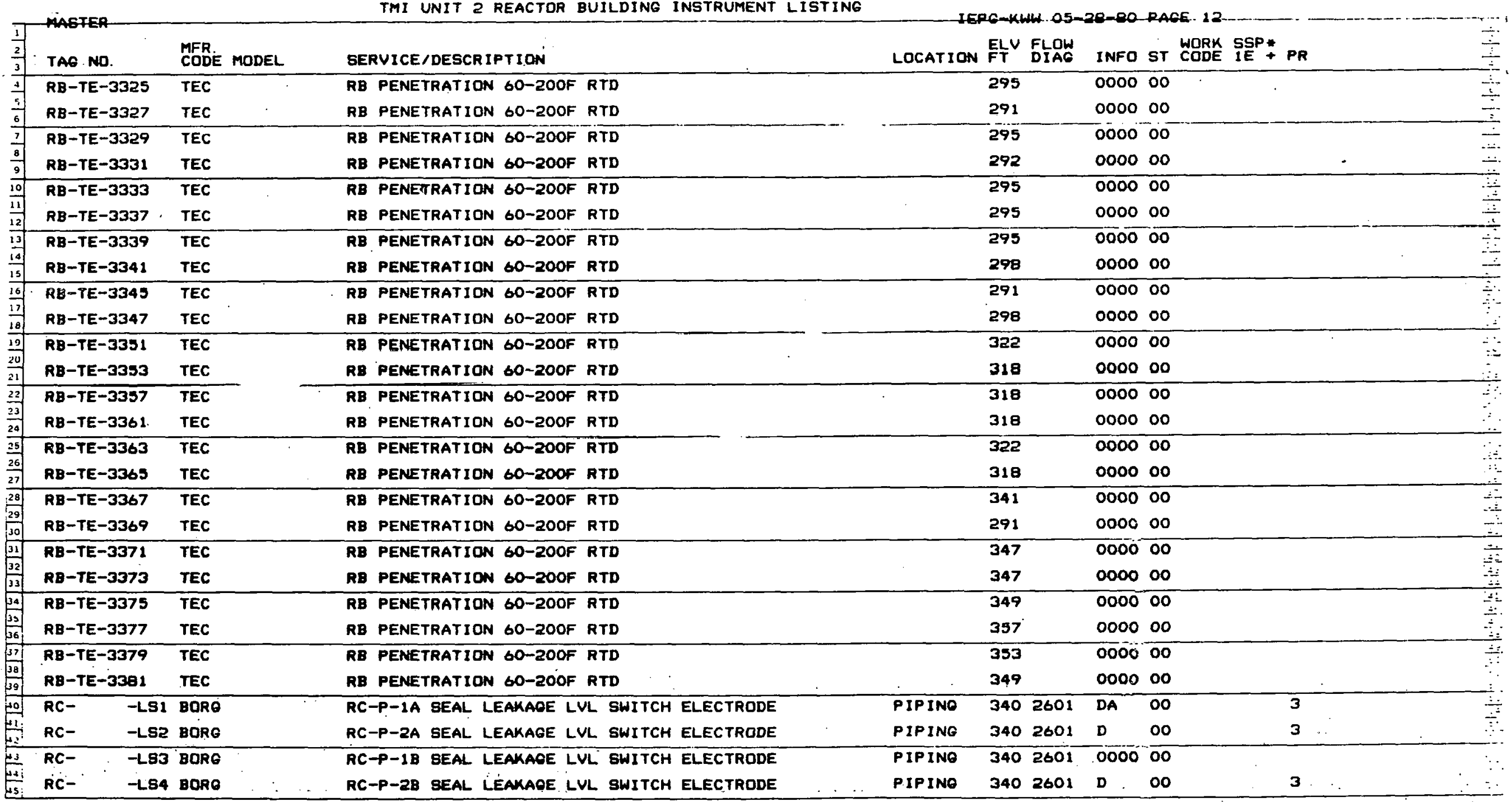


Appendix 5 (Cont'd)

TMI UNIT 2 REACTOR BUILDING INSTRUMENT LISTING

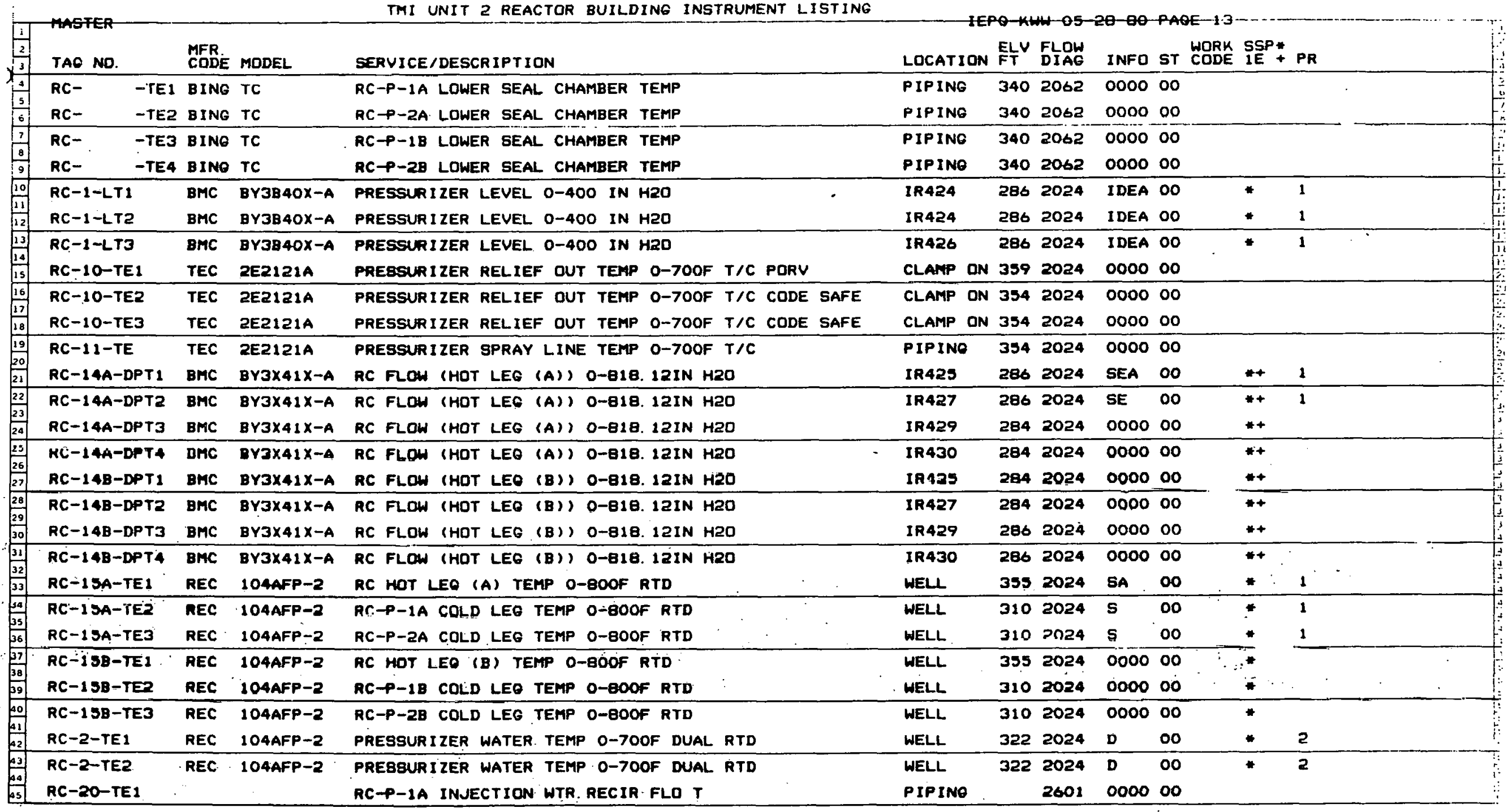


TMI UNIT 2 REACTOR BUILDING INSTRUMENT LISTING

\begin{tabular}{|c|c|c|c|c|c|c|c|c|c|c|c|c|}
\hline 2 & TAQ NO. & $\begin{array}{l}\text { MFR. } \\
\text { CODE }\end{array}$ & MODEL & GERVICE/DESCRIPTION & LOCATION & ELV & $\begin{array}{l}\text { FLOW } \\
\text { DIAG }\end{array}$ & INFO & ST & $\begin{array}{l}\text { WORK SSP* } \\
\text { CODE IE - }\end{array}$ & PR & \\
\hline 4 & RC-20-TE2 & & & RC-P-2A INJECTION WTR. RECIR FLD T & PIPING & & 2601 & onon & no & & & \\
\hline & HL-ZU-TEO & & & RC-H-I I INJECTION WTR. RECIR FLO T & PIP ING & & 2601 & 0000 & $\infty$ & & & \\
\hline 교 & RC-20-TE4 & & & RC-P-2B INJECTION WTR. RECIR FLO T & PIPINO & & 2601 & 0000 & 00 & & & ; \\
\hline$\frac{8}{9}$ & RC-21-TEL & & & RC-P-IA INU WTR FLO UP BL CHBR OT & PIPINO & & 2601 & 0000 & 00 & & & \\
\hline .0 & RC-21-TE2 & & & RC-P-2A"INJ WTR FLO UP SL CHBR OT & PIPINE & & 2601 & 0000 & 00 & & & 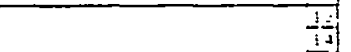 \\
\hline$-\frac{1}{2}$ & RC-21-TE3 & & & RC-P-1B INJ WTR FLO UP GL CHBR NT & PITINO & & 2601 & 0000 & oo & & & \\
\hline .3. & RC-21-TE4 & & & RC-P-2B INJ WTR FLO UP SL CHBR OT & PIPING & & 2601 & 0000 & 00 & & & $\therefore$ \\
\hline a) & $\mathrm{RC}-22-\mathrm{PT} 1$ & Fox & E11OH-INMR & RC-P-1A INJ WTR FLO UP SL CHBR IN 0-2500 PSIO & IR426 & 287 & 2024 & 0000 & $\infty$ & & & \\
\hline 6 & RC-22-PT2 & FOX & E12GH-INM2 & RC-P-2A INJ WTR FLO UP SL CHBR IN $0-2500$ PSIC & IR426 & 287 & 2024 & 0000 & 00 & & & $=$ \\
\hline : & RC-22-PT3 & FOX & E11GH-INM2 & RC-P-1B INJ WTR FLO UP SL CHBR IN $0-2500$ PSIG & IR42B & 287 & 2024 & 0000 & 00 & & & \\
\hline g) & RC-22-PT4 & FOX & E11OH-INH2 & KL-P-28 INJ WTR FLO UP BL CHBR IN 0-2500 P6IG & 18428 & 287 & 2024 & 0000 & 00 & & & \\
\hline 1) & RC-22-PTS & rux & EIIQH-RAEI & $\overline{R C}=P-1 A$ INJ WTR FLO MID BL IN PRE O-ZSOOU PGIG & IR424 & 286 & 2024 & 0000 & $\mathbf{0 0}$ & & & \\
\hline 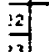 & $R C-22-P T 6$ & Fax & E110H-5AE1 & RC-P-2A INJ WTR FLO MID SL IN PRE 0-2500 PSIO & IR425 & 287 & 2024 & 0000 & 00 & & & \\
\hline$\frac{3}{24}$ & $R C-22-P T 7$ & FOX & E11OH-SAE1 & RC-P-1B INJ WTR FLO MID SL IN PRE 0-2500 PSIO & IR429 & 286 & 2024 & 0000 & 00 & & & $\because$ \\
\hline 5 & RC-22 $\Rightarrow P$ TO & Fox & C110H-8AE1 & RC-P=28 INJ WIR FLD MID SL IN PRE $0-2500$ PSIG & IR430 & 288 & 2024 & 0000 & oo & & & 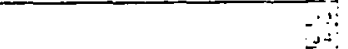 \\
\hline & RC-3A-PT1 & REC & $11520 p 9 A$ & RC HOT LEQ(A) PRES3-NARROW RANOE 1700-2500 PSIO & IR425 & 286 & 2024 & ISEA & 00 & + & 1 & : \\
\hline 38] & RC-3A-PT2 & REC & $11520 P 9 A$ & RC HOT LEG (A) PRESS-NARROW RANGE $1700-2500$ PSIO & IR427 & 286 & 2024 & ISE & 00 & + & 1 & 20 \\
\hline & RC-3A-PT3 & Fox & E11OH-INM2 & RC HOT LEG(A) PRESS-WIDE RANGE 0-2500 PSIO & IR425 & 287 & 2024 & SEA & $\infty$ & + & 1 & \\
\hline & RC-3A-PT4 & Fox & EI1OH-INMS & RC HOT LEQ(A) PRESS-WIDE RANOE O-2500 PSIO & IR427 & 287 & 2024 & SE & 00 & + & 1 & 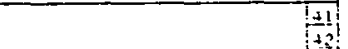 \\
\hline & RC-3A-PTS & Fox & EI1OH-INM2 & RC HOT LEO(A) PRESB-LOW RANOE O-500 PSIO & IR429 & 287 & 2024 & 0000 & oo & & & 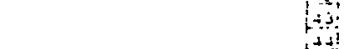 \\
\hline & RC-3B-PTI & REC & $11520 P 9 A$ & RC HOT LEO (B) PRESS NARROW RANCE $1700-2500$ PSIG & IR429 & 287 & 2024 & SE & 00 & + & 1 & +2 \\
\hline & RC-3B-PTZ & REC & $11520 \mathrm{P9A}$ & RC HOT LEG (B) PRESS NARRDW RAMGE 1700-2500 PSIG & IR430 & 286 & 2024 & 0000 & 00 & + & & ?. \\
\hline & RC-38-PT3 & FOX & E11OH-INM2 & RC HOT LEQ (B) PRESS WIDE RANGE 0-2500 PSIG & IR429 & 287 & 2024 & 0000 & 00 & + & & 19 \\
\hline & RC $\triangle 4 A-T E 1$ & REC & $177 H W-2$ & RC MOT LEO (A) TEMP 320-620F DUAL RTD & PIPINO & .952 & 2024 & SA & -o & *t & 1 & $\because \because$ \\
\hline & RL-4A-TE2 & REC & $177 H W-2$ & RC HDT LEQ (A) TEMP 520-620F DUAL RTD & PIPING & 352 & 2024 & SA & 00 & *t+ & 1 & 望 \\
\hline & RC- $4 A-T E 3$ & REC & $177 H W-2$ & RC HOT LEQ (A) TEMP' S20-62OF DUAL RTD & PIPING & 352 & 2024 & $\mathbf{s}$ & 00 & $\#+$ & 1 & 1 \\
\hline & RC-4A-TEA & REC & $177 H W-2$ & RC HOT LEQ (A) TEMP 520-620F DUAL RTD & PIPING & 352 & 2024 & $\mathbf{s}$ & 00 & *+ & 1 & $\therefore 1$ \\
\hline & RC-4B-TE1 & REC & $177 H W-2$ & RC HOT LEO (B) TEMP S20-62OF DUN RTD & PIPINO & 352 & 2024 & SA & $\infty 0$ & $*$ & 1 & : \\
\hline
\end{tabular}

$\leftarrow$
$\dot{0}$
$\stackrel{0}{o}$ 
TMI UNIT 2 REACTOR BUILDING INSTRUMENT LISTING

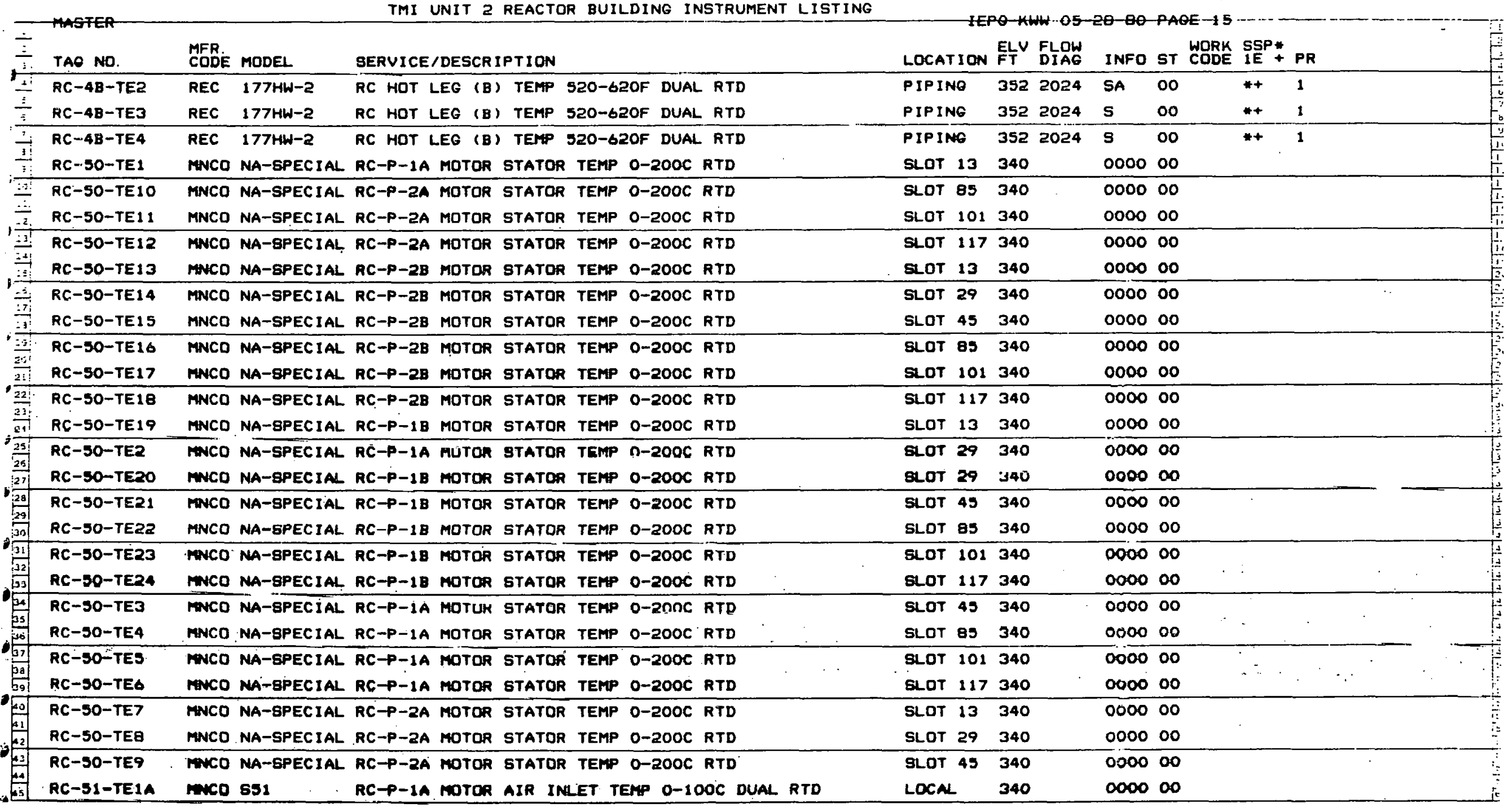


TMI UNIT 2 REACTOR BUILDING INSTRUMENT LISTING

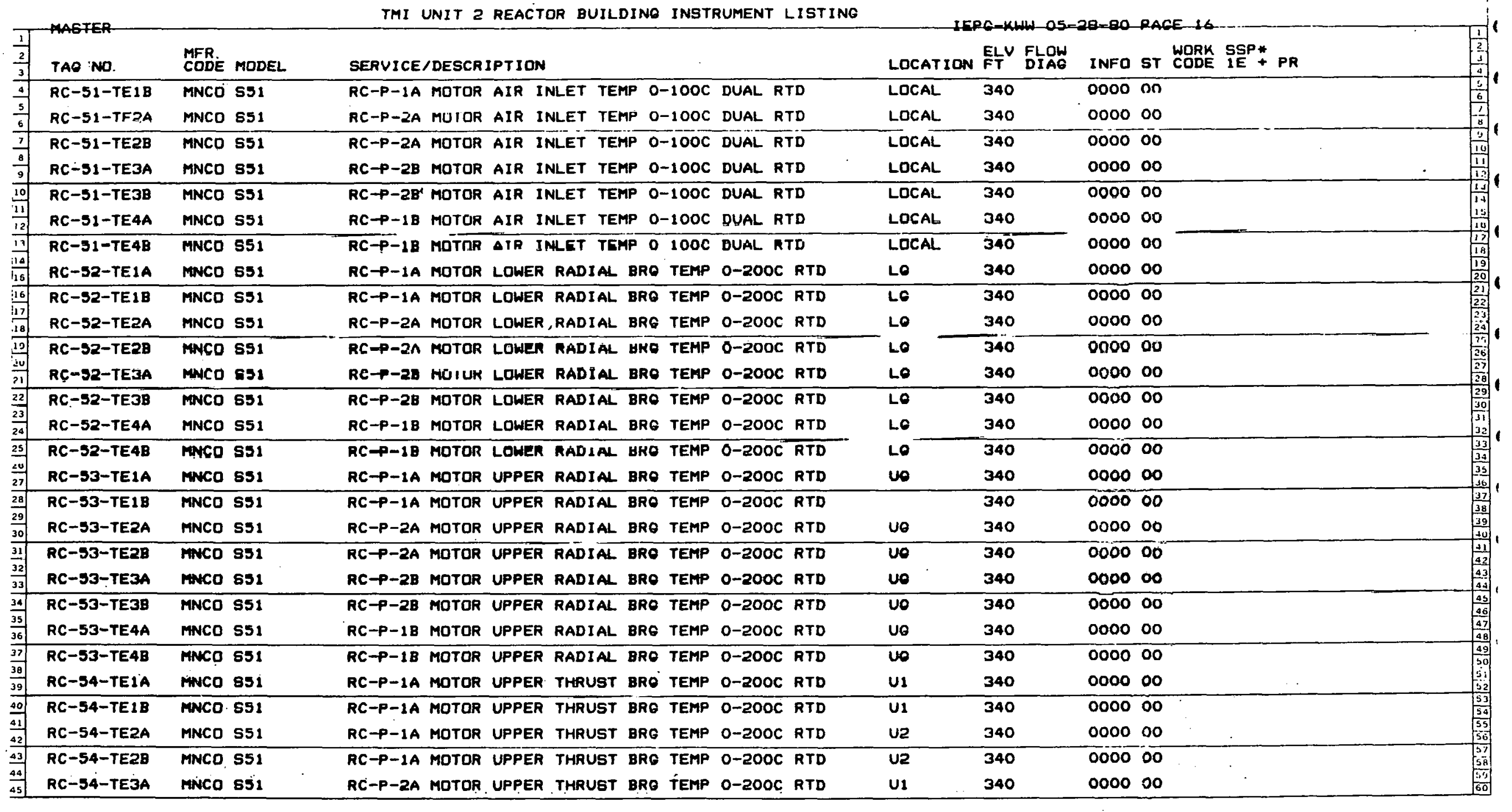


Appendix 5 (Cont'd)

1
0
1
$\infty$
0 


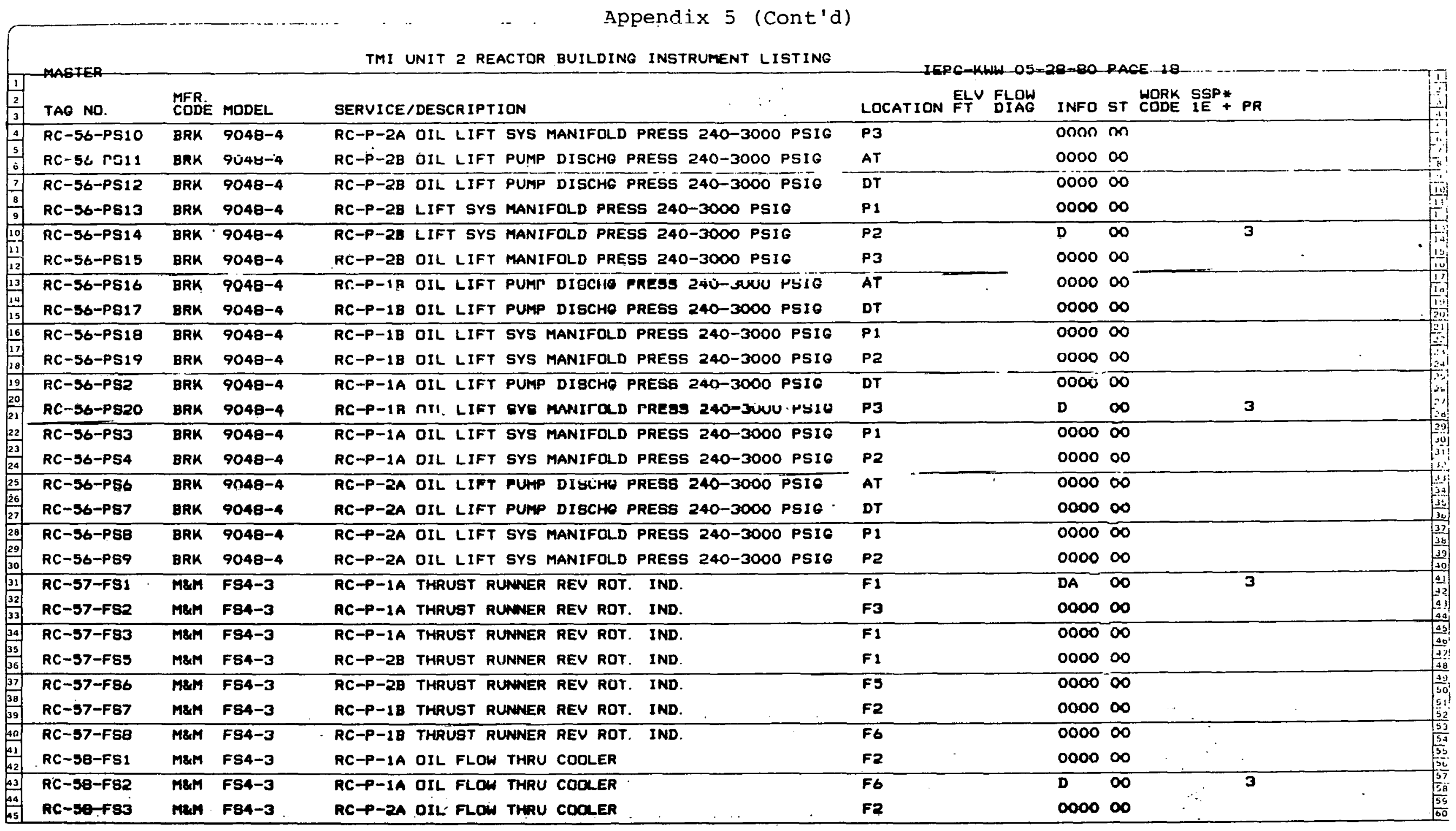

$:$
$\dot{0}$
1 
TMI UNIT 2 REACTOR BUILDING INSTRUMENT LISTING

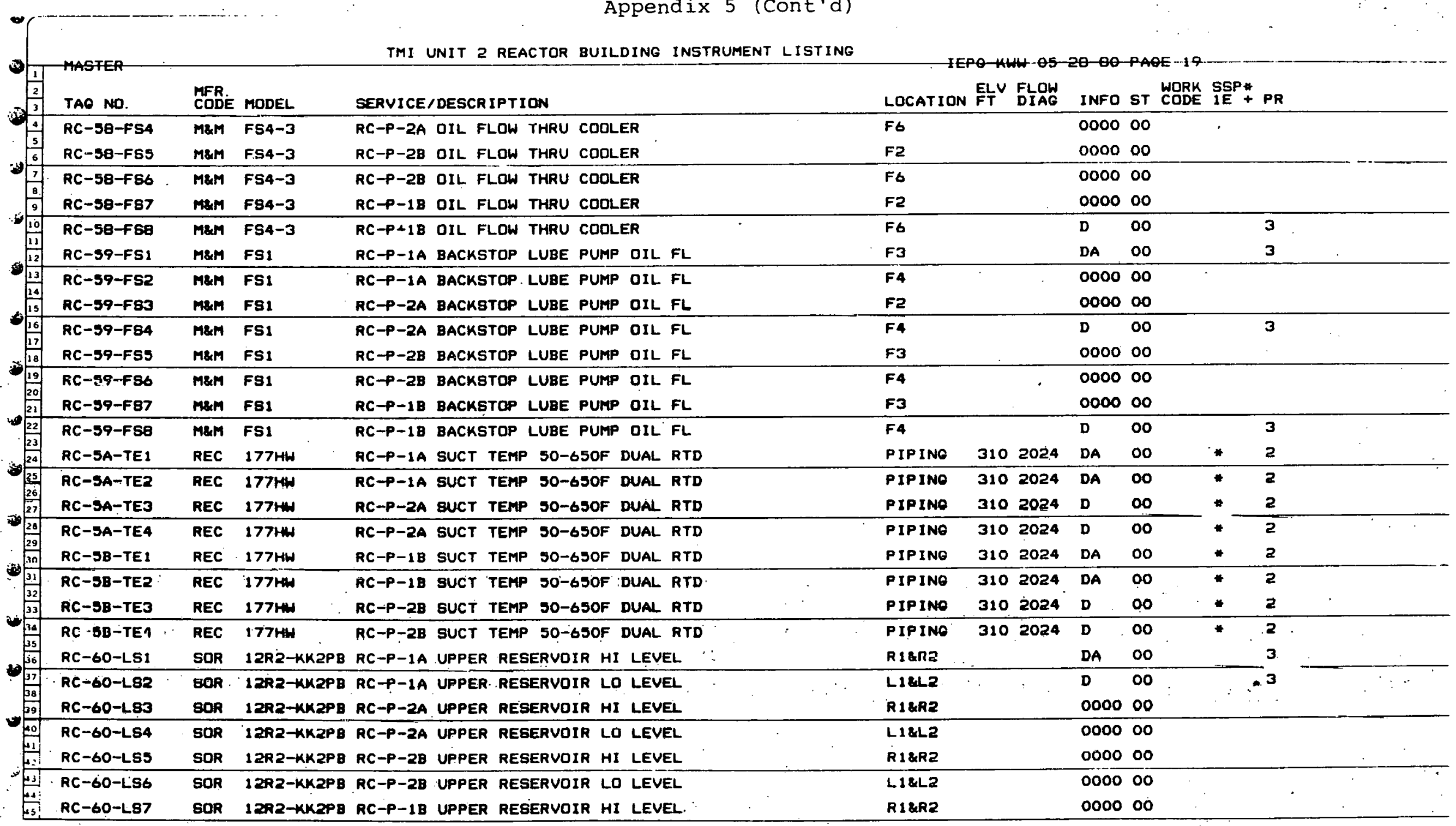


TMI UNIT 2 REACTOR BUILDING INSTRUMENT LISTING

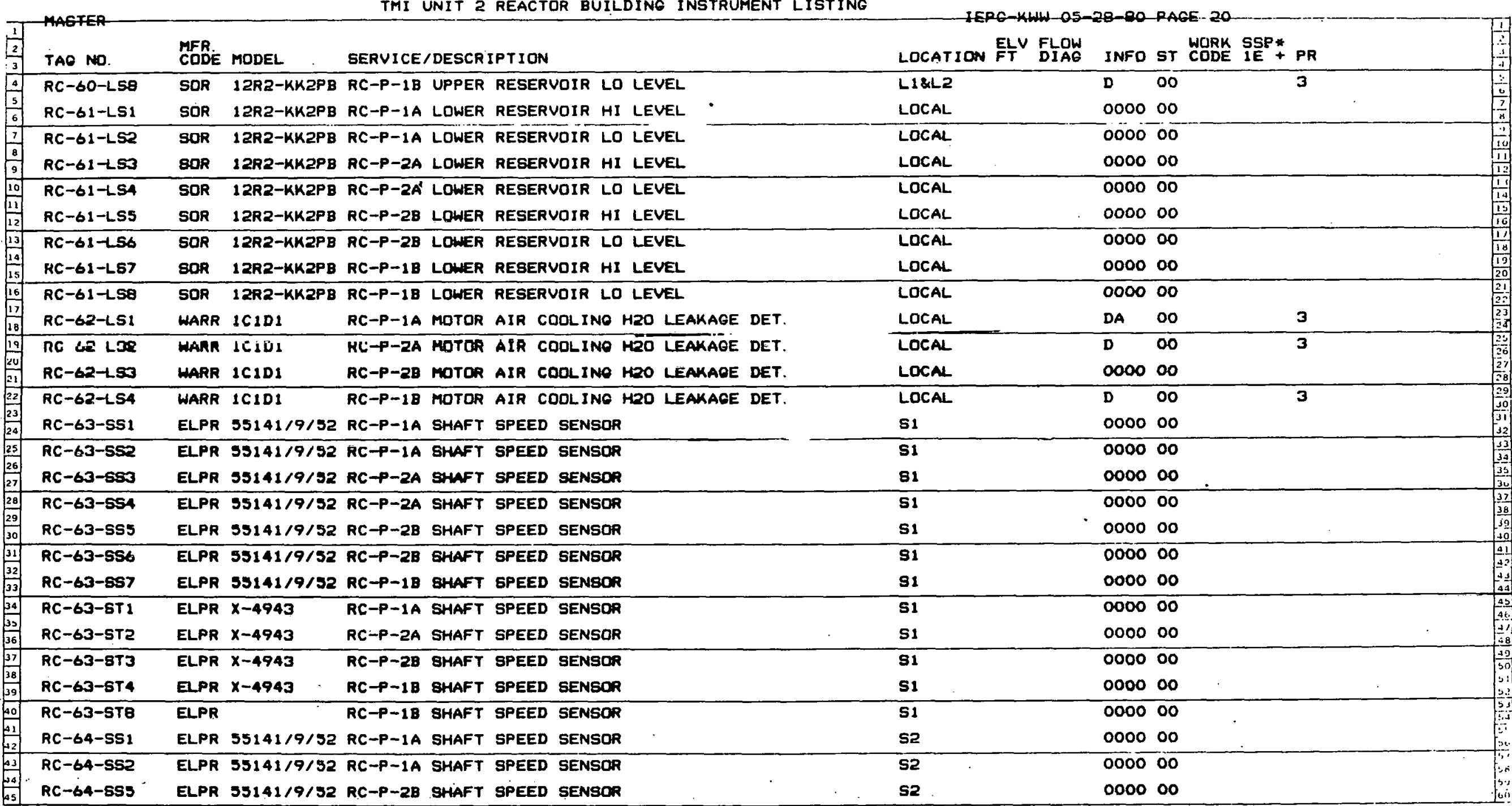




\section{Appendix 5 (Cont'd)}

TMI UNIT 2 REACTOR BUILDING INSTRUMENT LISTING

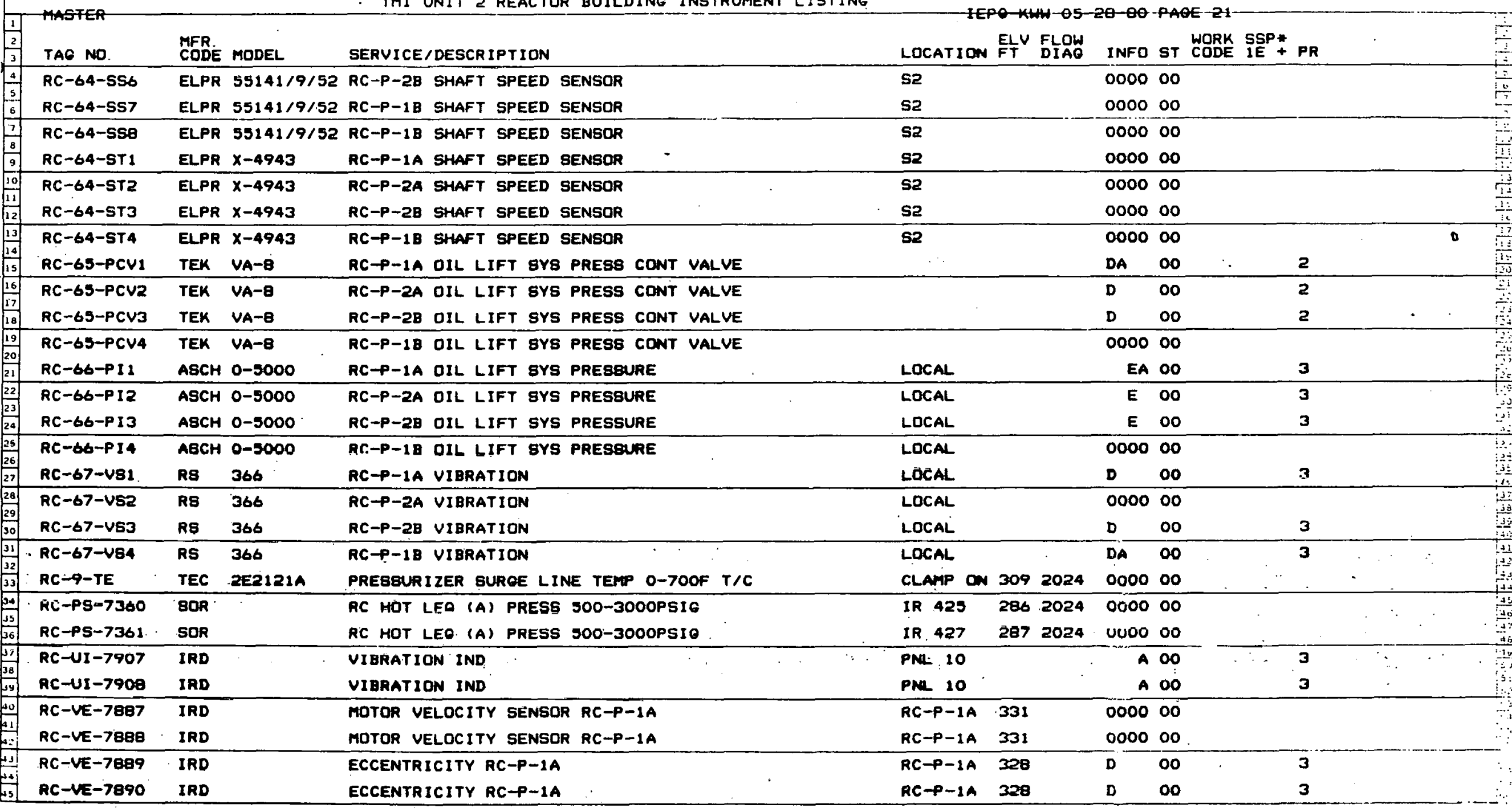

0
0
1
$\omega$ 


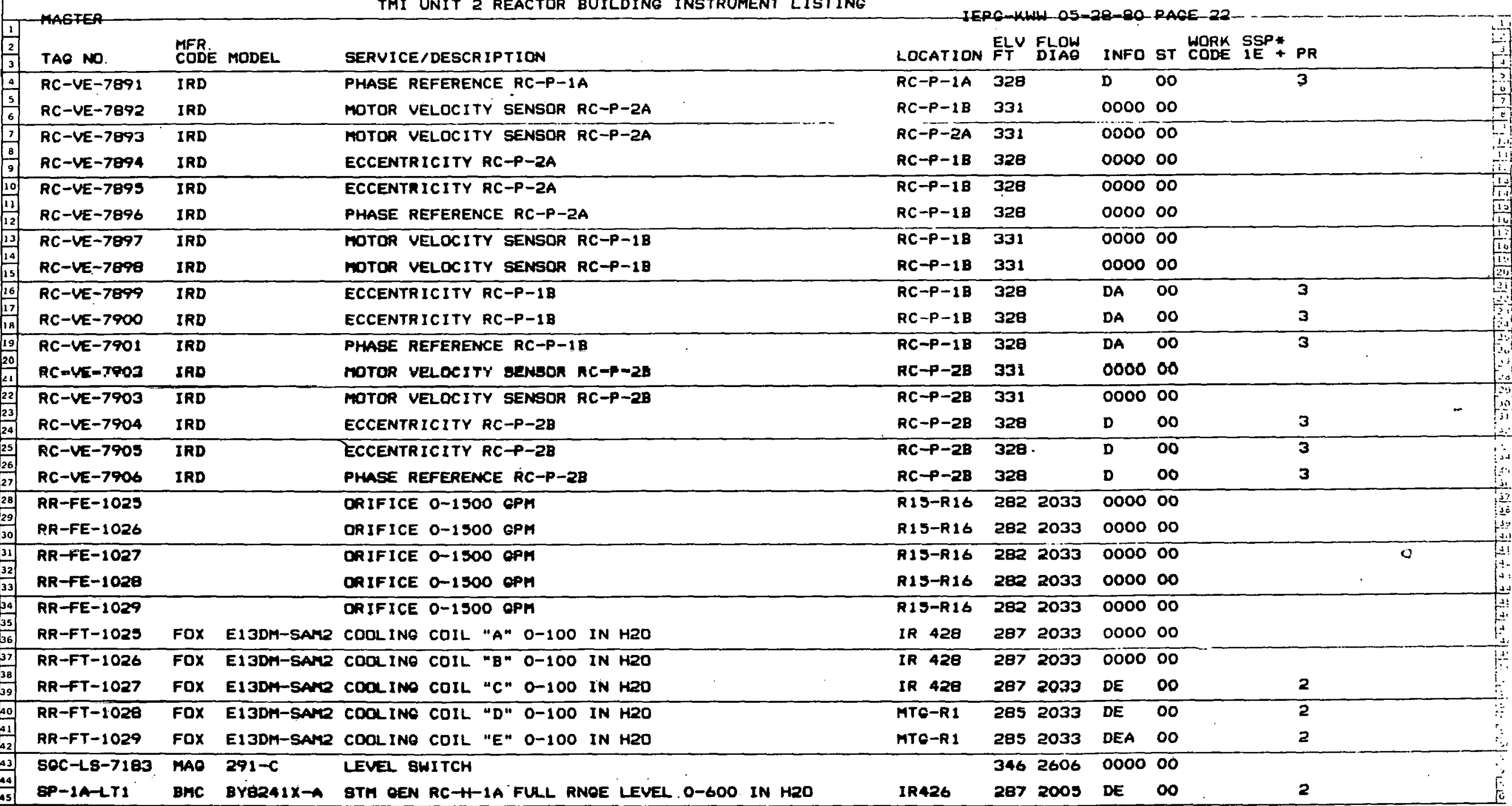


TMI UNIT 2 REACTOR BUILDING INSTRUMENT LISTING

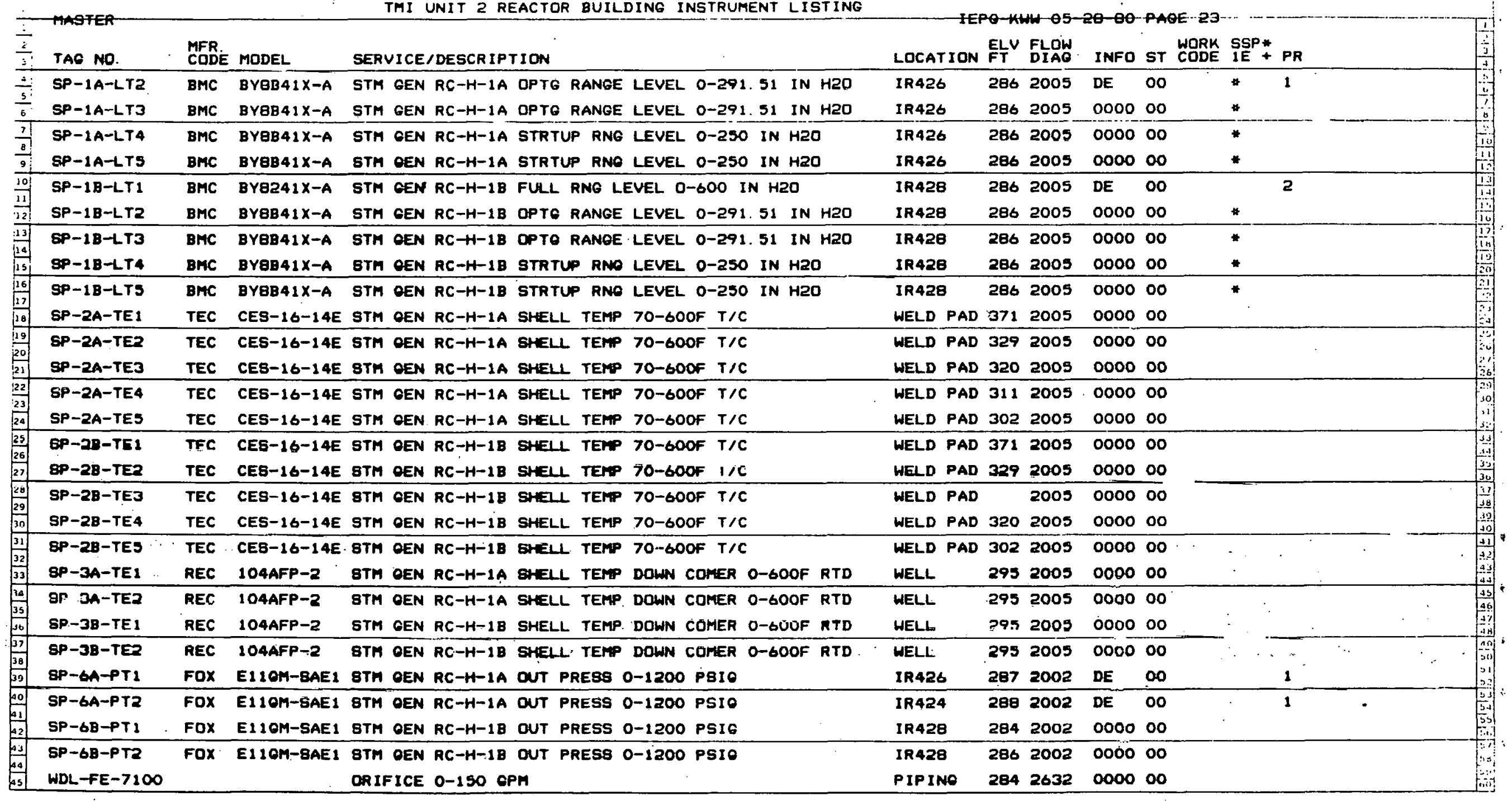




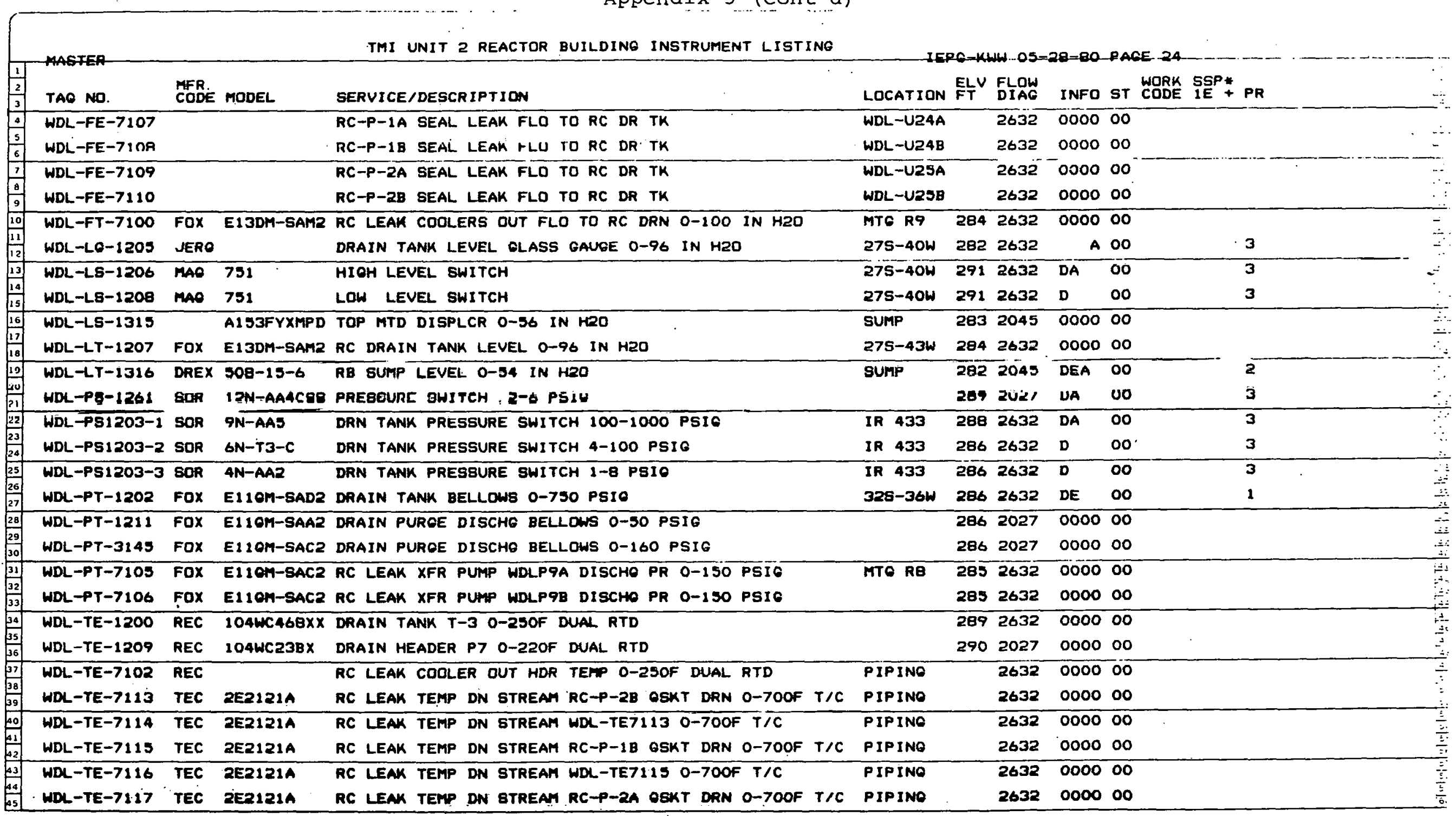


Appendix: 5 (Cont'd)

TMI UNIT 2 REACTOR BUILDING INSTRUMENT LISTING

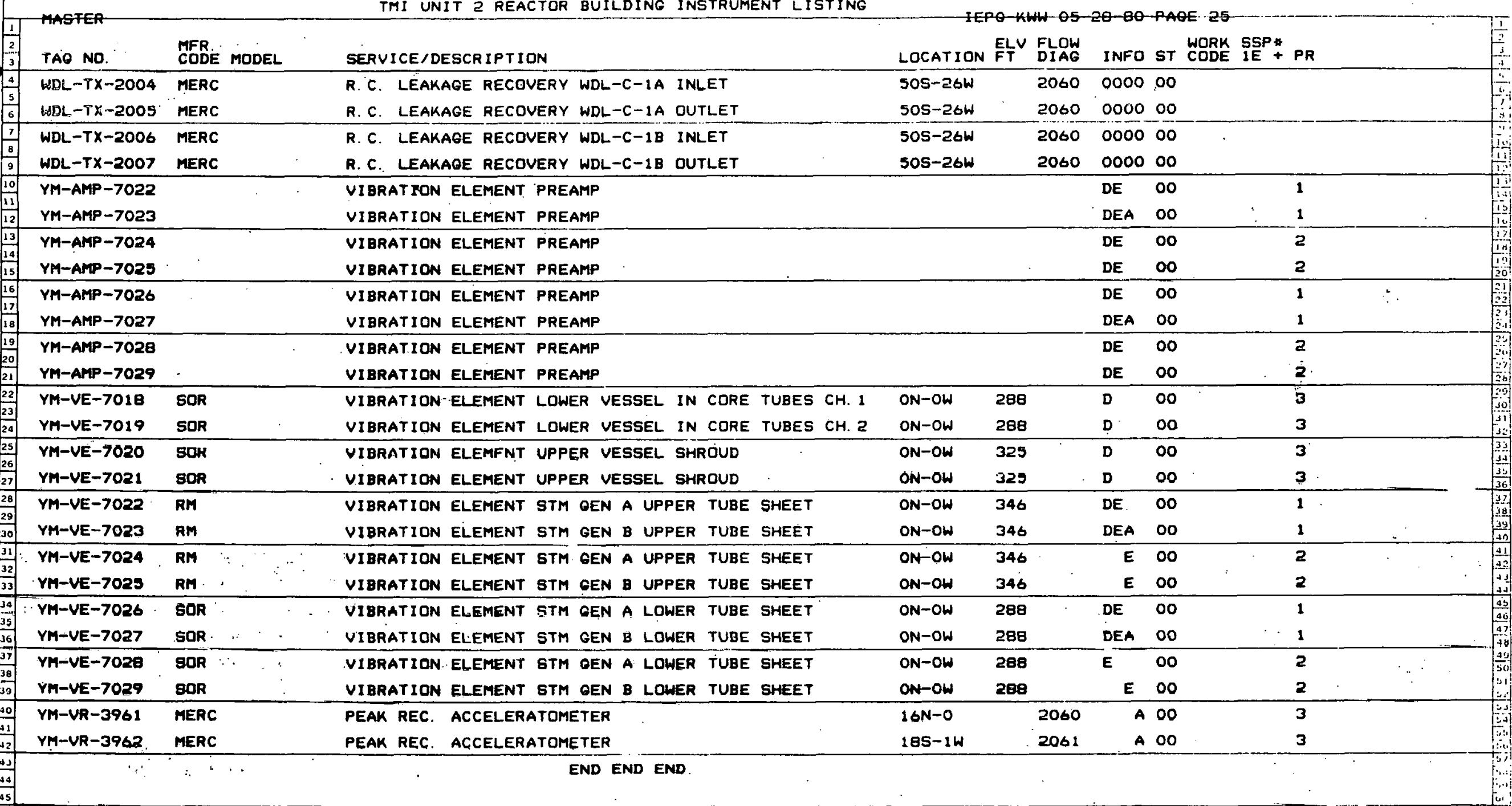




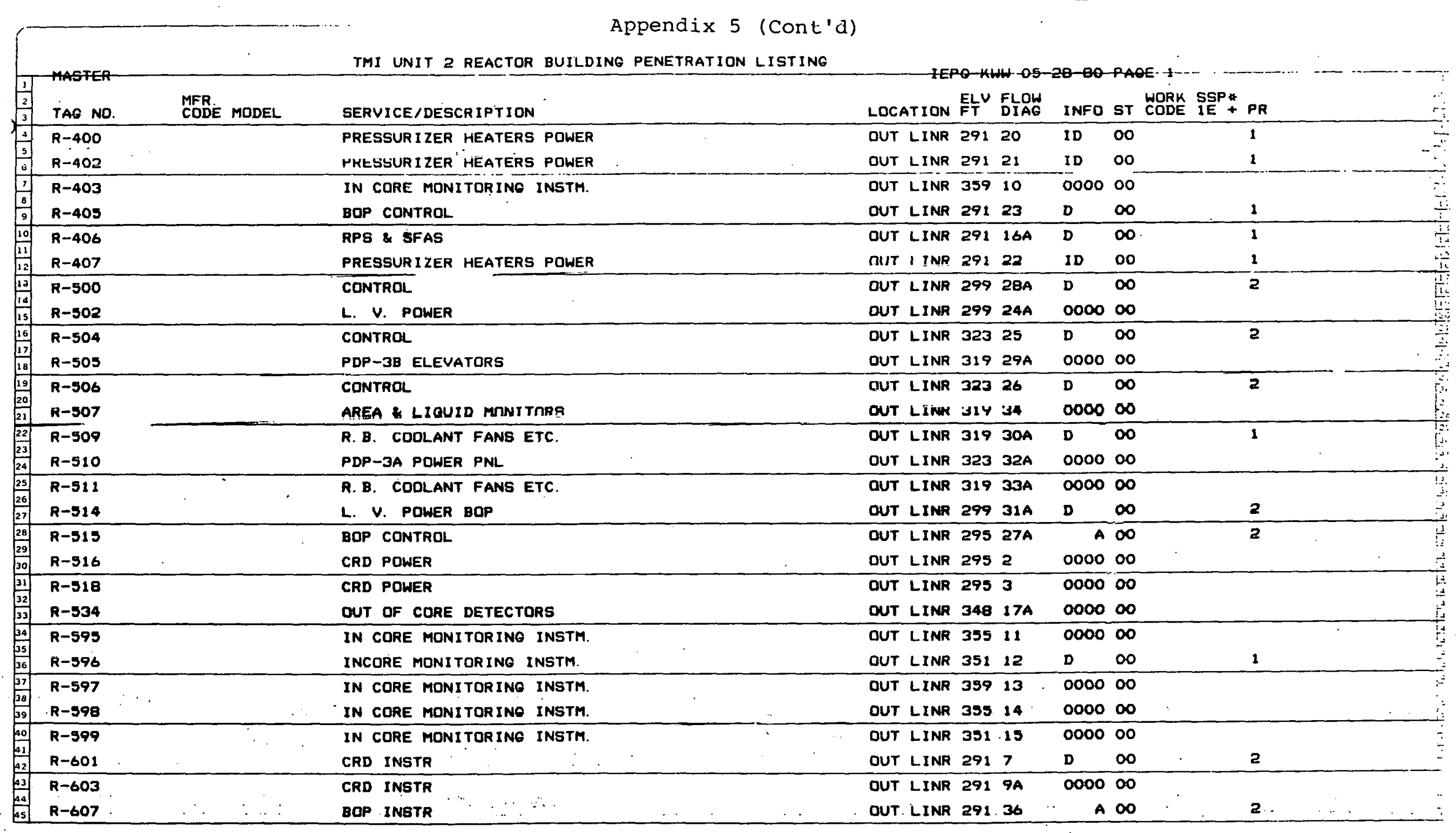

5
$\dot{0}$
$\dot{0}$ 


\section{Appendix 5 (Cont'd)}

TMI UNIT 2 REACTOR BUILDING PENETRATION LISTING

\begin{tabular}{|l|l|}
\cline { 1 - 1 } 1 & MASTER \\
\cline { 1 - 1 } 3 & TAQ NO \\
\cline { 1 - 1 } 5 & $\mathrm{R}-608$ \\
\hline 6 & $\mathrm{R}-610$ \\
\hline & $\mathrm{R}-611$ \\
$\mathrm{R}-612$
\end{tabular}

15 .15

$1 \frac{18}{19}$

29

31

24

í
IEPG- O5-28-PO PACE-3

LOCATION FLV FLOW ING INFO ST WODE SSP + PR

OUT LINR 29935 D 00

OUT LINR 292 19A. 000000

QUT LINR 29935000000

OUT LINR 29235 D 00
RC-P-2B FEEDER

RPS \& SFAS

BPARE

END END END 


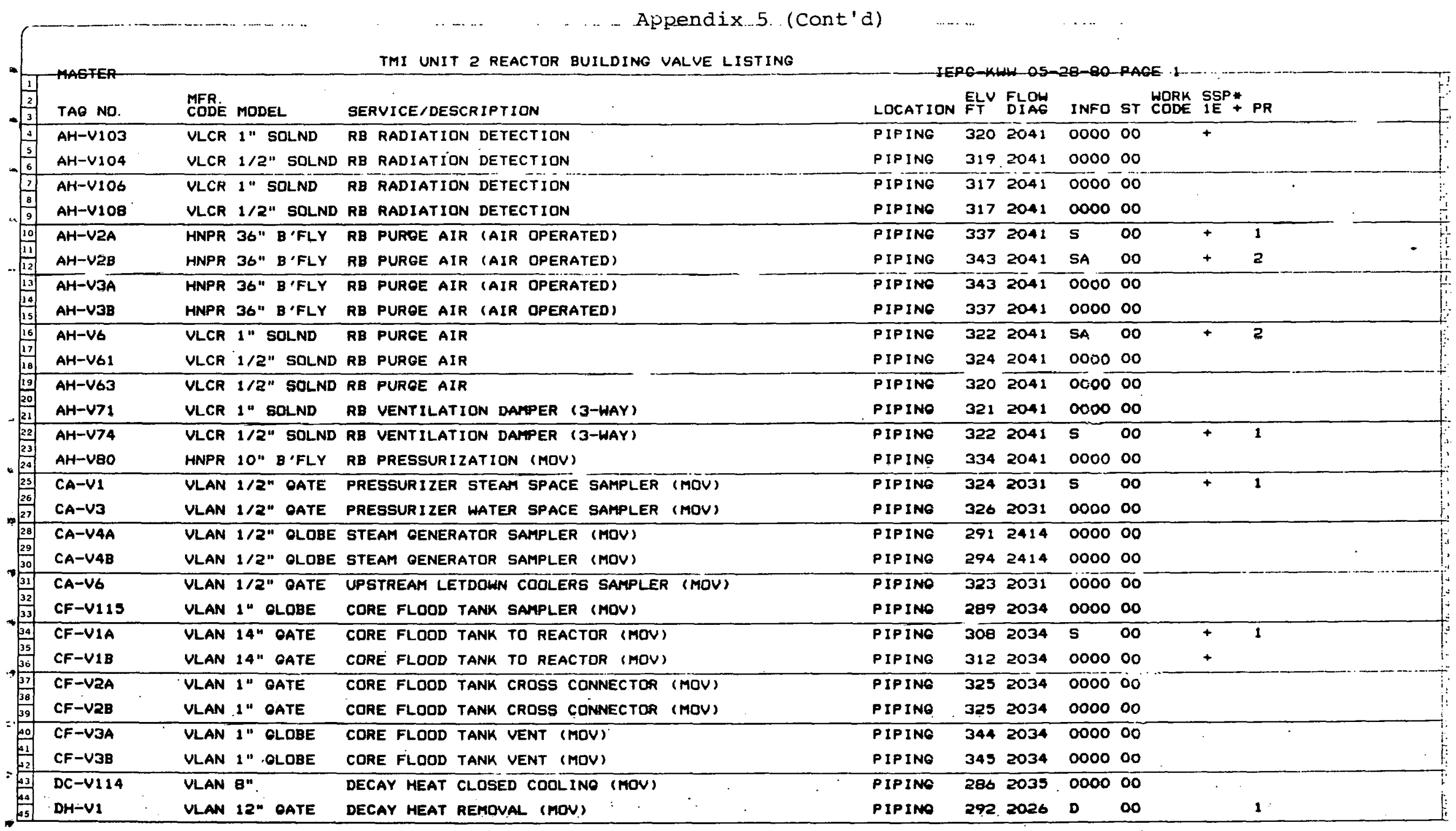




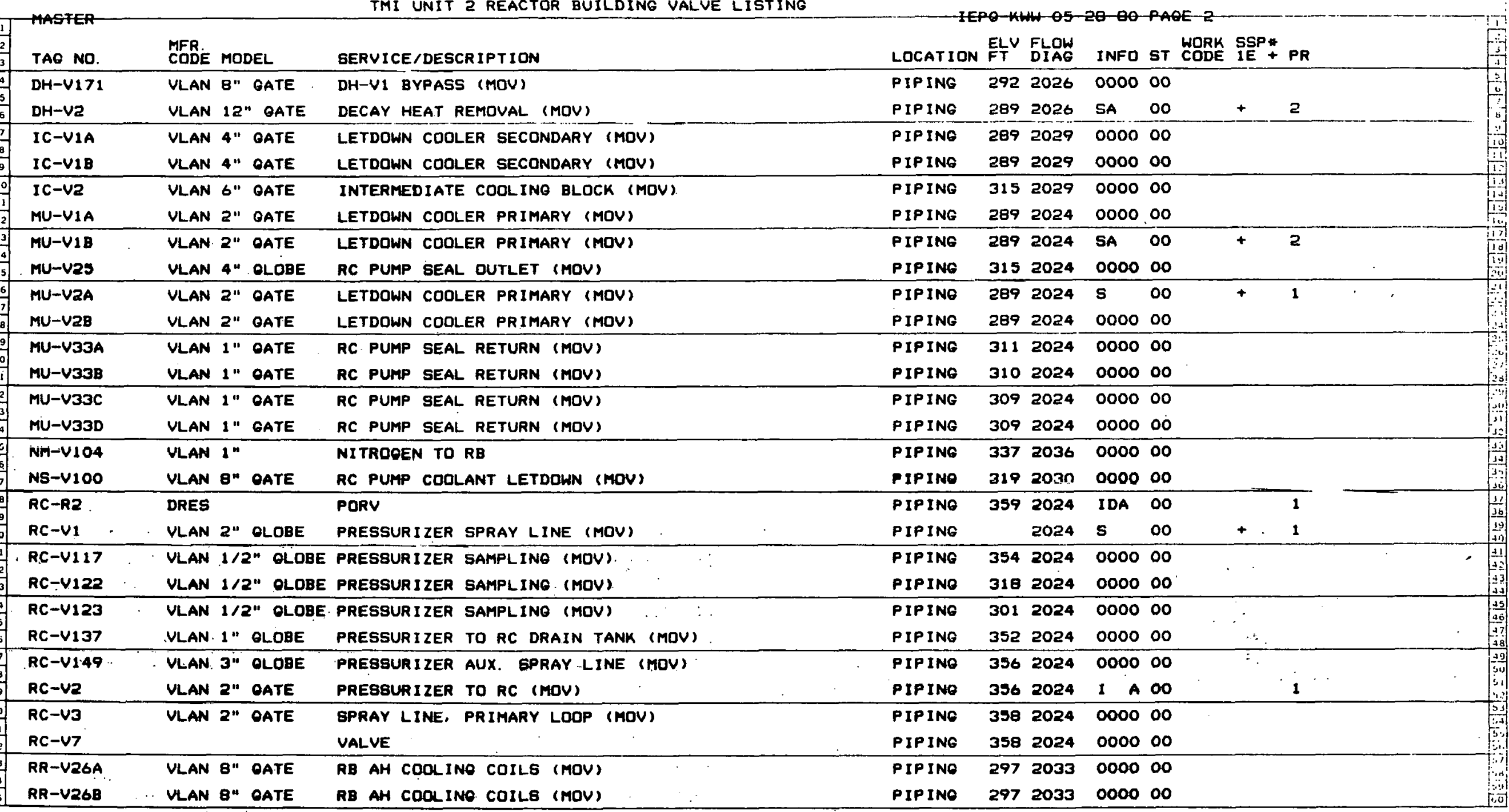




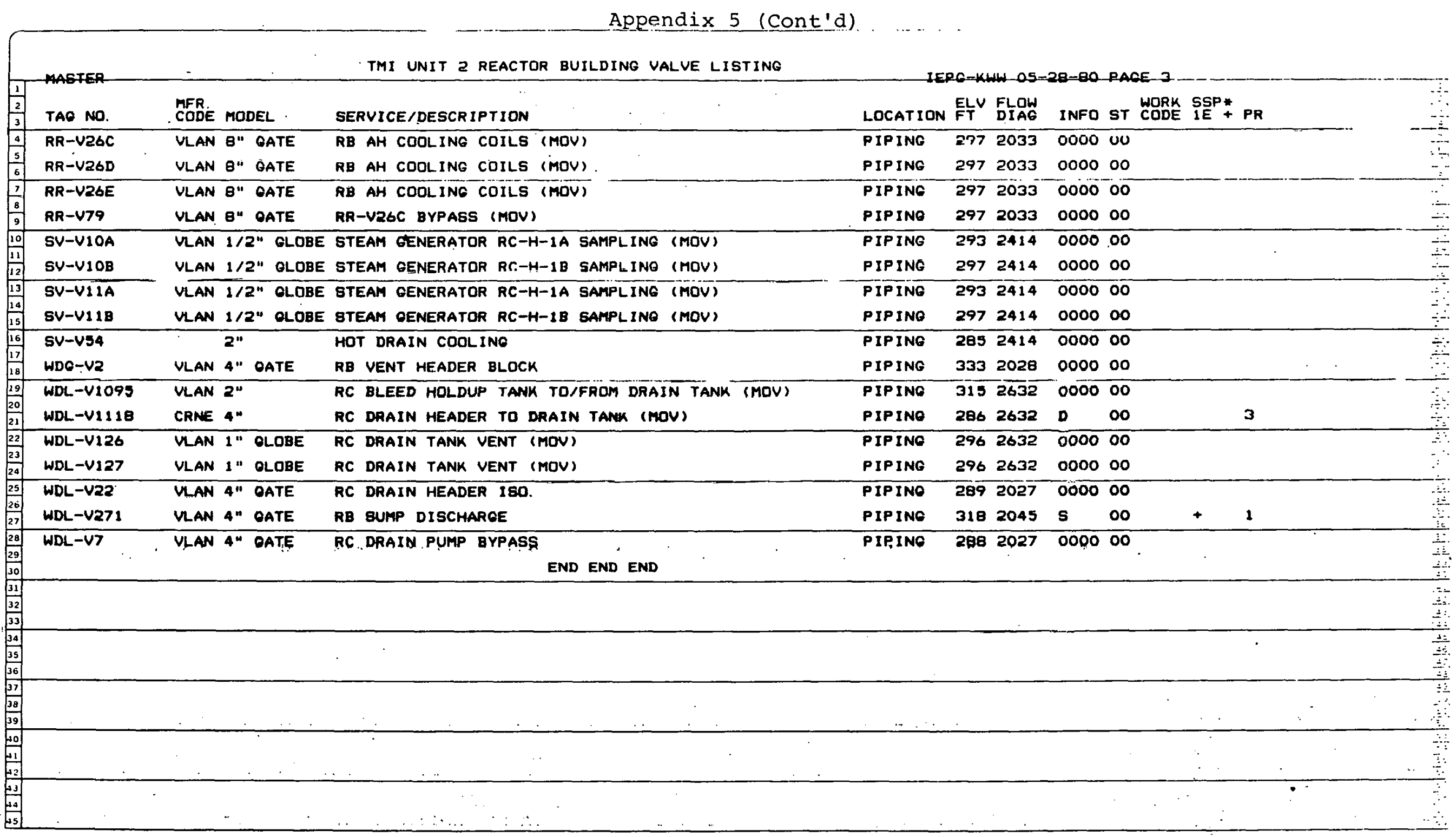

-
0
1
N 
Appendix 5 (Cont'd)

TMI UNIT 2 REACTOR BUILDING ELECTRICAL EQUIPMENT LISTING

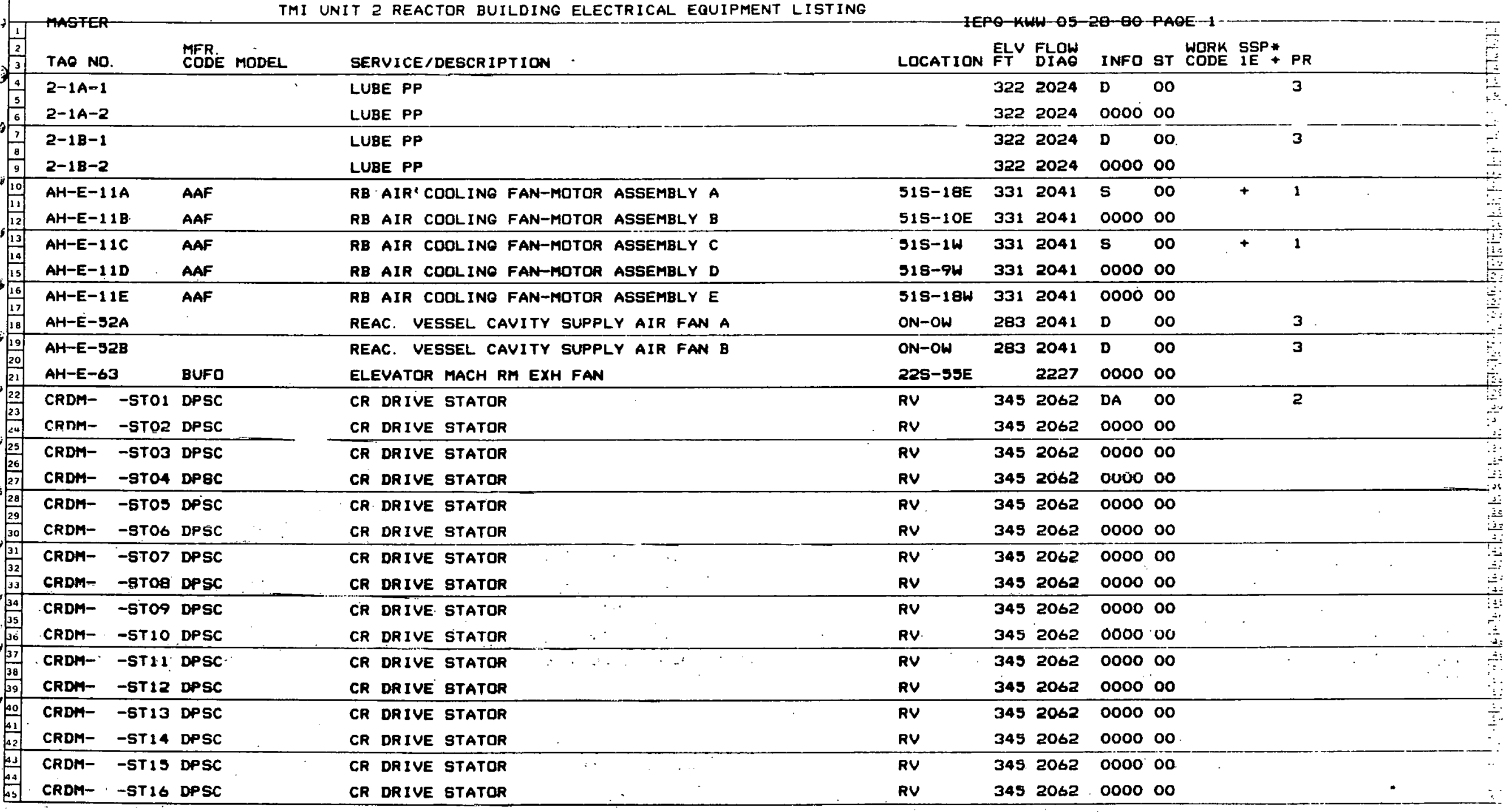


Appendix 5 (Cont'd)

TMI UNIT \& REACTOR BUILDING ELECTRICAL EOUIPMENT LISTING

W MATER

TAG NO. MFR. MODEL SERUICE/DESCRIPTION

1 CRDM- $-5 T 17$ DPSC

$\frac{5}{6}$ CRDM- $-5 T 18$ DPSC

, CRDM- -ST19 DPSC

I CRDM- -ST20 DPSC

CRDM- -ST21 DPSC

CRDM- -ST22 DPSC

CRDM- -5T23 DPSC

CRDM- -ST24 DPSC

CRDM- $-5 T 25$ DPSC

CRDM- -5T26 DPSC

- CRDM- -8T27 DPSC

20. CKLT- -5T2̌ DPBC

22 ORDH- -STKY DPSC

CRDM- -ST3O DPSC

CRDM- $-5 T 31$ DPSC

CKDH- -ST32 DPSC

CRDM- $-5 T 33$ DPSC

CRDM- -ST34 DPSC

1) CRDM- $-6 T 35$ DPSC

3 CRDM- -5 T36 DPSC

4) CRDM- $-5 T 37$ DPSC

CRDM- -ST3B DPSC

CRDM- -5T39 DPSC

CRDM- -ST4O DPSC

CRDM- -ST41 DPSC

CRDM- -ST42 DPSC

CRDM- -ST43 DPSC

CRDM- -6T44 DPSC
CR DRIVE STATOR

CR DRIVE STATOR

CR DRIVE STATOR

CR DRIVE STATOR

CR DRIVE STATOR

CR DRIVE STATOR

CR DRIVE STATOR

CR DRIVE STATOR

CR DRIVE STATOR

CR DRIVE STATOR

CR DRIVE STATOR

CR DRIVE STATOR

CR DRIVE STATOR

CR DRIVE STATOR

CR DRIVE STATOR

CR DRIVE GTATOR

CR DRIVE STATOR

CR DRIVE GTATOR

CR DRIVE STATOR

CR DRIVE STATOR

CR DRIVE STATOR

CR DRIVE STATOR

CR DRIVE STATOR

CR DRIVE STATOR

CR DRIVE STATOR

CR DRIVE STATOR

CR DRIVE STATOR

CR DRIVE STATOR

IEPG-KWW 05-20-00-PACE-

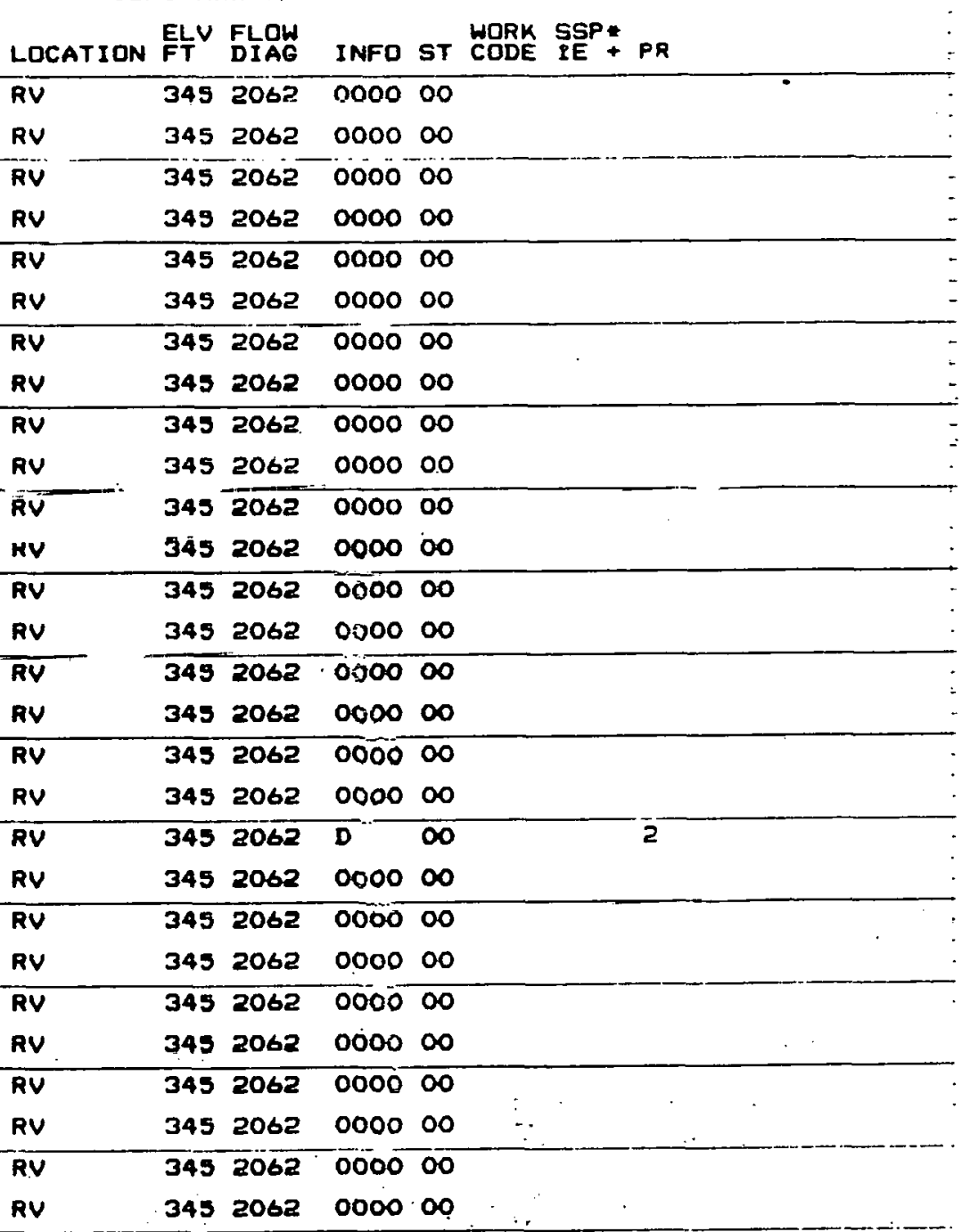

$\mapsto$
0
1
0 
Appendix 5 (Cont'd)

TMI UNIT 2 REACTOR BUILDING ELECTRICAL EQUIPMENT LISTING

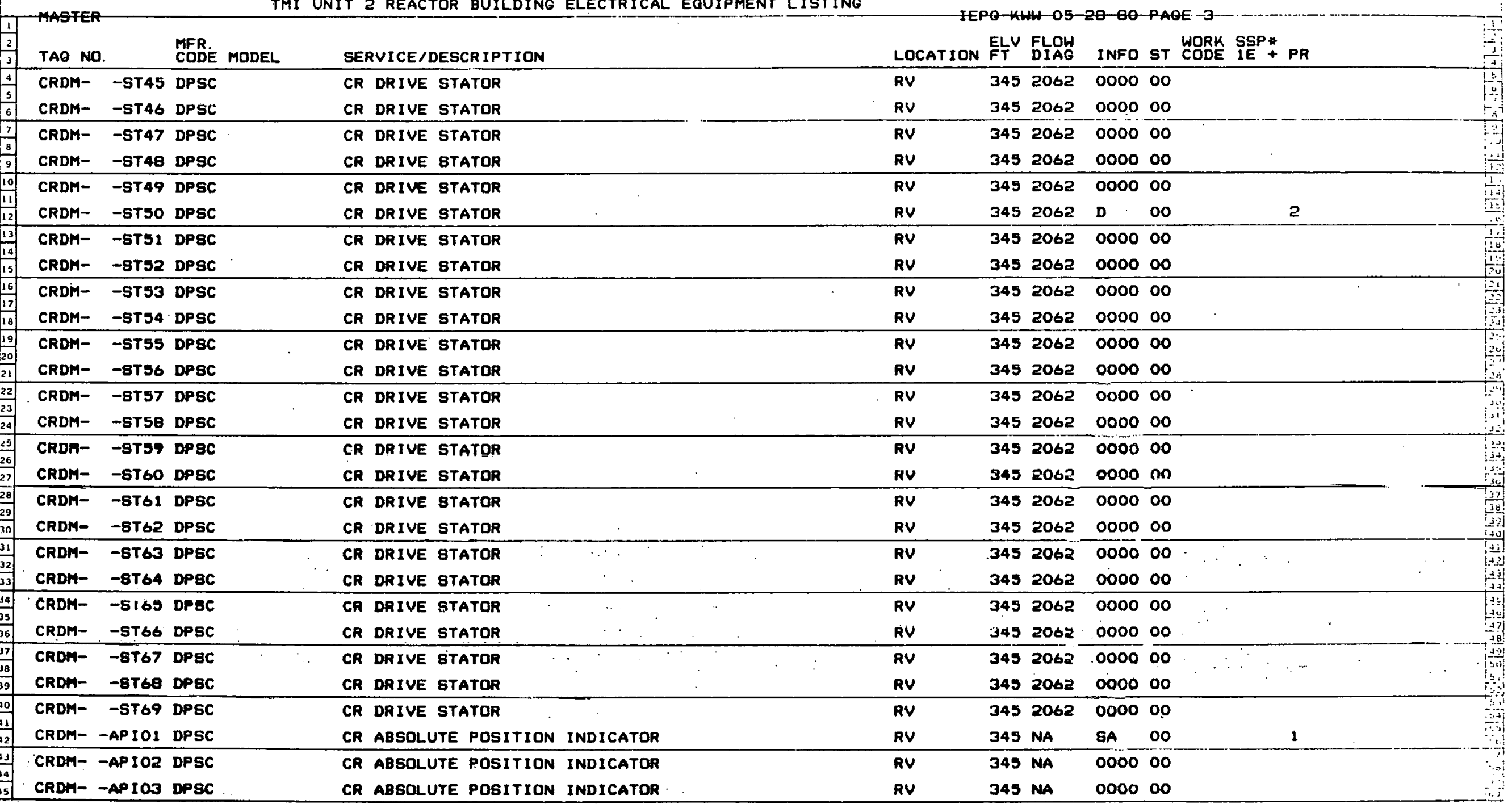


TMI UNIT 2 REACTOR BUILDING ELECTRICAL EQUIPMENT LISTING

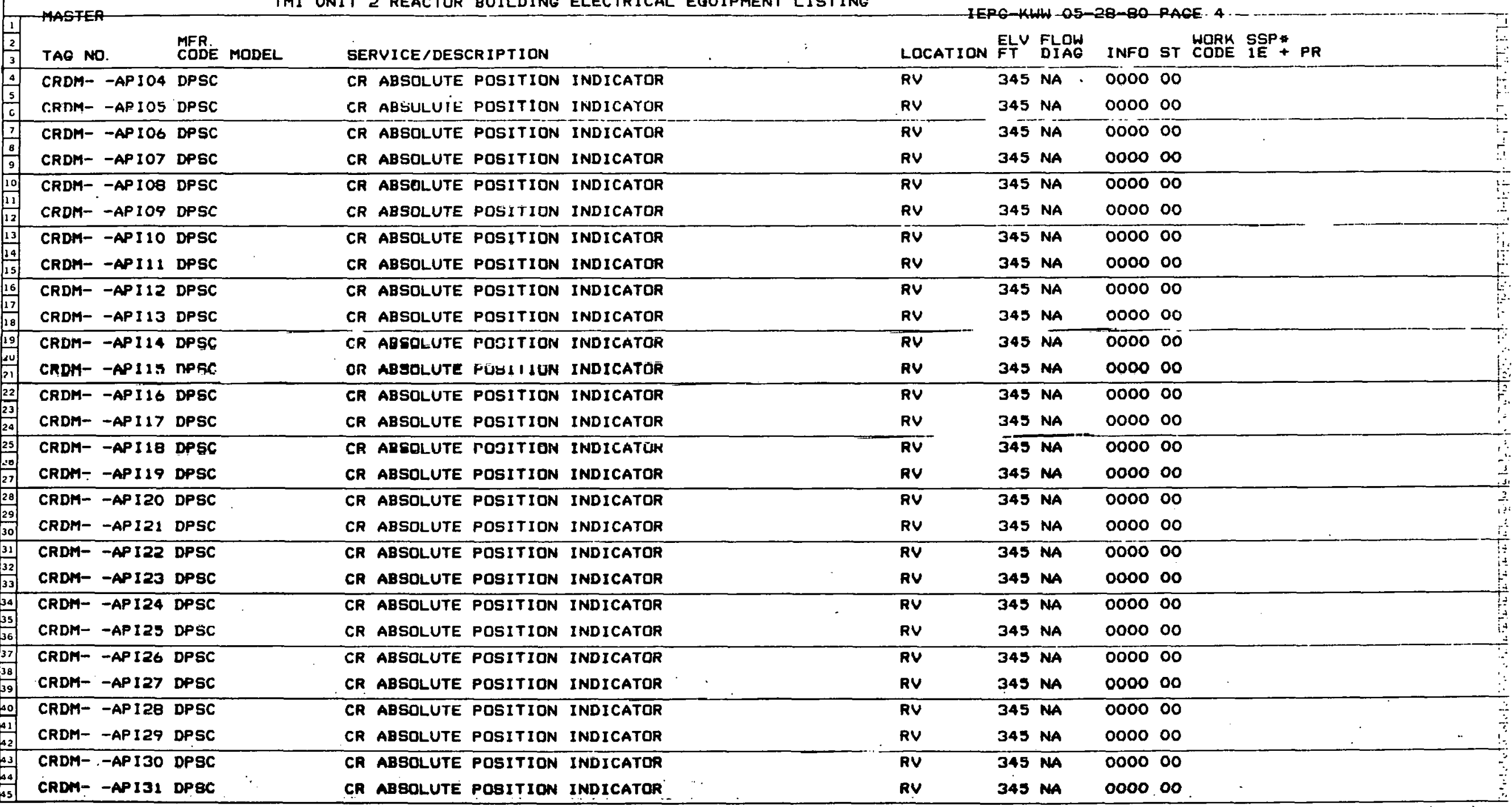

5
$\dot{0}$
5
8 
TMI UNIT 2 REACTOR BUILDING ELECTRICAL. EQUIPMENT LISTING

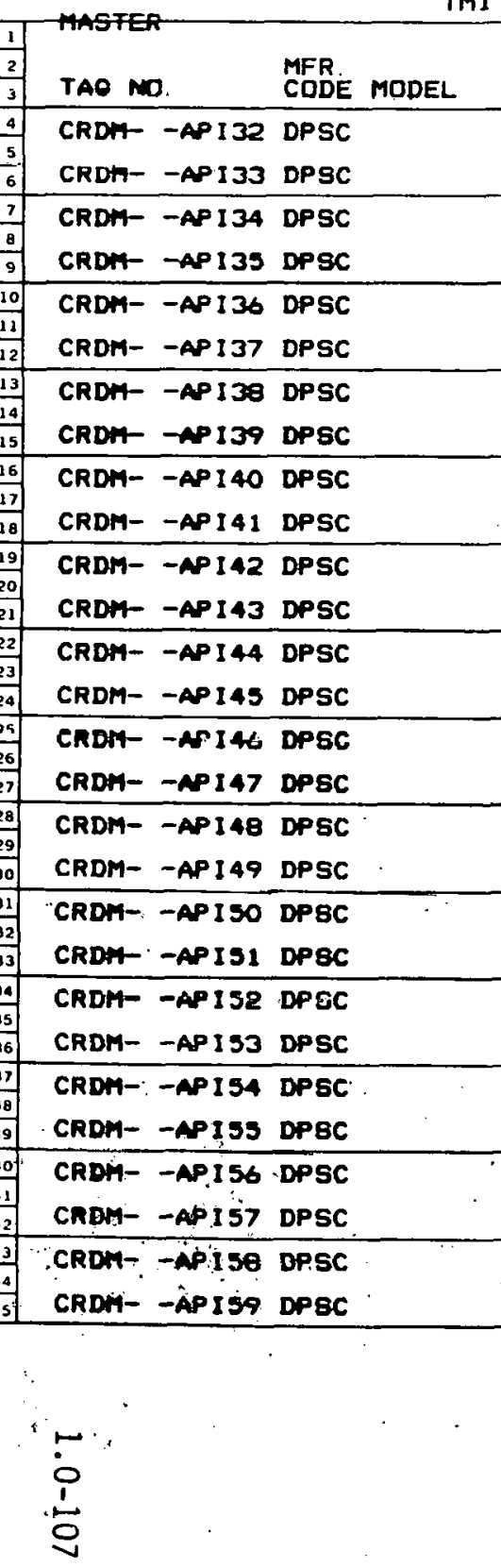

SERVICE/DESCRIPTION CR ABSOLUTE POSITION INDICATOR CR ABSOLUTE POSITION INDICATOR CR ABSOLUTE POSITION INDICATOR CR ABSOLUTE POSITION INDICATOR CR ABSOLUTE POSITION INDICATOR CR ABSOLUTE POSITION INDICATOR CR ABSOLUTE POSITION INDICATOR CR ABBOLUTE POSITION INDICATOR CR ABSOLUTE POSITION INDICATOR CR ABSOLUTE POSITION INDICATOR CR ABSOLUTE POSITION INDICATOR CR ABSOLUTE POSITION INDICATOR CR ABSOLUTE POSITION INDICATOR CR ABSOLUTE POSITION INDICATOR CR ARSOLUTE POSITION INDICATOR CR ABSOLUTE POSITION INDICATOR CR ABSOLUTE POSITION INDICATOR CR ABSOLUTE POSITION INDICATOR CR ABSOLUTE POSITION INDICATOR CR ABSOLUTE POEITION INDICATOA CR ABSOLUTE POSITION INDICATOR CR ABSOLUTE POSITION INDICATOR CR ABGOLUTE POSITION INDICATOR CR ABSOLUTE POSITION INDICATOR CR ABSOLUTE POSITION INDICATOR CR ABSOLUTE POSITION INDICATÓR CR ABSOLUTE POSITION INDICATOR CR ABSOLUTE POSITION INDICATOA
IEPO-KW-OS-20-OO PAOE-5-

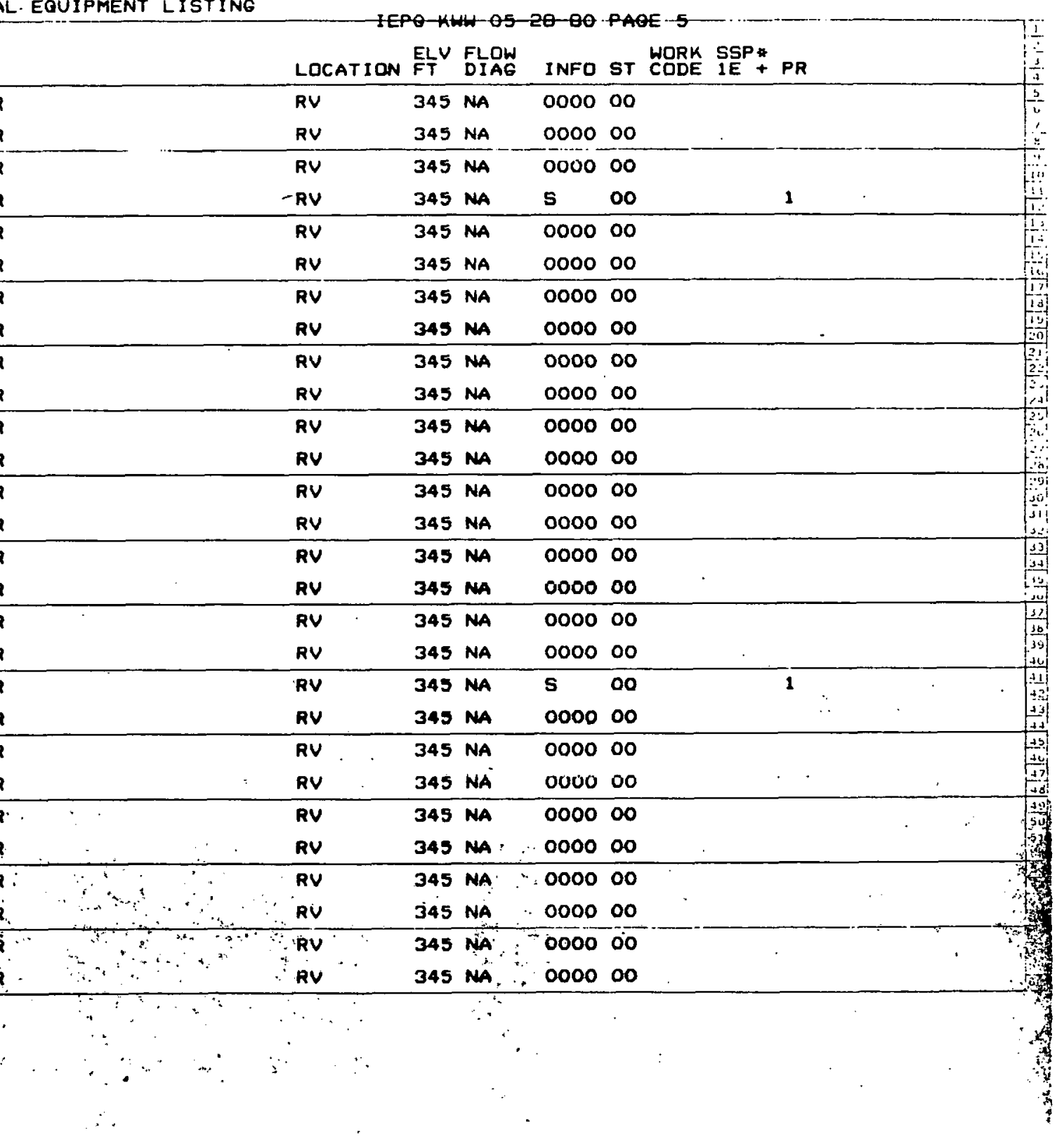




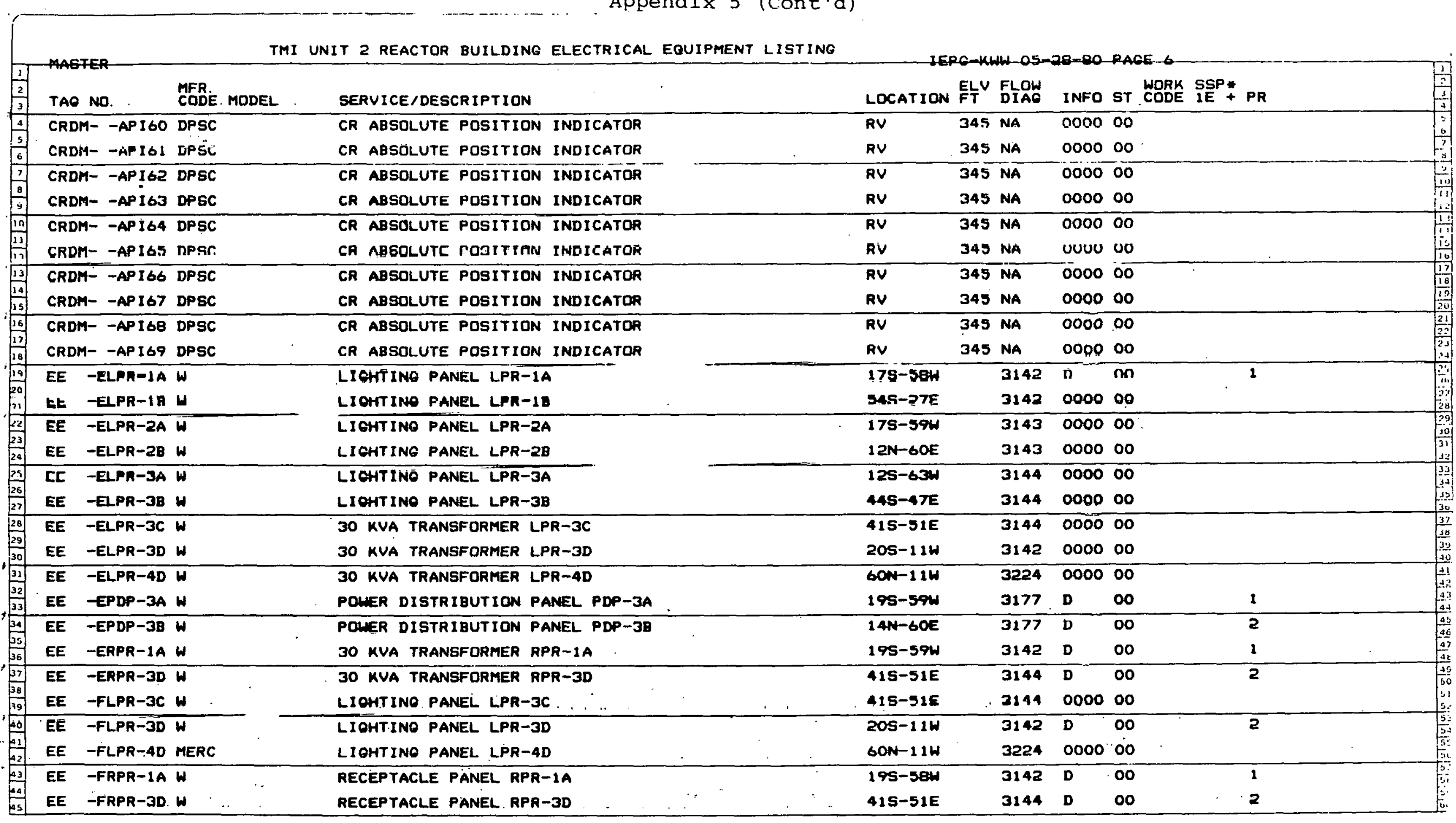

-
$\dot{1}$
1
$\circ$
0 
TMI UNIT 2 REACTOR BUILDING ELECTRICAL EQUIPMENT LISTING

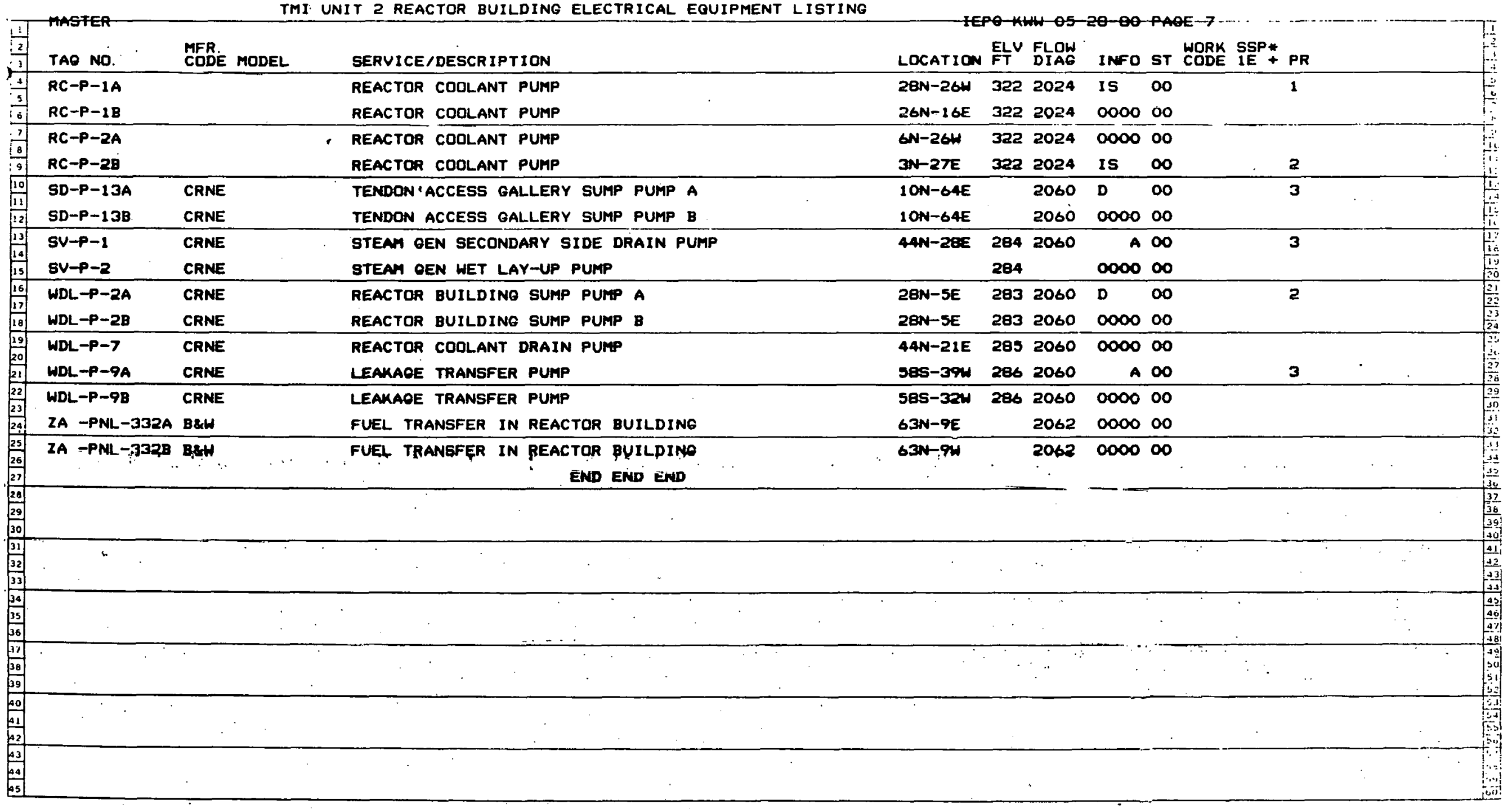




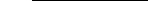


SAND 80-0699

Unlimited Release

March 1980

Recommended Data Acquisition Tasks at TMI-2

Relating to

Fission Product Transport and Deposition and Environments Characterization

Prepared by:

The TMI Examination Task 2.1 Planning Group

Final Report

P. Yarrington (SNL) - Coordinator

D. Dahlgren (SNL) - Person in Charge

LWR Safety R\&D Division - 4443

Sandia National Laboratories, Albuquerque, N.M. 87185

Prepared for:

Technical Working Group

TMI Information and Examination Program (Program sponsored jointly by DOE, EPRI, NRC, GPU)

Funded in part by:

Nuclear Power Development Division

U. S. Department of Energy 


\section{ABSTRACT}

This report presents a list of data acquisition tasks which are recommended for implementation during recovery of the TMI-2 nuclear power plant. The focus of this effort was the environment in containment during and after the accident. The data which would be collected under the recommended tasks were determined to be of significant value for improving current understanding of fission product dispersal processes during nuclear plant accidents. In developing these recommendations, several specific technical areas were considered which would benefit from such data. These technical areas are described in the report and data requirements in each area are identified and prioritized. A general discussion is presented of the sampling methods and analytical techniques which will be needed to implement the recommended tasks and obtain the desired data. 
This report was assembled and edited by F. Yarrington (SNL). All those 1 isted below as planning grcup members contributed text and/or suggestions for the present report. D. Dahlgren (SNL) had lead responsibility for the planning group effort.

(Organizations 1 isted alphabetically, asterisk

denotes TWG-designated planning group member)

Battelle Columbus Laboratory

Richard S. Denning*

James A. Gieseke*

Battelle Northwest Laboratories

Leo G. Faust*

Lyle D. Perrigo*

DOE/NPD

Andrew C. Millunzi

DOE/TMI Technical Integration office

Harold Burton

Greg Eidam

$\underline{E G \& G \text { - Idaho }}$

John W. Mandler *

EPRI/NSAC

Allan D. Miller*

Exxon Nuclear

Carl Crouthammel*

GPU/Bechtel

Bill Hopkins*

NRC/NRR

W. F. Pasedag*

Arlin Postma (NRR Conșultant) 
NRC/RES

Richard R. Sherry*

Oak Ridge National Labozatory

A. P. Mal inauskas*

Sandia Laboratories

Allan S. Benjamin* Dirk A. Dahlgren (Lead)*

Peter 0. Strom

Paul Yarrington (Ccordinator)*

Science Applications Inc.

Robert L. Ritzman* 


\section{CONTENTS}

Planning Group Membership List ..................... 2.1-3

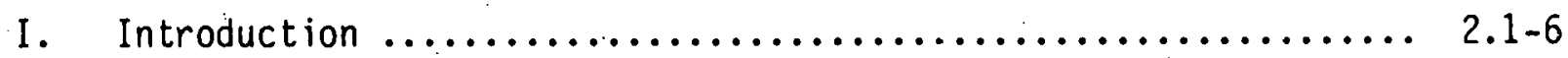

II . Recommended Tasks ................................ 2.10

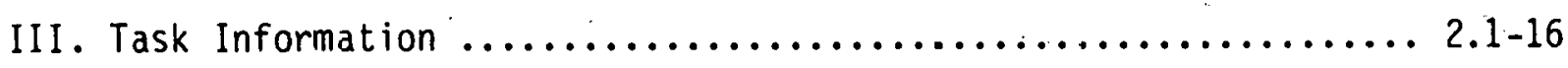

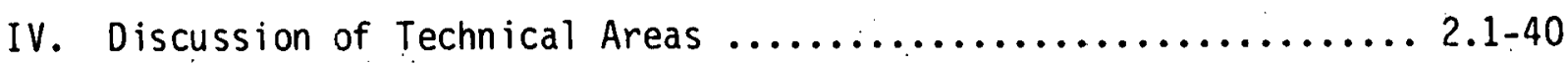

IV.A Licensing Assumptions ........................ 2.

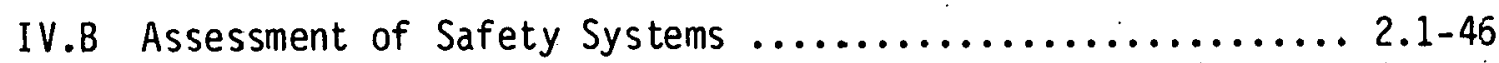

IV.C Model Testing and Development .................. 2.1-54

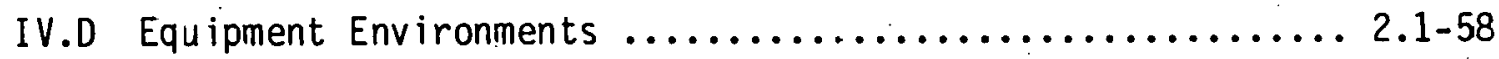

IV.E Accident Diagnosis ........................... 2.63

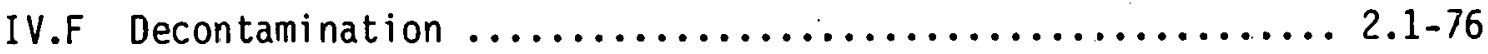

IV.G Mass Balance ............................ 2.1-79

IV.H Health Physics $\ldots \ldots \ldots \ldots \ldots \ldots \ldots \ldots \ldots \ldots \ldots \ldots \ldots \ldots \ldots \ldots .1-83$

IV.I Fuel Examination for Fission Product Release .......... 2.1-86

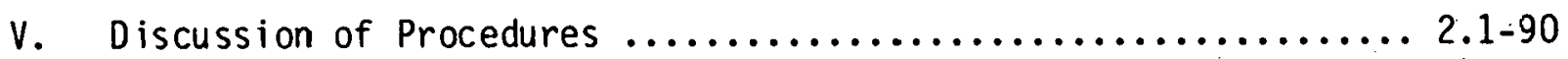

VI. Recommendations for Future Technical Support ............. 2.1-99 


\section{INTRODUCTION}

One of the primary objectives of the Technical Working Group (TWG) for the TMI Information and Examination program is to define the technical work that should be performed. The over-riding concern in this effort is to develop a program of data acquisition which has minimum impact on recovery operations while maximizing the value of reactor safety information which is obtained. To help develop such a program the TWG has assembled planning groups, comprised of industry, research and regulatory experts, to recommend actions in several specific technical areas.

This report presents TMI-2 data acquisition activities recommended by the Task 2.1 (Fission Product Transport and Dispersal and Environmen=s Characterization) planning group. The focus of this group was the post-accident environment in containment at TMI-2. The overall objective of this effort was to determine those data acquisition activities which could significantly improve current understanding of nuclear plant accident environmerts and the phenomena which contribute to those environments.

It was suggested by the TWG that the Task 2.1 planning group consider data acquisition tasks which would (1) improve current understanding of fission product dispersal mechanisms, (2) aid in the planning for dose reduction during decontamination operations, and (3) provide environmental data required by the equipment examination planning group (Task 1.0 ). 
Within this overall scope, data requirements were specified in a number of additional specialized technical areas. Data were identified which could be used to evaluate the adequacy of current Regulatory Guide source term assumptions and systems requirements. Data were also identified for evaluating the performance of existing safety systems, e.g., the containment sprays, or for developing design requirements for proposed safety systems, e.g., containment filtered venting systems.

Since much of the history of fission product transport during the accident will probably be inferred from current plant conditions, an effort was made to identify data which would still be available for accident diagnosis. . F specific technical area which would benefit from the environments history is the numerical modeling of radioactivity transport during accidents. Consideration was thus given to the data requirements which were known or expected to be of importance to model development or validation.

Data needs in the general area of plant decontamination were considered from two standpoints. First, data was sought which could help identify the generic decontamination problems (e.g., radio-chemical forms, hold up of contamination) presented by nuclear plant accidents. Secondly, the plant decontamination was considered from the health physics standpoint. Attention was given to the sampling or data analysis which would be. required to accomplish safe reentry, data acquisition and cleanup operations. 
Finally, consideration was given to the data requirements for performing a mass balance on significant radiologically toxic species. Completing a mass balance ensures that all important sinks and attenuation mechanisms have been identified for those species and thus that transport and deposition studies are accurate and complete. An important part of completing mass balances. will be the determination of fractional release and retention of species in the fuel. Thus attention was also given to the data which must be obtained from the fuel to achieve the objectives of the present task. Recommendations, for fuel analyses will be transmitted to the fuel examination planning group (Task 7.4)

The tasks which are recommended by the group are listed in Seczion II. Specific task information follows in section III. The task information includes a summary of the objectives and technical justification for each task. These summaries, in turn, refer to later sections of the report where more technical detail can be found regarding the purpose and usefulness of any given task. The data acquisition procedures and sampling strategy to be used for each task are also. included as task information in section III.

Section IV presents detailed discussions of the various technical areas which were considered in making the Task recommendations. The material addresses data requirements, suggested sampling and analysis procedures, and a priority assessment for the requested data in each technical area. 
A general discussion of sampling and analysis methods which will be required in executing the recommended data acquisition program is presented in section $v$. Dietails of the procedures are not specified since most will be specific for each task and many will be quite involved.

The need for continued technical guidance during the implementation phase of this program is discussed in section VI. Briefly, the procedures necessary to optimize the use of available time and maximize the quality of information obtained can be expected to depend on what is learned as the program proceeds. Thus, technical guidance will be needed to interpret these new data in terms of remaining work to be done. It is recommended that the present group be preserved, in some form, to perform this tecnical guidance function. 


\section{RECOMMENDED TASKS}

The data acquisition tasks recommended by the Task 2.1 planning group are presented below. Table II.l gives a simple priority ranking of the recommended tasks, where highest priority $=1$. The tasks which were assigned highest priority were considered to be very important to providing data needs in at least one of the technical areas studied by the group (bee section IV). In order to provide furthər guidance for implementing the tasks, this highest priority group was subdivided to indicate the relative importance of the tasks from an overall program standpoint. : The priority ranking used was as follows: $1-\mathrm{H}=$ highest, I-M. = intermediate, $\mathrm{I}-\mathrm{L}=$ lowest. Those tasks of rank $\mathrm{l}-\mathrm{H}$ are expected to provide maximum technical benefit from the acquired data. It should be emphasized, however, that tasks of rank $1-M$ or $1-L$ provide very important information in some technical area, and are thus highly recommended from a technical standpoint. Thus the present priority scheme is intended to reflect both the technical importance of the task, as well as, the overall benefit which is expected to be derivable from the data.

A summary of task information is presented in Table II.2. This table provices estimates of time involved for implementing tasks, expected radiation levels (environments) in areas where the work will be performed, and indication of whether major equipment development will be needed to complete the task. The information provided there gives. 
only rough estimates of times and environments, and is intended primarily for overall planning or for task comparison purposes. 
Table II.I

List of Recommended Tasks

Item

1 .

2 .

3.

4 .

5.

6.

7.

8 .

9.

10 .

11.

12.

13.

14.

15.

16.

17.

18.

19.

20 .

21 .
Task

Containment radiation mapping

Sump samples and inspection

RCS sludge samples

(fron vessel and primary system)

Equipment environments survey

Decortamination operations

Fuel examination for fission prcduct release

Samples from let down purification 1-M system

Samples from charcoal filters

Containment surfaces deposition samples

Contaïrment stainless steel surface swipes and samples

RCS samples (current)

Examination of let down coolers

Containment air samples

Primary system internals

examination and sampling

Air ccoling coil sample

Sample from containment stairwell concrete block

Operational histories

RCDT sludge samples

Gas decay tank swipe

Hydroger recombiner inspection

Samples from bleed tank
Priority*

$1-\mathrm{H}$

$1-\mathrm{H}$

$1-\mathrm{H}$

$1-\mathrm{H}$

$1-\mathrm{H}$

$1-\mathrm{H}$

$i-M$

$1-M$

$\cdot$

$1-M$

$1-M$

$1-M$

$1-M$

$1-M$

$1-M$

1-L

1-L

2

2

2

3 


\section{Table II.2}

Task

No.

Task

TASK SUMMARY

$$
\text { Priority }^{(a)} \text { Est. Time }^{(b)} \begin{aligned}
& \text { Expected } \\
& \text { Rad. Env. Equip. Dev. (d) }
\end{aligned}
$$

1. Containment radiation mapping $1-\mathrm{H}$

2. Sump samples (Thru pen.): 1-H and inspection (In cont.):

$1-\mathrm{H}$

3. RCS sludge samples (from vessel and primary system)

4. Equipment environment survey

$1-\mathrm{H}$

L

H

Y

M

L

L

$\mathrm{H}$

$Y$

M

$\mathrm{H}$

Y

5. Decontamination operations

6. Fuel examination for fission product release

1-H (Recommendations transmitted to separate

1-H $\} \quad\left\{\begin{array}{l}\text { TMI I\&E Program Task group - no further } \\ \text { action needed. }\end{array}\right.$ 
Tahle II. 2

Task

No.

7. Samples from let-down (filter): purification system (resin):

8. Samples from charcoal filters

9. Containumut surfaces deposition samples

10. Containment stainless steel surfaces swipes and samples

11. RCS samples (current)

12. Examination of let-down coolers

13. Containment air samples

14. Primary system intervals examination and sampling

15. Air cooling coil sample

\section{TASK SUMMARY}

Priority (a)

$1-M$

$1-M$

$1-M$

1-M

$1-M$

$1-\mathrm{M}$

$1-M$

$1-M$

$1-M$

$-M$ Est. Time ${ }^{(b)}$

Expected $(c)$
Rad. Env. Equip. Dev. (d) Required

$\mathrm{N}$

H

$\mathrm{N}$

M

L

$\mathrm{N}$

M

$\mathrm{H}$

N

M

H

$\mathrm{N}$

NA

NA

NA

NA

NA

$\mathrm{N}$

$\mathrm{H}$

H 


\section{Table II.2}

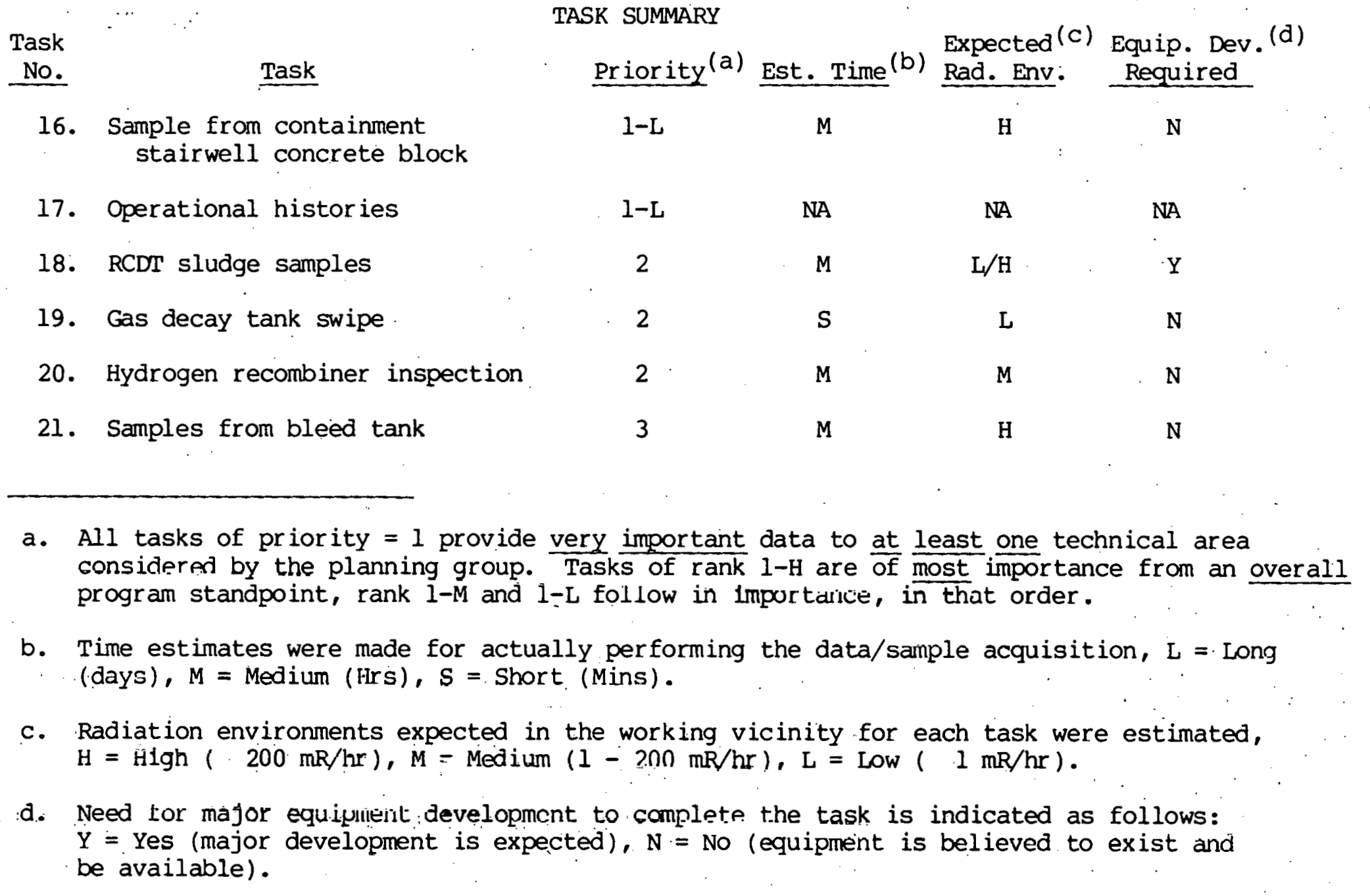




\section{TASK INFORMATION}

This section presents a summary of the objectives and technical justification for each task 1 isted in section II. A data acquisition plan is also recommended for each task. The data acquisition plan, in general, specifies the sampling techniques, number and size of samples, location of samples, dispasition of samples, estimate of required time, and sampling schedule relative to anticipated decontamination operations. 
- III. Task Information - Task No. 1

1. Task: Containment Radiation Mapping

2. Summary of Objectives: The objectives of this task are to acquire information about the distribution of radioactive materials in the containment structure and associated internal systems and measure radiation fields at various sites in the containment area.

3. Justification: Radiation mapping should be undertaken to assess the affects of component syszem and equipment layout, geometries and orientation on hold up of contamination and cleanup to remove contamination. The payoff is in better designs for future reactor systems and layouts that can be more readily cleaned be they for similar accidents or more routine operations necessary to control occupational exposure. Data obtained in this task will also supply needs in the areas of: Equipment Environments (IV.D), Accident Diagnosis (IV.E), and Health Physics (IV.H). In addition, the data will help define the scope and sampling strategy to be used in subsequent data acquisition tasks (e.g., Tasks 9 and 10 ).

4. Data Acquisition Plan and Schedule: All of the radiation mapping work must be done with special instruments before any decontamination is undertaken on any part of the containment or primary systems of the reactor. Field radiation measurements and adhesion samples acquired by the use of wetted cellulose acetate are needed from materials, equipment and surfaces at all levels in the containment system. To meet the objectives of acquiring comprehensive data on radioactive distribution, the identification of specific sites for these measurements and adhesion. samples must be done from detailed drawings and photographs of the system. Radiation level measures before and after TMI-2 decontamination can be used to measure DFs. This task should be completed before land provide guidance to) Tasks 9 and 10. In order to reduce dose to personnel, consideration should be given to the use of remote equipment for some of the work under this task. 


\section{Task Information - Task No. 2}

1. Task: Sump Samples \& Inspection

2. Summary of Objectives: Determine the quantity of radioactive material in the containment sump, and its chemical and physical form. Determine the height of the flocculent material of the sump and, if possible, the gradient of the flocculent's density. Determine the isotopic and chemical (elemental and compound) nature of the flocculent and the sediment on contact of the 282' elevation basement floor. Determine the size, location, and type of solid debris present in the sump water. Determine if there are any debris floating on the water.

3. Justification: See Data Requirements for the following sections:

IV.A Licensing Assumptions

IV.B Assessment of Safety systems

IV.C Model Testing \& Development

IV.D Equipment Environments

IV.E. Accident Diagnosis

IV.F Decontamination

IV.G Mass Balance

4. Data Acquisition Plan \& Schedule: This task will proceed in three phases. Phase one is to obtain quick data from existing penetrations before they are covered. The reasoning is that the man-rem and cost to obtain the data after penetration covering may be prohibitive. This phase should use the existing water sampling procedure to obtain samples of approximately $200 \mathrm{ml}$ volume of the water and several mixed water/sediment samples. Also, penetrations $\mathrm{R}-401$ and $\mathrm{R}-605$ to obtain a visual (submersible TV camera recording to tape) record of the size, location, and type of solid debris present on the $282^{\prime}$ elevation basement floor. Determine if there are any floating debris, its size, its location, and type.

Phase two consists of designing and implementing improved sampling schemes for the water and sediment. Core samples, totalling approximately $200 \mathrm{ml}$ volume, should be withdrawn from three different planar and depth positions. Depthwise, samples should be withdrawn from near the pool surface, from mid-depth, and from the bottom. Planar positions should be determined to achieve the best average composition, recognizing the limitations of sample withdrawal equipment.

The sampling procedure should not $\mathrm{mix}$ the liquid or suspended solids. Particularly for the bottom sample, 
suspended solids (floc) and settled particles (sludge) should.be removed from a known planar area so that the sump inventory can be calculated from sample data. In addition, it would be desirable to obtain a visual record of the optical density of the flocculent as a function of height. The sump sample apparatus should try to obtain a flocculent sample that preserves axial gradient of density and it should take the sample directly from basement floor. The analyses should look for any sediment due to unprocessed.water from containment air coolers. Analyze the sediment samples for isotopic, elemental chemical, and chenical compounds. Use state-of-the-art methods of analysis, e.g., Ge(Li)/multichannel analysis spectral stripping of data with a large scale spectral. stripping computer code; spark source mass spectra analysis of solids, x-ray fluorescence, etc.

Each of the water samples withdrawn should be divided into aliquots, with half of the sample being retained for archival purposes. Each aliquot would first be visually inspected for color, turbidity, floc, and sediment. Next, the sample would be centrifuged to separate liquid and particles. Each phase would then be analyzed for isotopic content. Liquid would be analyzed for pH, elemental composition, mineral content, and other chemical compounds: Solids would be analyzed for particle size, elemental content, and chemical compounds. The analytical procedures used should be selected after study of available option by an analytical chemist. It is anticipated that analysis method would include: emission spectroscopy, $x-r$.ay diffraction, electron microscopy, and $x-r a y$ fluorescence.

Sampling of the sump must be done prior to cleanup operations which would appreciably alter the quantity or form of material present in the sump.

Phase three consists of a post sump decontamination and draining analysis of the debris. The focus is on those debris which could effect the performance of safety systems relying on sump water. Care should be taken to differentiate accident debris from decontamination debris. 


\section{Task Information - Task No. 3}

1. Task: RCS Sludge Samples from Vessel and Primary System

2. Summary of Objectives: Acquire specific information about the identity and concentration of rupture debris, $f$ ission products and activated corrosion products in the primary system of TMI-2.

3. Justification: The sludge from the primary system is needed to proof test and specify decontamination processes for current efforts to cleanup TMI-2 and to acquire information on conditions that may be encountered in such systems following accidents such as occurred at TMI-2. Data from this task will supply needs for Model Testing and Development (IV.C), Accident Diagnosis (IV.E), Decontamination (IV.F) and Mass Balance (IV.G).

4. Data Acguisition Plan and Schedule: As soon as reasonably possible, after entry in to containment, samples should be drawn from the reactor vessel, the pressurizer vessel, the upper tube sheet and lower portion of steam generators, a lcw spot in the piping leading to the steam generators, and/ or a low spot in the return to the reactor vessel. Each sample should contain a reasonable amount of material up to ioog. Care should be exercised to ensure that sampling is down at the low spot in each of these test areas and that personnel exposure is minimized. 
III. Task Information - Task No. 4

1. Task: Equipment Environments Survey.

2. Summary of Objectives: Obtain samples and measurements in the vicinity of key equipment (specified in Task 1.0 planning group report) on or accessible from the $305 \mathrm{ft}$. floor

3. Justification: See data requirements for Equipment Environments (section IV.D)

4. Data Acquisition Plan and Schedule: For each piece of equipment included in the TMI-Examination Task 1.0 list of selected equipment (see Task 1.0 planning group report) obtain the following data and samples:

(i) Smears: One on equipment directly and one from supporting surface

(ii) Directional gamma dose rate:
A) Toward and away from equipment
B) In plane of supporting wall
C) Toward sump

(iii) Total dose samples: Remove length ( 8 in.) of insulated electrical cable near equipment (within $2 \mathrm{ft}$. of equipment, do not stretch during removal), remove glass samples (e.g., light bülbs)

(iv) Surface samples: If supporting surface is painted, remove a sample of the paint near the equipment (within 2 feet, but not at same location as smears) 


\section{Task Information - Task No. 5}

1. Task: Decontamination Operations

2. Summary of objectives: The purpose of this work is to cetermine the time, cost, and efficiency of various cecontamination steps and processes applied to the cleanup cf containment structure, primary system and associated components in TMI.

3. Justification: These data are needed to judge the relative merit of various canstituent steps in decontamination and to determine the cost-benefit incentives for various operations.

4. Data Acguisition Plan and Schedule: The first part of this effort is to prepare a detailed operating procedure that will specify the various decontamination operations that are to be conducted within containment at TMI. Following the preparation, acceptance and approval of such a plan, the next step is tc keep judicious records on the amount of time and manpower needed for various cleanup operations within containment. Additionally the cleanup DF for each sub operation should be measured to assist in the assessment of decontamination efficiency. With suitable records on the amount of waste generated in the specific decontamination operations, a cost-benefit analysis can be conducted from the data that are required. A more detailed recommendation for quantifying the effectiveness of decontamination activities (as outlined here) will be forwarded to the Tezhnical working Gzoup for consideration under TMI Examination Task 2.2 (Decontamination/Radiation Dose Reduction Technology).

** NOTE: Current plans call for recommendations regarding this task (Decontamination Operations) to be reported t.y Task 2.1 planning group member(s) at the review of the $11 / 27$ - 29/79 decontamination workshop report (Workshop and review coordinated by Task 2.2 - Decontamination/Radiation Dose Reduction Technology). 
III. Task Information - Task No. 6

1. Task: Fuel Examination for Fission Product Release.

2. Summary of Objectives: To determine the amount and type of fission products released from various regions of the TMI-2 core. To characterize this release as a function of fuel temperature, fuel grain morphology, fuel fragmentation, etc.

3. Justification: The information to be obtained under this task is necessary for areas IV-A through. IV-F. and is crucial for establishing the Fission Product Mass Balance $(I V-G)$.

4. Data Acquisition Plan and Schedule: The sampling strategy and measurements needed for this task are detailed in section IV.I of this report. These recommendations should be transmitted to the TMI-Examination Task 7.4 (Fuel Experiments and Examination) planning group Eor inclusion in their core examination plan.

** NOTE: Task 2.1 recommendations for fuel examination (as described above and in section IV.I) have been transmitted to the Task 7.4 planning group (letter, 12/18/79, P. Yarrington/SNL to A. Millunzi/DOE). 
III. Task Information - Task No. 7

1. Task: Samples from Letdown Cleanup System

2. Summary cf Objectives: Determine the radionuclide inventory retained on ion exchange resins and filters in the reactor coolant and purification (letdown cleanup) system. This incluces alpha-, beta-, and gamma-emitting radio-nuclides. Determine the chemical species of the particulate and colloidal material retained on the resins and filters.

3. Justification: This information is required for the 三ollowing:

1. Determination of the amounts of fission products and fuel released during the accident (see section IV.E Accident Diagnosis).

2. Mass balance (see Section IV.G).

3. Determination of the amount and chemical species of rupture debris (see Section IV.F - Decontamination).

4. Data Acquisition Plan and Schedule: Obtain and review the operating history of the letdown cleanup system during and after the accident. Identify which filters and resins were in use. If radiation levels permit, obtain section of makeup and purification demineralizer filter MU-F-SA or MU-F-SB (whichever was in use during and after the accident) and makeup filter $M D-F-2 A$ or $M U-F-2 B$ (whichever was in use during and after the accident). Divide each filter section into 5 pieces for: (1) isotopic beta-gamma analyses, (2) alpha analysis, (3) mass spec. analysis, (4) chemical analysis, and (5) archives. If high radiation levels prevent collection of samples of the filters, then back flush each filter, collect the back flush solution in a tank, recirculate the tank solution, and collect" 5 samples of the solution for $1-5$ above.

If accessible, collect 3 samples of resin from makeup and purification demineralizer $M U-K-1 A$ or $M U-K-1 B$ (whichever was in use during and after the accident), one each from the top, middie, and bottom of the demineralizer bed. If high radiation levels prevent collection of these samples, then collect 5 samples while the resin is being sluced from the demineralizer. Divide each sample into 5 parts for: (1) isotopic beta-gamma analyses, (2) alpha aralysis, i3) mass spec. analysis, (4) chemical analysis, and (5) archives. Use Ge(Li)/multichannel analysis for spectral stripping $0 \equiv$ data.

In collection of all samples (both filter and resin), care must be used to obtain representative samples. In performing analyses, special attention should be paid 
to determine. the quantities of $\mathrm{Ni}-59, \mathrm{Ni}-63, \mathrm{TC}-99$, $\mathrm{I}-129, \mathrm{Cs}-134, \mathrm{Cs}-137, \mathrm{Zr}-95 / \mathrm{Nb}-95, \mathrm{Sr}-89, \mathrm{sr}-90$, Ru-103, Ru-106, Ce-141, Ce-144, dranium, plutonium, and crud-associated radionuclides (e.g., Co-58, Co-60, $\mathrm{Cr}-51, \mathrm{Mn}-54)$, and the chemical somposition of particulate and colloidal material. 
III. Task Information - Task No. 8

1. Task: Samples from Charcoal Filters

2. Objectives: Deternine inventory of iodine and other radionuclides in charcoal and HEPA filters obtained from tie auxiliary building and fuel handling building ventilation exhaust cleanup systems. Determine I-129 inventory in charcoal filters and inventory plus chemical species of radionuclides on HEPA filters.

3. Justification: This information is required for the following:

1. Determination of the amounts of fission products released during the accident (see section IV.E Accident Diagnosis).

2. Mass jalance (see Section IV.G).

3. Determination of the amounts of radionuclides present in the air to aid in estimating partition factors (see Section JV.C - Model Testing and Evaluation).

$\leqq$ Determination of the amount of radionuclides removed from the air ty the cleanup systems (see section IV.B Assessment of Safety systems).

4. Data Acquisition Plan and Schedule: Obtain one section of HEPA fiiter and tray of charcoal filter from auxiliary building and one from fuel handling building ventilation cleanup systems. Obtain operating histories for these filters. Divide each HEPA section into 4 parts for: (1) isotopic beta-gamma analyses (inluding I-129 analysis), (2) chemical analysis, (3) mass spec. analysis, and (4) archives. Obtain 3 samples from each charcoal tray, one each from front, middle, and back relative to direction of air flow. Divide each sample into 2 portions for (1) I-129 analysis and (2) archives. It has also been suggested that the radiochemical analyses include Tc-99, $\mathrm{Ni}-59$ and $\mathrm{Ni}-63$. 
III. Task Information - Task No: 9

1. Task: Containment surface deposition samples

2. Summary of Objectives: Determine as much as possible the composition and distribution of radionuclides deposited on containment surfaces. This includes determination of the isotopic, chemical, and physical character of deposits including attached or settled particulates. Also determine general surface radiation dose rate patterns around the containment and obtain visual observations of the appearance and condition of various surfaces.

3. Justification: See data requirements for: Licensing Assumptions, Assessment of Safety Systems, Model Testing and Development, Equipment Environments, Accident Diagnosis, Decontamination, and Mass Balance (Sections IV-A, IV-B, IV-C, IV-D, IV-E, IV-F, and IV-G) .

4. Data Acquisition Plan and Schedule: All samples and observations should be obtained after $\mathrm{Kr}-85$ is removed from the containment atmosphere but before any surface decontamination work is begun. Work requires personnel entry into containment. Divide containment into quadrants at Elev. $347^{\prime}$ (sprayed) and Elev 305' (mostly unsprayed). Visually inspect for paint discoloration, metal corrosion, and/or equipment damage and obtain gamma radiation dose rate measurements at surfaces. Record observations and data. Obtain samples of both vertical and horizontal surfaces from all quadrants. Samples should include swipes and adhesion samples, portions of Epoxy paint paint, easily demountable pieces of hardware, pieces of giass, and perhaps drill or chisel chips (concrete). Estimated 50 to 100 samples, placed in protective containers and removed for laboratory analysis. Time requirement estimated at 2-4 man-hours. Laboratory analysis should include: quantitative gamma-ray spectrometry and radiochemical determination for Tc-99, Ni-59, Ni-63, Cs-137, Sr-90, Ru-106; Ce-144, and I-129; particulate characterization by emission/mass spectrometry and size classification by SEM and TEM; surface deposit chemical. characterization by reagent tests and perhaps $x-r$ ay diffraction; integrated dose determination using photo-luminescence techniques on glass samples. Save approximately 208 of the samples for archive storage. 
III. Task Information - Task No. 10

1. Task: Cortainment stainless steel surface swipes and samples

2. Summary of Objective: Determine as much as possible the isotopic and chemical composition of deposits on stainless steel surfaces and the accompanying radiation dose rate.

3. Justification: See data requirements for Licensing Assumptions, Assessment of Safety Systems, Model Testing and Developmen=, Accident Diagnosis, Decontamination, and Mass Balance (Sections IV-A, IV-B, IV-C, IV-E, IV-F, and (V-G).

4. Data Acquisition Plan and Schedule: All samples should be obtainec after $\mathrm{Kz}-\overline{85}$ is removed from the containment atmosphere but before any surface decontamination work is begun. Work requires personnel entry into containment and coordination with Task No. 9 efforts. Obtain gamma radiation dose rate measurements at stainless surfaces and then collect samples from vertical and horizontal surfaces in sprayed and unsprayed areas if possible. Samples shodld include swipes, adhesion samples, and easily demountable pieces of hardware or equipment. Estimated 1] to 20 samples, placed in protective containers and removed for laboratory analysis. Time requirement estimated at $1 / 2$ man-hour. Laboratory analysis using gamma-ray spectrometry and radiochemical analyses for TC-99, Ni-5 9 and $\mathrm{Ni}-53, \mathrm{Cs}-137, \mathrm{Sr}-90, \mathrm{Ru}-106, \mathrm{Ce}-144$, ard I-129 ar:d particilate characterization by emission/mass spectrometry and using SEM and TEM for particle size evaluation. Surface layer chemical characterization by reagent tests and perhaps $x$-ray diffraction. Save approximately $20 \%$ of the samples for archive storage. 
III. Task Information - Task No. 11

1. Task: RCS samples (current)

2. Summary of Objectives: Determine as thoroughly as possible the isotopic composition and the zhemical character of currently obtained reactor coolant samples.

3. Justification: See data requirements for: Licensing Assumptions, Assessment of Safety Systems, Model Testing and Development, Accident Diagnosis, and Mass Balance (Section IV-A, IV-B, IV-C, IV-E, and IV-G :

4. Data Acquisition Plan and Schedule: RCS samples are being taken and analyzed on a reasonably routine basis. The intent of this task is to review the procedares being employed and to recommend additions or modifications, if appropriate, for maximizing the information gained from this work. Sampling factors to consider include number, frequency, method of withdrawal, container used, and location of the sampling point. Analysis factors to consider are possibly more complete radioisotope analysis, analysis for dissolved gases, more complete chemical and physical property determinations, and inspection and identification of any suspended solids. This task work should begin immediately. 
III. Task Information - Task No. 12

1. Task: Examination of Let-Down coolers

2. Summary of Objectives: Examine coolers to determine the quantity and composition of radioactive materials plated out or collected in the coolers.

3. Justification: The coolers are a likely place for radioactive material released during and subsequent to core uncovery to be deposited. It is also possible that precipitation from the boric acid solution would have occurred in the coolers. The principal motivation for analysis of the coolers is to aid in the diagnosis of the accident (Section IV.E). The performance of the entire letdown system is under review, however, as it functions in severe accidents. Analysis of the letdown coolers could provide data to aid regulatory guidance (Section IV.A) in the future.

4. Data Acquisition Plan and Schedule: The letdown coolers would be expected to be $\mathrm{a}$ high radiation source which would probably be removed in tact. Gamma scanning should be used to identify deposited radioisotopes. Disássembly, physical inspection and the determination of chemical form would have to be performed in a hot cell. Archival samples of crud should be retained if possible. 
III. Task Information - Task No. 13

1. Task: Containment Air Samples

2. Summary of objectives: Determine the isotopic, elemental chemical and chemical compound form of all airborne species at the different elevations of the containment.

3. Justification: See Data Requirements for the following Sections:

IV.A Licensing Assumptions

IV.C Model Testing \& Development

IV.D Equipment Environments

IV.E Accident Diagnosis

IV.F Decontamination

IV.G Mass Balance

4. Data Acquisition Plans \& Schedule:

Using the following penetrations:

$$
\begin{array}{ll}
R-626 & \left(\mathrm{el} \cdot 347^{\prime}\right) \\
R-508 & \left(\mathrm{el} \cdot 305^{\prime}\right) \\
R-605 & \left(\mathrm{el} \cdot 282^{\prime}\right)
\end{array}
$$

obtain a direct containment air sample with a new apparatus that has a minimal run of piping to prevent plateout losses while maintaining containment integrity.

Analyze samples for specific radioisotopic activity, chemical elemental and chemical compounds. Particularly analyze for tritium, $\mathrm{Xe}, \mathrm{Kr}, \mathrm{H}_{2}, \mathrm{O}_{2}, \mathrm{~N}_{2}, \mathrm{H}_{\mathrm{e}}$, Organic Iodines, Particulate Iodine, Elemental Iodine and toxic gases.

Use state-of-the-art methods of analysis, e.g., mass spectrometry and, if indicated, gas chromatography.

Obtain samples before purge. 


\section{Task Information - Task No. 14}

1. Task: Primary System. Internals, Examination and Samples

2. Summary of Objectives: Determine isotopic, chemical and physical properties of plate-out on internal surfaces of primary system.

3. Justification: Measurements are needed to supply information related to mass balances, model testing and development, accident diagnosis, and decontamination. Analysis of activated products (activated poisons) in control rods will provide benchmark data for improved understanding of problems associated with waste (prim., low-level) disposal. Further definition of needs for tris task are provided in sections IV.C, IV.E, IV.F, and IV.G.

4. Data Acquisition Plan and Schedule: Sections of control rods, guide tubes, steam generator tubes, spacer grids, and upper grid plate should be collected for analyses. Samples of machined stainless surfaces should be obtained as well as samples of weld deposited stainless outside the reactor vessel (e.g., from steam generator manhole covers). Deposits and reactions with surfaces should be analyzed by gamma-scan as well as for chemical composition and physical properties. Solids deposits should be obtained preferably as swipes or scrapings or washing. All samples could be collected after purge and remote containment decontamination. Some samples may have to wait for core removal. However, it would be desirable to get some samples from the bottom of the pressure vessel and from system low points before disturbing the core. 


\section{Task Information - Task No. 15}

\section{Task: Air Cooling Coil Sample}

2. Objective: Make total dose measurements on air cooler units. Determine isotopic nature of plateout on representative sample.

3. Justification: See data requirements for: Licensing Assumptions, Assessment of Safety Systems, Accident Diagnosis and Mass Balance. (Sections IV.A, IV.B, IV.E and.I.V,G) .

4. Data Acquisition Plan and Schedule:

Individual. radiation surveys (total dose rates) should be made of each unit. Use gamma spectroscopy to examine a portion showing representative dose rates. In situ:techniques are recommended. Take at least cne smear fromi representative area. If sample must be removed for spectral analysis, roughly: $100 \mathrm{sq}$. cm. each of coil and, cooling, if in suriface, area will be required. Consult operational history! (l.Task:; No. 15) : to ensure that unit selected for sampling. iw.as: 1., operated during accident. Estimated times are as follows: Dose rate radiation survey ( $5 \mathrm{~min}$. ); in situ gamma spec., if possible, including smear $(10 \mathrm{~min}$.$) ; removing sample,".$ if necessary ( $1.5 \mathrm{min.})$. Samples and data should be taken before containment temperature or humidity is significantly altered. 
III. Task Information - Task No. 16

1. Task: Sample from containment stairwell concrete block

2. Summary of Objective: Determine the isotopic and chemical characteristics cf surface contamination on bare concrete not exposed to sprays. Evaluate the penetration profile of the contamination into the concrete.

3. Justification: See Jata requirements for: Licensing Assumptions, Model. Testing and Development, Accident Diagnosis, Decontanization, and Mass Balance (Sections IV-A, IV-C, IV-E, IV-F, and IV-G).

4. Data Acquisition Plan and Schedule: Samples of bare concrete from the stairwell area should be obtained vefore any surface decontamination work is begun. Personnel entry into the containment is necessary and the work should be coordinated with Task No. 11 efforts. obtain core samples (if possible) of unpainted concrete at three or four elevations in the stairwell from vertical and horizontal surfaces. Alternatively, knock off pieces of concrete wall or floor corners for samples. Estimated 5 to. 10 samples placed in protective containers and removed for laboratory study. Time requirement estimated at $1 / 2$ to 1 man-hour. Laboratory analysis using camma-ray spectrometry and radiochemical analysis for Cs-137, $\mathrm{Sr}-90, \mathrm{Ru}-106, \mathrm{Ce}-144$, and $\mathrm{I}-129$. Sectioning techniques (grinding, slicing, cr chipping) should be used to determine penetration depth and profile of the surface contamination. Autoradiography might be used to record uniformity of surface contamination. Save one or two samples for archive storage. 


\section{Task Information - Task No. 17}

1. Task: Operational histories

2. Objectives: Obtain operating histories for components/ systems which were operational during or following the accident and which have been selected for examination or evaluation by the Task 2.1 Planning group.

3. Justification: The performance of systems in containment can be expected to depend on the operational histories of those systems during the accident. The performance of interest includes both system effectiveness in performing intended functions, as well as effectiveness in controlling (or enhancing) fission product dispersal through unanticipated processes (e.g., deposition, resuspension, etc.)

4. Data Acquisition Plan and Schedule: Operational histories are required for the following systems:

1) Containment Air Coolers

2) BWST/NaOH Tanks (level histories)

3) Letdown Cleanup System

4) Hydrogen Recombiner

5) Auxiliary Building Ventilation Cleanup systems

The histories should extend from the present time back to times prior to the accident. Histories should also be obtained for any other equipment or system used to. add chemicals or water after the accident: 
III. Task Information - Task No. 18

1. Task: RCDT Sludge Samples

2. Summary of Objectives: To sample any sludge or particulates that may be in the botton of the Reactor Coolant Drain Tank.

3. Justification: The principal flow pathway for the transport of radioactivity and core debris to the containment during the accident was through the POV discharing into the RCDT. sludge and debris collected in the tank could provide information on the form of material released from the system as input to accident diagnosis (Section IV.E).

4. Data Acquisition Plan and Schedule: The RCDT is currently

under water. Sampling must therefore await cleanup and removal of the containment water. To be of value, the sample would have to be taken prior to flushing and aecontamination of the ta.zk. Access to the tank is available through a manhole but the sample would probably have to be collected remotely. The sample would be taken by scraping the surface. The material collected would undergo physical examination, radioisotopic analysis, and determination of chemical form. A few samples would be adequate including those for archival retention. 


\section{Task Information - Task No. 19}

\section{Task: Gas Decay Tank Swipe}

2. Summary of objectives: Determine the isotopic composition and chemical form of swipes from the waste gas decay tanks.

3. Justification: The waste gas decay tanks receive and accumulate gases released from the primary fliid through the Makeup and purification system. Scme volatile materials such as iodine and cesium could have been transported to the tanks and deposited on the tank surface. An approximate analysis of the quantity deposited and chemical form could assist in the analysis of the accident (Section IV.E) and in the modeling of radionuclide transport and deposition (section IV.C).

4. Data Acguisition Planning and Schedule: If possible, the swipes should be taken immediately following venting of the tank. Swipes would be desired from both the sides and bottom of the tanks. Analysis should be made of isotopic. mix and of chemical form. 
III. Task Information - Task No. 20

1. Task: Hydrogen recombiner inspection.

2. Summary of Objectives: Determine chemical and isotopic properties of plateout on internal surfaces of hydrogen recombiner system.

3. Justification: See data requirements for: Evaluation of Safety Systems, Mass Balance (Sections IV.B and IV.G)

4. Data Acquisition Plan and Schedule: Smear and gross gamma-scan should be obtained from internal surface of fore and aft spool pieces, fore and aft vent 1 ines, and innermost (toward zontainment) valve of hydrogen control vent 1 ine. Two one-foot sections of vent line taken near the forward containment penetration are required, one for archives and the other for mass spec. testing, laser-Raman spec. =esting, and $x$-ray fluorescence testing. Estimated time for smears and gross scans is about $10 \mathrm{~min} .$, time for obtaining vent 1 ine section should be less than $20 \mathrm{~min}$. All work could be done after purge and remote decon. 


\section{Task Information - Task. No. 21}

1. Task: Samples from Bleed Tank

2. Summary of objectives: Determine the quantizy of radioactive material in the bleed tank and its chemical and physical form.

3. Justification: Because an appreciable volume of reactor coolant is contained in the bleed tank, its content must be known to account for all of the fission products released from the core. This task supplies information to the following study areas: Licensing Assumptions (IV.A), Assessment of Safety systems (IV.B), Model Testing and Development (IV.C), Accident Diagnosis (IV.E), and.Mass Balance (IV.G).

4. Data Acquisition Plan and Schedule

Liquid in the bleed tank should be thoroughly agitated and then two samples of $100 \mathrm{ml}$ withdrawn. One of the liquid samples would be retained for archival purposes, and the other one divided into aiiquots for analysis.

After the liquid is drained from the bleed tank, surface deposits of representative areas facing upward, vertical, and downward, should be removed for analysis. Half of each sample should be retained in archives. Liquid samples would be visually observed for color, turbidity, floc, and sediment. Then the sample should be centrifuged to separate particles. In addition to isotopic content, each phase would be analyzed for elemental content: The liquid would further be analyzed for $\mathrm{pH}$ and boron concentration.

The surface samples (swipes) would be analyzed for isotopic makeup, with I-129 being of particular interest.

This task needs to be carried out in the near future because re-entry procedures will result in flushing of liquid currently held in bleed tanks. 
IV. DISCUSSION OF TECHNICAL AREAS

This section presents more detailed discussions of the various technical areas which were addressed by the planning group. Takes together, the data requirements identified in these areas were felt to provide a comprehensive data acquisition program an TMI accident environments. In general, the review of each individual area includes a technical justification for interest in the TMI data, a discussion of the data requirements in that area, and an assessment of the priority for data which might be obtained. 


\section{IV.A. LICENSING ASSUMPTIONS}

\section{Technical Justification}

The fission product source term for the design basis accident specified in Regulatory Guides $1.3^{a}$ and $1.4^{a}$, and associated assumptions concerning transport and deposition in containment were derived for the purpose of specifying a suitably conservative method of evaluating reacior sites. Therefore the primary purpose of the postulated source terms is to provide a conservative envelop of the maximum off-site doses resulting from a design basis accident.

Although these assumptions were not intended to realistically describe fission product behavior in containment, the lack of data concerning fission product release and transport under degraded core conditions has resulted in the use of these siting assumptions for such uses as: (1) specification of design parameters for fission product removal and retertion, (2) assessment of the radiation environment in post-accident containments, (3) providing the shielding criteria for shielding of cooling system components outside of containment, (4) setting criteria for instrumentation to follow the course of a LOCA, and (5) providing criteria for emergency planning. TMI-2 provides the opportunity to gather realistic data concerning certain aspects of fission product behavior ir. containment. Details of the fission product transport and. dispersal process probably cannot be deduced from the available data. Some information can be obtained, however, on dispersal patterns and deposition mechanisms. This information

\footnotetext{
TRegulatory Guides referred to herein are listed in Table IV:1.
} 
Regulatory Guides Relevant to TMI-Examination Task 2.1 Data Acquisition Recommendations

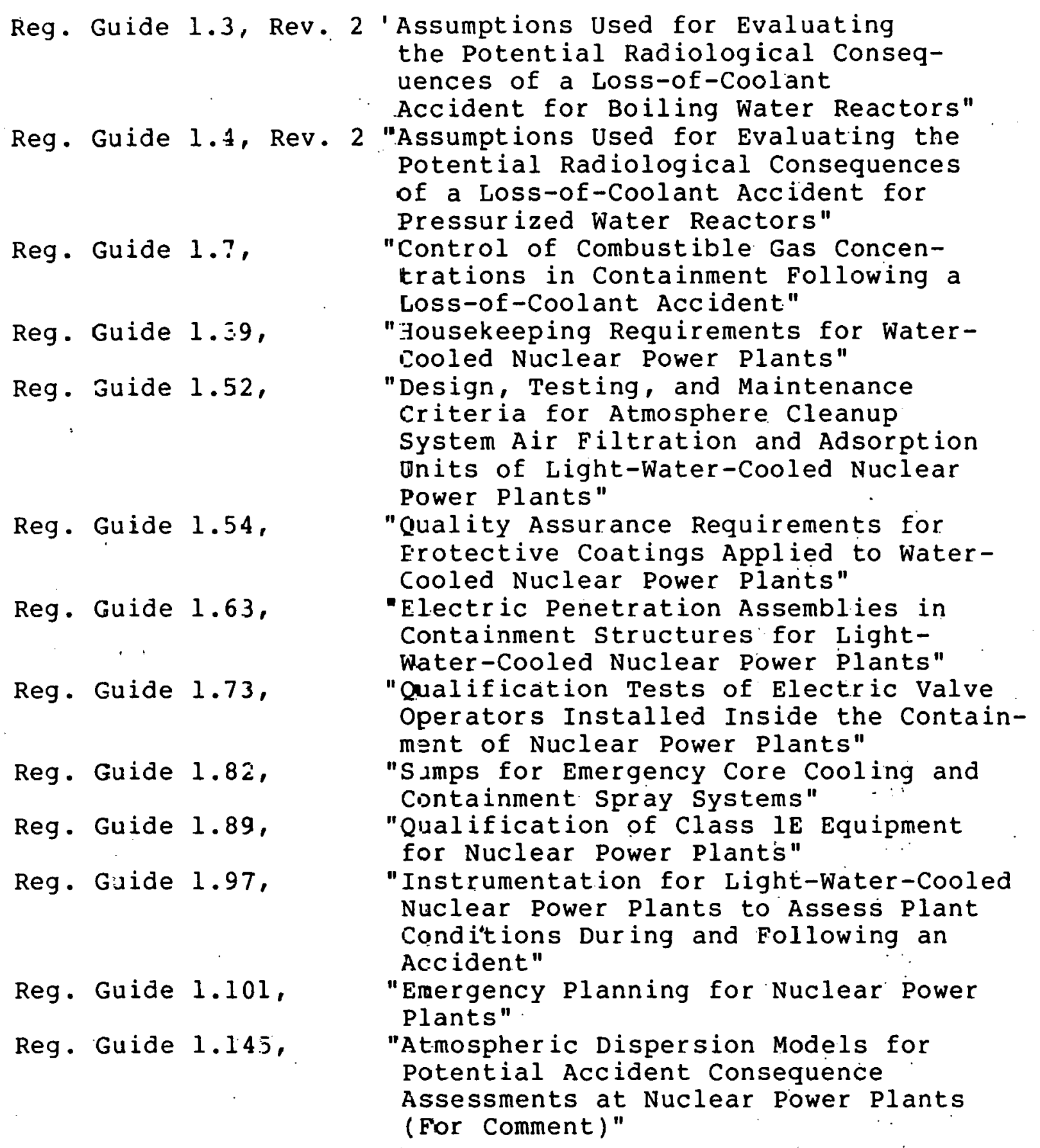


can provide a valuable basis for the re-examination of the fission product source terms and dispersal assumptions contained in these Regulatory Guides. After careful examination of these data, adjustments may be suggested to the assumptions in non-siting-related Regulatory Guides. Such adjustments, if warranted, could be expected to provide, for example: improvements in fission product trapping system designs, a more realistic specification of the postaccident environment, and/or more realistic criteria for emergency planning. Finally, the proposed data collection program would provide a valuable, realistic data base to support on-going efforts within NRC related to the classification of accidents, the revision of siting criteria, and the improvement of emergency prepardness.

II. Specification of Data Needs and Acquisition Procedures

In order to achieve these goals the data collection program must provide quantitative information on the physical and chemical state of the fission products, their partition between liquid and gas, and their location in the containment. The collected data need to answer the following, questions.

- How much radioactive material escaped from the fuel and over what time period did the release occur?

- How much radioactive material was airborne in the containment, and how long did it remain airborne?

- To what extent did the spray system function as designed? 
- Did fallout and plateout occur to the extent expected?

Based on these information needs, samples of surfaces, liquids, solids, and gases need to be analyzed for isotopic, chemical, and physical composition. (I-129 is the single most critical nuclide to be quantified). All of the sources which contain significant quantities of radioactive material need to be assayed. While the examination process will itself help identify the location and types of samples to be obtained, the following list identified the samples presently thought to be important.

- Surface Contamination

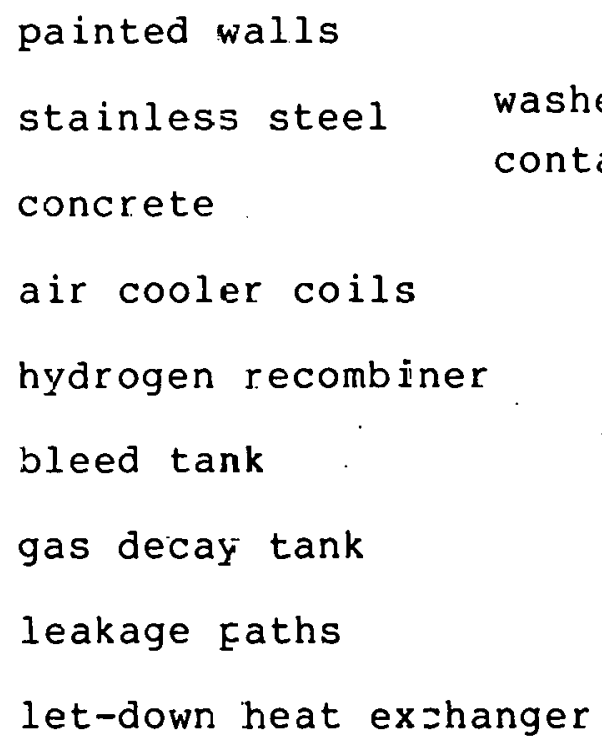

- Liquids

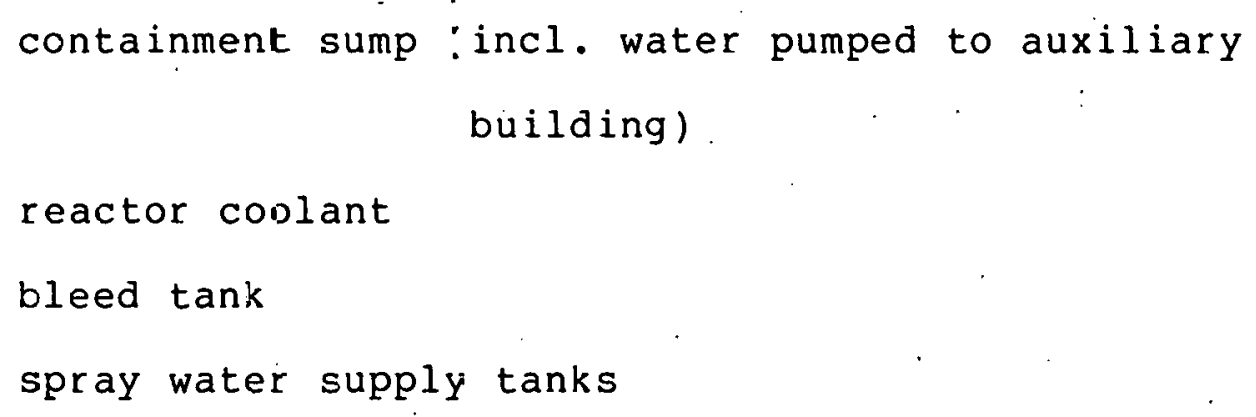


- Solids

charcoal used in auxiliary bldg. ventilation charcoal used in fuel handling bldg. ventilation floating or submerged in sump resins used in let-down system fuel and/or fuel debris

- Gases

containment atmosphere gas decay tanks

3. Priorities

The priority of information needs is related to the quantity of radioactive material at the location in question and the importance of the location in showing transport behavior. While all of the identified samples are important, the highest priority (1) should be given to:

- containment sump

- reactor coolant

- containment surfaces

- containment gas

- fuel and/or fuel debris

Next in priority (2) would be samples from:

- gas decay tank surface

- spray water composition

- hydrogen recombiner surface

Lowest in priority (3) are examinations of

- gas from gas decay tanks

- water from bleed tank

- surface of bleed tank 


\section{IV.B ASSESSMENT OF SAFETY SYSTEMS}

1. Technical Justification

Data on radioactivity transport and chemical specification of both fission product and nonradioactive chemical forms in the containment and auxiliary buildings can provide a basis for assessment of existing safety systems or proposed new safety systems. Eight areas have been identified where data from the Three-Mile Island plant may provide useful information for systems assessment. In order of likely benefit, these areas may be summarized as follows:

(a) Potential effect of sump water debris, sediment, and/ or sludge on cperation of. ECCS and containment spray recirculation pumps.

(b) Effect of containment sprays on surface deposition.

(c) Effectiveness of reactor coolant chemistry control.

(d) Establishment of design criteria for proposed containment venting systems.

(e) Effectiveness of air cleanup and ventilation systems.

(f) Effect on sump water chemistry caused by addition of river water.

(g) Interaction of fission product forms with containment air coolers.

(h) Establishment of design provisions for chemical makeup system to reduce personnel exposure in auxiliary building.

Specific justifications For each of these areas of 'interest are provided in the following paragraphs. 
(a) Sump Water Recirculation. The presence of debris, sediment, and sludge in the sump water could have affected the operational effectiveness of the ECCS and containment spray recirculation pumps in various ways. Solid debris such as wood pieces or large paint chips might have blocked the flow of water through the sump screens or, if the screens were torn or improperly placed, might have been sucked into the pumps. Sand-like sediments might also have presented a hazard to the pumps since they could have penetrated the screens. Sludge or other viscous liquid precipitants could have affected the operational characteristics of the pumps by increasing the viscosity and thermal conductivity cf the working fluid. Thus it is important to assess the physical state of the sump.

(b) Containment Spray Effectiveness: Fission product data from the TMI containment may be useful as a means of assessing the effectiveness of the containment sprays in promoting fission product deposition. The sprays are designed to enhance the rate of deposition for both iodine and particles. Since the sprays operated for 5 minutes following the hydrogen burning, surfaces that were exposed to the spray should exhibit different deposition characteristics than those that were not exposed to the spray. Analysis of the differences should shed light on the effectiveness of the spray in promoting fission product deposition and the mechanisms governing that deposition. 
(c) Reactor Coolant Chemistry Control. The effectiveness of systems designed to control the reactor coolant chemistry can be ascertained by examining the isotopic and chemical constitution of the primary system coolant and surface plateout. The concentration of boron in the primary system should be measured to determine whether a satisfactory delivery and subsequent maintenance of desirable boron levels were achieved. Concentrations of sodium hydroxide should be measured and fission product forms should be ascertained to determine whether fuel leaching contributed a significant source of fission products, and thus whether sodium hydroxide concentrations should be more carefully controlled.

(d) Vent System Design Criteria. Containment vent-filter systems for controlling pressures within containment and hydrogen control systems involving venting from containment are being proposed for the mitigation of consequences from core melt accidents involving severe core damage without melting. The design of certain aspects of these systems (e.g., the filtering media in vent-filter systems) will depend upon the physical and chemical forms of the fission products being vented. It is unlikely that extensive information can be obtained at this time on the fission products which were gaseous or airborn during the accident. Nevertheless, analyses of the plateout on containment surfaces may provide, at least, qualitative information on the forms and concentrations of certain of these fission products during the accident. It is recognized that processes (e.g., condensation, decay, 
flooding) have likely taken place since the accident which could interfere with the interpretaticn of plateout data for this purpose.

(e) Air Cleanup and Ventilation System Effectiveness. The effectiveness of various air. cleaning and ventilation exhaust systems in reducing the airborne fractions of radionuclides can be assessed by examining the amounts and chemical composition of fission products captured on the filters. The ventilation exhaust systems in the auxiliary and fuel handling buildings are pertinent.

(f) Sump Water Chemistry. During operation of the containment air coolers in the emergency mode (i.e., following the hydrogen burning), some 50,000 gallons of $r$ iver water are believed to have been pumped by the zeactor building emergency cooling booster pumps into the containment sump via the air cooler relief valve. The mineral impurities in the river water could have affected the chemistry of the sump water, which might have been important if emergency core cooling recirculation had been required. The concentration and chemical specification of mineral impurities in the sump should be ascertained.

(g) Air Cooler Effectiveness. The effeciveness of the containment air coolers might be influenced by deposition of fission products on the copper cooling coils. Significant amounts of deposition would (1) provide a heat source (through fission product decay) that might counteract the cooling function, and would (2) alter the surface state of the coils. Thus the 
isotopic and physical state of deposits on the cooling coils should be ascertained.

(h) Chemical Makeup System Design Criteria. A primary source of radioactivity exposure to personnel working in the auxiliary building was associated with radioactivity in the chemical letdown and makeup system. Methods of preventing these exposures in similar situations might include: (1) earlier isolation of the chemical letdown/makeup line, (2) upgrade of the system to the 'status of engineered safety features (ESFs), or (3) incorporation of a provision to remove fission products from the letdown line. The second and third measures might benefit from identification of the fission products and their chemical forms.

\section{Specification of Data Needs and Acquisition Procedures}

The nature of the sump debris (i.e., the quantity of the debris and its physical characteristics) should be examined by television cameras inserted through the available penetrations (R-401 and $R-605)$. These cameras should survey as wide an area of the sump surface as possible.

The depth of sludge and/or sediment at the bottom of the sump should also be established by either direct visual. observation (e.g., cameras) or by insertion and withdrawal of a sampling tube. Sluoge or sediment depth should be established for at least 3 different locations in the sump. Water samples should be taken from the sump at various depths and floor locations, including samples taken from the bottom of the sump where sludge and sediment could be collected. 
These samples should be analyzed (offs:te) to determine sediment grain size distributions and fluid properties such as density, viscosity, thermal conductivity, and specific heat. Chemical analyses should also be performed to datermine the concentrations and molecular forms of mineral impurities in the sump water.

Smear samples and gamma-scans should be obtained in the open stairwell area in regions that were exposed to spray (e.g., top of the stairwell) and regions that were not (e.g., lower portions of stairwell). Furthermore, smears and gamma scans should be obtained in the area where the 18-inch line from the reactor coolant drain tank exhausts into the stairwell area. In addition, surface samples should be collected (e.g., cutouts, boltheads, drill chip samples, paint patches), to be used for chemical analysis of isotopes and compounds and for archiving. These samples also should be collected in both sprayed and unsprayed regions in the stairwell area.

Reactor coolant samples should be taken from the primary system and subjected to isotopic and chemical analysis to determine (1) the concentration of boron, (2) the concentration of sodium hydroxide, and (3) the isotopic forms and concentrations of fission products. Swipes and scrapings should be taken from primary system surfaces in order to determine the amounts of deposited boric acid and the amounts and forms of Eission product species plated out on the surfaces. Smears and gamma scans should be obtained from internal 
surfaces of the hydrogen recombiner system, including the associated vent 1 ines and valves. In particular, smears and scans should be taken along the fore and aft vent lines, fore and aft spool pieces, and innermost (toward containment) valve of the hydrogen control vent line. Cutouts from the vent line should be taken near the forward (upstream) containment peretration for chemical analysis of isotopes and compounds and for archiving.

Sections of the HEPA and charcoal filters in the ventilation exhaust system of the auxiliary and fuel handling buildings should be obtained for purposes of isotope/compound analysis and for archiving.

Smears and gamma scans should be obtained from the copper coo:ing coils of the containment air cooler, and if possible, a 12-inch or longer cutaut should be extracted from the cooling coils. The cutout can subsequently be divided into several parts for purposes of isotopic/compound analysis, and for archiving.

Liquid and swipe samples should be taken from the chemical letdown and makeup system for purposes of isotope/compound analysis and for archiving. Samples from the resins and filters, the letdown cooler, and the letdown line would be useful.

\section{Priorities}

The data needs described above for safety system assessment are 1 isted below in order of decreasing priority:

(1) Camera examination of sump debris; camera examin- 
ation or sampling tube determination of sediment and/or sludge depths (first priority).

(2) Sump water samples for determination of sediment and/or sludge properties (first priority) and mineral impurities (second priority).

(3) Smear samples, gamma scans and surface samples from open stairwell area in sprayed and unsprayed (first priority).

(4) Primary system reactor coolant samples and surface swipes and scrapings (first priority).

(5) Smear samples, gamma scans, and cutouts from hydrogen recombiner vent lines, valves, and spool pieces (second priority).

(6) HEPA and charcoal filter sections from ventilation exhaust systems (second priority).

(7) Smear samples, gamma scans and cutout of copper cooling coil from containment air cooler (second priority).

(8) Cutouts and swipes from chemical letdown/makeup system and associated filters (third priority). 


\section{C MODEL TESTING AND DEVELOPMENT}

\section{Technical Justification}

A variety of correlations and computer codes have been developed to help evaluate the radiological consequences of nuclear reactor accidents by predicting the release, transport, and deposition of fission products within reactor primary systems and containments. Information derived from isotopic mass release and distribution data derivable from samples that can be now obtained from within the TMI-2 primary system and containment vessel could help determine the adequacy of such correlations and codes. Limitations exist, however, on the usefulness of the TMI data for validation purposes. The thermal and hydraulic conditions which have a controlling effect on release, transport and deposition cannot be estimated accurately for the transient period. In addition, much of the evidence regarding the early transport and deposition of radioactivity has been literally washed away by the subsequent hydraulic conditions. For these reasons, it is believed that the principal data of interest for quantitative model development or validation relate to the extent and timing of radioactivity release from the fuel rather than to transport and deposition behavior.

Because of the extent of fuel damage and radioactivity release in the accident, the data are probably not of particular value for codes that predict gap inventories or release in less severe accidents (e.g. DBA's). The types of release for which data may be inferred are core melt releases (as being examined 
with simulants in the SASCHA tests) or extended heatup releases at elevated temperatures below melting (as studied in the most recent series of tests at ORNL). In addition, release information for leaching from damaged fuel can be obtained.

It is unlikely that data can be csed directly in the verification of such computer codes as TRAP, CORRAL or NAUA which model radionuclide transport and deposition. Some qualitative information may exist, however, relating to. chemical reactions, physical form and chemical form which would be valuable for modeling purposes.

II. Specification of Data Needs

In general, data are needed to provide isotopic mass distribution and chemical and physical properties of radionuclides within the primary system and containment vessel. A primary goal must be to obtain data sufficient to close a mass balance relating the amounts calculated (e.g., by ORIGEN) to have been present in the fuel with the amounts now present in the fuel and elsewhere. The distribution or these materials released from the fuel is related to their transport, deposition and washout. Since physical form. (gaseous or vapor, particulate) determines predicted transport and deposition rates, it should be analyzed in the samples. Chemical forms of deposited materials are important in assessing sorption and possibly subsequent desorption rates for surface deposits. To analyze release from the fuel and to permit testing of transport and deposition models, sufficient data must be collected to specify the spatial 
distribution of radionuclides within the primary system and containment which are present in gaseous, liquid and solid forms. Specific samples should come from sprayed and unsprayed surfaces within the containment. 'To provide a means for determining chemical forms on surfaces, at least a portion of the samples must be obtained by surface removal. To provide a more complete map of deposition patterns, an extensive set of measurements of deposited concentrations should be obtained from surface activity.

Gas and water samples should be obtained from different locations if there is reason to believe that spatial inhomogeneities exist. This is probably not the case for the gas; however, water samples could exhibit significant spatial variations in concentration. Water samples should be collected from several. containment compartments and, if they exist, from sections of piping isolated at earlier times. Attention should be directed toward quantitatively sampling solids deposits or sludge on the floor of water-containing regions. Surfaces from within the primary system should be collected for isotopic mass, chemical form, and physical form determinations. Such surfaces should include sections of guide tubes, control rods, and steam generator tubes.

filthough examination of the fuel itself is not the responsibility of this effort to identify data needs, the importance of a thorough analysis to determine the inventory by radioisotopes remaining with the fuel.must be emphasized. 


\section{Priorities}

Information needed for testing and improvement of models is nearly identifical whether the goal is to determine releases from the fuel or to assess deposition mechanisms. The major additional requirements for evaluation of transport and deposition models include chemical and physical forms for deposited materials. Of highest priority is the direct measurement of the radioisotopes remaining within the fuel as a function of position in the core. High priority should also be given to the overall distribution of isotopic mass within the primary system and containment. of secondary importance would be thorough analyses of spatially distributed samples for the chemical and physical forms of deposited materials, although analyses for a limited number of samples should have high priority. 
IV.D. EQUIPMENT ENVIRONMENTS

\section{Technical Justification}

Proper functioning of equipment and instrumentation within containment under severe environmental conditions can be very important to the mitigation of accident consequences. The TMI accident offers the opportunity to obtain data on actual accident environments and to evaluate the performance of equipment subjected to those environments. The TMI Examination Task 1.0 planning group will be selecting specific equipment from containment for examination. In order to interpret the results of tests on that equipment it will be necessary to define the environments to which the equipment was subjected. The present planning group (Task 2.1) is identifying the data required to estimate these environments.

II. Specification of Data Needs and Acquisition Procedures

The important environmental conditions to be described are pressure, temperature, humidity, flooding (including caustic sprays) and radiation field as a function of time. The description of pressure, temperature, humidity and flooding must be obtained from plant records or inferred from analysis. The extent to which investigations of the existing conditions in containment can be used to infer the past thermodynamic conditions is limited.

Recordings of containment temperatures and pressures as a function of time in the accident are available and can, in general, be used to predict equipment environments. The 
relative humidity as a function of position within containmert can also be determined from analysis of these temperature and pressure data.

The available records, however, probably do not provide the local temperatures which were generated during the hydrogen burn. Evidence of the maximum temperatures might be obtained by visual examination. Scorched or blistered surfaces would provide this evidence and samples. should be collected of any such damage observed in the vicinity of key equipment. of the conditions affecting equipment performance, the most important from the viewpoint of data needs is the radiation environment. Both the gamma and beta fields must be described for each piece of equipment. The sources that must be examined are airborne, surface-deposited, shine from neighboring sources, and dissolved sources for submerged equipment.

At the time of reentry, the only data on radiation environments which can be obtained directly from containment samples will be current levels and, possibly, total dose. Smear samples and directional dose rate measurements or gamma surveys should be taken on and/or near each piece of equipment which is accessible and has been selected for study. The smear samples will help define the current radiation levels due to locally deposited contaminates. Smears should be taken directly from the equipment and its supporting surface or structure. The smears should be isotopically analyzed for data on both beta and gamma sources. 
The directional dose rate measurements or gamma surveys will help define the radiation environments due to airborne sources and neighboring sources, or hot spots. These measurements should at least be obtained in the direction of the sump and immediate horizontal and vertical surfaces.

Surface samples should be obtained in the vicinity of each piece of selected equipment. These samples should be subjected to laboratory analyses to determine isotopic concentrations of all contaminates present in significant amounts.

For determining equipment qualification limits, data on total dose can be very important. If radiation monitors were operative and recording accurately during the accident, then total dose (at the monitor location) can be determined easily: The monitor data, however, will probably not be sufficient to determine dose to various instruments if significant spatial dependence is found for the radiation levels. The smear samples recommended above will help determine the spatial uniformity of contamination.

Several indirect methods have been suggested for determining the local total dose. These methods are based on radiation induced degradation of (1) electrical cable elasticity, (2) semiconductor properties and (3) optical properties of glass. Samples, should be taken for these tests in the vicinity of equipment selected for study (and/or near smear sampling locations). Since these methods are indirect dosimetry techniques, it is recommended that the samples 
taken for these tests be placed in archives. In case more direct tests and/or analyses cannct provide the desired information, or in order to provide confirmatory data, these samples may be retrieved and tested.

III. Priorities

The sampling procedures recommended above should be implemented in the vicinity of equipment chosen for study by the Task 1.0, Equipment Survivability, planning group. That equipment will be prioritized according to its importance from a survivability standpoint. The priority of the environmental data for a given piece of equipment should be the same as the priority of that equipment for survivaility studies.

The data or samples which are recommended to be taken for each piece of equipment are summarized below:

1) smears: from equipment directly and from supporting. structure

2) Directional dose rates:
A) Toward and away from equipment
B) In plane of supporting wall
C) Toward sump

3) Total dose: 6 in. length of electrical cabie close to equipment, samples of glass (e.g., light bulbs)

4). Surface samples: from adjacent supporting structure The dome monitor $(\mathrm{BP}-\mathrm{R}-214)$ in the reactor building is a key instrument in reconstructing the radiological sequences of the accident. The accident began with a mis-interpretation of this instrument and its response continues to be a source 
of disagreement. Therefore, very careful and special attention must be paid to it during the recovery effort. A program consisting of, but not necessarily limited to, in situ testing and partial repair, and recalibration followed by more in situ testing should precede removal of HP-R214. A duplicate monitor should be procured for comparison purposes. Every effort should be made to reproduce the accident conditions in obtaining an empirical calibration for comparison purposes with theoretical calculations, e.g. gaseous or at least planar gamma ray sources (not point sources) should be used. Only by very careful attention to detail will the questions about this monitor's response be answered. Because of the importance of the dome monitor to the present Task 2.1 , it is requested that particular attention (high priority) be given to this instrument by the Task 1.0 planning group, whose responsibility it is to recommend reccivery, examination and testing of equipment from containment. 


\section{IV.E. ACCIDENT DIAGNOSIS EFFORTS AND NEEDS}

\section{GENERAL}

The suggestions offered here focus on the acquisition of information and data that should be of particular value for accident diagnostic purposes with respect to the jehavior of the fission products. Since the initial events of the TMI-2 accident occurred over seven months ago, it may appear somewhat doubtful that further sampling and data acquisition at this point and later will yield sufficient new information to justify the effort. However, many aspects regarding the behavior of the fission products throughout the accident and its aftermath remain unclear or unknown. Therefore, an attempt was made to identify work that could be done which might provide some useful insight in these areas so that we learn as much as possible from this unfortunate event. Some thought was given to data gathering procedures which would tend to minimize (1) interference with the plant recovery operations and (2) radiation exposures to working personnel. The following sections outline sampletaking efforts in several areas, including attempts to identify the need and the general features of the sampling and analyses strategies that might be used in each case.

\section{GAS SAMPLES - CONTAINMENT}

$\underline{\text { Need }}$

Numerous gas samples of the containment atmosphere have been taken since the early days of the accident, but apparently all of them have been extracted through a long sample line that runs from the dome region of the containment structure. Analyses 
have concentrated on noble gas radioactivities and the bulk gases $\left(\mathrm{H}_{2}, \mathrm{O}_{2}\right.$. and $\left.\mathrm{N}_{2}\right)$ although a few iodine species samples have been analyzed. At present the only fission gas activity expected to be left in the containment atmosphere is $\mathrm{Kr}-85$. However, it would be wise to characterize the containment gas composition as completely as possible before various decon procedures are used and/or before possible. purging and containment opening occurs.

\section{Sampling strategy}

1) Obtain duplicate gas samples from at least two widely separated regions of the containment space (if possible) using short sample lines (if possible) before the current "steady state" condition of the atmosphere is perturbed by any major action connected with plant recovery operations. The objective would'be to check uniformity of atmosphere composition and minimize effects (if any) of sample line surface area on composition measurements.

2) Gas samples should be collected in high integrity containers. such as glass break-seal tubes which are clean and outgassed before use. There is probably not much value in retaining any of these samples for archive purposes because sample integrity for a long period is hard to assure.

3) Similar gas samples might be taken after major steps in the containment decon effort to determine the effects of the decon procedure on the gas composition. However, these samples may not be of much value for accident diagnosis unless supersaturation of spray liquids with non-condensable gases were indicated. 
Analysis strategy

1) The samples should be analyzed by gamma-ray spectrometry for $\mathrm{Kr}-85$ content and/or other radioactivities.

2) The samples should be analyzed:by mass spectrometry in an effort to identify the components of the gas mixture; i.e., principal and trace gaseous and vapor species. Gas chromatography might also be used to help identify important molecular species.

\section{GAS SAMPLES - AUXILIARY BUILDING}

Need

No specific need for samples which could be of value in accident diagnosis were identified for this plant location.

$$
\text { WATER SAMPLES - CONTAINMENT }
$$

Need

The set of water samples obtained from the lower level of the containment (sump) in late August provided very valuable information about fission product releases during the TMI-2 accident. However, only one location. was sampled and only limited information was obtained about the character and composition of the sludge which lies on the bottom of the water pool. Therefore it would be very useful to have data from one or more other locations in the sump to determine the uniformity of the liquid composition. Also, some type of core sample (or a way of collecting the sludge from a known cross-sectional area) would be quite useful. In addition, samples from other possible water pools (maybe localized puddles in the refueling canal) might, on analysis, 
reveal different radionuclide compositions than those in the large sump volume. Such differences would probably provide some data, although indirectly, about the airborne radioactivities that occurred during the accident. It is likely, though, that interpretation of these latter results in terms of very early accident behavior might be quite difficult because of the time factor.

Sampling Strategy

1) Much of the sampling strategy is determined by limitations on adequate access to the containment sump. If Penetration 605 could be used, then a set of liquid samples should be extracted. A core type of sample should be taken via Penetration 401 if a technique can be developed to do so. So far, a good plan has not emerged for doing this. In either case sampling should be done before decon action is started.

2) Water samples from miscellaneous puddles at higher containment levels probably will require containment entry. However, these samples would be of no value if liquid decon or wash-down procedures are used before containment entry is made. This is not a high priority item, but if samples are taken the sampling should be done in duplicate. Sample volumes should be at least $50 \mathrm{ml}$ if possible. Analysis Strategy

1). Liquid samples should be analyzed as completely as possible for radionuclide content using gamma-ray spectrometry and radiochemical separations procedures to obtain samples for beta counting. In addition to cesium, strontium, 
iodine, and tritium radionuclides, efforts should be made to determine isotopic concentrations for tellurium, ruthenium, cerium, and zirconium-niobium species.

2) Chemical characterization of the composition of the liquid samples should be done using qualitative analysis procedures, emission spectrometry, and other standard measurements such as pH. If sludge samples can be recovered, the chemical composition of the solid should be identified through qualitative analysis and emission spectrometry. WATER SAMPLES - PRIMARY COOLANT

Need

Many primary coolant system samples have been taken and analyzed since the early pahses of the accident. The practice should be continued on a regular basis in order to accumulate further data which can be helpful in establishing the rates for radionuclide release by fuel leaching. Sampling strategy

Continue sampling on a weekly basis until the primary system is flushed or opened. Analysis strategy

Isotopic analysis by gamma-ray spectrometer and beta assay for cesium, strontium, ruthenium, and cerium isotopes. WATER SAMPLES - AUXILIARY BUILDING

Need

Since sampling of contaminated water stored in various tanks in and around the Auxiliary Building has been a routine procedure connected with plans to process this waste water, it 
does not seem necessary to suggest additional samples. The only recommendation that might be made would be to encourage as complete a radionuclide analysis of the samples as possible. This would include the use of activation analysis to determine I-129 concentrations and efforts to measure ruthenium and cerium radioisotopes.

\section{SUREACE DEPOSITION SAMPLES - CONTAINMENT}

Need

This is considered to be the most important series of samples that can be collected since only two such samples have been withdrawn up to now. Deposition samples have the potential for indicating how much airborne radioactivity existed, for telling us something about the timing of releases, for completing radionuclide activity balances in the system, and for indicating circulation paths in the containment space. The problem is obtaining enough samples and from the proper locations without severely impacting the plant recovery timetable and/or causing unwarranted radiation doses to working personnel. Sampling strategy

1) It is doubtful that meaningful samples could be obtained without entering the containment itself. In order for personnel to enter the reactor building (RB) it would seem imperative to first remove the airborne $\mathrm{Kr}-85$ from the RB. This might be accomplished by purging or by circulating the RB atmosphere through some KR-85 removal system and returning the cleaned gas back to the RB. Whatever approach is used, it is recommended that the moisture content of the RB atmosphere 
be maintained as close to the pre-treatment value as possible in order to avoid upsetting conditions at the air-surface interfaces which might trigger desorption of some deposited activity.

2) If or when the containment is free of $\mathrm{Kr}-85$, surface deposited radioactivity measurement work should begin. That is, these data must be obtained before any surface decontamination is done by injecting steam, water sprays, or chemical sprays. The opening of containment for possib-e personnel entry will likely be followed immediately by radiation surveys of varying extent. Only after the results of these surveys are available will it be possible to determine whetier personnel can enter, or if they can, how much working time will be available. If personnel entry before decon action were to be not allowed, then perhaps some remote method of acquiring surface deposition data could be used. The only idea at present would utilize something like a remote (cable or electronic signal) controlled traveling unit, equipped with a shielded and collimated detectcr, which could be positioned near walls or over various floor areas to record gamma-ray spectra of radioactivity fixed on these surfaces. Such a survey apparatus may not be feasible, too expensive, or too time consuming to be of practical-value. The concept and any apparatus that might result would have to be carefuily evaluated and checked out (calibrated, practice missions, etc.) before being used. Alternatively, the best strategy might simply be to abandon any idea of trying to obtain data under such conditions. 
3) If it should be determined that personnel could enter the RB for the purpose of obtaining measurements or samples then planning saould be done to minimize the time spent on such activities. The following suggestions are made with this restriction in mind.

a) Develop and test in an outside laboratory a semi-portable, calibrated, collimated survey instrument which could be positioned at a fixed distance from any surface in order to obtain rather rapidly a deposition profile throughout accessible regions of the containment. Ideally, the instrument could record gamma-ray spectra for subsequent unfolding analysis. Such data could be valuable in revealing circulation patterns in the vessel. Such surveys would also reveal "hot spots" on horizontal surfaces which might indicate residue from evaporated liquid puddles.*

b) Divide the containment into quadrants or 120 degree sectors and obtain deposition samples from a vertical and horizontal surface in each sector on each floor. Cover two floors the operating fioor (Elev. 347') since most of this region was sprayed, and the ground floor (Elev. 305') because large areas of this region were not sprayed (see TMI-2 FSAR). The depcsition samples could consist of easily removable pieces of hardware, equipment housings, and gratings or hatch covers (painted or unpainted). Such samples should be placed in specially designed containers with covers or spacers to protect the deposition surfaces prior to analysis. Samples of concrete (drill chips or chisel ships) should also be obtained. The whole sample gathering operation should be well planned and rehearsed to minimize the exposure time for workers. Vo-unteers might be requested to perform this particular work.

*However, possible external contamination of the detector in such an apparatus could render it essentially useless. 
c) Alternatively, surface samples from the sectors identified above might be obtained by using swipes or smears and adhesive (cellulose acetate) particle pickup samples. These would give only qualitative or perhaps semi-quantitative data because reproducible samples are hard to achieve, and because only a fraction of the surface activity is collected. The advantage is speed and small sample size. Perhaps a reproducible method could be devised for scraping the faint off a fixed area of flat surfaces.

Analysis strategy

1) The objective of analysis of deposition samples would be measurement of the total amount deposited on a known area of a surface for each measurable radionuclide. Large samples should be sectioned without raising material temperatures or disturbing the deposition surface except along the cutting Iines. Samples should be examined for particulate deposits and the particulate morphology should be characterized, if possible. Sectioned specimens might be radioassayed directly or subjected to chemical reagents (acids or complexing agents) to remove and dissolve the radionuclide deposits. The solutiors would then be radioassayed.

2) Radioassay should use gamma-ray spectrometry, beta analysis, and maybe activation analysis for iodine for quantitative determination of individual radionuclides such as Cs-134, Cs-137, Sr-90, Ru-106, Ce-144, and maybe I-129.

3) Archive specimens might be saved but careful storage practices would have to be followed to preserve the deposition surfaces. Also, some samples might be reserved for testing the effectiveness of various decon procedures that could be ussed for the general containment cleanup process. 


\section{EQUIPMENT REMOVAL FROM CONTAINMENT}

$\underline{\text { Need }}$

The removal and subsequent examination of certain pieces of equipment from the RB could provide valuable data for reconstructing radioactivity release and redistribution in the containment during the accident. Two pieces of equipment can be identified; the RB dome monitor (HP-R-214) and the containment cooler units. The dome monitor unit should be retrieved for inspection since it provided radiation level data during the early days of the accident. The integrity of the lead shield around the detector should be examined in order to better define the gamma-ray response (the attenuation factor) for the unit. The cooler units may contain a significant fraction of the iodine that was released from the core deposited on their internal surfaces, particularly the copper alloy cooling coils. If examination confirms this suspicion, the data would be valuable in closing the activity (material) balánce for radioiodine.

Sampling Strategy

1) It appears that dome monitor recovery could be done after containment decon procedures have been performed. Thus, timing is not an important factor for this item. The same may be true regarding removal of a $\mathrm{RB}$ cooler unit since the surfaces of the cooling coils are probably protected from decon sprays by the unit housing. However, if steam cleaning is performed, the coils will be exposed to the steam, and perhaps lose a portion of the deposited activity. Therefore, 
some reason exists for trying to remove a RB cooler unit before decon efforts start.

2) The advisability of removing a cooler unit can be assessed only after sufficient radiation surveys have been done to indicate the degree of hazard involved in the operation. If possible, this survey effort should include individual survey of each of the five units so that the results of any detailed examination of one unit can be extrapolated to the others to provide crude estimates of deposited radioactivity for the entire cooler system. The exact disposition of a cooler unit depends on several factors: the radiation level, the unit design, the demounting procedure; and whatever must be done to get a unit out of the RB itself. These issues have received no appreciable evaluation at this point. However, if containment entry by workers is possible, and if radiation surveys indicate the cooler units are important radioactivity deposition sites, and if removal and shipment of at least one unit for laboratory or hot cell exams is feasible, it is recommended that the project be strongly considered. Analysis Strategy

1) The dome monitor inspection should include study to attempt to determine when and why it may have stopped giving usable radiation readings and should include evaluation of the details of the lead shield around the detector.

2) The analysis of a RB cooler or its parts should attempt to determine how much radioiodine and other species such as cesium, strontium, etc., is deposited on the interior 
surfaces. Direct gamma ray radio-assay or analysis of chemical etch solutions might be used. Iodine deposition can probably be determined only by using activation analysis methods.

\section{FUEL SAMPLES}

Need

Eventually, samples of core material must be obtained and subjected to comprehensive radiochemical analysis to obtain measurements of the radionuclide inventory that still remains in the fuel. These data are essential for obtaining a material balance. for the fission products.

Sampling Strategy

Samples of fuel should be obtained from various locations in the reactor core and from regions below the core (if any fuel is found there) so that a sufficient set of fission product inventory data can be developed to help identify the spatial patterns or boundaries of the damaged fuel region(s). The exact sampling grid can only be determined after access to the core has been achieved and visual (TV or other) surveys have revealed the specific conditions that exist. Analysis strategy

Fuel samples from known locations should probably be dissolved (with collection of evolved gases) and then radiochemical analyses performed for fission products having different thermophysical and chemical properties. Examples are Cs-137, Sr-90, Zr-95, Ru-106, Ce-144, and Pu-238, although others may be possible. The gases collected during specimen dissolution should be analyzed for $\mathrm{Kr}-85$ content by radio- 
assay, or for stable noble gas ( $\mathrm{Xe}$ and $\mathrm{Kr}$ ) content by mass spectrometry. The objective of the analyses would be to establish the amount of fission product release that occurred from the damaged fuel for the various types of fission products and to map regions of the core having. similar radionuclide release values.

\section{PRIORITY RANKING}

The six areas of sampling and data work discussed above have been ranked roughly in terms of priority of information and also in terms of a judgment regarding the ease of obtaining the needed data. The results of the rankings are given in the following table.

Sampling Area

Containment Gas

Containment water

Primary Coolant Water

Surface Deposition in $R B$

Equipment Removal - RB

Core Fuel Debris

,

(a) Highest priority $=1$

(b) Easiest $=1$

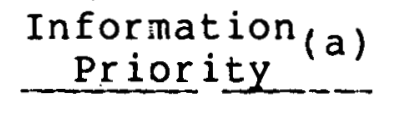

Ease of Data
Acquisition (b)

2

3

2

4

1

1

3

2

4

1

4 


\section{IV.F. DECONTAMINATION}

1. Introduction/Background

During the TMI-2 accident, the fuel was subjected to very high temperatures and such operation led to the rupture of the fuel cladding with resultant discharge and distribution of fuel rupture debris and fission products into the primary system and other parts of the containment system. The cleanup of the rupture debris and fission products that are in the containment structure and the primary system provides a unique opportunity to determine information that has a generic impact on the design of future reactors and component systems. It is essential therefore that the decontamination of this TMI containment and primary system be undertaken in such a way that a maximum amount of information can be acquired. This means that all allied operations as well as the cleaning activities be identified, planned, conducted and evaluated to achieve desired objectives. Especially important is the allied effort of thoroughly mapping radiation levels throughout the containment system prior to decontamination and following all major cleanup activities.

2. Technical Justification

The purpose of the proposed work is to provide generic information that should lead to better reactor containment and primary systems design. Such designs should make decontamination easier be it for accidents such as occurred at TMI or more routine cleanup activities to reduce occupational exposure. A careful assessment of the effectiveness 
of various decontamination techniques and analyses of the factors affecting each of the constituent steps will provide a. useful and much needed informaticn base on decontamination operations. Acquisition and analysis of sludge and adhesion samples specified in this work will also be useful in identifying and specifying cleanup procedures for TMI-2. The data acquired during the radiation mapping of the containment and primary systems will also hel? establish expected occupation exposure for other generic work in and around these systems. The TMI recovery operation should benefit from the detailed radiation measurements that are proposed for the mapping program. It is extremely important for the generic use of these data that they be acquired before containment system decontamination is started.

3. Speci.f ication of Data Needs and Acquisition

There are three types of tasks that should be undertaken t:o acquire the needed data for work in this area. These are listed below and were given more detailed treatment in sections II and III of this report:

Task 1: Data should be acquired on RCS sludge samples from the reactor vessel and primary system.

Task 2: Information should be acquired on radiation mapping and distribution in the containment system. Task 3: The decontamination operation should be carefully monitored to provide a maximun amount of information that can be of use to other operations 
of a similar or allied nature.

The data obtained in the first and second of these tasks will provide useful complementary or predecessor information for work proposed elsewhere in this report.

4. Priorities

The highest priority should be given to the radiation mapping of the containment systen and the acquisition of sludge samples from the reactor vessel and a low spot in the primary system. The monitoring of decontamination efforts, effectiveness and costs is also extremely important. The demanding need for these decontamination data will be supported by a position paper that will be forwarded to another task group to ensare that proper attention and emphasis is placed on this work. 


\section{IV.G MASS BALANCE}

\section{Technical Justification}

It is desirable to determine, as accurately as possible, the current mass balances of significant radiologically toxic species for two primary reasons: (1) such exercises guarantee the completeness of the studies, and (2) mass balance determinations ensure that all important sinks and attenuation mechanisins have been identified. Although it is perhaps intellectually satisfying to perform detailed mass balance determinations, considerable reduction in the effort required can be made on the basis of technical sufficiency. Thus, the species selected for mass balance considerations are to be selected based upon the following criteria: (1) significance from the standpoint of safety to the general public; (2) significance from the standpoint of personnel exposure during cleanup;decontamination operations; (3) significance from the standpoint of personnel exposure during normal plant maintenance; and (4) extent to which the species represents other species of similar chemical behavior.

II. Specification of Data Needs and Acquisition Procedures

Using the criteria presented, the chemical species which are to be given priority in mass balance determination exercises are as follows:

1. Helium - because of its direct relation to the number of failed fuel elements.

2. Krypton - because of its potential ease of release from the containment. Also, its chemistry is characteristic of xènon isotopes as well. 
3. Tritium - this nuclide provides a basis for comparison of all water scluble fission product species

4. Iodine - the dominant short term radiotoxic species in most postulated accident modes. Perhaps the most complex species chemically. Similar to bromine.

5. Cesium - a "semi-volatile" species which is easily released when defected fuel has attained high temperatures. It is highly soluble in the coolant and is the dominant radiotoxic species from the standpoint of cleanup/ decontamination and maintenance under many postulated accident scenarios.

6. Tellurium - a moderately volatile species which, unlike cesium, is not readily solubilized and can therefore become a dominant contaminant for specific equipment or locations.

7. Ruthenium - genezally released only under highly oxidizing conditions and very difficult to solubilize. Can be employed with cerium and uranium determinations to assess extent of fission product redistribution.

8. Strontium - Domirant beta-emitting species of low volatility but reasonably soluble (in oxide form) in water. Carbonate form is orders of magnitude less soluble. Possibly a major sludge component.

9. Barium - low volatility species but highly soluble (in oxide form) in water and therefore a good indicator of release by leaching of fuel. Carbonate is four orders of magnitude less soluble than the oxide. 
10. Cobalt - major activation product.

11. Silver - major component of control rods and has a high propensity for iodide and iodate, which causes precipitation of the dissolved iodine species.

12. Uranium - a typical refractory group species, it is also the primary carrier of most of the radioactivity in the core.

The system over which the mass balances are to be performed can be subdivided in many different ways, with varying degrees of detail. The final strategy is best developed later, however, as the tasks outlined in section II are reviewed and are considered within the context of the decontamination program. For example, it is possible to estimate fission product distributions on the surfaces of the primary circuit by examining the circuit component by component, with emphasis on the steam generator region. In all likelinood, however, it will not be possible to do this. Rather, the entire primary circuit will probably be subjected to decontamination as a unit. If such be the case, the sampling strategy will then involve determinations of the volumes and species concentrations of the decontamination solutions, rather than analysis of circuit surfaces.

III. Priorities

Sampling :strategy priorities are to be given to those features which, because of the volumes/surface areas involved (e.g., sump, fuel region, liquid storage tanks), or because the component performs a unique function which tends to concentrate released material (e.g., cooler units, purifi- 
cation/cleanup components, etc.), can be reasonably expected to contribute significantly to the mass balance result. priorities with respect to species vary because of the different nature of the samples selected for analysis. Moreover, it may be necessary to reexamine this aspect as the program proceeds. In general, however, highest priority should be given to accurate mass balances for iodine, uranium, krypton, and cesium, in approximately the order listed.

$2.1-82$ 


\section{IV.H. HEALTH PHYSICS}

\section{Technical Justification}

The bulk of the basic health physics needs will be generated by the other identified tasks. Hcwever, it must be stressed that no operation or activity will be conducted unless it can be done safely and at minimum personnel exposures. The potential surely exists for a maximum allowable exposure bank which cannot be exceeded but may effectively limit total activities, including sampling. We do not want to advocate such a bank but we must be cognizant that it may be established. The point is that we may be required to do essentially a cost-benefit analysis for many of the proposed activities. With that in mind, the following health physics data needs are presented.

II. Specification of Data Needs and Acquisition Procedures

1. Health Physics Data Needs. Quantity, quality and distribution of all radiation sources need to be determined. As a minimum, an isotopic determination of the fission products and any TRU's is necessary.

2. Personnel Dosimetry Program. There is a high probability that dosimetry needs will change as a function of decontamination processes and progress, areas and locations within the facility, and of the individual components. It is envisioned that in the beginning one will be worried about high dose rates typical of the gamma emitting components of the fission products. But as 
these are removed by decontamination efforts to much lower values, then one may only be concerned with residual beta contamination. If this turns out to be the case, then the same dosimetry program for personnel protection may change, including the needs for whole-body counting and bioassay procedures.

3. Records. Records must be a first-class system kept current and complete and must be defensible for a long period of time. These records must stand long after TMI recovery operations are completed. They must not only include the exposure records for each individual but should also include a running history of job-related activities.

4. Criteria. Establishment of criteria relating to the level of decontamination required must be generated. This is especially important for areas and components outside the primary system. Criteria relating to residual sources of contamination based on activity rather than exposure rate are probably less stringent than one normally employees. Methods and means of determining the residuals must also be established.

5. Waste. The development of waste handling plans, including transportation, for occupational exposure control must be developed. Most certainly onsite storage, at least temporarily, will probably be required. That could result in another source of personnel exposure which, if not treated adequately, 
could result in unnecessary restrictions.

6. Area and Effluent Radiation Monitors. A review of the location, accessibility, and usefulness of both area and effluent radiation monitors must be accomplished. Did they do what they were supposed to do or did they fail? Are they located correctly and were there enough of them for adequate coverage? Answers to these questions would and should be utilized for future instrument needs.

7. Eailures. Of the failures that did occur, were they the predicted ones? If not, can this be factored into the predictive models for future projections of failures. In addition, from what was detected, could one predict what failed, especially if the measurement was exposure rate.

8. Contingency Plans. Last but not least, plans must be in case of mishaps that might occur during sampling, decontamination, waste handling and recovery operations.

III. Priorities

The needs outlined in items. II. $1,2,3,6$, and 8 , above, are considered to be of highest priority. Items II. 4, 5, and 7 identity needs of a somewhat lower priority. 
IV.I. TMI FUEL EXAMINATION FOR FISSION PRODUCT RELEASE

I. Technical Justification

The purpose of this section is to recommend the types of data and measurements needed to characterize the release of fission products from the TMI-2 core. Another objective is to recommend analysis and experimentation that will be useful in understanding and interpreting the release data obtained during examination of the TMI-2 fuel.

The data described in this section are needed for the following purposes:

A. To develop a detailed time-dependent and location-dependent map of radionucludes.

B. To evaluate the release and transport of fission products from the core region to the containment.

C. To compare with regulatory guidelines, and to guide the development of new accident analysis source terms.

D. To verify/develop fission product source term models for use in risk assessment studies.

E. To gain insight into fission product release phenomena under full scale LWR accident conditions.

The technical working group has established a Fuel Experiments and Examination Planning Group to plan and oversee the acquisition of data.necessary to characterize the condition of the TMI-2 core, and to evaluate the type and amount of core damage. The recommendations included in this section should be transmitted to the Fuel Examination Group for inclusion in their core examination plant. 
II. Specification of Data Needs and Acquisition Procedures The following sampling strategy and measurements are recommended:

1. Reasonably intact fuel rods should be given full length gamma scans.

2. Fission product concentration (by isotope) by dissolvirg fuel samples and counting with a gamma spectrometer.

3. Using transmission and scanning electron microscopy (SEM): characterize the fission gas bubble concentration and distribution, porosity fraction, porosity interlinkage, cracking, grain separation, etc.

4. Using SEM, electron microprobe, and/or ion microprobe, characterize the distribution of solid fission product species (e.g., cesium and iodine).

5. Examine the fuel cross section for nonhomogeneous distribution of fission products (i.e., within the fuel matrix, within gas bubbles, at grain bcundaries, or associated with metallic or oxidic inclusions).

6. Determine, where possible, the chemical form of the fission products (i.e., oxide, metal, etc.).

7. For samples which have experienced cladding melting and fuel liquefaction, identify and characterize the partitioning of fission products between the oxidic and metallic phases.

8. Analyze metallic core components for fission product "gettering" (e.g., 1. exposed silver from the control rods for iodine gettering and, 2 . the amount of tellurium 
associated with the cladding as a function of cladding oxidation state.)

9. For each sample analyzed, a sibling sample should be transinitted to the TMI-2 sample archive. Each of the fuel sanples subjected to the above examinations should be characterized for:

a) fuel oxidation state,

b) cladding oxidation state,

c) fuel grain sizes, type, separation, etc.,

d) if fragmented, the size and surface area (BET) of the fragments;

e) condition of fuel sample (i.e., which of the seven classes described below does it fall into),

f) estimate of peak fuel temperature attained.

It can be expected that there exists an axial and radial gradation of fuel rod damage.

Moving radially inward from the fuel periphery to the core centerline, one may expect to encounter the following regions of fuel rod damage:

A) ballooned, but intact fuel rods,

B) ballooned and burst fuel rods,

C) bällooned, burst, and oxidized fuel rods,

D) flel rods with large segments of oxidized cladding missing, but with fuel still reasonably intact,

E) fuel rods where large sections of cladding and fuel are missing (i.e., fragmented fuel pellets and oxidized cladding rusble), 
F) regions of fuel rods which have experienced cladding melting and fuel liquefaction via the $\mathrm{zr}-\mathrm{zrO}_{2}-\mathrm{UO}_{2}$ eutectic formation, and

G) a region near the bottom of the core where unballooned and unoxidized fuel rod segments exist.

In order to characterize the total release of fission products from the TMI-2 core, samples must be taken and examinations perforined for fuel rod segment from all seven regions. A sufficient number of samples (3) from each of these regions should be taken to assure that fission product rælease from each region can be adequately characterized. In addition, the percentage of the total core within each of these groups must be determined in order that a fission product mass balance can be performed.

III. Priorities

Items $1,2,3,4,8$, and 9 are considered high priority. Items 5,6 , and 7 are of lower priority. 
V. DISCUSSION OF PROCEDURES

Four general classes of samples will be submitted for analysis during the course of this exercise; these include gas samples, liquid samples (possibly containing suspended matter), solids, and samples containing surface-deposited material: Analytical methods which can be employed to determine. the composition of each of the four classes of samples are identified below:

Gas Samples

1.. $\quad{ }^{85} \mathrm{Kr}$ counting

2. Mass spectrometry (species fractionation may be necessary to concentrate some of the components)

3. Gas chromatography

Liquid Samples

1. Gamma-ray spectrometry (before and after $\mathrm{F}(\mathrm{OH})_{3}, \operatorname{Pr}(\mathrm{OH})_{3}$, or other scavenging precipitation)

2. Radiochemical analysis for strontium, tritium, and iodine

3. Gross alpha.

4. Spark Source Mass Spectrometry (SSMS)

5. Isotopic Dilution Mass Spectrometry (IDMS), primarily for uranium and plutonium isotopic assays

Solids

1. Activation analysis

2. Autoraaiography

3. Scanning Electron Microscopy (SEM), X-ray fluorescence (XRF), Transmission Electron Microscopy (TEM), and optical microscopy 
4. Gamma-ray spectrometry

5. Dissolution, then selected analysis methods as described under "Liquid Samples"

6. Laser-Raman spectroscopy (Raman microprobe)

7. Infrared spectroscopy (Total reflectance)

\section{Surfaces}

1. Autoradiography

2. SEM and XRF

3. Gamma-ray spectrometry

4. Alpha particle track analysis

5. Leaching, then selected analysis methods as described under "Liquid Samples"

6. Laser-Raman spectroscopy.

7. Infrared spectroscopy

8. Secondary ion mass spectroscopy (SIMS)

9. Electron spectroscopy for chemical analysis (ESCA)

A general discussion of sampling and analysis procedures is presented below. Details of the procedures are not included since these will, in most cases, be specific to each sample. Collection of Liquid Samples:

Prior to sampling, the piping and instrument diagrams $\left(P \& I D^{\prime} S\right)$ of the tank or system should be examined to determine where samples should be taken, the lengths of sample lines, and tank volumes. Next, inspections of the systems should be made to verify the efficacy of the sample points and locations selected. Whenever samples are taken, the sample line must be adequately purged to assure that a 
representative sample is obtained. This involves purging the line for at least three line volumes before taking a sample. When sampling from a tank, the tank should be recirculated through two or three tank volumes, if possible.

If valve settings must be altered prior to sampling (e.g., if purging requires a high flow rate that must be reduced during sampling), the valve must be purged prior to obtaining the sample in order to remove the particulate matter loosened by altering the valve setting. Any change in a valve setting can cause a crud burst. This crud burst must be eliminated before a sample is taken, otherwise the sample . may not be valid.

All liquid samples should be collected in plastic bottles. Those collected for isotopic gamma analysis should be made slightly acidic through the addition of small amounts of concentrated $\mathrm{HCl}$ (about $2 \mathrm{cc}$ per $100 \mathrm{cc}$ of liquid). This is done to reduce radionuclide plateout on the surface of the bottle. Samples obtained for alpha-, beta-, or mass spec. analysis should be chemically untreated (i.e., no Hcl added). Collection of Gaseous Samples:

Prior to sampling the $P$ \& ID's of the system should be examined to verify that the sample points can be expected to yield valid samples. Whenever samples are taken, the sample line must be adequately purged to assure that a representative sample is obtained. This involved purging the line for at least three line volumes.

When obtaining grab samples, standard procedures 
utilizing either a flow-through container or an evacuated container should be used. When sampling for ${ }^{129} \mathrm{I}$, iodine species, or oxidized-oxidizable tritium, procedures such as those outlined in NUREG0384 can be used. Collection of Swipes and Smear Samples:

Standard procedures should be followed in obtaining smear and swipe samples. For example, the area sampled should be $100 \mathrm{~cm}^{2}$ and gauze or filters should be used for snears. Collection of Containment Surface Deposition Samples

It seems essential that such samples must be obtained by personnel who enter the containment for this purpose. Therefore the sample gathering operation should be well planned and rehearsed to minimize the exposure time for workers. The planning process should include persons who are very familiar with containment layout and contents. Initial entries into the containment should identify specific locations and items for sampling, particular tools needed, and the requirements for sample transport containers. Actually it would be helpful to do as much of this as possible on the basis o ledge before entry is made; then the entry could confirm or maybe modify the initial plans.

The essential item to observe in obtaining surface deposition samples is to preserve the integrity of the sample surface. This usually means detaching the specimens with care (minimum mechanical and thermal stress) and transporting them in protective containers which permit minimum agitation and no surface abrasion. The containers may have 
to be specially designed and fabricated, and the materials and construction should facilitate external decontamination operations after container removal from containment. Surface swipes, of course, spoil. the virgin nature of the surface they are taken from, but the swipes themselves should be placed in protective containers for transport. It is obvious that a sample identification scheme is needed so the position from where the sample was taken can be tracked. surface deposition samples should be taken from areas which were and were not exposed to the containment spray and from both vertical and horizontal surfaces in each area. Sample sizes should be at least $1 \mathrm{sq}$. in. in area and could range up to a sq. ft. or more depending on ease of transport. Efforts should be made to identify hardware items (nuts, small cover plates, flat-head bolts, etc.) or small pieces of equipment (metal insulation panels, equipment housing panels, etc.) that could be easily removed. Epoxy painted surfaces, as well as unpainted surfaces (stainless steel), should be sampled. Finally, some effort might be spent trying to. develop a technique for gently obtaining concrete samples. Perhaps a piece could be broken off the corner of a concrete ledge or square column by careful use of drills or chisels. Isotopic Gamma Analysis:

Samples should be gamma counted using current germanium detectar technology. The spectra should be analyzed using one of the currently accepted algorithms (e.g., GAMANAL, GAUSS-VI, SAMPO). Lower limits of detection should be determined for 
each radionuclide of interest that is not detected in a sample. Care must be taken when counting samples that contain particulates that could settle. This is especially important if counting is done using a vertical geometry.

Standard gamma-ray counting QA procedures should be followed. This includes efficiency calibrations using NBS standards or standards traceable to NBS, routine efficiency checks, and daily energy calibrations. The systems should be calibrated for each type and size of sample to be counted (e.g., bottles of liquid, gas bombs, smears,.swipes, paint chips, etc.). Laboratory Analysis of Surface Deposition Samples

The objective of the laboratory analysis is to identify what is on the surface, how much is on the surface, how it is distributed; and penetration into the base material (if any) on a radionuclide basis and on a chemical. or physical. basis; if possible. The laboratory analysis requires as much planning as the sample collection effort. It would be advisable to design an investigation sequence that would proceed in stages. The first stage might consist of visual observations of asreceived samples using optical microscopy to assess surface morphology and character. Particulate material characterization would be particularly useful. Radioassay by gammaray spectrometry might also be done at this stage to obtain initial radionuclide content data.

Following the initial examination, sample sectioning strategies might be developed to subdivide the sample so that various other analyses could be performed. Scanning 
electron microscopy (SEM) might be used to better resolve surfaze morphology and perhaps determine size distribution data for particulate material. Numerous destructive analyses could be performed such as:

-) use of chemical etchants and reagents to dissolve the deposition layer followed by quantitative gamma-ray spectrometry to determine radionuclide composition and amounts.

2) use of emission spectrometry and/or mass spectrometry on scrapings of the surface to identify chemical constituents of the layer of particulates.

3) perhaps use of $x$-ray diffraction analysis of scrapings of the surface in attempts to identify crystal structures in the deposition layer or the deposited particulates.

4) grinding or sectioning techniques might be used with radioassay of the substrate after each "slice" to determine the penetration profile of radioactivity below the surface of the sample.

Swipe samples should be analyzed for radionuclide species and content by gamma-ray spectrometry and beta-ray spectrometry if possible.

Chemical Compound Identification for Surface Samples

The most promising analytic technique for identifying fission product molecular forms on various surfaces (i.e., on concrete, steel, and swipe material surfaces) appears to be laser-Raman spectroscopy (i.e., the Raman microprobe). The Raman microprobe can be used microscopically to study particles as small as 1 micron in diameter, or can be applied macroscopically with a sensitivity of parts-per-million. The microprobe does not require a vacuum and does not perturb the sample. 
Of the seven or so Raman microprobe facilities in existence, none are set up to handle radioactive materials; however, the National Bureau of Standards may be interested in accommodating their laboratory to the task (Ref.: Private comm. Dr.E. S. Etz, NBS). The analytica- procedure would involve: (l) determining the Raman spectra 三or nonradioactive simulants of various selected fission product compounds, and. (2) observing which, if any. of thəse spectra can be identified in the overall spectral readings obtained from the radioactive samples.

Various other surface analytic techniques that may be useful in conjunction with laser-Raman spectrosccpy include: (1) secondary ion mass spectroscopy (SIMS), (2) infrared (IR) spectroscopy, and (3) electron spectroscopy for chemical analysis (ESCA). Scanning electron microscopy (SEM) would also be useful as a complementary characterization procedure. Other Analysis Technigues:

Standard analysis and $Q A$ procedures should be followed when performing other analyses such as mass spec., gas chromatography, $x$-ray fluorescence, radiography, radiochemistry, metallography, SEM, microprobe.

General QA:

Quality Assurance $(Q A)$ is a very important aspect of the sampling and analysis program. The EG\&G Idaho group coordinating the sample collection and analysis should be requested to provide their $Q A$ program to the TMI Examination Group for review. The TMI Examination Group should review this QA program and work 
with EG\&G Idaho, if needed, to insure an adequate QA program.

Due to the importance of the samples and the sampling and analysis program, it may be advisable to form a group to perform independent confirmatory measurements at TMI. This measurement group could report to either the NRC or the TMI Examination Group. 
VI . RECOMMENDATIONS FOR FUTURE TECHNICAL SUPPORT

It is recommended that the present planning group continue to provide technical guidance throughout implementation of the requested tasks. It may be desirable, from an efficiency standpoint, to organize subgroups with responsibility for specific sets of tasks. Overall guldance by the present group, however, should probably still be maintained.

The need for this continued guidance arises primarily from uncertainties in the containment environments, plant layout, and áccessibility of specific sample locations. These uncertainties make it impossible, at this time, to adequately specify details of the procedures to be used for the various tasks. These task details should, instead, be provided more near the time of implementation, and with the understanding of situations in containment at that time. The specific analytical techniques to be used are expected to change as data accumulates on the containment environments. Certain analyses may become unnecessary, based on results of earlier tasks, or particular analytical techniques may prove to be more appropriate for certain sets of tasks. Such considerations must be made as the data acquisition program progresses.

Finally, and most importantly, considering the uniqueness of the TMI data, an active quality assurance (QA) program must be implemented to guarantee the accuracy and validity of that data. This $Q A$ function could well be provided by members of the present planning group. 
N N 


\section{2}

DECONTAMINATION AND PERSONNEL EXPOSURE CONTROL

(issued under separate

cover as attachment 1) 

2.3 EARLY CONTAINMENT PENETRATION AND MONITORING

\author{
Prepared by
}

P. Yarrington

LWR Safety R\&D Division 4443

Sandia National Laboratcries

A Jbuquerque, New Mexico 87185

2.3-1 


\subsection{EARLY CONTAINMENT PENETRATION AND MONITORING}

\section{INTRODUCTION}

At the meeting of the TMI Technical Working Group on December 10-12, 1979, it was decided that a recommended plan would be developed for the use of remote equipment for early containment penetration and monitoring at TMI. The main purpose of this equipment would be to assist in performing various tasks under the MMI Examination and Infornation Program, thus minimizing the radiation dosage workers receive in carrying out the Program. We have identified several data acquisition tasks that could be performed remotely. The listing of these tasks in Enclosure 2 includes various TMI-Examination planning groups' recommendations and input from discussions at the December TWG meeting.

\section{SURVEY SUMMARY}

We contacted several organizations around the country that have had experience in designing, developing, or implementing equipment of the sort that would be needed for this program. (These organizations and their key personnel are listed in Enclosure 1.) Through inquiries to these organizations we sought to determine

o The sort of system/equipment that would be needed

o The availability of remote equipment for this project

- Which groups would be interested in participating in this project, if it is pursued

o Approximate costs and time scales for obtaining the necessary equipment.

Primary consideration was given to equipment that could be used to gather data during early entry into containment. 
To date, the survey on the availability and cost of equipment that would be suitable for TMI work has yielded the following findings:

- There is no available system that is totally adequate for the range of tasks desired to be performed.

- Most pieces of equipment needed to develop an adequate remote capability exist at various organizations around the country (see Enclosure 3). Some of the desired equipment, e.g., collimated radiation survey instruments and telemetry packages may require small development efforts.

- To develop adequate remote equipment for the range of tasks that can be reasonably expected of such equipment, the estimated funding and schedule commitments are:

$\begin{array}{ll}\text { funding: } & \$ 500 \mathrm{~K} \text { to } \$ 1000 \mathrm{~K} \\ \text { time: } & 6 \text { months to } 12 \text { months }\end{array}$

(A limited remote capability could probably be provided within 3 months at a cost of about $\$ 300 \mathrm{~K}$.)

A number of organizations have expressed interest in performing this system development work (see Enclosure 4).

- The WORM (Wireless Observing Remote Manipulator) vehicle (with attachments) at Lawrence Livermore Laboratory could be made avai lable within 3 months at a cost of $\$ 1 M$. The WORM is fairly 1 imited in its manipulative capabilities, but represents a very reasonable "early option" if time scales are suddenly shortened or do not permit development of a mare complete capability, as above. 


\section{General Recommendations}

All those contacted for this remote equipment survey generally agreed that in order to perform the desired tasks, a vehicle should be obtained and equipped with the following gear:

- Manipulating arm(s) to obtain swipes and surface/equipment samples, and do in-situ testing.

o TV and still cameras for inspection/navigation/permanent records.

- Radiation monitoring equipment for making dose rate and gamma-spectrum surveys.

The mobile capability was felt to be highly desirable due to:

1. Present uncertainties in the radionuclear and physical status in containment areas where tasks would be performed

2. The relatively large area in containment throughout which tasks are desired to be performed.

\section{Availability of Equipment}

Listed in Enclosure 3, by organization, is equipment that could be used directly, or integrated in a system design effort, and which could be made available immediately, i.e., probably in a matter of weeks at most. In most cases, the equipment is committed to programs and would, therefore, need to have backup systems identified before becoming available for the TMI work. Also, a backup capability would need to be identified and provided (the time scale for this varies) to cover the possibility of not being able to reuse (or decontaminate) the equipment after use at TMI. 
While the list of immediately available equipment is somewhat limited, all of the organizations which were contacted indicated interest in participating in some way to make appropriate equipment available for this program. In Enclosure 4, I have listed the areas/ activities in which each organization expressed interest. Actual participation wou'd, of course, depend on funding levels and upper management approval.

\section{Use of Remote Equipment for Plant Recovery}

It should be noted that, for planning and monitoring plant recovery operations, the utility will probably also have interest in much of the informaton obtained under TMI Examination tasks (1isted in Enclosure 2). Radiation surveys, for example, will be needed during decontamination to locate "hot spots" (particularly during early entries) and to assess the effectiveness of decontamination actions. Also, discussions with Bechtel personnel have indicated that remote equipment could be quite useful for doing actual decontamination work (in troublesome/inaccessible areas), as well as for performing minor repairs (e.g., seal packing to prevent recontamination of cleaned areas). Most of these recovery activities are not, of course, important from the standpoint of acquiring data or generic information under the TMI Examination program. The usefulness of remote equipment for these plant recovery activities should, however, provide some motivation for the utility to assist in obtaining the equipment.

FURTHER QUESTIONS TO BE ADDRESSED

In the very near future, and probably before recommending a definite course of action for "Early Containment Penetration and Monitoring," the following questions should be addressed:

0 What are the current time constraints on development and deployment of this equipment? 
For example:

1. When might building sprays be used?

2. When might the sump be drained?

3. When might systems, e.g., crane, be available?

(GPU/Bechtel input probably needed to answer most of these)

o What is the best estimate of containment status?

For example:

1. What spare penetrations will be available?

2. Will systems such as crane, elevator, electrical circuits be operative?

3. What are the current radiation levels - generally and hot spots?

(GPU/Bechtel input probably needed to answer these)

$0 \quad$ What level of funding is anticipated?

(Present estimated requirements:

Funds: $\$ 500 \mathrm{~K}-\$ 1000 \mathrm{~K}$,

Time to develop/del iver: 3 to 12 months

0 Would it be desirable to have the utility share in the cost of this effort?

o Would the utility be willing to share costs? 
o How will the project be funded, bid or sole source?

o Should this be an industry effort or national lab effort? 
TMI Examination Remote Equipment Survey

Organizations (and Personnel) Contacted

- Ergonne National Laboratory, Chicago, IL

Dr. A. Tevebcugh

Harry Youngquist

- Fermi National Accelerator Laboratory, Chicago, IL John Simon

John Grimson

- Programmed and Remote Systems, Inc., St. Paul, MN

Karl Neumeier

Ray Karinen

- MB Associates, San Ramon, CA

Lynn Foote

Carl Witham

- LGs Alamos Scientific Laboratory, Los Alamos, NM

Louis Rosen

Don Grisham

- Nuzlear Systems Associates, Brea, CA

Bill Thompson

- Lawrence Livermore Laboratory, Livermore, CA

Fred Jessen

Ira Morrison

- Sardia National Labcratories, Albuquerque, NM Dirk Dahlgren

Jim Gover

- Grumman Aerospace, Long Island, NY Russ Hagerman 
TMI Examination Remote Equipment Survey

Tasks Which Could Be Performed Remotely

- Radiation surveys of containment surfaces

(dose rate and spectral data)

- Collection of swipes/smears from containment surfaces

(from walls, floors, components)

- Collection of samples of containment surfaces

(from walls, floors, components)

- Collection of adhesion samples

(for "liftable" surface contamination)

- Removal of hardware samples

(concrete chips, cables, glass samples, etc.)

- In-situ testing of instruments

(electrical tests, calibration, etc.)

- Removal of small equipment/instruments

(thermocouples, transmitters, gauges, etc.)

Photography

(stills - records of physical condition, TVsurveillance) 
TMI Examination Remote Equipment Survey

Currently Available Equipment

(backup capabilities needed in some cases)

Argonne National Laboratory

Equipment :

Remote controlled vehicle with manipulator arm.

Notes:

Arm is lightweight but limited in dexterity.

Vehicle is basic PAR unit, 15 years old.

Fermi National Accelerator Laboratory

Equipment :

Manipulator arms

Notes:

Arms are rather heavy ( $300 \mathrm{lbs} / \mathrm{pair})$, but dexterous

Electrical, servo-controlled manipulation

Minor refurbishing needed

Los Alamos Scientific Laboratory

Equipment :

Manipulator arms

Notes:

Arms are lightweight ( $100 \mathrm{lbs} / \mathrm{pair}$ ), highly dexterous

Electrical servo-controlled and/or hydraulic manipulation

Many specially adapted tools available

Routinely used, reliable equiment

LASL personnel continually upgrade this equipment

MB Associates

Equipment :

Remotely controlled, wheeled vehicle

Notes:

Vehicle designed to be highly intrepid (stair

climbing capability, negotiates rough terrain)

Vehicle must be retrieved from owner

Minor refurbishment probably needed 
- Programmed and Remote Systems

Equipment :

Manipulator arms, video equipment and remote control equipment

Notes:

This equipment is new, "on the shelf" hardware

Reliability seems to be well established

Manipulator arms limited in dexterity

- Lawrence Livermore Laboratory

Equipment:

WORM (Wireless Observing Remote Manipulator) This is a tracked, remote controlled vehicle equipped with video system, manipulating arm, radiation detection equipment

Notes:

Unit is relatively new ( 3 years old)

LLL has substantially upgraded the equipment as originally received from Rocky flats (manufacturer)

Manipulating arm has 1 imited dexteriry

Vehicle is fairly intrepid (climbs moderate-grade stairs, etc.)

Equipment is currently dedicated to NEST (Nuclear Emergency Search Team) and kept in good rapair 
TMI Examination Remote Equipment Survey

Areas of Participation Desired by Contacted Organizations

Argonne National Laboratory

Participation Desired:

Make resident vehicle available

Consult on operations and system design

Fermi National Accelerator Laboratory

Participation Desired:

Make resident manipulators available

Consult on operations, system design, video equipment

Programmed and Remote Systems

Farticipation Desired:

Supply manipulator $\operatorname{arm}(s)$, vehicle hardware

Do complete or partial system design/

development/integration

MB Ass Jciates

Participation Desired:

Supply vehicle manipulator arms, video equipment

Do complete or partial system design/development/

integration

Los Alamos Scientific Laboratory

Participation Desired:

Make resident manipulator(s) available and supply

(limited time) technicians to operate/train

Do complete or partial system design/development/ integration

\section{Lawrence Livermore Laboratory}

Pa-ticipation Desired:

Make resident WORM vehicle (with "attachments") available and supply (limited time) technicians to operate/train

Consult on operations or system design 
Enclosure 4, page 2

- Sanãia National Laboratories

Participation Desired:

Do instrument/subsystem design and development

Consult on system design

- Grumman Aerospace Corporation

Participation Desired:

Do complete system design/development/integration

- Nuclear Systems Associates

Participation Desired:

Do complete or partial system design/development/ integration

Consult on system design 
ט 
$=$ 



$$
\begin{gathered}
4.0 \\
\text { PHYSICAL PLANT EXAMIMATION } \\
\text { (combined with section 2.0) }
\end{gathered}
$$





\section{0}

ARCHIVE SAMPLE REPOSITORY

(archive facility requirements under review by DOE) 
$6: 1$

JOINT TMI-2 INFORMATION AND EXAMINATION PROGRAM - TASK 6.1 PR IMARY SYSTEM PRESSURE BOUNDARY CHARACTERIZATION

RECOMMENDED ACTION PLAN

\section{INTRODUCTION AND SUMMARY}

Principal bases for the subject program, briefly stated, are that (1) there should be significant amounts of generically useful. technical information uniquely available at TMI-2 beyond that which must necessarily be extracted in the course of plant cleanup, and (2) there may be generic technical information of significant value to plant cleanup and recovery which is not immediately availabie but the development of wich could be accelerated so that it could be used there. In the area of primary system pressure boundary characterization, there is no doubt that significant information is uniquely available at TMI-2. Because primary system components are expensive to replace, particularly the reactor vessel and head, there is great iriterest in obtaining information on how to determine (and demonstrate) capability for continued useful service after an unusual operating incident or accident and, although not exactly a well-controlled experiment, exanination and sesting of TMI-2 components can provide real data that would be difficult and expensive to get any other way. Because of the potential impact on overall recovery strategy and plans, it has been assumed that GPU's base program for determining whether the TMI-2 reactor vessel and head are still usable will have to be very comprehensive and thus include acquisition of essentially all the significant information potentially available. A possible exception is in regard to the vessel head where it has been recognized that the decision might be made to order a new one because of the relative cost and uncertainty involved in trying to demonstrate reusability. In such a case, considerable information of generic value could be obtained from detailed examination of the discarded head. Otherwise, it has been assumed that the joint program in this area would consist mainly of accelerated effort on generic research and development programs which can provide needed input data and more advanced methods and techniques for use in TMI-2 pressure boundary component qualification assessment and confirmation.

It was planned initially to utilize existing channels and resources (EPRI's Pressure Vessel Study Group, cognizant code bodies, etc.) to develop action recommendations for this aspect of the joint information and examination program. In addition, because of their substantial impact on the opportunities and needs appropriate to Task 6.1, GPU base recovery program plans in this area were the subject of early discussion with designated GPU representatives and related EPRI/NSAC projects also were reviewed wi th responsible staff members to identify technical areas of particular interest. Potential Task 6.1 work items which appear to merit detailed consideration are in the following categories: Technical Input and Integration with the Base Recovery Program; Analytical Support to the Base Program; Advanced Methods Development and Demonstration; Data Acquisition and Analysis, and Compilation and Dissemination of Results. Examples of work items expected to be included in these various categories are cited in the Task Description section below.

Costs assignable to this task, i.e. not covered by GPU's base program or by independent EPRI or NSAC projects, are roughly projected to total $\$ 2.3$ million. Considering necessary interactions with the recovery program 
schedule and other constraints as currently perceived, it is expected that completion of the task will take about 42 months. According to current agreements among the joint program participants, this task will be funded by EPRI. With the exception of field data acquisition at the plant site, it is expected that subtask implementation will be managed directly by EPRI/NSAC rather than going through DOE's Technical Integration office.

\section{TASK DESCRIPTION}

Technical Input and Integration: Specific effort will be required to assure effective exchange of technical information and support among various parallel efforts related to (a) assessment of conditions to which TMI-2 primary system pressure boundary components, particularly the reactor vessel and head, were exposed (NSAC, GPU, etc.) and (b) procedures for demonstrating the qualification of pressure boundary components for continued use after experiencing conditions beyond those considered in their design (EPRI, GPU, code bodies). This is expected to include discussion of technical topics of general interest such as the relative merits of al ternative analytical or test methods, and al so more specific items such as proposed program or project plan review and comment. For implementation of this subtask, it is recommended that an appropriately sized and qualified group of representatives of the various organizations and functions be established. Those represented should include GPU (Lee Rochino), selected contractors (e.g. Steve Brown, B\&W; Bill Cooper, Teledyne), NSAC (Garry Thomas) and EPRI (Ted Marston, Sam Tagart). A representative of DOE also might be included but, because of potential conflict of interest, NRC representation probably should not.

Analytical Support: The GPU base program approach to characterization of reactor vessel and head condition calls first for a thermal-hydraulic analysis to determine the temperatures and pressures attained in these components. Analysis then will be made of resulting stresses in various areas, particularly those which thermal analyses show may have exceeded design temperature conditions. Because of the importance of calculated temperatures to the overall evaluation, it has been agreed that an independent analysis would be in order. As part of their effort to provide an accurate reconstruction of the TMI-2 event, NSAC has a related project underway that should meet this requirement. It is recommended that the parallel NSAC and GPU programs be integrated to this extent and that Task 6.1 of the joint information and examination program provide for orderly comparison of results and resolution of differences including utilization ultimately of temperature "bench marks" that should be available from examination of TMI-2 core components, upper plenum internals, vessel and head specimens, etc. It also is recommended that the joint program task provide analytical support to GPU's assessment of imposed stresses, material properties, etc. to which there are parallel EPRI projects (Nuclear Systems \& Materials Department Research Program Plan-1980) or other independent but technically related efforts that could provide useful input. Such longer-term generic development activities in turn would benefit from the information and insights gained from this "real life" example.

Advanced Methods Development and Demonstration: As noted earlier, one of the bases for the joint information and examination program is that there may be technical advances in development wich would be of value at TMI-2 and which might be made available in time for use there if provided appropriate initiative and support. This is certainly so in regard to methods for determining 
the condition of primary system pressure boundary components, particularly the reactor vessel and head. GPU's base program will include $100 \%$ inspection of these major components, detailed examination of in-vessel surveillance specimens, an archive sample test program and, finally, acquisition of actual material samples from critical component locations for detailed characterization and confirmation. There are advances being made in methods and techniques which may be of significant value in each of these program elements. Not all such advances are ready for field application under the conditions existing or expected at TMI-2, however. For example, there are a number of NDE methods and techniques being worked on under EPRI's Nuclear Systems and Materials Subprogram 424, "NDE Development and Application," which might improve TMI-2 primary system pressure boundary inspection capability if they can be adapted to the particular requirements and constraints of concern. Another advance conceived for other purposes but potentially of substantial value in this area is the "micro-specimen" approach to the direct characterization of component materials. Development of this approach for possible use in nuclear system requalification is a longer term objective of EPRI Subprogram 405, "Pressure Boundary Technology." In both these cases it appears that effort could be increased and focused to provide timely results for application to essential TMI-2 recovery progran functions. Other similar needs and opportunities probably exist for accelerating development of generically useful methods and/or for establishing the validity of such advanced methods for determining whether pressure system components are qualified for continued use after experiencing emergency or faulted transients, as defined by code. Therefore it is recommended that the joint program Task 6.1 include provisions for accelerating development and application of advanced methods and techniques in the two areas described above and in other areas identified under the Technical Input and Integration Subtask where timely results should be obtainable at costs commensurate with their value to the industry.

Data Acquisition and Analysis: As noted earlier, GPU's base program on primary system pressure boundary characterization probably will have to include acquisition of essentially all the significant data potentially available. However, a prescribed part of the Technical Input and Intégration Subtask of 6.1 should be to identify additional data of generic value that may not be included in the base program as finally detailed. The decision as to whether to provide for the acquisition of such data under this subtask would still be subject to the broader program constraints of compatibilty with GPU's recovery program schedule and value sufficient to justify the cost of acquisition. Similarly in regard to data analyses, joint program bases and objectives may indicate justification for more extensive or generic consideration than required by the base recovery program. The existence of such needs and opportunities will have to be determined as data availability and base program scope are more fully defined.

Task 6.1 Action Plan Summary

Early Items:

- Establish PSPBC Task Implementation Group to communicate and discuss parallel program plans and results.

- Initiate orderly comparison of independent analyses of component themal 
histories and proceed to resolution of significant differences, if any.

- Follow up with support of subsequent stress analysis, etc., as appropriate. (Also provide specific input, consultation and support to the decision on head disposition, if requested).

- Develop detailed plans for accelerating availability of advanced NDE and micro-specimen methods and techniques; initiate effort in other areas agreed to be of potential value and capable of timely results.

- Identify potential requirements for data acquisition or other site activities in support of this task so that they can be transmitted to the TlO for consideration of potential costs, schedule conflicts, operational feasibility, contractual provisions, etc.

Rough Cost Projections:

- Technical Input and Integration

$50 \mathrm{~K}$

- Analytical Support

$1,050 \mathrm{~K}$

- Thermal analysis comparison and reconciliation

- Coordination with.GPU on their elastic analysis

- Detailed elastic-plastic analysis of key areas

- Advanced fracture mechanics analysis

- Development of appropriate flow properties from archival materials

- Advanced Methods

$1000 \mathrm{~K}$

- NDE: Materials Property Measurement

Nozzle and Pressure Vessel NDT Techniques

Inspection Methods Device Development

NDE Center Support

0 : Direct Assessment of Material Properties

Micro-specimen acquisition techniques

Micro-specimen qualification and codification

Micro-speciman confirmation for TMI -2 materials, EPRI archival

o Other samples (irradiated, aged), etc.

- Data Acquisition and Analysis

$150 \mathrm{~K}$

- Compilation and Dissemination of Results

$50 \mathrm{~K}$ 


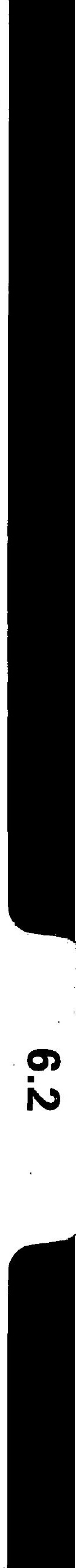


6.2

\author{
DRAFT LIST \\ OF \\ NUCLEAR STEAM SUPPLY SYSTEM MECHANICAL COMPONENTS \\ RECOMMENDED FOR EXAMINATION \\ DURING RECOVERY OF THE TMI-2 PLANT
}

Prepared by

TMI-2 RECOVERY MECHANICAL COMPONENTS PLANNING GROUP

R.N. Whitesel (EPRI) - Chairman

\author{
Prepared for \\ TECHNICAL WORKING GRDUP. \\ TMI-2 RECOVERY R\&D PROGRAM \\ (JOINTLY-SPONSORED BY DOE, NRC, GPU \& EPRI)
}

April 1980

$6.2-1$ 


\section{PREFACE}

This report has been prepared at the request of the Technical Working Group for TMI-2 Recovery R\&D Planning. The purpose of this report is to recommend a number of mechanical components for study. This report is one of several which, when taken together, comprise the scope of research activities presently envisioned by the four sponsoring organizations - DOE, NRC, GPU and EPRI. 


\section{CONTENTS}

PREFACE $\ldots \ldots \ldots \ldots \ldots \ldots \ldots \ldots \ldots \ldots \ldots \ldots \ldots \ldots \ldots \ldots \ldots \ldots \ldots \ldots \ldots .6 .2-2$

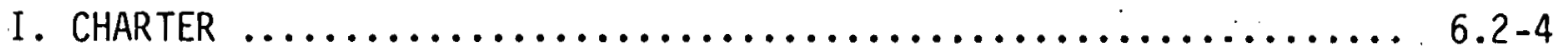

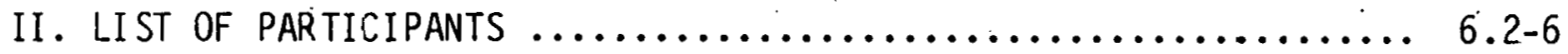

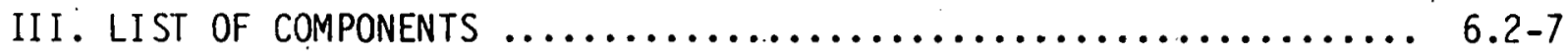

IV. JUSTIFICATION FOR SELECTION $\ldots \ldots \ldots \ldots \ldots \ldots \ldots \ldots \ldots \ldots \ldots \ldots .2 .14$

V. RECOMMENDED TESTING $\ldots \ldots \ldots \ldots \ldots \ldots \ldots \ldots \ldots \ldots \ldots \ldots \ldots .6 .2-19$ 


\section{CHARTER}

\section{TASK 6.2 Mechanical Components}

Examination programs for ectrical equipment and instruments and for the primary system pressure toundary are described under Tasks 1.0 and 6.1. The goal of the Planning Group for Mechanical Components is to determine the research required related to the safety and engineering aspects of the performance of the other components, most of which are mechanical.

The mechanical components inside the containment building were subjected to temperatures, pressures, chemicals, radiation, and moisture conditions not normally encountered. For many of these components these unusual conditions can be judged to have negligible effect on the component's performance or continued useability. The Planning Group will determine which of these mechanical components is deserving of study or inspection, using the following criteria:

1. Is it likely that the accident conditions could have affected the component's performance in such a way as to impact plant safety?

2. Would inspection or testing of the component yield information on the quality of component design, leading, perhaps, to design improvements?

A tentative list of mechanical components which may be judged worthy of examination is listed below:

1. Reactor Coolant pumps

2. Relief valves 
I. CHARTER, cont.

3. Isolation valves inside containment

4. Control Rod Drive Mechanisms

5. Pressurizer Heaters

6. Containment Air Coolers

7. Letdown system

8. Gas Compressor

9. Auxiliary Bldg. Filters

The tasks of this Planning Group will consist of the following:

1. Make the list more complete.

2. Develop specific reasons why information from a given component will be useful and/or interesting.

3. List the techniques for obtaining the information, e.g., in-situ testing, bench testing, off site disassembly, etc.

4. Prioritize the list. 


\section{LIST OF PARTICIPANTS}

The following individuals took part in developing the information contained in this report:

NAME

H. Alexander

T. Bump

J. Crawford

M. Cross

S. Dam

R. Dietz

J. Drab

G. Echlbarger

J. Ferrel1

M. Hauck

R. Hol zworth

R. Lyons

F. Manning

C. Paolino

T. Patterson

J. Richardson

1. J. Schlereth

T. Skogland

L. Stanek

W. Tacy, Jr.

A. Telfer

R. Whitesel

J. Zudans
AFFILIATION

Ontario Hydro

ANL

Combustion Engineering

"I

Burns \& Roe

Byron Jackson

Limitorque Inc.

Diamond Power Spec. Corp.

Velan Engineering

Bingham-Willamette Co.

EG\&G/TIO

Ontario Hydro

US NRC

Westinghouse

Bechtel Power

Dresser Ind.

Anchor-Darling

GE

B\&W

Dresser Ind.

Ontario Hydro

EPRI

US NRC
PHONE NO.

416-592-6046

312-972-5898

203-688-1911 ×3313

203-688-1911 x3982

201-262-8800

213-587-6171

804-528-4400

614-653-6540

514-748-7743

503-226-5335

717-782-3933

416-592-4924

301-492-8377

412-373-5042

301-258-4150

318-640-6120

215-667-9090

20ิ6-292-6905

804-384-5111 x2611

318-640-6128

416-592-5392

415-855-2083

301-492-7363 


\section{LIST OF COMPONENTS}

The components recommended for study under this program are listed in Table 1. These have been prioritized using the numbers 1 through 6 , with a "1" assigned to the most important items. Generally, the priorities were determined by the importance of the item to the functioning of a safety or. safety-related system, and by the usefulness of the information to be gained. from study of the component.

It is assumed that much general decontamination of the interior of the containment building will take place before extensive study of these components gets underway. For this reason, some protection from the fluids and processes to be used may be necessary in order to preserve the data to be obtained from certain components, especially valve actuators. 
TABLE 1

\begin{tabular}{|c|c|c|c|c|c|c|}
\hline $\begin{array}{l}\text { ITFM } \\
\text { NO }\end{array}$ & PRIORITY & COMPONENT NAME & TAG NUMBER & SYSTEM & FLOW DIAGRAM & DECON PROTECTION REQTS \\
\hline 1 & 1 & Reactor Coolant Pumps & $\begin{array}{r}R C-P-1 A \\
B \\
-2 A \\
B\end{array}$ & $\begin{array}{l}\text { Reactor } \\
\text { Coolant }\end{array}$ & 2024 & None \\
\hline 2 & 1 & $\begin{array}{l}\text { Reactor Coolant Pump } \\
\text { Motors }\end{array}$ & $\begin{array}{r}R C-P-1 A \\
B \\
-2 A \\
B\end{array}$ & $\begin{array}{l}\text { Reactor } \\
\text { Coolant }\end{array}$ & 2024 & None \\
\hline 3 & 1 & $\begin{array}{l}\text { Electromatic Relief } \\
\text { Valve (mfd. by Dresser) }\end{array}$ & $R C-R 2$ & $\begin{array}{l}\text { Reactor } \\
\text { Coolant }\end{array}$ & 2024 & $\begin{array}{l}\text { Plastic bag prior to } \\
\text { large scale decon }\end{array}$ \\
\hline 4 & 1 & $\begin{array}{l}\text { Pressurizer Block Valve, } \\
\text { (mfd. by Velan) } \\
\text { including Limitorque } \\
\text { operator }\end{array}$ & $\mathrm{RC}-\mathrm{V} 2$ & $\begin{array}{l}\text { Reactor } \\
\text { Coolant }\end{array}$ & 2024 & Protect from sprays \\
\hline 5 & 1 & $\begin{array}{l}\text { Pressurizer Relief Valve } \\
\text { Piping and Supports }\end{array}$ & & $\begin{array}{l}\text { Reactor } \\
\text { Coolant }\end{array}$ & & None \\
\hline 6 & 1 & $\begin{array}{l}\text { Major Component Supports } \\
\text { - steam generator } \\
\text { - pressurizer } \\
\text { - reactor vessel } \\
\text { - core flood tank }\end{array}$ & & & & . None \\
\hline
\end{tabular}


TABLE 1 cont.

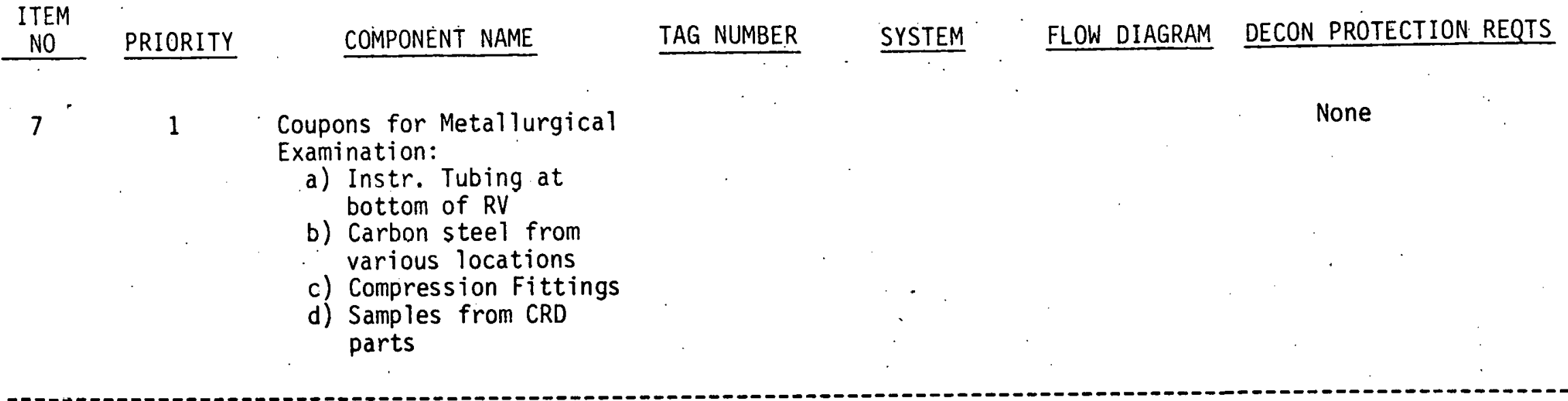

8. 1 Steam Generators $\quad \begin{array}{rlll}\text { RC-H-1A } & \text { Reactor } & 2024 & \text { Noolant }\end{array}$

2 Hydraulic Snubbers

Protect during decon

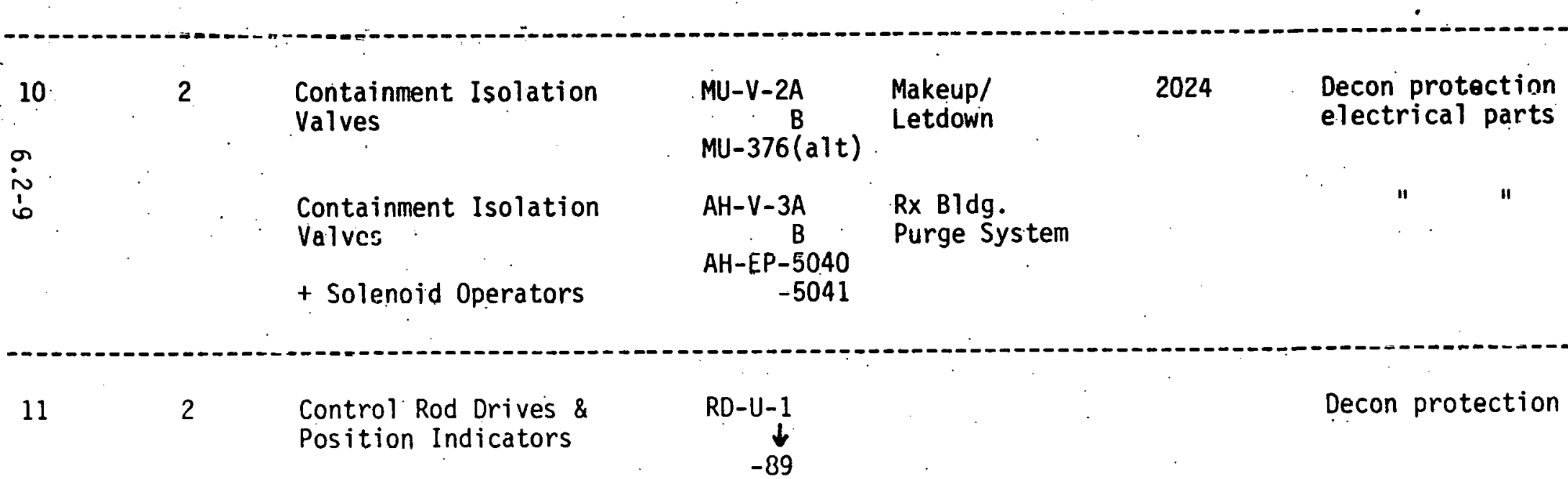


TAGLE 1 cont.

\begin{tabular}{|c|c|c|c|c|c|c|}
\hline $\begin{array}{c}\text { ITEM } \\
\text { NO }\end{array}$ & PRIORITY & COMPUNENT NAME & TAG NUMBER & SYSTEM & FLOW DIAGRAM & DECON PROTECTION REQTS \\
\hline 12 & 2 & $\begin{array}{l}\text { Pressurizer Safety } \\
\text { Relief Valve }\end{array}$ & $\begin{array}{r}R C-R-1 A \\
B\end{array}$ & $\begin{array}{l}\text { Reactor } \\
\text { Coolant }\end{array}$ & 2024 & Protect from sprays \\
\hline
\end{tabular}

13

2 Concrete \& Piping

None

a) 3 coat application

incended for submergence (basement walls \& floor)

b) 2 coat application on other concrete surfaces

c) Applications on

piping, misc. steel

surfaces \& core

flood tank

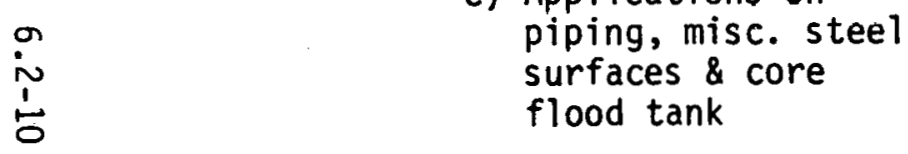

142 Inner Door Seals on None

Air Lock \#2

None

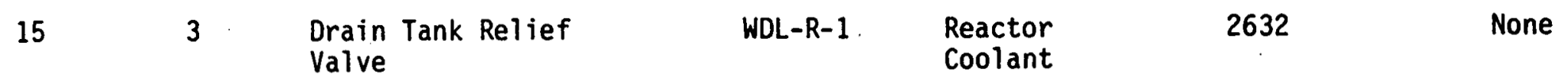

16

3 Mechanical Snubbers
MUH-281-S
MUH-282-S
$\mathrm{RCH}-4-\mathrm{S}$
RCH-5-S
RCH-21-S
FWH-124-S
FWH-125-S


TABLE 1 cont

\begin{tabular}{|c|c|c|c|c|c|c|}
\hline $\begin{array}{c}\text { ITEM } \\
\text { NO }\end{array}$ & PRIORITY & COMPONENT NAME & TAG NUMBER & SYSTEM & FLOW DIAGRAM & DECON PROTECTION REQTS \\
\hline 17 & 3 & Makeup System Filters & $\begin{array}{r}M U-F-5 A \\
B\end{array}$ & $\begin{array}{l}\text { Makeup/ } \\
\text { Letdown }\end{array}$ & 2024 & None \\
\hline
\end{tabular}

Polar Crane (preferred)
Fuel Handling Bridge
(alternate)

Reactor Vessel Closure
Head Seals \& Holddown
Bolts $\quad \begin{aligned} & \text { Makeup Pump } \\ & 20\end{aligned}$


TABLE 1 cont

\begin{tabular}{|c|c|c|c|c|c|c|}
\hline $\begin{array}{c}\text { ITEM } \\
\text { NO }\end{array}$ & PRIORITY & COMPONENT NAME & TAG NUMBER & SYSTEM & FLOW DIAGRAM & DECON PROTECTION REQTS \\
\hline 24 & 5 & $\begin{array}{l}\text { Damper (plus actuator) \& } \\
\text { Valve in Rx. Bldg. } \\
\text { Cooling System }\end{array}$ & $D-5127-B$ & $\begin{array}{l}\text { Reactor B1dg. } \\
\text { Cooling (AH) }\end{array}$ & 2041 & Protect during decon \\
\hline
\end{tabular}

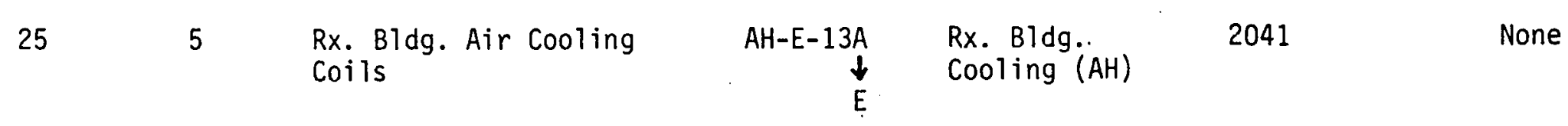

-

$5 \quad$ Rx. Bldg. Ductwork

Rx. Bldg.

Cooling (AH)

2041

None

$$
-
$$

$27 \quad 5 \quad$ Rx. Bldg. Fan Inlet

Gravily Dampers

Rx. Bldy.

Cooling (AH)

2041

None

$\cdots$

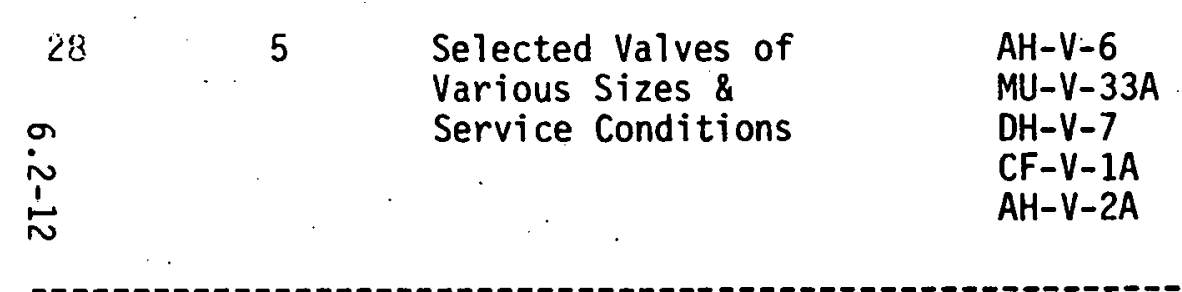

$29 \quad 6 \quad \begin{aligned} & \text { Control Rod Drive } \\ & \text { Service Structure }\end{aligned}$

Cooling Fans

$A H-E-52 A$
$B$

2041

Protect during decon

Protect during decon

MU-V $-33 A$

DH-V-7

$C F-V-1 A$
$A H-V-2 A$

Protect during decon.

\begin{tabular}{|c|c|c|c|c|}
\hline 30 & 6 & Letdown Coolers & $\begin{array}{r}M U-C-1 A \\
B\end{array}$ & $\begin{array}{l}\text { Makeup/ } \\
\text { Letdown }\end{array}$ \\
\hline
\end{tabular}


TABLE I cont.

\begin{tabular}{|c|c|c|c|c|c|c|}
\hline $\begin{array}{l}\text { ITEM } \\
\text { NO. }\end{array}$ & PRIORITY & COMPONENT NAME & TAG NUMBER & SYSTEM & FLOW DIAGRAM & DECON PROTECTION REQTS \\
\hline 31 & 6 & $\begin{array}{l}\text { Pipe \& Cable Tray } \\
\text { Supports }\end{array}$ & & & & None \\
\hline
\end{tabular}

$62 \begin{aligned} & \text { Concrete Surfaces in } \\ & \text { Contact w/ radioactive } \\ & \text { fluid }\end{aligned}$
$63 \quad \begin{aligned} & \text { Block Orifice in } \\ & \text { Letdown System }\end{aligned}$

$34 \quad 6 \quad$ Selected Electrical

Distribution, Lighting

Protect during decon

Panels \& Transformers

$n$
1
1
$\omega$ 


\section{JUSTIFICATION FOR SELECTION}

The justifications for the selection of the components listed in Table 1 are summarized in Table 2. The order and numbering of the components is the same in both tables. 
ITEM NO. COMPONENT NAME 1 Reactor Coolant Pumps

2 3

4

5

6

7
Reactor Coolant Pump Motors

Electromatic Relief Valve

Block Valve

Pressurizer Relief Valve Piping and Supports

Major Component Supports

Coupolis for Metallurgical Examinations

\section{JUSTIFICATION}

- The pumps operated for long periods of time with coolant conditions which were considerably different from those for which they were designed.

- Information to be gained from this examination will help determine the ability of reactor coolant pumps to perform acceptably in off-normal conditions.

- See Item 1 above.

- Incident related: Opened and failed to close.

- Possibly leaking before accident.

- Subjected to conditions outside its designed parameters.

- Incident related.

- Was cycled to maintain pressure control: Did this cycling cause wear, or did the accident service conditions affect it?

- Used for service outside design parameters.

- The seals in the valve will be useful in studies of materials perfornance.

- Incident related.

- Could have a bearing on valve performance.

- Experienced severe transients during the incident.

- Subjected to extreme transients from the fluid system.

- Look for evidence of chemical attack.

- Look for evidence of hydrogen embrittlenent.

- Look for corrosion product hideout.

- Look for effects of high radiation environment and temperature. 
ITEM NO. COMPONENT NAME

8

Steam Generators

9

10

11

13

14 Inner Door Seals

15

16

17 Makeup System

18 for Air Lock \#2

Drain Tank Relief

Valve

Filters

Hydraulic

Snubbers

Containment

Isolation Valve

Control Rod Drives and Position

Indicators

Pressurizer Safety

Relief Valve

Concrete and

Piping Coatings

Mechanical

Snubber

Polar Crane

\section{JUSTIFICATION}

- Subjected to thermal shock after the generator boiled dry.

- Evidence of leaking in the "A" steam generator: Was this caused by the accident, and what was the nature of the leak?

- Component motion caused by the incident may have degraded snubber integrity; data are required to assess snubber performance during such an incident.

- Are the valves. operable?

- What is the post-incident condition of the valve, the motor operator and the valve seat?

- What is the condition of magnets and other electrical parts following exposure to radiation and high temperature and humidity?

- Incident related.

- May have lifted during the incident.

- Subjected to prolonged high radiation environment and extended time under water.

- Looking for performance under these conditions.

- Suspected of leaking during incident.

- Was this leak incident related?

- Incident related.

- Appears to have lifted early based on pressure sensor in the drain tank.

- Interested in post-incident operability.

- No known data exists on performance of snubbers following exposure to such incidents.

- Examine for load of radioactive debris.

- Are they plugged?

- Subjected to high radiation, humidity, and relatively high temperatures.

- Source of elastomers and lubricants for materials testing. 
TABLE 2 cont.

\begin{tabular}{|c|c|c|}
\hline :EM NO. & COMPONENT NAME & JUSTIFICATION \\
\hline 19 & $\begin{array}{l}\text { Reactor Vessel } \\
\text { Closure Head Seals } \\
\text { and Holddown Bolts }\end{array}$ & - Post-incident condition following thermal transient. \\
\hline 20 & Makeup Pumps & $\begin{array}{l}\text { - What is the present condition of the pump which passed } \\
\text { the most flow of radioactive coolant? }\end{array}$ \\
\hline 21 & $\begin{array}{l}\text { Pressurizer } \\
\text { Heater }\end{array}$ & $\begin{array}{l}\text { - Some have failed. } \\
\text { - Interested in post-incident condition. }\end{array}$ \\
\hline 22 & $\begin{array}{l}\text { Reflective } \\
\text { Insulation } \\
\text { on Selected } \\
\text { Piping and } \\
\text { Components }\end{array}$ & $\begin{array}{l}\text { - Interested in general post-incident condition. } \\
\text { - Identification of any damage that indicates piping } \\
\text { moved. }\end{array}$ \\
\hline 23 & $\begin{array}{l}\text { Reactor Building } \\
\text { Air Cooling Fan. }\end{array}$ & $\begin{array}{l}\text { - Interested in how present performance compares with } \\
\text { data from environmental qualification tests, vendor } \\
\text { performance tests and startup test program results. }\end{array}$ \\
\hline 24 & $\begin{array}{l}\text { Damper (Plus Actu- } \\
\text { ator) \& Valve in } \\
\text { Reactor Building }\end{array}$ & $\begin{array}{l}\text { - This damper opens to provide circulation of air to } \\
\text { the top of the containment building (solenoid } \\
\text { valve operated). }\end{array}$ \\
\hline & & $\begin{array}{l}\text { - Interested in post-incident condition: and present } \\
\text { operability of this representative safety-related part. }\end{array}$ \\
\hline 25 & $\begin{array}{l}\text { Reactor Building } \\
\text { Air Cooling Coils }\end{array}$ & $\begin{array}{l}\text { - Incident related. } \\
\text { - Safety related. } \\
\text { - Evidence of leakage. }\end{array}$ \\
\hline 26 & $\begin{array}{l}\text { Reactor Building } \\
\text { Duct Work }\end{array}$ & $\begin{array}{l}\text { - Verification of the performance of relief panels used } \\
\text { for over pressure protection. }\end{array}$ \\
\hline 27 & $\begin{array}{l}\text { Reactor Building } \\
\text { Fan Inlet Gravity } \\
\text { Dampers }\end{array}$ & $\begin{array}{l}\text { - Safety-related item - prevents bypassing of the fans. } \\
\text { - Potentially incident related. }\end{array}$ \\
\hline 28 & $\begin{array}{l}\text { Selected Valves } \\
\text { of Various Sizes } \\
\& \text { Service Conditions }\end{array}$ & $\begin{array}{l}\text { - Interested in post-incident condition and operability } \\
\text { after exposure to incident environment. }\end{array}$ \\
\hline 29 & $\begin{array}{l}\text { Control Rod Drives } \\
\text { Service Structure } \\
\text { Cooling Fans }\end{array}$ & - Interested in post-incident operating condition. \\
\hline
\end{tabular}


TABLE 2 cont.

ITEM NO. COMPONENT NAME

JUSTIFICATION

$30 \quad$ Letdown Coolers

- Interested in post-incident condition.

31

Pipe \& Cable Tray

Supports

- Post-incident condition.

- Did anything move?

32 Concrete Surfaces in Contact with

- If coating systems fail, how much penetration of Radioactive Fluids radioactivity occurs?

33 Block Orifice in the Letdown System

34

Selected Electrical Distribution Lighting Panels \& Transformers

- Interested in possible blockage.

- Post-incident condition.

- Survivability in accident environment. 
V. RECOMMENDED TESTING

A list of recommended testing is shown for each component in Table 3. These are intended for use as guides in developing detailed examination work plans and procedures. 
TABLE 3

ITEM NO.

COMPONENT NAME

TEST RECOMMENDATIONS

\section{Activities}

Reactor Coolant Pumps

1. Remove and inspect mechanical shaft seals (visual \& pressure breakdown checks).

2. Inspect primary cover-to-case gasket enclosure for leakage.

3. Attempt to determine radiation exposure to the thermal shield.

4. Inspect main closure stubs by:
a. Visual
b. U1trasonics (as required)
c. Dye penetrant (as required)

5. Perform visual inspection and dimensional checks on:
a. Bearings
b. Impellers
c. Thermal barrier (and a bolt securing the thermal barrier)
d. Shaft

6. Inspect pump instrumentation

7. Performance tests (including vibration)

Test Units

Perform tests $1,2,3 \& 4$ on all four pumps.

Perform tests $5,6 \& 7$ on one unit only.

Test Sequence

1. Tests to be performed after RCS is drained and the pumps decontaminated.

Data

1. Gather'data from previous operation and record it for comparison with results of performance tests. 
IIEM NO. COMPONENT NAME

2

Reactor Coolant Pump Motors
Electromatic Relief Valve
TEST RECOMMENDATIONS

Activities

1. Perform visual inspection and dimensional checks of bearings.

2. Examine lubricating oil for concamination and breakdown of lubricating qualities.

3. Performance test on the motors.

Test Units

1. Examine one motor per test and determine usefulness of further testing of other units.

\section{Test Sequence}

1. Perform tests after removal of pump spacer.

In-situ Tests

1. Note axial fosition of soienoid plunger.

2. Note position of pilot valve operating lever.

3. Perform static electrical checks of solenoid.

4. Determine operability of solenoid and valve control. system.

\section{After Removal}

1. Note corrosion products in or near valve.

2. Note position of main valve disc upon removal of outlet pipe.

3. Note pipe movements (cold spring at inlet and outlet).

\section{Bench Testing}

1. Check operability.

2. Disassembie for corrosion product search, dimensional checks and wear measurements.

3. Look for plastic deformation in the valve bod!. 
ITEM NO. COMPONENT NAME

4

Pressurizer Block Valve
Pressurizer Relief Valve Piping \& Supports
6

7
Major Component Supports

Coupons for Metallurgical Examination

Steam Generators

Hydraulic Snubbers

\section{TEST RECOMMENDATIONS}

In-situ

1. Visual examination.

2. Perform static electrical checks of actuator.

Bench Tests

1. Examine for wear of parts and check operability.

2. Check condition of seals.

In situ

1. Make visual inspection of piping installation supports, attachments, welds, snubber connections \& pressurizer nozzles from the three connections on the pressurizer to the drain tank.

2. Following visual inspection, additional modeling, testing \& metallurgical \& stress analysis may be required.

Visual Inspection.

Perform standard metallurgical tests for hardness, ductility, brittleness, etc.

1. Perform eddy current testing on selected groups of tubes.

2. Remove some number of tubes and destructively examine.

3. Perform visual examination of secondary side.

1. Visually inspect for leakage and integrity of seals.

2. Perform functional tests for activation level, release rate, drag, spring rate and dead band.

3. Make dimensional inspection to confirm structural integrity.

4. Visually inspect for impacting of restraints on motor stand.

NOTE: It is suggested that consideration be give to draft NRC Reg Guide SC708-4 in the planning o: the test procedures. 
TABLE 3 cont.

IICM NO. COMPONENT NAME

10

11

12

13

14

15

16
Containment Isolation Valve

Control Rod Drives

\& Position Indicators

Pressurizer Safety

Relief Valve

Concrete \& Piping Coatings

Inner Door Seals

Drain Tank Relief Valve

Mechanical

Snubbers

\section{TEST RECOMMENDA-IONS}

1. For the makeup system valves, perform visual \& electrical checks in-situ, and bench tests for both operability and wear measurements.

2. For the valves in the reactor building purge system perform the following:
a) visual and electrical checks in-situ
b) determine tightness of the valve seat
c) destructive analys is of elastomers (ASTM D2882, D2670, \& D2625)
d) disassemble and inspect solenoid and operator for condition

1. Standard electrical checks.

2. Destructive analys is of CRDM Closure Seals:

1. Visual inspection.

2. Possible bench examination for leakage and chatter.

Evaluate adhesion, durability and penetration of radioactivity.

1. Visually inspect to determine cause of leak, i.e., damage or misinstallation.

2. Perform destructive analysis of elastomer seal.

1. Bench test for operability.

2. Check valve seat faces.

1. Visually inspect.

2. Perform functional tests on 3 to 5 samples to measure activation level, release rate, drag, deadband and spring rate.

NOTE: Again, NRC Draft Reg Guide SC708-4 may be helpful in detailed test procedure planning. 
ITEM NO. COMPONENT NAME

17

18

1 19

20

21

22

23

24

25

26

27

28

25
Makeup Sys tem

Filters

Polar Crane

Reactor Vesse1

Closure Head Seals

\& Holddown Bolts

Makeup Pumps

Pressurizer Heater

Reflective

Insulation on

Selected Piping

\& Components

Reactor Building

Air Cooling Fans

Damper \& Valve in Reactor Building

Cooling System

Reactor Building Air Cooling Coils

Reactor Building

Duct Work

Reactor Building

Fan Inlet Gravity

Dampers

Selected Valves

Control Rod Drive Service Structure Cooling Fan

\section{TEST RECOMMENDATIONS}

1. Sample filter material for analysis of debris.

2. Attempt to characterize isotopic content.

Note conditions of lubricants and parts (mostly electrical).

1. Visually inspect "0" ring seal.

2. Perform metallurgical tests of one or more head bolts.

No specific recommendations.

1. Visually inspect for evidence of operation dry.

2. Perform standard electrical checks for continuity, etc.

Visuar inspection.

1. Check operability.

2. Disassemble for seal and bearing inspection.

1. Visual inspection.

2. In-situ operability check.

1. Perform visual inspection and check for leakage.

Visual inspection for damage.

1. Visual inspection.

2. In-situ operability check.

1. Visual inspection of valve and operator.

2. Disassembly and inspection for condition of seats, packing, elastomers, and electrical parts.

In-situ operability check. 
TABLE 3 cont.

ITEM NO. COMPONENT NAME

30

Letdown Coolers

31 Pipe and Cable

Tray Supports

32 Concrete Surfaces

in Contact with

Radioactive Fluids

33 Block Orifice

34 Selected Electrical

Distribution Lighting

Panels \& Transformers

\section{TEST RECOMMENDATIONS}

1. Visual inspection.

2. Leak test.

Visual inspection.

Follow results of GPU test borings for requalification

Attempt to measure pressure drop.

1. Visual inspection.

2. Standard electrical checks. 
7.1

CRITICALITY CONTROL STUDY

(to be supplied

at a

later date) 
N 


\title{
7.2
}

\author{
RECOMMENDATIONS ON IN-PLACE \\ TMI-2 CORE DAMAGE EXAMINATIONS
}

PREPARED BY

TMI-2 EXAMINATION PLANNING GROUP 7.2

FOR THE

JOINT DOE/EPRI/NRC/GPU

TECHNICAL WORKING GROUP

APRIL 1980 


\section{PREFACE}

This Jocument represents the compilation of suggestions, recommendations and ideas presented by the various members of the TMI-2 Examination Planning Group 7.2. The planning group held two formal meetings to first discuss the assignment and later to review the draft report.

The following is a list of the organizations and personnel who participated in the discussions and preparation of this document.

\begin{tabular}{|c|c|}
\hline Argonne National Laboratory & - G. Chisholm \\
\hline Babcock \& Wilcox Co. & $\begin{array}{l}\text { G.E. Kulynych } \\
\text { M.H. Montgomery } \\
\text { R.A. King }\end{array}$ \\
\hline Battel le Northwest Laboratory & - E.L. Courtright \\
\hline Bechtel Power Corporation & $\begin{array}{l}\text { - W.C. Hopkins } \\
\text { T.E. Morris }\end{array}$ \\
\hline Conbustion Engineering & - A. Stathoplos \\
\hline $\begin{array}{l}\text { EGRG-Idaho } \\
\text { - TI0 office }\end{array}$ & $\begin{array}{l}\text { - D.D. Owen } \\
\text { - H.M. Burton }\end{array}$ \\
\hline EPRI/NSAC & $\begin{array}{l}\text { - R.H. Leyse } \\
\text { - A.B. Carson } \\
\text { - R.N. Whitsel } \\
\text { - G.R. Thomas } \\
\text { - L.C. Oakes } \\
\text { - R. Oehlberg } \\
\text { - D. Cain }\end{array}$ \\
\hline Nuclear Regulatory Commission & $\begin{array}{l}\text { - M.L. Picklesimer } \\
\text { - R. Meyer }\end{array}$ \\
\hline Oak Ridge National Labs & $\begin{array}{l}\text { - R. Blumberg } \\
\text { - D.0. Hobson } \\
\text { - J.T. Mihalczo }\end{array}$ \\
\hline Rockwell (AI) & - L.M. Maki \\
\hline U.S. Dept. of Energy & - A.C. Millunzi \\
\hline Westinghouse Electric Co. & $\begin{array}{l}\text { - D.L. Burman } \\
\text { - L.E. Hochreiter }\end{array}$ \\
\hline
\end{tabular}




\subsection{INTRODUCTION}

2.0 DESCRIPTION OF REACTOR AND CORE

2.1 Reactor Vesse1

2.2 Reactor Internals

2.3 Fuel Assemblies

2.4 Control Rod Assembly

2.5 Burnable Poison Rod Assembly

2.6 Neutron Source Holder

3.0 RECOMMENDED INSPECTION \& EXAMINATION PROGRAM

3.1 Before Head Removal

3.2 Before Plenum Removal

3.3 Before Fuel Removal

3.4 During Fuel Removal

3.5 After Fuel Removal

4.0 SUMMARY DISCUSSION \& TASK DESCRIPTION

4.1 Task Group 7.2 Recommended List of Key Early Projects APPENDIX A - AXIAL GAMMA SCAN OF EACH FUEL ASSEMBLY PRIOR TO REMOVAL FROM THE REACTIDR VESSEL

APPENDIX B - STATE OF THE ART OF EXAMINATION TECHNIQUES FOR USE ON THE TMI-2 CORE 


\subsection{INTRODUCTION}

The TMI-2 incident is the only significant commercial power accident that has occurred since the beginning of electrical generation using nuclear power. Since it is the only accident, effort should be made to accurately investigate the reactor system and particularly the core to obtain data. on the core behavior and the results of degraded core cooling.

The most important data include the core configuration, local fuel damage, damage to other core components including control rod thimbles, grids, control rods and burnable poison rods.

Information can be developed from the TMI-2 core to be used to assess the margin between current licensing criteria and degraded core cooling situations. TMI-2 proved that the core remained coolable at elevated temperatures far in excess of the current licensing limit even though significant core damage occurred.

There are three areas of effort that can benefit from an extensive examination of the TMI-2 core damage:

- An understanding of the specific condition of the fuel and other core components in the TMI-2 reactor.

- Data that will be useful in better benchmarking the analytical studies in the area of degraded core cooling.

- Data that will enable better planning of research programs in the area of damaged fuel behavior.

Some of the specific questions to be studied related to the TMI-2 accident include:

(1) How much fission product activity was released?

(2) . Degree of eutectic formation and/or fuel melting?

(3) Degree of metal-water reaction?

(4) General core configuration and location of core debris?

(5) Location of the thermocouples junctions? 
These questions and others related to the TMI'-2 core damage can be answered by an examination of the TMI-2 core during the reactor disassembiy and core removal program, it is appropriate that this assessment be performed as part of the R\&D program to obtain vital industry data from the TMI-2 accident.

In the area of analytical and experimental studies related to damaged fuel performance, TMI-2 can be considered as an important prototypical experimentation and may yield many important answers. Various study groups have recommended that the behavior of severely damaged fuel should be better linderstood in order to know more clearly what margins existed at TMi-2 and in nuclear power plants in general.

The third area of examination is less directly related to TMI-2 than core damage assessment and the behavior of severely damaged fue1. The TMI-2 accident probably also has some fuel damage of the kind that is considered in the present design-basis accidents (e.g., fuel cladding ballooning and rupture). Inasmuch as research programs are currently being funded in these areas to reduce existing uncertainties, TMI-2 examinations would provide an expedient way of making significant progress in these areas. The scientific value of these tests should not be underestimated since it is very difficult in even the most expensive' test-reactor experiemnts to achieve the accident conditions of a commercial reactor environment that were present in TMI-2.

In addition to measurements on fuel and core materials, it is desirable to undertake a program of examination of other components inside the reactor vessel. These include examination of some of the internals structures, control rod guides, vent valves, and other similar components to obtain information relative to degree of oxidation, significant deformations affecting flow paths, performance of key components under severe conditions, etc.

Many of the individual TMI-2 core measurements will apply to a.l1 three of the above areas of interest.

While it is not expected that core or fuel designs will change significantly in light of TMI-2, the information from the core examination will improve understanding of reactor safety and thereby improve safety through improved operating and emergency procedures. 


\subsection{DESCRIPTION OF REACTOR AND CORE}

The purpose of this section is to present a summary description of the TMI -2 reactor and core that may be important in the examination program. The areas of interest include the reactor vessel and closure head, the internal structure, and the various core components.

\subsection{REACTOR VESSEL}

The reactor vessel consists of a cylindrical shell with a spherically dished bottom head, and a ring flange to which a removable reactor closure head is bolted. A cylinder attached to the vessel's shell supports the vessel and extends downward to a flanged base ring which is bolted to the foundation of the building. The reactor closure head is spherically dished and welded to a ring $\mathrm{fl}$ ange which mates with and is bolted to the vessel with large-diameter studs. All internal surfaces of the vessel are clad with stainless steel weld deposit. The reactor vessel and internals general arrangement are shown on Figure 2-1, and a cross section is shown in Figure 2-2.

Two concentric metallic 0-rings provide the pressure integrity seal Detween the closure head and the vessel's flange. Pressure taps at the annulus between the two 0-rings are used to monitor seal leakage and to hydrotest the seal after closure. The reactor vessel's ring flange includes an internal ledge to support the core and the internal structural components.

The vessel had two outlet nozzles through which the reactor coolant is transported to the steam generators, and four inlet nozzles through which reactor coolant re-enters the reactor vessel. Two smaller nozzles between the reactor coolant nozzles serve as inlets for decay heat cooling and emergency cooling water injection (core flooding and low-pressure injection).

The bottom head of the vessel is penetrated by small in-core instrumentation nozzles. The closure head is penetrated by sixty-nine flanged nozzles for the control rod drive mechanisms and eight small flanged nozzles originally designed for vent valve thermocouples. 


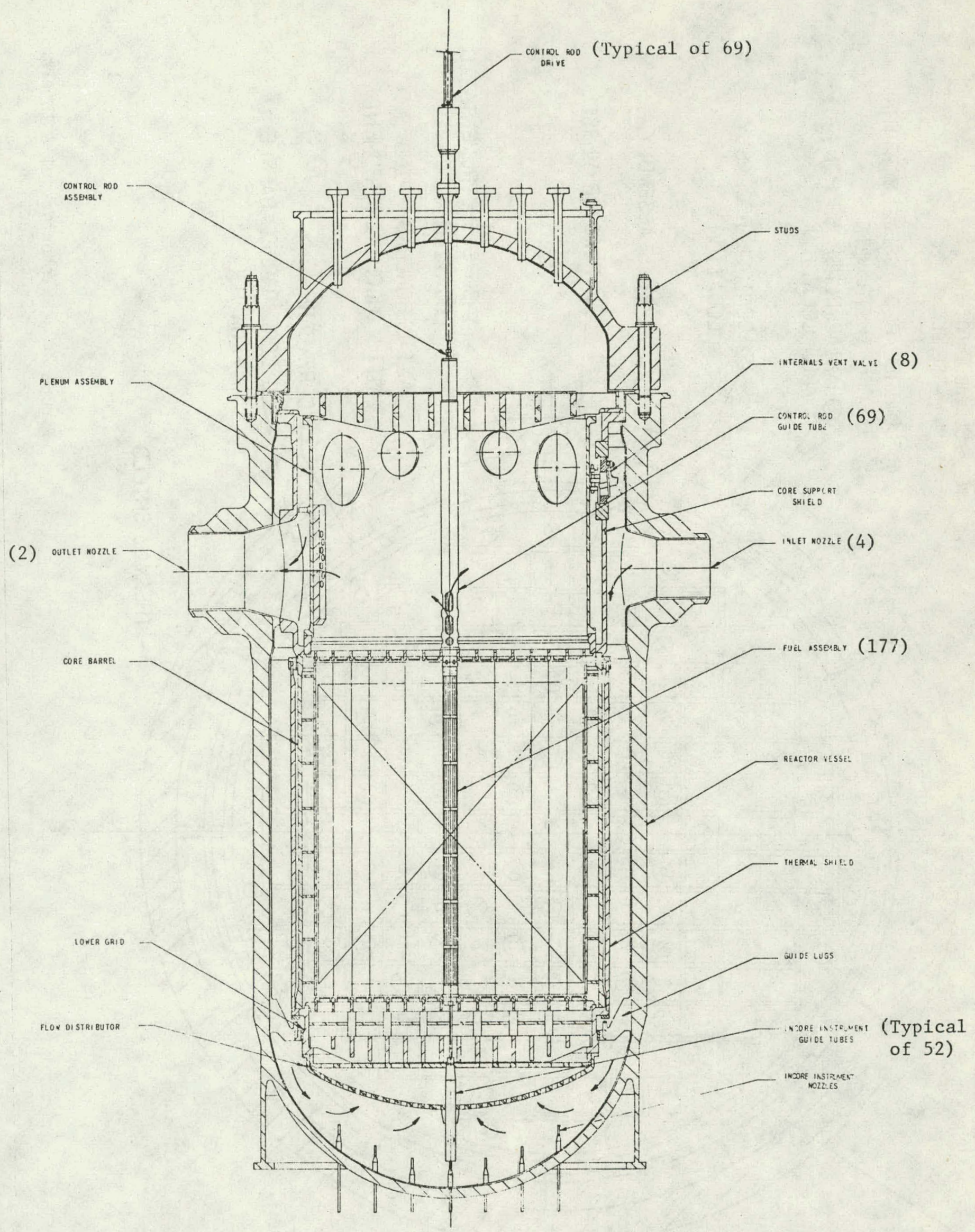

Figure 2-1 Reactor Vessel \& Internals- 


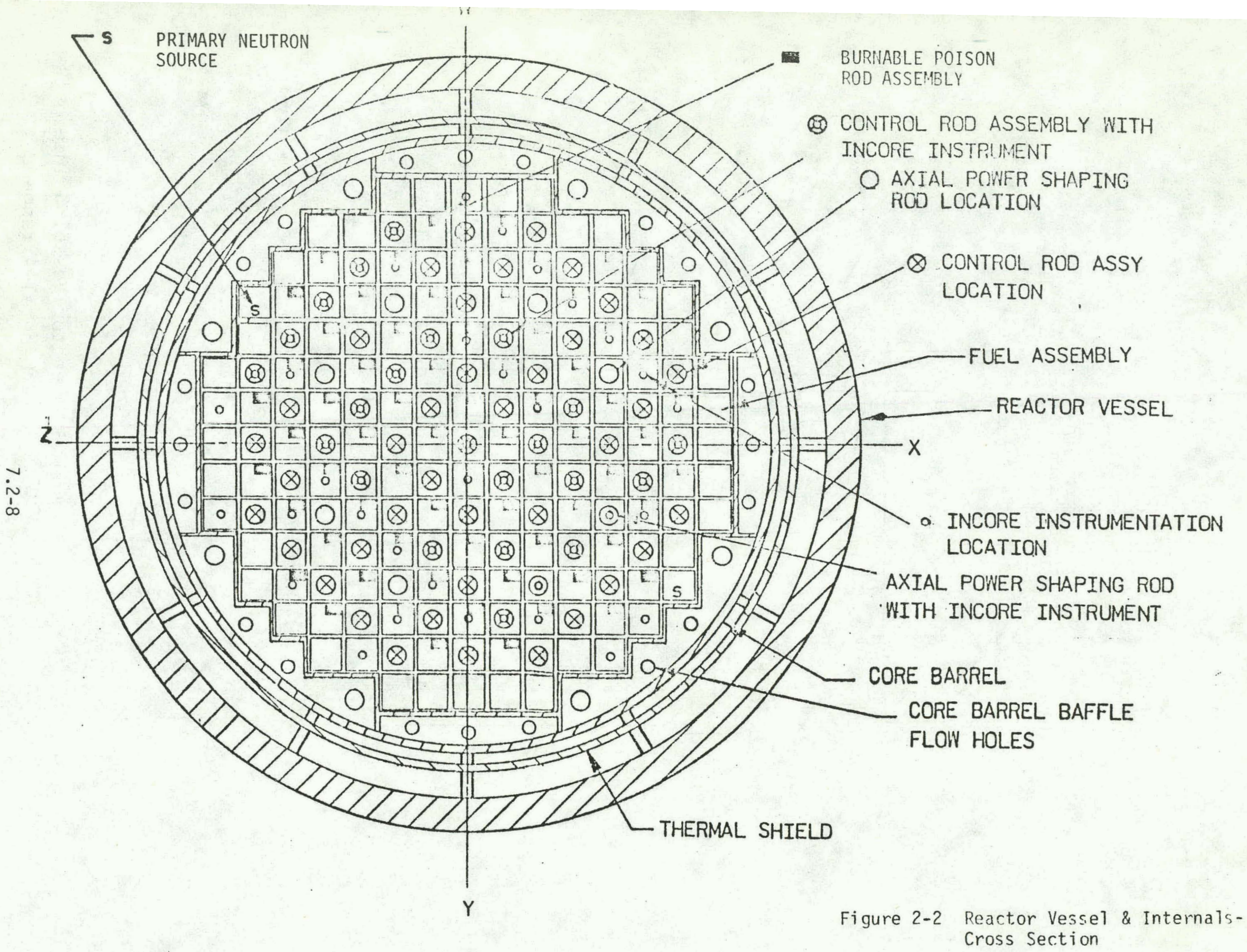


Core guide lugs welded to the reactor vessel inside wall limit the vertical drop of the reactor internals and prevents rotation about the vertical axis in the unlikely event of major internals structural failure.

Surveillance specimen holder tubes are located between the reactor vessel wall and the thermal shield.

Metal reflective insulation is used on the exterior of the vessel from the closure flange down to the support cylinder, on the exterior of the bottom head dome, and on all insulated surfaces of the support skirt. Removable metal reflective insulation panels enclose the top head closure flange and studs. Metal reflective insulation is used on the closure head dome fitted around the control rod drive mechanism nozzles.

The incore instrument penetrations are field-weld-joined to pipes that contain two 90 degree bends and terminate in bolted sealing flanges in a shielded area at a higher elevation in the reactor building. These tubes contain incore detector assemblies for measuring the neutron flux and temperatures in the reactor core.

\subsection{REACTOR INTERNALS}

The reactor internals include two major sub-assemblies: the plenum assembly and the core support assembly. The core support assembly is made up the core support structure, the core barrel, lower grid, flow distributor, incore instrument guide tubes, thermal shield and surveillance holder tubes. Figure 2-1 shows the reactor vessel and reactor internals arrangement. Figure 2-2 is a cross section through the reactor vessel. The internals structure can be removed from the reactor vessel by lifting from above.

\subsubsection{Plenum Assembly}

The plenum assembly is located above the reactor core and is removed as a single component before refueling. It consists of a plenum cover, upper grid, CRA guide tube assemblies, and a flanged plenum cylinder with openings 
for reactor coolant outlet flow. The plenum cover is a series of parallel thick plates intersecting to form square lattices with a perforated top plate and flange; it is attached to the plenum cylinder's top flange. The perforated top plate is $1 / 2$-inch thick and has sixty-nine 8.520-inch-diameter holes. The plenum cover is attached to the top flange of the plenum cylinder. Three lifting lugs are provided for handling the plenum assembly. These lugs, 17-1/4 inches high, 3 inches thick, and 3 inches wide, are welded to the lattice systems of the cover. The CRA guide tubes are welded to the plenum cover top plate and bolted to the upper grid. CRA guide assemblies provide guidance and protect CRA from the effects of coolant crossflow, and provide structural attachment of the grid assembly to the plenum cover.

The plenum cylinder is a 130-inch-OD, 1-1/2-inch thick cylinder with flanges on both ends to connect the cylinder to the plenum cover and upper grid. Six 34-inch-diameter holes and four 22-inch-diameter holes in the plenum cylinder provide a flow path for the coolant water.

The upper grid is a machined plate with openings that locate the lower end of the individual CRA guide tube assembly relative to the upper end of a corresponding fuel assembly. The grid is bolted to the plenum cylinder's lower flange. Locating keyways in the plenum assembly cover flange engage the reacotr vessel's top flange locating keys to align the plenum assembly with the reactor vessel, the reactor closure head and the core support assembly. The bottom of the plenum assembly is guided by the inside surface of the lower flange of the core support shield.

Alignment between fuel assemblies is provided by pads bolted to the lower surface of the upper grid.

\subsubsection{Core Support Assembly}

The core support assembly consists of the core support shield, core barrel, lower grid assembly, flow distributor, thermal shield, incore instrument guide tubes, surveillance specimen holder tubes, and internals vent valves. 
The core support shield is a large, flanged cylinder which mates with the reactor vessel opening. The forged top flange (146 inches ID) rests on a circumferential ledge in the reactor vessel top closure flange. The core support shield lower flange (136 inches ID) is bolted to the core barrel. The inside surface of the lower flange guides and aligns the plenum assembly relative to the core support shield. The cylinder wall has two nozzle openings for coolant flow. These openings are formed by two forged rings, which seal to the reactor vessel outlet nozzles by the differential thermal expansion between the stainless steel core support shield and the carbon steel reactor vessel. The nozzle seal surfaces are finished and fitted to a predetermined cold gap providing clearance during core support assembly installation and remcval. At reactor operating temperatures, the mating metal surfaces are in contact to make a seal without exceeding allowable stresses in either the reactor vessel or the internals. The cylinder wall also has eight holes in which vent valve mounting rings are welded.

The core barrel supports the fuel assemblies, lower grid, flow distributor, and incore intrument guide tubes. The barrel consists of a flanged cylinder, a series of internal horizontal spacers bolted to the cylinder, and a series of vertical plates bolted to the inner surfaces of the horizontal spacers to form an inner wall enclosing the fuel assemblies. The upper flange of the cylinder is bolted to the mating lower flange of the core support shield assembly, and the lower flange is bolted to the mating flange of the lower grid assemb1y. All bolts are lock-welded after final assembly.

The lower grid assembly provides alignment and support for the fuel assemblies, supports the thermal shield and flow distributor, and aligns the incore instrument guide tubes with fuel assembly instrument tubes. The lower grid consists of two lattice structures separated sy short tubular columns and surrounded by a forged flanged cylinder. The top flange of the forged cylinder is bolted to the lower flange of the core barrel. A perforated flat plate, midway between the two lattice structures aids in distributing coolant flow through the core. Alignment between fuel assemblies is provided by pads bolted to the top surface of the upper lattice structure. 
The flow distributor is a perforated, dished head with an external flange which is bolted to the bottom flange of the lower grid. The distributor supports the incore instrument guide tubes and distributes the reactor coolan: entering the bottom of the core.

The thermal shield is a stainless steel cylinder 151 inches in OD, installed in the annulus between the core barrel cylinder and the reactor vessel's inner wall.

Surveillance specimen holder tubes are installed on the core support assembly's outer wall to contain the surveillance specimen assemblies. The tubes extend from the top flange to the core support shield to the lower end of the thermal shield.

\subsection{FUEL ASSEMBLIES}

The fuel for the reactor is in the form of sinteted pellets of lowenriched uranium dioxide clad in Zircaloy-4 tubing. The ends of the tubing are sealed by Zircaloy-4 end caps attached to the tubing by welding. The cladding, fuel pellets, end caps, and fuel support components form a "fuel rod". Twohundred and eight fuel rods, sixteen control rod guide tubes, one instrumentation tube assembly, seven spacer sleeves, eight spacer grids, and two end fittings form a structural cage which contains the rods and tubes in a $15 \times 15$ array. The center position in the assembly is reserved for instrumentation, and the remaining 16 positions are provided for the guide tubes which guide the control rods. Figure 2-3 shows the construction features of a fuel assembly. Fuel assembly components, materials, and dimensions are listed below.

\section{Item \\ Fuel Rod}

Fue 1

Fue 1 Cladding

Fuel rod pitcl

Active fuel length

Ceramic spacer

Spring

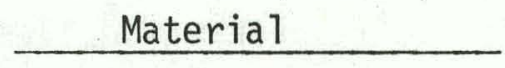

$\mathrm{UO}_{2}$ sintered pellets

Zircaloy-4

Dimensions, In.

0.370 dia. 0.430 OD X 0.377 ID $\times 153-1 / 8$ long

0.568

144

$\mathrm{ZrO}_{2}$

SS, Type 302

0.368 dia.

4 in. 


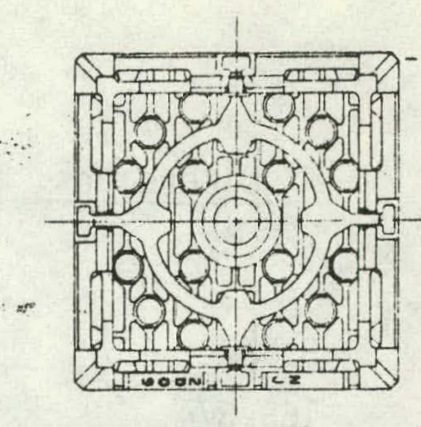

TOP VIEW
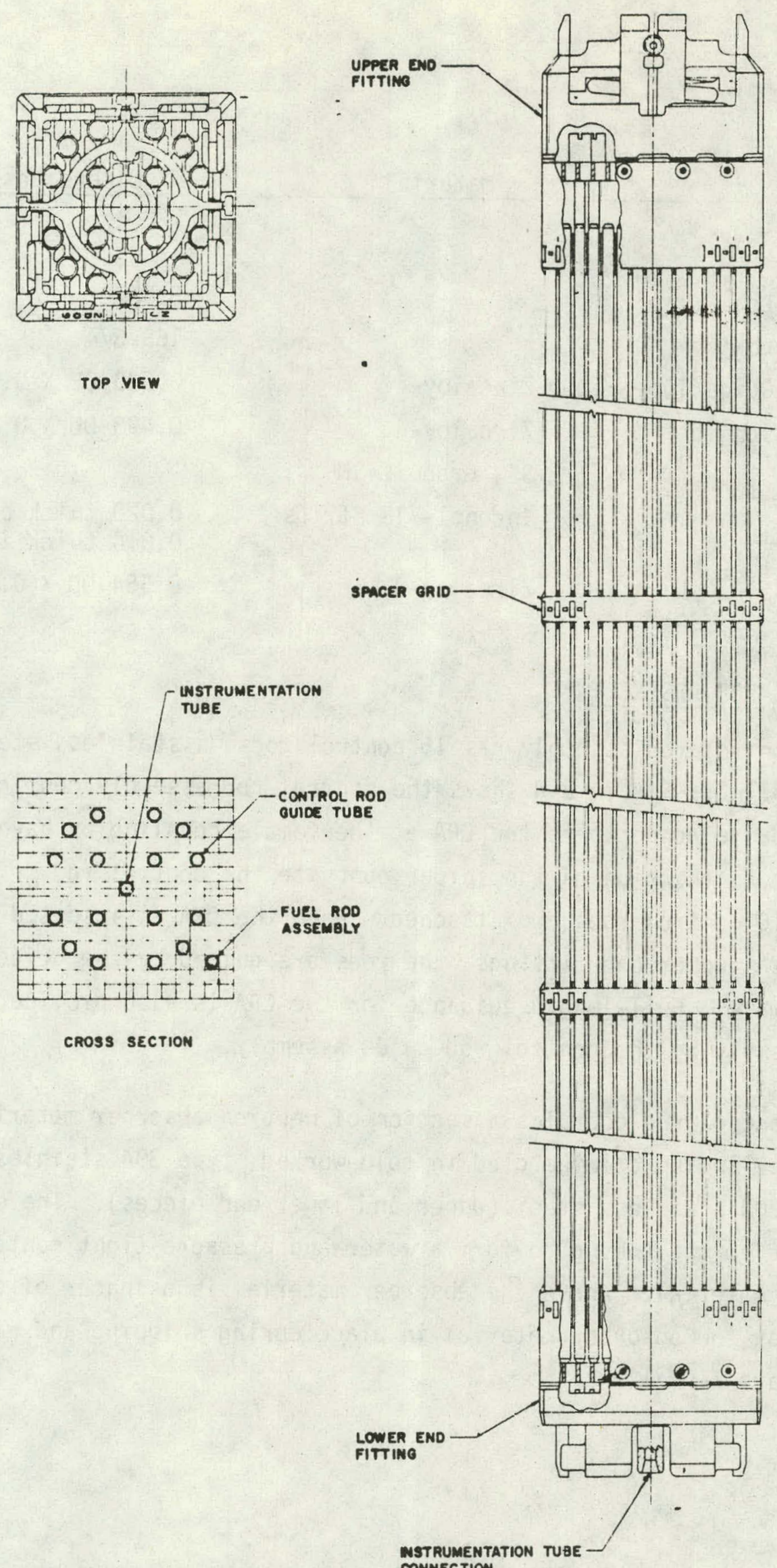

CONNECTION

Figure 2-3 FUEL ASSEMBLY 
Fuet Assembly

Fuel Assy Pitch

Overal1 Length

Control Rod Guide Tube

Zircaloy -4

$165-3 / 4$

Instrumentation Tube

Zircaloy-4

0.530 OD $\times 0.498$ ID

End Fittings

SS, Grade CF3M

Spacer Grid

Incone1-718 Strips

0.493 OD $\times 0.441$ ID

Spacer Sleeve

Zircaloy -4

0.020 thick exteriors

0.016 thick interiors

0.554 OD $\times 0.502$ ID

\subsection{CONTROL ROD ASSEMBLY}

Each control rod assembly has 16 control rods, a stainless steel spider, and a female coupling. Figure $\imath-4$ shows the control rod assembly configurations. The control rod drive connects to the CRA at the female coupling by bayonet type connection. On the side of the sjider opposite the control rod drive connection, the 16 control rods are attached. When the CRA is inserted into the fuel assembly's upper end fittings, the rods are guided by the guide tubes of the flel assembly. Full-lencth guidance for the CRA is also provided in the upper plenum assembly by the control rod guide assembly.

Each control rod contains a section of neutron absorber material an alloy of silver-indium-cadnium clad in cold-worked, type 304 stainless stee 1 tubing and type 304 stainless steel (upper and lower end pieces). The end pieces are welded to the tubirg to form a water-and pressure-tight container for the absorber material. Atove the absorber material is a spacer of corrugated tubing which keeps the absorber materici in place during shipping and handling. Data on the CRA are as follows: 


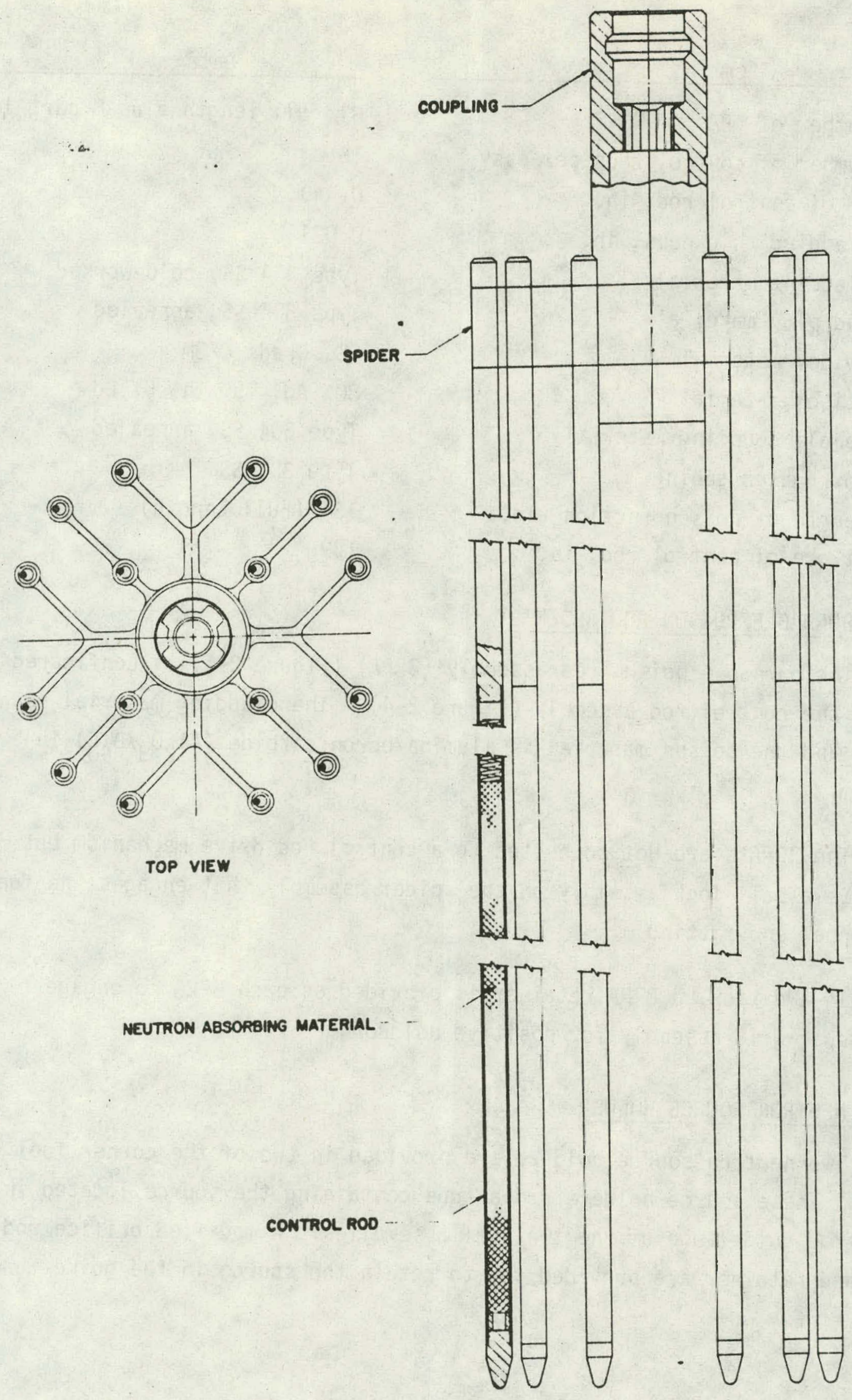

Figure 2-4 CONTROL ROD ASSEMBLY 
Item

Number of CRAs

Number of control rocs per assy

$O D$ of control rod, in.

Cladding thickness, in.

Cladding material

End plug material

Spider material

Poison material

Female coupling material

Corrugated tubing

Length of poison section, in.

Stroke of control rod, in.
Data

61 Full length plus 8 part length

16

0.440

0.021

Type 304 SS, cold-worked

Type 304 SS, annealed

SS, Grade CF3M

$80 \% \mathrm{Ag}, 15 \% \mathrm{In}, 5 \% \mathrm{Cd}$

Type 304 SS, annealed

Type 321 SS

134 (Ful1 Length)

139

\subsection{BURNABLE POISON ROD ASSEMBLY}

The burnable poison rod assembly (BRPA) (Figure 2-5) is configured similar to the control rod assembly (Figure $2-4$ ). The cladding material is Zircaloy -4 and the poison material is alumina/boron carbide $\left(\mathrm{Al}_{2} \mathrm{O}_{3} / \mathrm{B}_{4} \mathrm{C}\right)$ in pellet form.

The BPRA's are not connected to a control rod drive mechanism but instead have a ball-lock assemtity on the spider assembly that engages the fuel assembly upper end fitting.

In addition, a BPRA retainer is provided on each BPRA to engage with the upper grid assembly for positive holddown.

\subsection{NEUTRON SOURCE HOLDEF}

Two neutron source holders are provided in two of the corner fuel positions. These source holders are a tube containing the source located in a control rod guide tube of the two fuel assemblies. A modified orifice rod assembly and retainer are provided for to retain the source in the guide tube. 


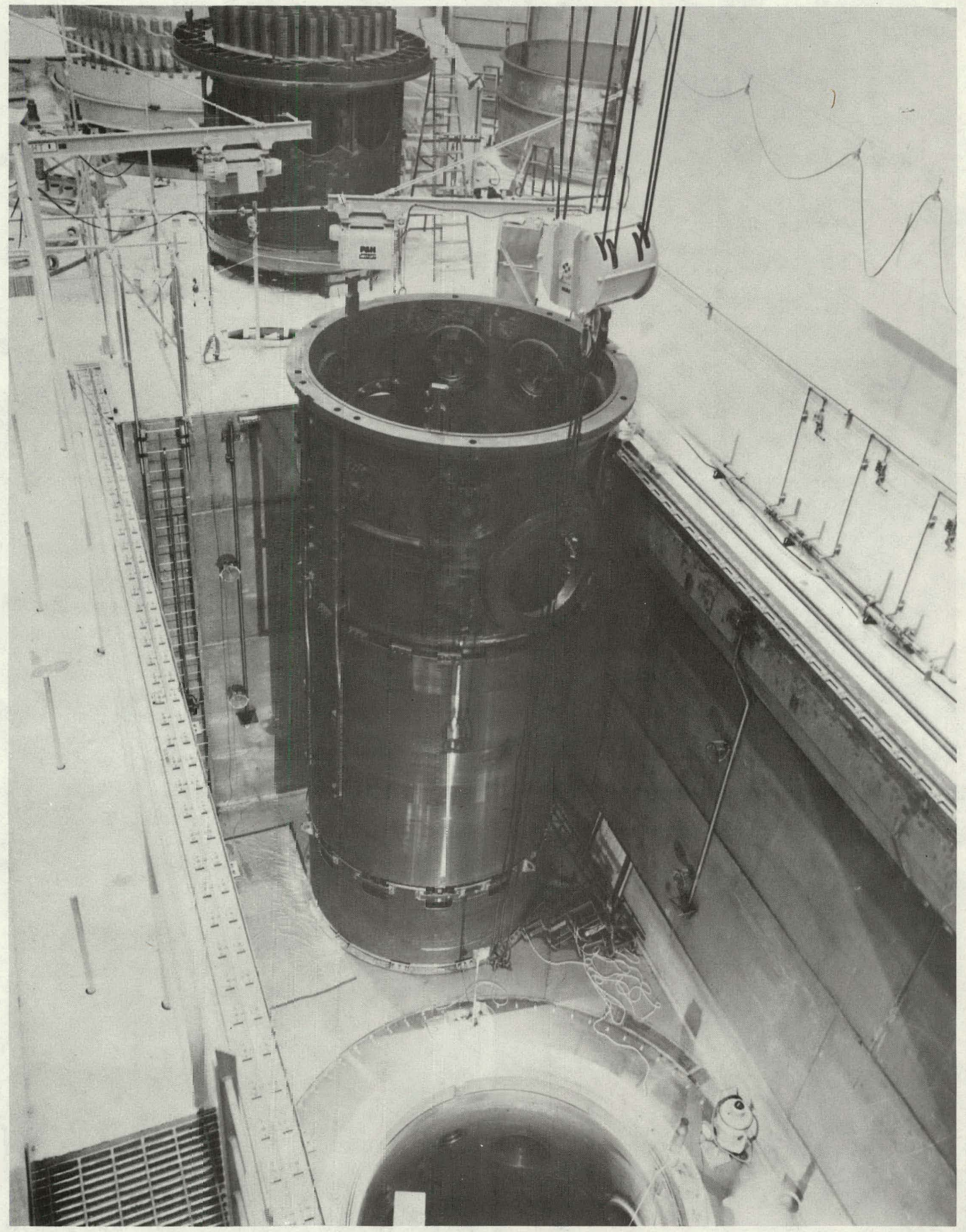

REACTOR INTERNALS CORE SUPPORT ASSEMBLY 


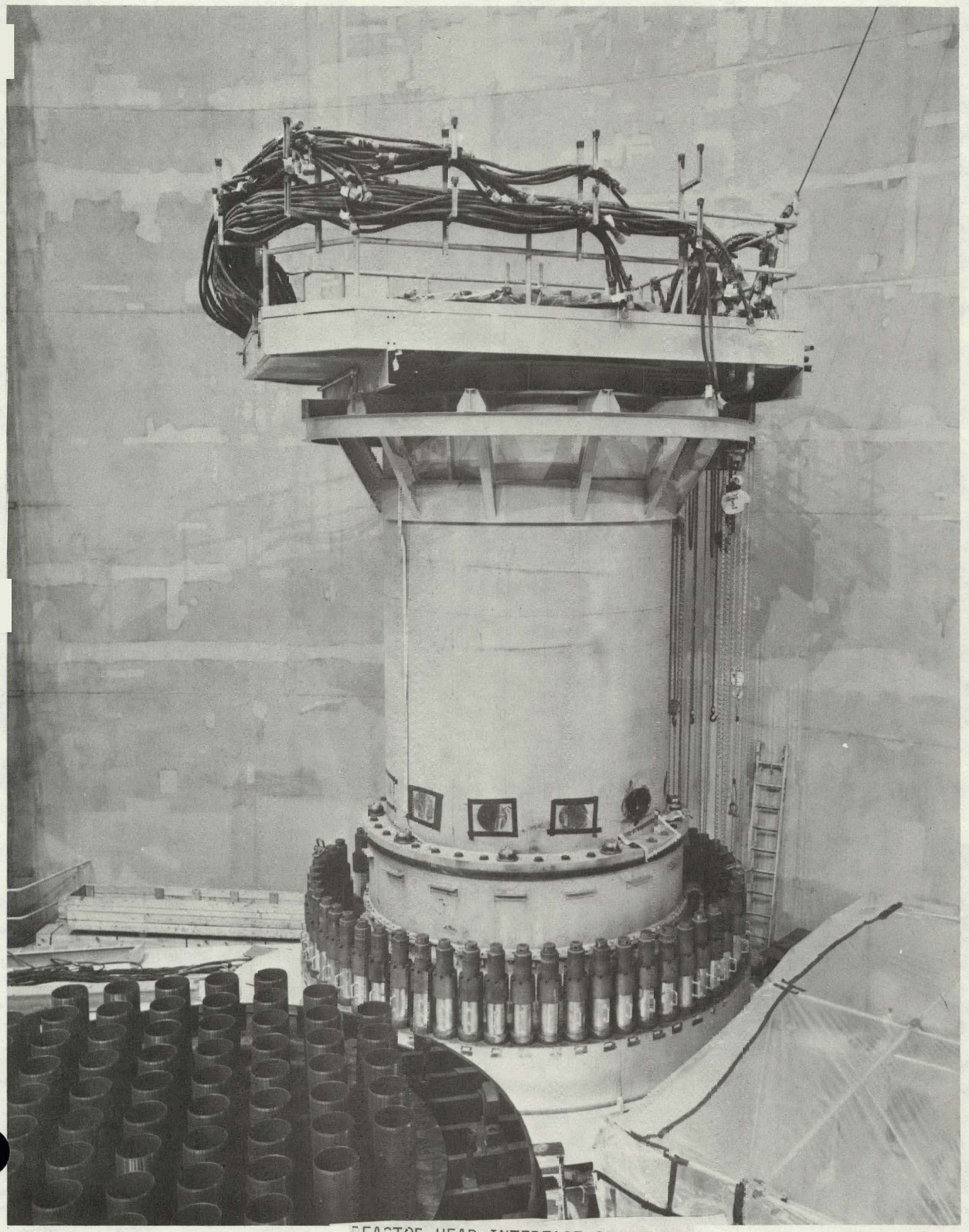




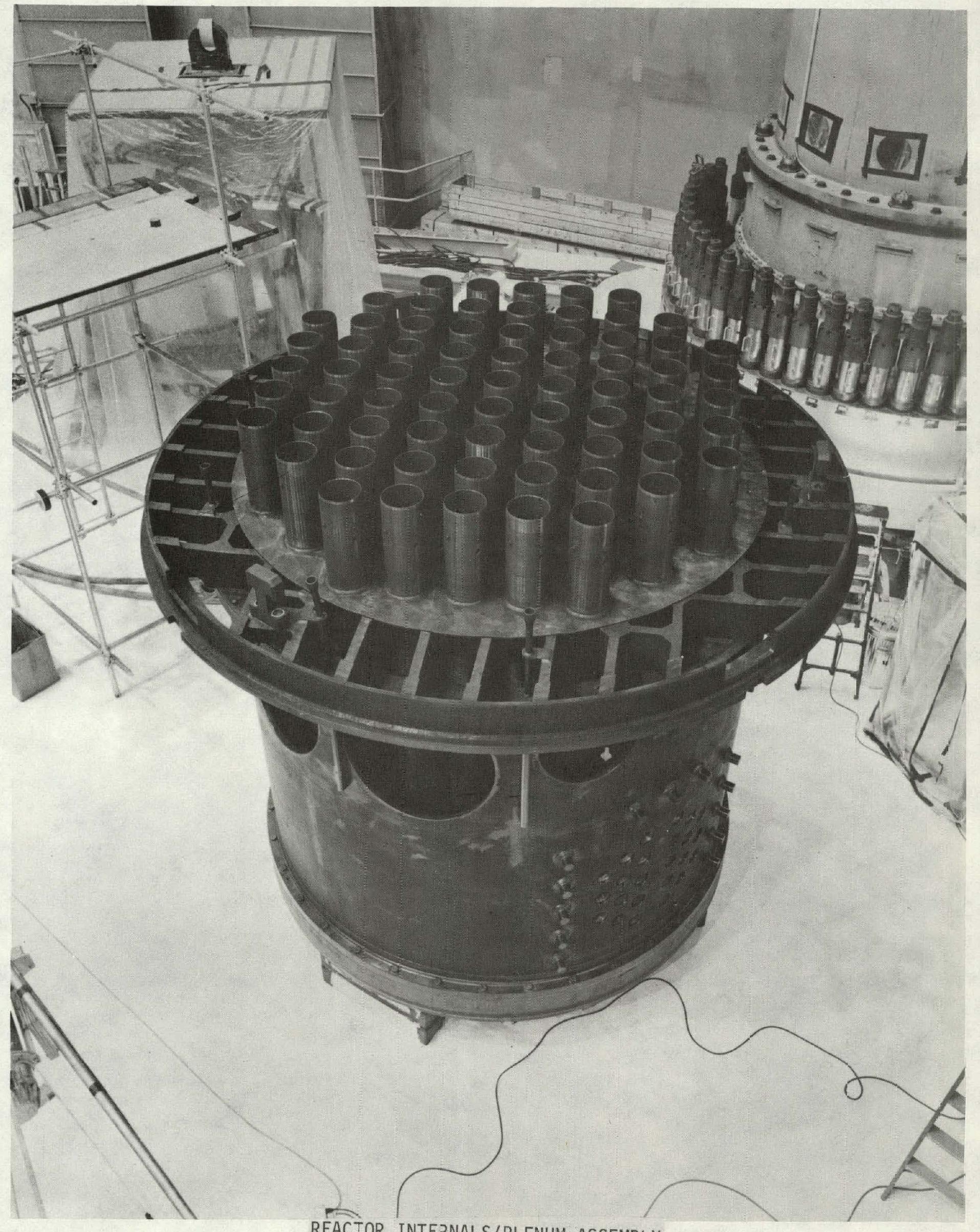


Figure 2-5 Burnable Poison Rod Assembly

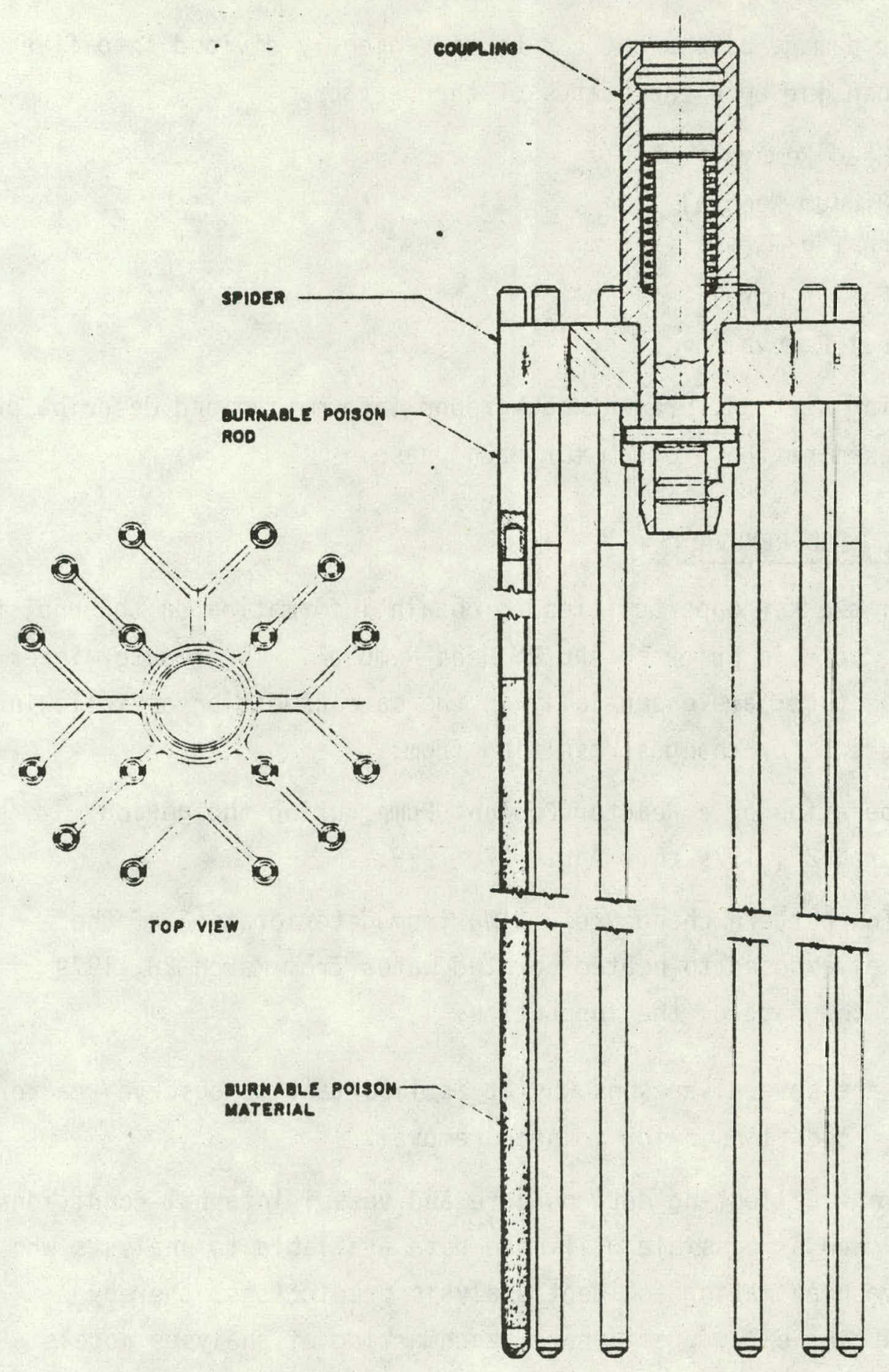


The reactor damage assessment can be conveniently divided into five major phases depending upon the status of the reactor.

- Before Head Removal

- Before Plenum Removal

- Before Fuel Removal

- During Fuel Removal

- After Fuel Removal

The following sections present background information and describe proposed inspection and examination program for each phase.

\subsection{BEFORE HEAD REMOVAL}

One of the key opportunities to obtain information on the undisturbed condition of the core is prior to the RV Head Removal. The reactor internals and core, at this time, are essentially in the same condition as following the transient except for changes resulting from:

a) Operation of a Reactor Coolant Pump during the period March 28, 1979 thru Apri1 27, 1979.

b) Flow pattern change resulting from deterioration of the fuel exposed to heated borated water from March 28, 1979 to the date of the inspection.

There are several reasons why it is important to observe reactor coolant internal conditions prior to head removal.

- Time - Collecting data on core and vessel internal conditions as soon as possible will make data available to analysts who have been making accident analysis predictions, thereby enabling early preliminary benchmarking of analyses models and assumptions. 
- Evaluation of Fuel Inspection \& Removal Methods - Both the R\&D inspection programs and the Fuel Removal Programs will have been planned based upon predictions of core \& internals conditions. Actual measurements and observations will serve to add validity to the assumptions or suggest modifications are necessary, thereby enabling better advance planning of R\&D and core removal activities.

- Acquire Vital Samples for Offsite Examination - The process of obtaining access for inspections may result in obtaining key samples of selected materials which may be analyzed for information of core conditions.

- Obtain Undisturbed Data - The process of removing the reactor vessel head and upper internals has the potential for disturbing the components inside the vessel. Data obtained from the undisturbed core condition will allow better characterization of the detailed data obtained during the later core examinations.

Several complementary techniques could be carried out to assess core conditions. They can be characterized approximately as follows:

A. Direct Visual Observation

B. Indirect Measurements

C. Component Movement

D. Collect Samples

The following proposed programs outline selected projects which encompass one or more of these techniques.

\subsubsection{Core Access Thru CRDM Nozzle}

This project would involve removing one or more of the control rod drive mechanisms from the vessel head and utilize the vessel opening for viewing into the vessel, the upper internals (control rod guides) and the top of the fuel assembly. 
This project would:

- Demonstrate uncoupling of a CRDM extension rod from a control rod.

- Demonstrate the ability to remove a CRDM from the vessel head.

- Provide a sample of material (CRDM coupling) that was located immediately above the fuel during the transient for laboratory examination to enable assessment of temperature history based upon metallurgical examination plus other examinations.

- Other data on conditions of upper plenum assembly including the upper grid and the Control Rod Guide columns. Of particular interest is the degree of slumping, if any, of the upper grid structure.

- Provide information on the potential separation of the fuel upper end fitting from the upper grid structure.

- Provide an insight into the general condition of the upper parts of the fuel assemblies.

The observation and measurements that could be made would utilize some combination of visual (optics, TV, etc.) and other (UT, EC, etc.) techniques.

\subsubsection{Core Access Thru TC Nozzles}

Another access to the RV interior is through one or more of the small ( 1" dia) flanged penetrations outside the cluster of CRDM's on the vessel head. Inserting a viewing device through these holes would provide information on the conditions in the upper head region, the plenum cover, and potentially provide the ability to look into the plenum assembly and the top of the upper grid structure. It may also be possible to extend the observations to reactor vessel annulus area and obtain information from the bottom of the reactor vessel. This would require development of a long $\left(40^{\prime}\right)$ viewing device that could be inserted into a 1 inch diameter opening. 


\subsubsection{Incore Detector Calibration Tube}

The general condition of the incore detectors may be determined prior to removal of the detectors using several techniques.

- Make an assessment based upon visual observation and dose measurements to see if the center calibration tubes have failed. Water in the calibration tube is an indication of tube failure.

- Insertion of a wire probe into the calibration tubes would be an indication of the level in the core where gross. damage may have occurred.

- May be able to obtain neutron flux profile with a wire scan of the core thru the calibration tubes.

\subsubsection{Incore Instrumentation}

The incore instrumentation guide tubes provide direct access to the core prior to head removal from the lover portion of the reactor vessel. These access holes can provide considerable irsight into the radial and axial damage configuration.

Incore instrumentation strings should be removed from selective assemblies to provide a representative number and type of damaged assemblies. The ability to remove an incore detector gives valuable information as well as providing samples of core material for laboratory examination. With the detector removed from the incore guide tubes, other measuriements could be. made utilizing the incore guide tube access to the fuel assemblies.

The mechanical strength of the fuel assembly instrumentation tubes can be measured. This can be accomplished by a mechanical loading head inserted after removal of. the detector. Measurements could be made at various heights to determine residual tube strength. The diametral load could be empirically related to tensile properties. This direct measurement could be coupled with oxide thickness measurements using eddy current methods to give indications of the mechanical properties. 
The lower reactor vessel head can be checked using gamma-scan techniques to survey for gross fuel debris in this region. The sensing elements could be calibrated to provide rough measurements of curies content of mixed insoluble fission products characteristics of fuel, residual.

A neutron flux profile may be obtained by inserting a Dy-Ae wire through the guide tube if the incore detector is removed. An evaluation must first be made to verify that sufficient neutron flux exist from spontaneous and $(\gamma, n)$ reactions.

The use of a neutron source, and a fission chamber in an adjacent location or use of the existing source level instrumentation may provide information of the subcritical multiplication of the core.

\subsubsection{Control Rod Movement}

The eight APSR's are located near the periphery of the core. A program should be developed to attempt to move selected APSR's to determine if they can be moved. If the APSR's can be moved, this would indicate minimum ' damage/distribution to the guide tubes.

The movement of the APSR's with neutron detectors located in nearby incore guide tubes could provide information or reactivity distribution in that region of the core.

\subsection{BEFORE PLENUM REMOVAL}

fifter removal of the RV Head, the plenum cover is exposed. This affords an opportunity for several measurements, samples and observations that are inportant relative to core configuration.

\subsubsection{Photograph and Sample Any Loose Material on the Plenum Cover}

The area above the plenum cover is a "low" flow area in a PWR.

It is possible that even with single pump operation, sufficient fluid velocities were achieved in the core and plenum areas to transport some luose fuel or clad segments to the area above the plenum cover. This material would then settle on the plenum cover. 
TABLE 3.1

SUMMARY - INSPECTION AND TECHNIQUES

BEFORE HEAD REMOVAL

\section{RECOMMENDED INSPECTION}

1. Visual examination thru Head Penetrations

- TC Nozzles

- CRDM Nozzles

2. Gamma Scan internal

- vessel penetration

- incore detector tubes

3. Gamma Scan external

4. Neutron Flux Profile

5. Movement of Core Components

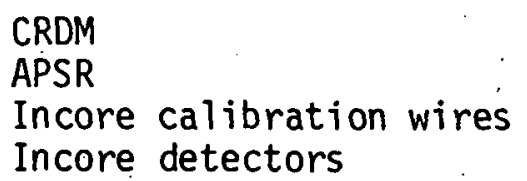

6. Samples for examination

CRD coupling

Incore detector

\section{TECHNIQUE/EQUIPMENT DEFINITION}

Visual examination techniques

- Video

- Fiberscope

Potential for EC or UT examination

Gamma probe thru vessel penetrations or incore detector tubes.

Gamma detectors for external gamma scan.

Neutron detector inserted thru incore tubes

- Systematic program of attempting to remotely move core components to determine if they can be moved

- Tooling and containers to remove samples to hot cell 
Photographing and sampling the material on the plenum cover will preserve data on potential fuel and clad segments that may have come loose $i$ the core. It would also serve to indicate the types of fuel segments that maj be located in other parts of the system. Samples of material could be shipped off-site for detailed examinction.

\subsubsection{Measure Contral Rod Location Relative to Plenum Cover}

Measurement of the lacation of the top of the control rod coupling will provide a set of data relative to the degree of dislocation of the fuel upper end fittings. Separation of the fuel end fitting from the upper grid would indicate degree of core slump - if any.

\subsubsection{Measure Key [limensions of Plenum}

Several key measurements of the plenum cover can be used to obtain supporting data on core condition:

- Measurement af flatness of plenum cover may give some indication of the loads imposed by the fuel on the plenum.

- Jistortion of control rod guide tubes may indicate cemperature in the plenum.

- Measurement of oxide thickness in the guide tubes or plenum structure may indicate temperatures in the plenum area. Instrumentation to determine oxide thickness may have to be developed.

\subsubsection{Program of Selected Control Rod Measurement}

A carefully controlled selected program of attempts to move control rads would provide some cata on the conditions of control rods and associatec guide tubes. This program would supplement the program of APSR movement suggested in Section 3.1.5. These control rod movements would be done by hand with direct measurement of force required to move the rod. 


\subsubsection{Visual Observations}

A program of visual and photographic observations should be conducted prior to and during the plenum movement. These observations and records will provide data on:

a) Inspection of top of peripheral fuel assemblies by inserting the viewing device through the openings in the plenum.

b) If any end fittings are below the upper grid, observations of the surrounding upper end fitting areas can be made by inserting the viewing device through the CRD guide tubes.

c) It may be possible to drill through the plenum cover and obtain access to the top of fuel assemblies containing LBP's.

d) It may be possible to obtain access to the control , rod guide tubes of peripheral fuel assemblies. Eddy current measurement or a tydraulic mandrel could be used to determine wall thickness of the control rod guide tubes in the peripheral fuel assemblies.

e) Visual monitoring and recording during the plenum remova: will provide:

- evidence of fuel assembly hang-up in the plenum

- early data on the general conditions on the top of the core.

\subsubsection{Sample Debris from Inside Plenum}

Pieces of fuel or clad may have migrated into the plenum region above the core or into the guide tubes. Samples of this material would supplement previous samples from above the plenum.

\subsubsection{Visual Examination of Nozzle Gap}

With the use of viewing devices and/or thickness gage, the inspection of the gap between the core support assembly and the outlet nozzle on the reactor vessel may be practical. This would give a good early indication of the degree of distortion of the internals during the transient - hence, a benchmark for internals temperature. 
TABLE 3.2

SUMMARY - INSPECTION AND TECHNIQUES

BEFORE PLENUM REMOVAL

\section{RECOMMENDED INSPECTION}

1. Visual Examination of Plenum Cover

2. Collect samples on plenum cover, inside plenum assembly

3. Measurement of location of components

- flatness of plenum cover

- location of fuel upper end fittings

4. Visual observation of top of core

5. Movement of control rods

6. Zircaloy oxidation measurements

7. Visual examination of nozzle gap

\section{TECHNIQUE/EQUIPMENT DEFINITION}

Visual examination techniques

- Video, color photography

Sample containers for shipment to hot cell

- Tooling to retrieve samples

Tooling for underwater measurements

Viewing device thru plenum (TV, fiber optics)

Systematic program to determine load required for movement of control rods

Techniques to determine oxide thickness

- Eddy. current

- strength of zircaloy tube

Viewing device \& thickness gage 


\subsection{BEFORE FUEL REMOVAL}

Following removal of the TMI-2 reactor vessel head and the upper plenum assembly, there are a variety of inspections that should be made to ascertain the in-situ core condition.

A number of assumptions were used to form the framework with in which the inspection recommendations are made;

1. Assume reactor vessel head and upper plenum assembly has been removed and the top of the core is accessible.

2. Movement/removal of core instrumentation presumed to be minor; no significant perturbation of core material has occurred.

3. Sequential inspections provide foundation for evaluating applicability of subsequent efforts.

4. Focus of core examinations on those required to characterize core condition that could not be performed after fuel movement.

5. Limit man-rem exposure.

6. Inspection program has been limited to core/fuel examinations; inspection of other components such as core barrel, vessel nozzles, etc. assumed covered elsewhere.

7. Inspection program predicated basically upon availability of existing equipment techniques with adaptations to TMI environmental requirements.

Two key assumptions are that operations prior to uncovery of the top of the core have not significantly perturbed the core condition and that the program would depend on essentially available inspection techniques and equipment.

The recommended inspections are limited to core components defined by the fuel assembly and control components prior to fuel movement. Examination of other core internals (e.g., core support assembly, flow nozzles) may also be desirable to perform prior to fuel movement, but are not addressed here. The 
type of examinations proposed are considered to be generally within existing design or overall NDT capabilities although the adaptability of any system to the TMI environment must be evaluated. The specific inspection sequence must be flexible based on the observed core condition. All observations should be thoroughly documented using as much electronic recording instrumentation as possible along with the more conventional manual logging of unusual observations.

The electronic recording capability will permit subsequent computerized data processing and retrieval for more efficient analysis.

The overall objectives of the proposed core inspection program prior to fuel shuffling areas follows:

- Assess the radial and axial extent of damage to fuel assemblies and related core components. Specific consideration will be given to identifying and characterizing those features that may be severely disturbed or destroyed once fuel movement begins.

- Provide a bases for evaluating alternate techniques for removing core components.

-. Provide technical bases for selecting components (e.g., peripheral fuel assemblies, CRAs, APSRAs) for early transfer out of the core to permit more exhaustive examinations elsewhere.

Any floating debris in the cavity pool water resulting from plenum removal should be cleaned up before beginning the "over the core" inspections. to improve visibility and reduce man-rem exposures. The examinations are intended to be sequential and provide information about the core which cannot be reconstructed later. For example, evaluating the degree of fuel assembly independence prior to fue 1 movement will be important in selecting the techniques for core removal and also make it possible to locate and identify a condition between assemblies (e.g., bowed rods or grids, reaction products) which may be destroyed during removal. The initial activities listed in Table 3.3 will be employed on a core wide basis to characterize the extent and nature of any damage that may be present. The precise method in which later inspections are applied 
would then be established after review of the initial inspection results. In any case, however, priority would be given to characterizing regions of the core with varying degrees of damage as well as peripheral assemblies in the preparation for moving selected assemblies earlier in the program.

Control Rods and Axial Power Shaping Rods will be examined to determine if they are intact and the extent of any damage that may be present. Several of the CRAs and APSRs inspected would then be removed from the core and transferred to the spent fuel pool. This operation will permit inspection of the guide tube ID in various core regions to assess the integrity and axial damage profile.

Examination of the instrumentation tube could potentially be done by removing the "mixing cup" from the fuel assembiy upper end fitting to give access to the instrument guide tube at the top of the core. The axial position and condition of the top of selected instruments can be determined prior to instrument removal. After these instruments are removed, the ID of the guide tube can then be examined to evaluate condition and presence of any reaction products. This would also make it possible to remotely inspect the lower core as well as the lower core support structure.

After all of the proposed exams have been completed and the results reviewed, then one or more peripheral or moderately damaged assemblies can be removed from the core. This improved access would make it possible to perform additional inspections, if necessary, to the fuel remaining in the core as well as the lower core support structure.

\subsubsection{Recommended Inspections}

The recommended inspections are listed in Table 1 with the applicable equipment/technique for obtaining the information correspondingly identified in a side-by-side format. Although the table is large self-explanatory Section 3.3.2 provides an expanded description of the inspection techniques/ equipment. 
TABLE 3.3

SUMMARY-INSPECTIONS AND TECHNIQUES

\section{BLTORE FUEL REMOVAL}

Recommended Inspection

1. Document and map top of core to assess

o extent of observable damage functions of radial position

- location and inventory of debris

- determination of observable component integrity (i.e., corrosion and/or oxidation film characteristics, RCC cluster upper end fitting integrity)

2. Relative elevation profile of core components to evaluate severity of fuel assembly slumping.

3. Sampling of debris and loose material from top of core to ascertain composition of loose products or any reaction products that may be present

- provide early direct indication of type of reactions

- debris analysis and estimation of amount of deposit
Technique/Equipment Definition

Visual inspection (forward to right angle viewing direction)

o high resolution TV system with computer enhancement and hard copy reproduction capability.

- periscope for high magnitifcation, high resolution, color photography of top of vessel

- mechanical probe manually operated in conjunction with remote TV

- alternate-acoustical imaging or high frequency ultrasonic equipment for mapping

- continuous visual monitoring capability by remote TV

- vacuum pumping and filtration system for loose product

- technique for obtaining sample of fixed or agglomerated material (basic mechanical removal.) 


\section{TABLE 3.3 (contd)}

4. Evaluation of convective coolant flow characteristics to determine coolant flow paths through core and identify potential blockage locations over entire core cross section along with characterization of flow velocity.

5. Incore residual temperature measurements to provide axial and radial profiles of core and potentially assess locations of large masses of high source material resulting from core damage.

6. Gross and specific isotope counting measurements to locate regions and identify source isotopes associated with high fields

- axial and radial core profiles using results of previous exams to locate regions of high interest

7. Evaluation of Control Rod Assembly damage

- cracks, oxidation/corrosion of lifting

- time lapsed infrared photography

- addition of dye or tracer element to coolant

- flow velocity measurement

- standard thermocouple recording and instrumentation hub and vanes.

- integrity (i.e., cracks, ballooning, chemical interactions) on individual fingers

- CRA removal

- mixing cup removal or penetration to allow access to central thimble tubes

- self powered detectors

- collimator system with spectrometric analysis capability

- ultrasonics (plate wave generator)

- ECT

- lifting device with calibrated load cell 


\section{TABLE 3.3 (contd)}

8. Thimble tube probing to characterize condition and debris sampling

- free path measurements

- ECT for evidence of oxidation/corrosion, or cracks

- visual inspection and photography

9. Axial position of instrumentation within the thimble

10. Expansion of CRA thimbles at bottom of predetermined strain to evaluate integrity prior to movement of fuel assembly

11. Assessment of physical independence of fuel assemblies prior to movement

12. Lift peripheral and/or "undamaged" fuel assemblies for more detailed evaluations

elsewhere

- one assembly or group of assemblies are moved then additional inspections can be performed due to increased access.
- mechanical probe with simultaneous ECT capability

- borescope/photographic system with light enhancement capability

- cutting device to remove $T / C$ mixing cup

- depth probe

- hydraulic expansion head with "U" cup seals

- mechanical spreading device

- gaging technique to quantify assembly to assembly spacing

- spears

- fuel assembly basket

- calibrated load cell device 


\subsubsection{Technique and Equipment Descriptions}

The following is a summary of the various techniques that could be used to perform the inspections summarized in Table 1 . The numbered items correspond to the similar item numbers in Table 1.

1. Visual techniques would be employed to document and map the top of the core. The equipment used would depend upon the exact purpose of the inspection. The basic equipment would consist of a high resolution, underwater TV system and a periscope with high magnification and photographic adaptability. Both systems would have forward to right-angle viewing capabilities.

Extensions of the basic capabilities would include:

a. Computer enhancement and hard copy reproduction for the TV system.

b. Stereoscopic viewing for both periscope and TV. sys tems .

2. Measurements taken from a fixed reference elevation to an established feature at the top of the core would constitute the basic technique for obtaining the relative axial core profile.

. The equipment would cons ist of a manual mechanical probe, in conjunction with an underwater TV system. Development of an acoustical imaging system to map the core profile as an extension of present capabilities.

3. The techniques to obtain samples of loose or fixed material from the top of the core involve manual manipulation of basic tools. The tools/ equipment for this operation include:

a. Remote Underwater TV to provide continuous visual monitoring.

b. Vacuum pumping and filtration system for collection of loose products.

c. Mechanical removal system for fixed or agglomerated materia1. 
4. The techniques for evaluation of convective coolant flow are considered state of the art, but would require adaptation for specific applications. Some of the techniques deemed suitable for this purpose are:
a. Infrared Photography
b. Flow Velocity Measurements
c. Tracer Element or Dye Addition
d. Temperature Profiling with Thermocouples

5. The technique for obtaining incore residual temperature measurements involve manual probing with fixtures utilizing state of the art thermocouples in conjunction with standard recording instrumentation.

\section{Measuring gamma and/or neutron flux involves manually} positioning the detectors at points of interest and through appropriate read-out/recording equipment the levels and/or spectra could be obtained. This technique is an extension of the state of the art and would require a developmental effort.

7. Techniques for Evaluation of control rod damage involves visual inspection and NDT Methods, which are currently state of the art, with adaptation for the specific applications.

The equipment used would depend upon the specific NDT technique and/or the item/area being evaluated.

8. Two techniques could be used for thimble tube probing The first is a mechanical interference probe with simultaneous Eddy Current capability. The second is a visual system, involving the use of a boroscope with photographic capability.

These techniques involve state of the art equipment with suitable development for the application.

9. A technique for Evaluation of the control rod quide tube integrity prior to movement of a fuel assembly involves the use of expansion techniques along the tube and/or evaluation by Eddy Current testing methods. 
10. A technique for assessing the physical independence of fuel assemblies involves the insertion of a "feeler gauge" between assemblies. Basic mechanical devices such as spreading device and a gauge are required.

11. A technique for moving an "undamaged" fuel assembly involves the use of spears, which provide a structural link between the top and bottom of the fuel assembly and/or the use of a "basket".

12. A technique for determining the axial frosition of the instrumentation thimble involves a mechanical proble after anj obstructions at the top of the fuel assembly are removed by whatever cutting device is required. The cutting device requires development for the specific application.

\subsection{DURING FUEL REMOVAL}

Following the removal of the TMI-2 upper head and plenum assembly, the top of the core is exposed. A series of measurements and data taking will be performed before actual fuel removal is started. These measurements and techniques are discussed in Sections 3.1 through 3.3 of this report. This section of the report discusses the data needs and the measurements methods which could be used while the damaged fuel is being removed from the core.

Several analytical predictions have been performed to assess thermalhydraulic effects and the resulting core damage which may have occurred during the TMI-2 accident. Post accident examination of the core and reactor hardware should provịde sufficient data to address the assumptions and models used to characterize the core during the accident, and to provide data on the modes of fuel failure. Specific data needs include:

1. Overal1 Core Configuration;

- The degree of core blockage-assembly by assembly.

- The size of the blockage zones.

- The radial and axial distribution of core blockage.

2. Local Fuel Damage

- Evidence of fuel melt.

- Evidence of fuel slumping due to melting. 
- Evidence of clad "drip" or "flow" or did it completely oxidize first.

- Evidence of eutectic formation.

- Fuel particle size and distribution.

- Fuel rod ballooning and burst characteristics.

3. Core Hardware Examination

- Evidence of stainless steel melting.

- Degree of control rod guide tube oxidation or other damage.

- Degree of damage or melting of the inconel fuel assembly grids.

4. Estimate of Core Temperature History

- Preliminary estimates of local core temperature history as a function of elevation and radial position.

- Distribution and degree of clad oxidation.

5. Core Fuel Distribution

- Distribution of fuel in the core, the reactor vessel and throughout the remainder of the systems.

- Mass balance.

Many of the above data needs can only be met by a combination of in-situ observations and detailed hot-cell examinations. The in-situ measurements will give preliminary results, however, they will later be confirmed by more detailed off-site measurements. Integration of the in-situ program and the off-site program will be required to optimize data gathering.

\subsubsection{Pre-Defueling Preparation}

Prior to core removal, a good deal of data will be obtained on the structural. state of the core. This information will be used during defueling planning and is also part of the identified data requirements. Before core examination begins, a file should be developed for each fuel assembly. In this file should go key manufacturing, fuel loading and assembly inspection and sperational' data. The intent is to have each of the fuel assembly's history 
documented. A detailed sketch of the fuel assembly should be made with each grid, rod, guide thimble given an identification number. The crientation of the assembly with respect to the core should also be noted. fs data is obtained on each assembly it can also be recorded and filed with the before accident fuel assembly records.

Before removing fuel, metal impact detectors should be attached to the internals and/or the reactor vessel lower plenum. Several sensors could be attached $90^{\circ}$ apart and other sensors on the hot legs. These sensors can be used to hear if large quantities of rubble fall onto the lower grid or reactor lower plenum. The detectors probably cannot pick up a single pellet however.

\subsubsection{Photographs and Other Visual}

The first approach should be to immediately photograph the top of the core using TV camera in high quality color. Conventional and periscope TV cameras should be used. In addition, mechanical measurements of the length from some fixed reference point like the top flange, to each fuel assembly nozzle should be made to see if any slumping of the core top has occurred. What is of interest is whether the core damage can be assumed to be symetrical and similar to the before accident power distribution. It is assumed that the control rods can not be moved in the center of the core. If they can be moved, then small gamma detectors should be inserted into the inccre detector locations to obtain a gamma map. The results from a gamma map should indicate the degree of symmetry of the core damage.

The TV camera scan should also indicate the state of the top fuel nozzles, and should indicate whether any: stäinless steel melting occurred. TV scans and the mechanical measurements should also indicate the structural differences between an edge. fuel assembly and the center fuel assemblies. It is believed that the edge fuel assemblies should be less damaged and may be removable in a conventional fashion. If the edge assemblies appear structurally sound, then eight of the outside assemblies should be removed in symmetrical locations as dictated by the before accident power distribution. 


\subsubsection{Study of Outer Assemblies}

With eight outside assemblies removed, detailed axial TV camera scans can be made in these locations. This will help determine the axial extent of the fuel damage as well as to determine the symmetry of the damage. Mechanical measurements should also be made from the vacated assembly position on rod spacing, grid locations, and relative rod spacing on those assemblies which can be viewed. Detailed photograph studies can also be made of selected fuel damage after reviewing the TV camera data. The objective of the detail photographic study would be to obtain local fuel damage information using high resolution photographs. Scales or reference dimensions should also appear on the photographs for each reference.

\subsubsection{Removal of Upper End Fittings}

After removing the eight outside assemblies, it is recommended that the upper end fittings of the interior fuel assemblies be removed without causing any fuel movement. It is believed that these end fittings could not be successfully used to lift the inner fuel assemblies and removing them will allow more detailed viewing of the core damage. Detailed TV camera scans should be made of the core top (without end fittings). Once these scans are reviewed, more localized TV camera and photographic studies can then be made of local areas to obtain local fuel damage, clad damage, fuel particle sizes, and the presence of fuel or clad melting. The degree of core wide fuel damage symmetry can be examined. Mechanical measurements of fuel particle sizes, clad damage, and flow blockage can be made by insertion of calibrated feeler gauges from the top of the core. Also selected samples of clad, fuel, and core structures can be obtained for preliminary hot cell analysis.

\subsubsection{Removal of Control Assemblies}

After the mechanical and photographic data has been obtained, attempts should be made to remove selected control rods or LBP rods to open the guide tube thimbles such that small gamma detectors can be inserted. The gamma detector technique should indicate fuel density and, therefore, blockage (on an assembly wide basis) as a function of axial position for each assembly measured in the core. Some R\&D will be needed to help develop the computer 
software to translate the gamma data into flow b ockage. Some bench tests will also be needed to calibrate the measurement technique. If symmetry is tested, and found to exist from assembly to assembly, not all assemblies wi:l require gamma scans: The gamma scans can also help select thase assemblies which will be saved for hot cell Post Irradiation Examination work.

\subsubsection{CR Guide Tube 0xidation}

Prior to movement of fuel, it is desirable to determine the degree of control rod guide tube oxidation for selected fuel assemblies. Most of the peripheral assemblies do not contain control rods or burnable poison rocis and therefore the guide tube thimbles may be open. This condition may be achieved on other assemblies by removing the control rod or burnable poison rod assembly.

Special instrumentations to measure the strength (hence the thickness of zircaloy) can be inserted into the guide tubes to give a preliminary measurement of the degree of residual zircaloy metal remaining at various radia: and axial locations in the core.

R\&D will be required to develos the instrumental and benc? testing for calibration of oxide thickness.

\subsubsection{Individual Assembly Movement}

After the gamma scans and oxide measurements are complete and reviewed, a decision can be made on how much of the core can be removed with a minimum of data. Each fuel assembly should be photographed, sketches made, and particular irregularities noted. The assemblies selected for hot cell PIE work should be removed so that the PIE assemblies can be handled with less fear of damage and distortion due to handling.

A pie shaped wedge such as that shown in Figure 3-1 would remain after the remainder of the fuel has been lifted out of the core. Since there does not appear to be any practible way to "cast" the fuel in epoxy cr other compounds, this fuel should be removed axially. such that more accurate photographs can be made of the fuel damage at each elevation to obtain the resulting axial flow blockage. Samples of fuel clad, fuel particles, grids, guide tubes, control 
TABLE 3.4

SUMMARY - INSPECTION AND TECHNIQUES

DURING FUEL REMOVAL

\section{RECOMMENDED INSPECTION}

1. TV and Photographic mapping of fuel assembly during removal

2. TV and photographic axial mapping of in-place fuel via vacated assembly areas

3. Gamma scan axial mapping of in-place rue 7 vid vacated assembly

4. Control rod guide tube zircaloy strength measurement

5. Fue 1 rod measurement to determine extent of rod bow, ballooning, etc.

6. Encapsulated Fuel Assembly(ies)

7. Collect samples of fuel, control rod, burnable poison rod, etc. for off-site examinations.

\section{TECHNIQUE/EQUIPMENT DEFINITION}

- Visual Inspection (forward to right angle viewing direction)

- High resolution TV system

- Color Photography - periscope for magnification

- Visual Inspection

- High resolution

- Color photography

- Movable collimated gamma detector

- Calibrated mechanical or hydraulic expander to measure residual $\mathrm{Zr}$ tube strnegth

- Calibrated rod gap measurement device

- Method of encapsulation to remove damaged fuel assemblies intact.

- Sample collection tools and containers 
FIGURE 3-1

TMI-2 PROPOSED FUEL REMOVAL

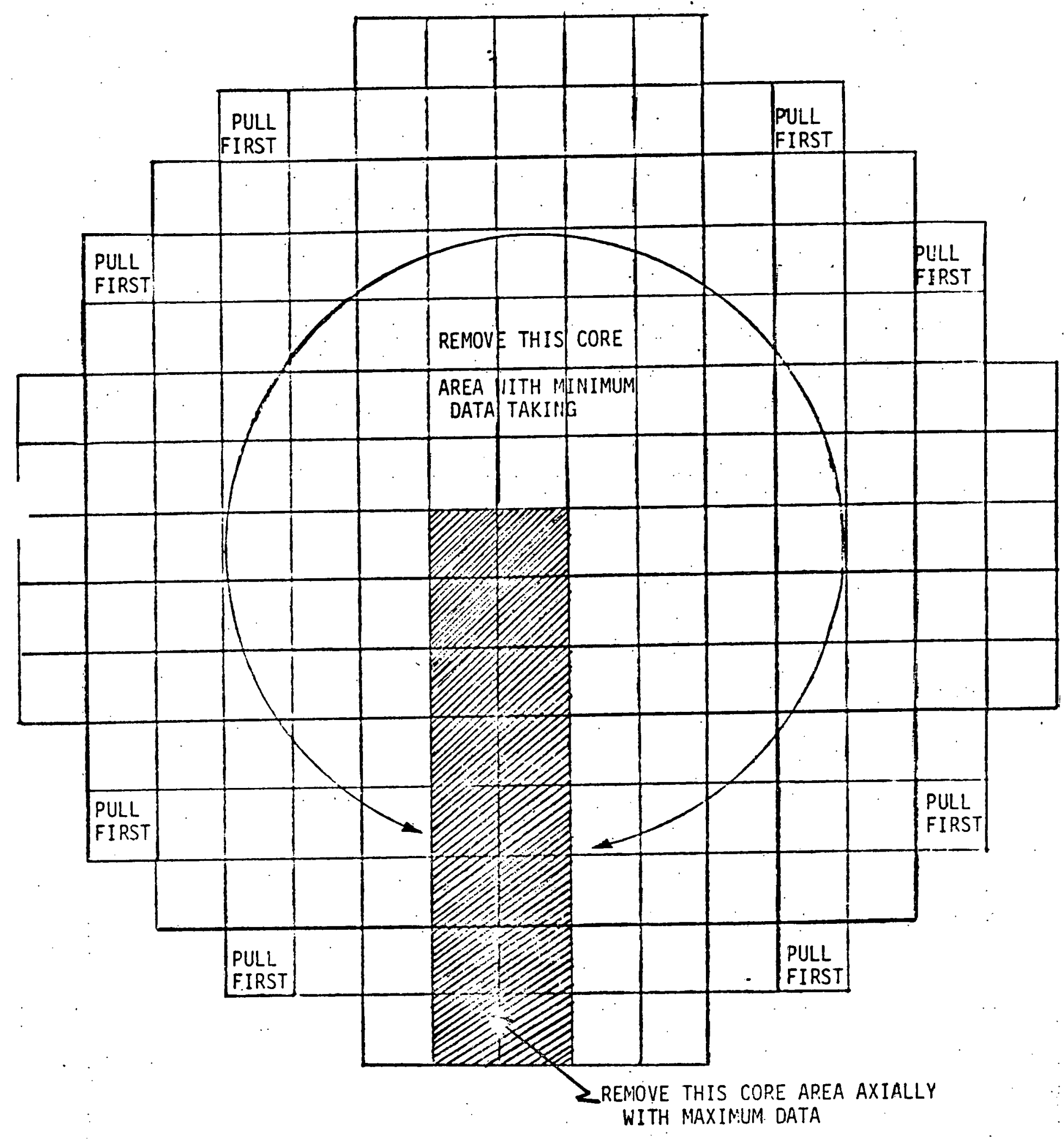


rod materials; can be taken for additional PIE work. Mechanical measurements of fue 1 rod distortion, bowing, oxidation, and clearances should also be made along with the photographic study to help infer the localized flow blockage. These measurements should continue downward into the undamaged fuel zone.

After all the fuel has been removed, TV scans can be made of the lower internals, lower plenum, and the fuel particle distribution which is present in the lower plenum. The distribution of the fuel rubble in the lower plenum can be inferred with mechanical measurements of the height of the debris as function or radial and angular position. It is assumed that similar measurements of fuel rubble which could have collected on the top of the internals has been made such that a complete fuel mass balance can be made. Samples should be obtained at different points to obtain the particle sizes and packing density. The fuel inventory in the lower plenum can then be calculated and conveyed to the final measurement when the lower plenum is cleared out. The same type of measurements may also be needed for fuel rubble in the lower guide support structure.

\subsection{AFTER FUEL REMOVAL}

After the core is removed, a number of key activities are required to complete the cleanup. During these activities, measurements and observations. should be made to supplement the previously gathered data.

Some of these key activities include:

- Inspection of lower internals

- Search for loose fuel \& hardware

- Inspection of Reactor Vessel

\subsubsection{Inspection of Lower Internals}

Physical measurements of the reactor internals should be made according to a pre-planned program. This should be a part of the overall effort to provide a post accident geometry of the reactor vessel and its internals. Drawings of the internals showing the key as-built and post accident geometry should be prepared. Changes in dimension should provide the following information: 
a) Peak temperatures and differential, temperature of core and reactor vessel,

b) the reuseability of core structural components, and

c). indication of changes in flow passages during the accident.

The measurements can be made by working at the level of the reactor closure flange, using measurement techniques that are existing technology These techniques include optical and laser surveying instruments, as well as direct measurements using rods and tapes.

In addition to physical measurements, visual and color TV observations should be made to record color changes, distortions, failed fasteners, etc. that will give added information on the temperatures reached during the transient:

Following removal of the internals from the reactor vessel, visual and TV records can be made of external conditions of the core support structure, the thermal shield clamps, and the lower flow distributor assembly.

\subsubsection{Search for Loose Fuel \& Hardware}

Using an underwater television camera with several live monitors plus continuous video tape recording, the in-vessel area stall be photographed. All features that appear to be worthy of attention are to be photographed. These include discolored, disfigured, mechanically damaged areas, as well as collections of debris or items of unknown origin. Video recordings will be identified as to location in the core.

The television camera viewing operation can be followed by adaptation of the camera with small bore extensions, right angle viewing heads, and special lighting to view the areas under the core support plate to search for more loose fuel pieces and parts.' 
It would seem logical that the reactor could not be returned to service without assurance that all fuel and loose debris were cleaned up. At this point it would seem that this criterion would require full view of the lower head of the reactor vessel and any surfaces that could be suspected of holding up material. It may also require replacing the reactor upper head, establishing circulation through the vesse1, filtering out debris and then examining the vessel once again. This process may determine that the best times for gaining access for the various viewing and gamma scanning operations.

Traverses should be made with gamma detectors over as much of the interior of the reactor vessel as possible. Traverses are to be done in the $x-y$ direction (plan view) as well as in the $z$ direction (up and down), if possible. Gamma spectroscopy provides indication of gross amounts of radioactive material as well as specific identification of the isotopes that are present. These readings can:

a) Tocate fuel remaining in the vessel after the cleanup operations,

b) provide information on the surface condition of the reactor internals such as the support shroud; core support plant, flow diffuser, etc., this information can possibly be utilized as an indication of the reusability and/or the disposal technique to be used on individual components,

c) provide information on the transport of radioactive species during the accident; these readings would have to be interpreted and analyzed along with other operating data such as flow, cleanup operations, etc., that would affect transport and deposition of materials.

The technique of gamma spectroscopy is quite sophisticated and has many applications including the examination of both operating and shutdown reactor components. The program proposed above would probably require some development work. The geometry of aiming a collinator/detector assembly at a vertical wall would have to be examined. Looking straight down would require very little, if any, development. The levels of radiation to be encountered 
would have to be estimated. It may also require some advanced planning and preparation and perhaps some experiments to calibrate and adjust the instruments. Also, there may be some amount of computer programming and software developnent" required for the analysis of the data. It would require, in addition, a small staff of knowledgeable technicians to use the instrumentation and to interpret the data at the time the operation to assure that the date is useful.

\subsubsection{Inspection of Reactor Vessel}

Following removal of the internals, the reactor vessel should be examined for damage resulting from the transient. Some key areas include:

- damage to outlet nozzle seals due to excessive radial thermal expansion of the core support structure

- damage to the lower guide lugs due to excessive axial thermal expansion of the core support structure

- position and verticality of the incore detector nozzles

- Evidence of local "hot spots" due to accumulation of fuel fragments.

These observations and measurements will require develcpment of an integrated measurement program. The data will be used to characterize the temperature response of the reactor internals during the transient.

\subsubsection{Metallurgical Specimens (Archive).}

Metallurgical specimens should be gathered. Those comonents that may have some further useability will not be cut up, but any areas where there are obvious indications of damage should have metal specimens removed. The techniques for removal can include mechanical metal cutters, such as hole saws or nibblers, or thermal devices such as an underwater plasma torch. These specimens can be taken at any time in the process of rebuilding the plart or of disposing of the damaged components. Examination of these specimens off-site can provide evidence of temperature history, and any metallurgical changes to the physical properties of the materials due to the accident. 
TABLE 3.5

SUMMARY - INSPECTION AND TECHNIQUES

AFTER FUEL REMOVAL

\section{RECOMMENDED INSPECTION}

1. Photography \& Visual Examination of Internals \& Vessel

- damage

- deformation

- color (temperature)

2. Search for Loose Fuel \& Debris (loose fuel in internal baffle plates, lower plenum, etc.)

3. Internals \& Vessel Measurements - deformation.

4. Samples for examination and archive

\section{TECHNIQUE/EQUIPMENT DEFINITION}

Visual monitoring techniques

- Video with image enhancement

- Color photography

Visual and Gamma Scan

- collimated movable gamma scan

- isotopic discrimination methods

Remote measurement tooling

- Imprint casting for local deformation

- Optical \& laser techniques

Sample cutting equipment 
The February 1980 meeting of Task Group 7.2 reviewed the draft inspection and examination program and recommended a list of key early projects for consideration by the TWG.

Summary Task Descriptions have been prepared to describe in more detail the scope of the proposed Tasks and the estimated key milestone schedule data.

It is recognized that much detail planning and evaluation is necessary to eventually evolve the comprehensive program. Task 17 ists preparation. of "event trees" for the program. This preparation of "event trees" is part of the detail planning necessary to define the program and properly integrate the inspection and examination program into the overall Reactor Disassembly and Core Removal Plan. 


\section{1 TASK GROUP 7.2 RECOMMENDE[ LIST OF KEY EARLY PROJECTS}

1. (I). Penetration of Reactor Vessel from Top

2. (I) Ex-Vessel Radiation Examination

3. (I) Examination Via Incore Instrumentation Tubes

4. (II) Plenum Penetration \& Mockup Design

5. (II) Pre-Head Removal Access to Annulus and Lower Plenum

6. (I) Topography of Top of Core

7. (II) Technique for Measuring Core Porosity

8. (II) Technique to Access Physical Independence of Damaged Fuel

9. (I) Techniques for Debris Retrieval and Characterization

10. (II) Core Gamma Scan Through Fuel Assembly Control Rod Guide Tibes

11. (I) Casting (Encapsulation) of one or more Fuel Assemblies

12. (II) Technique of TV and Photo Scênning

13. (I) Technique for Removing Damaged Fue 1

14. (I) Techniques for Measuring Strength of Fuel Bundle Structural Members

15. (I) Data Needs (identification)

16. (I) Specifications of Core Status

17. (II) Summary of Event Trees

18. (I) Special Tooling Development

NOTE: (I) = Proiect should get started immediately

(II) $=$ Project can be started later 
Objective

Inspection of Reactor Internals and Fuel prior to RV Head Removal.

Discussion

The degree of equipment and fuel damage in the TMI-2 reactor is not precisely known. It is expected that significant fuel damage has occurred. that debris is present in the core and the reactor internals and that some fusing together of core components has occurred. Reactor internals damage resulting from the transient may impede disassembly of the reactor and upon removal, may disturb the core configuration.

It is important that as much information as practical be obtained on the conditions inside the reactor prior to reactor disassembly. This information will serve to benchmark the various analyses already completed or underway. It will also guide the development of programs to cbtain more data on the TMI-2 core and other experiments planned or underway. The early look into the reactor vesse i will also provide data for detailed planning of the exarnination programs to be conducted during and after defueling.

\section{Scope of Project}

The scope of the project will involve:

- Identify methods of providing the desired inspection.

- Evaluation of inspection alternatives.

- Selection of primary approach.

- Engineering of equipment and procedures.

- Integration into the TMI-2 Reactor Disassembly Plan.

- Assembly of equipment.

- Demonstration of equipment and procedures.

- Conduct inspection at TMI-2

- Report results of Development Program and Inspection

Schedule (Key Milestone)

Demonstration of Equipment and Procedures ready for field operations by March 1981 . 
Objective

Examination of the radiation streaming from the reactor vessel to assess the potential fuel distribution in the TMI-2 reactor.

\section{Discussion}

The degree of equipment and fuel damage in the TMI-2 reactor is not precisely known. One method of obtaining information on the location of fuel (and hence, extent of fuel damage) is to perform an accurate scan of gamma radiation coming from the reactor vessel. By measuring the gamma radiation levels at selected axial and circumferential positions around the reactor vessel, then comparing these measurements with various postulated fuel geometries, it may be possible to correlate the measurements with calculations, thereby obtaining an assessment of reactor core damage.

of particular interest, it may be possible to determine if the peripheral fuel assemblies are significantly damaged (i.e., slumped) as predicted by some studies or if they are relatively undamaged. This information would be useful in guiding other NRC fuel behavior experiments and analyses code development.

Scope

The scope of the project will involve:

- Identify methods to provide the desired measurement.

- Evaluation of alternatives and selection of primary approach.

- Engineering of equipment and procedures.

- Assembiy of equipment and demonstration.

- Conduct inspection at TMI-2.

- Analyses of radiation leakage from the reactor for various fuel geometries.

- Correlation of measurements with analyses.

- Report results and conclusions of project.

Schedule (Key Milestone)

Equipment ready for site work - March 1981. 
Objective

Obtain information on TMI-2 core status prior to removal of reactor vessel closure head and internals.

Discussion

The degree of fuel damage and the physical configuration of the TMI-2 zore is not precisely known. One method of obtaining information on the core status is to utilize the incore instrumentation guide tubes to perform physical measurements of the core. The types of physical measurements that could be performed include:

- The force required to remove an incore detector assembly.

- Examination of the physical condition of the incore detector.

- Installation of gamma detectors to monitor fuel radiation levels.

- Installation of neutron source and neutron detector to assess the degree of criticality.

Some combination of these measurements witr appropriate evaluation will provide early assessment of the core damage.

\section{Scope}

The scope of the project will involve:

- Identify and recommend an integrated program of measurements.

- Engineering of equipment and procedures.

- Assembly of equipment and demonstration.

- Direct incontainment measurement program.

- Correlate measurements and evaluate results.

- Report results and conclusions on project.

Schedule (Key Milestone)

Equipment and procedure demonstration - ready for site work - March 1981 
4. PLENUM PENETRATION \& MOCKUP DESIGN

Objective

To establish a preliminary plan for making penetrating thru the plenum assembly and preliminary plenum mock-up design.

\section{Discussion}

Removal of the reactor closure head permits access to the top of the plenum assembly. The plenum assembly effectively inhibits much direct observation of the top of the core; thereby making it difficult to obtain much data about the condition of the core prior to removal of the plenum.

The plenum assembly contains the upper grid plate which engages the upper end fitting of the fuel assemblies. It is important to known the status of the upper end fittings prior to and during the lift of the plenum. One way to obtain this information is to cut holes in the plenum cover and insert viewing devices down to and beyond the upper grid plate (if possible).

Performing such an operation at TMI will possibly require a mock-up of the plenum for demonstration and training. This project would identify cases for a mock-up and prepare a preliminary design.

Scope of Project

The -scope of the project will involve:

- Identify methods to obtain core assessment data thru the plenum; including need to cut access holes in the plenum.

- Identify location and size of access holes (if required).

- Preliminary Design of Mock-up (if required) to check ability to make penetration and inspections.

- Integration into TMI-2 Recovery PIan Schedule.

- Report results.

Schedule (Key Milestone)

Complete study by December 1980. 
5. PRE-HEAD REMOVAL ACCESS TO ANNULUS AND LOWER PLENUM

Objective

Perform a study to evaluate methods, risks and benefits of inspecting the RV annulus and lower plenum region prior to head removal.

Discussion

Inspection of the RV annulus and bottom plenum may disclose the presence (or absence) of fuel and other core debris. T'is information is beneficial in understanding the potential condition of the core prior to removal of the head and upper internals.

A method suggested to benefit these inspections is to cut the core flood nozzle and insert viewing devices (TV, fiber optics, etc.) into the annulus region and to the lower plenum. This is a fairly significant operation and would involve some hazard and considerable man-rem exposure. A study should therefore be conducted to evaluate. this approach and to determine if this inspection is justified.

Scope of Project

The scope of this project will involve:

- Evaluate potential program of annulus and lower plenum inspection.

- Identify conceptual designs.

- Identify alternate means to obtain data.

- Estimate cost (dollars, schedule, man-rem).

- Perform preliminary safety assessment.

- Evaluate benefits versus cost and risk, and make recommendations.

Schedule (Key Milestone)

Study completion - December 1980 
6. TOPOGRAPHY OF TOP OF CORE

Objective

To assist in establishing the degree of core damage by measurement of the profile of the top of the core following Plenum Assembly removal.

Discussion

Unrestricted inspection access to the top of the core occurs following plenum assembiy removal. Subsequent fuel movement activities may result in loss of information and evidence important to core damage evaluation. . It is therefore important to establish the "as is" condition of the core after plenum assembly removal and before fue 1 movement activities. Measuring and recording the profile of the top of core is one method of capturing "as is" data.

The topography of the top of the core may provide confirmation evidence regarding areas of minimum and maximum damage. It will provide qualitative evidence of core time-temperature relationships during the accident.

The topography examination will also assist in establishing reliable methods of retrieving debris and core parts for further inspections, evaluations and anatysis.

\section{Scope of Project}

The scope of the project will involve:

- Identify methods of performing the measurements and/or recording the evidence.

- Evaiuation of methods described above.

- Integration into TMI-2 fuel Removal P1an.

- Priority selection of methods for measurement and/or recording.

- Equipment and system specification and selection.

- Method demonstration arid equipment/system tests:

- Procedure development.

- Integration into TMI-2 schedule.

- Execute work on TMI-2.

- Report results.

Schedule (Key Milestone)

Equipment and procedure ready for site work - August 1981. 
7. TECHNIQUE FOR MEASURING CORE POROSITY (II)

Objective

Develop a method for in-situ determination of core porosity prior to or during the fuel removal program.

Discussion

Various scenarios of the TMI-2 accident have postulated varying degrees of core damage. Whatever the actual degree of damage, the core retained a "coolable" geometry in the post-accident recovery period. It is of interest to obtain a "Macro" scale determination of core porosity as a function of radial position in the core.

Following removel of the fuel, it may be possible to reccnstruct empirically the geometry of the core. The macro-scale measurement would be useful in correlating hydraulic behavior to the core geometry.

Several methods have been suggested:

- Fluid velocity measurements (fixed or natural circulation).

- Penetration of dyes.

- Thermar measurements.

Scope of Project

The scope of the project will involve:

- Identify potential means to determine core porosity distribution.

- Evaluate practicality of the suggested methods.

- If any methods appear practical, identify deveropment needs, schedule \& cost.

- Prepare recommendations on whether to pursue the program.

Schedule (Key Mi lestone)

Complete study by December 1980 . 
8. TEC:INIQUE TO ACCESS PHYSICAL INDEPENDENCE OF DAMAGED FUEL (II)

Objective

Develop a technique (tooling) that can be readily used to assess if the damaged fuel assemblies of the core are physically independent or are metallurgically and mechanically interconnected.

\section{Discussion}

The various core damage scenarios have estimated that the TMI-2 fue 1 assemblies experienced a wide range of damage. The degree of damage can range from slight overheating and oxidation and perforation of the clad where the physical geometry of the assembly is relatively unchanged to complete disintegration of the upper parts of the fuel assembly with some degree of melting and eutectic formation, forming a metallurgical bond between adjacent fuel assemblies.

Upon obtaining access to the top of the core (i.e., removal of upper plenum) it is important to learn which of the fuel assemblies are physically independent and which may be either mechanically and/or metallurgically bonded together.

This information is useful in understanding the degree and nature of core damage as well as being important to the planning of subsequent fuel removal and inspection program.

\section{Scope of Project}

The scope of this project involve:

- Identify conceptual techniques for determining fue 1 assembly independence.

- Evaluate techniques for practicability, cost, risk, probability of success, etc. and recommend referenced technique.

- Detailed design of a prototype development.

- Derronstration of prototype.

- Development of procedure.

Schedule (Key Milestone)

Demons ‘rate prototype tooling - December 1981 
9. TECHNIQUES FOR DEBRIS RETRIEVAL AND CHARACTERIZATION

Objective

Develop methods for core parts debris removal during Reactor Disassembly and Fuel

Removal. Prepare methods which document the physical conditions of this debris.

Discussion

Debris from damaged fuel and control components is expected to be dispersed throughout the Reactor Coolant System. Some of this deoris will be collected for further examination and analysis. It is important to characterize the debris (to the extent that physical conditions permit) with regard to location, size, shape, colcr and any other physical condition which may assist in establishing the progress of the accident and resultant core damage.

Due to Reactor Coolant Pump operation after core damage occurred, it is expected that debris will be found throughout the RCS and interconnected auxiliary systems; particularly in dead legs and low flow areas. Methods are needed to locate, retrieve and document the conditions of this outs ide the reactor debris.

Debris is also expected to be in the Plenum Assembly, the Core Support Assembly and in the Core Area. As Reactor Disassembly proceeds, part of this debris will. be exposed and easily retrieved. If it is not retrieved, some debris will likely be disturbed during subsequent Reactor Disassembly activities. Other debris will be lodged in the Reactor Components in varying degrees of accessability. Methods to remove some portion of this debris are needed and a means to document debris conditions is needed.

Scope of Project

The scope of this work will involve:

- Identify potential Pocations for debris of interest to the R\&D plan.

- Identify potential physcial configuration of debris.

- Establish characterization criteria and methods.

- Identify and evaluate retrieval methods for each potential location and physical configuration.

- Establish equipment and tooling needs for above methods.

- Integrate into TMI-2 Reactor Disassembly and Fuel Removal Plan.

- Equipment procurement and assembly.

- Equipment and procedure demonstration.

- Execute work at TMI-2.

- Prepare final report.

Schedule (Key Milestone)

Preparation for on site work complete by August 1981. 
10. CORE GAMMA SCAN THROUGH FUEL ASSEMBLY CONTROL ROD GUIDE TUBES

Objective

Development of equipment and techniques to perform core gamma scan through fuel assembly control rod guide tubes.

Discussion

One technique for establishing in-situ fuel geometry is to perform a comprehensive core gamma scan. This technique involves inserting a small gamma detector into control rod guide tubes and performing an axial gamma scan of selected fuel assemblies thhroughout the core.

To obtain access to the control rod guide tubes may require removal of core control components (individual rods or assemblies). The peripheral assemblies do not contain control components and if relatively undamaged would provide easy access for the gamma scan.

\section{Scope of Project}

The scope of this project involves:

- Review core damage estimates and evaluate potential information to be obtained by core gamma scan.

- Identify development required (analytical and hardware) for the gamma scan program.

- Develop instrumentation.

- Develop procedures.

- Conduct gamma scan

- Evaluate data and report results.

Schedule (Key Milestone)

Demonstrate technique of prototype equipment - December 1981 
11. CASTING (ENCAPSULATION) OF ONE OR MORE FUEL ASSEMBLIES

Objective

The objective of this project is to develop a tecinnique to encapsulate a section of the TMI-2 core (one or more fuel assemblies).

\section{Discussion}

Several analytical studies have been performed to assess the thermal-hydraulic effects which have occurred during the TMI-2 accident and the resulting core damage. Post-examination of the core and reactor hardware shculd provide sufficient data to assess the assumptions and models used to characterize the core during the accident. Since significant portions of the core may be severely damaged, and removal of the fuel will disturb the "as-found" condition it is of interest to preserve at least a portion of the core in the "as-found" condition for ultimate detailed examination off site. One postulated method is to encapsulate some portion of the core (one or two fuel assemblies) thereby retaining the "as-found" physical condition during the removal process.

The encapsulated fuel column could then be shipped to a laboratory for detailed examinations to obtain information on the core damage sequence. which could later be used for fuel damage modeling studies.

\section{Scope}

- Identify conceptual encapsulation techniques.

- Identify interface 1 imitations for fuel encapsulation.

- Evaluate practicality of encapsulation vs potential benefit of information.

- Recominend encapsulation feasibility.

Schedule (Key Milestone)

Recommendation of encapsulation feasibility by October 1980 . 
12. TECHNIQUE OF TV AND PHOTO SCANNING

Objective

Prepare a program of TV and photo scanning, using state-of-the-art technique, to record the condition of the TMI-2 core.

\section{Discussion}

Perhaps the most significant record of the in-situ condition of the TMI-2 core can be made by a program of video and photo mapping using state-of-the-art underwater techniques. This program will provide a necessary reference documentation of the overa11 as-found condition.

To successfully carry out such a program requires knowledge of the dates technology is available in this area. Techniques such as color, photo-enhancement, infra-red photography, 3-D video or photo should be evaluated.

Engineering and development efforts to apply these technologies to the TMI-2 environment should be specified and planned.

Scope of Project

The scope of the project involves:

-. Identify techniques that are available.

- Evaluate alternatives and recommend primary approach.

- Identify development and/or engineering for specific application.

- Integration into TMI-2 core removal plan.

- Procurement of equipment and/or services

- Demonstration of equipment.

- Conduct inspection at TMI-2 and deliver records.

Schedule (Key Milestone)

Demonstration of equipment by December 1981 
13. TECHNIQUE FOR REMOVING DAMAGED FUEL (I)

Objective

Development of techniques to remove damaged fuel from the TMI-2 reactor for further examination.

Discussion

In order to establish the specific core damage that occured during the TMI-2 accident, damaged fue 1 must be retrieved for further off-site examination and experiments. The fuel selected for examination should be representative of the varying degrees of damage. Other criteria for selection would include benchmarking damage to analytical predictions of core damage based upon data recorded during the accident.

In order for subsequent examination of damaged fue 1 to be meaningful in establishing specific core damage which occurred during the accident, the fuel to be examined must be removed without causing further damage.

The technique for damaged fuel removal should include steps to inspec: fuel in-place, select final means of handling, movement to an inspection station and movement to a suitable container.

Scope of Project

The scope of this work will include the following:

- Establish preliminary target fuel assemilies for removal for subsequent examination.

- Prepare a plan to finalize target fuel assemblies after access to the fuel.

- Define the houndary damage conditions for fuel to be removed for subsequent examination.

- Prepare methods for removal of the spectrum of damaged fuel expecied per the selection process above and the boundary damage conditions above.

- Integrate project into TMI-2 Fuel Removal Plan.

- Evaluate and select methods to be pursued.

- Procure equipment and material and fabricate tooling.

- Demonstrate tooling and procedures.

- Prepare final report.

Schedule (Key Milestone)

Preparations for on-site work complete by October 1981. 
14. TECHNIQUES FOR MEASURING STRENGTH OF FUEL BUNDLE STRUCTURAL MEMBERS

Objective

To measure strength of a representative number of fuel element structura 1 members in order to:

1) Assist in evaluation core damage

2) Establish load bearing capability of fuel to support removal methods while minimizing further damage.

Discussion

The structural members of interest in this project are the control rod guide tubes and attachment of these tubes to the fuel end fittings.

Although the degree of core damage is not precisely known, it is believed that degrees of structural weakening exist up to failure, thus leaving portions of some fuel assemblies in a rubblized condition. In order to assist in evaluating both radial and axial damage, it is desirable to measure the strength remaining in a representative number of fuel assemblies. Strength comparison radially (fuel assembly to fuel assembly) and axially (single-fuel assemblies) and an overall comparison to as-built strength should provide input to the overall evaluation of the fuel damage caused by the accident.

It is desired that some number of fuel assemblies be removed carefully so that additional examinations can be performed. The strength measurement would provide data in establishing the residual strength in fuel assemblies and assist in fuel removal method selection to minimize further damage during removal.

Scope of Project

The scope of this project will involve:

- Identify methods of measurement of control rod guide tube strength.

- Identify methods of inspection for/or measurement of strength of guide tube attachment to upper end fitting.

- Evaluation and selection of methods to pursue.

- Equipment procurement and system assembiy.

- Demonstration of equipment and procedures.

- Integration of project into TMI-2 Fuel Removal Plan.

- Execute work at TMI-2.

- Prepare final report.

Schedule (Key Milestọne)

Preparation for'on-site work complete by October 1981. 
15. DATA NEEDS (IOENTIFICATION)

Objective

Define the requirements for data from the TMI-2 Core Damage Assessment Porgram.

Discussion

The TMI-2 Core Damage Assessment Program may involve a substantial number of measurements and observations. Each step in the reactor defueling program will result in the accumulation of observations and data on the conditions in and around the reactor.

It is important that a pre-planned analys is of data requirements be conducted to guide the development of the examination and inspection programs. Such an analysis will assist in performing cost trade-off evaluation studies as well as minimize the potential of overlooking significant data during the process.

Scope of Project

This project can be carried out in parallel with the Technical Planning of specific projects including the definition of event trees (See Project 17). The scope of the project involves:

0 Identify type of data required

- Identify alternatives for obtaining data

- Define how data will be reported and stored. 
16. SPECIFICATIONS OF CORE STATUS

objective

Prepare a specification which can serve as the reference core status description for the design and development of equipment and procedures for Core Damage Assessment.

Discussion

Various analytical studies of the TMI-2 accident have been performed to assess the thermal-hydrualic transient and the resulting core damage.

Most of these studies agree trat parts of the core have been severely damaged to the extent that conventional fuel handling methods will not be possible for. the entire defueling and core inspection operations.

It is necessary therefore to prepare a specification document that describes in some detail the "reference" core description that would be used as a guide in the various Planning and Developinent Frograms associated with the Core Damage Assessment Plan.

This task is not intended as an analytical task to perform an independent assessments, but it would review the assessmants made to date with the objective of describing the rarige of core damage assessments. In addition, it would establish a "reference" description for the different degrees of damage that will have to be inspected and removed from the core. These could vary from essentially undamaged fuel assemblies to assemblies that have only 2-3 ft of undamaged structure with the remainder in some specified configuration above the undamaged section. This specification would then form a common bas is for the development of equipment and procedures for core inspection and defueling.

\section{Scope}

- Collect availabie core damage assessments.

- Prepare a description of the different types of damaged fuel and non-fuel componerits in the core.

- Define the "reference" types of damaged fuel and non-fuel components in the core.

- Prepare drawings, sketches, etc., to visually describe the types of damaged fuel and non-fuel components.

- Compile the above information in a document, "Specification of Core Status."

Schedule (Key Milestone)

The document should be available to guide designers by October 1, 1980 . 
17. SUMMARY OF EVENT TREES (II)

Objective

Develop "event trees" to aid in the planning and execution of the TMI-2 Core Damage Assessment Program.

Discussion

The TMI-2 Core Damage Assessment Program involves a number of measurements and inspections during the program for disassembly and defueling of the TMI-2 reactor. The specific condition of the reactor is not currently known, therefore the planning for the program is based upon assumed conditions that will. exist during the defueling.

Since it is likely that actual conditions and/or actual disassembly and defueling steps will be different from those currently assumed, it is of beneift to establish a series of "event trees" to define the inspection and examination program both under base-case conditions and with assumed alternative conditions.

Scope of Project

Scope of project will involve:

- Prepare "event trees" defining sequence of inspection and examination program.

- Identify alternative inspection and examination programs.

These "event tree" preparations can be done in parallel with the technical planning for the inspection examination program and the identification of data needs (See Project 15). 
18. SPECIAL TOOLING DEVELOPMENT (I)

Objective

Develop generic special tooling needed for potential applications on the core damage assessment of TMI-2.

Discussion

To carry out the core damage assessment tasks at TMI-2 will require the development of specialized tooling.

Examples of the types of tooling that may require development include:

- Tests and tooling to enable CRDM uncoupling.

- Tooling to cut CRDM's to allow head removal in the event that CRD uncoupling cannot be accomplished.

- Tooling to cut samples of materials from inside the reactor vessel.

- Other special tooling to handle semi-remote operations in high radiation areas.

This tooling, developed as part of the TMI-2 Core Damage Assessment Program is the type of tooling required on a generic basis for special maintenance application in high radiation fields on other reactors. In addition, this tooling will have application in dismantling and decommissioning of power reactors.

Scope of Project

The scope of this project involves:

- Identify special tooling required for core damage assessment.

- Identify required developments

- Design, and development of tools including fabrication and/or procurement

- Demonstration testing

Schedule (Key Milestone)

Depends upon specific tooling required. Identification of special tools by December 1980 . 


\section{APPENDIX A}

\section{AXIAL GAMMA SCAN OF EACH FUEL ASSEMBLY PRIOR TO}

REMOVAL FROM THE REACTOR VESSEL

\section{OBJECTIVE}

To measure the distribution of fuel with in the reactor vessel and quantify the extent, in a three dimensional sense, of fuel dislocations resultant from the accident.

METHOD

By locating a miniature gamma sensitive detector in one of the fingers of the fuel assembly removal tool, axial gamma scan data can be acquired as the tool is being inserted for assembly removal. By combining the gamma detector with the removal tool, the need for special gamma scan equipment is eliminated. By performing the data acquisition in parallel with core disassembly, virtually no. critical path time would be required and hence minimum impact on the overall schedule would result. Recording of the data could be done remotely and automatically.

DISCUSSION

Because of the high electron density of uranium, the mean free path of fission product gamma rays within a spent fuel assembly is considered to be short, approximating the radial dimensions of the fuel assembly. Hence, a gamma sensitive probe, running along the length of the assembly somewhere near the assembly center, would receive a signal proportional to the fission product density in the area of the detector. Since the fission product derisity is expected to be smoothly varying and proportional to the most recent local power density, discontinuities or abnormalities in the expected gamma signal, can be interpreted as discontinuities or abnormalities in the local fuel density. Axia' surveys in each fuel assembly (or half, quarter, or eighth core segment) could be used to construct a three dimensional gamma plot from which equivalent fuel content contour plo:s could be constructed.

The proposal to locate a gamma sensitive detector in the fuel assembly removal tool is based on the proposal that this tool be capable of lifting intact fuel assemblies from the bottom assembly nozzle. It is assumed that, to accomplish this, several "fingers" would have to be inserted into the fuel assembly control rod guide tube reaching to the bottom nozzle which would be grasped for assenbly withdrawal. 
Since al1 16 control rod guide tube locations will probably not be used, it is proposed that one or possibly two additional "fingers" be added to the fue 1 assembiy removal tool (for the first interior ring of guide tubes) and be used to house a miniature gamma detector at the bottom tip. The gamma detector could be one of several commercially available of the size and sentitivity to acquire the required data. (W Elmyra division makes a miniature neutron detector . 188" dia. which is sensitive to gamma rays. Increased sensitivity of this detector could be achieved by increasing the gas pressure, thinning the dimension of the center electrode and/or filling with a high $Z$ gas such as Xenon). If time and developmental money were available, a gamma sensitive crystal (NaI or equivalent) mounted on the end of a fiber optics light pipe could possibly be developed and used in place of the gas filled ion chamber.

Whatever detector is used, the necessary power supplies, preamplifiers, amplifiers, cables, connectors and recorders would be required to complete the measuring/ recording process. Data analys is would require correlation of the detector position (axial location of the fuel assembly removal tool), with detector output signal. 


\section{APPENDIX B}

STATE-OF-THE-ART FOR EXAMINATION TECHNIQUES

FOR USE IN THE TMI-2 CORE

\section{Compiled by}

David 0. Hobson

Metals and Ceramics Division

Oak Ridge National Laboratory 


\section{PREFACE}

The following compilation is the result of the cooperation of many people and several institutions who provided the compiler with their expertise, suggestions and help. A probably highly inadequate effort is made in the text to give these persons and institutions due credit for their help. Names are placed jarenthetically under various text headings to indicate input into the report. In several instances the compiler has quoted verbatim from written submissions; in other instances he has taken the input and distilled it into his own words. Where no names are included, the compiler is responsible. 
1.0 INTRODUCTION

1.1 Basic Concerns

2.0 MECHANICAL

2.1 Sampling

2.2 Probing

2.3 Actuating

2.4 Measuring

3.0 INSTRUMENTATION

3.1 Visual and Photographic

3.2 Nondestructive Testing

3.3 Nuclear Techniques

3. 4 Reactivity Measurements

3.5 Gamma Spectrometry

3.5 Continuous-Gamma-Ray Spectrometry

3.7 Solid State Tract Recorder Methods for Neutron Dosimetry

3.8 Thermal Measurements

3.9 Flow Measurements

3.10 Portable Microcomputer Aid to TMI Investigation 


\subsection{INTRODUCTION}

Among the examinations and measurements proposed for use before, during, and after removal of the TMI-2 core are many that represent high technology techniques. In addition to the sophistication inherent in the techniques themselves, there is the need to implement them in a hostile, unwieldly environment. It should be emphasized at the outset that the needed techniques do exist to do the required examinations.

Mechanical examination methods for remote surveillance of reactor components have been available for years. Specific early examples include the techniques used with the Homogeneous Reactor Experiment (HRE-2) core tank and with the Boiling Nuclear Superheater Reactor (BONUS) in the early and late 1960s. Both of these examples involved mechanical manipulation to allow visual, photographic, and videographic examination of in-reactor components. In addition, the mechanical operations of cutting, handling, and part removal had to be accomplished. Similar operations have been carried out at CANDU, Enrico Fermi, Windscale, and SRE. State of the art advancements in the above areas lie in more sophisticated methods for manipulating equipment and in advances in miniaturization, resolution, and durability of the components.

In addition to the mechanically-oriented methods, there are instrument-based measurement technologies that should be employed in the TMI-2 situation. These include the areas of neutron and gamma ray transport in both complex and deep penetration geometries. In fact, a substantial portion of this compilation is devoted to these areas. These will be among the first areas to be explored on TMI-2 and are among the most complex, technologically, to be utilized in the examination.

Finally, there exists much expertise in the area of computer science relative to radiation transport methods, reactor physics applications, and other computer-based analytical methods. This is useful for interpreting data from measurements taken both before the pressure vessel is opened and during all phases of measurements related to the nuclear configuration of the core. Various computer codes are available to handle the complex analysis problems involving reactivity and other radiation transport problems. 
The following pages of this report will describe suggested examination procedures, discuss their requirements, and describe the apparent states of the art that exist for them. Time limits prevented an exhastive search for methods and techniques, so any description contained herein is subject to updating.

The proposed examination procedure is broken down into the five chronological stages outlined below. Subsequent discussion will refer to the various stage numbers.

Stage 1. Before pressure vessel head removal.

Stage 2. Before removal of plenum and upper internals.

Stage 3. Before fue $\hat{\imath}$ movement.

Stage 4. During fuel removal.

Stage 5. After fuel removal.

\subsection{BASIC CONCERNS}

The techniques to be discussed in this section are all intended for use in a high-radiation level, aqueous environment which will require remote operation at all times. This presents two major problems that must be addressed for.each technique:

1. How does one manipulate, lift, cut, photograph, measure, or. do whatever else has to be done at a distance, under water, and out of sight, and still know exactly where the manipulating, lifting, cutting, etc., is actually being done?

2. What measures are necessary in order to be able to do the above. tasks in a high radiation and contamination environment?

A third consideration that permeates the entire task is that of reactivity in the core. Any movement of fuel in the core will have an effect. on reactivity and, if the core is disrupted as a result of the accident, reactivity changes resulting from core removal may not be predictable. Because of this, reactivity measurements will be necessary throughout the examination. 


\subsection{MECHANICAL (P. Holz, R. Blumberg - ORNL)}

The mechanical activities of sampling, probing, actuating, and measuring require a wide range of manipulative operations. Many can be performed around the top and sides of the reactor vessel prior to head removal and from above the vessel after head removal. Others must be accomplished through existing tubes, penetrations, and accessory components. Table 2-1 outlines the proposed mechanical activities according to type and stage number.

In addition to the above activities, almost all of the other observation techniques (photography, TV, eddy current, reactivity, etc.) involve mechanical methods for getting the observing instrument into position.

Techniques presently exist for implementing all of the necessary mechanical activities. However, the amount of operational complexity will ultimately depend upon the radiation levels present in the working area. For the purpose of this report, it is assumed that working personnel have access to the containment building and are able to work, with whatever radiation or contamination protection is required, over the reactor vessel. (If such access is unavailable, then the area of robotics must be explored). A further assumption is made that facilities are available for lifting heavy equipment (500t polar crane, auxiliary hook, etc.), for positioning and holding manipulation equipment, and for removing the reactor head bolts under water.

\subsection{SAMPLING}

Manipulator technology is well advanced and includes capabilities for delicate, well controlled movements as well as high force applications. Master/ slave manipulators, as well as motor driven or hydraulically operated remote arms, are available. The long operating distances, possibly 50 to $60 \mathrm{ft}$., may require space technology techniques involving force compensation to allow lateral or torque forces to be applied.

Small underwater cutting torches, as we11 as saws, shears, drills, tongs, etc., are all adaptable for sampling activities. Properly instrumented, for sight and nuclear detection capabilities, these should allow sampling of any portion of the core, core vessel, and internais. 


\begin{tabular}{lll}
\hline ACTIVITY & \multicolumn{1}{c}{ DESCRIPTION } & STAGE NUMRER(s) \\
\hline Sampling & Obtain samples of material by cutting, & $1,2,3,4$, and 5 \\
& scraping, picking up, breaking off, etc. & \\
\multirow{2}{*}{ Probing } & Mechanical probing of core & 1 \\
& Dept' probes through CR guides & 3 \\
& Core height measurements & 5 \\
& Search for loose fuel and other parts & 1 \\
Actuating & Movements of in-core instruments & 1 \\
& Exercise CR and APSR components & 3,5 \\
& Control component movement experiments & \\
\hline
\end{tabular}




\subsection{PROBING}

This activity is srincipally one of seeing how far a wire, rod, detector, or light will go into a tube, orifice, or cavity in the reactor. Its principal duty is to determine blockage locations, but ancillary duties could involve boroscopy, and the insertion of activation sources into the core. Although engineering drawings of the pressure vessel and reactor core exist and show the various places into and through which a probe can be inserted, it is probable that core disruption will necessitate exploratory probing to discover existing pathways into the core.

\subsection{ACTUATING}

This activity involves careful movement of existing components within the pressure vesse1, such as control rods, axial power shaping rods, in-core instrumentation, etc. Such movements will supply different types of information, such as:

1. Mechanical feeciback
a. ease of movement
b. extent of movement

2. Nuclear feedback
a. neutron flux changes
b. reactivity distribution

\subsection{MEASURING}

This activity has both manipulative and visual requirements whereby mechanical measurements are made of various key dimensions in the reactor vessel. of the various mechanical activities discussed in this section, this probably has the most stringent requirements for precise movement and placement. Among the measurements to be made are:

1. Distance from top of core to a reference plane

2. Reactor Vessel diameters

3. Warpage of vessel internals

Present manipulator technology is adequate for any foreseeable requirements of the TMI-2 examination. 
3.0 INSTRUMENTATION MEASUREMENTS (R.L. Shepard - ORNL; E.L. Courtright - FNL)

TMI-2 examination techniques will involve the use of various items of instrumentation of a widely desparate nature. These techniques are summarized in Table 3-1.

Many of the techniques in Table 3-1 will require computer backup, either as a data acquisition service or as an analytical tool for processing the data obtained from the instruments.

In the following sections a brief description of the state of the art will be given for each technique. It must be recognized that any reference to a specific brand name or specific method is for illustrative purposes only, anc that comparable alternatives may exist.

3.1 Visual and Photographic (G.J. Bolfing, R.L. Moore, and R. Blumberg - OP.NL)

In general, the states of the art for visual and photographic examination of the TMI-2 core are well advanced. Miniaturization of video cameras has progressed, along with development of radiation resistant components and availability of: adequate lighting sources, to a point where exploration within the core is possible.

A 1977 brochure by Westinghouse Electric Corporation describes a camera as follows:

The equipment to be demonstrated is a miniatrue self-lighted W camera designed specifically for nuclear reactor inspection. This camera consists of a camera head, a multilead interconnecting cable and a camera control unit. Special features are a small, lightweight, radiation tolerant camera head of $1.25 \mathrm{in}$. diam and $13 \mathrm{in.}$ length, more than 550 TV lines resolution, remote optical focus and an internal light source power supply. A wide range of viewing and lighting attachments make the camera very versatile and thus suitable in many remote veiwing applications. Attachments include forward, fixed, and rotating right angel viewing with and without internal lights usable in air and underwater. 
Table 3-1 Examination Techniques and Instrumentation Requirements

\section{Technique}

Type of Instrumentation or Measurement

1. Visual and Photographic

1. Video
a. standard
b. stereoscopic

2. Camera (photographic)
a. wide angle
b. miniaturized
$\approx$ stereoscopic

2. Nondestructive Testing

1. Eddy Current
a. oxide thickness
b. gap measurements
c. swelling measurements

2. Ultrasonic

$\dot{c}$. distance measurements

t. acoustical imaging

3. Nuclear

1. Reactivity

a. source neutron multiplication measurement

b. boron displacement measurement

c. ${ }^{252} \mathrm{Cf}$ source drive neutron noise analysis

2. $\gamma$ Measurements
a. $y$ scanning
$i$. gross configuration
ii. specific isotope counting
b. Compton Recoil $\gamma$-ray Spectroscopy

4. Thermal

1. Thermometry
a. thermocouples
b. resistance thermometers

2. Infrared Scanning

5. Flow

1. Ultrasonic Flow Measurements

2. Pitot Tube

3. Hot Film Anemometry

4. Tracer Techniques

5. Time-lapse Infrared Photography

6. Turbine Flowmeters

7. Day Techniques 
This camera is designed and tested for operation up to $60^{\circ} \mathrm{C}$ (which might necessitate development of a method for cooling) and at gamma dose rates of $2 \times 10^{6} \mathrm{R} / \mathrm{hr}$ up to a fluence of $10^{8} \mathrm{R}$, according to $\underline{W}$ literature. More recent information indicates that camera sizes less than 1 inch diameter are available.

Stereoscopic viewing capabilities are available in video equipment manufactured by several companies. One example utilized polarization coding for separation of left and right video channels and the wearing of simple viewing glasses. Another uses a multiplexing technique whereby the video from two cameras is displayed and viewed through glasses with electronically controlled shutters for each eye. The latter system is stated to be compatible with both color and blackiwhite cameras. Both systems have video recording capabilities.

\subsection{Nondestructive Testing (C.V. Dodd - ORNL; E.L. Courtright - PNL;} R.L. Brown and E.M. Sheen - HEDL)

Both eddy current and acoustical examination methods are well developed and applicable to the TMI-2 task. Eddy current techniques have been used to measure oxide film thicknesses in a range from 0-0.005 in. $(0.13 \mathrm{~mm})$ with an accuracy of $+0.00005 \mathrm{in} .(+0.001 \mathrm{~mm})$. The probe consists of a small, flat coil spring loaded against the part. The oxide surface will keep the coil from resting flat on the metal surface, and the distance between the coil face and the matal (which is the oxide thickness) is measured. This type of measurement has been performed in high radiation and thermal environments (up to $1400 \mathrm{~h}$ in an operating reactor) with no degradation of the probe. The probe will have to be constructed and calibrated, using well established design rules, but the instrumentation is on hand.

Eddy current techniques have been used to measure small dimensional variations to within \pm 0.00001 inch $(0.3 \mu \mathrm{m})$ in high radiation environments and in narrow places [coolant channels as small as 0.032 in. (0.3 MM) have been measured. The probe consists of a fixed coil that measures the distance to a spring-loaded feeler or directly to the metal surface. Additional coils have been used to measure the distance to different points on a tube surface and a profile of the tube has been measured. Instrumentation to drive a 24 coil array is on hand, but the probes would have to be constructed to fit inside the 
the tuke bundle.

Multiple-frequency efdy-current techniques are available that could with appropriate calibration and adaptation, simultaneously measure oxide, oxygen-stablized $\alpha$. and prior $\beta$ phase thicknesses on intact fuel rods and other Zircaloy surfaces. A three-frequency instrument is capable of measuring six independent parameters such as thickness, conductivity, etc.

Ultrasonic techniques are presently available to perform both distance measurements and acoustical imaging.

A possible use of ultrasound in the core damage assessment for TMI is in measuring distances from $z$ reference plane to points in a rubble pile or specific object in the core. Small displacements can be readily measured. Sound velocity in water is approximately $0.06 \mathrm{in}$. per microsecond yielding range measurements within a few mils without difficulty, using standard commercially available ultrasonic test instruments and transducers. This type of data could greatly supplement optical images and may be used in confined areas where TV imaging is not possible due to cifficulty in illuminating the area or size of the TV camera. UTtrasonic transducers $1 / 2$ inch in diam or smaller are commonplace.

Standard commercial ultrasound transducers are available for operation to $400^{\circ} \mathrm{F}$, well above the boiling point of low pressure water. Specific application at TMI will require advanced planning and possible construction of special scanners and probes to enter available apertures and make specific range measurements or ultrasonic images.

An advantage of ultrascund gauging measurements over mechanical measurement is the application of neglicible force on the object, which may be important in measuring distance to loose parts or fuel pieces.

In addition, acoustical imaging techniques are available capable of providing images of objects submerged in opaque liquid media. Such a technique could provide information to supplement video or photographic results. 


\subsection{Nuclear Techniques (D. E. Bartine - ORNL)}

As stated earlier, reactivity of the core is an imporzant consideration during any examination or removal operation. Because the core is probably disrupted or displaced to some extent by the accident, its response to that examination or removal may not be predictable. It was suggested by the Technical Staff Report prepared for the President's Commission on the Accident at Three Mile Island that recriticality in the disrupted core dces not have a high potential for occurrence. This conclusion should not be interpreted to mean that reentry and recovery operations at the reactor are free from the possitilty of criticality. With this in mind, it can be seen that the examination techniques discussed below have a special importance; one that extends beyond the acquisition of data for curiosity's sake.

Several techniques exist for determining the reactivity of the core. Three of them will be discussed: (1) Source neutron multiplication measurements, (2) Boron displacement measurements, and (3) ${ }^{252} \mathrm{Cf}$-source-driven neutron noise analysis. These techniques, in general, require computer backup to analyze the data that will be generated. Appropriate computer programs exist to do the required analyses.

In addition to the above, significant neutronics analyses will be required to interpret the data obtained from the various neutron and gamma measurements. This analysis should include $1-D, * 2-D, *$ and possibly $3-D$ models.

*1-D and 2-D TMI models have been developed at ORNL and used to calculate both neutron and gamma fluxes during and after the accident. Thus, the models to be used are available.

\subsection{Reactivity Measurements (J.T. Mihalczo and J.T. Thamas-ORNL)}

Neutron Source Multiplication Measurements. By inserting physically small ${ }^{252} \mathrm{Cf}$ neutron sources into the core and measuring the resulting count rate in neutron-sensitive detectors placed around the core, the source neutron multiplication can be determined. The results of these measurements should be compared with the results of calculations. Because of their small size, the ${ }^{252} \mathrm{Cf}$ sources 
could be moved in reentrant holes or along the outer surfaces of the core to provide the spatial variation of the neutron multiplication. Existing detector systems may or may not be adequate. Top-to-bottom asymmetry of the measurement would give some indication of the extent of the damaged core region.

This type of measurement could be performed before head removal, before plenum removal, and before and during fuel movement.

Source neutron multiplication measurements depend on the source-coredetector configuration and do not determine the reactivity directly. However, if detection efficiency and neutron source changes are properly accounted for, the sign of any reactivity increment (introduced, for example, by control rod or fuel movements) can be determined but not the magnitude. These changes are usually calculated, and so depend on some knowledge of the core configuration. When this method is used to determine reactivity, reference measurements at a known reactivity configuration are required.

Boron Displacement Measurements. Reactivity determination by boron displacement measurements involves changing the boron content of the coolant by a known amount and then measuring the corresponding change in the neutron count rate. In the interpretation of these measurements it is assumed that the count rate is proportional to the source neutron multiplication which, in turn, is inversely proportional to the departure of the neutron mulitplication factor, $k$, from unity. Given measurements of the count rate at two boron concentrations:

$$
\left[C R_{1} \propto\left(1-k_{1}\right)^{-1}, C R_{2} \propto\left(1-k_{2}\right)^{-1}, k_{2}=k_{1}+\Delta K\right]
$$

the multiplication factor $k_{1}$ can be determined if the value of $\Delta k$ is known from previous measurements or from calculations of the change in the multiplication factor with boron concentration. Since the count rates are proportional to both the detection efficiency and neutron source strengths, changes in these quantities must be accounted for in interpreting the measured count rates.

Boron displacement measurements should be made before head removal. Repeating the measurement after head removal with a detector close to the core 
would also be usefur, since the effects of changes in detection efficiency with changes in the neutron attenuation through the borated water would be reduced.

Like the previous method, boron displacement results may be sensitive to the source-core-detector configuration. The value of $\Delta k$ associated with the change in boron concentration must be known. If this value is based on previous measurements with an undamaged core or on calculations for some assumed core configuration, then an uncertainty in the value of the reactivity is introduced.

$252 \mathrm{Cf}$-Source Driven Neutron Noise Analysis. The ${ }^{252} \mathrm{Cf}$-driven neutron noise method of subcriticality monitoring has some unique advantages for application at TMI-2, namely that the reactivity is determined from measurements only at the subcritical state of interest and the interpretation of the data does not require knowledge of either the absolute or relative vales of the detection efficiency or the core's inherent neutron source strength. In this measurement method a ${ }^{252} \mathrm{Cf}$ neutron source in an ionization chamber (detector 1 ) is placed in the core or near the core boundary, along with a pair of high-sensitivity fission chambers (detectors 2 and 3 ) that contain up to $100 \mathrm{~g}$ of $235 \mathrm{U}$ each. These detectors could be placed on top of the core when the upper plenum has been removed. The crosspower spectral densities between the two fission chamgers $\left(G_{23}\right)$, as well as the crosspower spectral density between each detector and the ${ }^{252} \mathrm{Cf}$ ionization chamber $\left(G_{12}\right.$ and $\left.G_{13}\right)$ are obtained from the detector signals. The multiplication factor, $k$, depends on the following ratio of these spectral densities:

$$
\frac{1-k}{k}=\frac{G^{*}{ }_{12} G_{13}}{G_{11} G_{23}} C,
$$

where the constant $C$ depends on known or measured properties of the reactor and the ${ }^{252} \mathrm{Cf}$ ionization chamber and * means complex conjugation. Preliminary considerations indicate that measurements might be technically feasible with detectors at the core boundary. Fortunately, fission chambers are insensitive to $\gamma$ rays (chambers exist which can detect individual fission in gamma ray fields of $10^{7} \mathrm{R} / \mathrm{hr}$, thus $\gamma / \mathrm{n}$ discrimination ratios of $\gg 10^{8}$ are possible) and highintensity ${ }^{252} \mathrm{Cf}$ sources can also be fabricated inside ionization chambers. 
Preliminary calculations indicate that detection efficiency with high-sensitivity fission chambers would permit measurements to be made with around $15 \%$ accuracy in $20 \mathrm{~h}$.

${ }^{252} \mathrm{Cf}$-driven noise measurements should be made only after the upper plenum has been removed, prior to fuel movement. The reactivity thus determined could be used as a reference measurement for the source neutron multiplication method described earlier. If access to the core through instrument guide tubes or control rod guides is possible then the quantities required to obtain the reactivity can be measured and specific knowledge of core configuration is not needed.

This method of reactivity determination requires further experimental and theoretical evaluation prior to application to a large reactor; furthermore, it requires construction of a high intensity $252 \mathrm{Cf}$ ionization chamber. Theoretical evaluation must ascertain the influence of spatial effects or the subcriticálity determination. Measurements by this method on an undamaged LWR core woulc be highly desirable before attempting measurements with the TMI-2 damaged core. For multiplication factors lower than 0.8 this method may only be capable of determining that the neutron multiplication factor is less than 0.8 and not the actual value.

In addition, these noise analysis measurements obta in the prompt neutron decay constant by two methods: (1) from the breakfrequency of the cross power spectral densities, $G_{12}, G_{12}$, and $G_{23}$ (three determinations) and (2) from the ratio of the real to the imaginary part of the cross power spectral densities with ${ }^{252} \mathrm{Cf}$. The prompt neutron decay constant can also be obtained from pulsed neutron measurements in the time domain but these frequency domain noise analys is measurements do not require the use of an elaborate pulsed neutron source. In effect, the ${ }^{252} \mathrm{Cf}$ is the equivalent of a randomly pulsed neutron source and was initially used for pulsed neutron measurements before the advantages of the frequency domain measurements were realized. Although this method obtains the same information (the prompt neutron decay constant) as the pulsed neutron method, in addition to the ratio of spectral densities which is proportional to the 
reactivity, it may not be as sensitive to spatial ef-ects since the ratio of spectral densities is independent of frequency.

Comparative Measurements. Performance of simultaneous measurements in a similar, but undamaged core (for example, TMI-1) and comparison with measurements on TMI-2 would establish the accuracy of the methods. This, of course, depends on the availability of TMI-1 for such measurements.

General Comment. All three methods described rely to some extent on knowledge of the core condition. If the core is accessible through guide tubes, the parameters required for reactivity determination by the ${ }^{252} \mathrm{Cf}$ source driven noise method that depend on the core configuration can be measured. Multiple methods of reactivity determination are highly recommended. In these measurements it must be assured that the reactivity of the entire core is determined.

\subsection{Gamma Spectometry (K.H. Valentine and F.F. Dyer - ORNL)}

Gamma spectrometry has been used routinely in the postirradiation examination of irradiated fuel assemblies and fuel samples from both commercial and experimental nuclear reactors. The exceptional energy resolution of the lithium-drifted germanium detector (Ge-Li) provides the capability for efficient and accurate evaluative identification of radionuclides. In cases where the source geometry is known or can be inferred, quantitative analys is can also be performed, often with accuracies exceeding $1 \%$.

The TMI-2 core will have cooled for approximately two years by the time the damage assessment measurements commence. Thus, the more active, short-1ived fission products will have decayed, resulting in nore easily interpretable gamma ray energy spectra. After two years of cooling, the fission products that will still be present in detectable quantities include ${ }^{136} \mathrm{Cs},{ }^{154} \mathrm{Eu},{ }^{125} \mathrm{Sb}, 134 \mathrm{Cs}$, and ${ }^{144} \mathrm{Ce}$.

Before Head Removal. Gamma spectroscopy may be useful in determining the position of fission products (which presumably will be strongly correlated with the position of fuel material) within the reactor vessel. A major complication is that the core must be viewed through a minimum of approximately 12 inches 
of steel ( $8 \mathrm{in}$. in the reactor vessel and 2 in. in each the thermal shield and core barrel). Assuming that the measurement is possible, it could be performed by posit:oning a shielded, collimated Ge-Li detector in the $3 \mathrm{ft}$ wide annular region between the reactor vessel and the biological shield with the collimator entrance as close to the reactor vessel wall as possible. The collimator would then by angularly scanned in a horizontal plane between tangents to the inner wall of the reactor vessel. By repeating the measurement at differenc elevations and different azimuthal locations on the reactor vessel wall, it should be possible to construct a fairly accurate outline of the fuel mass.

As previously mentioned, radiation originating in the core must pass through a ninimum of $12 \mathrm{in.}$ of steel to reach an ex-vessel detector and at the limits of the angular scan, the effective thickness will be about twice this value. The measurement should therefore be based on a high energy photon which has a larger probability for penetrating the steel with no interaction. A likely candidate would be the $2186 \mathrm{keV}$ gamma-ray of ${ }^{144} \mathrm{Ce}$ which is easily detectable in a typic $\equiv 1$ fission product energy specturm. At $2186 \mathrm{keV}$, the transmission coefficient for $12 \mathrm{in}$. of steel is about $6 \times 10^{-5}$ and for $24 \mathrm{in}$. it will be about $4 \times 10^{-9}$. In addition, water between the core and reactor vessel will further reduce the unscattered photon intensity by a factor of 10 to 100 . Although the total attenuation is large, the core is a very intense source so useful data rates may still be obtainable. The feasibility of this method could be demonstrated by some relatively simple hot cell measurements.

Before any core components have been removed, it is doubtful that useful information could be obtained from a spectrometer lowered into the vicinity of the core. A typical Ge-Li spectrometer will tend to saturate in ambient radiation fields exceeding $10 \mathrm{mR} / \mathrm{h}$. Therefore, until enough fuel assemblies have been renoved to provide space for an adequate shield, in situ gamma specrometry of the core is not feasible. However, the sensitivity of gamma ionization chambers can be adjusted for a wide range gamma dose rates so it would be possible to jower such a chamber down any vertical core penetration to obtain an axial profile of the total gamma dose rate. 
During Fuel Movement. If individual fuel assemblies are removable, then it would be possible to obtain accurate, on-site axial fission product profiles, for single assemblies. Such a procedure was performed during the gamma spectroscopic examination of Peach Bottom HTGR core components. This work was performed by placing a shield-collimator assembly over the core; data were acquired by a Ge-Li spectrometer as each fuel element was withdrawn from the core into the shield-collimator assembly. One of the major goals of this work was to determine the relative release and redistribution of fission products, particularly the isotopes of Cs.

\subsection{Continuous-Gamma-Ray Spectrometry (R. Gold - HEDDL)}

In addition to the gamma-ray spectrometry with a Ge-Li detector described in the previous section, a method called Compton Recoil Gamma-Ray Spectroscopy has been developed by HEDL and has been employed for the measurement of the continuous gamma-ray component of the mixed radiation field of the pressure vessel and for determining the absolute gamma dose rate.

Measurement of this gamma-ray component is important, not only as the major radiation constituent of a reactor field, but also to provide further studies of the interdependent relationship between neutron and the gamma-ray components of a power reactor environment. A high-resolution, lithium-drifted solicon solid state detector ( $\mathrm{Si}(\mathrm{Li})$ detector) was used in this work. Rather than the customary application of solid state detectors (i.e., the direct detection of discrete photo-peaks) this method uses the detector response for measurement of the Compton recoil electron distrijution induced by the continuous gamma-ray spectrum of interest. The compton electron distribution and the continuous gamma-ray spectrum are related by a integral equation, based on the well known Klein-Nishina formula for Compton scattering. In terms of the measured Compton recoil electron distribution, the continuous gamma-ray spectrum can be determined with appropirate unfolding techniques.

\subsection{Solid State Tract Recorder Methods for Neutron Dosimetry. (R. Gold - HEDL)}

This is a nondestructive method that can be applied directly to assemblies to provide comprehensive measurements of spontaneous neutron generation from 
spent fuel. Neutron dosimetry measurements with spent fuel assemblies can serve two broad purposes; namely, to characterize the composition and the radiation environment. This first purpose includes the need to understand and quantify spent fuel fissile isotopic compositions for reasons of economics, safeguards, or reactor physics. The second purpose encompasses health and safety requirements created by the spent fuel radiation environment.

\section{'Compiler's Comments:}

Following review of the original draft of this document, several possible technical problem areas were pointed out by Gold and Kaiser of HEDL. These areas are enumerated and addressed below.)

1. Interference from a $2.23 \mathrm{MeV}$ gamma ray arising from neutron capture on hydrogen.

The total neutron source strength of the TMI-2 core 300 days after the accident is less than $10^{9}$ neutrons $\mathrm{s}^{-1}$.* If it is assumed that every neutron is captured by hydrogen, then the resulting source of $2.23 \mathrm{MeV}$ gamma rays in $10^{9}$ photons $\mathrm{s}^{-1}$. However, the TMI-2 core contains about 82 metric tons of fuel with an average burnup of 3100 MND/T.* This should have resulted in a cumulative yield of about $9 \mathrm{~kg}$ of ${ }^{144} \mathrm{Ce}$, of which perhaps $10 \%(\sim 1 \mathrm{~kg})$ will be present by the time the measurement is made. One $\mathrm{kg}$ of ${ }^{144} \mathrm{Ce}$ provides source of $2186 \mathrm{keV}$ photons with a strength of approximately $10^{15}$ photons $\mathrm{s}^{-1}$. Thus, the ${ }^{144} \mathrm{Ce}$ to neutron capture photon ratio should be the order of $10^{6}$. Even if the $2.23 \mathrm{MeV}$ capture gamma were detectable, it would not interfere with the measurement since its full energy peak is about $40 \mathrm{keV}$ above the $3186 \mathrm{keV}$ full energy peak (and therefore separately resolvable) and the continuous (Compton) portion of the response only extends up to about $2000 \mathrm{keV}$ (excepting some lower-probability multiple interactions).

*Personal communication with D. Selby, ORNL Engineering Physics, dated February. 15, 1980. 
Additional sources of gamma background may include airborne ${ }^{85} \mathrm{Kr}$, contaminated water, and displaced fuel material in the filenums or primary piping. The most troublesone source of background will be exvessel sources of ${ }^{144} \mathrm{Ce}$ since $2186 \mathrm{keV}$ photons emitted by ex-vessel cesium sources will be indistinguishable from the useful signal consisting of unscattered, $2186 \mathrm{keV}$ photons originating in the core. The problem is completely independent of detector type and one of the major objectives of the hot cell scoping measurements would be to insure that adequate shielding can be provided in the allowable space.

2. Non-interpretability of Ge-Li spectra due to existence of a complex, highly degraded gamma continuum.

This measurement does not require analysis of the continuum. The choice of the $2186 \mathrm{keV}$ gamma ray emitted by ${ }^{144} \mathrm{Ce}$ was made not only to maximize penetrability of the core barrel, thermal shield and reactor vessel but also to place the useful response of the detector beyond the continuum.

3. Possibility of detector saturation from low energy events. Low energy photon events constitute a noise source for this measurement regardless of the type of detector that is employed. The hot cell scoping measurements will indicate whether or not adequate shields, filters, and collimators can be provided in the allowable space.

4. Applicability of the Ge-Li detector is severely restricted due to the need for collimation, shielding and liquid nitrogen cooling.

The collimator is necessary to limit the solid angle viewed by the detector to a limited portion of the core and thus is an integral part of the system regardless of detector type. Also, for reasons mentioned previously, any detector will probably have to be shielded against background. Liquid nitrogen cooling is a proven technique for maintaining cryogenic temperatures and a small dewar (containing a 5-7 day supply of liquid nitrogen) should not restrict applicability for the proposed measurement. 
The Compton recoil probe has been suggested as a possible alternative to the Ge-Li detector. Its advantage lies mainly with its larger usieful cross section for high energy photon interactions resulting in an inherentiy greater capability for low energy photon rejection. However, whereas the Ge-Li detector concentrates the useful signal from $2186 \mathrm{keV}$ photons into a $3 \mathrm{keV}$ wide region about the full energy, the Compton recoil probe will distribute the useful information between $0 \mathrm{keV}$ and the Compton edge ( $1957 \mathrm{keV})$. In practice, this means that full energy peaks are directly observable in a Ge-Li spectrum but must be mathematically unfolded from a Compton recoil spectrum. In the interest of supplying the best possible piece of hardware to TMI-2, it would probably be advisable to subject both types of detectors to identical scoping measurements, preferably at the same site. (End of answer to HEDL comments).

Before Plenum Removal. After the head is removed, a view of the core From above the reactor vessel will still be obstructed by the upper internal structure. However, gamma spectrometry might still be useful to scan the inside surface of the head for condensation of volatile fission products.

After Plenum Removal. If the water covering the core is sufficiently transparent, the core should be observable with remote TV cameras at this point. If the water is opaque, than a Ge-Li detector with a vertical collimator could be used to determine or verify the extent of lateral fuel displacement (if any).

The measurements described above might also be deferred until hot cell examination if the fuel assemblies do not appear to be susceptable to handling damage.

After Fuel Removal. After the core is removed, gamma spectrometry colld be used to examine the inside of the reactor vessel for condensed or plated out fission products. 


\subsection{Thermal Measurements}

It is probable that the core thermocouples, originally situated approximately 6 in. above the top fuel level, have melted and that the junctions have remade at some lower level with in the care body. The readings presently being obtained are certainly out of calibration and are in indeterminate positions. It should be possible, following head removal, to perform temperature scans across the top of the core and, with suitable modifications, to probe down into the core.

Two basic thermometry methods exist, thermocouples and resistance thermoneters: These can be obtained in a variety of materials and output ranges. Techniques exist to measure temperatures at any point that can $b 2$ reached by the measuring devices.

Infrared scanning techniques are available whereby temperature gradients across the core can be detected. These same techniques may be applicable during core removal to provide cross-sectional mapping through the core.

\subsection{Flow Measurements (M.H. Fontana, P. Garrison, and H.W. Hoffman - ORIL; E.M. Sheen - HEDL, and K.G. Therp - EG\&E Idaho)}

The amounts and positions of blockage with in the core are of prime interest in the examination of Three Mile Island. One can assume at this time that two types of flow are available in the reactor core, convective flow due to the thermal gradients in the core and pumped flow up through the core. In either case techniques must be available to measure very low flowrates. Such techniques could include: (1) ultrasonic flow meassurements, (2) pitot tube, (3) hot film anemometry, (4) tracer techniques, (5) time-lapse infrared photography, (6) turbine flowmeters, and (7) dye techniques.

If local hotspots are present or if discrete flowpaths exist in the disrupted core, then convection currents can exist in the absence of pumped flow. Measurement of these currents (their rates and directions) can give important clues to the state of core. Such currents should be quite slow, on the order of a few tenths of a foot per second or less, and will necessitate high sensitivity in the measuring methods. 
Ultrasonic flowmeters are presently available commercially from a number of vendors and have gocd sensitivity in the 0.1 to $0.2 \mathrm{ft} / \mathrm{sec}$ range. Some development wors may be required to adapt them for insertion into a bulk liquid medium that has no well defined flow boundaries.

Both pitot tube and hat film anemometry techniques are available which could provide measurements within well defined positions over the core. The pitot tube method is perhaps somewhat less sensitive than the hot film anemometer.

Tracer techniques can provide flow distribution information when used in conjunction with small amounts of pumped flow up through the core. Arrays of detectors with discreze energy w'ndows would be used to detect the emergence of the tracer. at the top of the core.

Time-lapse infrared phctography can be used to delineate flow patterns above the core. If the water flows at different rates through different paths in the core, then thermal gradients will be introduced and should be detectable. Commercial infrared equipment exists to handle the task.

In conjunction with the various flow rate detectors, it should be possible to insert stringers into different radial and circumferential positions to measure pressure drops as a function of various forced convection flowrates. Instrumentation and data acquisition techniques exist to perform the experiment.

\subsection{Portable Microcomputer Aid to TMI Investigations (J.T. West - ORNL)}

Small microcomputer technology and capability is changing at a very rapid rate. The microcomputer systems available now are small, portable, economical, self-contained, and offer a significant resource tool to the field engineers. at reactor sites during normal operation and during post-accident evaluation. There exists a need for computational compatibility at reactor sites which take advantage of the technological tools now available.

The hardware cost of such a system could be less than $\$ 6,000$. It could be self-contained and portable. Its operation would not be dependent on 
any external device such as phone lines or an off-site computer. Its space requirements would be minimal and it would be easily transportable.

The most straightforward approach to utilize this resource would be to consider making several small computer codes operational on a small microcomputer. These codes could be made interactive and the input could be limited to simple engineering-type parameters. The codes which are suitable for immediate application are as follows:

1. SDC - Shielding Design Code, gives several different geometry configurations of source, shield, and point detector as options. It employs Rockwell's shielding equations. Capabilities include:

1) Dose at a point, given the source

2) Shield thickness; given source and dose at a point requirements

3) Could be made to iterate - given dose at a point and shield thickness to determine a source density.

2. NAC - Neutron Activation Code - for zero dimensional systems, this code with a given flux, material description, irradiation time, decay time generates gamma source densities due to neutron activation. It can perform cyclic activation with differing irradiation and decay periods. Source output could be used in SDC.

3. PHOEBE - Beta and gamma source from $235 \mathrm{U}$ fission products given fuel composition, burn-up, and decay time. Source could be used in SDC.

4. To be developed - A simplified materials composition processor capable of generating isotopic atom densities for several standard compositions.

5. To be developed - A spectrum unfolding code to compute source strengths and material composition given input from a gamma spectroscopy analyzer.

6. To be developed - An off line interactive input processor to generate input for a larger standard computer code to run on a large standard computer off-site. This gives the microcomputer smart remote terminal capability as well as off line capabilities. 
It is very cifficult to estimate the time requirements to implement the above proposals. Estimates are:

\begin{tabular}{c}
$\begin{array}{c}\text { Proposal } \\
\text { No. }\end{array}$ \\
\hline 1 \\
2 \\
3 \\
4 \\
5 \\
6
\end{tabular}

\begin{tabular}{|c|}
\hline $\begin{array}{l}\text { Time Requirements } \\
\text { (man-months) }\end{array}$ \\
\hline 4 to 8 \\
\hline 2 to 4 \\
\hline 2 to 4 \\
\hline 3 to 5 \\
\hline 10 to 12 \\
\hline 8 to 14 \\
\hline
\end{tabular}

These proposals represent only a few applications of microcomputers. 


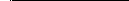


AGNS $-35900-1.5-79$

Distribution

Category UC-85

\author{
SCOPING STUDIES OF THE ALTERNATIVE OPTIONS \\ FOR DEFUELING, PACKAGING, SHIPPING, AND \\ DISPOSING OF THE TMI-2 SPENT FUEL CORE \\ Robert T. Anderson \\ Project Engineer \\ September 1980
}

ALLIED-GENERAL NUCLEAR SERVICES

POST OFEICE BOX 347

BARNWELL, SOUTH CAROLINA 29812

\footnotetext{
PFEPARED FOR THE

DEPARTMENT OF ENERGY

WASTE AND FUEL CYCLE TECHNOLOGY OFFICE

UNDER CONTRACT DE-AC09-78ET35900
} 


\section{FOREWORD}

This study was requested $b_{y}$ the Department of Energy as part of its continuing efforts to assure that experience gained and lessons learned from the accident and recovery of the Three Mile Island (TMI) nuclear plant are made generally available to the nuclear community thereby promoting improved safety in the future. These efforts and studies are in accordance with recommendations made by the president's Commission to investigate the accident at TMI, the President's December 7,1979 , action on these recommendations, and recent Congressional authorization for DOE to conduct a TMI infornation and examination program. It should be noted that the analyses, conclusions, and recommendations herein are solely those of the contractor and are neither endorsed nor opposed by the Department. Further, the responsibility for decisions on defueling, packaging, shipping, and disposing of the TMI-2 core rests with the General Public Utilities (GPU) Corporation under their license and subject to the regulations of the Nuclear Regulatory Commission (NFC). It is hoped that the study will prove useful to both NRC and GPU as one source of technical input to their decisicn. 


\section{ACKNOWLEDGMENT}

This report was prepared by a team of AGNS technical personnel. The general areas of responsibility are noted below.

Kenneth J. Anderson . . . . . . . . . . Dry Storage Robert T. Anderson . . . . Wet Storage, Transportation W. Lynn Godfrey . . . . . . . . . . . . . . . Economics John H. Gray . . . . . . . . Chemical Reprocessing Lawrence A. Kean . . . . . . . . . . . . . Design Paul N. McCreery . . . . . . . . . . . Shipping Casks George A. Townes . . . . . Fuel Handling \& Packaging William R. Waltz . . . . . . . Fuel Characteristics 


\section{ABSTRACT}

This report presents the results of a study performed by Allied-General Nuclear services under contract to the Department of Energy to examine the means of packaging, shipping, and eventually disposing of the failed fuel from the TMI-2 reactor core. A portion of this fuel will be shipped to nuclear facilities to perform detailed physical examinations. These diagnostic tests will research the behavior of power reactor fuel under "loss-of-coolant" accident conditions. The resulting information will be of benefit to nuclear power plant owners and designers, to the governmental regulatory agencies, and to nuclear fuel designers.

Removal of this fuel from the TMI-2 core is also a significant step in the eventual cleanup of this facility. The report presents a scoping study of the technical operations required for defueling and canning. The TMI fuel when canned could be stored in the spent fuel storage pool. After a period of on-site storage, it is expected that the bulk of the fuel will be shipped off-site for either extended storage or possibly, chemical reprocessing. The final disposition of this fuel, as is common to the expectations for all spent high-level nuclear waste, is geological burial.

Evaluation is made of the technical, economic, and institutional factors associated with alternate approaches to disposition of this fuel. Recommendations are presented concerning future generic development tasks needed for the defueling, packaging, on-site storage, and possible off-site shipping of this fuel.

The alternative actions proposed are typically the same as those projected for the disposition of spent fuel from all private nuclear power plants. Obviously, the mechanical condition of the fuel will dictate the exact techniques to be employed for defueling and canning of the fuel components. The various technical operations required for fuel handling have individually been performed at other nuclear installations. Even though the expected scale of the operations presented are large, they are not expected to present insurmountable problems in terms of operational difficulty. 


\section{CONTENTS}

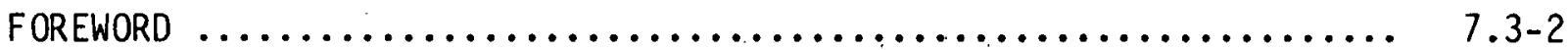

ACKNOWLEDGMENT $\ldots \ldots \ldots \ldots \ldots \ldots \ldots \ldots \ldots \ldots \ldots \ldots \ldots \ldots \ldots \ldots \ldots \ldots \ldots . \ldots \ldots$

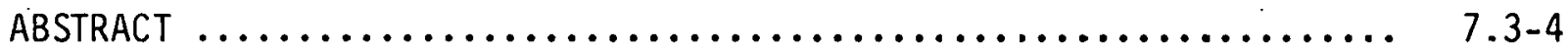

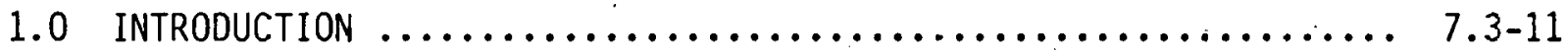

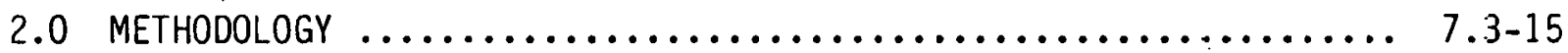

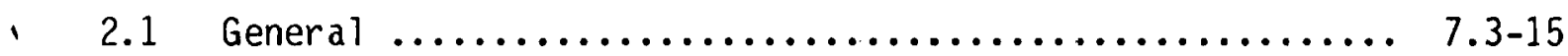

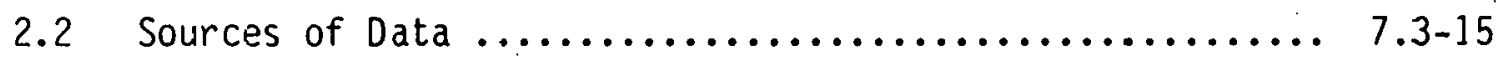

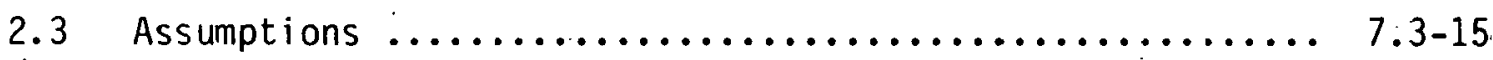

2.4 References $\ldots \ldots \ldots \ldots \ldots \ldots \ldots \ldots \ldots \ldots \ldots \ldots \ldots \ldots \ldots . . \ldots \ldots \ldots$

3.0 . SUMMARY OF RESULTS AND CONCLUSIONS $\ldots \ldots \ldots \ldots \ldots \ldots \ldots \ldots \ldots \ldots . \ldots . . \ldots 7-21$

3.1 Fuel Handling, Packaging, and Shipment off-Site ....... 7.3-21

3.1.1 Fuel. Characteristics .................... 7.3-21

3.1 .2 Fuel Packaging and Handling ................ 7.3-22

3.1 .3 Fuel Can Design ........................ 7.3-24

3.1.4 Operational Procedures ................... 7.3-25

3.1.5 Operational Space Requirements ............. 7.3-25

3.1.6 Shipping and Logistic Considerations ......... 7.3-25

3.2 Technical Evaluation of TMI-2 Fuel Core

Disposition 0ptions .......................

3.2 .1 Chemical Reprocessing .................. T.3-8

3.2 .2 Wet Storage of Spent Fuel ............... $7.3-30$

3.2.3 Dry Storage ..........................

3.3 Economic and Schedule Studies .................. $7.3-52$

4.0 RECOMMENDATIONS $\ldots \ldots \ldots \ldots \ldots \ldots \ldots \ldots \ldots \ldots \ldots \ldots \ldots \ldots \ldots \ldots \ldots . . \ldots \ldots$

4.1 General .............................. $7.3-33$

4.2 Discussion of Near-Term Needs $\ldots \ldots \ldots \ldots \ldots \ldots \ldots \ldots \ldots . . \ldots .3 . \ldots 3$

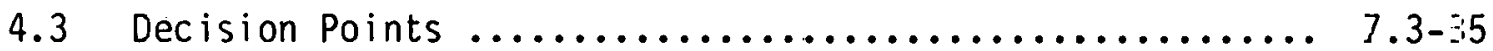

4.4 Key Action Items $\ldots \ldots \ldots \ldots \ldots \ldots \ldots \ldots \ldots \ldots \ldots \ldots \ldots \ldots$

4.5 References $\ldots \ldots \ldots \ldots \ldots \ldots \ldots \ldots \ldots \ldots \ldots \ldots \ldots \ldots \ldots . . \ldots \ldots \ldots$

5.0 FUEL REMOVAL, ON-SITE HANDLING AND OFF-SITE SHIPPING $\ldots \ldots \ldots . .7 .3-5$ 
5.1 Introduction $\ldots \ldots \ldots \ldots \ldots \ldots \ldots \ldots \ldots \ldots \ldots \ldots \ldots \ldots \ldots \ldots \ldots, 7.3-58$

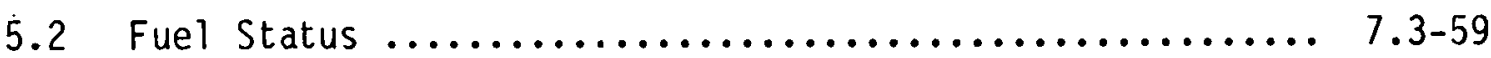

5.2.1 General ................................. 7.3-59

5.2.2 Initial Fuel Lcading Data ..................... 7.3-60

5.2.3 Fuel Burnup Data ............................ 7.3-60

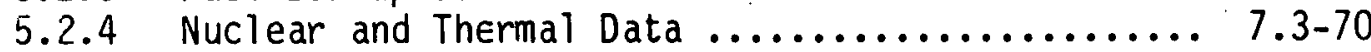

5.2.5 Damaged Fuel Canditions $\ldots \ldots \ldots \ldots \ldots \ldots \ldots \ldots \ldots .7 .3-73$

5.2.6 Criticality Conditions for Fuel Debris ......... 7.3-75

5.3 Fuel Handling and Packaying Evaluation .............. 7.3-77

5.3.1 General ................................ 7.3-77

5.3.2 Fuel Handling Interface Evaluation ........... 7.3-78

5.3.3 Prel iminary Package Design .................. 7.3-85

5.3.4 Prel iminary Scoping of Operational Procedures ... 7.3-105

5.3.5 Conclusions and Recommendations .............. 7.3-113

5.4 Shipping Cask Evaluation for Off-Site Fuel Movement .... 7.3-115

5.4.1. General ............................... 7.3-115

5.4.2 Description of Licensed LWR Shipping Cask

Systems ............................... 7.3-116

5.4.3 NRC Licensing Criteria for Shipping Casks ....... 7.3-122

5.4.4 Fuel Loading Location Options ................ 7.3-123

5.4.5 Conclusions and Recommendations ............. 7.3-132

5.5 Spent Fuel Shipping Logistic and Safety

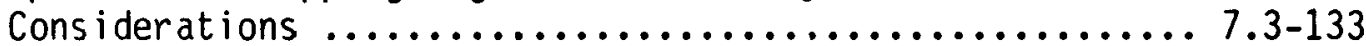

5.5.1 General .............................. 7.3-133

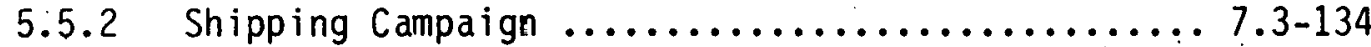

5.5 .3 Shipping Economics $\ldots \ldots \ldots \ldots \ldots \ldots \ldots \ldots \ldots \ldots .7 .3 .134$

5.5.4 Institutional Problems Involved with

Spent Fuel Shipping ....................... 7.3-139

5.5.5 Safety and Envircnmental Aspects of

Spent Fuel Shipping $\ldots \ldots \ldots \ldots \ldots \ldots \ldots \ldots \ldots \ldots, 7.3-143$

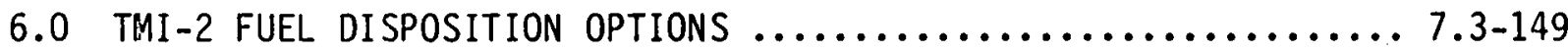

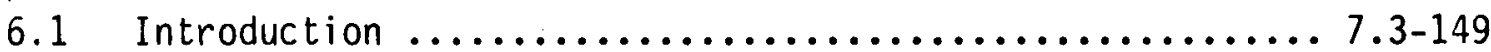

6.2 Chemical Reprocessing of TMI-2 Fuel $\ldots \ldots \ldots \ldots \ldots \ldots \ldots . . .6 .3-150$

6.2.1 General ............................... 7.3-150

6.2.2 Technical Review of Selected Reprocessing

Facilities ................................ 7.3-152

6.2.3 Preliminary Facility Assessment ............. 7.3-154

6.2.4 Core Parameters Affecting Reprocessing ......... 7.3-155

6.2 .5 Safety Considerations $\ldots \ldots \ldots \ldots \ldots \ldots \ldots \ldots \ldots . .7 .3-158$

6.2.6 Product and Waste Handling Alternatives ......... 7.3-158

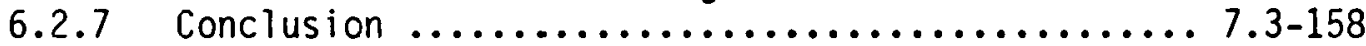




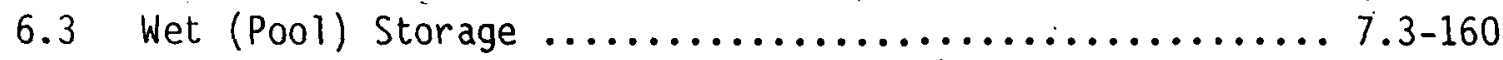

6.3.1 Introduction .......................... 7.3-160

6.3.2 Technical Considerations ..................... 7.3-162

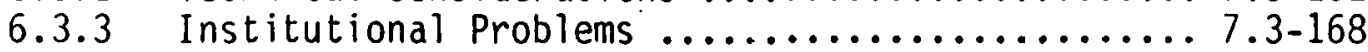

6.3 .4 Facility Licensing ..................... 7.3-172

6.3.5 Implementation Considerations $\ldots \ldots \ldots \ldots \ldots \ldots \ldots .7 .3-172$

6.3.6 Advanced Wet Storage Techniques .............. 7.3-173

6.4 Dry Storage Concepts for TMI-2 Reactor Fuel ........... 7.3-174

6.4.1 General ................................ 7.3-174

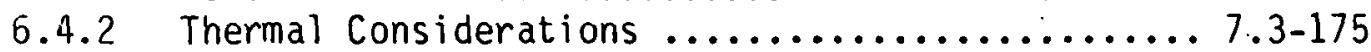

6.4.3 Vault Storage Concepts ...................... 7.3-176

6.4.4 Caisson (Silo) Storage Concepts ............... 7.3-181

6.4.5 Engineered Berm ......................... 7.3-192

6.4.6 Cost Estimates ........................... 7.3-195

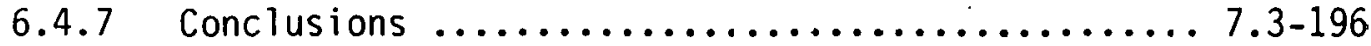

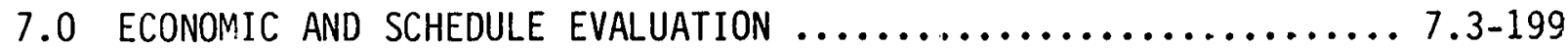

7.1 General .................................... 7.3-199

7.2 Economic Study Results $\ldots \ldots \ldots \ldots \ldots \ldots \ldots \ldots \ldots \ldots \ldots . . \ldots \ldots .3-199$

7.2.1 Chemical Reprocessing ..................... 7.3-199

7.2 .2 Pool Storage $\ldots \ldots \ldots \ldots \ldots \ldots \ldots \ldots \ldots \ldots \ldots \ldots, 7.3-202$

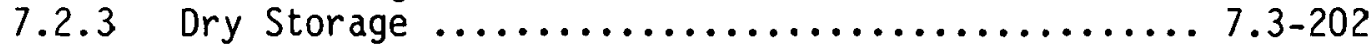

7.3 Cost Assumptions .............................. 7.3-204

7.3.1 TMI On-Site Cost (Cost Center 1.0) ........... 7.3-206

7.3.2 Shipping Costs $\ldots \ldots \ldots \ldots \ldots \ldots \ldots \ldots \ldots \ldots \ldots . . \ldots \ldots .3 .206$

TABLES

2-1 Sources of Study Data $\ldots \ldots \ldots \ldots \ldots \ldots \ldots \ldots \ldots \ldots \ldots \ldots, 7.3-16$

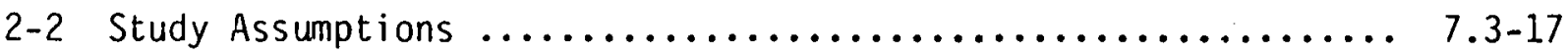

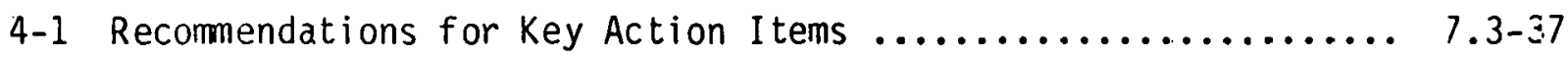

5-1 B\&W - TMI-2 Fuel Data .............................. 7.61

5-2 Core Totals - At Shutdown (Average) ................... 7.3-66

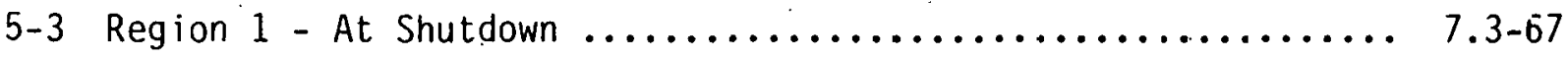

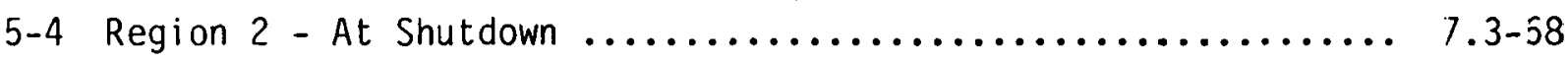

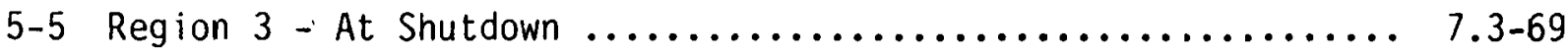


5-6 TMI-2 Core Region 2 Fuel - Gammas per Second per Metric Ton of Uranium at Various Decay Times .................... 7.3-71

5-7 Comparison of Gamma Source Terms - TMI-2 Fuel Versus High Burnup Fuel (One Year Decay -ime) ................... 7.3-72

5-8 Selected Packaging System Dimensions for Candidate Truck Cask (in Inches) $\ldots \ldots \ldots \ldots \ldots \ldots \ldots \ldots \ldots \ldots \ldots \ldots . \ldots \ldots$ 7.3-87

5-9a Defueling Manpower Estimates ..................... 7.3-112

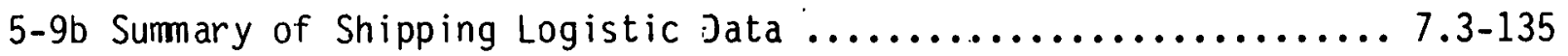

5-10 Characteristics of LWR Spent Fuel Casks ............... 7.3-136

5-11 Spent.Fuel Shipping Assumptions .................... 7.3-137

5-12 Summary of Spent Fuel Shipping Institutional Factors ....... 7.3-140

5-13 TMI-2 Spent Fuel Shipment Accident Probability Summary ....... 7.3-145

6-1 Disposition Alternative Comparison ...................... 7-151

6-2 Summary of Pool Storage Study zesults .................. 7.3-161

6-3 Spent Fuel Pool Interface Criteria (Summary) ............ 7.3-169

7-1 TMI-2 Fuel Disposition Cost Centers .................. 7.3-200

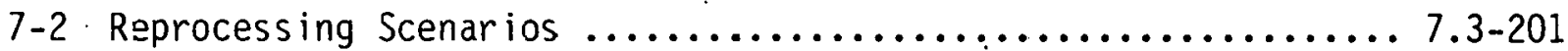

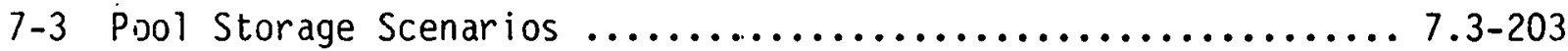

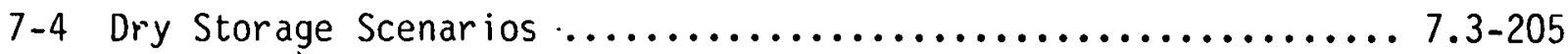

FIGURES

1-1 TMI-2 Core Disposition Options $\ldots \ldots \ldots \ldots \ldots \ldots \ldots \ldots \ldots \ldots . \ldots \ldots .12$

2-1 Representative TMI-2 Cleanup Schedule ................ 7.3-18

3-1 Illustration of TMI Defueling and Canning and Storage Areas ................................ 7.3-23

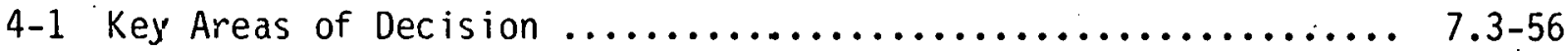

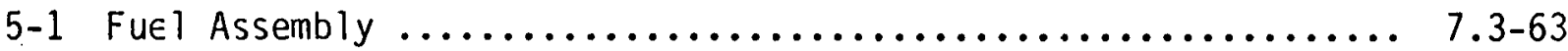

$5-2$ Control Rod Assembly ........................... 7.3-64

5-3 Burnable Poison Rod Assembly ..................... 7.3-65 


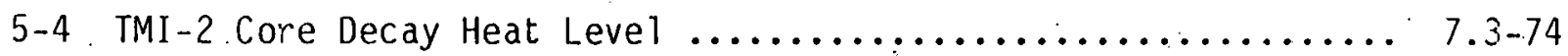

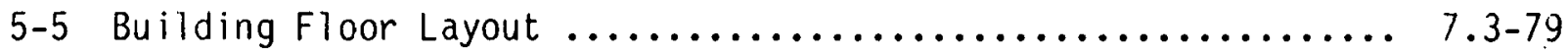

5-6 Truck Cask Cavity Dimensions (Inches) $\ldots \ldots \ldots \ldots \ldots \ldots \ldots . . \ldots .6 .63$

5-7. Packaging Dimensional Interfaces $\ldots \ldots \ldots \ldots \ldots \ldots \ldots \ldots \ldots, 7.3-88$

5-8 Bottom Loading Shroud ............................ 7.3-90

5-9 Shroud Bottom-Loading Sequence $\ldots \ldots \ldots \ldots \ldots \ldots \ldots \ldots \ldots \ldots . . \ldots .3-92$

5-10 Grappling Cap Detail .............................. 7.3-93

5-11 Side-Loading - Fuel Handling Shroud ..................... 7.3-94

5-12 Loaded (Two Part) Handling Shroud Concept ............... 7.3-95

5-13 TMI-2 Reactor Vessel \& Internals-General Arrangement ........ 7.3-9i

5-14 Elevation View Debris System ...................... 7.3-98

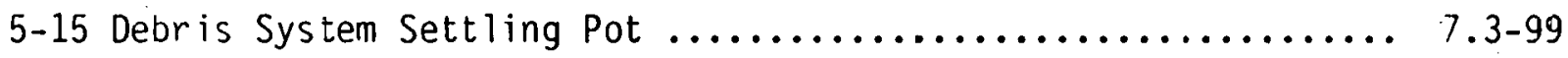

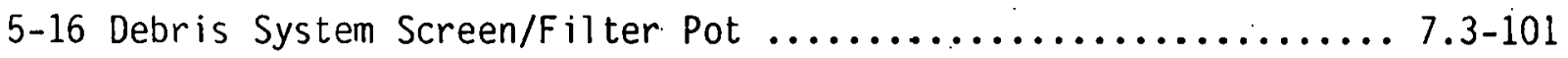

5-17 Fuel Canister Loaded with Debris System

Elements for Transfer ................................. 7-102

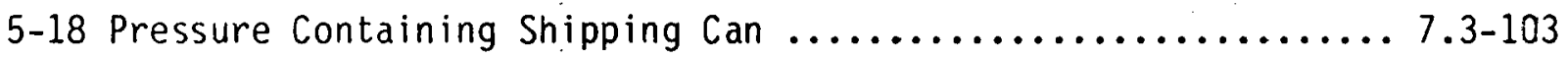

5-19 Mechanically Sealing Cap for Shipping Vessel Closure ........ 7.3-104

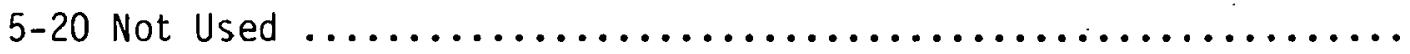

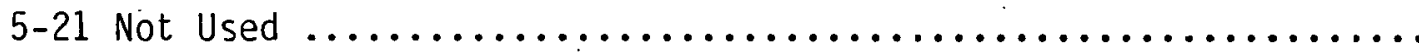

5-22 Legal Weight Truck Casks ............................ 7.3-117

$5-23$ Cask Handling at TMI................................ 7. $3-127$

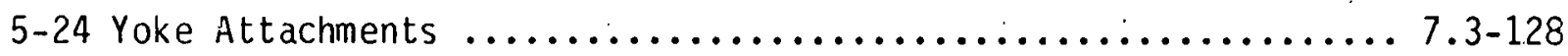

5-25 Shelf for Cask Loading Pool .............................. 7-129

5-26 Current Estimates of Spent Fuel Shipping Costs

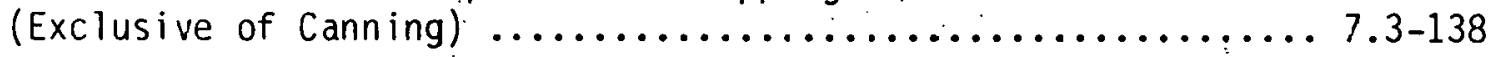

5-27 NRC Restricted Cities ........................... 7.3-141

5-28 Highway Weight and Dimensional Limitations $\ldots \ldots \ldots \ldots \ldots \ldots .7 .3-142$

6-1 Movable LWR Fuel Storage Racks ...................... 7.3-163 
6-2 Typical PWR Spent Fuel Storage Rack Poison-Fixed Design $(7 \times 8) \quad \ldots \ldots \ldots \ldots \ldots \ldots \ldots \ldots \ldots \ldots \ldots \ldots \ldots \ldots \ldots \ldots .3 .167$

6-3 Radiation Levels for PWR Fuel in Pool ................... 7.3-165

6-4 Square (Nine-Inch) Can Loaded with Fuel Pins

from Two Complete PWR Fuel Assembly Dummies ................ 7.3-174

6-5 Vault with Forced Air Cooling ......................... 7.3-177

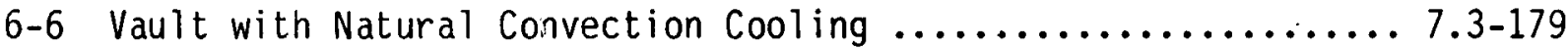

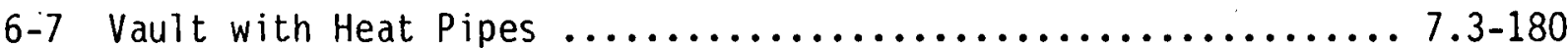

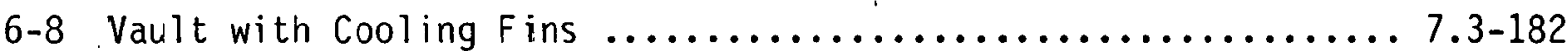

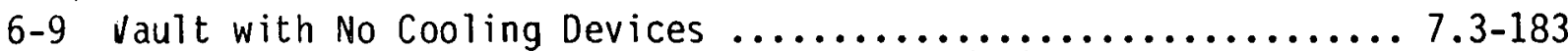

6-10 Caisson - Natural Convection - Aboveground ............... 7.3-185

6-11 Caisson - Natural Convection - Below ground ................ 7.3-186

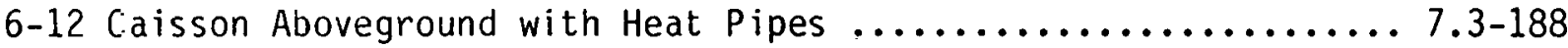

6-13 Caisson Below ground with Heat Pipes $. . . \ldots . . . . . . . . . . .7 .3-189$

6-14 Caisson Aboveground with No Cooling .................... 7.3-190

6-15 Caisson Below ground with No Cooling ..................... 7.3-191

6-16 Engineered Berm Concept Caisson Storage ................. 7.3-194 


\subsection{INTRODUCTION}

This report presents an evaluation of technical, institutional, and economic considerations related to the disposition of the fuel from the TMI-2 reactor core. This work was performed under contract to the Department of Energy (DOE) by personnel of Allied-General Nuclear Services (AGNS). The study takes the form of a scoping evaluation of the various technical options open to the TMI-2 recovery team and the problems associated with the implementation of these same options. Due to the uncertainty of the physical condition of the fuel and its removal from the core, as well as the nationwide uncertainty related to spent fuel disposition, a number of different approaches are covered. Discussion of the different alternative approaches or options relates to the probable implications in the expected time frame for the reactor defueling and fuel canning (1983 to 1985).

The ideal sequence of operations for movement of the fuel from the core to its ultimate disposition is shown schematically in Figure 1-l. Technically, there are three phases which must be addressed. These include the removal of the fuel from the core and canistering of the fuel in a form suitable to maintain its integrity during further handling, storage, or shipping operations. The fuel may remain in storage on-site until a permanent disposition is identified. A possible second phase is the shipment of the fuel off-site. This could be to either an interim facility for diagnostic evaluation or for storage. Finally, the nuclear fuel waste material must be disposed of, probably by subterranean burial. The latter step is common to all spent fuel or nuclear waste. A possible technical option preparatory to burial is reprocessing to recover and recycle useful fuel material.

As illustrated in Figure 1-1, the fuel condition could range from intact to pieces of "debris," or even large "fused" segments of the core. The canning operation must accommodate this wide spectrum of possible conditions. It is expected that the TMI reactor spent fuel pool will be used as a storage area and for other possible handing and testing operations preparatory to shipment off-site. The cannistered fuel will be stored in a spent fuel pool until there is an identification of a means for disposition of the fuel. A small portion of the fuel will be sent to hot cell areas at national laboratories or commercial facilities to permit a diagnostic evaluation. An evaluation of the fuel condition will permit a greater understanding of the TMI reactor accident sequences and consequences. The remainder 


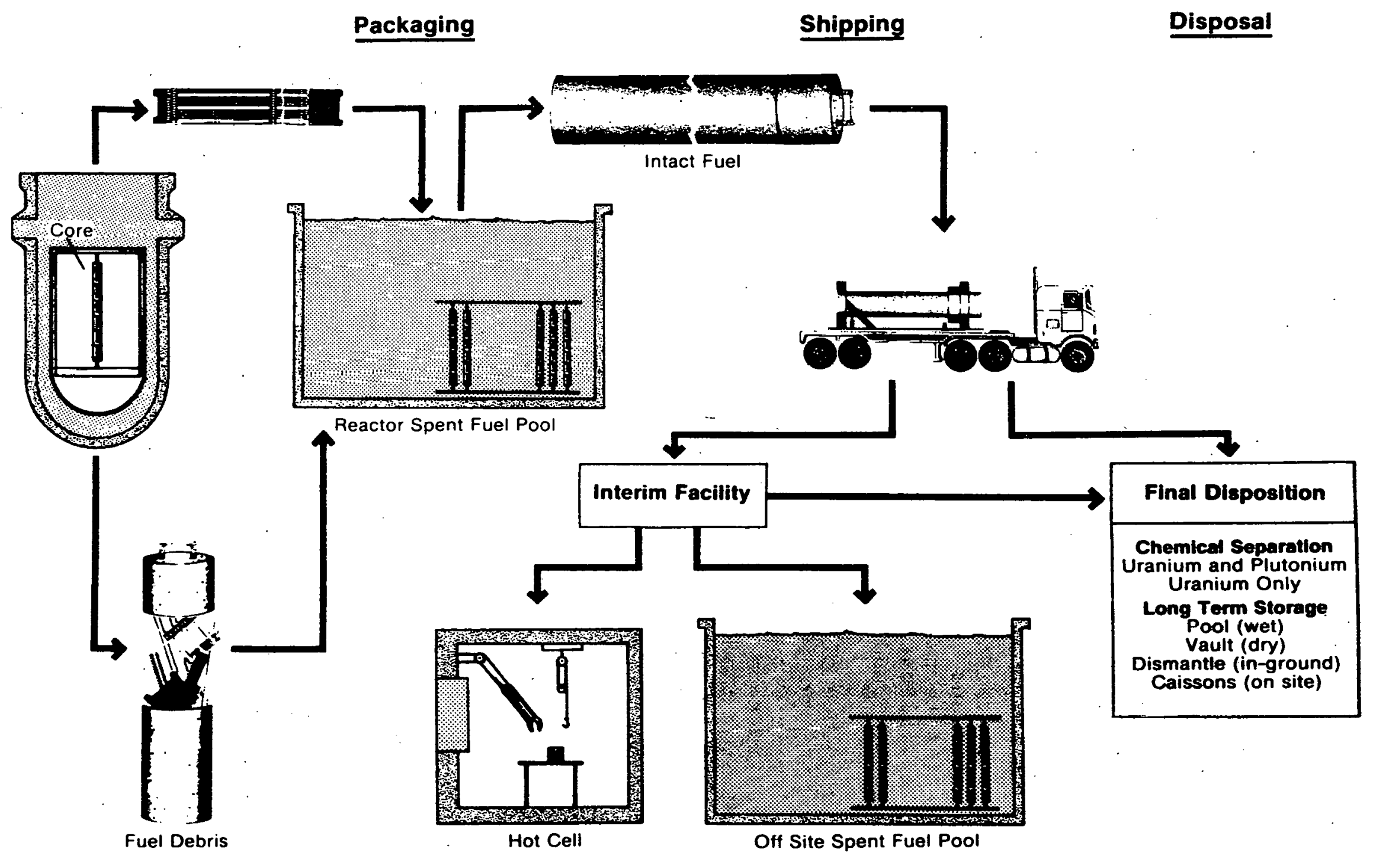

TMI-2 CORE DISPOSITION OPTIONS

FIGURE 1-1 
of the fuel will eventually be shipped to another location for either interim storage or for final disposition.

The first four sections of the report summarize the methodology, results, and recommended areas of further work to be performed. The remainder of the study was divided into three detailed areas of emphasis: (1) the technical aspects of packaging and shipping; (2) the technical aspects of various disposition alternatives; and (3) the economic and schedular examination of the various disposition alternatives. These are summarized in the foliowing report sections.

(1) Chapter 5.0 - Fuel packaging and Shipping Considerations - Section 5.2 examines the status of the fuel, and section 5.3 examines the packaging of the fuel preparatory to being placed into a shipping cask. This includes the various considerations associated with removal from the region of the reactor core itself,. handling between the reactor building and the auxiliary building, the canning of the fuel and related operations preparatory to placement in a spent fuel cask, and the loading of the spent fuel cask. Section 5.4 examines the shipping cask operations at the TMI-2 facility and alternative unloading facility, and the various aspects of selecting a cask to handle the fuel or fuel debris which has been canned. Section 5.5 examines the logistics considerations related to shipping the fuel off-site including availability of equipment, routing, and current uncertainties in shipping.

(2) Chapter 6.0 - Disposition Options - The various options examined are wet and dry storage of the fuel and chemical reprocessing. Section 6.2 examines the technical feasibility of eventually reprocessing the fuel to recover useful uranium and possibly plutonium. Examination was made of all current domestic reprocessing processes as related to performing this at both government laboratories and at domestic commercial facilities specifically designed for LWR power reactor fuel. Section 6.3 examines the technical factors associated with storage of canned fuel in a wet pool. Section 6.4 similarly evaluates the dry storage options available, including storage within a hot cell. These options were evaluated for technical feasibility and relative safety. Implementation considerations including facility availability were also covered.

(3) Chapter 7.0 - Comparative Economics - This is a summary of the comparative economics of ultimately disposing of this fuel based on the various alternatives studied. 
These estimates allow for variations in the actual date of implementation.

The report results presented allow sufficient latitude to accommodate a wide variety of fuel conditions. In a similar vein, the TMI recovery group will have the option of determining such areas as shipping mode and disposition technique based on the facility options open or expected to be open within the time frame. The conclusions and recommendations are also covered in a like manner.

We recommend that an operational plan for defueling be formulated in the near future to permit more definitive plans to be made. This involves examining the recommended areas for future studies and determining a projected plan of action. This will be needed to arrange for the fabrication, leasing, licensing, etc., of the various equipment to be utilized. In this line, the most evident need to permit detailed planning is that a preliminary examination of the actual fuel condition be made as soon as possible. 


\subsection{METHODOLOGY}

\subsection{General}

This section of the report discusses the means utilized to accumulate meaningful data and to develop specific information. Also included are the technical assumptions used which lead to the various conclusions and recommendations. Where specific conclusions are based on tentative or unproven data, and where the recommendations are extremely sensitive to these factors, specific notation is made within the body of the report. In these situations, alternative paths of actions are presented with the resulting consequences.

\subsection{Sources of Data}

The specific progression of development of information for this report and the sources of data are noted on Table 2-1. Maximum use was made of information Iurnished $b_{y}$ DOE and GPU related to reconstruction of the accident scenario and the initial design of TMI-2. Fuel data were obtained from the Babcock \& Wilcox Company ( $B \& W$ ) and from the plant Final Safety Analysis Report (FSAR). In certain specific areas related to fuel movement between the reactor and the auxiliary building, as-built drawings were supplied by GPU and the DOE-TMI Technical Integration office.

Utilization was made of in-house AGNS data related to spent fuel transportation, spent fuel canning for failed fuel and shipment, chemical reprocessing, spent fuel pool (wet) storage, and hot cell (dry) storage. AGNS data and hardware experience on these various areas were found to be directly applicable in developing technical data and factors related to implementation at similar type facilities.

\subsection{Assumptions}

A listing of the major report and study assumptions is presented in Table 2-2. The rationale for these assumptions is presented in greater detail following the table:

- Mid-1983 Initial Fuel Removal Date - This cate was postulated on the abstracted published schedule shown in Figure 2-1. The date is not crucial to the results of this study; however, it permits evaluations of fuel source strength to be developed and to portray the time factors associated with the implementation of shipping and various disposition options.

- Fuel Condition - The Rogovin report (Reference 2.1) and the NSAC study (Reference 2.2) present divergent views on 
TABLE 2-1

SOURCES OF STUDY DATA

- VISIT TO TMI-2 SITE, DUKE OCONEE POWER PLANT (B\&W NSSS) TO OBSERVE REFUELING

- CONTACTS WITH DOE TECHNICAL INTEGRATION OFFICE, B\&W, BECHTEL, SANDIA-TTC, OAK RIDGE NATIONAL LABORATORY (FUEL INFORMATION)

- TECHNICAL REVIEWS OF TMI FSAR, APPLICABLE SITE DRAWINGS, B\&W FUEL INFORMATION, BECHTEL STUDY ON TMI-RECOVERY

- teCHNICAL EVALUATIONS OF PUBLISHED STUDIES AND DATA:

- FUEL STATUS AND CURRENT CHARCTERISTICS

- SHIPPING CASK STATUS

- SHIPPING LOGISTICS

- TECHNICAL EVALUATIONS OF "STATE-OF-THE-ART" OF:

- CHEMICAL REPROCESSING

- WET POOL STORAGE

- DRY STORAGE

- ECONOMIC MODELS BASED ON PUBLISHED STUDIES OF WET STORAGE COSTS, REPROCESSING COSTS, SHIPPING COSTS, AND GEOLOGICAL REPOSITORY COSTS 
TABLE 2-2

STUDY ASSUMPTICNS

- FUEL - HAS BEEN FREED FROM THE REACTOR CORE BASKET

- FUEL STATUS - FROM INTACT (POOR SHAPE) TO DEBRIS (RUBBLE)

- CANNING REQUIRED FOR HANDLING/ShIPPING/STORAGE

- FUEL MOVEMENT - THROUGH UPENDER (REACTOR BUILDING) TO AUXILIARY BUILDING (SPENT FUEL POOL)

- NRC LICENSING OVERVIEW. OF ALL ON-SITE OPERATIONS AND SHIPPING

- APPROXIMATELY 3 TO 5\% OF. FUEL GOES TO HOT CELL FOR DIAGNOSTIC EVALUATION

- DATES: START OF DEFUELING AND CANNING 1983

INITIAL SHIPPING

AT LEAST 6 MONTHS AFTER START OF DEFUELING

"OFF-SITE" SHIPPING DURATION

1 TO 2 YEARS

- EARLIEST GOVERNMENT-AFR

1984 AVAILABILITY

- EARLIEST REPROCESSING $\quad 1990$

- GEOLOGICAL REPOSITOR̈Y

AVAILABLE.

1995

- ECONOMICS - 1980 DOLLARS

- COSTS ESTIMATED THROUGH FINAL DISPOSAL OF FUEL 


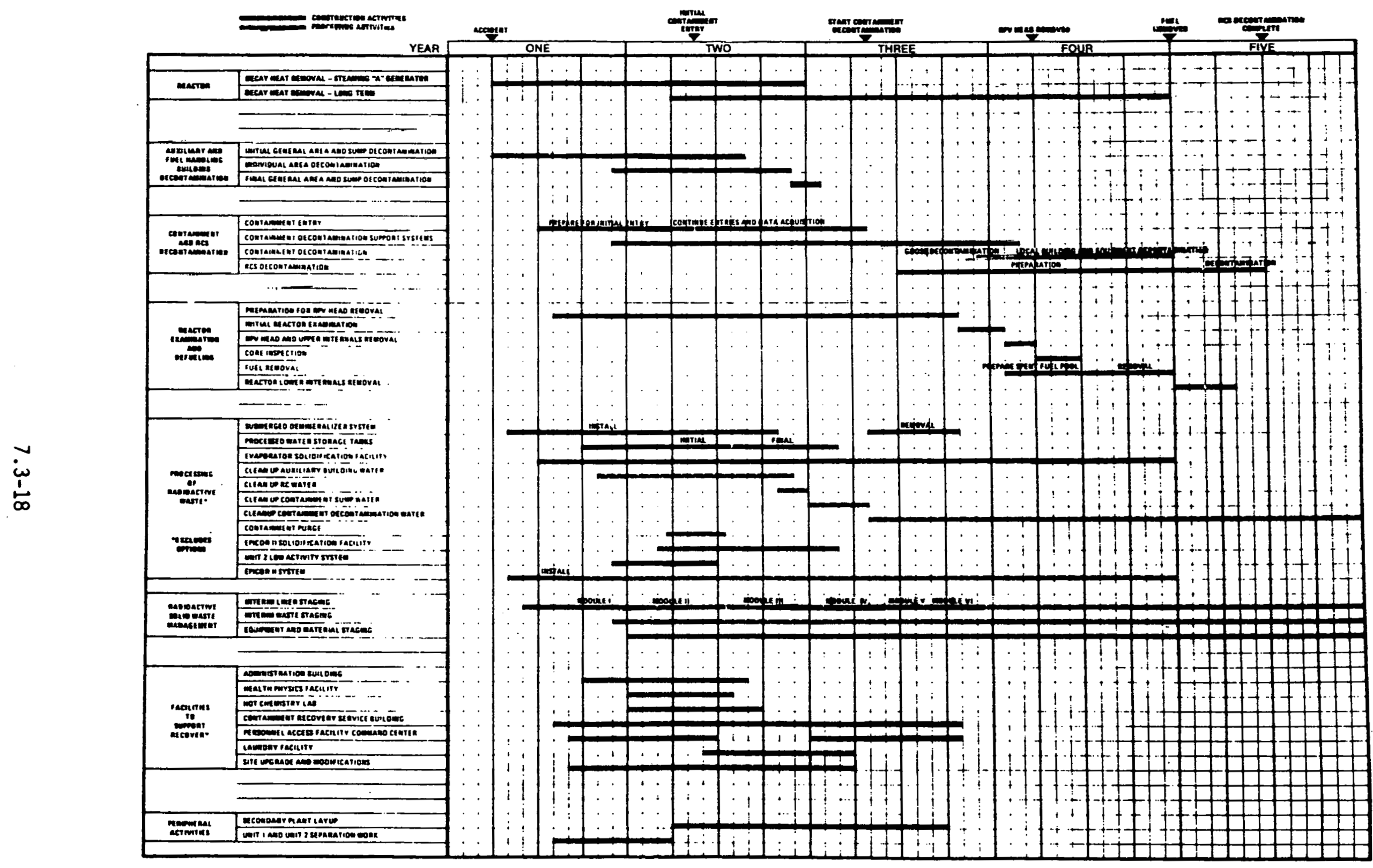

REPRESENTATIVE TMI-2 CLEANUP SCHEDULE

(FROM REFERENCE 2.5)

FIGURE 2-1 
the expected condition of the damaged fuel. These two studies present a spectrum of opinions on the fuel status. Plans must be made to accommodate a gamut of possible fuel conditions.

- 1980 Cost Data - The cost data presented in this report are based primarily on generic estimates of various operations and the equipment and facilities where these would be performed. Many of these operations have never been performed. As a result, the reported information is based on speculative data without recourse to any actual experience. However, the purpose of the cost estimates is merely to portray the relative magnitude of cost for the various options. It is not meant to be utilized for detailed planning purposes. As a result, scphisticated analyses considering the effect of the time value of money, or inflationary factors were not employed.

- NRC Licensing Overview - All of the operations currently being performed in the recovery of the TMI core are being separately and individually evaluated by a team of NRC personnel. Since the TMI facility is a commercial operating reactor and the fuel is in the possession of Metropolitan-Edison (Met-Ed), it seems plausible to assume that all of the operations related to defueling, canning of the fuel, shipment off-site, and probably any disposition alternatives, will come under the NRC overview. However, fuel cousd be moved utilizing government leased casks or other shipping equipment; fuel could be stored at governmental facilities such as national laboratories preparatory to further examination or storage; and governmental ownership of the fuel or the recovered products is a possibility. These are special situations; however, DOE authority related to recovery of spent fuel from other commerical facilities may establish precedents in this area.

- Facility Availability - Current projections are that the earliest available date for away-from-reactor storage facilities is projected to be 1984 (Reference 2.3). Similarly, AGNS in-house evaluations of the earliest possible date for domestic reprocessing of commercial LWR fuel is assumed to be the late 1980's and more likely 1990. Recent policy statements by president Carter (Reference 2.4) indicate that selection of a suitable site for final disposition of nuclear waste, either commercial or defense, is not expected until 1985. Hence, it is expected that a repository for high-level nuclear spent fuel or other waste material would not be operational until the 1995-2000 period. 


\section{REFERENCES}

2.1 Rogovin, M., et al, Three Mile Island - A Report to the Commissioners and to the Public, TMI 18:52, Volume 1 (April 1979).

2.2 Supplement to Analysis of Three Mile Island - Unit 2 Accident, NSAC-1 (October 1979).

2.3 Department of Energy Study on Spent Nuclear Fuel storage, DOE/SR-0004, Volume 1 (March 1980).

2.4 "Statement on Nuclear Power Policy," President Carter (April 1977).

2.5 Nuclear News, page 56, (March 1980). 


\subsection{SUMMARY OF RESULTS AND CONCLUSICNS}

\subsection{Fuel Handling, Packaging, and shipment off-Site}

The major uncertainty in the defueling and canning of the TMI-2 core is, of course, the physical status of the fuel itself. It will be necessary to develop several alternative techniques for the packaging and handling of the fuel. In all cases, fuel canning is seen as a necessary condition both for the storage of fuel, and the possible off-site shipment of fuel. In the former cases, packaging is needed for structural integrity, collection, and to prevent fuel pool contamination due to loose, nonsoluble debris, and soluble fission products. To the largest extent possible, procedures should assure that a standard can size is developed and fuel handling operations minimized. Canning will also be required for shipment to assure containment during transport.

The problems associated with the assumed poor physical shape of the fuel are to some degree counterbalanced by the low burnup of the fuel and the aging period prior to removal from the reactor. These factors result in low nuclear source strength and in very low values of thermal decay heat levels. In addition, most of the free, volatile fission gases normally found in the fuel plenum are believed to have been released during the accident. Hence, the handing equipment designed and the procedures developed must primarily consider the physical form of the fuel. To a lesser extent than normal, are the operations bounded by fuel radiation levels.

\subsubsection{Fuel Characteristics}

(1) Three general fuel configurations were assumed. In the first, the fuel is intact but badly weakened and probably bowed in the upper regions of the assemblies. In the second, the fuel is assumed to be debris. Debris may be further divided into two types. The first consists of relatively large pieces which can be handled by mechanical handling devices. The second type consists of smaller pieces which must be vacuumed and filtered. A third fuel configuration assumes that portions of adjacent fuel assemblies may be "welded" to each other in an undefined physical configuration. In this case, techniques will be required to physically separate these larger pieces into sizes which can be canned.

(2) In the central core region, the nonfuel-bearing components such as control rod spiders, axial power shaping 
rods, etc., may be badly distorted and nonseparable from the remainder of the fuel assembly.

(3) The radiological source strength, and the decay heat level, as noted previously, will be extremely low due to low burnup and aging of the fuel. These source terms, after three years of aging, will be comparable to those normally considered for 10- to 15-year old fuel.

(4) The maximum and average enrichments of the fuel are approximately 3.08 and 2.28 , respectively. The plutonium quantity is approximately $154 \mathrm{kilograms.}$

(5) The high average enrichment of the failed fuel underscores the economic value of the remaining fuel material.

(6) Criticality limitations appear to limit the can crosssectional size to approximately 9.4 inches for a square can, or a 10.6-inch diameter for a round can. The use of a can with neutron poisons integral with the wall structure should be examined, if a larger can size is required.

3.1.2 Fuel Packaging and Handling

(1) Fuel Movement Route - The fuel can be removed from the containment building and transported to the auxiliary building via two routes. The more practical route is a movement directly between the two buildings using the fuel transfer carriage/upender and the fuel canal to place the fuel in the auxiliary building pool. (See Figure 3.1.) This option presents certain geometrical (transporter can cross section) restraints, and limits the size of the package to 11.48 inches square cross section or 15.25 inches in diameter and 15.1 feet long. Another route would be the loading of larger or fused pieces into a transport cask, and moving this cask through the containment hatch external to the reactor and back into the spent fuel pool for further packaging and handling. Due to the need to design specialized transport packages and the potential difficulties in moving this larger cask through the reactor building, it is not recommended unless disengagement of fused fuel pieces proves to be of overwhelming difficulty.

(2) Fuel Removal Alternatives and Procedures - Three alternative defueling and canning procedures are briefly summarized below. Their usage depends on the physical condition of the fuel. 


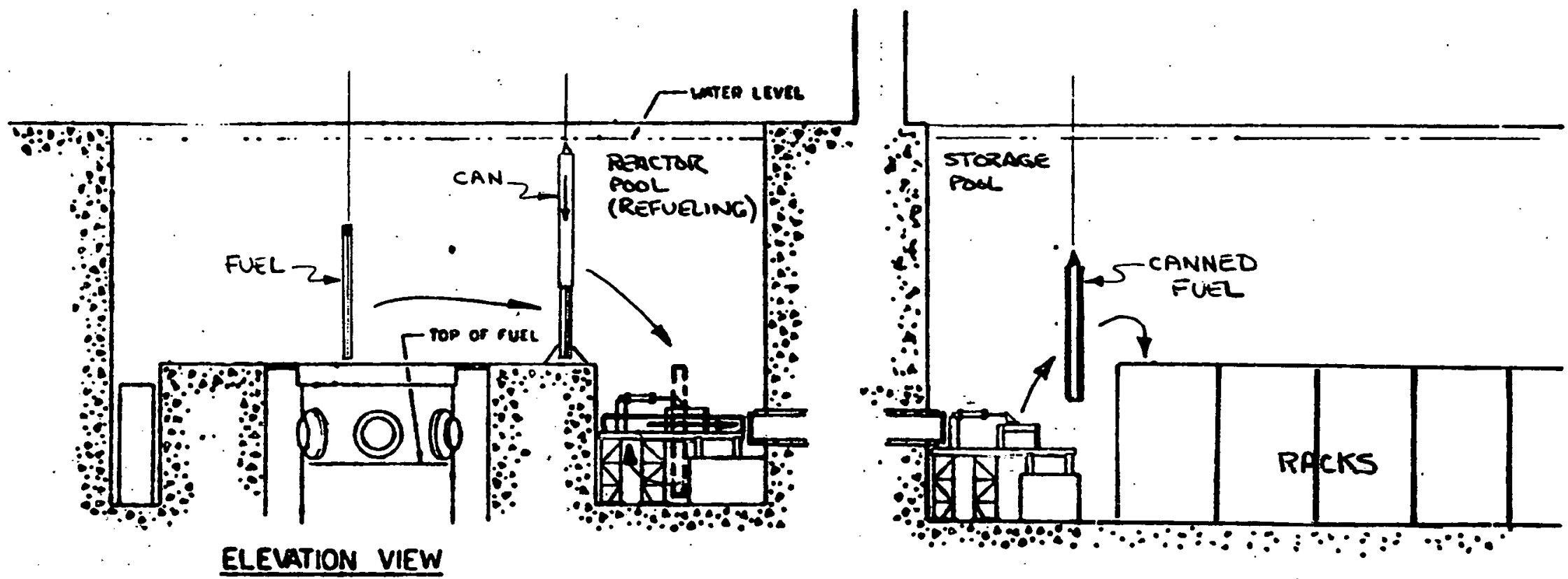


- Intact Fuel - This fuel is assumed to be severely weakened and to have perforations in the upper region of the assembly. A thin-walled can is recommended as a structural shroud for the handling of this fuel. This can would be used immediately upon removal from the reactor core. It would be utilized to provide both vertical and lateral support of the fuel assembly as it is being moved via the upender to the auxiliary building. It would also function to prevent the spread of major contamination to the radioactive water, or material from the fuel.

- Debris - It is expected that the fuel debris will be remotely removed from the reactor core, ir a twostep process. The larger pieces, such as damaged fuel, end fittings, and grid spacers, will be removed utilizing mechanical tongs or similar devices. These pieces would be placed into a can which functions as a "bucket." The remaining smaller pieces such as fuel shrouds, cladding, and fuel fines/corrosion products, would be "vacuumed" from the core. A vacuuming system would utilize a combination of a centrifugal action and filtering screens to further segregate the pieces by size. These smaller pieces would be canned in a similar manner to the larger pieces. These cans would be capped and again moved via the fuel transporter to the auxiliary pool.

- Large "Fused" Pieces - Some fuel assemblies may be fused together preventing their separation into a normal geometry utilizing conventional techniques. Hence, it will be necessary to either place these larger segments in a transporter can as noted above or to mechanically separate these pieces into sections which are suitably sized to fit the handling cans required to move the fuel via the upender.

\subsubsection{Fuel Can Design}

A major near-term action required is the design of the can utilized for fuel handling, storage, and possibly, shipment of the fuel. Interfaces between the reactor fuel handing equipment, fuel storage racks, shipping casks, storage and unloading facilities, must all be considered. Evaluations must be made of geometry, criticality, containment, environmental, and corrosion effects. Some of the design features of the canning are:

- Fuel Handling - Major considerations are handling during both reactor defueling and storage operations, and later 
utilization with the shipping cask, and reopening of the package for examination purposes.

- Debris and Large Fuel pieces - The can must be sized to accommodate a variety of fuel component sizes. Simultaneously, the can must be compatible with the storage rack and shipping casks. A problem is the criticality uncertainty as it relates to the cross-sectional dimension of the can. The can size could be increased by using neutron poisons built into the can.

- Storage Conditions - The fuel can must be compatible with the pool water. Containment to prevent dispersion of radioactivity and subsequent decontamination problems must be assured.

- Shipping - The can functions as the containment of the fuel material required for use with a shipping cask. This can must have connections for venting and draining water. It must also be compatible with existing shipping casks from a dimensional standpoint.

Optimally, a single can design would be developed that is adaptable to all projected uses. These canning operations would be coordinated with defueling of the core to minimize handling operations. The can would be sized to accommodate the entire spectrum of fuel conditions expected. In the case of possible large fuel pieces, a separation technique would be utilized pricr to canning.

\section{1 .4 Operational procedures}

It was not within the scope of this report to define in detail the operational procedures required for defueling. The scoping procedures presented in the report indicate that there are means for handling the fuel or debris and for canning this fuel. It is expected that many of the procedures will be unique to the TMI defueling situation and will also be subject to extensive regulatory overview and approval. Development time should be allocated for this reviewing approval cycle. A major consideration is the prevention of contamination spreading during handing and canning operations between the various pools. A contamination barrier is noted within this report which could serve in this capacity. It would make the decontamination of a shipping cask, which is loaded in the TMI cask loading pool, a simpler operation.

\section{1 .5 Operational Space Requirements}

The extent of decontamination of the reactor building and the potential conflicts between various cleanup operations, which require the need for the pool storage space, mandate 
that priorities be established for these defueling and fuel canning operations.

\subsubsection{Shipping and Logistic Considerations}

(1) Shipping Cask Utilization - A review of the TMI facility indicates that probably all of the available and licensed LWR shipping casks (a total of about 16 casks) could be utilized for operations at TMI if required. Specifically, both legal and overweight truck casks and rail casks could be handled at this facility. (The facility was designed to accommodate the GE-IF300 rail cask.)

(2) Selection of the Most Suitable Cask - The realities of the TMI situation stress operational flexibility and ease of licensing for this situation. For this reason, we have recommended the use of a legal weight truck cask for off-site shipment. The weight of these casks (approximately 25 tons) facilitates ease of handing. These casks are normally smooth-walled, and will result in easier decontamination. In addition, questions related to cask content, in particular "debris", can be more readily accommodated from a licensing framework. There are three separately licensed cask models noted within the report, and there are a total of 10 casks which could be made available.

The rail casks offer a larger internal volume (typically 36-inch cavity I.D. versus 13- to 17-inches for truck casks) but handling, licensing, and availability considerations, are all expected to be far more difficult to resolve (based on historical precedents in the licensing of rail casks.)

(3) Shipping Can Interface - It is expected that a separate shipping can will be required as an additional containment barrier during any off-site move. The design of this can must be accommodated within the fixed dimensions of the available shipping casks. Ideally, the can used for fuel handing and storage would also function as the containment during shipping.

(4) Cask Handling - This report discusses several operational improvements at the TMI facility related to the interface between the facility crane and the cask loading. A shelf is proposed to simplify operational steps and eliminate the crane contamination.

(5) Cask Requirements - It is estimated that there will be 200 to 250 shipments off-site. To accomplish this over approximately 2-years time, a minimum of two casks for 
relatively short distances and upwards of four casks would be needed. If rail cask movement were selected, the same shipping campaign could be handled with one cask.

(6) Institutional problems - In recent years a number of cities and other regions have precluded movement of radioactive materials through their boundaries. In addition, recent NRC and DOT regulations have sought to embargo routes through cities with populations greater than 100,000 people. Transportation movements of spent fuel have been minimal to date; however, local and federal regulations have been enacted to safeguard against possible terrorist attack and accidents within urban areas. Guarded shipments, secret (unpublished) routings, vehicle immobilization techniques, and constant en-route communications are required by the newest governmental regulation (10 CFR Part 73 ). The result has been to considerably increase the cost and difficulty associated with the shipment of spent fuel.

(7) NRC Licensing of Casks - The specific cask(s) selected for the TMI spent fuel shipments will probably require a licensing amendment for TMI failed fuel. This will require a period of time to portray the specific characterization of the fuel, debris, shipping container, and handling techniques.

3.2 Technical Evaluation of TMI-2 Fuel Core Disposition Options

It is known that a certain fraction of the fuel will be shipped off-site to permit examination and evaluation of this severely damaged fuel. However, it is expected that the bulk of the fuel must be stored prior to ultimate disposal or perhaps reprocessing. Technical evaluations were made of wet storage as either a short-term or long-term option, dry storage at either hot cell vaults or in caissons, or chemical reprocessing for recovery of uranium and possibly plutonium. These evaluations considered whether the process could be performed, how the option would be implemented, and the institutional-economic problems associated with its implementation. Final disposition (not projected to be available until at least 1995), was assumec to be a canning of an immobilized form of either reprocessed high-level waste (HLW) or the spent fuel itself, at a federal repository.

The evaluations performed showed each option is technically feasible. Institutional and/or perhaps licensing restrictions may be the major deterrent to implementation. pool storage, probably at the TMI facility, was felt to be the 
obvious near-term option available for most of the fuel. The only non-standard technical consideration associated with pool storage is related to the need to can most or all the fuel prior to storage. Canning is needed for fuel structural integrity and to prevent the spread of contamination. The major problem is the possible lack of available space. This is a nationwide problem in the nuclear industry.

Chemical reprocessing could be performed both domestically and overseas if need be. No technical problem appeared insoluble. However, the international perspective of institutional problems related to separated plutonium is unclear. The availability of domestic reprocessing is not expected until the late 1980's at the earliest.

Dry storage of spent fuel could be the most flexible alternative due to the amount of fuel to be handled. However, to date, there has been no major demonstration of dry storage of LWR fuel. Canned TMI fuel in a dry environment should present few technical problems, due to the extremely low heat load and the probable release of most of the free $f$ is sion gases from the fuel during the accident.

The ultimate step, geological disposal of HLW or spent fuel, is of course a major impediment, to the nuclear industry relating to the resolution of the nuclear power issue. Current pronouncements by the president indicate that resolution of this problem is not to be expected until the late $1990^{\prime} \mathrm{s}$.

A summary of the major conclusions for each of the alternative disposition options studied is presented below.

\subsubsection{Chemical Reprocessing}

(1) The reprocessing option is technically performable at any facility designed and constructed for the handling of commercial LWR fuel, and could probably be performed at certain governmental facilities. In the latter case, concerns arise to the availability of a chopleach, headend process to dissolve the fuel, and to the NRC licensing considerations related to the emission of gases and liquid waste from the process. Conversion of the uranyl stream to uranium hexafluoride ( $\mathrm{UF}_{6}$ ) may present criticality problems, since plant limits are normally held to maximum uranium enrichments of between 1.2 to 1.88 (depending on the facility). However, this could be accommodated by process modifications or a blending of the fuel to attain lower enrichment levels. 
(2) Uranium Value - The economics of reprocessing are enhanced by the low fuel burnup which results in an average enrichment of 2.28. This is much higher than baseline economic studies of reprocessing which postulate a fuel enrichment of less than 1 \& remaining in the fuel.

(3) Plutonium Disposition - The technical options available include separation of the plutonium:stream, permitting the plutonium to remain with the uranium product. (coprocessing), and the mixing of the plutonium stream with the high-level waste. Each one of these options is technically possible. However, the economic and political considerations vary drastically. Economically, the separation of plutonium is a better alternative since it potentially has value as a feed for advanced fuel (breeder) cycles. From a safety standpoint, it is desirable to reuse or "reburn" the plutonium rather than disposing of it due to its long radioactive half-life. The above areas have been argued and disputed for several years and near-term resolution is not forthcoming.

(4) Facility Availability - A total of six chemical reprocessing facilities were evaluated. Detailed evaluations of three were discussed within the report. The current governmental facilities were deemed to be far less suitable for this application. They were not specifically designed for this fuel and would require plant modification to accommodate the fuel. Licensing considerations related to enissions might preclude their use. In certain cases, the facilities are currently inoperative or their processing capacity was too low. However, it also is assumed that the current commercial reprocessing facilities could never operate in a private mode. They would require governmental ownership to proceed. Hence, the largest impediment to implementation of chemical reprocessing would be the transfer of these facilities to governmental ownership or the resolution of political impediments to reprocessing.

(5) Foreign Reprocessing - This is a technical possibility and could be implemented in the 1980's. The cost of fuel shipment overseas would be a significant portion of the total cost of reprocessing. In view of United States Executive'Branch concerns relating to international nuclear proliferation, this does not appear to be a practical alternative. 
3.2 .2 Wet Storage of Spent Fuel

(1) Feasibility - Some fuel will be stored at a spent fuel pool, whether at TMI or elsewhere, for a limited duration. Intact fuel and fuel with minor cladding failure has been stored under wet pool conditions for over 20 years with no major technical or licensing problems.

(2) Technical - The only nonstandard technical undertaking is the canning of the core and the design of storage racks to accommodate canned fuel. Canning and storage of nuclear fuel has been performed heretofore. The major concern is assuring that handling operations are optimized from a safety concern and that the can is compatible with future shipping and disposition alternatives. The cans must be designed for both intact fuel and debris. The cans must operate in pool environments where the water is either heavily borated or demineralized. The cans must prevent the spread of badly fragmented fuel and minimize pool contamination. The new spent fuel racks must be designed to accommodate the fuel can size. Criticality evaluations must be performed for the debris since it has an unknown composition and configuration. Prior experience with reactor license amendments to accommodate fuel pool reracking has shown that an average of 2 to $2-1 / 2$ years is required for review and approval.

(3) Pool Space and Volume Conditions - Utilizing state-ofthe-art fuel storage rack technology, the floor space required to store the entire core is relatively small (approximately 250 square feet). In addition, due to the low source strength of the fuel itself, the amount of water cover required over the fuel for shielding purposes can also be limited.

(4) Eacility Availability - Four possible alternatives for wet storage of spent fuel are presented within the report. The most obvious would be to retain an area within the TMI fuel pool itself. This is probably the most economical alternative. Also posible is the transshipment to another reactor pool, preferably at a site resulting in the shortest transport distance. There have been several transshipments between reactor Fools within the United States. This alternative is subject to the availability of space at other reactors, and this appears to be a tentative assumption at best. fnother possible alternative is the movement to an independent or away-from-reactor (AFR) pool location. This regionalized facility, operated under proposed governmental ownership, would store fuel from a number 
of different reactors, with supposedly each having no available space. The storage period at the AFR would be limited, pending resolution of disposition of highlevel waste and spent fuel within the United states. The difficulty with this option is that the earliest operational date for an AFR may be later than that desired for off-site shipment. Current projections are that an AFR could be operational in the 1983 to 1985 time period and operated by DOE. This is a highly speculative assumption. A folirth alternative is the storage of fuel at a pool located at a governmental facility.

\section{$\underline{3.2 .3 \text { Dry Storage }}$}

(1) Technical Feasibility - An evaiuation was made of several different dry storage options including vault storage and caisson storage (several assemblies within a heavily shielded container). Both above- and belowground storage was considered. The primary generic limitation to dry storage of spent fuel is the need to filter the off-gas coolant and to assure cooling resulting from the decay heat load of the fuel. In the case of TMI fuel, it is expected that the free fission gases normally contained in the fuel rcd plenum were released during the accident. Also, the fuel decay heat level will be extremely low (in the vicinity of 20 kilowatts) for the entire core after five years of aging. As with wet storage, the fuel must be canned. However, the can will function as the containment during storage.

(2) Technical precedents - The technical attractiveness of this option is clear. However, dry storage has never been practiced for quantities of LWR spent fuel. Neither has dry storage ever been 1 icensed by the NRC for this fuel type. However, there is no major or insurmountable safety problem which could not be overcome with proper design.

(3) Convenience - If the availability of a hot cell were demonstrated or caissons couid be fabricated within the requisite time period, this particular option could have merit for limited on-sice storage. Little operational support is required and only minimal facilities are needed.

(4) Facility Availability - There are several governmental hot cells located at national laboratories which could be utilized to store portions of this fuel. The storage volume required is not great. Questions involving safety and licensing would have to be evaluated for 
each facility on a case basis. These questions could be time consuming if a near-term solution is desired. Caisson storage is advantageous since it is siteindependent.

\subsection{Economic and Schedule studies}

Stucies were performed related to the cost factors associated with:

(1) Shipment off-site.

(2) Disposition of spent fuel by:

- Chemical reprocessing

- Pool storage

- Dry storage.

(3) Final disposition cf failed fuel as HLW or canned spent fuel in a geological repository.

The details of nine specific scenarios are presented in section 7.0. This section presents individual costs as a function of the technical options selected for study, and the appropriate date of implementation for each step. In general the following conclusions were needed:

- The economic value of the low burnup fuel core (average enrichment of 2.28 ) is sufficient to justify reprocessing strictly to recover tre uranium. This is true irrespective of the status of utilization of plutonium. However, this assumption is predicated on the availability of an established and mature reprocessing industry; which is now an indeterminate factor.

- Pocl storage of canned fuel at the TMI Facility is the most economical near-term choice until resolution or definition of the nualear high-level waste picture becomes available.

- Dry storage of fuel in dedicated vaults or caissons is the most costly disposition alternative if the entire core is handled in this manner. Of these two options, caisson storage is more economical. Caissons would be used to handle portions of the core which require special handling. 
, 4.0 RECOMMENDATIONS

$\underline{4.1}$ General

A series of recommendations was developed based on the results of this report. These recommendations are to be used as general guidance for programmatic plans related to the ultimate usage by concerned parties such as the TMITechnical Integration office under DOE sponsorship and the TMI-2 recovery team from GPU. These recommendations are intended to be a part of the integrated research program being developed for the nuclear community through a coord:nating agreement of the following parties: Department of Energy (DOE), Electric Power Research Institute (EPRI;, Nuclear Regulatory Commission (NRC), and General Public Utilities (GPU). The study contained herein was performed to comprise the requirements for sub-Task 7.3 of a joint agreement between the four cooperating parties (Reference $4.1)$.

Table 4-1 presents a listing of near-term, priority recommendations for this program. The following guidelines were utilized to develop the items categorized as near-term requirements :

(1) Schedule Limiting. - A major impact on the overall TMI recovery schedule.

(2) TMI-2 Cleanup Need - An area which must be technically resolved prior to defueling, fackaging, or shipment off site.

(3) Safety/Licensing - A technical area which has an overall impact on the operational safety involved in the recovery and is expected to be given close scrutiny by the NRC preparatory to the operation proceeding.

(4) Nuclear Community Interest - An area of interest to the nuclear community (both industry and government) from the standpoint of near term operational and safaty evaluations related to fuel performance during accidents and potential recovery operations.

4.2 Discussion of Near-Term Needs

The following discussion broadly summarjzes the items considered to be of greatest importance for near-term studies, design, or testing related to the defueling and associated operations of the TMI-2 core. 
(1) Fuel Examination and Core Removal procedures - These items are noted here because of the close relationship they bear to the planning of the total defueling operation. The near-term examination of the core should be of the highest priority. Only through at least a preliminary examination can any detailed planning be performed related to procedures. Preliminary plans will, of necessity, be diverse due to the lack of knowledge of the degree of fuel damage. Of equal importance is the preliminary definition of fuel removal techniques. Contained within this report are tentative techniques for removing debris. However, techniques for removing intact fuel and fused sections of fuel will have to be developed to the same extent. Item 14, Reference 4.2 (the Sub-Task 7.2 Report) denotes a tool which penetrates the central guide tube and permits a go/no-go evaluation of the structural strength of the fuel assembly. This type of tool would be valuable in the defueling operations.

As a portion of the above development studies, various tooling and observation techniques must be developed to permit remote examination and handing of the fuel in the reactor core. This equipment should be tested in a simulated operational environment. This would be preparatory to usage in a nuclear pool environment. Operator training and checking of necessary tools should be performed utilizing mock-ups. Another possibility is videotaped simulations of the operations as they are expected to be performed, which can be used as a reference for future operations.

(2) Canning (Design, Analysis, and Testing) - In this report, a number of different types of canning requirements are described. It is readily noted that the number of interfaces between the reactor, spent fuel pool handing conditions, shipping casks conditions, and ultimate or interim storage requirements must be consistent and compatible. The canning definition presented is preliminary. The prime requisite is determining can dimensions which are compatible with handling, storage, and shipping restraints. The can design must also assure that containment and structural problems are resolved, that criticality is precluded and that the equipment can be utilized operationally at the TMI-2 facility.

A single can design and canning procedure is desired. It is possible, that due to the variation in observed fuel condition, that more than one can should be developed. A thorough interface study is necessary to assure that there are no loose ends concerning handling 
restrictions. Criticality limitations associated with debris collection may place restraints on the operational requirements.

\section{(3). Shipping Cask Selection and Testing}

Interface questions related to both on-site and receiving site handling options are strongly affected by the shipping cask(s) to be used. It will probably be necessary to have the cask owner process a license amendment to the certificate-of-compliance (COC) to accommodate the fuel "debris." In addition, certain modifications may be required.to the TMI handing facility, the receiving facility(ies), and the cask itself. It is recommended that a detailed selection study be made of the spent fuel casks and initial negotiations be arranged with a cask owner to permit "Eormal" plans to be made.

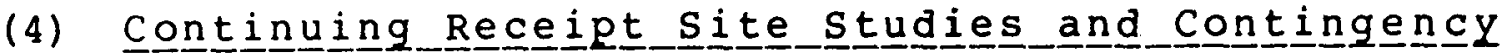
planning

of necessity, this report focused on the "generic" characteristics of possible sites for off-site receipt and temporary (long- or short-term) storage of the TMI-2 fuel. It is very important that "focused" studies for these receipt locations be developed in order to permit planning of technical interfaces, shipping campaigns, and possible modifications to either the shipping cask or the receipt site handling facilities and equipment.

In addition, the possibility of only "limited" fuel movement (in the near-term) from the TMI plant site must be considered. Two alternatives which should be considered are:

(a) Pool storage - The provision of spent fuel racks suitable for canned fuel to accommodate the entire TMI-2 core within either the Unit 1 or Unit 2 spent fuel pool should be evaluated.

(b) Caisson Storage - As a contirigency, caissons similar to concrete vessels or possibly similar in design to rail - spent fuel casks could be employed to serve as caissons on the island.

\subsection{Decision Points}

Figure 4-1 categorizes the specific items or locations that would be involved in making key decisions. Specifically covered are: 
Failed Fuel Categories - Six different fuel states are noted which impact canning and fuel handling considerations.

On-Site Handling - Two different alternatives for the movement of the spent fuel and debris from the reactor containment to the auxiliary building are presented.

On-Site storage - Four possible on-site locations are denoted related to possible means of temporarily storing the fuel on the island.

Canning Options - Seven can categories for alternative approaches to handling, collection, storage, and shipping of the fuel are presented.

Off-site storage - Eight possible types of facilities could be considered for near-term receipt of all or part of the TMI-2 core.

Spent Fuel Cask - Four types of spent fuel casks could be utilized for shipment of the TMI-2 fuel off-site.

\subsection{Key Action Items}

This section of the report presents fourteen action items which identify and comprise simplified work scopes for the major near-term areas of study related to core defueling and disposition. Items $A$ and $B$ are repeated from Reference 4.2 since they are of great importance to the accomplishment of the remaining twelve items. Item $C$ is a preparatory study of operational factors and other general areas related to unique concerns such as radiation fields safeguards and accountability of the fuel. As noted previously, it will be desirable to combine functions of the various canning alternatives proposed in order to minimize handing and cost. Items 4-8 may be combined as additional development in this area commences. Similarly, Items $1-3$ which relate to defueling operations could be amalgamated into a single larger work area. 
TABLE 4-1

RECOMMENDATIONS FOR KEY ACTION ITEMS

ITEM

A Penetration of Reactor Vessel and Examination of Core Condition

B Fuel Bundle Strength "Go/No-Go" Test

C Preparatory studies of Operational Factors

1 Fused Fuel separation Techniques

2 Handling Techniques for "Large" Fuel Pieces

3 Fuel "Fines" Vacuuming Techniques

4. Intact Fuel Handling Shroud Development

5 Debris Can Development

6 Handing and storage of Fused Fuel

7 Development of Fuel "Leaker" Cans and Storage Racks

8 Operational Procedures for Canned Fuel

9 Shipping Cask Selection and Testing

10 Receiving Criteria for Research Fuel

11 Continued Studies of Fuel Disposition sites

12 "Caisson". Storage studies 
ITEM A - PENETRATION OF REACTOR VESSEL FROM TOP (REFERENCE 4.2)*

\section{Objective}

Inspection of Reactor Internals and Fuel prior to reactor vessel (RV) Head Removal.

\section{Discussion}

The degree of equipment and fuel damage in the TMI-2 reactor is not precisely known. It is expected that significant fueI damage has occurred, that debris is present in the core and the reactor internals, and that some fusing together of core components has occurred. Reactor internals damage resulting fron the transient may impede disassembly of the reactor and., upon removal, may disturb the core configuration.

It is important that as much information as practical be obtained on the conditions inside the reactor prior to reactor disassembly. This information will serve to benchmark the various analyses already completed or underway. It will also guide the development of programs to obtain more data on the TMI-2 core and other experiments planned or underway. The early look into the reactor vessel will also provide data for detailed planning of the examination programs to be conducted during and after defueling.

\section{Project Scope}

The scope of the project will involve:

- Identify methods of providing the desired inspection

- Evaluation of inspection alternatives

- Selection of primary approach

- Engineering of equipment and procedures

- Integration into the TMI-2 Reactor Disassembly Plan

- Assembly of equipment

- Demonstratio. of equipment and procedures

- Conduct inspection at TMI-2

- Report resulis of Development Program and Inspection.

\section{Schedule}

Demonstration of Equipment and Procedures ready for field operations by March 1981 .

\footnotetext{
*Sub-Task 7.2 Report, "Recommendations on In-Place TMI-2 Core Damage Exaninations."
} 
ITEM B - TECHNIQUES FOR MEASURING STRENGTH OF FUEL BUNDLE STRUCTURAL MEMBERS (REFERENCE 4.2)

("Go/No-Go" Test of Intact Fuel)

Objective

To measure strength of a representative number of fuel element structural members in order to:

(1) Assist in evaluation of core damage.

(2) Establish load bearing capability of fuel, to support removal methods while minimizing further damage.

Discussion

The structural members of interest in this project are the control rod guide tubes and attachment of these tubes to the fuel end fittings.

Although the degree of core damage is not precisely known, it is believed that degrees of structural weakening ex ist up to failure, thus leaving portions of some fuel. assemblies in a "rubblized" condition. In order to assist in evaluating both radial and axial damage, it is desirable to measure the strength remaining in a representative number of fuel assemblies. Strength comparison radially (fuel assembly to fuel assembly) and axially (single fuel assemblies) and an overall comparison to as-built strength should provide input.

Schedule

Preparation for on-site work complete by October 1981. 


\section{ITEM C - PREPARATORY STUDEES OF OPERATIONAL FACTORS}

Objective

Evaluation of operational factors and constraints related to the core defueling and carning procedures.

\section{Discussion}

The exact nature of the operational procedures for defueling ard cannery will be determined to a large extent by various environmental and regulatory factors which are unique to the TMI-2 reactor facility and circumstances. Among these are nuclear-related factors and the radiation field surrounding the areas where the operations are to take place and special studies of means of determining and accounting for the core material itself.

The radiation field will determine the number of operators required, operational steps and precautions, the types of personal equipment required, and the work locations. Materials accountakility will be required to assure that provisions are made for proper safeguarding of the cannistered fuel. This is particularly true for fuel collected and canned in the debris form.

\section{Project Scope}

- Perform survey of radiation field in various portion's of the reactor containment and auxiliary building. Use this data to estimate operational working tones and other radiation safety precaution.

- Develop preliminary operational procedures defining operating areas ard remote equipment requirements.

- Evaluate material accountability requirements canned for debris and failed fuel.

- Prepare safeguards plan for canned fuel.

Schedule

- Radeological factors study required by early 1981.

- Safeguard plans required by mid 1981. 
ITEM 1 -- "FUSED" FUEL SEPARATION TECHNIQUES

Objective

Tooling and equipment to permit packaging of large fuel pieces which will have compatability with storage cans and off-site shipment.

Discussion

There is a possibility that portions of the core are "fused" together. These pieces may be too large to place in a can and moved via the "normal" route to the auxiliary building. simultaneously, this fuel will be uncapable of being placed in "normal" fuel storage racks and of being shipped offsite. It is important that a tool(s) and equipment be developed that could "Pry" the fuel apart. The fuel would be separated into a size suitable for packaging.

\section{Project Scope}

- Examine potential fuel damage scenarios and identify a range of possible fusing mechanisms and configurations.

- Evaluate possible areas of operational "danger" in separating and means of precluding damage to adjacentintact fuel

- Define the amount of remote handling required and develop "viewing" techniques

- Develop several conceptual designs and evaluate, in terms of function, operational flexibility, safety, and reliability

- Select design and fabricate a prototype

- Test prototype tool in non-contaminated environment

- Demonstrate equipment and train selected operational personnel

- Develop final operational procedures.

Schedule

Equipment and procedures should be ready for field operations by early 1982 . 


\section{ITEM 2 - HANDLING "LF.RGE" FUEL PIECES}

\section{Objective}

Tooling and equipment for collecting and removing large (normal package size) fuel pieces from the reactor.

\section{Discussion}

It is possible that there will be large (but manageable) fuel pieces which must be removed from the reactor vessel. This could include fuel end fittings, shrouds, spacer grids, control-rod assembly pieces, etc. These pieces may have occurred as a result of the accident or from separation of fused pieces.

It is expected that these tools would include long-handled grapples and tongs of a specialized design. The tools, equipment, and procedures developed to handle these pieces could have "generic" applicability to the nuclear industry. There are instances where it is necessary to remove and collect similar-sized pieces of highly radioactive material.

\section{Project Scope}

- Characterize the size, weight, and configuration of possible fuel pieces

- Identify the possible location(s) of this fuel, within the reactor vessel

- Identify the degree of remote operation and the need and type of visual surveillance equipment required

- Identify areas of operational inconvenience and potential safety problems related with equipment handling

- Survey the availability of existing equipment and the possible purchase of commercially available equipment

- Develop several conceptual designs of tooling for the specialized tasks and evaluate in terms of function, operational flexibility, safety, and reliability.

- Fabricate prototype equipment as necessary

- Test prototype equipment in a non-contaminated environment

- Demonstrate equipment and train selected operational personnel 
- Develop final operational procedures.

Schedule

Equipment and procedures should be ready for field operations by early 1982 . 
ITEM 3 -- FUEL "FINES" VACUUMING TECHNIQUE

Objective

Equipment to remove and segregate fuel "fines" and other highly radioactive particles from the reactor vessel.

\section{Discussion}

A portion of the fuel (and other highly radioactive debris) may be in the form of fine particulate matter that cannot be removed using conventional handling tools. A "vacuuming" technique which removes the "fines" and segregates the particulate by straining, settling, or filtering for ultimate collection in a "debris" can is required.

This type of equipment is o generic interest to the nuclear community for decontamination of areas with extensive loose corrosion product deposition, possible cleaning of the exterior of stored fuel assemblies, and similar applications.

\section{Project Scope}

- Identify the areas of the reactor vessel containing "fines" and the degree of remote operation of the equipment which is required

- Survey the "available" equipment (if any) and commercial components with similar applicability

- Identify remote surveillance equipment needed

- Develop equipment performance specifications and preliminary conceptual designs

- Evaluate equipment in terms of operational safety (including possibility of nuclear criticality), function, operational flexibility, and reliability

- Purchase or fabricate prototype "skid-mounted" vacuuming system

- Test prototype equipment in a non-contaminated environment

- Demonstrate equipment and train selected operational personnel

- Develop final operational procedures.

\section{Schedule}

Equipment and procedures should be ready for field operations by early 1982 . 


\section{ITEM 4 -- "INTACT" FUEL HANDLING CAN DEVELOPMENT}

Objective

To provide physical support and structural augmentation to intact fuel assemblies of questionable integrity during handling movements within the reactor containment building.

\section{Discussion}

There is a possibility that a large portion of the fuel will be physically intact, and capable of normal movement from the reactor and containment to the auxiliary building. However, the structural integrity would be suspect. There is a need to place a "shroud" around the fuel to permit safe handling within the reactor building. This shroud would provide the structural and handing envelope for the fuel. It would also serve to prevent the spread of damaged fuel and particulate matter attached to the fuel structure.

\section{Project Scope}

- Examine cannister handling from the standpoint of. minimizing operations, safety, and assuring operational flexibility and interface with handing tools

- Assure that visual surveillance equipment has been developed and can function in this environment

- Fabricate prototype equipment

- Test equipment on "dummy fuel" in a simulated, nonradioactive environment

- Demonstrate final design and train selected operational personnel

- Prepare operational procedures.

Schedule

Equipment and procedures should be ready for field operations by early 1982 . 
Objective

Collection and handiing of fuel debris in a safe geometry to prevent external release of contamination:

\section{Discussion}

It is expected that a portion of the fuel material will be removed from the TMI-2 reactor vessel as debris. This debris is of unknown size, and the source strength/ criticality parameters cannot be estimated in advance. It is important that a can be designed to collect this debris in a safe manner.

The can design parameters should consider safe geometry and preclude contamination spreading during the handing operations.

\section{Project Scope}

- Preparation of design and performance specification

- Evaluation of can interfaces with reactor handing equipment, leaker can (if separate), fuel upender, shipping cask, etc.

- Evaluation of maintaining "safe-geometry," nuclear source evaluation, etc.

- Preliminary design of can, considering facility operational interfaces, material selection, etc.

- Fabrication of prototype can

- Develop criticality and source strength measurement devices

- Perform can loading and handing tests using simulated fuel "debris" in a wet pool environment

- Preparation of design specifications for procurement of cans

- procurement and fabrication of the number of cans needed.

\section{Schedule}

- Prototype can developed and tested by late 1981

- Cans procured and at TMI-2 by second quarter 1982. 
ITEM 6 -- HANDLING AND STORAGE OF FUSED FUEL

Objective

To provide procedures and equipment (possibly) for handling "on-site" storage of large "fused" fuel assemblies which cannot be separated (see ITEM 1 ).

Discussion

Based on prior studies, it is possible that portions of the core will be "fused" together. These pieces may not be separable into.sizes which can be positioned in a "safe-geometry" debris can or readily moved to the auxiliary fuel pools using the fuel upender/transporter.

It is important that operational procedures and equipment be, available for collection of this fuel for on-site movement, (both within and out of the reactor containment) and for eventual on-site storage.

\section{Project Scope}

- Definition of performance criteria and necessary operations, including large "pieces" and possible handling routes

- Selection (or development) of a transporter cask

- Preliminary development of operational techniques and procedures for on-site handling of fuel

- Decision to lease spent fuel rail cask, either to fabricate or lease a special purpose transporter cask, or decide that procedure is unnecessary

- Develop on-site storage location considering pool and dry caisson options - possible movement off-site to a research facility

- Develop operational procedures and tests based on demonstrated need.

Schedule

- Decision for fabrication of on-site transporter needed by $\operatorname{mid}-1981$

- Availability of demonstrated procedures and equipment by second quarter 1982 (if needed). 
ITEM 7 -- DEVELOPMENT FUEE STORAGE CANS AND STORAGE RACKS

Objective

To provide cans and pool storage locations for the handing and storage of fuel assemblies or (manageable) debris.

Discussion

It is expected that most of the fuel and fuel material will be canistered to permit long-term storage and containment of the fuel. The fuel canister will have to be stored "on-site" with spent fuel racks for a period of time. The can must be compatible with fuel storage racks (both at TMI-2 and possibly at an off-site location), shipping casks, and TMI-2 handling equipment.

The design of a can for failed fuel has broad generic applicability to the world-wide nuclear community due to the current need of storing spent fuel for extended periods of time.

\section{Project Scope}

- Develop performance specification for storage can, defining all related interface considerations

- Develop performance specification for fuel storage rack considering the can interface and the TMI-2 pool requirements

- Prepare preliminary design of a can and fabricate prototype

- Evaluate can design in terms of the shipping cask (on-site and off-site) handing limitations

- Test can in wet (nonradioactive) environment and perform loading tests with simulated (damaged) intact fuel

- Prepare operational procedures for canning

- Prepare cannister procurement specifications

- Prepare procurement specifications for the detailed design and fabrication of fuel storage racks

- Determine number of storage rack slots and cans required

- Evaluate storage pool modifications to accommodate rack 
- Procure storage cans and racks based on required projections

- Install storage racks in pool storage area.

Schedule

- Prototype can designed and tested by mid-1981

- Spent fuel rack design completed by mid-1981

- Leaker cans and fuel storage racks procurement initiated in third quarter 1981

- Leaker cans available and fuel storage racks in-place at TMI- 2 by mid-1982. 
ITEM 8 -- OPERATIONAL PROC

Objective

To develop procedures for handling in-reactor movement of canned fuel assemblies and debris.

Discussion

It will be necessary to plan the techniques necessary for moving canned fuel from the reactor containment building to the auxiliary building for storage. In addition to testing of the fuel handling equipment, it will also be necessary to plan (a) where the canning will take place, (b) the need for inter-pool contamination barriers, (c) possible safety and equipment reliability probiems during handling, (d) where the fuel will be stored, and (e) where the fuel will be loaded into a spent fuel cask for off-site shipping.

\section{Project Scope}

- Evaluate canning, storage, and shipping schedules in view of overall plant recoverg operation and needed decontamination areas

- Examine the effects of fuel canining on the handing, storing, and shipping operations

- Develop preliminary operational procedures and evaluate technical, safety, and licensing interfaces

- Perform "on-site" tests using dummy fuel and prototype $\operatorname{can}(s)$

- Train operators in selectæd procedures

- Prepare final procedures.

Schedule

Equipment and procedures required in-place by mid-1982. 
ITEM $9--$ SHIPPING CASK SELECTION AND TESTING

Objective

To provide means of shipping fuel from the TMI-2 reactor.

Discussion

Off-site shipment of TMI-2 fuel must be coordinated with the operation and use of the specific shipping cask to be used. It is important that the operations related to on-site canning, loading of the spent fuel, and off-loading at a receipt site consider the specific design and handing aspects of the cask.

Shipment of TMI-2 fuel will probably require an NRC license amendment to the cask $\mathrm{COC}$ to accommodate the "uncertaindebris" fuel form. It is also recommended that a special shipping can be utilized with the cask.

\section{Project Scope}

- Evaluate truck cask characteristics and select cask(s) to be used

- Negotiate cask usage with owner

- Evaluate cavity dimensional and operational interfaces between the cask and the leaker can

- Develop debris "source". term for shipping cask license amendment to $\mathrm{COC}$

- Cask owner prepare cask/site interface plan and preliminary operational procedure

- Cask owner prepare and process license amendment to the - cask COC

- Fabricate prototype shipping can

- Test interfaces and operational procedures in a noncontaminated pool with the can and the spent fuel cask

- Verify (with dummy fuel) cask handling procedures at TMI-2 pool

- Train plant operators in loading techniques. 
Schedule

- Finalize negotiations with cask owner by early 1981

- Submit cask COC license amendment by third quarter 1981

- Procedure and equipment in field and demonstrated by third quarter 1982 . 
Objective

To provide interface criteria at receiving site for cask handling and fuel package handling.

\section{Discussion}

Approximately 2 to 58 of the canned fuel will be shipped to hot cells for diagnostic examination purposes. The hot cells may require modification or special handing procedures to unload the shipping cask and to "decan" tine fuel in the hot cell.

An informational package will be required at the receiving facility concerning the details of the package and the shipping cask. In addition, it may prove necessary to modify the internal leaker cans to permit handing or loading at this research facility.

\section{Project Scope}

- Prepare interface criteria document. on shipping cask

- Prepare interface criteria document on leaker can

- Perform interface studies with selected research sites

- Test cask unloading procedures (with empty cask).

Schedule

- Criteria package required by early 1982

- Cask and equipment required in field by third quarter 1982 
ITEM $11--$ CONTINUED STUDIES OF FUEL DISPOSITION SITES

Objective

To determine the location of an off-site nuclear storage facility for the TMI-2 fuel zore.

Discussion

It will probably be necessary to ship the TMI-2 fuel core to an off-site location for secure storage. The AGNS report develops the basic technical and institutional criteria for comparing alternative locations. It is important that a study of actual locations and possibilities be made directly by either a governmental agericy or GPU (or both).

Site "specific" studies are reeded to complete further plans related to on-site (TMI) storage needs, shipping cask definition and routing, and the length of time needed to totally defuel the reactor preparatory to recommissioning.

Project Scope

- Develop specification on total quantity of fuel to be shipped, expected date of shipment, fuel configuration, and means of shipping off-site.

- Prepare (or abstract) listing of all potential domestic pool, hot-cell, storage-vault, etc., locations at nuclear facilities.

- Develop shipping cask and fuel package interface criteria.

- Compare site criteria with cask/package interface limitations and "refine" list of possible sites.

- Visit selected sites and rank alternates on basis of both technical and nontechnical (political, economic) implementation possibility.

- Initiate implementation plans for selected facility(ies).

- Develop operational sequences - interface limits - site modification requirements.

Schedule

The implementation plan should be completed by mid-1981. 


\section{ITEM 12 -- "CAISSON" STORAGE STUDIES}

Objective

To provide an alternative means of storage for a portion of the TMI-2 fuel core.

Discussion

The uncertainties of the TMI-2 core defueling and off-site shipment are sufficiently great that a backup alternative is required for fuel storage (either on-site or off-site). Caisson storage is a means whereby a heavily sicielded, (dry) container could be utilized for safe and secure storage.

These caissons (which could approximate rail - spent fuel casks) can be manufactured on demand. They are site independent which provides flexibility in location. In addition, these caissons could be utilized for the "on-site" transport. of large fused pieces (see Item 6).

This study has generic interest to the nuclear industry in the event of an AFR not being available for storage at the required time.

Project scope

- Examine use of modified rail cask design, foreign "Stone henge" equipment, and new design concepts

- Preliminary contacts with owners/designers of current equipment

- Develop cost and implementation schedule

- Develop operátional criteria based on canned TMI-2 fuel

- Develop implementation plan.

Schedule

Implementation plan required by fourth quarter 1981. 

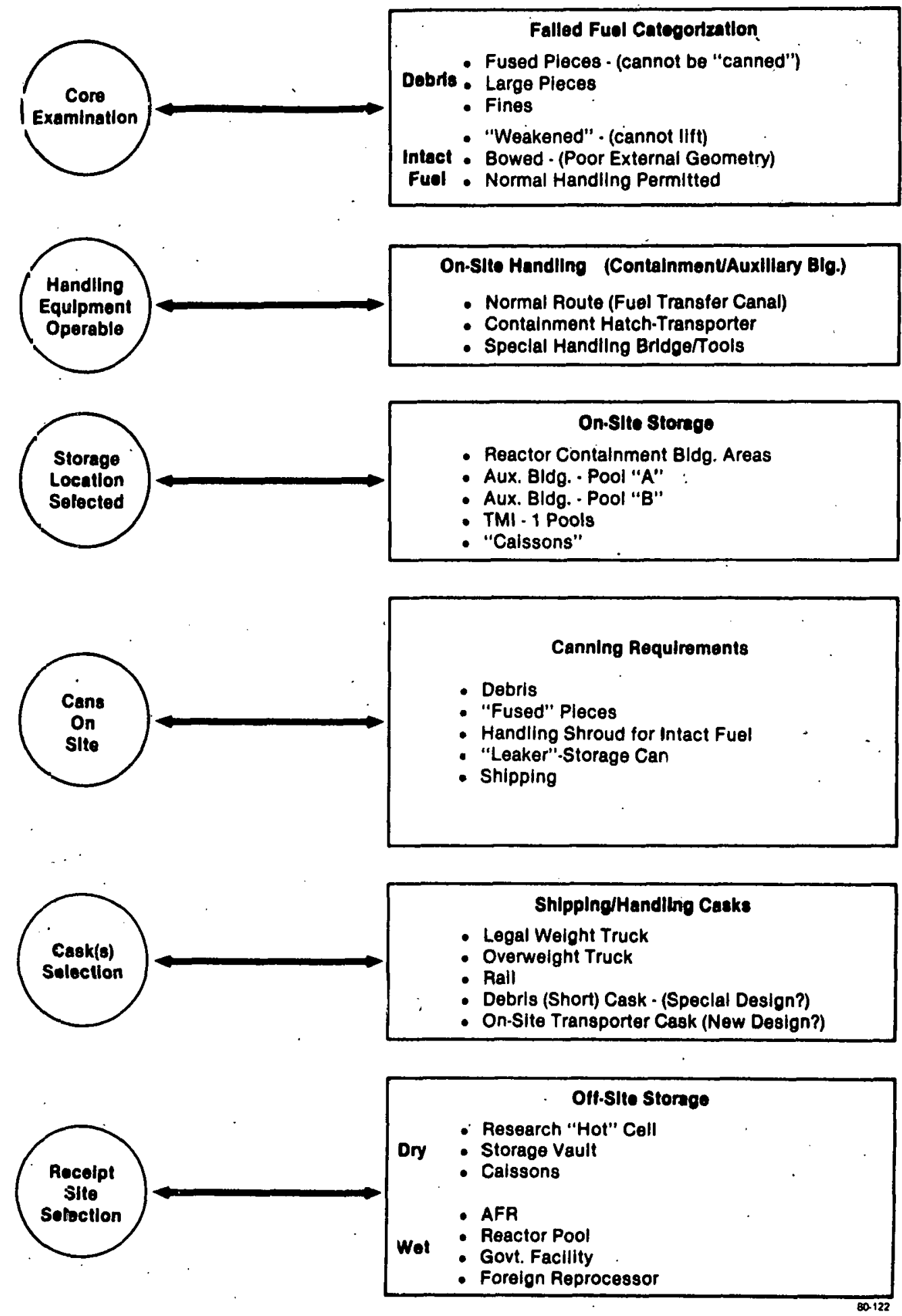

\section{KEY AREAS OF DECISION}

\section{FIGURE $\quad 4-1$}




\subsection{References}

4.1. Meeting Report of TMI Examination Technical Working Group on Recommendations for the DOE/NRC/EPRI/GPU Joint Coordinating Committee, (September 1979).

4.2 Recommendations on In-Place TMI-2 Core Danage Examinations, (April 1980). 
5.0 FUEL REMOVAL, ON-SITE HANDLING AND OFF-SITE SHIPPING

\subsection{Introduction}

The purpose of this section of the report is to examine the technical details associated with (1) the removal of the fuel from the reactor core basket, (2) the handling of the fuel within the reactor containment and spent fuel buildings, (3) the necessary fuel canning to permit long-term storage and off-site shipment, and (4) the technical and logistic aspects of moving fuel off-site via a shipping cask. As noted previously, the techniques associated with the initial movement of the fuel from the reactor were not within the scope of this particular study. Certain general assumptions and recommendations are included and are presented in the applicable section (5.3).

Presented in this section is scoping information pertaining to the technical alternatives which considers a variety of fuel conditions and institutional limitations. The data presented is not meant to define the exact procedures which are necessary. However, it is clear that normal fuel handling techniques typically employed at similar reactors will probably be precluded due to the damaged nature of the fuel. To this end, concepts for handling both intact fuel and debris are included. A variety of possible shipping alternatives are presented on a parametric basis. Also included is a discussion of the fuel itself. The comparison between the fuel in its normal configuration, at the expected condition or ranges of conditions, and the nuclear composition, source strength and thermal decay heat loads at the expected time of shipment were also developed.

In summary, the following key points are noted in this section:

(1) The uncertain nature of the fuel's condition forces planning to be made for both intact fuel and fuel debris.

(2) The fuel nuclear source strength and the thermal decay heat levels are expected to be very low at the time of core defueling due to fuel aging and the low burnup experienced.

(3) It is highly probable that the fuel will require canning for on-site storage and handling. The canning design function can be combined to provide both containment and structural strength to the fuel. 
(4) Fuel shipment off-site will require individual fuel containment within the cask cavity. This can probably be accomplished in common with the fuel storage can.

(5) The use of legal weight truck (LWT) casks appears most attractive for off-site shipment. The preferred use of this lighter weight-small capacity cask is due to greater ease in handling, the availability of more casks, and application at the maximum number of receiving sites. Licensing of the cask by the NRC for this application is also expected to be more readily accomplished.

(6) A major area of development is the design and sizing of the fuel cannister to assure compatibility with the onsite fuel transporter/upender, spent fuel racks, and spent fuel shipping casks.

\subsection{Fuel Status}

\subsubsection{General}

The intent of this study was to categorize the fuel for the purposes of evaluating the various handling, packaging, and shipping techniques to be discussed in later sections. It is also convenient to group, for study purposes, the design characteristics and the burnup characteristics of the TMI fuel as it was initially. loaded into the reactor core and utilized. Three specific factors related to this fuel are developed in later paragraphs and have considerable impact on this study:

(1). The gamma, neutron, and thermal decay heat levels after three years of aging, and, as associated with the relatively low burnup of this fuel, are far less than has normally been postulated for most fue? shipping and wet storage scenarios. This extends the range of possible casks which can be utilized for shipping and minimizes some of the pool and supporting equipment limitations normally required for wet storage.

(2) The general assumption that there is almost total fuel failure (in terms of cladding integrity) will force the requirement for canning. Canning will limit contamination of the storage area due to leaching and crud falloff.

(3) The canning of debris presents an uncertain situation related to exact material composition and estimated source strength. The sizing of the can must preclude any possibility of a fuel loading which could lead to an accidental criticality. In this study, conservative 
assumptions have been made related to the interrelationship be=ween criticality and can size. These assumptions place restraints on the dimensions and configurations for the can itself. With proper can sizing, it can readily be shown that criticality is not a problem with debris. If these limitations present unworkable restrictions on the packaging, it is possible to utilize a neutron poison in the structure of the can.

\subsubsection{Initial Fuel Loading Data}

Table 5-1 presents data on the 177 assemblies which were initially placed into the TMI-2 fuel core. These data were obtained directly from the Babcock and Wilcox company and represent the baseline condition for removal. The core comprises a total of 82.7 MTU and three separate enrichment regions. This table also defines and presents the nonfuel components that were included with this core. It is believed that most of these components which fit within the guide tubes of the spent fuel assembly are not removable from the fuel. Hence: it is necessary to evaluate the input of these components on the fuel geometry and nuclear characteristics when considering shipping, canning, and possibly at a later date, storage or chemical reprocessing options. Figures 5-1 through 5-3 show the geometrical cross section of the fuel including a control rod assembly, and an axial shaping rod assembly.

\subsubsection{Fuel Burnup Data}

In order to determine the actual nuclear levels contained within the fuel, it was necessary to perform several computer analyses directly related to the operating history of this fuel. The burnup of this fuel is approximately $3200 \mathrm{MWd} / \mathrm{MTU}$, which is 108 of projected equilibrium fuel burnup for normal fuel management. To get exact values of the fuel isotopic concentration, data was made available from the Babcock and Wllcox Company based on their in-house calculations. AGNS made similar calculations using the NULIF computer program (Reference 5.2) to determine these fuel burnup characteristics and to further corroborate the Babcock and Wilcox data. Tables 5-2 through 5-5 contain the values for the three individual core regions and the core average. Each table has four parts: Part A lists the mass of each of the uranium, plutonium, and neptunium isotopes; Part $B$ shows the elemental quantities of uranium and plutonium; Part $C$ displays the uranium enrichment (i.e.. percent $U-235) ;$ and Part $D$ shows the percent of $\mathrm{Pu}-240$ in total plutonium. 
TABLE 5-1

B\&W - TMI-2 FUEL DATA

(Initial Core/Loading) Reference 5.1

Fuel Loading

Initial Enrichment Zone - 1 1.98 (1.65) \#Assemblies (current enrichment

$$
\begin{array}{ll}
\text { zone - } 2 & 2.64(2.27) \\
\text { zone - } 3 & 2.96(2.66)
\end{array}
$$

Total: $256(2.21)$

( avg)
56

61

60

177

Burnup - average $3156 \mathrm{MWd} / \mathrm{MTU}$

Kilogram Uranium/Assembly: 463 (avg)

Total Core Loading: $82 \mathrm{MTU}$

1. Fuel Assembly Characteristics

$15 \times 15-$ matrix

208 fuel tubes

16 control (guide) tubes

1 central instrument tube

$\begin{array}{ll}\text { Pitch } & 8.536 " \\ \text { O.A. length } & 165.62^{\prime \prime} \\ \text { fuel tube length } & 153.12^{\prime \prime} \\ \text { active fuel length } 144^{\prime \prime}\end{array}$

fuel tube diameter instrument tube diameter guide tube diameter

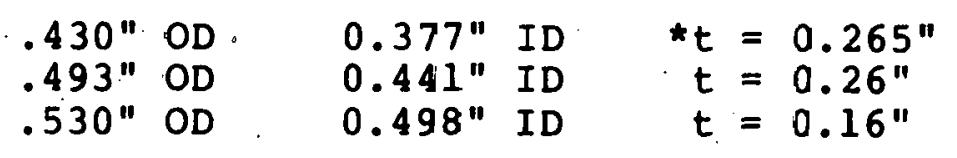

2. Fuel Numbers

Spent Fuel Assemblies

Control Rod Assemblies (CRA)

177

Axial Power 61

Burnable Posion Rod Assemblies (BPRA) 68

Orifice Rod Ássemblies (ORA)

$\frac{0}{177} \quad$ (40 assemblies

* $t=$ tubing wall thickness 


\section{TABLE 5-1 (CONTINUED) \\ B\&W - TMI -2 FUEL DATA \\ (Initial Coreloading) Reference 5.1}

3. Fuel Assembly Composition

$\mathrm{UO}_{.2}-92.58$ theoretical density

fuel clad guide tubes spacer grids zircalloy 4 end fittings Zircalloy 4 Incone1-718 Stainless Steel, grade CF 3M

4. Control Rod Assembly (CRA)

$16 /$ assembly

guide tube material length - 134"

dimensions

Type 304 stainless steel $0.440 "$ OD

composition - Ag-In-Cd, 80-15-58 ratio

$0.21^{\prime \prime}-t \mathrm{clad}$

5. Axial Shaping Rod Assemblies

$16 /$ assembly

$0.440 "$ OD $0.021 "$ clad thickness

composition - Ag-In-Cd 80-15-58 ratio

Type 304 stainless steel

length 36" (poison)

6. Burnable Poison Rod Assemblies

$16 \%$ assembly

$0.430 "$ OD $0.35 "$ clad thickness

poison - Al O - B C

Zircalloy 4

poison length $126 "$ 

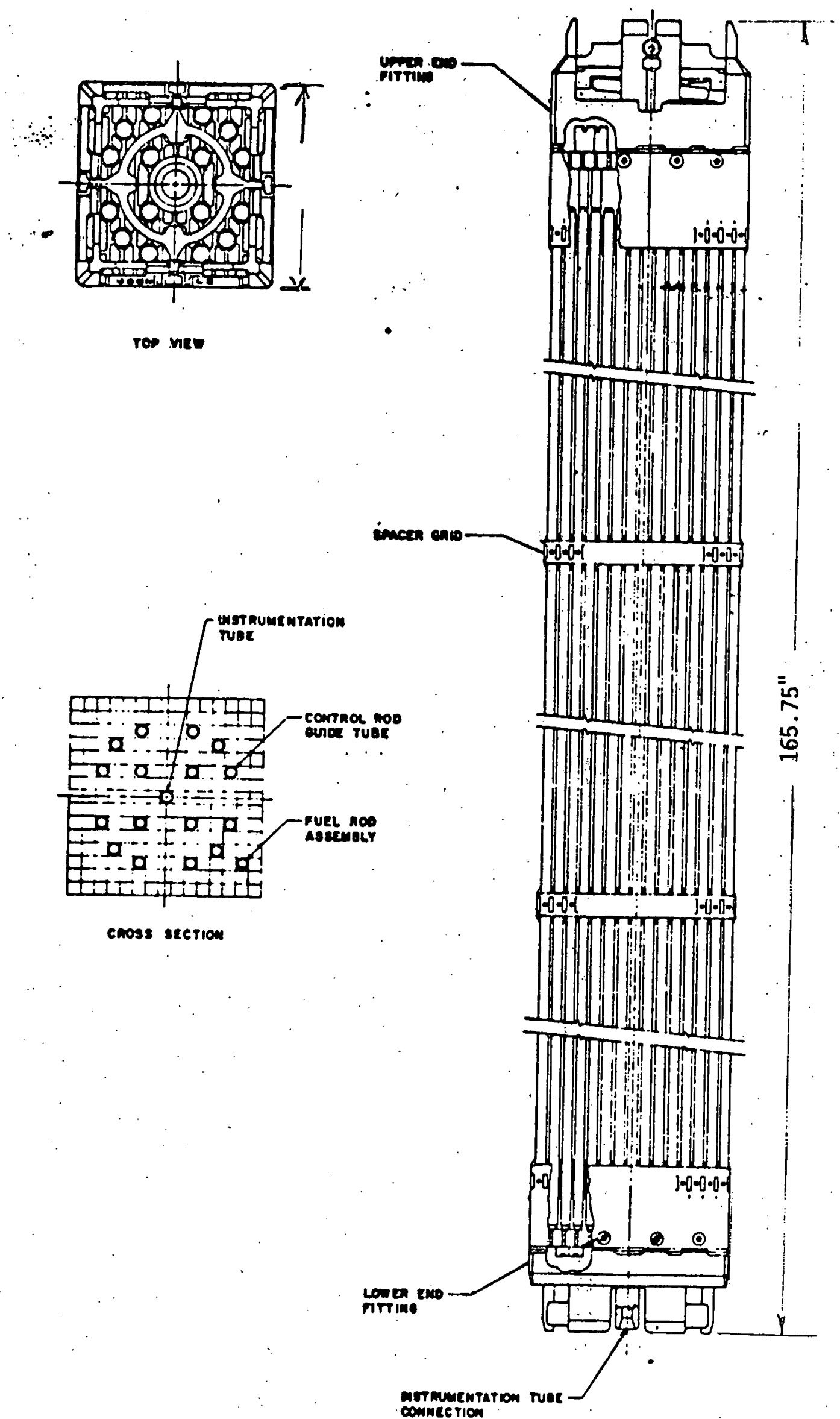

FUEL ASSEMBLY

FIGURE 5-1 


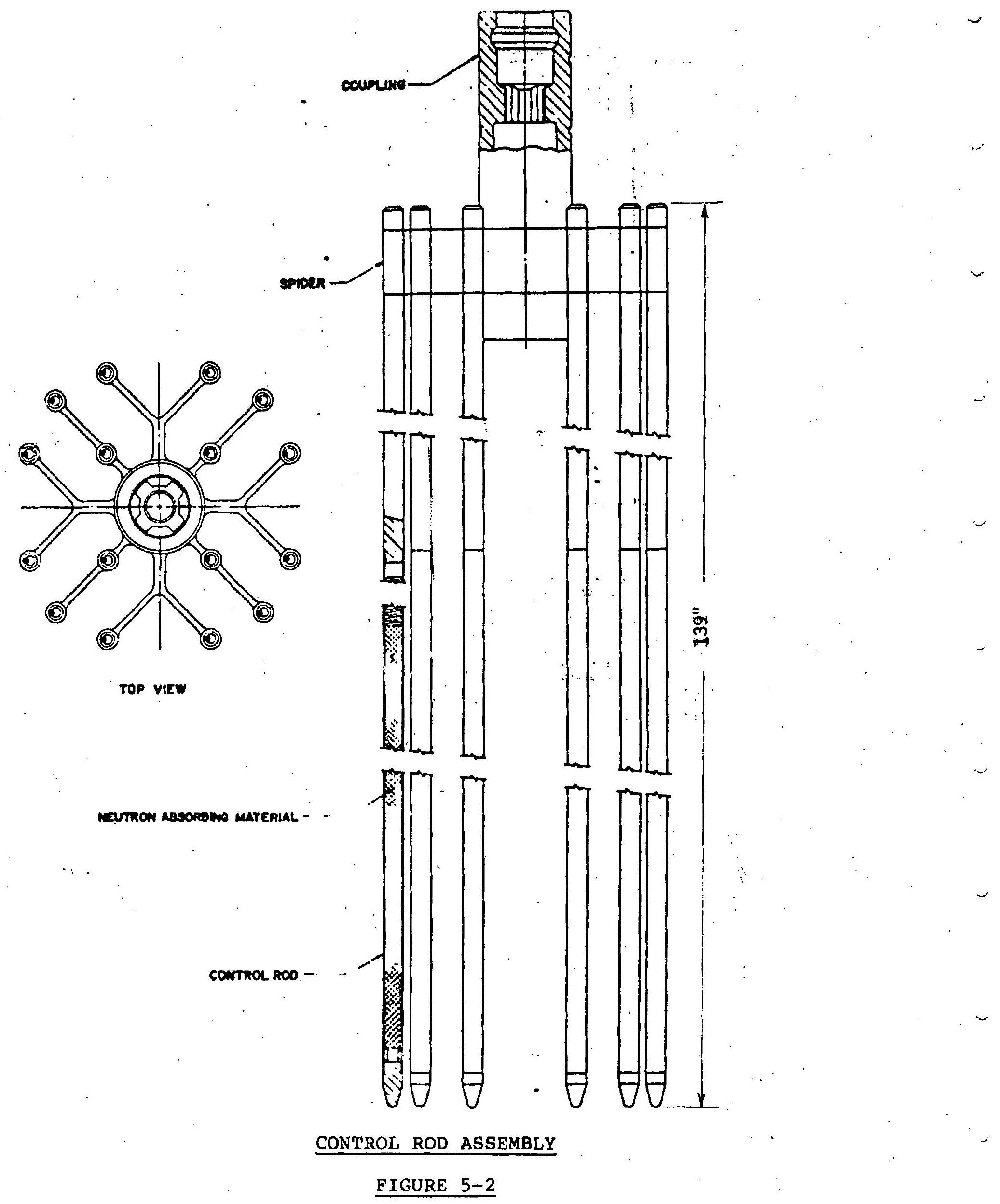




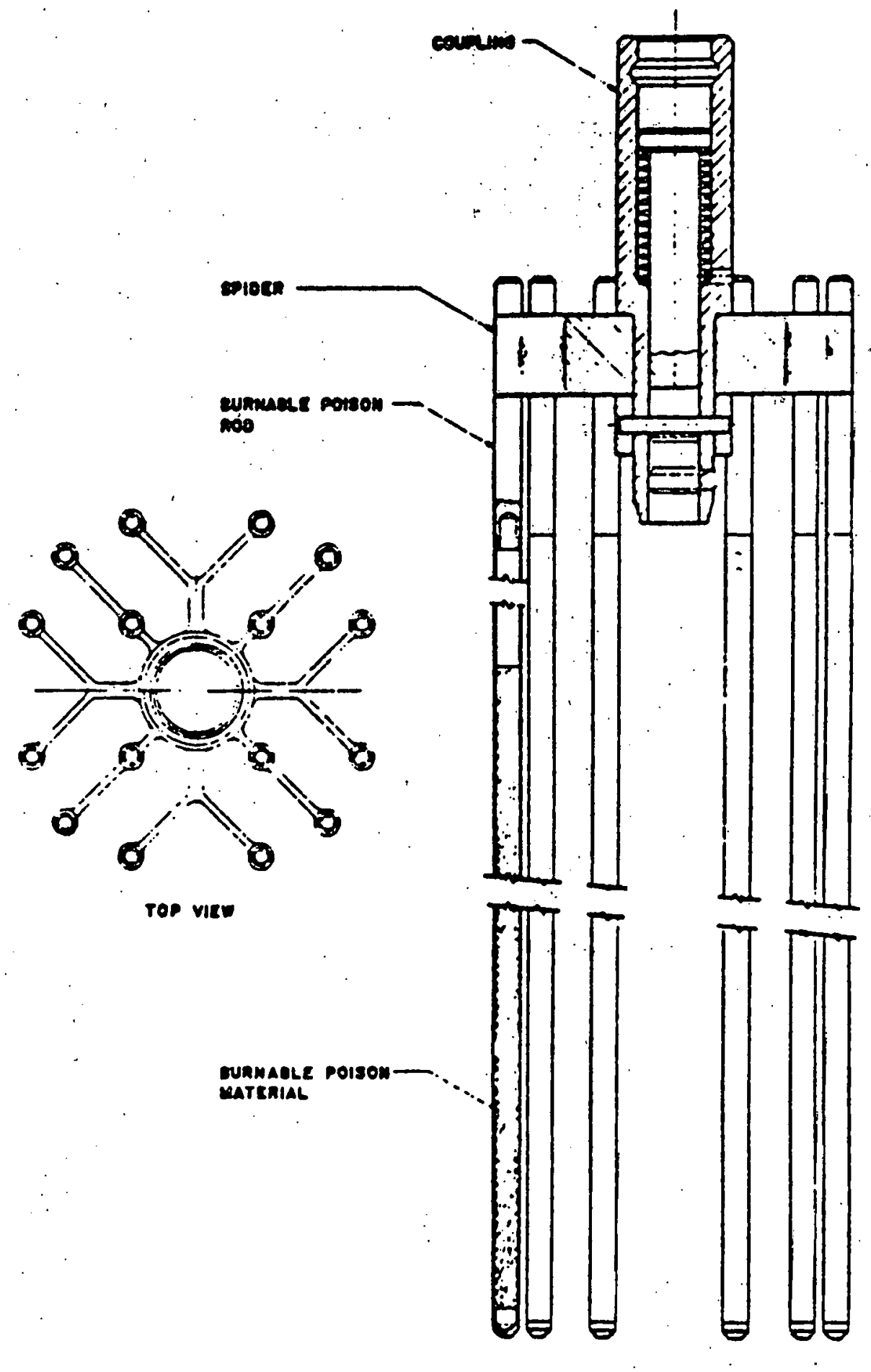

BURNABLE POISON ROD ASSEMBLY

FIGURE $5-3$ 
TABLE 5-2

\section{CORE ISOTOPIC TOTALS - (AT SHUTDOWN - AVERAGE)}

A. Isotope

$\mathrm{U}-235$

$\mathrm{U}-236$

$\mathrm{U}-238$

$\mathrm{Pu}-238$

$\mathrm{Pu}-239$

Pu-240

$\mathrm{Pu}-241$

$\mathrm{Pu}-242$

$\mathrm{Np}-239$

$\mathrm{Np}-239$

B. Element

U.

$\mathrm{Pu}$
Mass (AGNS)

(Kilograms)

1828.8

52.25

79761

0.041

130.02

10.09

1.923

0.059

1.247

6.165

AGNS

Tilograms

81641

142.2
Mass (B\&W)*

(Kilograms)

$\therefore \quad 1825.2$

55.31

80866

.140 .39

10.96

2.323

0.084

6.608

$\frac{\text { B\&W }}{\text { (Kilograms }}$

82746

153.7

C. Uranium Enrichment

$\begin{array}{ll}\text { AGNS } & 2.248 \\ \text { B\&W } & 2.218\end{array}$

D. Percent Pu-240 in Total Plutonium

$\begin{array}{ll}\text { AGNS } & 7.18 \\ \text { B\&W } & 7.18\end{array}$

* Refers to data supplied by B\&W to AGNS in March 1980. 
TABLE 5-3

REGION I FUEL ISOTOPIC CONTRATION - (AT SHUTDOWN)

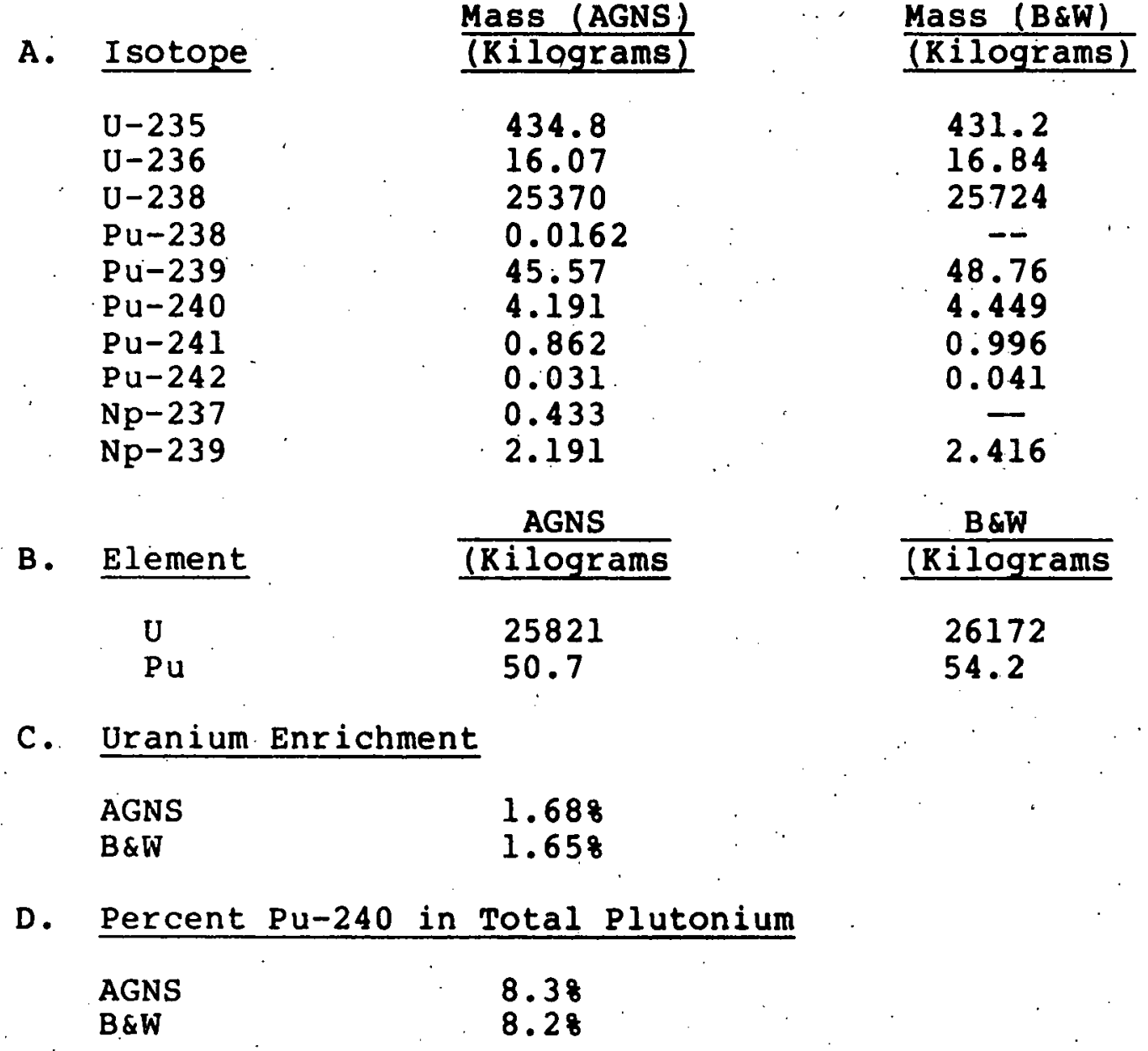




\section{TABLE 5-4}

REGION 2 FUEL ISOTOPIC CONCENTRATION - (AT SHUTDOWN)

A. Isotope

$$
\mathrm{U}-235
$$

$\mathrm{U}-236$

$\mathrm{U}-238$

$\mathrm{Pu}-238$

$\mathrm{Pu}-239$

$\mathrm{Pu}-240$

$\mathrm{Pu}-24]$

$\mathrm{Pu}-242$

$\mathrm{Np}-237$

$\mathrm{Np}-239$

B. Element

U

$\mathrm{Pu}$ $\frac{\text { Mass (AGNS) }}{\text { (Kilograms) }}$

645.5

20.13

27457

0.0167

48.07

3.807

0.753

0.022

0.494

2.062

\section{$\frac{\text { AGNS }}{\text { (Kilograms }}$}

28122

52.7
Mass (B\&W)

(Kilograms)

646.4

21.61

27832

54.04

4.173

0.885

0.030

2.563

$B \& W$

(Kilograms

28500

59.1

C. Uranium Enrichment

$\begin{array}{ll}\text { AGNS } & 2.308 \\ \text { B\&W } & 2.278\end{array}$

D. Percent Pu-240 in Total Plutonium

AGNS 7.28

$B \& W$

7.18 


\section{TABLE 5-5}

REGION 3 FUEL ISOTOPIC CONCENTRATION - (AT SHUTDOWN)

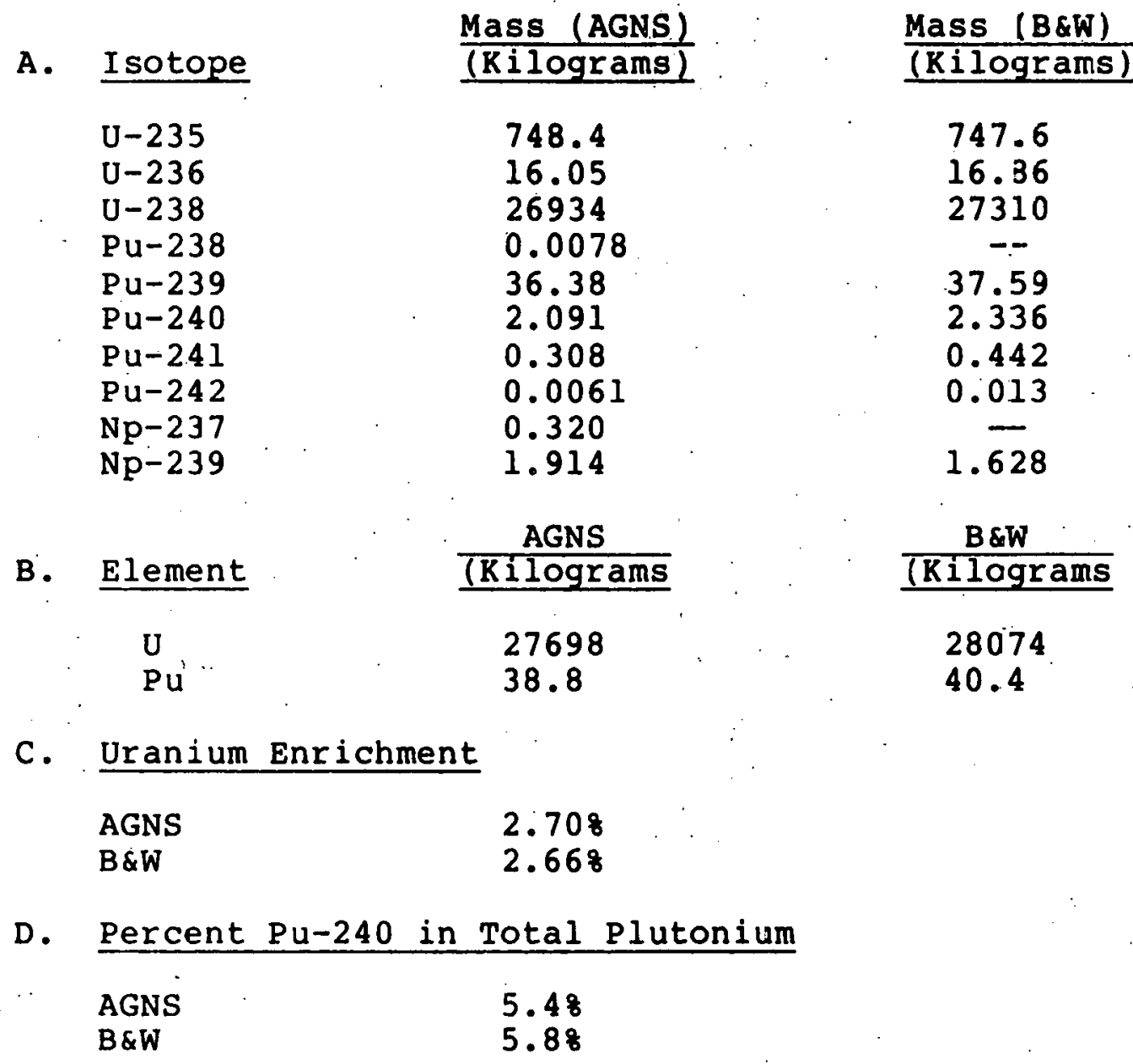


- AGNS-B\&W Agreement - There is reasonably good agreement between the AGNS and 'B\&W evaluations.' Important quantities such as the uranium enrichment and percent Pu-240 in the plutonium are virtually identical. It is noted that the $B \& W$ results show somewhat larger quantities of plutonium. The typical variation is less than 108. As an illustration, the $B \& W$ value for total core plutonium is 153.7 kilograms versus the AGNS value of 142.2 kilograms (see Table 5-2, Part $B$ ). The $B \& W$ values were utilized as the baseline for this core study.

- Uranium Enrichment - The value of the enrichment of U-235 of the existing "burned-up" fuel is 1.658 in Region 1 , 2.278 in Region 2, and 2.668 in Region 3 . The average enrichment is 2.28. The enrichment in Regions 2 and 3 is higher than that allowed in facilities utilized to convert the uranyl nitrate from reprocessing plants to uranium hexafloride (UF). Typical upper limits on enrichment from a criticality standpoint are 1.88 .

- Pu-240 Content - The percent of Pu-240 in plutonium is less than that of usual "reactor-grade" plutonium due to low fuel burnup. The Pu-240 content ranges from 5.88 in Region 3 to 8.28 in Region 1 . In a reprocessing plant, typically, pu-240 limits must be greater than 5 to prevent criticality problems. Also, the Pu-240 content in the 5 to 88 range is in the vicinity of that considered to be weapons-grade. Note that the plutonium content is approximately one-sixth of that expected for full burnup fuel.

\subsubsection{Nuclear and Thermal Data}

An initial assumption was that the fuel would not be removed from the core and canned, preparatory to shipping until mid1982. Current expectations are that this represents at best an early limit. Due to the relatively low burnup of the TMI-2 fuel, it was expected that the nuclear source strength would be relatively low at this time. To corroborate this, an evaluation was made to determine the values after three years or more of aging. Shipping at any later date will result in still lower values of nuclear source strength and decay heat.

The computer code ORIGEN (Reference 5.3) was used for this purpose. The values presented are those for Region 2 of the core which received the highest burnup and, as such, would present the limiting case. Table 5-5 shows the gamma source strengths at selected decay times from one to five years after the shutdown of this core in March 1979. The values presented represent the number for each of twelve specific energy groups. Tables 5-6 and 5-7 are a comparison of the 
TABLE $5 \div 6$

TMI-2 CORE REGION 2 FUEL SOURCE STRENGTH (2)

GAMMAS PER SECOND: PER METRIC TON OF URANIUM AT VARIOUS DECAY TIMES

Mean

Energy

$(\mathrm{MeV})$

0.30

0.63

1.10

1.55

1.99

2.38

2.75

3. 25

3. 70

4.22

4.70

5.25
$1.12+15$

$4.83+15$

$9.99+13$

$2.94+13$

$4.63+13$

$1.68+12$

$1.18+11$

$3.67+9$

3. $77+3$

$2.38+3$

1. $12+3$

$7.08+2$
$4.86+14$

$9.21+14$

$4.69+13$

1. $38+13$

$1.94+13$

8. $32+11$

$5.96+10$

$1.86+9$

$3.61+3$

$2.28+3$

$1.08+3$

$6.78+2$
Decay Time

\begin{tabular}{lll}
$\begin{array}{l}\text { Three } \\
\text { Years }\end{array}$ & $\begin{array}{l}\text { Four } \\
\text { Years }\end{array}$ & $\begin{array}{l}\text { Five } \\
\text { Years }\end{array}$ \\
\hline
\end{tabular}

\section{$2.23+14$}

$6.51+14$

$2.36+13$

$6 \cdot 79+12$

$8.16+12$

$4.14+11$

3. $0: 1+10$

$9.43+8$

$3.57+3$

$2.25+3$

$1.07+3$

$6.71+2$

$1.11+14$
$5.42+14$
$1.27+13$
$3.54+12$
$3.44+12$
$2.06+11$
$1.52+10$
$4.78+8$
$3.56+3$
$2.25+3$
$1.06+3$
$6.69+2$

6. $22+13$

4. $77+14$

$7.47+12$

$1.98+12$

$1.45+12$

$1.03+11$

$7.96+9$

$2.48+8$.

$3.56+3$

$2.24+3$

1. $06+3$

$6.68+2$

NOTES: (1) Earliest shipping date.

(2) Fuel assembly loading $=0.467 \mathrm{MTU}$. 
TABLE 5-7

COMPARISON OF GAMMA SOURCE TERMS -

TMI-2 FUEL VERSUS HIGH BURNUP FUEL

(One Year Decay Time)

(1)

Mean

Energy

(MeV)

0.30

0.63

1.10

1.55

1.99

2.38

2.75

3.25

3.70

4.22

4.70

5.25
(2)

\section{Gammas per second} per Metric Ton

TMI-2 High Burnup Fuel

$1.12+15$

$4.83+15$

$9.99+13$

$2.94+13$

$4.63+13$

$1.68+12$

$1.18+11$

$3.67+9$

$3.77+3$

$2.38+3$

$1.12+3$

$7.08+2$
$5.82+15$

$3.14+16$

$1.22+15$

$4.10+14$

$2.02+14$

$2.09+13$

$1.59+12$

$5.01+10$

$8.42+6$

$5.31+6$

$2.51+6$

$1.58+6$
(4)

Ratio of Column (3) to

Column (2) 
gamma source terms for the low burnup TMI fuel after one year of decay time with those of high burnup fuel $(33,000$ MWd/MTU versus approximately $3200 \mathrm{MWd} / \mathrm{MTU})$. These tables 4 to 15 , below $3.7 \mathrm{MeV}$ (a high energy cutoff). For the high energy gamma groups, the source terms are larger by a factor of 2000. Hence, the total gamma source strength for TMI fuel is considerably lower than fuel of normal burnup.

A similar evaluation was made of the neutron source term in the TMI fuel. This source strength is from spontaneous fissions and $(\alpha, n)$ reactions, at a typical neutron energy level of $5.0 \mathrm{MeV}$. During the period of time of interest, the source strength for the TMI fuel is approximately 1000 times less than that for typical high burnup fuel. The neutron source strength is virtually negligible from a shielding standpoint. This is of importance in recognizing that a separate neutron shield would not be required in a spent fuel cask.

Also of importance is the thermal power of the fuel occurring from decay heat emanating from gamma emission. Again, the computer program ORIGEN was used to determine this thermal power for each of the three core regions as a function of decay time. The resuits shown in Figure 5-4 depict the corresponding thermal power of fuel with high burnup. The decay heat level, after three years of burnup, is approximately an order of magnitude less than that expected for high burnup fuel. The average values for the entire core and for a typical assembly are given below:

- Total core heat load after three years - 30 kilowatts

- Maximum fuel assembly heat load - 190 watts.

These heat load values are approximately two orders of magnitude less than the design values normaliy used for spent fuel shipping or wet pool storage for individual assebmlies. In the normal design case, high burnup fuel and approximately 120 days of fuel aging are assumed. It is not expected that there will be any. thermal limitations associated with either dry storage, dry shipment within a spent fuel cask, or storage in a wet pool. In the latter case, the natural circulation present in the pool should be adequate to assure cooling (if sufficient water volume is present.).

\subsubsection{Damaged Fuel Conditions}

There have been two separate and independent assessments made of the TMI-2 fuel core after the accident itself. These assessments were based on operating records, instrument readings (primarily pressure drops and thermocouple 


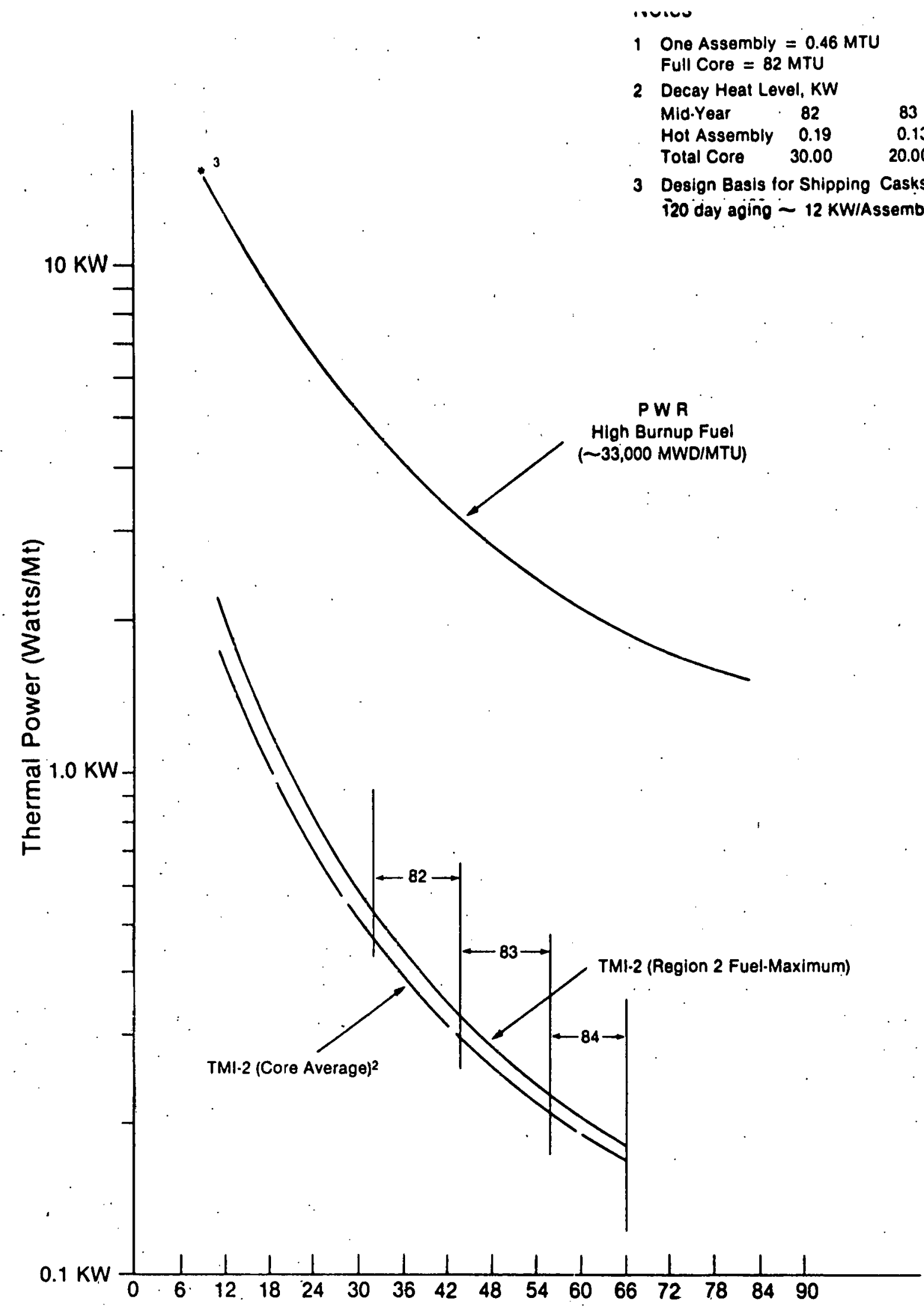

Decay Time (Months)

TMI-2 CORE DECAY HEAT LEVEL ${ }^{1}$

FIGURE 5-4 
evaluations\}, and computerized reconstructions of the accident. These two evaluations represent an optimistic and a pessimistic boundary on the core conditions. The more optimistic evaluation was performed under the auspices of the EPRI-NSAC study team (Reference 5.5); this evaluation focused on most of the major structural damage occurring in the central region of the core and extending approximately one-half of the axial length down into the fuel (this corresponds to the amount of the fuel that was uncovered during the accident). In this study, it was assumed that the outer peripheral fuel assemblies were relatively undamaged from a structural standpoint, with the possible exception of the fission gas plenums. These were assumed to have been breached due to the thermal creep of the cladding. (Note: PWR fuel rods are typically pressurized to 600 to 800 psi with helium during manufacture. Fission gases would migrate from the fuel to the plenum gap during the accident thermal transient. During the accident, the gas pressure in the fuel plenums and axial gaps of the fuel may have exceeded 2000 to $3000 \mathrm{psi.)}$ The more pessimistic study, referred to as the Rogovin report analysis (reference 5.4) predicts major fuel damage and perhaps melting of the fuel cladding itself. The Rogovin study assumed that a sufficient amount of the core reached a temperature where a solid "almost welded" structure may have formed between various fuel components. It was also expected that a portion of the fuel had dropped to the lower regions of the reactor vessel.

Based on these conflicting opinions and lacking observational information, it is obvious that provision and planning must be made for handling both intact fuel and debris (fuel fittings, cladding, $\mathrm{UO}_{2}$ powder, etc.). Provision must also be made for handling or separating fused formations of fuel which are larger in cross section than the fuel assembly. Even with the "intact" assemblies, there will be considerable damage and embrittlement of the cladding. There will probably be some bowing of the individual fuel rods. The failed rod plenum (or some other portion of the fuel) may project out into the flow passage. Fuel handing and packaging techniques must be well thought out to minimize any possible further damage of the fuel. Canning is required to prevent the spread of fuel material to other portions of the containment vessel and pools during handling.

\subsubsection{Criticality Conditions for Fuel Debris}

There is no commercial reactor experience related to loading pieces (debris) of LWR fuel into spent fuel cans. Ultimately, this debris must either be stored within racks in a spent fuel pool or be placed within a can and shipped offsite in a spent fuel cask. The operational safety questions 
relate to the nuclear source strength within the container and the precluding the possibility of criticality. As noted in section 5.2.3, the source strengths will be very low. However, the capability of assessing them prior to actual loading is not possible due to the segregated nature of the fuel.

Criticality concerns are not straightforward. A separate evaluation was made of this question. The analysis evaluated a single, water-filled can approximately 9 inches square in cross section and 15 feet long located in an unborated fuel storage pool. Pool water boration reduces the probability of criticality; however, accommodation for unborated water will probably be required, since off-site storage pools will probably be unborated. Both the fuel geometry and the fuel enrichment are unknown. From the standpoint of geometry, it can be conservatively assumed that the fuel will be in virtually any shape and size corresponding to the can dimension, and that any metal to water ratio may exist within the can. A detailed evaluation of this question requires a series of parametric studies equating various fuel sizes, shapes, and various metal-towater ratios so that a worst case configuration could be established. A study of the literature (Reference 5.6) contains information that is useful for this purpose. specifically, an infinitely tall cylinder 10.6 inches in diameter can be shown to be slightly subcritical containing a heterogeneous oxide fuel at 3.08 enrichment. In this reference case, the $\mathrm{UO}_{2}$ could be in any shape and size, as well as the metal-to-water ratio. The infinite water reflector can be assumed which is the worse case. Using hand techniques, the 10.6-inch diameter was determined to be equivalent from a criticality standpoint to a square cross section approximately 9.4 inches on a side. Evaluations of the can indicate that the can will be fabricated from stainless steel and will have a wall thickness typically between 0.08 and 0.10 inches thick. The effect of the stainless steel wall on reactivity is essentially negligible in this case.

The preceding evaluation was conservatively based on the 3.08 maximum initial enrichment in Region 3 of this core. This upper (no burnup) limit was utilized due to the fact that there are many regions which are blanketed by control rods during the short burnup history. As a result, there are axial regions of the core that would have,experienced very little burnup. Hence, this upper limit is not an unrealistic assumption, particularly in view of potential licensing questions.

The criticality concerns related to the canning of debris are of sufficient importance that it is recommended that 
further analyses be performed. These problems would be minimized to some extent at the TMI-2 site where the pools are borated. However, at unloading facilities and in the licensing of spent fuel casks, a more comprehensive evaluation would be required.

The total uncertainty in the physical status of the TMI-2 core requires that a number of alternative paths be developed for the handling scenarios. These paths could be simplified to a certain extent if initial information and testing techniques were developed early on. Hence, it is recommended that at the earliest practical time a means be developed for obtaining a visual scan of the core. In addition, it is recommended that a measurement tool be developed for determining (in the case of visually intact fuel) structural integrity. A possible tool concept for this purpose is noted as Item 14 Reference 5.7. This tool tests the central guide tube of the fuel assembly and determines the relative strength of the guide tube. This can be used to estimate the axial strength of the assembly.

\subsection{Fuel Handling and Packaging Evaluation}

\subsubsection{General}

The purpose of this specific study is to evaluate the technical aspects of fuel handling within the containment and spent fuel (auxiliary) buildings, and the provisions for packaging the fuel in a form which is suitable for either extended pool storage or for shipment off-site. This study is divided into three specific technical areas which are listed below:

- Handling Interface Evaluation - This involves the physical movement of the fuel or debris from the reactor area and containment building, through the fuel transfer tunnel, to the pool areas in the auxiliary building. It includes discussion of temporary storage in storage racks and the isolation of the contaminated from the noncontaminated during handling operations. It also includes a preliminary evaluation of the loading and unloading of the fuel package from the spent fuel cask at a receiving facility.

- Canister Design - This is an evaluation of the canning requirements for both "intact" fuel and debris. It also includes a discussion of canning techniques. A considerable number of interfaces exist between handling requirements for on-site movement of the intact fuel and debris handling. The can must function as a containment boundary for use with the shipping cask and also permit long-term, on-site storage in the TMI pools: 
Scoping of Operational procedures - Included is a preliminary evaluation of the types of techniques and operational factors which must be examined prior to the removal of the fuel from the core. Included in this are the transport of the fuel from the core. Included in this are the transport of fuel between buildings and the canning operations. These procedures are defined in sufficient detail to allow a preliminary evaluation of potential hazard areas to be developed.

\subsubsection{Fuel Handling Interface Evaluation}

\subsubsection{Handling Operations at the Reactor}

\section{(1) Fuel Hoisting (Removal from the Reactor Fuel Basket)}

If the fuel bow or twist is excessive (typically $1 / 4$ inch), the fuel handing bridges probably cannot be utilized without modification. Also, if the structural integrity of the assemblies is not adequate for vertical lift with the normal top-end grappling, a different grapply system must be provided on these units. The fuel assembly must be provided with additional structure of verified integrity. (This additional structure could reduce the bow and twist allowance discussed above.) The auxiliary crane units also provide little flexibility for grappling and for working around mechanical lifting interferences that could develop from distortion of the fuel. Obviously, the standard lifting gear cannot be used to lift debris.

If the fuel handling bridges in the Fuel Transfer Pool (FTP) and the Fuel Storage pools (FSP) cannot be utilized (see Figure 5-5 which is schematic of the three pools), auxiliary service hoist trolleys to replace the existing trolleys and designed to accommodate potentially damaged fuel handling are an acceptable alternate. These crane hoists should be of the hook and cable type versus telescoping tubes in order to provide great hoisting flexibility and to accommodate tooling to fit between the hook and the fuel assembly.

Another alternative for hoisting an intact assembly would be the use of the existing overhead cranes with modified auxiliary hoist control. Special systems would be required to ensure adequate water cover over fuel at all times (interlocki and hoisting control flexibility such as extended. slow speed operation, small vertical and horizontal positioning tolerances, and overload sensing capabilities. However, auxiliary service hoist trolleys installed on the fuel handling bridge would decouple existing crane functions and fuel handling. An auxiliary hoist trolley should better 


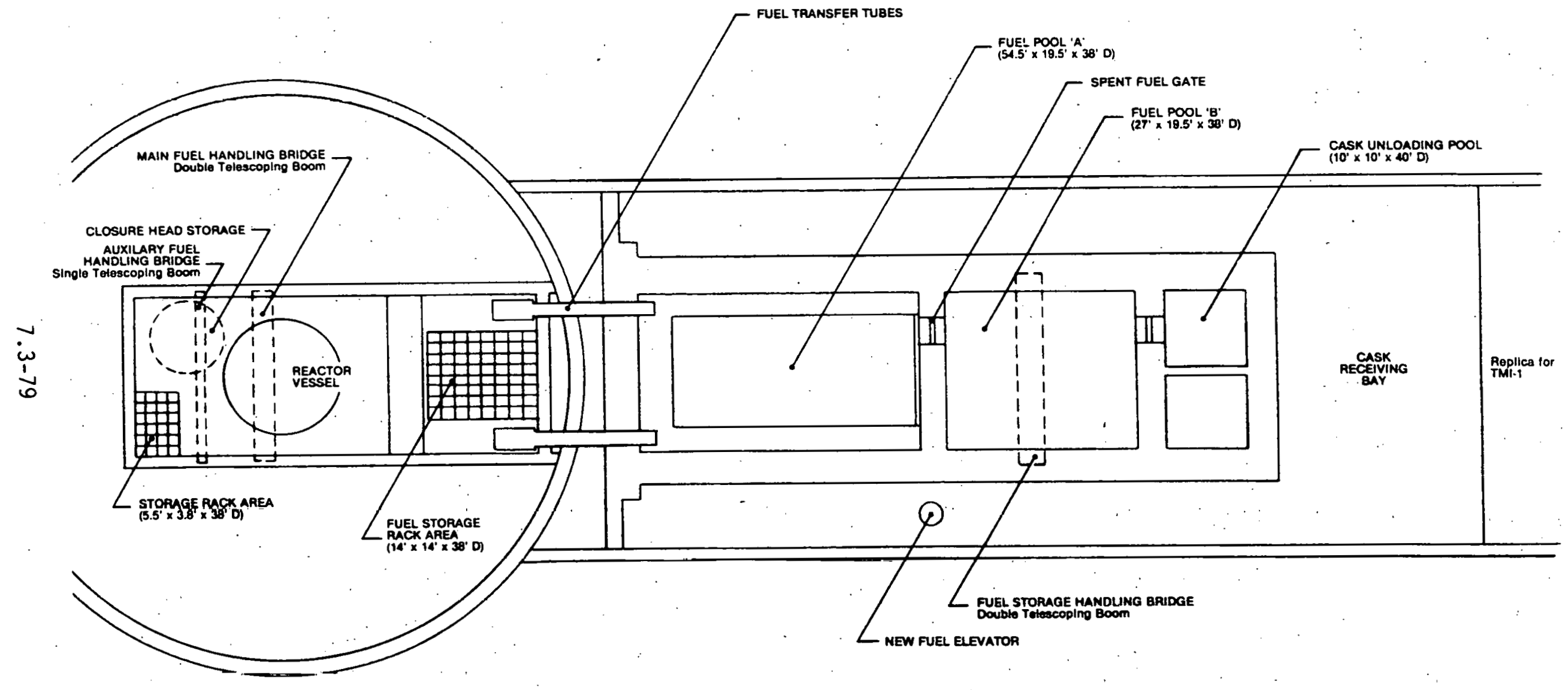

BUILDING FLOOR LAYOUT

FIGURE $5-5$ 
accommodate the anticipated manual operations in the defueling operation.

Communications with GPU personnel (Reference 5.9) indicate that operating experience with these existing fuel handiing units by itself would suggest alternative fuel hoisting end fitting distortion, fuel could be packaged and the paskages handled by intermediate hoisting tools suspended from crane hooks. Final engagement should allow for manual positioning corrections. Hoisting and translation should be preceded by visual verification of a positive engagement of the fuel assembly being grappled.

(2) Fuel Transfer Carriage/Upender (Movement Between the Containment and the Auxiliary Building)

An evaluation of the interfaces between packaged fuel assemblies and the fuel transfer carriage basket was performed by reference to Babcock and Wilcox drawings of the fuel transfer system: (Reference 5.9).

$$
\begin{aligned}
& 44-54-009-03 \\
& 44-54-011-02 \\
& 44-54-017-08 \\
& 44-54-040-30
\end{aligned}
$$

The evaluation was used to develop any limiting or potential dimensional interfaces such as the maximum canister length or largest allowable cross section.

The maximum packaged fuel length that could be accommodated by the carriage basket was investigated. Assuming the stop assembly (Ițem 1170, 44-54-011-02) could be relocated, the limiting interference would be the flange of the transfer tube (Item 3367, 44-54-040-30) on the reactor side. Use of this maximum length might require removal of the pool emergency cable system (Item 0022, 44-54-017-08), but insufficient detail was available to say with certainty. The maximum; uninterfered length would be 15 feet $1 / 8$ inch. The maximum square cross section that could be contained within the 15.25-inch octagonal inside diameter of the basket would be 11.48 inches. However, since the "worstcase" criticality (square) cross section has been calculated at 9.4 inches (see Section 5.2.6), this 11.48-inch dimension is not 1 imiting and is noted for reference purposes. Therefore, a square fuel assembly structural packaging cavity should not exceed 9.4 inches. Assuming the 9.4-inch dimension (13.3-inch diagonal) is limiting, it appears, so far as the basket is concerned, that cylindrical packaging of the fuel for containment purposes (already within a structural package) could be performed in the fuel transfer 
pool and still utilize the carriage for transfer to the fuel storage pool. (See Figure 5-5).

The required structural capacity of the unit was qualitatively evaluated. The added weight of providing the packaging for the fuel assembly, as discussed above and in detail later, would not increase the fuel-plus-control-rod

packaging for the fuel assembly, as discussed above and in detail later, would not increase the fuel-plus-control-rod weight of 1700 pounds (which the carriage routinely accommodates) by more than 358. For the present study, it was assumed that conservatism in the structural design of the unit could accommodate this, but this should be verified when the packaging system design is finalized.

(3) Fuel storage Rack (Storage in the Auxiliary Building Pool)

Specially designed packaged fuel racks will be required for the failed fuel (Burns and Roe Drawing W.0. 2555/2066 R.15). Typically, for seismic reasons, racks are limited to $3 / 8$ to $1 / 2$ inch maximum cross-sectional clearance between the fiel and the rack. In this case, the packaged, damaged fuel would not be expected to fit existing slots in the Burns and Roe design. However, new racks could be designed to accommodate any packaging systems envisioned using state-of-treart technology.

(4) Storage Pool Isolation (Prevention of Contamination Between Pools)

Contamination control could be a significant challenge during the defueling and canning operations. Bour areas could be potentially isolated from each other (see Figure 5-5). They are the following:

- Fuel Transfer Pool (FTP)

- Spent Fuel Storage Pool A (FSP A)

- Spent Fuel Storage Pool B (FSP B)

- Cask Loading Pool (CLP).

However, from preliminary evaluation, it appears that the FTP and FSP A would not be easily isolated during defueling. The potential to isolate the FTP and FSP A from FSP B and FSP B from the CLP appeared more feasible. These isolations could be accomplished by a module canal lock system installed in the area where the isolation gates now fit (see Figure 5-5). The locks would permit isolation and transfers; whereas, the existing gates permit only one of those functions. The locks would be relative low volume, temporary water barriers that could be isolated via gate closings 
and piped for multiple water inventory changeouts when so isolated. The changeouts would affect decontamination. Mechanical or chemical decontamination capabilities could also be added to the lock systems. One-tenth decontamination factors at each of the two envisioned locks would provide a hundred-fold decontamination factor for the packaged fuel and, as a result, would prevent excessive contamination of the shipping cask exterior during loading.

With this two-lock system, there is only provision for structural packaging. This should be done to the fuel in the higher background area of the FTP and all contamination containment packaging should be done in FSP A.

Our evaluation indicates that the above isolation/ decontamination concept would be compatible with the existing water cleanup/cooling systems of the reactor facility. However, temporary submerged demineralizer systems in these pools might be preferred to the facility systems based on radiation exposure experience associated with use of these units as reported by GPU. (Reference 5.0).

\subsubsection{Fuel Packaging and Spent Fuel Cask Interfaces}

\section{Cask Evaluations}

Interface evaluations with the three candidate truck casks are more easily visualized by reference to Figure 5-6. Obviously, the NLI $1 / 2$ cask is most restrictive with its smaller 13.375-inch diameter. The fuel cavity length could be increased to 176.37 inches using elliptical heads (an unlicensed modification) as envisioned for pressure integrity of the containment packaging if canning considerations mandate extra length. Scaled and more detailed drawings of these casks are shown in section 5.4. The Fort st. Vrain cask with its larger cavity offers the most flexibility in this regard.

As explained in Section 5.4, various considerations with rail casks cloud consideration of their use in defueling. However, a large rail cask could conceivably be utilized as a "bucket" to move fused pieces of debris directly through the containment hatch and outside to the auxiliary building. In addition, a special handling cask with inside cavity diameters in excess of 40 to 45 inches could be developed for the same function. A cask such as this would weigh in excess of 100 tons. Care in moving these casks near safetyrelated items must be evaluated while in the containment building. 


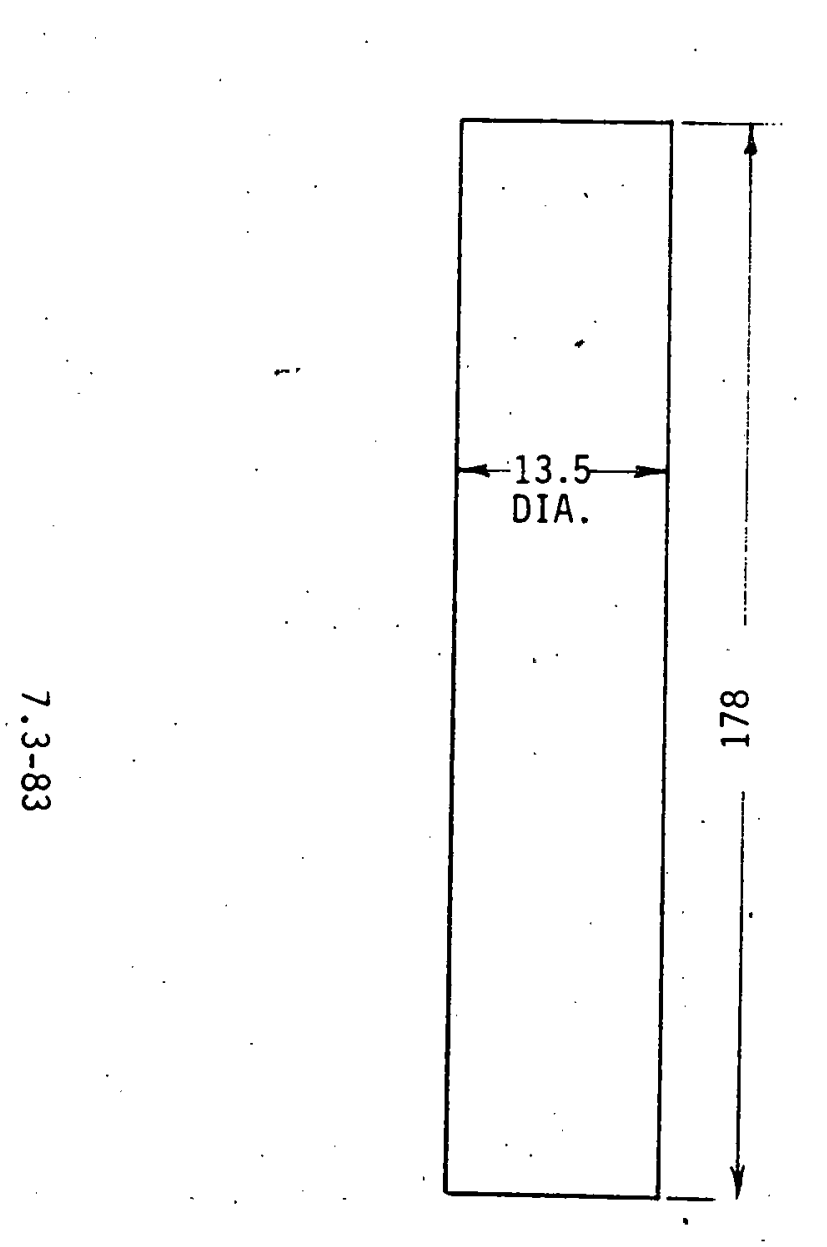

NUCLEAR ASSURANCE CORP.

(NAC-1)

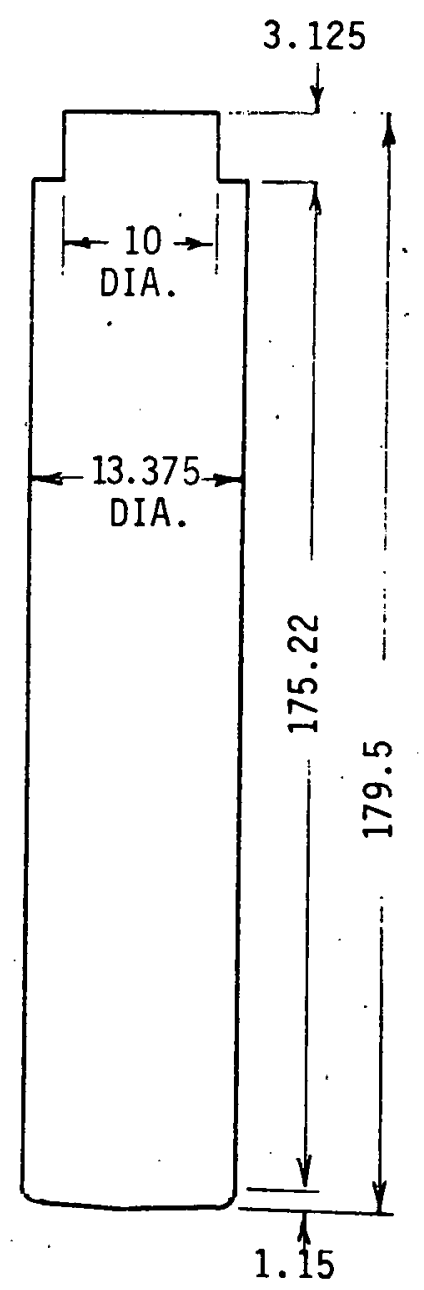

NL INDUSTRIES

(NLI-1/2)

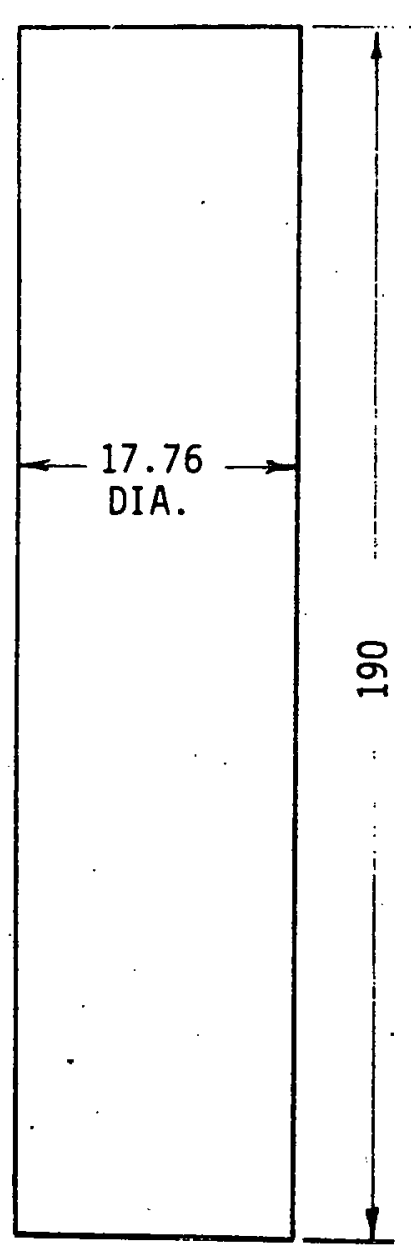

FT. ST. VRAIN (FSiV)

TRUCK CASK CAVITY DIMENSIONS (INCHES)

FIGURE 5-6 


\subsubsection{Off-Site Receiving Facility Considerations}

A generic interface evaluation of the packaged fuel with an off-site receiving facility was made. The receiving facility could be assumed to provide some of the following furctions for the fuel:

(1) Analysis of Fuel Condition

(2) Fuel Storage

(3) Chemical Reprocessing

(4) Ultimate Disposal as Waste.

The facility must be able to receive and unload the shipping cask. As a result, the design of the fuel packaging system should be small enough to minimize hindrance with implementation of the above four functions and to permit the cask receipt to be made. The following minimal package criteria were established. The packaging system should:

- Frovide pressure retaining capability and contamination control

- Provide internal sampling capability to the package

- Fermit a coarse "dryout" of a wet container and alternately a water filling via a fill and drain system.

A pressure retaining cylindrical can with a welded end cap providing vent, fill, and drain capability for the enclosed fuel would meet the first three requirements. It would not accommodate these possible requirements:

- To provide an alternative for remote mechanical wet or dry unpackaging

- To provide alternative can cross sections to preclude mandatory repackaging for more efficient storage, or to accommodate handling preparatory to reprocessing, or for hot cell analysis and examinations.

Space and equipment limitation present at potential receiving facilities could require mechanical uncapping and/or packaging that opens or disassembles mechanically to expose the iuel. For example, a research facility that must unpackage may not have remote, metal-cutting equipment. Also, it is probably more likely that the facility lacks the room or has insufficient size (typically a length equal to twice the can length is needed to permit axial unpackaging). Here, a specially designed can that parted on an axial centerline would be desirable. However, this design would compromise the degree of containment integrity provided by 
the welded cap on the cylindrical can. This can could be placed within the storage can.

Reprocessing and storage facilities which can handle cans with square cross sections could also experience difficulty if cylindrical cans were employed. However, for storage facilities complete interface evaluations would be required on a site specific basis. Interface items to be addressed for these proposed facilities would include among other things:

- Dimensional suitability

- Structural and seismic integrity

- Criticality evaluation

- Thermal environment

- Corrosion environment.

Since facilities selection is not feasible at this stage of the study, the first three requirements for the can will be assumed controlling. In any case, the handling flexibility desired in the packaging system must be decided before the design of the package is finalized.

5.3.3 Preliminary Canning Design

5.3.3.1 Introduction

Any TMI-2 fuel for which there is doubt concerning structural adequacy for lifting should be provided structural integrity for further handling. Augmentation of the axial structural strength of the fuel should be provided as soon as feasible during the defueling operation. One possible concept is the use of a handling shroud to provide structural integrity. This shroud would be a thin-walled can which fits around and holds or "girdles" the assembly somewhat like a "second skin." Variations in the design of the shroud could accommodate a spectrum of fuel damage. For the present considerations, confinement of contamination is not possible except to preclude further structural failure. However, if it is necessary to remove debris, an underwater vacuum system could be used to provide an alternative for removing the fuel from the reactor and packaging the fuel pieces.

Contamination confinement is an obvious final requirement of the packaging system. To provide this function, a second packaging unit would be provided over the shroud. It is referred to in this report as the "can."

A common feature of any packaging system design is the following material and bail features. The material must be compatible with both dry and wet potential storage and 
shipping environments. Notably, the TMI pools are borated whereas many pools utilize demineralized water. Austenitic stainless steel (e.g., 304 or 316 stainless steel) has historically served well in similar applications and will be assumed in all following designs.

Quastions could arise regarding stress-corrosion cracking of these austenitic stainless steels in the presence of caustics and chlorides. Caustic could appear from the combination of water and rubidium, the decay product of Kripton 85. Chlorides could appear from contamination of the water systems by chloride-containing substances inadvertently introduced to the pool water. However, both mechanisms are dependent on stress, temperature, and concentration. In addition, packaging systems would have to be exposed to temperatures in excess of about $120^{\circ} \mathrm{F}$ regardless of concentration for either contaminate to be of concer $n$ in this context. This is not anticipated due to the low therma- heat load of the fuel. If higher temperatures must be considered, then quantifying the concentrations is necessary to determine if the combinations of temperature and concentration would require stress-relieving of welds or other accommodations. The level of oxygen, chlorides, or other pool contaminants would influence pitting concerns that could suggest consideration of titanium or other corrosionresistant ailoys for the packaging system. However, until chloride levels are shown to be a problem, the austenitic stainless steels would be preferred in light of the lower cost.

The design of the handling bail for the can should allow simple engagement of a tool that links the crane hook. This tool would permit avoidance of submergence (i.e., contamination) of crane hook block and cables while maintaining the required water cover (typically 8 feet) over the fuel. It also would provide the opportunity to design both manual positioning and visually verified engagement capabilities into the tooling. Finally, bail design should minimize the can axial length due to the limited spent fuel cask cavity lergths (see Figure 5-6) that packaging units must eventually fit within. Common to all packaging designs are the dimensional constraint of the various candidate casks that could be used to ship the fuel. Various options are presented in Table 5-8. Figure 5-7 clarifies the dimensions listed in the table.

The required clearance between concentric cans (if double containment is desired) are expected to be approximately $1 / 8$ j.nch to 1./4 inch total. This range is based on the fabrication tolerance and operating experience with spent fue racks and casks. Listed wall thicknesses are based on structural calculations that were used to size minimum 


\section{TABLE $5-8$}

\section{SELECTED PACKAGING SYSTEM DIMENSIONS FOR CANDIDATE TRUCK CASK (IN INCHES)}

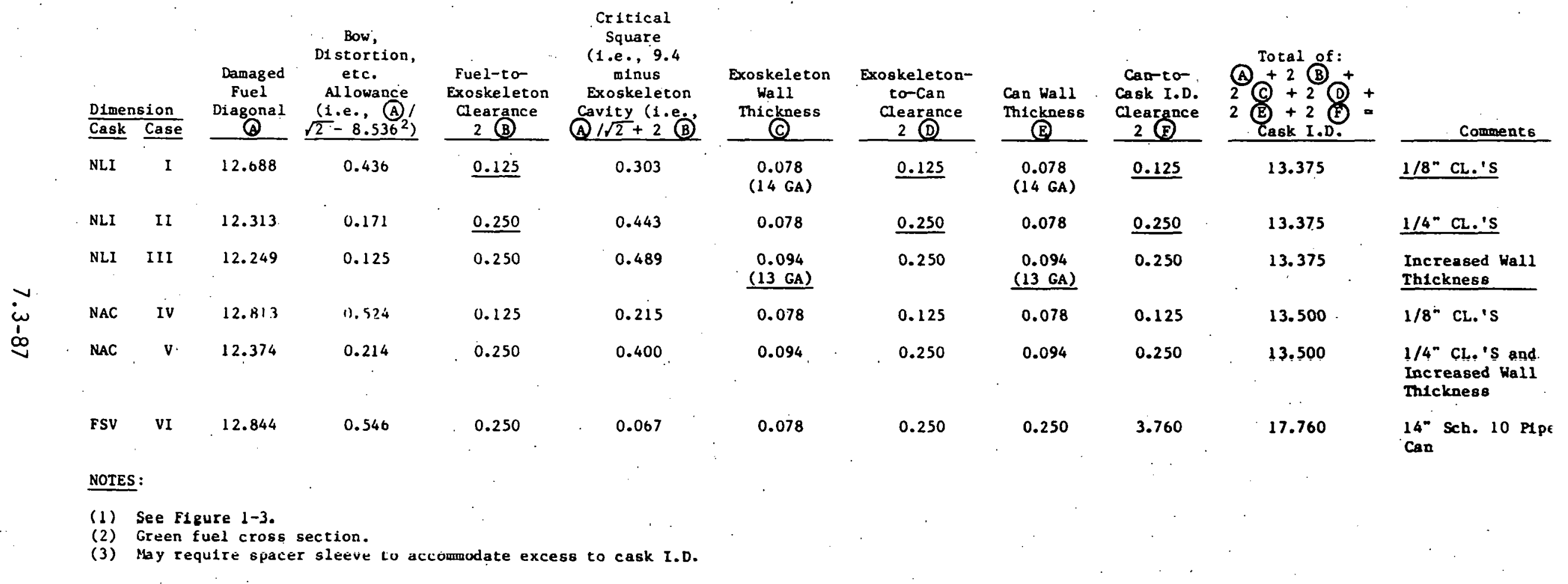




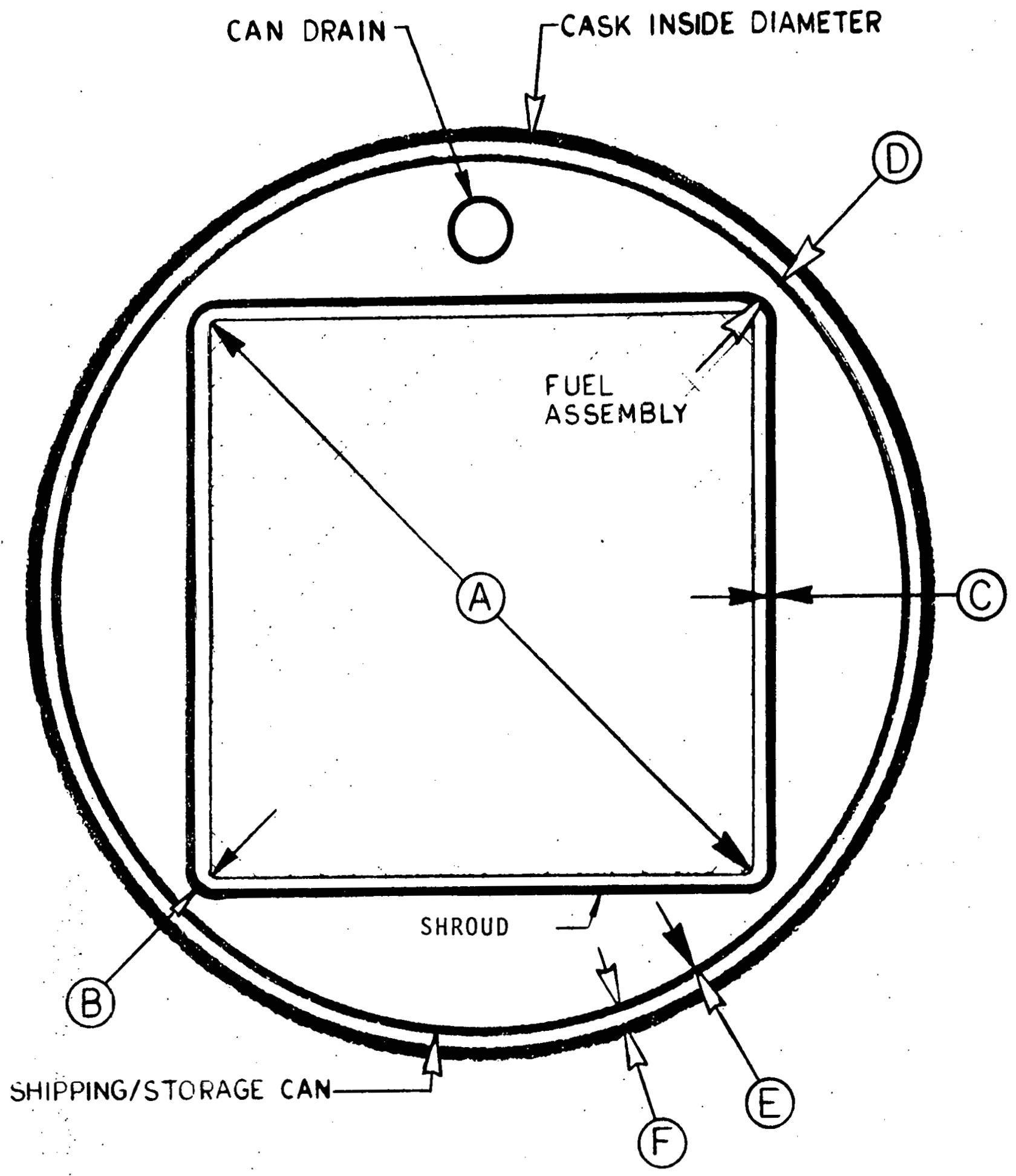

PACKAGING DIMENSIONAL INTERFACES

FIGURE $5-7$ 
values. These were necessary to provide reasonable fits within the smaller cavities. However, fabrication and handling difficulties using these minimum wall thicknesses could preclude their use. Development and demonstration of candidate systems would resolve these concerns.

Resulting fuel distortion allowances (column 2) were derived by subtracting the sum of these clearances and thicknesses from internal cavity dimensions of the candidate casks. More disposition definition should be developed before either selecting from these alternatives or creating additional ones. Note that column 4 shows that all of the cavity diameters are critically safe.

\subsubsection{Handling Intact Fuel}

An optimistic scenario wherein (some of the fuel is intact and capable of handling) must be anticipated. This fuel is probably located at the outer periphery of the core. As a worst case situation regarding packaging system design, the accident could have rendered the nonfuel components (i.e., control rod assemblies, axial power shaping rod (see Figures 5-2 and 5-3) as a fixed component of the fuel assembly. Therefore, the "design" fuel for this case would be as follows:

\section{NOTES}

- Normal Length: $1655 / 8$ "

- Maximum Length: $171^{1 "}$

- Normal Cross Section: 8.536"

- Maximum Cross Section: 9.4-9.5". (estimate)

Includes allowances for irradiation growth, thermal expansion at $212^{\circ} \mathrm{F}$, and added length of nonfuel bearing component.

Maximums that could be accommodated by candidate casks are shown in Table 5-7.

- Weight: 1682 lbs.

Narmal green fuel plus control rod assembly.

The fuel canister design concept was influenced by anticipated methods of lifting the damaged fuel. These lifting techniques include:

- Grappling of fuel via empty instrumentation tube(s) below the suspected structural damage level.

- Grappling of fuel assembly via side access below the suspected structural damage level li.e., after removal of neighboring assembly(ies)]. 


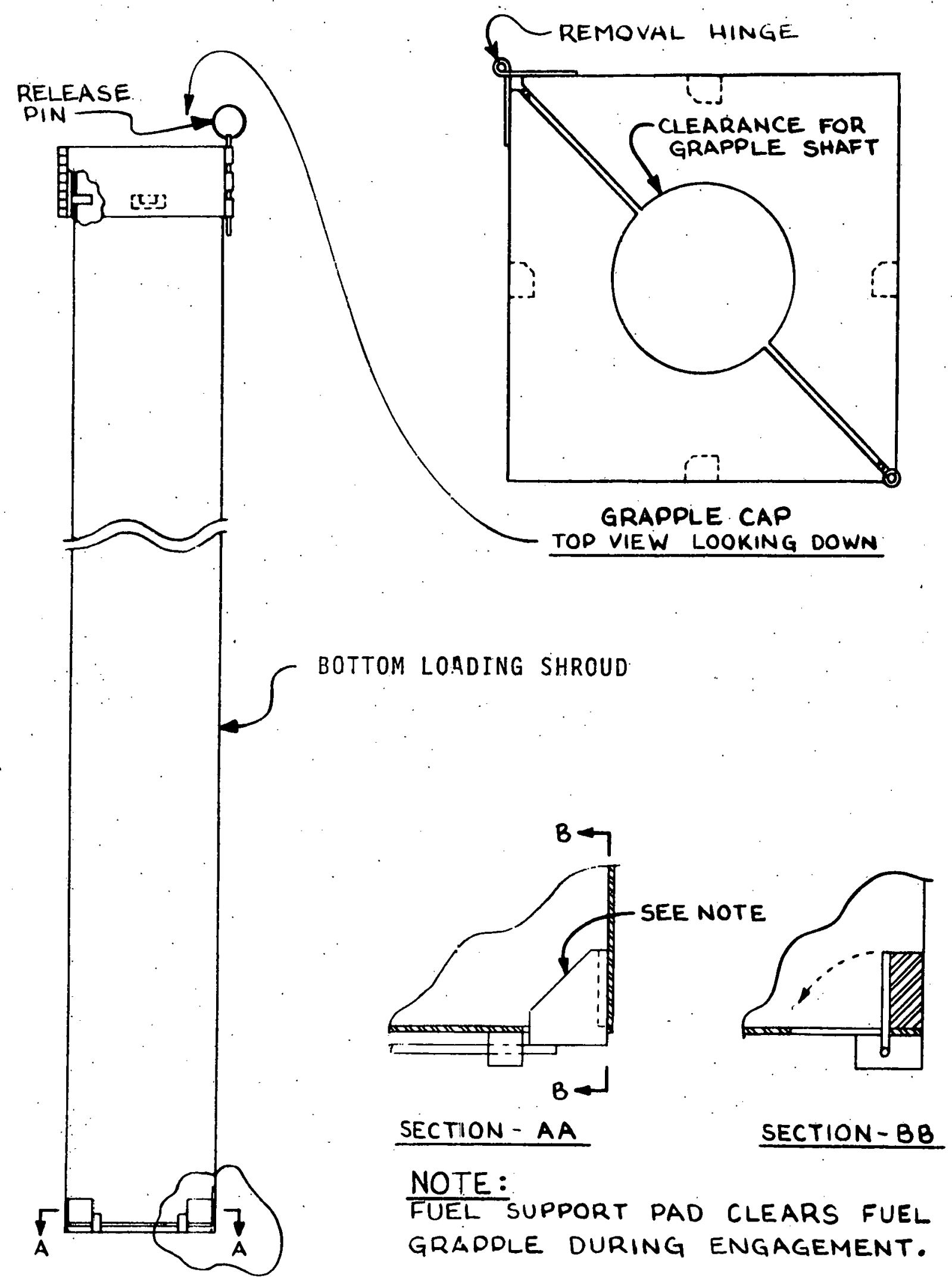

BOTTOM LOADING SHROUD CONCEPT

FIGURE 5-8 
A handling shroud concept shown in Figure 5-8 would accommodate the first two methods. The shroud would be "bottom loaded." Once the fuel was lifted completely, the pads (see Sections $A A$ and $B B$ of Figure 5-8) would drop down and could support the fuel if needed. The need would arise if axial failures of the fuel occurred during further handling.

The intended handling sequence is shown in Figure 5-9. The grapple head would be placed within the shroud. The grapple cap would be secured and the grapple would move the shroud into its holder for loading. This would permit the grapple to lower and to engage the fuel. Meanwhile, the shroud is being supported by the holder. Sequence (1) to (3) shows shroud loading.

It might be desirable to let a portion of the grapple remain with the fuel. Th.is would require a disconnect designed into the grapple. But, at this stage of the study, a reusable grapple was assumed. The next step would require setdown of the shroud. Sequence (4) of Figure 5-9 shows the shroud after setdown in the fuel transfer carriage basket. In sequence (4), the grapple cap is shown being removed, thus freeing the grapple which would likewise be removed. The grapple cap detail can be seen in Figure 5-10 showing the release pin and removal hinge. The handing cap (Figure 5-10) is then installed (sequence (5)). Note the screwed retaining clip shown on the left side of section $A$, Figure 5-8. This permits remote mechanical unpackaging at a receiving facility, if required, by removal of a screw. Also, in this figure, note that the design of the bail would minimize the shroud's axial length and permit visual verification of the handling tool engagement from above. Finally, in Figure 5-9, Sequence (6), the fuel shroud is shown after being loaded into a can and ready for shipment.

A side access shroud which would accommodate the fuel lifting method for badly distorted fuel as shown in Figure 5-11. The fuel would be side loaded into the $90^{\circ}$ half-section shown on the left. A holding frame would incline this section to ensure that the fuel did not topple when released from the grapple that removed it from the core. With the grapple clear, the mating half (on right) would be positioned. Figure 5-12 shows the fuel loaded in the shroud. Note that end overlaps would prevent lengthwise displacement and resulting disengagement of the halves. Then the springloaded latches would be remotely set. This would mechanically lock the halves together.

The preceding procedure is conceptual in nature. If possible, it is desirable to combine all of the canning operations and to have one basic canning procedure and can 


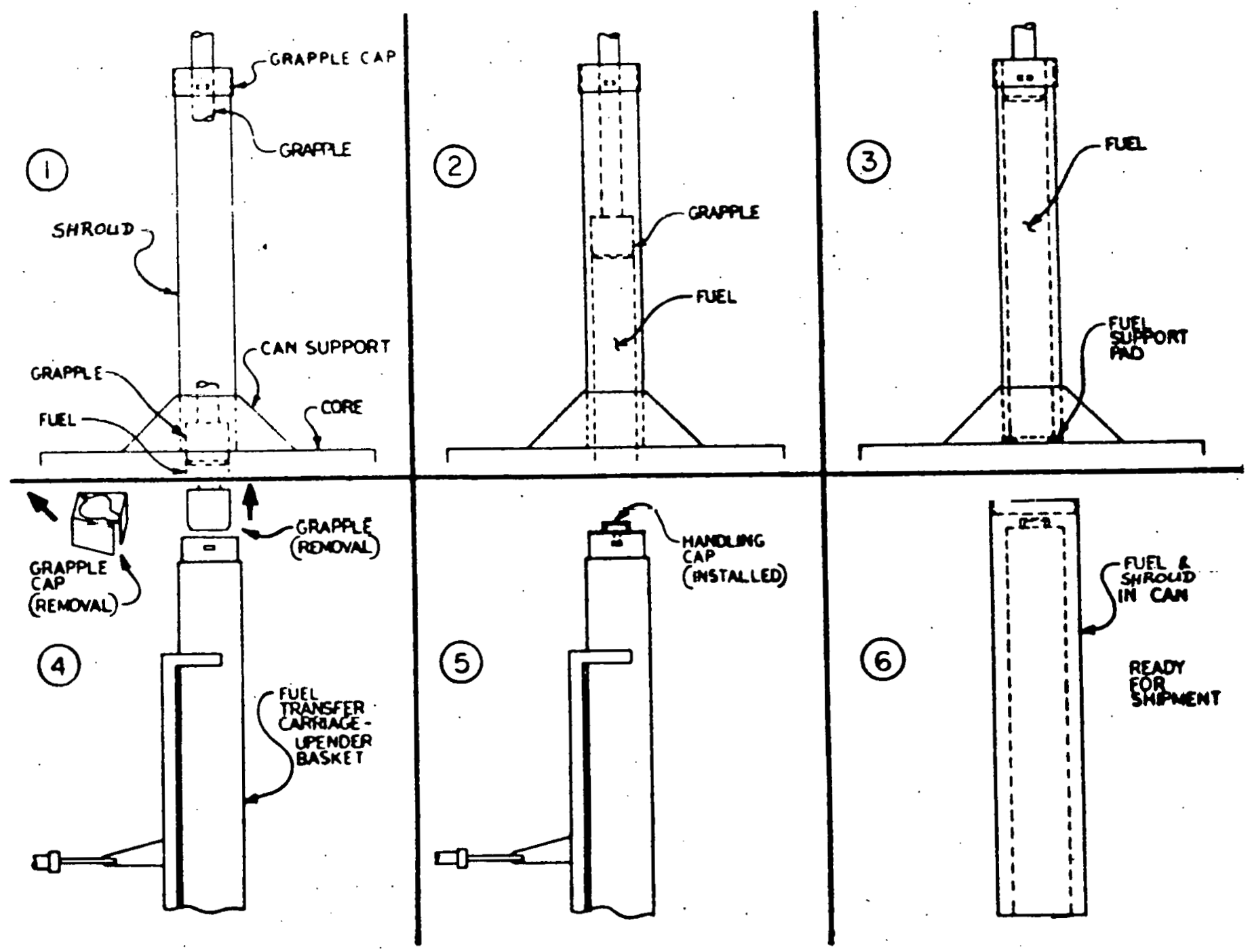

SHROUD BOTTOM-LOADING SEQUENCE

FIGURE 5-9 

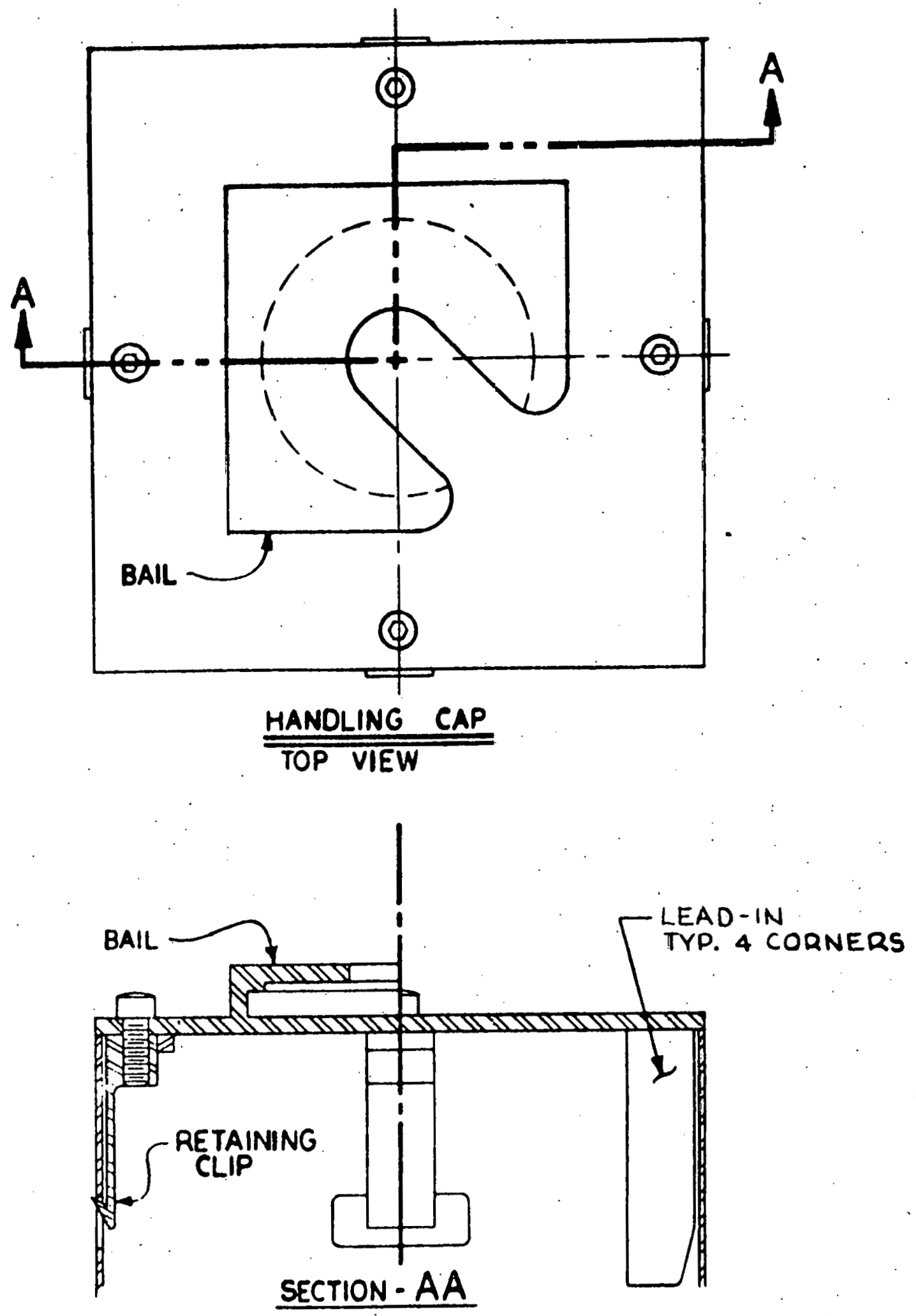

GRAPPLING CAP DETAIL

FIGURE $5-10$ 


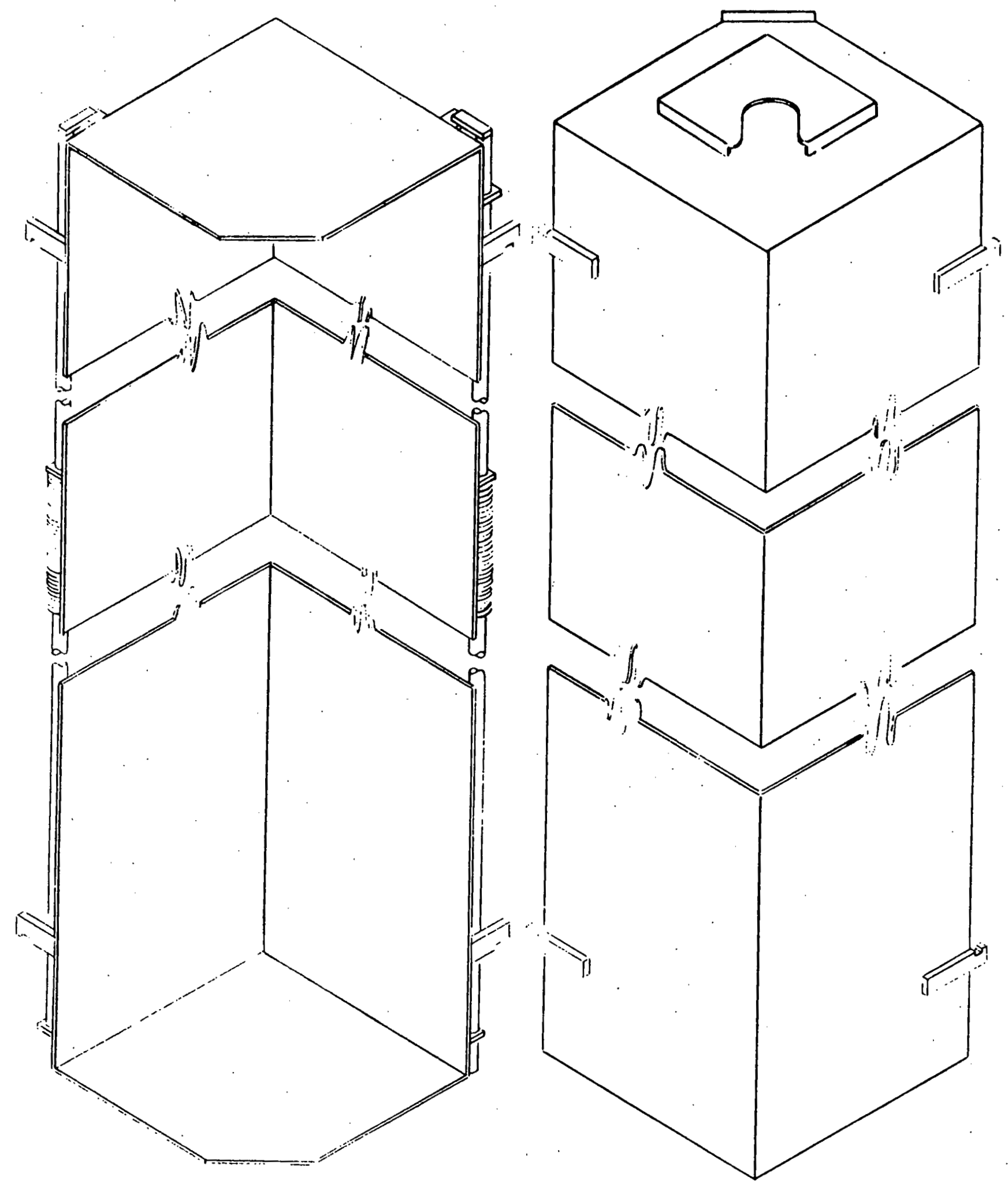

SIDE LOADING - FUEL HANDLING SHROUD (CONCEPT)

FIGURE 5-11 

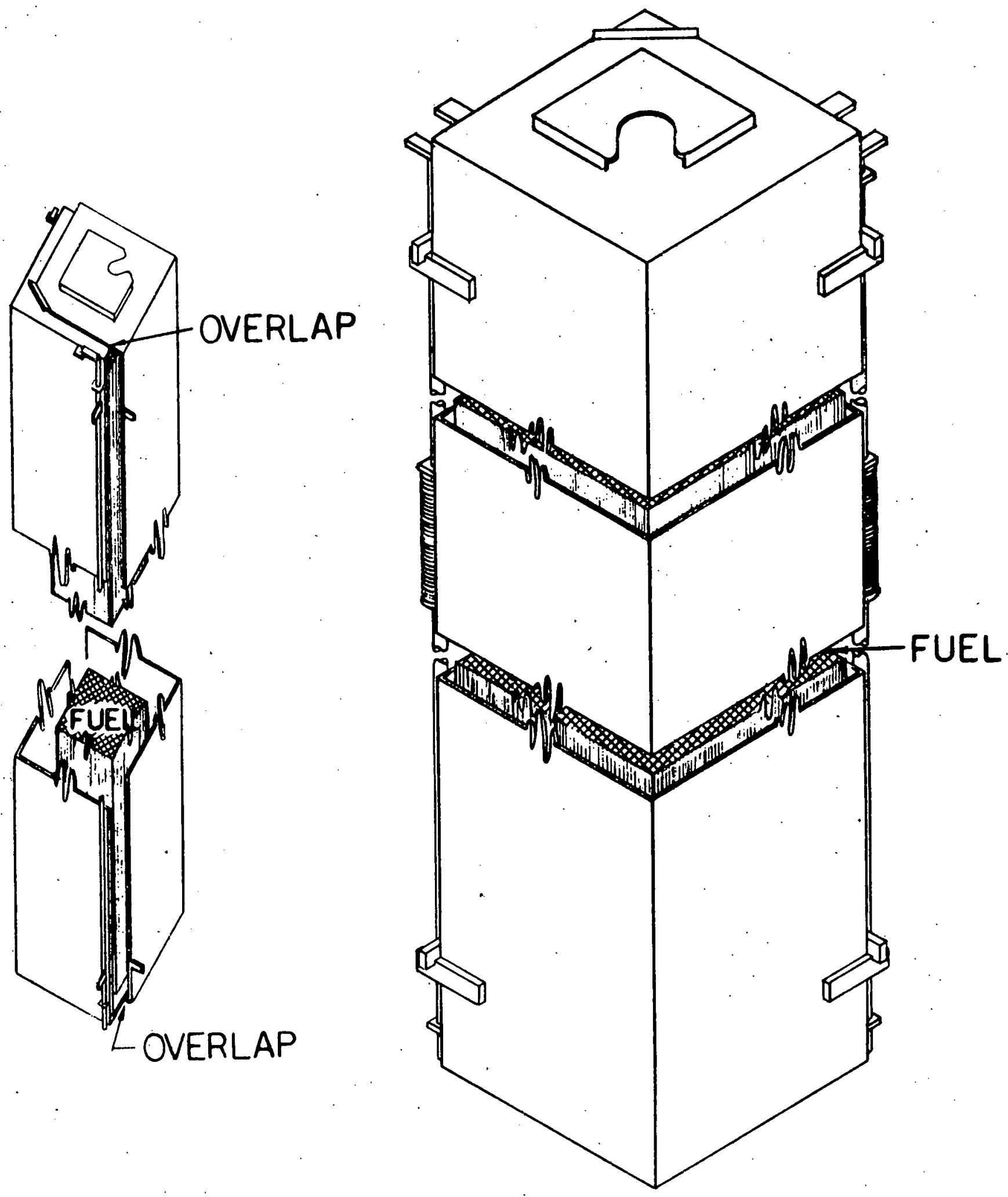

LOADED (TWO PART) HANDLING SHROUD CONCEPT

FIGURE $5-12$

$7.3-95$ 
design. This canning operation would logically be combined with the core defueling to minimize handing.

\subsubsection{Handling Debris}

Some of the fuel may be disintegrated to the point of debris. This debris might consist of:

- $\mathrm{UO}_{2}$ pellets

- cladding

- $\mathrm{UO}_{2}$ powder

- Cladding fines

- Other fuel hardware fragmenzs in a size spectrum ranging from visually discernible to fine particulate.

Debris would probably be located in the upper, central core region, or on the reactor vessel bottom (see Figure 5-13). Frozen core sections are not directly addressed but some form of remote sectioning wou:d be assumed to render them dimensionally equivalent to either intact fuel or debris.

The first steps of the approach to package debris would possibly be to utilize remote underwater tools (hook and tongs). They would be used to manually segregate and free, large-scale fragments into cans for collection. Once the larger fragments were cleared, an underwater vacuum debris system would be placed on the core periphery (see Figure 5-14). Alternatively, the vacuuming of fines could be performed prior to removing large fuel component pieces. The vacuum system would be operated remotely and should include a:

- Vacuum

- Remotely positioned vacuum intake hose

- Settling pot and filtering pot(s) with replaceable element( $s$ )

- Discharge hose or pipe and other interconnecting hose or pipe.

The settling pot would serve as a coarse filter. By utilizing turbulence in the settling region and centrifugal action provided by baffling, it would disentrain the coarser debris. Determination of the number of pots required would require better definition or estimation of the particle size distribution (this is a recommended development area). The 


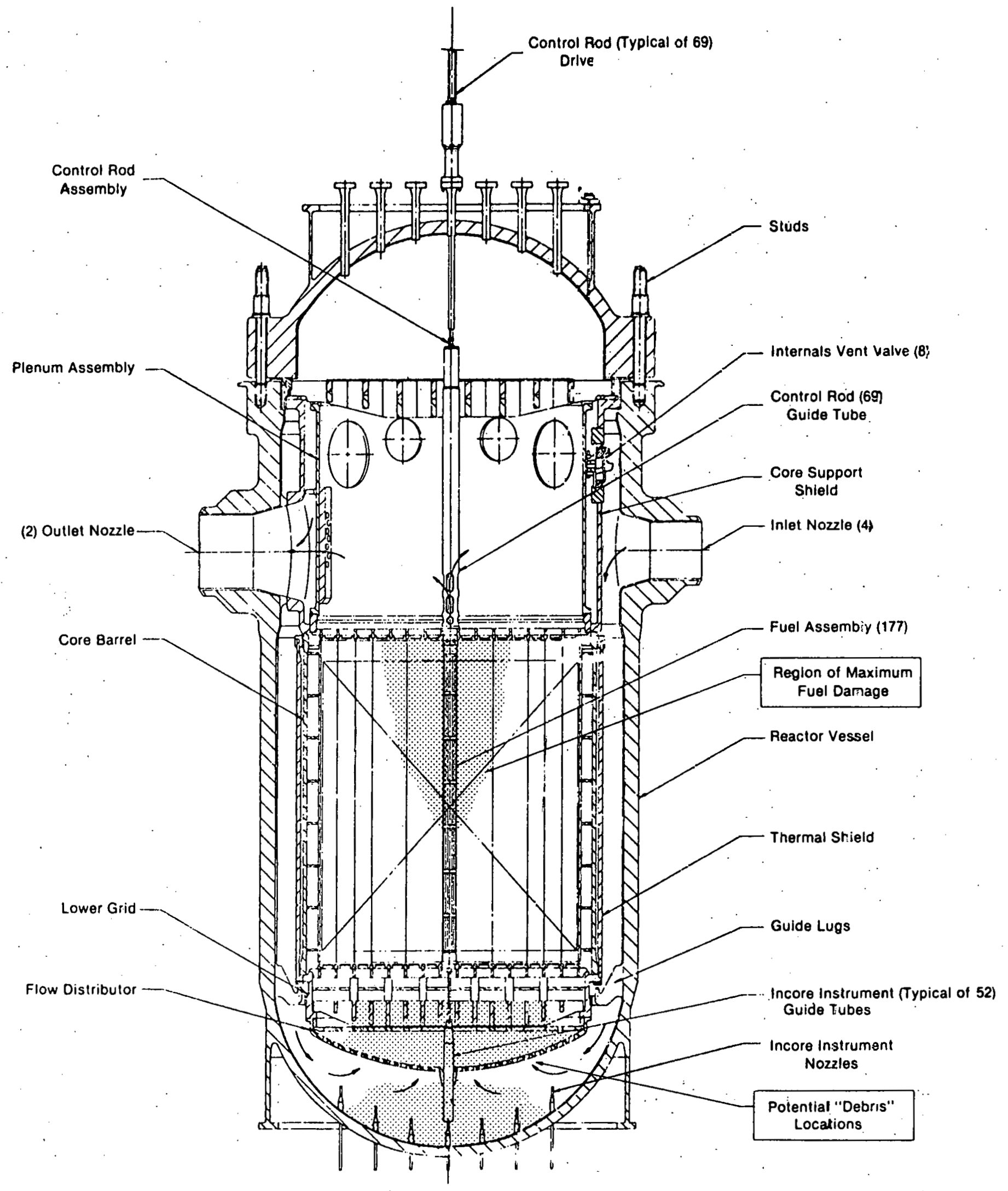

TMI-2 REACTOR VESSEL AND INTERNALS-GENERAL ARRANGEMENT

FIGURE 5-13 


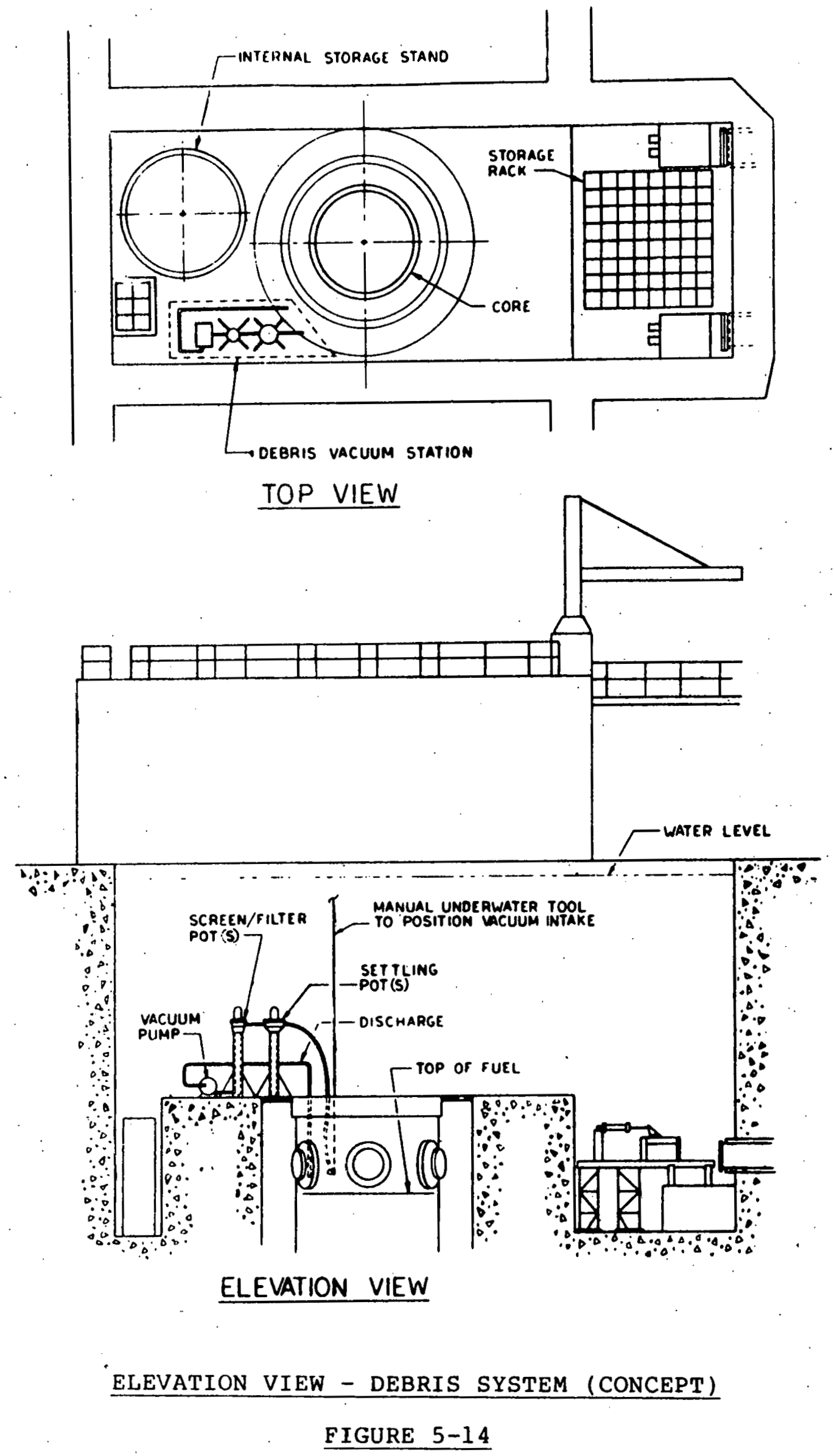




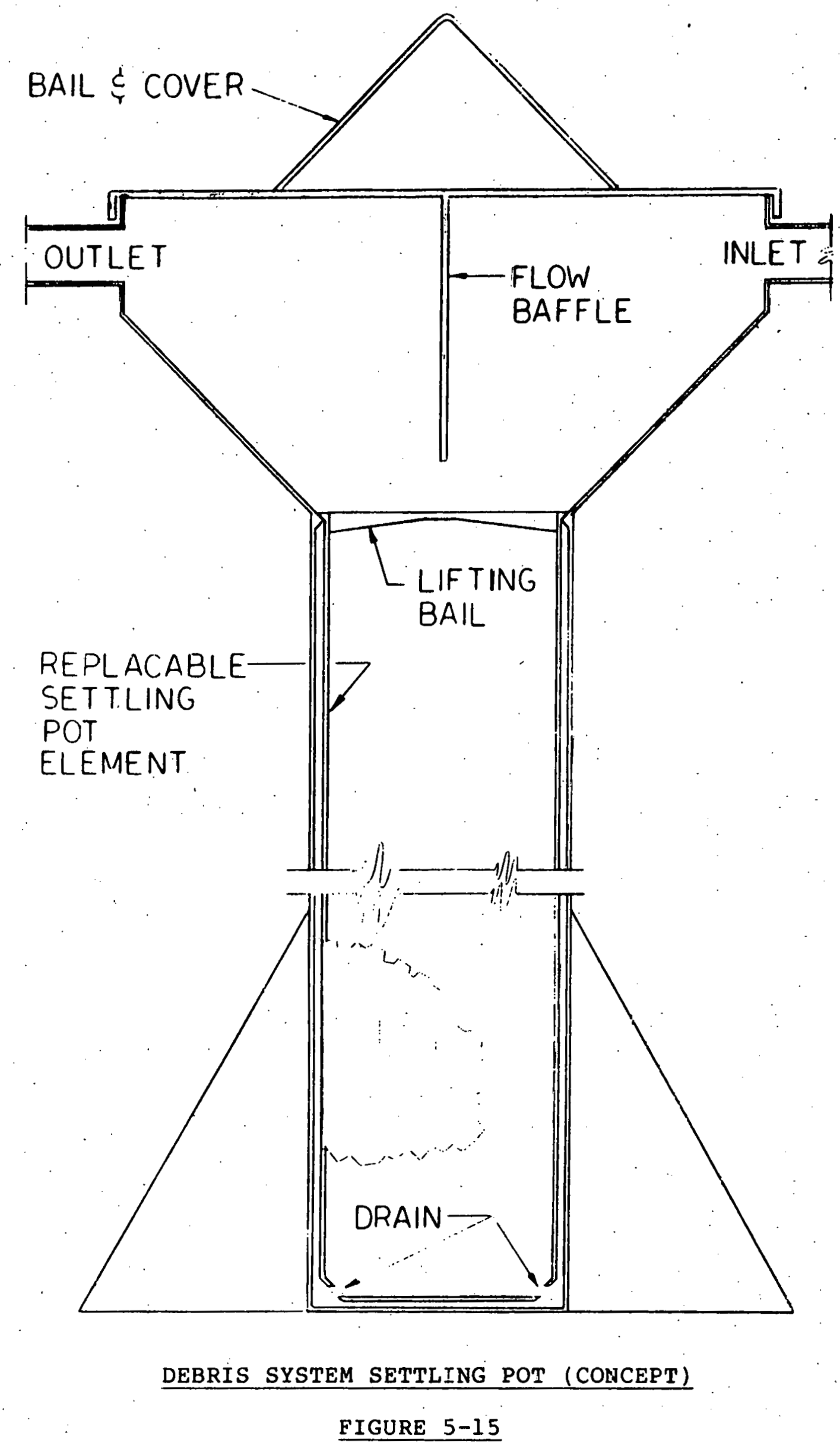

7.3-99 
final stage of this system should require filtration as provided by the filtering pot or pots for the fine particulate. These units are illustrated in Figures 5-15 and 5-16. The vacuuming system is illustrated as discrete components but, after a design definition, an integrated skid package unit would be intended. Maximum use should be made of operationally proven equipment which is commercially available. Critically safe geometries should be incorporated throughout the design. The boron concentration in the pool water should preclude criticality. However, a neutron multiplication counter measuring channel is shown on the filter pot. Licensing concerns could require verification that $K_{e f f}$ of the debris is within acceptable limits during loading. Note that it would be desirable to utilize a commercial vacuuming system if one were available.

After loading of the replaceable filter elements, they would be capped and the capped unit transferred for shipment canning. Elements within the fuel can units are shown in Figure 5-17.

\subsubsection{Conceptual Design of the Shipping Can}

It is felt that all of the fuel will require canning to prevent leakage and pool contamination during storage. Also, if the shroud concept is employed for handing, (note that it is not a pressure vessel nor does it provide absolute confinement of fuel contamination). Placing both the fuel and the shroud into an additional can, provides contamination confinement. This is shown in Figure 5-18. A square cross section to facilitate various disposition options would be possible. However, disposition decisions would be required as well as design decisions relating to the pressure-retaining requirements of the can.

A drain for the can would permit a pressurized draining of the can interior. This in turn would drain both the exoskeleton and fuel. All shroud concept designs should be either inherently free draining or should be provided with separate drains. However, fuel and can displacement weights of approximately 2000 pounds would preclude buoyant floating of drained and packaged fuel. The drain system could consist of a drain leg and a pressure leg. The drain leg would be affixed to the can cap and be the length of the can. It would fit jnto the clearance between the can cross section and the sidewall during cap installation. Both legs would be provided with remote valves and remote quick disconnects. This system would permit both connections to be on the can upper Iid.

The can cap nay require welding to the pressure vessel depending on the pressure retaining requirements. (Also, a 


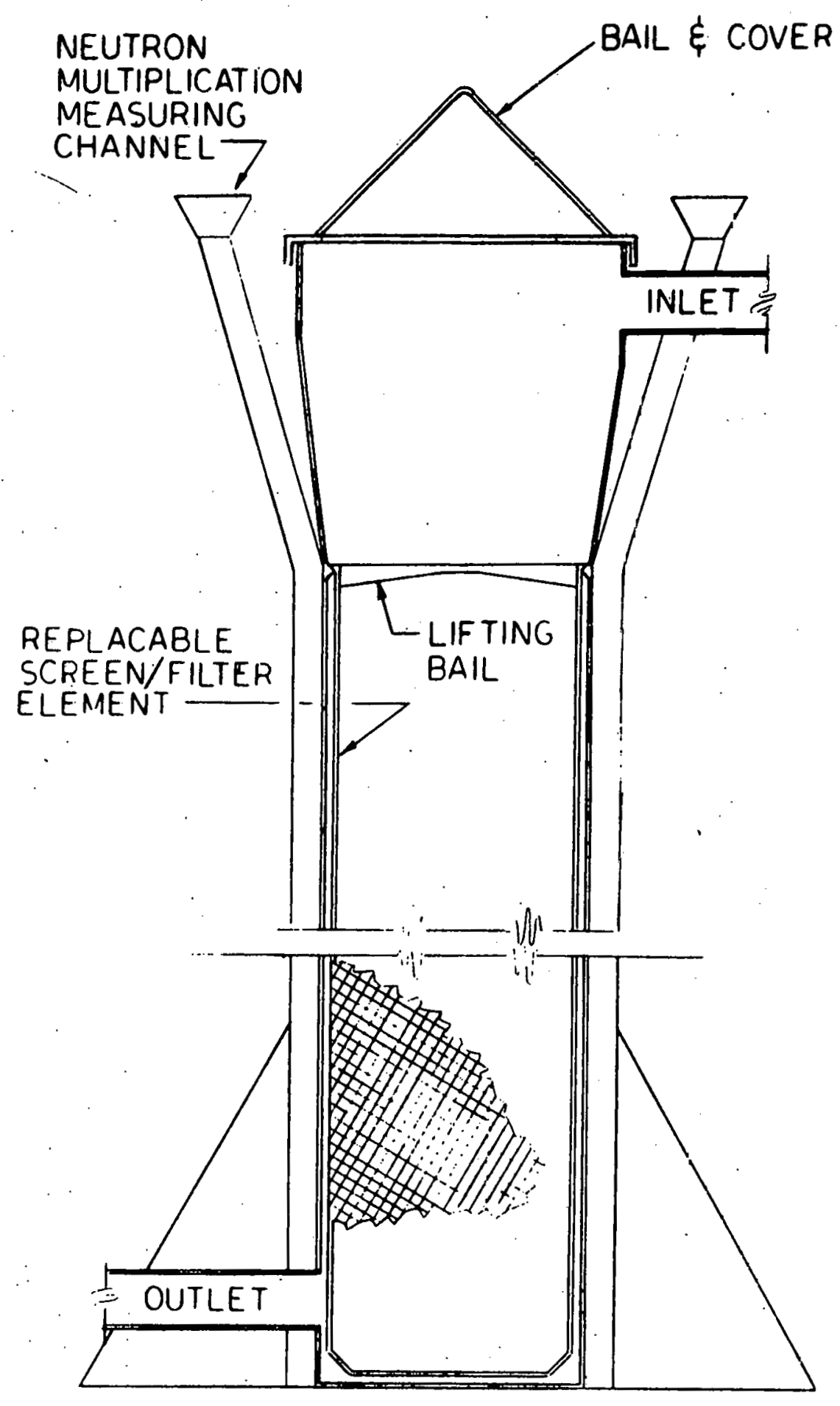

DEBRIS SYSTEM SCREEN/FILTER POT (CONCEPT)

FIGURE $5-16$ 


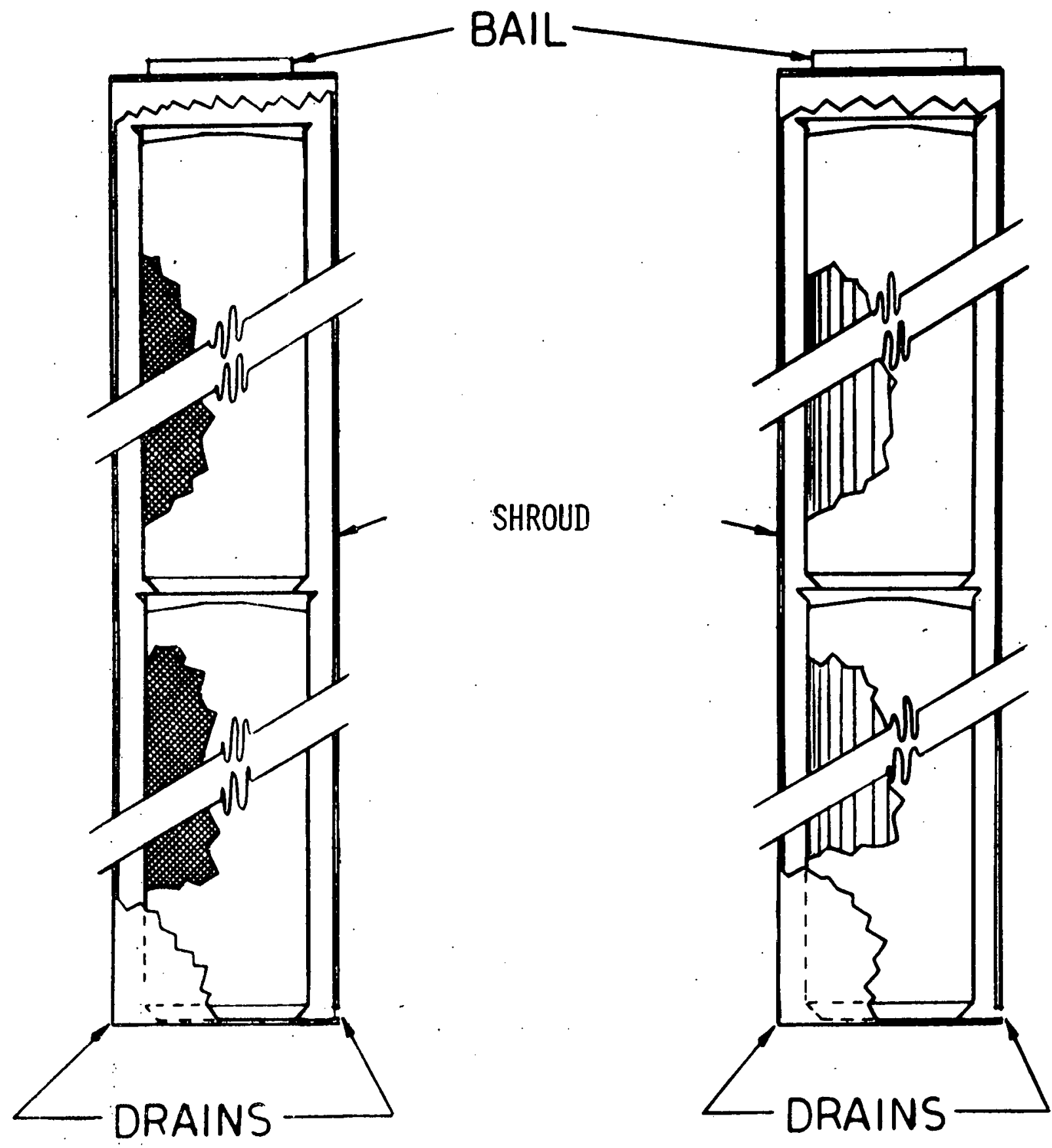

FUEL CANISTER LOADED WITH

DEBRIS SYSTEM ELEMENTS FOR TRANSFER

FIGURE $5-17$ 


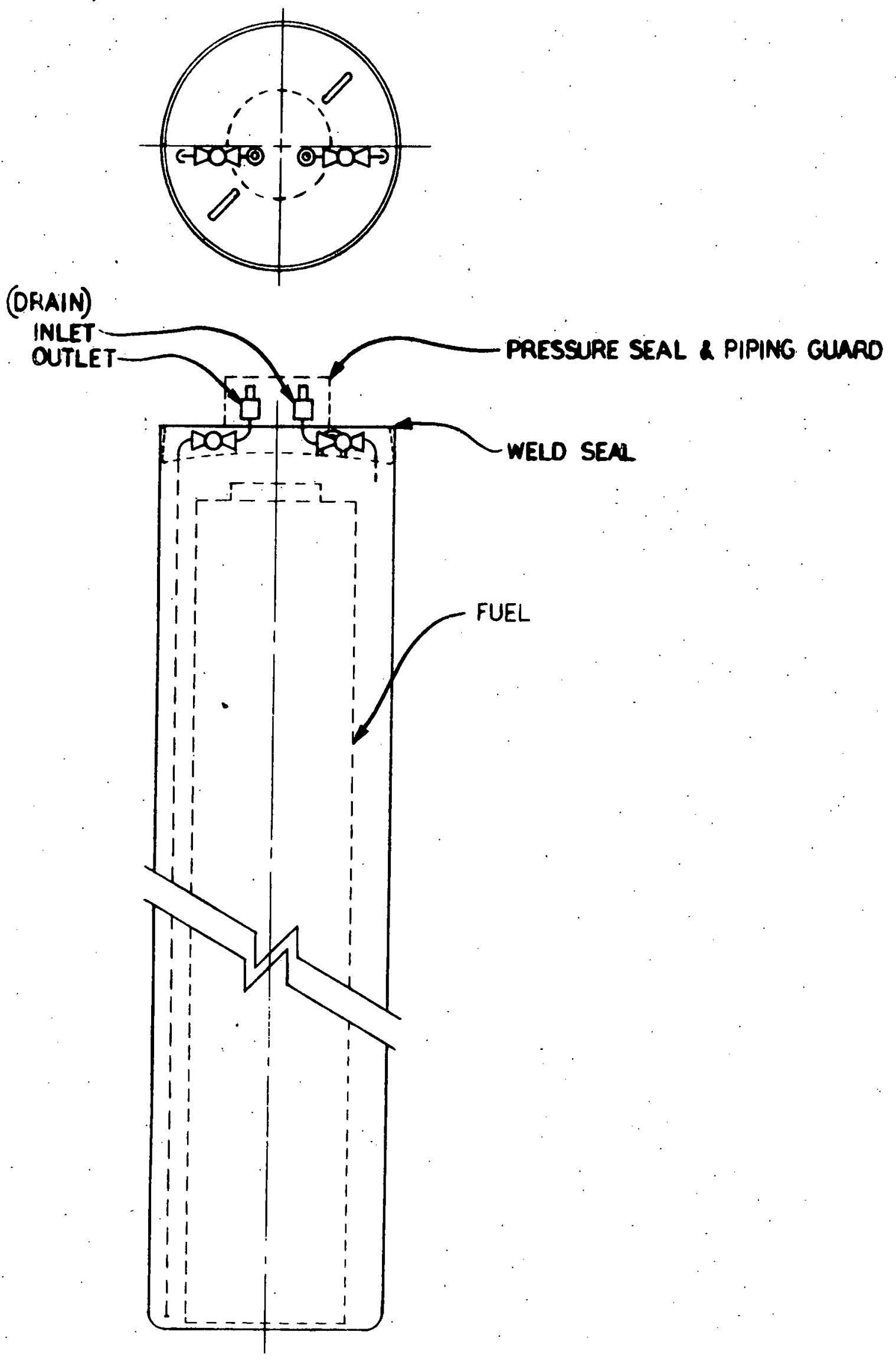

PRESSURE CONTAINING SHIPPING CAN (CONCEPT)

FIGURE $5-18$

7.3-1J3 


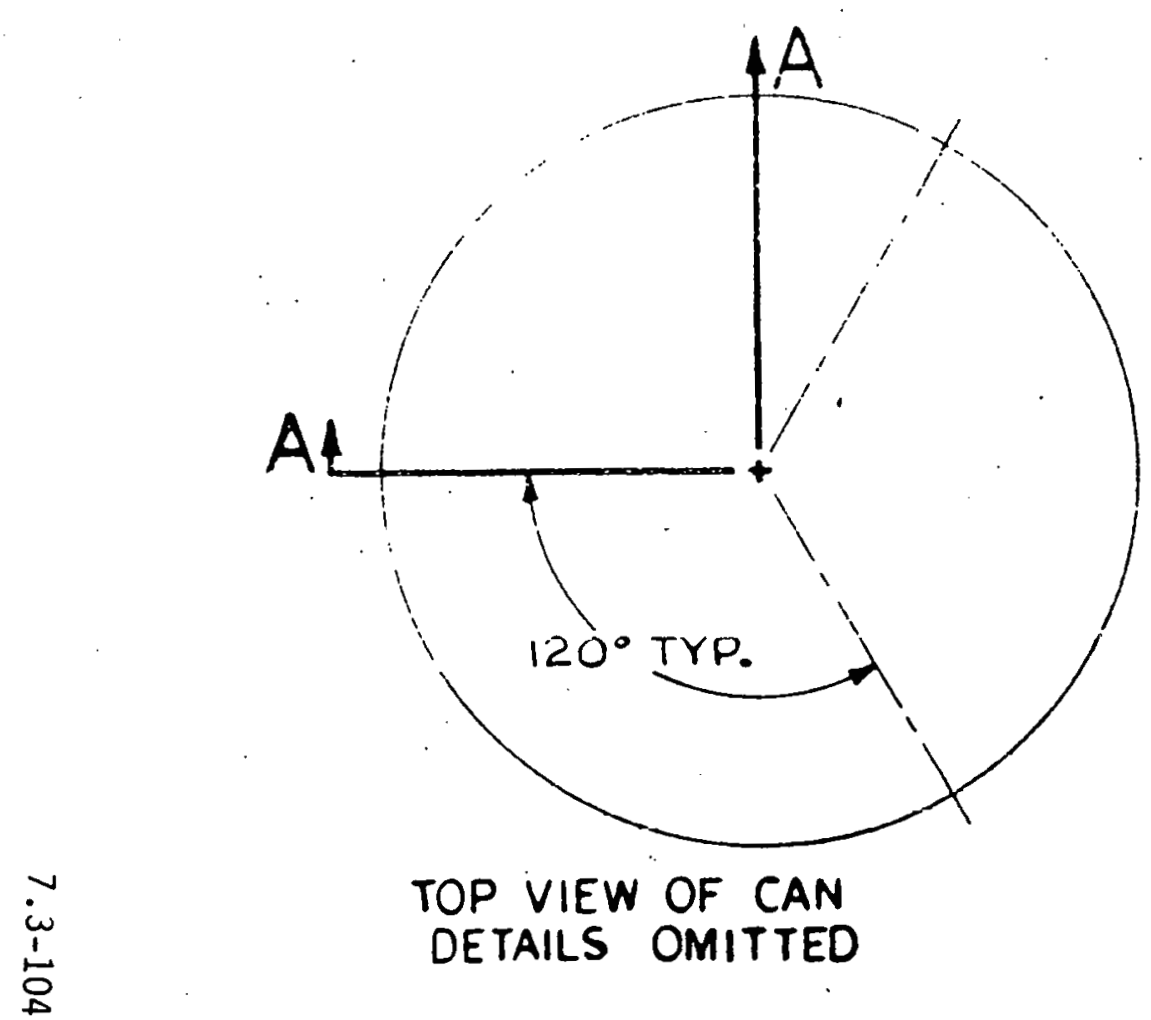

$$
\underset{\text { (ALTERNATE) }}{\text { MECHANICAL CAP }}
$$
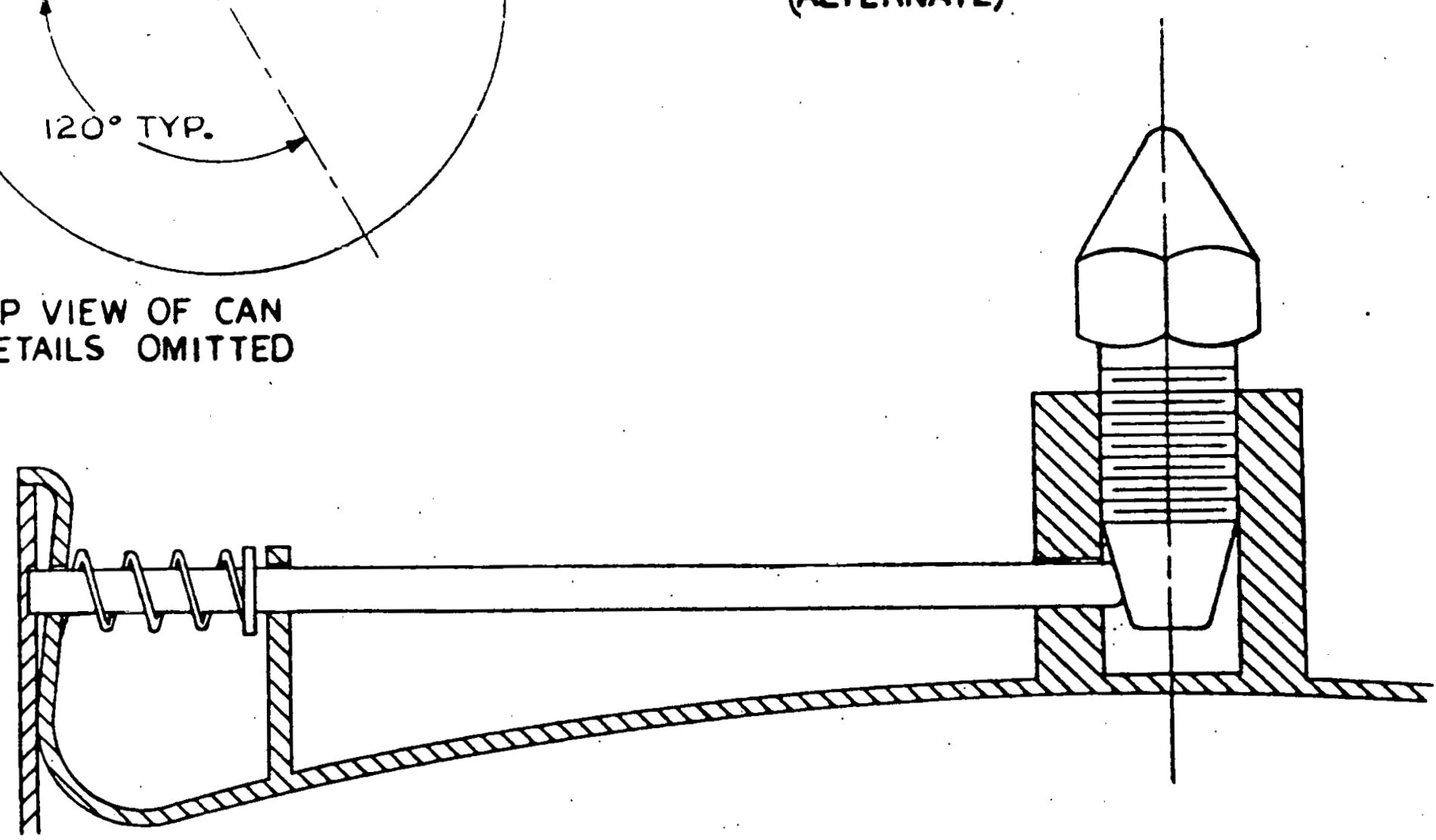

MECHANICALLY SEALING CAP FOR SHIPPING VESSEL CLOSURE FIGURE 5-19 
valve pressure seal and piping guard could be necessary as shown [dotted] in the figure.) As an alternative, a mechanically secured cap is shown in Figure 5-19. This would facilitate unpackaging at a receiving facility if shipping requirements would accept the low internal pressure capability of this can. Lower pressure retaining requirements seem probable if it is confirmed most, or all, of the free fission gas was released during the accident. Also, the low decay level of the fuel results in pressure retaining requirements. Note that temperatures of the aged fuel will also be comparable to the temperature of the can itself (probably less than $200^{\circ} \mathrm{F}$ ).

\subsubsection{Preliminary Scoping of Operational procedures}

\subsubsection{Packaging}

It is expected that the normal reactor defueling operations will be impossible because of the fuel condition. Two obvious alternatives exist as to the location for canning the fuel. As discussed earlier, criticality limitations on can geometry fix a can diameter that should fit in the fuel transfer carriage/upender "as-built." Therefore, canning could be done either in the FTP or the FSP's. The following factors related to each of the areas are listed for canning locations (see Figure 5-5):

\section{FUEL CANNING LOCATIONAL FACTORS}

Canning in the

Fuel Transfer Pool

- With additional isolation system(s), contamination

can be confined to a smaller area.

- Canning operations can be combined and handling operations minimized.
Canning in the Fuel storage Pool

Lower background radiation and contamination potential to canning personnel.

More operational space.

Lower contamination of can exterior.

Reduced operational restrictions (due to proximity of surrounding critical equipment).

Lower operator fatigue since personnel protective clotring requirements should be reduced 
Based on the above list, canning in the fuel storage pool appears advantageous from the standpoint of reduced operator exposure. Canning in the FTP permits a more efficient operation and can reduce contamination. of course, as the fuel condition is defined, the degree of confinement will be better known. If the conceptual shroud previously defined is used, definition of the fuel condition will fix the degree of confinement that could be expected. If it is decided to perform the canning operation in the fuel transfer pool an effective isolation barrier should be provided between the two pools (where none now exists) to prevent the spread of contamination.

One method of confinement when using the transfer tube as a lock could be utilized between the FTP and FSP A. Additional equipment required would be the installation of support piping and a 30-inch gate valve on the reactor side of the transfer tube. Another modification would be the provision for a remote mechanical disconnect between the two fuel transfer carriage subassemblies in place of the permanent bolted connections that now exist. Also required would be an additional drive on the reactor side that could remotely connect to the basket half of the carriage assembly in the transfer tube and move it into the reactor side upender (and reverse the procedure).

The system would then operate as follows:

(1) Shut both välves.

(2) Flood FSP, transfer tube, and FTP.

(3) Load damaged fuel in FTP carriage.

(4) Open FTP valve.

(5) Move fuel into tube and disconnect drive.

(6) Shut FTP valve.

(7) Flush tube.

(8) Open FSP valve.

(9) Connect drive and move fuel to FSP.

(10) Unload fuel from carriage into FSP.

(11) Return carriage basket to FTP providing isolation via valves.

(12) Repeat defueling transfer steps as above as required. 
Since this approach would require blind remote connections and substantial equipment modification, it will not be pursued further until better contamination control criteria are developed. It does appear though to have generic application to future reactor or retrofit designs to better accommodate the potential for damaged fuel defueling. Therefore, the FTP will be considered only for fitting the shroud.

Using the FTP for fitting the exoskeleton and the FSP A for canning has been previously discussed. Locks could be fitted into the gate clearances between the FSP A and B and between FSP $B$ and the CLP. This scheme will serve as the selected candidate to develop one alternative defueling procedure.

\subsubsection{Defueling Procedures}

Preliminary to any procedure definition, these parameters were qualitatively reviewed for their impact on the details of the procedures:

- Criticality control

- Contamination control

- Protective clothing requirements

- Radiation environment

- Humidity and temperature

- Degree of mechanization of defueling equipment

- Visibility

- Special nuclear material accountability and safeguarding control

- Preservation of accident data unique to core condition:

If investigation of the the core condition shows it to be composed of badly damaged (but intact) fuel and debris, the following procedure could be used. The following procedure implicitly recognizes requirements resulting from the above parameters.

First, the handling tools, dummy failed flel, fuel cans, debris vacuuming system, and the shipping cask(s) and ali other planned equipment should be "checked out" at an uncontaminated pool or facility (see section 4.0, "Recommendations"). This procedure has a precedent in the testing 
operations of the TMI submerged, demineralizer system. Checkout should address all design functions and maintenance capabilities of the equipment. Checkout should be completed with both direct viewing as well as with underwater television viewing. This checkout should be done by operators who would perform the defueling as a portion of their training. The overall objective of this checkout would be to familiarize operators with streamlined procedures on reducing total operator exposure during the actual defueling. The next, or parallel, step would involve operator training. It would formally address in the classroom and, then, in practice and demonstration:

(1) All nine parameters above.

(2) Facility, facility procedures, and equipment and tooling familiarization.

(3) Emergency procedures.

(4). Step-by-step defueling from core to shipping cask.

The defueling and canning procedure could be expected to proceed in a generic manner with steps similar to the following procedure. Various operational concerns related to the exact sequence of operation must include decisions on:

(a) using single or double canning (or handing shroud)

(b) the sequence of collection ( $i . e$. when vacuuming, intact fuel removal, etc.) is performed

(c) location of canning operations (i.e. containment building auxiliary building)

This generic procedure will undoubtedly vary in many ways from the final method selected due to factors in defueling which have not yet been determined. However, the procedure is presented to illustrate the types of steps which must be taken.

DRAFT HANDLING PROCEDÜE

Step No. Description

(1) Establish and implement all access and administrative control subsystems of the packaging procedure.

(2) Expose core for defueling by removal of plenum assembly and incore detectors (if possible). 
Position a cannister in FTP to package relative large debris fragments.

(4) Using manual tooling and underwater television, inspect, inventory, and package large debris fragments. Capping of the canister and transport to FSP will be repeated as required until all large debris fragments are removed.

(5) Inspect to confirm that no large debris fragments remain.

(6) Install core debris system.

(7) Install and check out neutron multiplication measuring system in core debris system.

(8) Manually position vacuum intake to remove core debris.

(9) As the vacuum system is loaded, replace with fresh elements and stack loaded elements into the canister. Cap and transport to FSP.

(10) Inspect to confirm that no core debris remains and remaining fuel is accessible to hoisting.

(11) (Assuming conformance to the criticality control plan), select one radius on which assemblies touch side-to-side toward core center (i.e., at $0^{\circ}, 90^{\circ}$, $180^{\circ}$, or $270^{\circ}$ ).

(12) Position the handing shroud over peripheral assembly on radius selected in step No. 11 .

(13) Inventory fuel, grapple, and load into the shroud.

(14). Inspect removed fuel during loading. Inspect adjacent fuel in core after loading to guide selection of the handling shroud and grapple method (i.e., top end, instrumentation tube internal, or side) for removing next assembly toward center.

(15) Move fuel to fuel transfer carriage/upender.

(16) Free grapply, secure canister, and transport fuel to FSP. Store fuel. Complete inventory. prepare to load next assembly in accordance with step No. (14). 
(17) Proceed toward center, repeating Step Nos. (13) to $(16)$.

NOTE: Major revision in the procedure should be anticipated as core physical data is obtained during step No. (17). The degree of "free-standing ability" of fuel assemblies progressing toward the core center could impact the removal sequence (i.e., a "radius-at-the-time" or "concentric rings") and would determine whether "lateral support shims" (i.e., mechanical structures to replace the sidewise support to a remaining damaged assembly formerly provided by the removed assembly) must be inserted. Also, distortion and interference may require freeing two, three, or even four sides of an assembly to clear interferences before the assembly itself can be removed.

(18) Reactivate core debris system as required during defueling.

(19) Complete defueling.

(20) Prepare core for repair work.

NOTE: With all fuel/canisters in FSP now, the FTP can be isolated. Storage or canning for storage or shipping can proceed in the FSP.

(21) To can fuel-position can vertically on FSP bottom in "loading holder."

(22) Hoist shroud into can.

(23) Position and secure can cap. Store and complete inventory.

(24) If required, drain can interior.

(25) Move to first decontamination lock and decontaminate.

(26) Move to FSP $B$ and store or proceed to second decontamination lock in preparation for cask loading. Decontaminate in second lock.

(27) Move to CLP. 
Load can into cask and position can spacer insert, if required.

(29) Secure cask and drain, if required. .

(30) Drain cask loading pool, decontaminate cask, and load cask on truck trailer in preparation for shipment.

Others have shown that with poisoned higher density racks and storage of 250 containers of damaged fuel in the existing pools, that 10 years of normal reactor operation is possible at TMI-2 following recovery.

\subsubsection{Operations Evaluation}

\section{Manpower Requirements}

Other manpower estimates were reviewed at this stage of scoping the defueling. Four shifts could be staffed yielding a total of 87 persons. The staffing projections are listed in Table 5-9a. Obviously this staffing level could be varied to reflect local radiation limiţs and to maximize operational efficiency.

A shielded area should be provided where personnel could go if one phase of the operation outpaced the other and where the second team could stand by. Hands-on work would require two teams if conditions require the use of respirators, which would limit the continuous work span.

Operations Hazards Analysis

In analyzing the impact that core damage determination will have on definition of the packaging system, a frozen eutectic is assumed as the worst potential case, pending actual core inspection. A packacing system for this case could result in special casks used for containment building defueling. In between this extreme and 1008 rubble would be damaged fuel assemblies and fuel debris with defueling Eollowing original design routings. Under the most favorable of conditions, the packaging syszem could be no more than leaker cans used with routine defueling.

Various personnel, equipment, and institutional hazards will effect the success of the recovery operation. They will all require complete definition of level or potential for NRC licensing concurrence prior to a final design of the defueling scheme. Those that affect the packaging and which must be evaluated are listed in Table 5-10. 
TABLE 5-9a

DEFUELING MANPOWER ESTIMATES*

One program manager. ( 1 total)

One video specialist and a helper. To maintain the video squipment and perform repairs as necessary. (2 total)

One coordinator or shift supervisor per shift. . (4 totall).

Two teams of two people per shift for each series operation (i.e., $2 \times 2$ per operation per shift) of (I) placing damaged fuel in exoskeleton or debris vacuuming and movement of fuel exoskeleton to transfer tube, (2) support of movement through tube, canning and movement of fuel in spent fuel area, and (3) loading cask and preparing cask for shipment. Radiation control and tracking to maintain accurate records of the location of assemblies and wastes which may be generated would be the responsibility of the various teams. (48 total)

Health Physics personnel. 3 two-man teams for each operation above (i.e., six per shift), should also be familiar with the equipment and procedures. (24 total)

Fuel engineering personnel. Two per shift, would monitor the operations and fuel condition from the control center. ( 8 total)

TOTAL CENSUS - 87 *

*Actual crew size may be $2-3$ times larger to minimize operator radiation dose. 
Once the damaged fuel conditions and the institutional factors are defined, the other hazards can be addressed by training, equipment design, redundancy, and administrative control.

\subsubsection{Conclusions and Recommendations}

\subsubsection{Fuel Handling}

Detail development of these procedures and equipment will require better definition of the core (and its fuel) condition. Therefore, the importance of timely information retrieval on the actual core condition cannot be overemphasized. However, if confirmation is not possible, alternates must be available. Therefore, alternate defueling procedures and equipment will be discussed in the following. As a minimum, due to fission gas releases recorded, leaker fuel must be assumed.

Normal defueling procedures and fuel handling equipment as originally conceived could be used for defueling, if core inspection and fuel damage assessment can confirm that:

(1) The fuel deformation external to the design envelope (bow) is less than $1 / 4$ inch.

(2) The fuel twist is less than $3-4^{\circ}$.

(3) The structural integrity is adequate for a vertical lift.

(4) The structeral integrity is adequate for horizontal positioning of the fuel (with only bottom side fully supported).

(5) Top end fitting distortion does not preclude grapple er:gagement.

(6) Debris and/or distortions have not created mechanical interferences between top end fittings and 1 ifting grapple, between assemblies in the core, or between assemblies and the core support structure.

If, however, fuel damage creates a fuel envelope requirement in excess of 8.8 -inches square or precludes top-end grappling, auxiliary service hoist trolleys replacing the: existing fuel handling bridge trolleys would provide a fuel. hoisting and handling alternative. Modification of existing crane hoist controls for damaged fuel handling is another alternative but would preclude other recovery work during fuel handling. 
- Criticality considerations (i.e., 10.6-inch diameter or 9.4-inch square cross-section) force any potential packaging system to easily fit within the existing fuel transfer carriage basket. Minor modifications would permit a maximum packaging system length of 15 feet $1 / 8$ inch to fit the carriage. The cross section dimensions could be increased using a poisoned canning system.

- New pool storage racks will probably be required that will accommodate the new packaging system.

- Contamination control between fuel pools could be provided by pool isolation locks and still permit poolto-pool transfers if contamination control requirements justify.

- The NLI $1 / 2$ legal weight truck cask is the limiting licensed shipping cask with a 13-3/8-inch inner diameter by 175.22-inch long cavity. Potential packaging system alternatives appear feasible that would fit within this cask. Cask size definition is a major factor in selecting an appropriate can size.

- Generic requirements of potential receiving facilities that would "study, store, or process" the fuel could be accommodated by potential packaging systems.

\subsubsection{Preliminary Package Design}

- Of primary importance in development of the can design and resulting operational procedures is to minimize to total number of canning operations and hence the number of cans required. However, certain canister concepts, such as the shroud may be required to augment the weakened state of the fuel.

- Fuel for which structural integrity cannot be confirmed could be provided with an external metal shroud. This would be fitted as soon as possible after initial defueling fuel movement. If debris is present, underwater vacuum systems provide an alternative for packaging the fuel. If frozen or large fused sections are present, remote sectioning could be done to render them dimensionally equivalent to intact fuel or debris. Alternately, a transfer casket or "bucket" could be employed. The can material would be an austenitic stainless steel unless future chemical contamination potential was verified that would preclude its use. Simple, low-axial height and easily viewed handling bails for grappling appear desirable for potential packaging system alternatives. Dimensional constraints appear to allow several alternative systems. The use of a handing shroud could 
accommodate either reasonably intact fuel, fuel that was excessively bowed, or fuel that required other than topend grappling.

- A vacuum system for debris could consist of both settling and filter pots. These pots would have replaceable elements that could be remotely packaged after loading. The system would be designed as a packaged unit for submergence in the core shelf area. The pots and the elements would be integral with or capable of being fitted into the storage can.

- The shipping can would provide contamination confinement as required by the degree of fuel damage. It would permit draining and water filling. Configuration and pressure retaining alternatives are envisioned to accommodate various disposition options. The storage can may be designed to serve as an additional containment within the slippery cask.

\subsubsection{Operational Procedures}

If fuel damage warrants either a vacuum system for debris, cans, and contamination control locks between the storage and cask loading pools as noted in this report, then defueling procedures would involve:

- Training

- Cold Checkout

- Fragment Removal

- Debris Removal

- Fuel Assembly Removal

- Transfer to Auxiliary Building

- Canning

- Contamination Control

- Shipping.

If contamination control is required, the fuel transfer tube could be modified to a contamination control lock by modifying the transfer carriage and adding a gate valve at the reactor end of the tube. Also, if fuel damage required, "lateral support shims" (i.e., box-like structures simulating fuel envelope) could be replaced for removed fuel assemblies to provide lateral support to remaining fuel.

\subsection{Shipping Cask Evaluation for off-Site Fuel Movement}

\section{$5: 4.1 \quad$ General}

Included in this section is an evaluation of the spent fuel casks which could be utilized for moving the core off-site. Included are the considerations related to the interfaces 
between the casks and the TMI-2 site, other unloading (receiving) areas. Also developed are preliminary cask hanciling procedures for handling canned fuel. This evaluation takes into account that the fuel movement will probably not occur until 1983 or later based on current projections. As noted previously, there is a possibility that fuel will be stored on-site for an extended period. Preliminary indications are that it would probably take one to three years to ship all of the fuel from the site. This stucy examines the available casks that are currently licensed or could be licensed within the United States for this fuel movement, the licensing of these casks in light of current and projected NRC requirements, and the operational procedures necessary to handle the spent fuel casks.

Viable transportation modes for most of the TMI fuel ordinarily would include legal weight truck (LWT), overweight trucix (OWT), and rail systems. The LWT systems are the NAC-1 (NFS-4), the NLI 1/2, and the GA FSV (Fort St. Vrain) casks. Overweight truck systems include the TN-8 and possibly the HTGR Peachbottom (Vandenberg) casks. The possible licensed LWR rail systems are the NLI $10 / 24$ and the GE IF-3C0 systems which are covered in this section. It is also possible that smaller casks (not normally employed for LWR Euel) could be used for moving fuel debris to a research facility. The potential availability of all the casks must be reviewed in light of the anticipated requirements for their use during the 1983-85 time period.

The report conclusions present a preference for LWT casks. This is due to easier handling, licensing, simplified operational procedures, and greater potential for operation at other unloading sites. Nevertheless, an effort has been made to define and describe the two rail casks which are applicable. The larger fuel cavities available with these casks permit them to be utilized for both off-site use and potertially for on-site handling. However, it is believed that licensing, particularly for the packages handling debris, will be extremely difficult. The OWT casks are not considered to be a viable option for utilization in Pennsylvania, both due to their lack of availability and due to the need for overweight permits. Summary data on all of the licensed LWR casks is presented in Table 5-10.

\subsubsection{Description of Licensed LWR Shipping Cask Systems}

\subsubsection{Legal Weight Truck (LWT) System}

The LNT casks considered for this application are shown in their relative proportions in Figure 5-22 (refer to Figure 5-6, Section 5.3 for cavity dimensions). A minimum of three dedicated casks would be required to move all of 


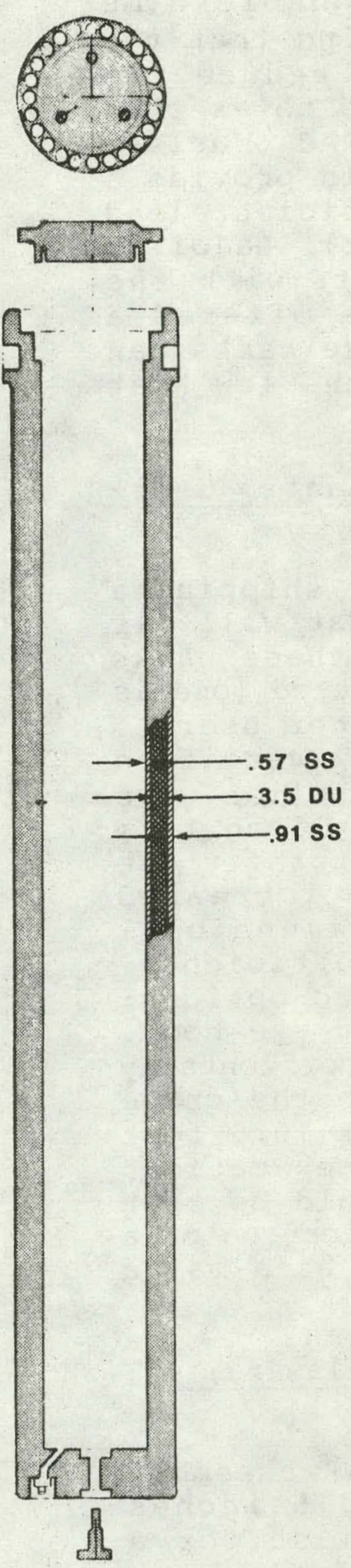

FSV-1
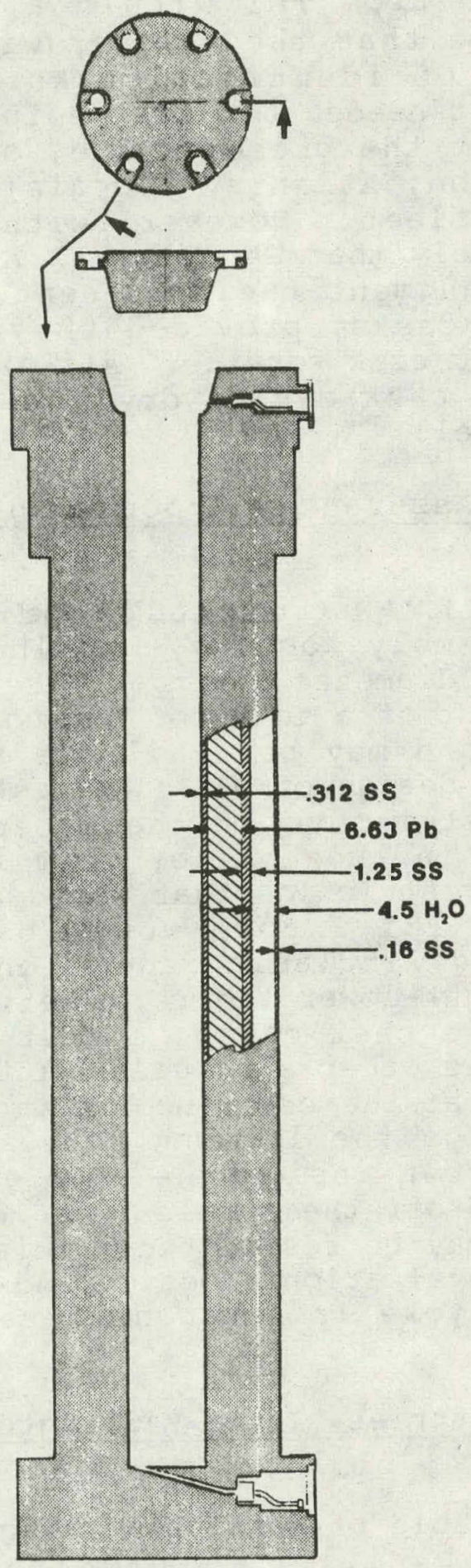

NAC - 1
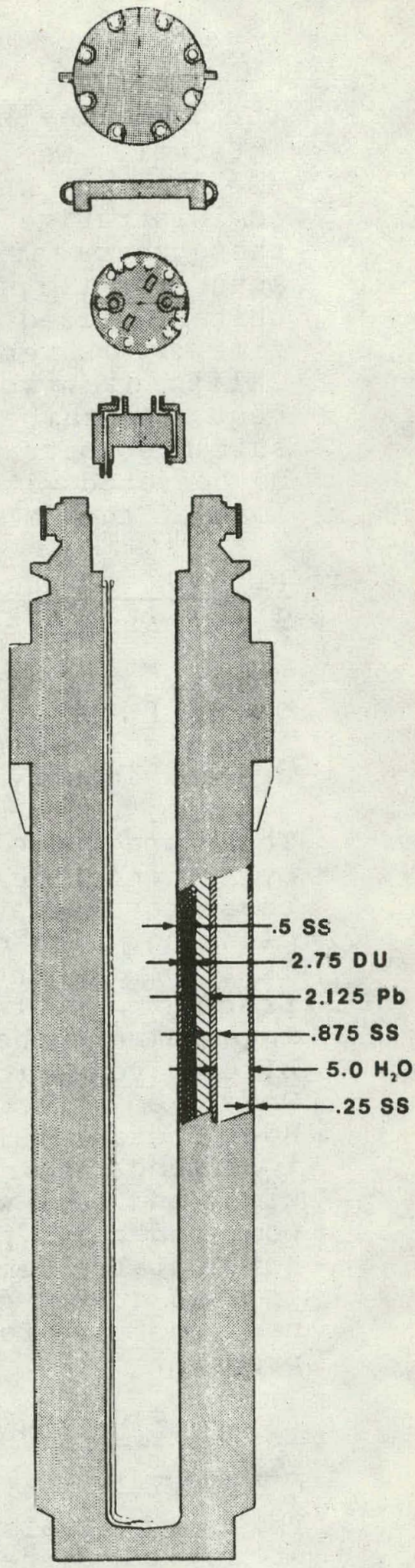

NLI -1/2

LEGAL WEIGHT TRUCK CASKS

FIGURE $\quad 5-22$ 
the fuel envisioned from TMI within a reasonable time interval. We believe that the shipper will find that the use of this number of identical casks will reduce the administrative effort needed to prepare for and to execute these shipments. At the present time, only NAC (Nuclear Assurance Corporation, Atlanta, Georgia) could provide a suitably sized cask fleet. However, with sufficient lead time, it is very likely that GA (General Atomic), LaJolla, California, would augment their present fleets with the required number of casks, provided they were offered a suitable contract for cask service. All of these casks can be operated with the cask cavity dry, due to the low heat load of the TMI-2 fuel.

(1) NAC-1 (Nuclear Assurance Corporation, Atlanta, Georgia) - LWT Cask

This is a legal weight LWR truck cask capable of shipping a sirgle PWR fuel assembly, or any container that will fit into its 13.5 inches diameter cavity. Three of these casks are now in service out of a total of six constructed (one is owned by Duke Power and may be available to other users). These are the oldest design of all LWT casks now certified by NRC and recent restrictions on their certification give cause to hesitate to include all of them in any long term planning. The only restriction that would affect their use in transporting the TMI-2 core would be the increased frequency of testing, including additional dimensional measurements that might lower their in-service efficiency. Lifting yokes for this cask can be either of two options: One is a "J" hook; the other a manually attached eye hook. Both hooks require that the crane hook be moved horizontally to disengage or engage the lifting yoke. With the crane block and hook well down inside the 10.5 foot $\times 10.5$ foot pool and cable played off the spools to a wide separation, horizontal movement may be restricted. This should be kept in mind during cask evaluation. Design and fabrication of a new remote-operating yoke is not considered to be a major problem.

(2) NLI $1 / 2$ (NL Industries, Inc, Wilmington, Delaware) LWR Cask

This cask is also capable of shipping a single PWR assembly or any container that will fit into its 13.375 inches diameter cavity, provided accommodation is made for a 1/2-inch drain line running down the inside edge of the cavity. Five of these casks are currently available. In the spring of 1980, the certification was renewed by NRC for another five years. This cask is estimated to have the least licensing difficulty of any of the casks. It also has the smallest cavity cross section. The lifting yoke system 
for these casks has good remote operation capabilities; however, a yoke adaptor for single palm hook engagement would expedite handling. The adaptor is almost mandatory from an operational convenience standpoint, since the procedures require that the yoke be attached to and removed from the hook frequently.

This dry cask has its drain outlet at the top. It is drained by pressurizing the fuel cavity and forcing the water up through the drain line (bottom to top). When the last of the water is expelled, it is followed by a surge of compressed air ( 10 to 15 psig). Provision must be made in the cask work area to handle this pressurized, wet gas through an effluent control system.

The metallic o-ring seal on the cask inner head is susceptible to being damaged. We would recommend, as a part of the NRC licensing amendment review for this operation, that a head modification be proposed as a licensing amendment to allow replacement of the metallic "O" ring head seal with an elastomer "O" ring. This change would prove to be practical as a time saver for the user. The problem is that if the metallic seal is damaged, it is not discernible until the cask is out of the pool. If the head seal will not hold pressure, then the cask must go back into the pool, the head lifted off, and brought back to the surface and the seal replaced. All of this amounts to an extra cask handling cycle. Replacement of this $\$ 150$ seal before each use will not preclude damage during seating. An elastomer seal can recover from bumps that will ruin a metal seal. The metallic seal is necessary when this cask is being used at its rated thermal capacity, 10.5 kilowatts. It probably is not necessary at the low decay heat loads of tire TMI-2 fuel. This cask is considered to be a prime candidate for the TMI job.

(3) Fort St. Vrain (FSV) Cask (General Atomic, La Jolla, California)

This is a legal weight HTGR fuel cask, not an LWR cask. However, GA is in the process of reviewirg its design applicability for hauling low burnup or aged LWR fuel. Its cavity is over 10 inches longer and twice the crosssectional area (17.76 inches diameter) of the NAC-1 and the NLI 1/2. This could be useful for larger fuel pieces. The current design revisions include inner head seating and sealing and a revised lifting system. There is no neutron shield on the cask. However, none should be needed for TMI-2 fuel due to its low neutron source strength. Three of these casks now exist. Two are used infrequently for HTGR (Ft. St. Vrain) fuel movements and are owaed by Public Service of Colorado. The other is owned by General Atomic. 
The large cavity capacity and volume of this cask makes it especially attractive. The certification uncertainty is cause for caution in committing to its use; however, it is likely that this uncertainty could be resolved in a timely manner. Once the licensing uncertainties are resolved, this cask will be a very viable candidate for this job. (Particularly since it is the only cask designed to directly load and unload into a hot cell.)

5.4.2.2 Overweight Truck Casks

(1) TN-8 (Transnuclear, White Plains, New York) - OWT Cask

This is an overweight truck cask (approximately 35-ton cask weicht) with a capacity of three PWR assemblies. The three fuel channels are an integral part of the cask structure, thus the envelope cross section is limited to 8.5 inches square. We believe that this is too restrictive to allow this cask to be a viable candidate for TMI-2 use with the present failed fuel dimensional uncertainty. As a point of information, $T N$ is preparing a design revision to the ir $\mathrm{TN}-9$ cask model (this is similar in size and appearance to the $\mathrm{TN}-8$, but is dedicated to BWR fuel) to provide the option of a cavity with removable basket.* The cavity diameter will be about 18 inches. TN projects that casks could be available in the United States by 1982 .

Any potential user of an overweight system is cautioned to consider the system's vulnerability to local regulatory agencies' arbitrary discussions. This is particularly true in the State of Pennsylvania which is especially restrictive to overweight truck shipments (see Figure 5-28).

(2) Peachbottom (or Vandenberg Cask) one Unit Owned by Chem-Nuclear Systems, Barnwell, South Carolina; One other Unit in Existence) - OWT

This is a 35-ton OWT cask with cavity dimensions of 116 inches in length by 36 inches diameter. Both cavity and payload must be shipped dry. This cask would be a viable candidate only if it became necessary to ship short, large (up to 36 inches diameter) canisters which have to be loaded in the spent fuel pool. This situation is not very likely because of criticality considerations. The certificate on this cask expires December 31,1980 . Its operational usage for spent fuel is tentative beyond that.

\footnotetext{
*Personal Communication with K. Goldman, Transnuclear.
} 


\subsubsection{Rail Shipping Cask System}

\section{(1) IF-300 (General Electric, Morris, Illinois)}

This is a 70-ton LWR rail cask designed to haul 7 PWR assemblies or 18 BWR's. The cask cavity is 37.5 inches in diameter. This cask system has been in service since 1975 and is operationally proven. The TMI site has a rail spur capability and was designed for shipping fuel off-site using these GE casks.

The lifting yoke is a " $J$ " hook so, like the NAC-l, block clearances for horizontal travel would have to be verified before this cask could be considered for use.

Like all multi-element casks, we are concerned regarding the possible licensing delay if the IF-300 should be selected to haul TMI-2 fuel from the present core. We doubt that rapid approval would be forthcoming. The IF-300 would be more time consuming to decontaminate after loading than any of the legal weight truck casks because it does not have a smooth outer surface and no contamination barrier-type skirt has been developed for it.

\section{(2) NLI 10/24 (NL Industries, Wilmington, Delaware)}

This is a 100-ton rail cask designed to haul 10. PWR assemblies or 24 BWR assemblies. Its cavity diameter is 45 inches. Like the IF-300, we doubt that the advisability of requesting the NRC to consider licensing it to haul TMI-2 fuel canisters. Two casks could be made available but are not now in operation.

This cask is not considered to be viable for the TMI-2 application because of its large size. A silhouette of the $10 / 24$ is shown (Figure 5-23) in the TMI decontamination pit. Decontamination work obviously would have to be done elsewhere. Handling this cask in the 10.5-foot $\times 10.5$-foot pool would be a tedious operation. We believe also that a special mobile head-handling gantry would be required.

\section{(3) General Rail Considerations}

A preliminary evaluation was made of rail handling possibilities at the TMI site. It appeared that either the GE IF300 or NLI $10 / 24$ could be used. A point of caution concerns the rail track/rail car interface leading into the site. Rail cars with three-axle trucks (such as the NLI 10/24) may have potential problems in negotiating the curve at the start of the siding as the track leaves the main line and enters a bridge approach that is shared with road traffic. under these conditions, rocks, etc., that lodge in the track 
recess intended for the wheel flanges, can cause derailment. The curve radius is within that allowable for these trucks; and if the recesses were cleaned prior to each use, there should be littlee problem, but administrative controls in this regard usually are weak. Two-axle rail trucks (such as employed on the IF-300 car) would be less susceptible to this type of derailment. An incoming (empty) cask would be headed toward. the river if it derailed.: An outgoing (loaded) cask car might come to rest interfering with the main line traffic of the railroad.

\subsubsection{NRC Licensing Criteria for Shipping Casks}

We have previously assumed that all off-site shipments of TMI-2 fuel would be made in NRC certified casks. We further assume that any casks now certified for the shipment of spent nuclear fuel will require a certificate amendment prior to shipping severely damaged fuel. This amendment would be requested by the cask owner (who would be under contract for this movement) even though there may be no structural modifications to the cask system. There are good reasons for the cask owner to do the processing of the NRC licensing amendment. The best is that it is the most expeditious way and the owners are most familiar with the NRC SAR and licensing restrictions. The owner may agree to do this work as a condition for selling his services. The 1 icensing amendment cannot be initiated until the internal shipping container is well into the design phase. Since the fissile material cannot be assumed to have any structural integrity, a fuel particle size for optimum reactivity probably will need to be considered. Similarly, it should be assumed that at some time during loading, draining, shipping, filling, and unloading, the system will experience optimum water-to-metal ratio and maximum neutron reflection. Unless the above assumptions can be shown to be unwarranted, the fuel container design almost certainly will be based on an always-safe (criticality) geometry. Further consideration of these individually safe geometries must be made for interactions between them during canister loading and storage. Packaging inefficiencies will be expected with multi-element casks. It is expected that not as many TMI-2 canisters could be included in the package as the cask design can accept as PWR assemblies. If this is the case, the economics of the transport service will swing in favor of the LWT mode.

A final problem is cask availability. The shipper (TMI) must be certain that the cask(s) selected for canister capability and NRC certificate amendment will be available in the quantity needed whenever the core is ready to be moved. If the opening of an AFR coincides with the TMI shipping schedule, available casks may be in short supply. 


\subsubsection{Fuel Loading Location Options}

There are two possible locations for loading the spent fuel. cask at the TMI site. These are the spent fuel cask loading pool and the fuel service pool. Operational considerations at both locations are presented here.

\subsubsection{Spent Fuel Cask Loading Pool}

The TMI fuel pool is a specific-purpose (dedicated) design and is the better of the two possible available options for cask loading. Its use would be denied only if, for some reason, the packaged fuel cannot be moved from the reactor service pool into the spent fuel storage pool via the transfer tunnels or that it is being used for other TMI recovery operations. Although no standard operating procedures now exist for loading spent fuel at TMI, these can readily be developed from the generic procedures furnished by the cask owner. Before developing these procedures based on the $A E$ 's cask handling design concept, it will do well to consider some changes that will:

- Eliminate handling the cask closure heads with special tools

- Permit greater lateral movement of the hock within the confines of the pool boundary.

These will, in turn, decrease turnaround time.

In our interpretation of the present concept, the pool is dry whenever the cask is lowered into the pool or removed from the pool. This is done to avoid wetting the block and ropes and preventing the spread of contamination as pool water drips from them. Since the pool must be full of water until the head is set into place, the design technique requires that the head be attached to a special tool - - a grapple on the end of a segmented pole which, in turn, is suspended from an auxiliary hook. The most expedient method of handling a head in this manner is to leave the head and grapple in the pool all the time, from a tie-off point at least 2 feet (for LWT) from the edge of the pool. The upper section may have to be removable to allow the fuel bridge crane access directly over the cask. In practice, the head would be removed from the cask for inspection before the cask goes into the pool. The head is then replaced and the cask lowered into the dry pool, anc the pool is filled with water. Next, the head is grappled using the special tool, lifted from the cask and set aside, still in the pool. After loading the cask with fuel, the head is replaced; released from the grapple and the grapple is returned to its pool-edge holder. 
Using the above system in the TMI pool with a rail cask is impossible; there is not sufficient room in the 10.5-foot square cross section. Removal of the head before going into the pool and replacing it later is possible, but not practical. With truck-cask size closure heads, the procedure will work, but it wastes time because it requires that operational procedures be performed in series that could be done in parallel. Also, if a seal ring protector is to be used, it, too, would require remote handling in the pool rather than simply placing it on top of the cask before the cask is submerged. In addition, placement of the closure head should not be attempted using nonrigid (cable) slings. The torsional pendulum effect on the slings is too much to control adequately. The preferred method of head placement is to suspend it by slings from the lifting yoke and seat the head just prior to latching onto the cask to return it to the surface. To accomplish tis without wetting the block (wetting the hook should be of little concern), two additional fixtures are needed. One is a yoke extension about 20 feet long (see Section 5.4.4, Appendix B), and the other is a shelf about half the depth of the pool on which the cask can rest (see Section 5.4.4, Appendix C). This concept is in use at various sites (e.g., the Duke oconee pools), and the only thing out of the ordinary for this application would be the folding capability of the shelf. with this system, the pool does not need to be drained to facilitate cask handling. The cask is picked up by the yoke and placed on the shelf at a level 22 feet below the curb. The lift pin of the yoke adaptor will be just above the water surface. Next, the yoke is released from the hook. The extension is attached to the hook and the yoke to the shelf is folded against the walls of the pool, then lowered to the floor of the pool. Now the cask is at the -44 foot level with respect to the curb, the yoke lifting ete, or bottom of the extension, at the -22.5 foot level. The hook is at the -1.5 foot level, just below pool water level with the block still dry. Exact dimensions for the above will vary depending on the cask model selected, but the system should be workable with any of them. When the fuel is loaded, simply attach the head to the yoke in the normal manner, seat the head, latch on to the cask, and return it to the shelf. Remove the extension and then complete the removal of the cask from the pool to the decontamination area. A step-wise summary loading procedure is given in Appendix A (Section 5.4.4).

The use of a shelf for loading will eliminate the need to empty and then to refill the loading pool, which has a capacity of 35,000 gallons of water. Also, use of the extension on the hook eliminates the need for the block to travel about 22 feet down into the pool, where its 
horizontal travel may have to be restricted to prevent cables from contacting the pool curbing.

The feasibility of using the decontamination pit as a cask shelf was investigated and rejected. The floor elevation is too high (several feet). to permit use of hook extensions of the necessary length. It would have entailed the installation of a gate between the two pools. The use of a 5-foot thick crash pad in the pool might resolve the extension length problem, so this may be a possibility to keep in mind for the future when ordinary spent fuel will be shipped from the site.

Even if smooth exterior surface truck casks are used (rail casks typically have cooling fins on their outer surface), it is recommended that a jacket (contamination-barrier) be fitted to the cask body prior to each loading. This jacket functions as a barrier to the spreading of contamination from the pool water and will reduce decontamination time and measurably improve turnaround time.

\subsubsection{Fuel Service Pool}

It is possible that canistered fuel could not be moved via the normal route through the transfer tunnel (a situation where the "fused" fuel assemblies cannot be separated into suitable sizes). In this case a transfer cask will be needed. The "fused" fuel must be loaded into the transfer cask inside the containment building. There is adequate crane capacity (500-ton polar crane) to handle the cask. It appears that the cask movement could be routed through the containment building to the building hatch. This movement needs further study when the area becomes accessible for operational needs and the recovery equipment is in place. space is available in the containment building to decontaminate the cask, but it is said to be limited and wash water may recontaminate other areas. Access by highway vehicle is available but would require repeated opening of containment into a yet-to-be-built air lock. Rail cars cannot be accommodated because there is no siding planned into this new air lock. A siding, if used, would be a very short branch off the rail line into the turbine building. A large cavity rail cask could be used to move the fuel or fuel segment to the auxiliary building.

A clearance study indicates that, if casks were to be loaded in this area, the present fuel handling crane (bridge) could not be used because the bottom of the masts would not clear the top of any LWR cask in the canal. The top of the fuel, if still intact, would have to be brought to within 5 to 6 feet of the surface (elevation 346 feet) to clear a 16-foot cask sitting at the 308.5-foot elevation. However, 
with the low fuel burnup condition, that much water might suffice. It appears that the use of an existing "shelf" at the 322-foot level could serve as a cask resting location, so that a yoke extension could be used in lieu of wetting the hook.

5.4.4.3 Appendix A - Summary Loading Procedures Using Recommended Modifications

(1) Bring the cask from the transport vehicle to the decontamination pit.

(2) Fill and flush the cask with demineralized water.

(3) Remove the head and inspect the pressure boundary seals. Set head aside.

(4) Move the cask to the shelf* in the loading pool. Detach the hook** from the yoke**.

(5). Attach the extension* to the hook; then attach the yoke to the extension.

(6) Lift the cask a few feet to allow clearance for the shelf sections as they fold away.

(7) Lower the cask to the bottom of the pool. Disengage the cask.

(8) Remove the yoke from tre pool and attach the cask head to the yoke slings while the fuel canister is being loaded into the cask.

(9). Return the yoke to the pool and place the head into position before engaging the yoke to the cask. Engage the cask.

(10) Raise the cask above the cask shelf level.

(11). Move the shelves into piace.

(12) Lower the cask onto the shelf.

(13) Release the extension from the yoke.

(14) Remove the extension from the hook.

\footnotetext{
* See Appendix $C$, following.

${ }^{*}$ See Figures 5-23, 5-24, and 5-25.
} 


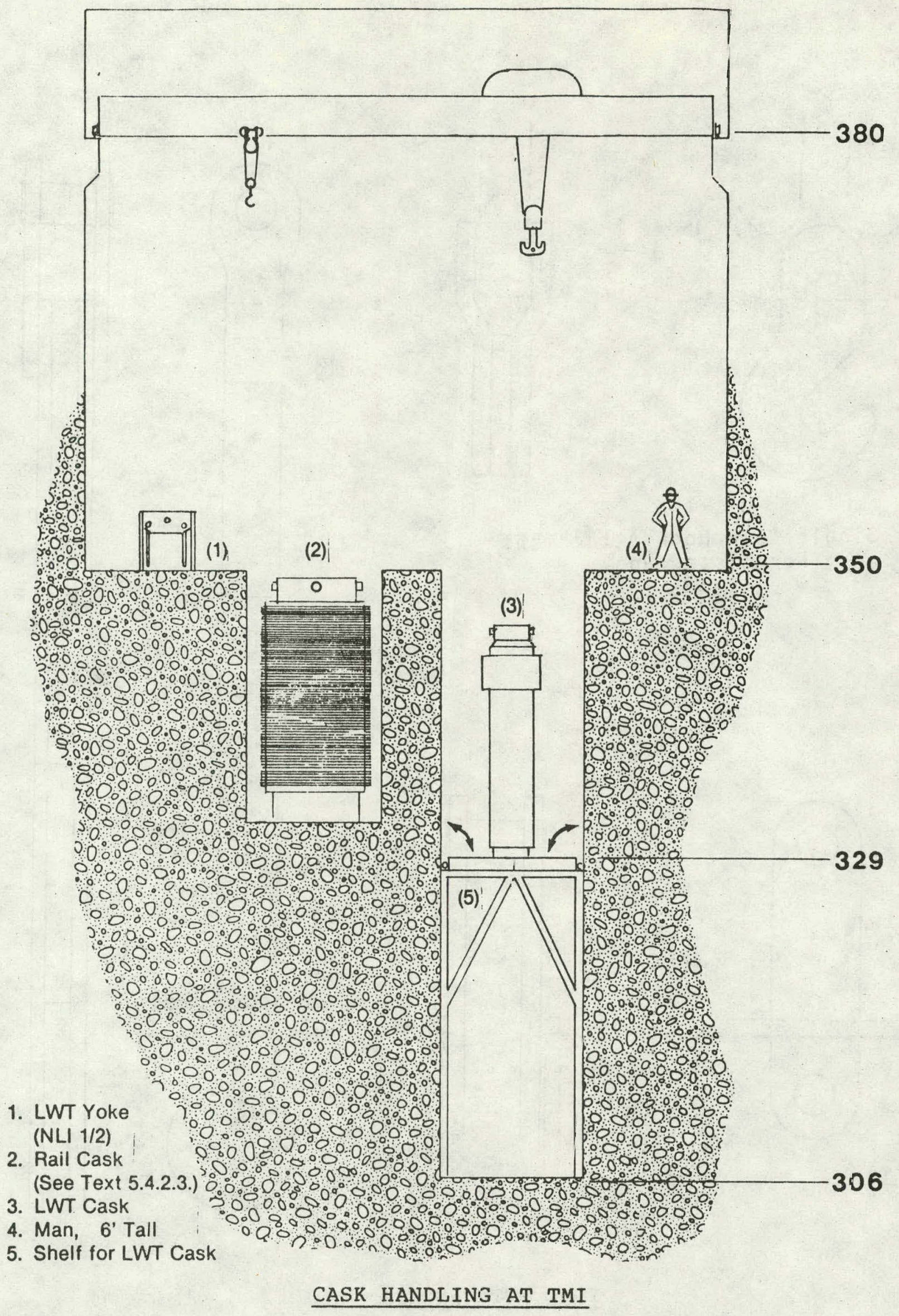

FIGURE $5-23$ 


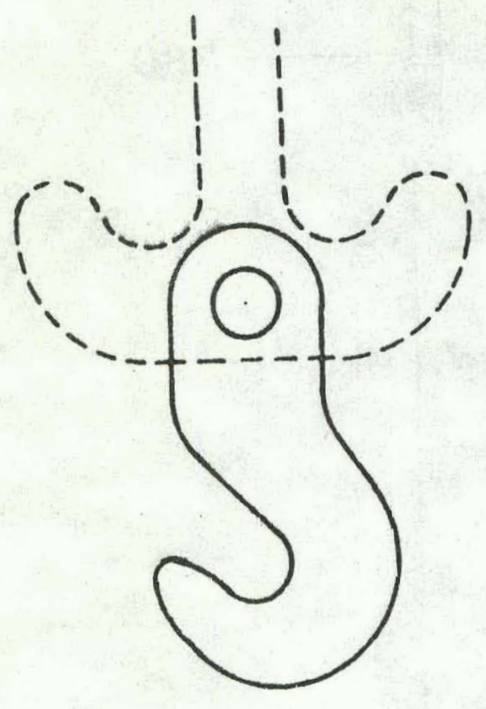

Single Palm Hook Attachment For Sister Hook

Figure B-1
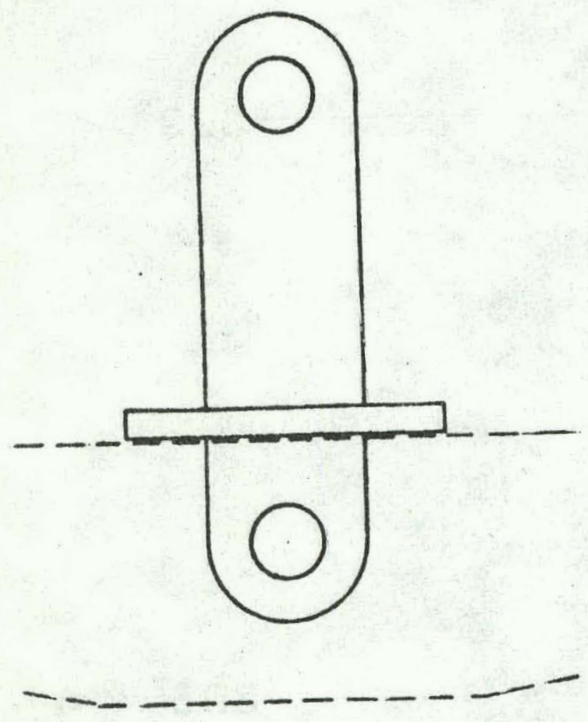
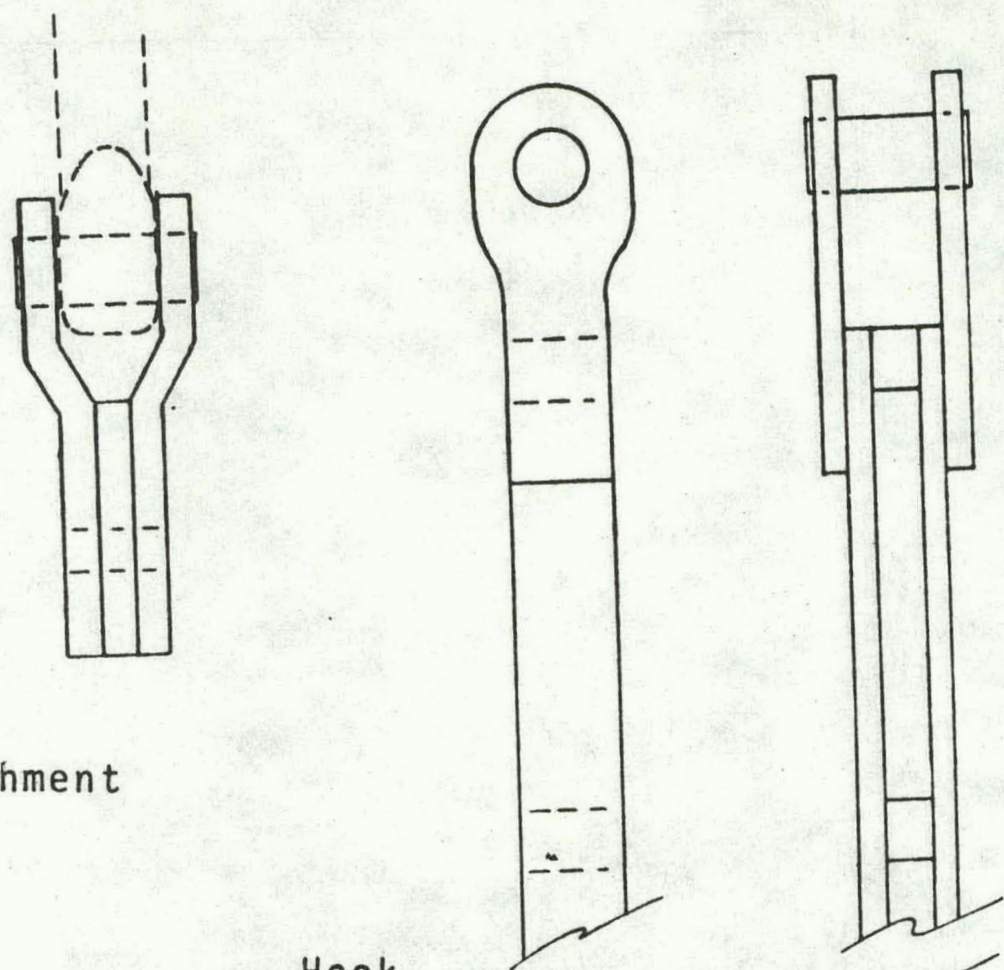

Hook Extension

Figure $B-2$

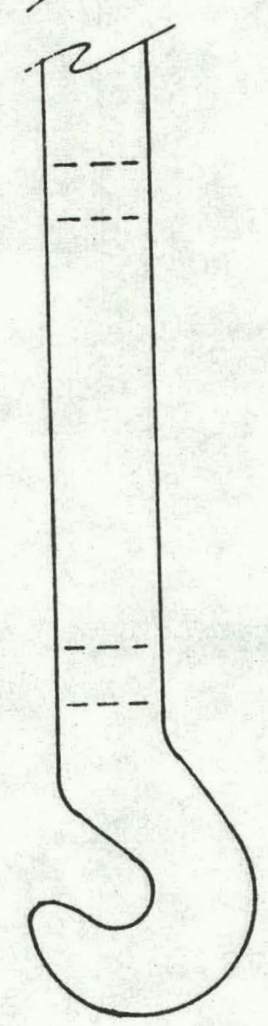

YOKE ATTACHMENTS

FIGURE $\quad 5-24$ 


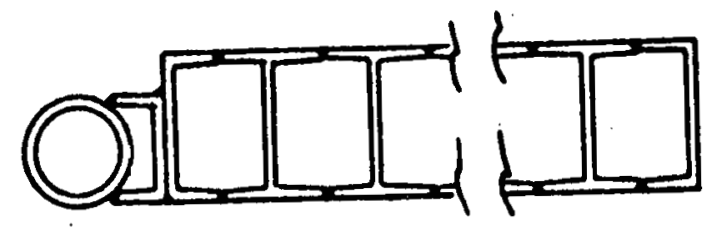

Shelf Cross Section

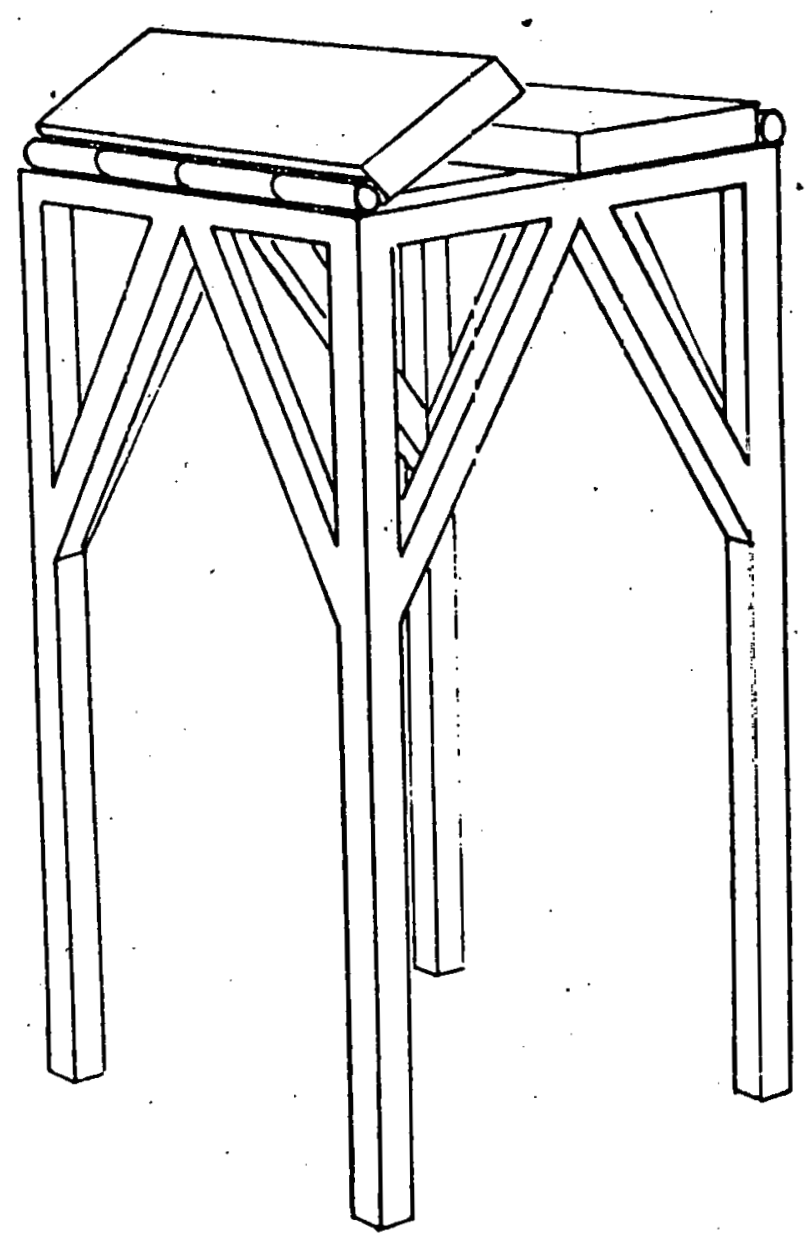

SHELF FOR CASK LOADING POOL

FIGURE 5-25 
(15) Reengage the main hook to the yoke and move the cask to the decontamination area.

(16) Perform the functions for preparing the cask for shipment.

(17) Move the cask to the vehicle.

5.4.4.4 Appendix B - Lifting Adaptors (See Figure 5-22)

Figure B-1 Sister. Hook Single Palm Extension - The sister hook of the cask handling crane is not particularly suited. to mating with cask lifting yokes in use today. Some of the available yokes, for specific casks, can use the lifting eye in the sister hook. If these yokes must be removed and attached very often, a lot of time will be used just in getting the pin in and out. An adaptor of the type, illustrated in Figure 5-24, will allow expeditious use with most of the existing yokes and with the other adaptors. If it is decided to used the folding shelf concept for cask handling, this adaptor should be used.

Figure B-2 Yoke Extension - The extension is used in lieu of wetting the block when placing the cask into the cask loading pool. The hook-to-yoke-tocask arrangement is used to lower the cask to a level that about covers the yoke. Then the extension is used between the hook and yoke to lower the cask the remaining distance to the bottom of the pool. The procedure is reversed for cask removal.

Figure B-3 NL Yoke Adaptor - The NL yokes use a lift pin through the strongbacks to mate with the palm of a hook or the lifting eye if that is what is available. The NL "A" yoke also features optional double pins, the spacing of which is designed specifically and exclusively for the sister hook of a whiting redundant lifting system. The lifting pin through the strongback is not a satisfactory system if the yoke must be detached and replaced even occasionally. A simple adaptor left permanently in place (but removable) allows the yoke to be engaged by the single palm hook without withdrawing and inserting the lifting pin. 


\subsubsection{Appendix C - Cask Sit-Down Shelf (See Figure 5-25)}

This structure is a framework of $\varepsilon$-inch box sections with a split platform on top hinged at opposite edges. The platform is made of 10 inch, 22 pound "I" beams welded edge to edge, about 13 beams on each side. All four edges of this weldment are closed by welding 10-inch channel, as shown. A piano-type hinge is used. The platform, or shelf, is airtight and the buoyancy effect is about $85 \%$, so little effort is needed to raise them. This would be done by hydraulic (water) cylinders, controlled from the surface. The structure fits as snugly into the pool cavity as construction tolerances will permit. Any three beams will support the 25-ton cask, assuming independent end supported beams and center loading. The cask bottom diameter will always cover at least 7 beams.

When folded, the opening into the bottom of the pool will be just under 9 feet square instead of 10.5 feet square. This is adequate for handling LWT casks and their lifting yokes.

When in use, the pool will be full and the shelves will be in the closed position. The cask will be lowered to the shelf level and the yoke released. An extension will be added between the hook and the yoke, and the yoke reattached to the cask. The cask will be raised 4 or 5 feet to allow the shelves to fold back against the pool walls. Gravity actuated safety latches will hold the shelves against the pool walls, even though the hinge placement of each shelf is such that the shelf center of gravity is nearer the wall than is the centerline of the hinge. The shelves remain in this position until the cask is brought out of the pool. The cask is raised to about 4 to 6 feet above the shelves, then the shelves are made to close. The cask is set down and the extension is released from the yoke. The hook is reattached to the yoke and the cask is removed. The shelves remain closed awaiting the next loading cycle.

The diagram (Figure 5-25) is conceptual. Load distribution at the bottom of the pool must be taken into account, particularly for drop-accident conditions.

Judging from the depth of the pool., it is likely that the plant architect-engineer intended eventually to install a 4to 5-foot thick crash pad on the floor of the pool. Not only would this affect the design of the base of the support structure but also would affect the length of the proposed extension for the hook (Figure 5-24). 


\subsubsection{Conclusions and Recommendations}

\subsubsection{Cask Selection}

Shipping cask certification for damaged fuel should be directed initially toward casks of the single assembly truck cask (LWT) design, because:

- The cask certification amendment at the NRC should be more readily accomplished.

- Fuel being shipped to examination facilities for research facility must move by truck casks due to facility limitations.

- The remote handling of the heavy closure heads on rail casks is very difficult, even with special handling tools.

- Information developed for the single-assembly cask will be useful in certifying multi-assembly casks if it becomes apparent that certification of the latter is warranted.

Regulatory controls and inconsistencies at the local level weigh heavily against the use of overweight truck casks.

\subsubsection{Recommended Actions}

- The selection of a specific highway cask may be limited by the size of the canister within which the fuel is shipped. Cask selection and canister design should proceed with the responsible parties in close communication.

- When the cask selection is finalized, prepare detailed casks handling procedures for each. This will cause potential operating problems to surface, including the need for additional special tools.

- Perform "dry runs" at the Tír facility with the cask selected. It would be particularly desirable to do this before the cask handling area becomes excessively contaminated. A dry run will help train the operators and will provide a final check on the adequacies of the operating procedures.

- If the cask is to be loaded in the fuel service pool, and if the fuel handling bridge is to be used, the fuel masts will have to be shortened to clear the top of the cask. (Neither "if" is considered very likely.) 
5.5 Spent Fuel Shipping Logistic and Safety Considerations

$\underline{5.5 .1 \quad \text { General }}$

It has previously been assumed that all of the fuel from the TMI-2 reactor will eventually be canned and moved off-site in a shipping cask to either an interim hot cell location for laboratory-type examination, or to an interim pool storage location. The pool storage may be preparatory to reprocessing or wet storage.

In the current "millieu" associated with the shipment of nuclear spent fuel, there is almost as much uncertainty with attaining the requisite approvals for shipping as there is in finding a suitable storage location. It is recognized that the fuel itself will probably not be moved until 1983 or later. However, the problems which have been fomenting in the area of spent fuel transportation have existed and perhaps become worse in the last 3 to 5 years. As a result, a current evaluation of the shipping considerations concerning cask availability, routing, estimates of the shipping duration, and costs which are incurred can probably be accurately projected.

The specific areas examined are:

- Can fuel be shipped off-site?

- How many canisters will be shipped and how much fuel can be shipped in a given spent fuel cask?

- What are the possible or likely shipping distances?

- What is the expected duration of the shipping campaign?

- What type of routing problems and restrictions can be expected?

In the preceding portion of this study (Section 5.4), it was noted that both truck and rail shipments are theoretically possible. Also, it is believed that the TMI site could accommodate all of the NRC-licensed casks. However, truck shipment (LWT) was shown to be the preferred alternative because of its greater operational flexibility and due to our belief that it's NRC Certificate of Compliance could be more easily amended for this type of failed fuel shipment. The logistics studies to be presented show that rail shipments, while requiring less operational time and fewer shipments, will probably involve a more lengthy shipping campaign. Under ideal conditions, the comparative costs between rail and truck shipments are expected to be within 
$10 \%$ of each other when the shipping distance exceeds 500 miles.

\subsubsection{Shipping Campaign}

From two to four truck shipping casks should be made available for this operation. Whereas there is no specific constraint concerning the length of time to remove all of the fuel from the TMI-2 site, a reasonable period is 18 to 24 months. For scoping purposes, four transport distances 300 miles, 750 miles, 1500 miles, 3000 miles - were examined. Table 5-9b presents shipping cycle times and the total shipping campaign duration utilizing either two; three, and four truck casks or one 7-element rail cask. Even though there are 177 assemblies to be shipped, it was assumed that failed fuel considerations would lead to approximately 308 more packages (225 to 235 shipments). As can be seen from this table, a bare minimum of either two truck casks or one rail cask would be required to accomplish these shipments within a reasonable time period for even movement to a nearby reactor. For shipping distances in excess of $1500 \mathrm{miles}$ from the TMI site, at least three to four truck casks or again one rail cask would be desirable.

A further question to be examined is the requisite number of casks available. Table 5-10 is a listing of the currently licensed spent fuel casks now available. The current commitment for utilization of these casks is not known. It will probably depend upon the national availability of an AFR location or the widespread transshipment of spent fuel between reactors with suitable storage space. As noted previously, we recommend that new casks be identified and reserved for this purpose at the earliest practical time after a date for possible shipment is determined. Our opinion is that this equipment could definitely be made available if a firm commitment is made with the cask owner.

\subsubsection{Shipping Economics}

There are a number of uncertain factors involved with estimating spent fuel-shipping costs. Table 5-11 presents the assumptions used to scope the approximate costs to move all of the fuel from the site. The only remaining factor to be evaluated parametrically is the actual distance from the site to the receipt location. Figure $5-26$ is an estimate of the approximate unit cost $(\$ / \mathrm{kgu})$ of shipment, and the total cost for shipping the fuel core. This data was factored into the various possible disposition economics scenarios discussed in sections 6.0 and 7.0. Shipping costs from an interim storage site to a final or ultimate location was also considered in that evaluation. The cost of shipping can represent a significant portion of the near-term cost of 
TABLE 5-9b

SUMMARY OF SHIPPING LOGISTIC DATA

$\frac{\text { DISTANCE }}{\text { (MILES) }}$

300

750

1500

3000 $\frac{\text { CYCLE TIME, DAYS }}{\text { TRUCK }}$

$3 \quad 7.3$

$4.6-12.3$

$7.2 \quad 20.7$

$12.4 \quad 37.3$

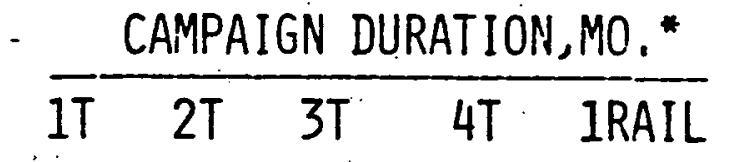

$23.0115-\quad-8.0$

- $17.311 .5-13.5$

- $27.618 .413 .8 \quad 22.7$

$\therefore \quad-31.723 .840 .8$ 
TABLE $\quad 5-10$

\section{CHARACTERISTICS OF LWR SPENT FUEL CASKS}

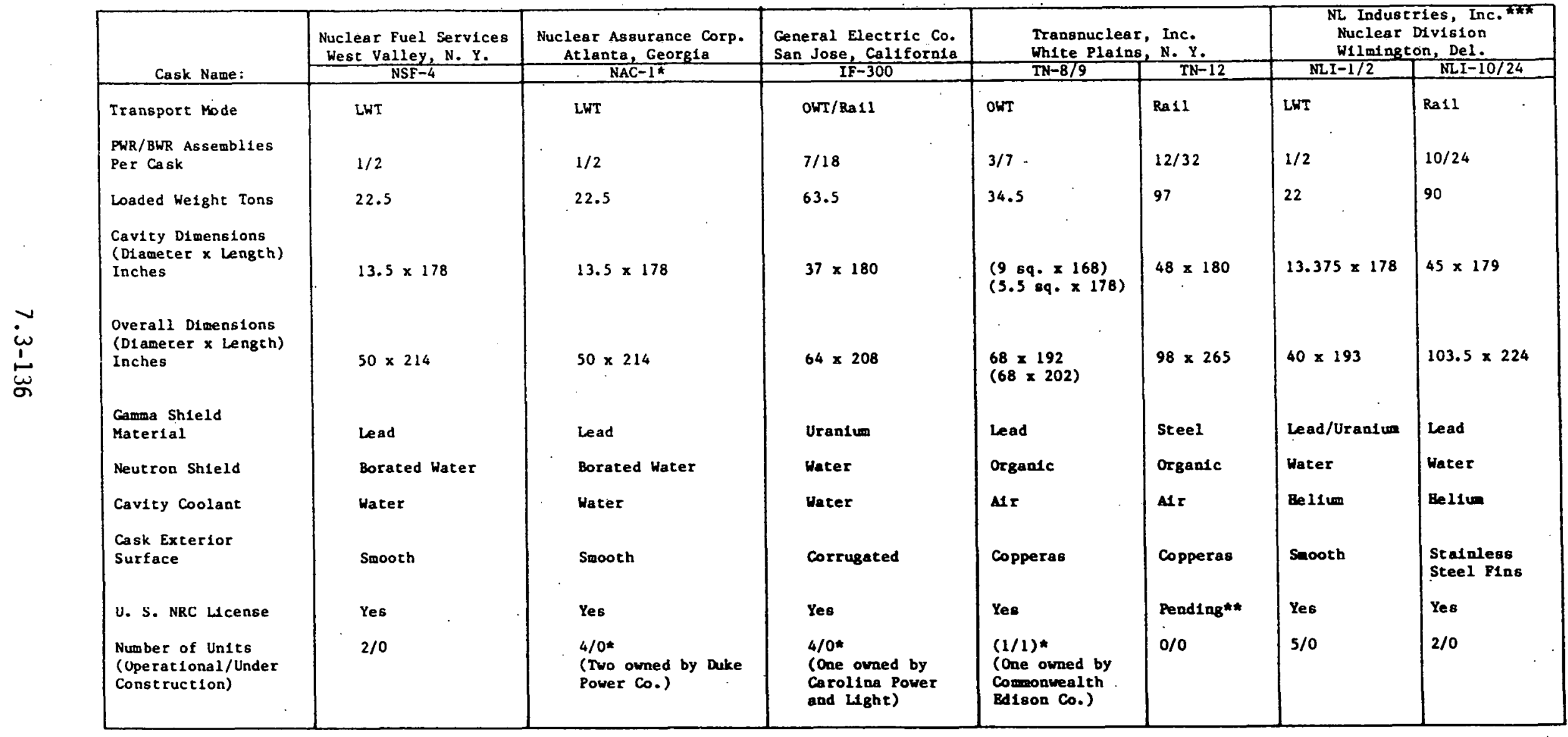

LWT - Legal Weight Truck

OWT - Overweight Truck.

*NAC- 1 and the NFS-4 cask designs are essentially Ident1cal.

$\star \star \star N L$ Industies $1 \mathrm{~s}$ not in this business $(9 / 30 / 80$ ) and is considering leasing equipwent to NAC (At lanta, (ieorgia). 


\section{TABLE 5-11}

\section{SPENT FUEL SHIPPING ASSUMPTIONS}

1. CASK LEASE COSTS

TRUCK CASK

$\$ 1000 /$ day

RAIL CASK

2. AVERAGE TURNAROUND TIMES AND SPEEDS

TRUCK - SPEED

TMI TURNAROUND

RECEIVING FACILITY TURNAROUND

$30 \mathrm{MPH}$

24 Hours

24 Hours

RAIL - SPEED

TMI TURNAROUND

$6 \mathrm{MPH}$

RECEIVING FACILITY TURNAROUND

48 Hours

48 Hours

3. SHIPPING TARIFFS *

TRUCK

$\$ 2.25 / \mathrm{Mile}$

RAIL $\$ 16.00 / \mathrm{Mile}$

4. AVERAGE SHIPPING CASK CAPACITY (TMI-2 FUEL CANNED)

TRUCK

$0.4 \mathrm{MTU}$

RAIL

$2.5 \mathrm{MTU}$

5. SHIPPING DISTANCE IS $15 \%$ GREATER THAN NORMAL-ROUTE MILEAGE .

*With special safeguard provisions. 


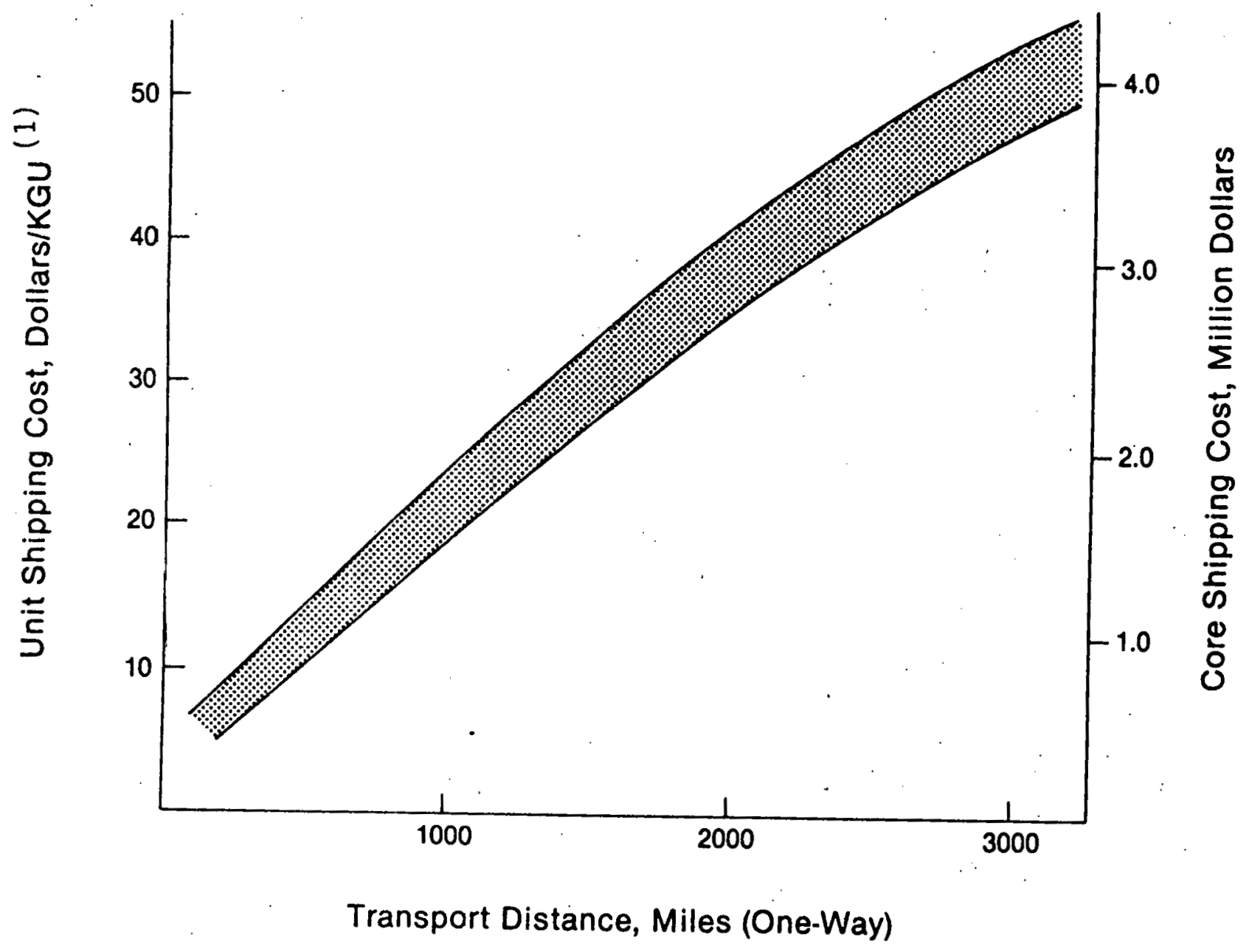

(1) TMI-2 Core Inventory is $82,700 \mathrm{KgU}$.

CURRENT ESTIMATES OF SPENT FUEL SHIPPING COSTS (EXCLUSIVE OF CANNING)

FIGURE $5-26$ 
disposition of the TMI core. This is particularly true if the fuel were to be shipped to a distant location.

5.5 .4 Institutional problems Involved with spent Fuel Shipping

There are currently many factors at work within the United States to make shipping of spent fuel, either by truck or rail, a difficult and time consuming operation. References 5.11 and 5.12 list some of the current problems for socalled "normal" spent fuel shipments. Table 5-12 highlights the major areas to be considered.

- Cask Licensing - The NRC Certificate of Compliance for any of the casks noted in Table 5-10 will have to be amended to handle the TMI fuel. It is expected that only canned fuel will be shipped.

- Routing Considerations - Reference 5.13, the NRC regulation guide on safeguards, and Reference 5.14 proposes DOT routing provisions*, delineates a number of provisions related to the movement of spent fuel. These provisions include specialized routing, the use of surveillance vehicles, routing preapproval by state officials, and embargoes of major population centers.

The most problematic concern for the TMI core disposition will probably be, related to routing of shipments around the periphery of major population centers. Several case studies were performed for this study by Mr. David Jcy of Oak Ridge National Laboratory looking at possible receipt locations of the TMI fuel. Figure 5-27 illustrates (a non-TMI routing plan) the number of cities which must be bypassed to accommodate the NRC (and possibly DOT-MTB) regulations. The typical routing expected is longer than the normal route distance by about 15-25\%. This will be reflected in the relative inconvenience of the operation and perhaps the overall duration of the shipping campaign.

There are also shipping restrictions related to the weight of the package being shipped and the size of the vehicle. Currently, the legal weight truck minimum in the united States is 73,280 pounds GVW (gross vehicle weight). Since 1977, there has been a "push" to extend this to 78,000 to 80,000 GVW for federal highways. Figure 5-28 illustrates the states that have not increased to 80,000 pounds and

\footnotetext{
*DOT-MTB Docket HM-164, Highway Routing of Radioactive
} Materials. 
TABLE 5-12

\section{SUMMARY OF SPENT FUEL SHIPPING INSTITUTIONAL FACTORS}

IRUCK (LEGAL HEIGHI)

- MOST SUBJECT TO LOCAL INTERVENTION DUE TO VISIBILITY ON HIGHWAYS

- ROUTINGS - MUST BYPASS URBAN AREAS > 100,000 PEOPLE

- DOT/NRC - INTERFACE WITH RESPECT TO USE OF HIGHWAYSSECONDARY ROADS

- DOT HEARINGS CURRENTLY UNDERWAY CONCERNING ROUTING-LOCAL EMBARGOS, ETC. (DOT-MTB, HM-164)

- LARGE NUMBER OF SHIPMENTS ( 225 - 12-24 MO. DURATION REQUIRED)

- WILL PROBABLY NEED 3-4 IDENTICAL CASKS - ARE THEY AVAILABLE?

- HANDLING AND LICENSING EASIER

OVERWEIGHI IRUCK

- SPECIAL hIGHWAY PERMITS NEEDED

- ONE CASK AVAILABLE

- UEDICATED FUEL CAVITIES (MODIFICATION BEING EXAMINED)

RAll

- IESS VISIBLE, LESS SHIPMENTS

- OPERATIONAL haNDLING MORE DIFFICULT LEADING TO MORE SITE INTERFACE PROBLEMS

- LICENSING DIFFICULTIES ARE GREATER

- RAILROAD-NUCLEAR INDUSTRY CONFLICTS STILL POSSIBLE

GOVERNMENT CASKS

- NOT SPECIFICALLY DESIGNED FOR COMMERCIAL LWR FUEL OR FACILITIES

- POTENTIALLY USABLE FOR SHIPPING DEBRIS IN SHORT CANISTERS 


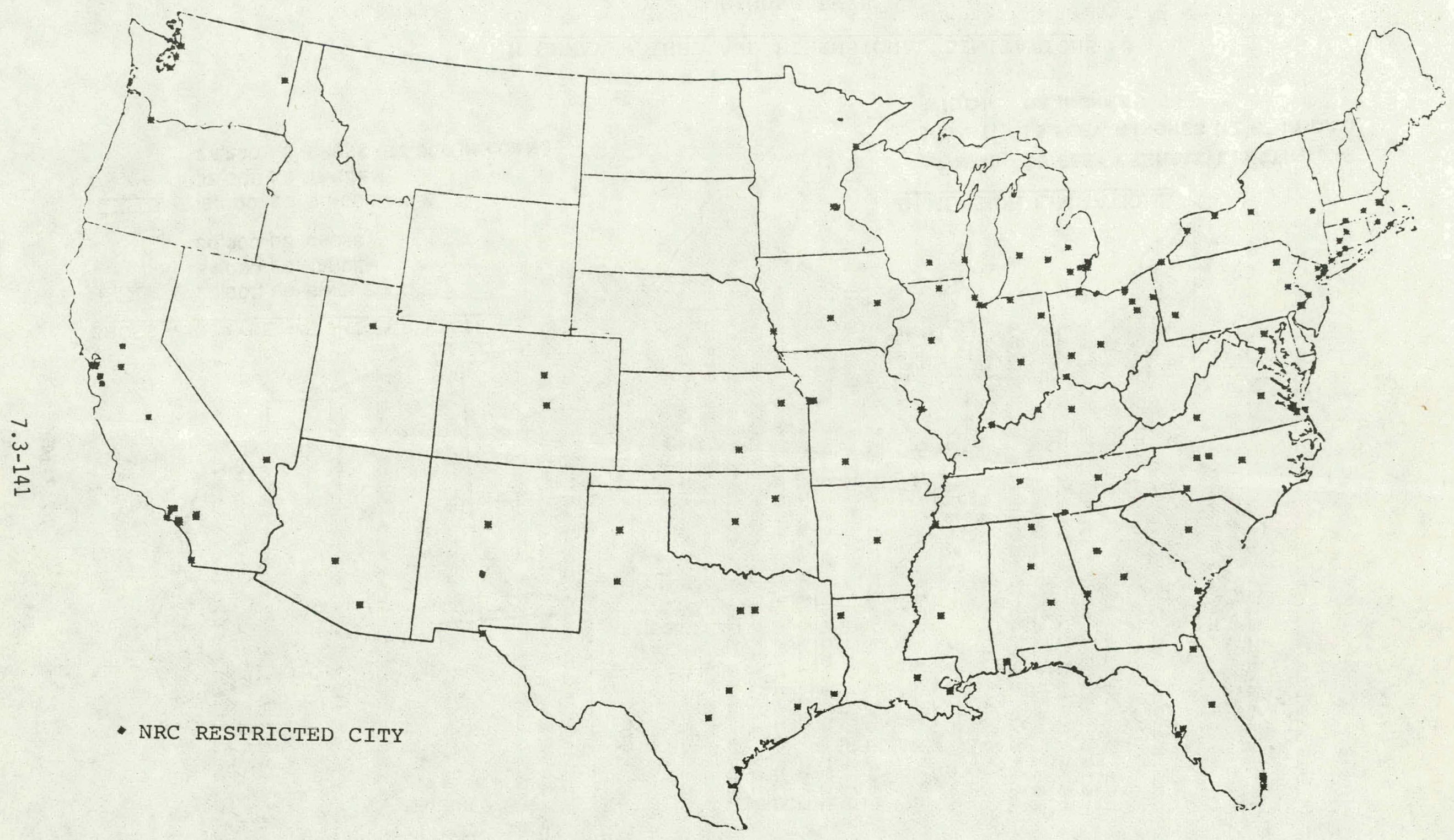

NRC RFSTRICTED CITIES

FIGURE $\quad 5-27$ 


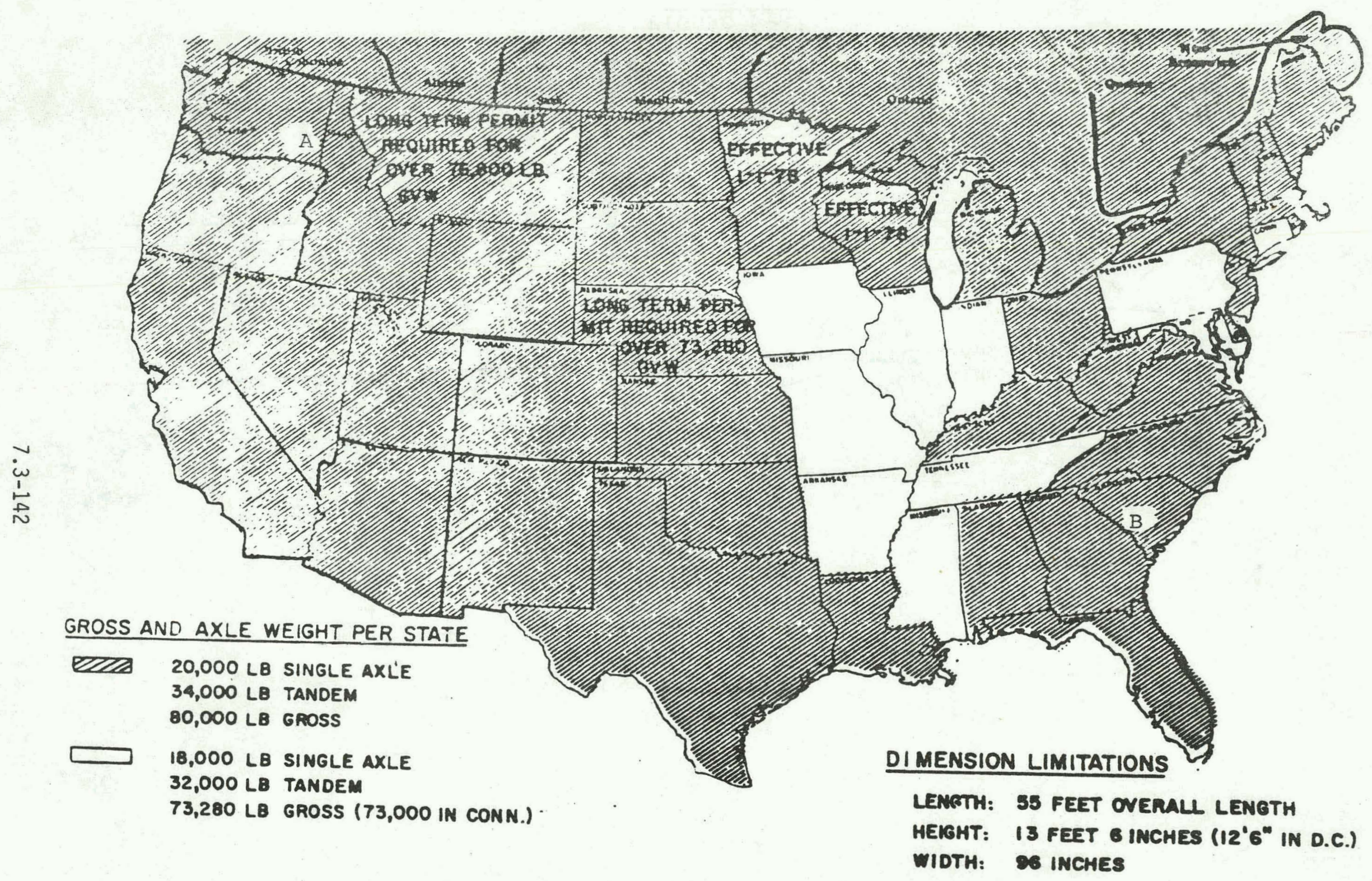

HIGHWAY WEIGHT AND DIMENSIONAL LIMITATIONS

FIGURE $5-28$ 
shows that a literal "chain" stretches across the "midU. S." This limits any major overweight "East-to-west" traffic flow. Also limiting on the East coast (for North-to-South movement) are the states of Connecticut, Maryland, and Pennsylvania. This consideration further emphasizes the difficulty in emplcying overweight truck shipments (typically 100 to 105,000 GVW). from TMI where special highway permits would be required. [Note: spent HTGR fuel has been moved in the early $1970^{\prime}$ 's from the Peach

Bottom reactor (also in Pennsylvania) using a 105,000 GVW shipping cask system.] Most LWT spent fuel casks are designed to be very close to the 73,280 pound weight limit. Canned fuel and shipping containers may cause this 1 imit to be exceeded. (This should be examined as a portion of a study of cask/shipping can interface study.)

Routing 1 imitations are affected by local ordinances and prohibitions against radioactive material shipments. At present, there are over $100 \mathrm{such}$ local ordinances. oak Ridge National Laboratory maintains a listing of these localities. Since the "picture" is changing constantly, there is no need to present that data here. It should be noted that the proposed DOT regulations in HM-164 would assert federal "authority" over interstate shipments and, while not simplifying the logistic problems, should at least present a national coordination of radioactive material routing.

5.5.5 Safety and Environmental Aspects of Spent Fue 1 Shipping

There has been widespread movement of radioactive material throughout the united states for over thirty years. However, the majority of the material shipped has been low level (in particular, waste material) and small quantities (short-lived medical isotopes). High-level shipments such as spent fuel have been far less, and rarely have amounted to more than one million miles per year over the road, including both defense wastes and fuel and commercial power reactor spent fuel. To date, although there have been accidents with radioactive material shipments, there has been no release of high-level nuclear material. The principle underlying shipping safety is containment of the radioactive material during a sequence of extreme "hypothetical" accidents which in summation are far more severe and improbable than any naturally occurring accident. These scenarios are described in 10 CFR 71 and 49 CFR 171.

To look at actual safety or environmental impact of any given shipment, it is necessary to examine the specifics of the situation. This can be accomplished in three stages: 
- Accident probability

- Accident scenario

- Shipping cask/radioactive material design and interface.

These areas are developed in the following paragraphs.

\subsubsection{Accident Probability}

Feference 5.16 examined the probability (based on statistics cf billions of highway transport miles and thousands of accidents) of a loaded truck being in (1) any accident and (2) being involved in a serious accident. The latter category does not equate to the "hypothetical". accidents previously discussed. In fact, they are typically far less severe. The exact definition is presented in Table 5-13. The probabilities are:

(1) Any Highway Accident -- $2.5 \times 10^{-6} / \mathrm{mile}$

(2) A Severe Accident $-7.2 \times 10^{-9} / \mathrm{mile}$.

Table 5-13 evaluates the probabilistic frequency of (1) any accident and (2) a severe accident for the TMI-2 shipping scenario. This table indicates that a minor accident (fender-bender) could occur. However, the probability of a highway accident of any major damage to the transport vehicle (other than to flame anti-nuclear hysteria) is very small. Statistics for rail transport are in the same order of magnitude, but since there are less shipments to move the same amount of material, a lower probability of an accident would result.

\subsubsection{Accident Scenario}

Reference 5.17, NuReg/CR-0811, is a scoping study which examines the generic technical aspects of spent fuel cask transportation accidents. The study looks at the following factors:

(1) How is the radioactive material released from the fuel cladding?

(2) How is the material released from the spent fuel cask?

(3) What accident scenario is required to breach the cladding or the cask containment?

(4) How much and what type of material would be released?

(5) What are the mechanisms for material dispersion (i.e., wind, fire, environment, etc.)?

(6) What is the biological effect? 
TMI-2 SPENT FUEL SHIPMENT ACCIDENT PROBABILITY SUMMARY

1. SEVERE ACCIDENT DEFINITION

$\frac{\text { Collision }}{\text { Vehicle Speed, MPH }}$

$$
\begin{gathered}
0-50 \\
50-70 \\
>70
\end{gathered}
$$

$$
\begin{gathered}
\frac{\text { Fire }}{\text { Fire Duration, Hour }} \\
\begin{array}{c}
>1 \\
1 / 2-1 \\
0-1 / 2
\end{array}
\end{gathered}
$$

2. SHIPPING MILEAGE ESTIMATE

- Shipment to interim storage from TMI (750 miles $\times 225$ shipments) $=168,750$ miles

- Shipment to geological repository from interim storage (1500 miles $\times 225$ shipments) $=337,500$ miles Total $=506,250$ miles

3. PROBABILITY CALCULATION (BASED ON 225 SHIPMENTS)

(A) Any Accident $(506,250$ miles $) \times\left(2.5 \times 10^{-6}\right)=1.25$

(b) Severe Accident $(506,250$ miles $) \times\left(7.2 \times 10^{-9}\right)=$ .0036 . 
The results of this study are abstracted below:

"Calculations were performed to estimate cask and fuel assembly failure thresholds when faced with various challenges, mainly impact environments. The calculations indicated that gross cask failure is unlikely, and that such a conclusion can be demonstrated. It was also seen that the failure threshold for fuel rods subjected to impact is probably much lower than the cask itself.

Calculations were also performed to develop a range of radionuclide source term estimates for several "extra severe" scenarios which would reflect the inherent uncertainties that exist in data or models concerning fuel and fission product behavior. Possible effects on public health of the variability of these source terms were examined by performing radiation dose calculations using standard environmental transport and dose evaluation models. The results were used to indicate which portions of the radiological source terms have the greater influence on estimated public health impacts. Findings showed that the potential release of respirable fuel dust has the greatest effect on radiation dose estimates, particularly with respect to the lung and bone organs. In addition, if the cesium and/or ruthenium fission products should exist as volatile species, these materials would also become significant dose contributors. The other volatile fission products (krypton, iodine, and tellurium) were found to be minor dose contributors under nearly all release variation conditions."

This study defines the low probability of a severe accident breaching the cask containment occurring and the relatively minor radiological impact. The study also shows that the form of the fuel is relatively unimportant from a structural standpoint, except where the release of "fine" particles is possible. This latter factor emphasizes the need of separately canning fuel debris.

5.5.5.3 Specific Shipping Cask/Radioactive Material Form

Even though the specific cask and fuel form are not known, certain "limiting" assumptions can be made for the TMI-2 situation:

(1) I. legal weight truck spent fuel cask is employed. 
(2) An additional container or shipping can is employed.

(3) The fuel cladding is failed. Some of the fuel debris is in a "fine" particulate form. However, all of the free $\mathrm{Kr}-85$ and other volatile fission products have been released. Also, most of the cesium has been leached out by the water in the core primary. loop.

(4) The fuel is shipped "dry."

(5) The fuel has aged for over three years and has a decay heat load of about 0.2 kilowatt per cask or less.

In this scenario, clearly the greatest accidental danger (biological) is the release of the "fines" present in the debris. It is generally. felt that the primary cask cavity could prevent the release of this material. However, a sealed can would serve as an additional barrier to release. The inner container or can is also required for fuel storage and handling purposes. We feel that the NRC would require canning of damaged fuel shipped off-site. 
REFERENCES

5.1 Three Mile Island - Unit 2, Final Safety Analysis Report.

5.2 Nuclear Safety Guide - TID-7016, p. 44, Rev. 2 (June 1978).

5.3 ORIGEN - Computer Program, (AGNS Memorandum - TMI-2 Fuel Characteristics - Gamma and Neutron Source Items, NT-80-63 (March 1980).

5.4 Rogovin, M., et al, Three Mile Island-A Report to the Commissioners and to the Public, TMI 18:52, Volume 1 (April 1979).

5.5 Supplement to Analysis of Three Mile Island - Unit 2 Accident, NSAC-1 (October 1979).

5.6 AGNS Internal Memorandum: Waltz, W. R. , to Anderson, R. T., TMI-2 Fuel Characteristics - Decay Heat and Criticality Considerations, NT/80/86 (March 1980).

5.? Recommendations of TMI Examination Technical working Group Task 7.2 for the DOE/NRC/EPRI/GPU Joint Coordinating Committee (April 1980).

5.8 Telephone Communication - P. N. McCreery (AGNS) to G. Kunder (GPU).

5.9 TMI-2 Initial Planning Study for Contaminant Entry and Decontamination, Bechtel Power Corp. (July 1979).

5.10 TMI-2 Planning Study for Phase II - Reactor Internals Disassembly, Fuel Removal and Cleanup, Bechtel Corp. (May 1980).

5.11 Anderson, R. T., Nuclear Spent Fuel Transportation Studies, AGNS 35900-1.4-59 (November 1979).

5.12 Nuclear Shipments - The Going is Rough, Nuclear Industry (November 1979).

5.13 Physical protection of Shipments of Irradiated Reactor Fuel, NuReg-0561 (September 1979).

5.14 Proposed Legislation - Department of Transportation HM-164 on Shipment of Radioactive Materials.

5.15 Clarke, R., et al, Severities of Transportation Accidents, SLA-74-0001, Sandia Laboratories (July 1976). 


\subsection{TMI-2 FUEL DISPOSITION OPTIONS}

\subsection{Introduction}

- There are a number of potential alternatives for disposition of the damaged TMI-2 core. After canning is completed, the overall questions regarding disposition of TMI fuel are not significantly greater than for any other current-generation LWR fuel. Some of the possible options are interim solutions, since final disposition of LWR spent fuel in the United States is not expected to be resolved until 1990 or later. Temporary storage at the TMI fuel pool, preparatory to either the proposed diagnostic evaluation of selected fuel segments or to total off-site shipment, is expected. This study examines the technical aspects of alternative short- and long-term disposition options. Specific focus is directed towards the current state-of-the-art, industry, and technical problems, and possible variations in operations and techniques at various sites. Also included are generic institutional problems related to these alternatives. The study is generic and does not examine specific sites other than to portray possible technical problems.

Three disposition alternatives were studied and are noted below:

(1) Chemical Reprocessing - Until 1977, it was generally assumed that all spent LWR fuel would be chemically reprocessed to recover the useful uranium and plutonium. This is obviously not the current situation. However, reprocessing at a later date is certainly a possibility. This study examined the current status and technical factors associated specifically with reprocessing of TMI-2 fuel. Discussion is made of both the variations in chemical processes developed for domestic governmental and commercial facilities. The study also examined variations to plutonium recovery such as coprocessing or partitioning of the plutonium with the waste. We project that domestic reprocessing would not be available until 1990; hence, an interim period of wet storage would be required for the 5 to 6 years prior to the initiation of reprocessing. Another possible option is foreign reprocessing.

(2) Wet Storage - Most spent LWR fuel is currently being stored in pools at operational reactors and governmental facilities. The TMI-2 Euel will be stored under pool conditions at the TMI facility awaiting possible off-site shipment. Long-term wet storage of fuel either at a reactor or an AFR, preparatory to final disposition in the spent fuel form, is also likely. 
Wet storage of spent fuel has been common practice since the mid-1950's, and few technical problems are foreseen. The damaged TMI fuel will, however, require canning and storage in special spent fuel racks.

(3) Dry storage - This option consists of taking canned TMI-2 fuel and locating it within either a storage vault or hot cell, specially designed spent fuel or concrete caissons. There is little domestic experience with this type of storage. Due to the low decay heat load of the fuel, the small quantity of fuel which has to be handled, and the relatively low source term. The possibility of interim dry storage of at least portions of the fuel core is a real consideration. In addition, the research examination of some of the fuel will be performed at various hot cells under dry conditions.

Each of these options is examined in this section, and each appear to have certain merit. Table 6-1 summarizes the most important factors for each. In the near term, actual disposition options must consider the wet and dry storage alternatives.

The evaluations presented in this section focus primarily on the technical details of storage and reprocessing. In all off-site conditions, the interface with the spent fuel casks being utilized to deliver fuel and remove fuel from these facilities must be considered. In addition, the effect of the canning operations at the TMI-2 fuel pool must also be included. Due to the uncertainty in the site(s) to be utilized, these interface studies have of necessity been generic. However, it is believed, based on prior experience, that the major technical factors have been highlighted.

\subsection{Chemical Reprocessing of TMI-2 Fuel}

6.2 .1 General

One of the alternatives available for the disposition of the damaged core at the TMI-2 reactor involves chemical reprocessing. By reprocessing fuel, the value associated with uranium and plutonium, separative work, and conversion steps is recovered and economic benefits realized when these materials are reused through recycle. Since the fuel value for IMI-2 level with low burnup is greater than the estimated costs associated with reprocessing, an economic incentive exists for the reprocessing option. The cost associated with domestic commercial reprocessing has been estimated to be approximately $\$ 265 / \mathrm{kilograms}$ uranium $(1980$ dollars). This estimate includes costs for the separation and purification processes and the uranyl nitrate conversion 


\section{DISPOSITION ALTERNATIVE COMPARISON}

1. CHEMICAL PROCESSING

adVANIAGES - ECONOMIC VALUE OF $2.2 \%$ ENRICHED U-235

- IMPROVED WASTE FORM

DISADVANTAGES - EFFECT OF PLUTONIUM SEPARATION ON U.S. PROLIFERATION POLICY

- NOT AVAILABLE UNTIL 1990

2. POOL STORAGE

ADYANTAGES $\quad-$ PROVEN TECHNOLOGY

- LOW STORAGE SPACE REQUIREMENTS

DISADYANTAGES - PROJECTED LACK OF STORAGE SPACE BY 1983

- TEMPORARY SOLUTION

3. DRY STORAGE

ADVANTAGES - COULD MAKE USE OF AVAILABLE HOT CELL SPACE

- tMI fUEL heAt LOAD IS LOW AND fREe fISSION GASES RELEASED

- CAISSON STORAGE IS SITE-INDEPENDENT

DISADVANIAGES - NOT A LICENSED TECHNOLOGY

- TEMPORARY SOLUTTION 
to uranium hexafluoride $\left(U F_{6}\right)$. It was also assumed that these costs are incurred curing a period when the facility is fully operational and has processed light water reactor fuel. This approach is compatible with possible government ownership of a domestic reprocessing facility. The schedules which include fuel availability after 1985, following interim storage, would be on a similar ecconomic basis.

For each metric tonne of heavy meral recovered, cost savings are realized in areas of uranium not mined, purified, and converted to oxides, in enrichments above natural which results in less separative work, and in conversion to uranium hexafluoride $\left(U_{6}\right)$. This particular study assumes the recovered plutonium will be used in breeder fuel.

Initial estimates indicate that current domestic reprocessing costs will be approximately 20 to 25 million dollars for the 83 MTU of TMI-2 fuel. The value of this fuel when recycled is about $\$ 55$ to $\$ 60 \mathrm{million}$.

There are six reprocessing facilities within the United States which were examined and included as candidates for processing the TMI-2 fuel. Three of the candidate facilities were studied in detail, the other three having been eliminated by considerations of size, condition of plant, etc.

Reprocessing of spent fuel has been successfully demonstrated in overseas facilities located at Windscale and La Hague. They were not technically evaluated as potential candidates. This is partially due to our lack of detailed knowledge of their process, and the uncertainty related to resolution of differences between the United States proliferation position and international agreements. Preliminary data indicate that their reprocessing charges would be high and that overseas shipments and return of recovered product and waste would be both extremely costly and present institutional problems. However, because both foreign facilities are equipped with chop-leach headend and utilize the Purex process, they should be able to process the damaged core, with some headend modifications possible.

\subsubsection{Technical Review of Selected Reprocessing Facilities}

Government-owned facilities are available to handle either highly enriched or depleted to slightly enriched uranium. In the zirflex process, the zirconium cladding around the metal fuel is first removed in an ammonium nitrate-fluoride mixture followed by uranium metal dissolution in nitric acid. Fuel decladding by this method significantly reduces the processing rate. This rate could be improved if modern 
chop-leach equipment used for commercial fuel were incorporated into the facility. Subsequent processing operations, including the basic PUREX extraction, partitioning, and heavy metal purification steps, were also restricted for criticality control. The large process tanks and pulse columns limited uranium enrichment to 1 to 1.28 (note that TMI fuel enrichment is expected to range from 1.7 to 3.08 ). To process fuel with higher fissile content, significant modifications to process equipment would be required. The product forms have been uranyl and plutonium nitrate solutions which were converted to their respective oxides or plutonium metal.

Since the fuel from TMI-2 contains uranium eiriched up to ,38, processing would be accomplished using the HM flowsheet specifically modified to handle enriched fuel.

A chemical method involving mercury-catalyzed acid dissolution of the aluminum-uranium fuel is the normal approach for obtaining the heavy metal process solutions. This method would be suitable for uranium fuel contained in stainless steel or Zircaloy provided the claciding was ir small pieces to maximize the surface area of the fuel exposed to the dissolving solution. However, none of the government-owned process lines are equipped with a chop-leach headend which will require the alternate method of electrolytic dissolution to be used for the dissolution of integral fuel rods and assemblies. Since most experience with electrolytic dissolution has been with stainless steel c-adding, additional development work would be required before dissolution of zircaloy by this approach could be considered practical.

Once the heavy metals are in solution, the capabilities for performing any type of PUREX operation using any number of potential flowsheet conditions at government facilities are posisible. The only process related restriction is due to the lack of geometrically favored equipment which limits the process concentrations of $U-235$ to below 6 grams/liter. During dissolution specially designed inserts have been placed in the dissolver vessel to hold the fuel tubes in a critically safe configuration.

Purified product solutions of uranyl and plitonium nitrate can be converted to a variety of product forms. Both metal and oxide forms of plutonium are routinely made. However, only solutions of depleted to slightly enriched uranium can be converted to the oxides. Enriched uranyi nitrate solutions are shipped off-site before conversion to uranium hexafluoride.

Commercial reprocessing facilities are different from existing government facilities in that they were designed 
specifically to handle light water reactor fuel. This has necessitated compliance during process design with NRC regulations and criteria regarding design, construction, and release of liquid and gaseous effluents. Release limits are normally not as restrictive for government facilities although they may be required if commercial light water reactor fuel is processed in those facilities.

The canned fuel from the TMI-2 core would be remotely transferred from the storage pool to the feed mechanism of a shear where fuel segments are chopped into 2- to 5-inch lengths for maximum exposure to the acid dissolvent. After dissolution, feed solutions are clarified and adjustments made in acidity and heavy metal concentration to permit fuel processing using different flowsheet conditions. Partition of uranium from plutonium is performed electrolytically which reduces the concentration of contaminants in the product streams and lowers the volume of solid waste. Additional purification cycles are used in both the uranium and plutonium cycles with the products being purified uranyl and plutonium nitrate solutions.

There are no commercial facilities to convert the liquid plutonium nitrate stream to a solid form (plutonium oxide) or waste conversion facilities. The plutonium facility may not be needed if this stream is included with the waste or is coprocessed with the uranium.

\subsubsection{Preliminary Facility Assessment}

Nontechnical factors have had a significant impact on the reprocessing of commercial LWR spent fuel and could affect the selection of a facility for the processing of the TMI-2 core. Because commercial reprocessing of fuel has been deferred indefinitely, government ownership of a private facility appears to be a prerequisite. similarly, the reprocessing of privately owned fuel in a government facility, built and operated with public funds, would be difficult to resolve.

In terms of government facilities, the Hanford PUREX Plant is not currently operational and would require expensive equipment modifications to satisfy current operational and emission regulations. It would probably have to be licensed by the NRC for commercial light water reactor fuel. Modification and use of a currently operating government production facility would necessitate a reduction in weapons production capacity until the expensive process modifications could be implemented. This would probably be unacceptable. 
In order to properly evaluate the viability of the reprocessing option, specific information is required concerning fuel characteristics, reactor operating conditions, and the condition of the core. This in turn defines the final properties of the fuel and enables the canning, shipping, and storage alternatives to be compatible with the reprocessing requirements. Where necessary fuel condition information is not available, best assumptions have been made in this study.

6.2.4.1 Fuel Characteristics

Approximately 142 metric tons of solid material will be removed from the damaged core for reprocessing. Included are 83 tonnes of heavy metal actinides and 59 tonnes of cladding and nonfuel bearing components. The average enrichment of the uranium now in the three core regions is 2.28 with a maximum of 2.78 existing in Region 3. The maximum burnup occurred in Region 2 (3518 MWd/MTU) which produced approximately 2.1 kilograms of plutonium/MTU. The average core concentration of plutonium is $1.85 \mathrm{kilograms}$ of plutonium/MTU. Because of the low burnup, most of the plutonium exists as Pu-239 (91.3\% average), with remaining average isotopic concentrations of 7.18 for Pu-240, 1.58 for Pu-241, and 0.058 for Pu-242. Both the low burnup and long cooling period before reprocessing will result in optimum handling and processing conditions for the spent fuel.

Cladding materials and most of the nonfuel-bearing components associated with the fuel have low solubility in nitric acid solutions. Their concentrations in the product solutions will be insignificant compared to contributions from processing conditions and chemical additives. Most of the silver-indium-cadmium contained in the control rods will dissolve during the dissolution step. However, their low distribution coefficients in tributyl phosphate will result in removal with the high level liguid waste stream.

6.2.4.2 Assumptions

The following list of assumptions are those which have been made. Besides assumptions pertaining to fuel properties, those associated with core, canning, and storage operations are included when they have an impact on the reprocessing alternative.

1. The damaged core contains. Doth integral fuel rods/ assemblies and randomly mixed debris containing uranium fuel. 
2. Both types of fuel will be canned in containers suitable for a chop-leach dissolution headend.

3. A nondestructive analysis method will be used to determine the uranium content of the canned fuel prior to shipment. This information would be helpful but is not mandatory for the reprocessing option.

4. Interim storage of the canned fuel will take place until an acceptable facility is identified and a schedule approved.

5. Plutonium could make a positive contribution to the fuel worth through eventual use in breeder reactors.

6. Estimation of reprocessing costs for the TMI-2 core at a commercial facility is based on a schedule which projects imminent hot operation or is during a period when the facility is already operating.

7. The possibility of a commercial facility in a processing mode which would produce a purified plutonium product solution has not been eliminated.

\subsubsection{Fuel Reprocessing Alternatives}

The PUREX process is considered "standard" for spent fuel reprocessing. The basic steps include actinide extraction into a solvent, tributyl phosphate (TBP), which eliminates most of the fission products with the high-level aqueous raffinate. Next, uranium and plutonium are partitioned using chemical reductants or an electrolytic process of the tyce installed at the BNFP. The separated streams for the partition step undergo additional purification cycles with the final purified products being separate, uranyl nitrate and plutonium nitrate, liquid solutions.

For proliferation reasons, this approach to chemical reprocessing is not presently considered to be acceptable. Alternatives have been suggested and studies conducted at the BNFP and worldwide which have demonstrated a high degree of Elexibility for changing the basic PuREX process flowsheet to meet proposed weapons proliferation criteria.

\section{$6.2 .4 .3 .1 \quad$ Coprocessing}

This approach to chemical reprocessing involves partial partitioning where the uranium to plutonium ratio is controlled in the aqueous stream from the partitioning step. For the TMI fuel, this would involve all of the plutonium remaining with the uranium product, resulting in only one prodict stream. A typical ratio for breeder application is 
20 to 258 plutonium. This coproduct may then undergo additional purification from fission products, depending on whether the low or high decontamination flowsheet is used. For the low decontamination flowsheet, a controlled amount of fission products is extracted with the actinides and remain with the coproduct stream without further decontamination. With both the low and high decontamination flowsheets, the organic stream containing the bulk of the uranium from the partitioning step undergoes additional fission product purification. Therefore, the product forms from the coprocessing alternative are a pure uranyl nitrate solution and a uranium-plutonium coproduct solution with or without a controlled level of fission product impurities.

\subsection{Plutonium with High-Level waste}

The final alternative for the plutonium would be to discard it with the high-level waste. This could be accomplished using the high decontamination coprocessing flowsheet. After the plutonium is partitioned from the uranium, the aqueous stream would be sent directly to waste.

The process high-level waste typically consists of a nitric acid solution containing the fission products and particulate matter removed from the process streams. The major point of origination is the dissolver in the chop-leach portion of the process. The plutonium nitrate stream would be separately partitioned and then diverted and mixed with the dissolver liquid. The mixture of the two solutions would be stored in waste tanks pending national resolution of the disposal of nuclear high-level waste material.

\subsubsection{Plutonium Questions}

The major question which must be resolved before the damaged core at TMI-2 is reprocessed concerns the "fate" of the plutonium. Since the economics for reprocessing are improved if the plutonium could be used in breeder fuel, this is the preferred position and offers the greatest economic incentive. Even if plutonium is not recovered, the value associated with the uranium alone is considerable. This is particularly true for the TMI fuel where the enrichment value is high (2.28 average). A suitable product containing plutonium would be obtained from the coprocessing alternative or could result using a reprocessing flowsheet where no partitioning between uranium and plutonium occurs. This would involve a series of extraction and stripping cycles to remove the fission products, resulting in a purified uranium-plutonium coproduct solution in the same concentration ratio as the feed solution. In this form.the uranium-plutonium mixture would have little value. A significant conversion effort would be necessary to produce the 
20 to $25 \%$ plutonium mixture needed for breeder application. This approach is too expensive to justify further consideration. The economics for the reprocessing option would also improve if the government were to purchase the recovered plutonium. This acquisition currently seems unlikely.

The final alternative for the plutonium would be to discard it with the high-level waste. This could be accomplished ising the high decontamination coprocessing flowsheet. After the plutonium is partitioned from the uranium, the aqueous stream would be sent directly to waste.

\subsubsection{Safety Considerations}

With each of the candidate reprocessing facilities and with each of the alternate reprocessing flowsheets, safety considerations were evaluated. The major concern inside the reprocessing facility is criticality control. External to the operations is control of the gaseous and liquid enissions.

\section{2 .6 Product and waste Handling Alternatives}

Each of the candidate facilities has different capabilities for handling the products and wastes generated during reprocessing.

At the present time, however, suitable facilities do not exist for the long-term storage of coproduct solutions or mixed oxide which would eventually be converted to breeder fuel. Use of a government-owned facility would require resolution of the question as to whether wastes from commercial fuel can be solidified along with wastes from weapons production.

\section{2 .7 Conclusion}

Chemical reprocessing of the damaged core at $\mathrm{TMI}-2$ is a possible disposal alternative. Favorable economics could be realized solely through recycle of uranium and do not depend upon the recycle of plutonium. Even if the plutonium were to be discardea into the high-level waste, the fuel worth of the enriched uranium could justify its recovery. However, the economics would be improved if the plutonium were eventually used in breeder reactors.

The technical aspects associated with reprocessing of the TMI fuel have been studied, and it has been verified that the fuel could be reprocessed. Any of the three following scenarios are possible: 
- Normal Reprocessing - Separation ¿nto distirict uranium, plutonium, and high-level waste streams.

- Coprocessing - The plutonium stream remaining with the uranium, and the high-level waste streams as normal reprocessing.

- Plutonium as Waste - The uranium waste stream is distinct (as in normal reprocessing) and the plutonium remains with the high-level waste stream (high-DF flowsheet).

The processing or chemical separation process can accommodate the damaged physical form of the TMI fuel and the incorporation of nonfuel material (control rods, burnable poisons, etc.) with the fuel stream. The canning of the fuel of the TMI reactor must be such as to accommodate the mechanical "headend" of the Separations Facility to permit the "chop-leach" to be performed.

Institutional problems related to the following questions prevail:

1. When will domestic reprocessing be available (if ever)? This is estimated to be 1990 at the earliest.

2. How will the waste form be handled? Solidification of process wastes has been shown on laboratory or pilot scale processes. There is considerable question as to when the process will be available for waste solidification and as to what form the waste will be permitted to be converted to.

3. Plutonium disposition? The plutonium can be either separated for breeder or military uses, remain with the uranium, or remain with the waste. Each of these options pose technical, political, and safety questions which will not be resolved in the next few years.

4. Foreign reprocessing? It appears foreign reprocessing plants could handle the TMI fuel. Again, certain technical questions involving fuel form and facility enrichment limits are not known by AGNS. However, consideration of the United States "exporting" waste material and the shipping overseas would be highly controversial.

5. Facility selection? Only a facility specifically designed for̀ commercial LWR fuel could satisfy licensing requirements for reprocessing and be able to conform with government regulation regarding emission standards. Two of the three facilities are capable of reprocessing the enriched fuel using any of the candidate flowsheets 
once the heavy metals are in solution. However, for the coprocessing alternative producing a mixture of uranium and plutonium, suitable facilities do not exist for either interim storage of coproduct solutions or conversion to mixed oxide.

The important facility requirement for reprocessing the TMI-2 core is believed to be the possession of a chop-leach headend.

\subsection{Wet (Pool) storage}

\subsubsection{Introduction}

The most practical near-term means of storage of the TMI fuel would appear to be wet storage at the TMI facility. storage of spent fuel in a pool is a well-proven technology. Th:e majority of spent fuel in the United States is now being stored in these pools. The only clear distinction between the TMI fuel and the vast majority of other LWR spent fuel assemblies is the badly failed condition of TMI fuel. As a result, as noted in preceding sections of this report, this fuel will be canistered. This appears to be a prerequisite for this failed fuel to assure that it is isolated from the pool water to prevent contamination.

The options open for the disposition of TMI spent fuel in addition to the TMI pools are as follows:

- At another reactor pool (preferably at a nearby facility)

- At a regional Away-From-Reactor (AFR) storage pool

- In pool spaces at national laboratories.

The availability of off-site spent fuel pools is difficult to predict at this point in time. This is due to the projected nonavailability of wet pool storage space within the United states projected in the 1983 to 1985 period. Using state-of-the-art, high-density "poison" rack design, results in relatively small storage space requirements. Pre?iminary estimates of the space required indicate an area of approximately 250 square feet, which does not represent a major portion of existing fuel pool areas.

Table 6-2 summarizes the key results and recommendations for this study. Reference 6.2 indicates that over 50 domestic LWR applications have been received by the NRC since 1974 for reracking spent fuel pools. Current studies by AGNS indicate that a number of companies are now involved in the design, licensing, and fabrication of high-density racks for reactor pools. Licensing difficulty is compounded when there is outside intervention and a requirement for public hearings. The remainder of the technical considerations are normally "straightforward." 


\section{IABLE 6-2}

\section{SUMMARY OF POOL STORAGE STUDY RESULTS}

\section{GENERAL}

- WET STORAGE PROBABLE FOR SOME FUEL (EITHER SHORT- OR LONG-TERM)

- FUEL WOULD BE CANNEd (STAINLESS STEEL) AND STORED VERTICALLY IN SPECIAL HIGH-DENSITY STORAGE RACKS

- TECHNICAL PROBLEMS RELATEd TO FUEL CANMING INCLUDE CAN SIZE, CRITICALITY. PRECAUTIONS, AND POSSIBLE CAN CORROSION IN BORATED POOLS

2. EACILITY REQUIREMENTS

- STORAGE - APPROX. 250 SQUARE FEET FLOUR SPACE

- APPROX. 21 TO 22 FEET HEIGHT (MINIMUM)

- CASK UNLOADING - PIT APPROX. $8^{\prime} \times 8^{\prime} \times 34^{\prime}$ DEEP

- CRANE APPROX. 25+ TONS (TRUCK CASK)

- SPENT FUEL RACKS - FUEL SLOT DIMENSION TO FIT 10- TO 11-INCH SQUARE CAN - MINIMUM SPACING APPROXIMATELY 12 INCHES (CENTER-CENTER)

3. POSSIBLE STORAGE LOCATIONS

- TMI SPENT FUEL POOLS

- NEARBY REACTOR (SPACE AVAILABLITY PROBLEMS)

- AFR (1983 EARLIEST DATE OF STARTUP)

- gOVERNMENT FACILITY (LICENSING/COMMERCIAL FUEL FACILITY MODIFICATIONS REQUIRED)

4. RECOMMENDATIONS

- eXamine SeVERAL POSSible loCations (INTERFACE StUdy)

- UESIGN LEAKER CAN/STORAGE RACK CONFIGURATION 


\subsubsection{Technical Considerations}

The primary technical areas to be considered are listed below:

- Space - How much pool floor space is required to store the spent fuel?

- Fuel Can Dimensions - The physical geometry of the fuel can and its compatibility with available fuel racks.

- Fuel Pool/Fuel Can Compatibility - The effect of the canned fuel on the fuel pool water must be evaluated.

- Nuclear Criticality/Structural/Seismic/Thermal Considerations - These areas are evaluated for any reactor spent fuel pool when a reracking modification is planned.

- Pool Depth - A depth of water is required over the fuel itself for shielding and handling purposes.

- Spent Fuel Cask Handling Capability - There is a need to have suitable equipment and spaces to receive the spent fuel casks which have shipped the fuel from the TMI reactor facility.

- Handling Accident Problems - Evaluation of handiing cranes and racks for accidents, such as cask drops or fuel element dropping.

Preliminary evaluations indicate that the technical factors noted above would not. normally preclude fuel storage at any reactor or currently proposed AFR facility. However, pools at government laboratories or facilities, which were not designed specifically for commercial LWR fuel, would have to be evaluated on a "case" bases.

The amount of fuel to be handled or the number of cans to be stored has previously been assumed to be approximately 225 to 250. To comply with shipping considerations, we can assume that the overall envelope per can will probably be less than 10 inches in square cross section (as noted in Section 5.2, criticality considerations could limit this to 9.4 inches) by approximately 15 feet in length. Spent fuel racks are typically designed in a "honeycomb" fashion, with stainless steel walls comprising the structure of the rack and serving to segregate (or position) adjacent assemblies. Figure 6-1 shows a typical design of a "fixed" rack using a neutron poison to minimize spacing. The poison is not a structural material and is typically "vented" to the pool water to permit "outgassing" of the poison material. Racks may either be "free standing on the pool floor" or mounted 


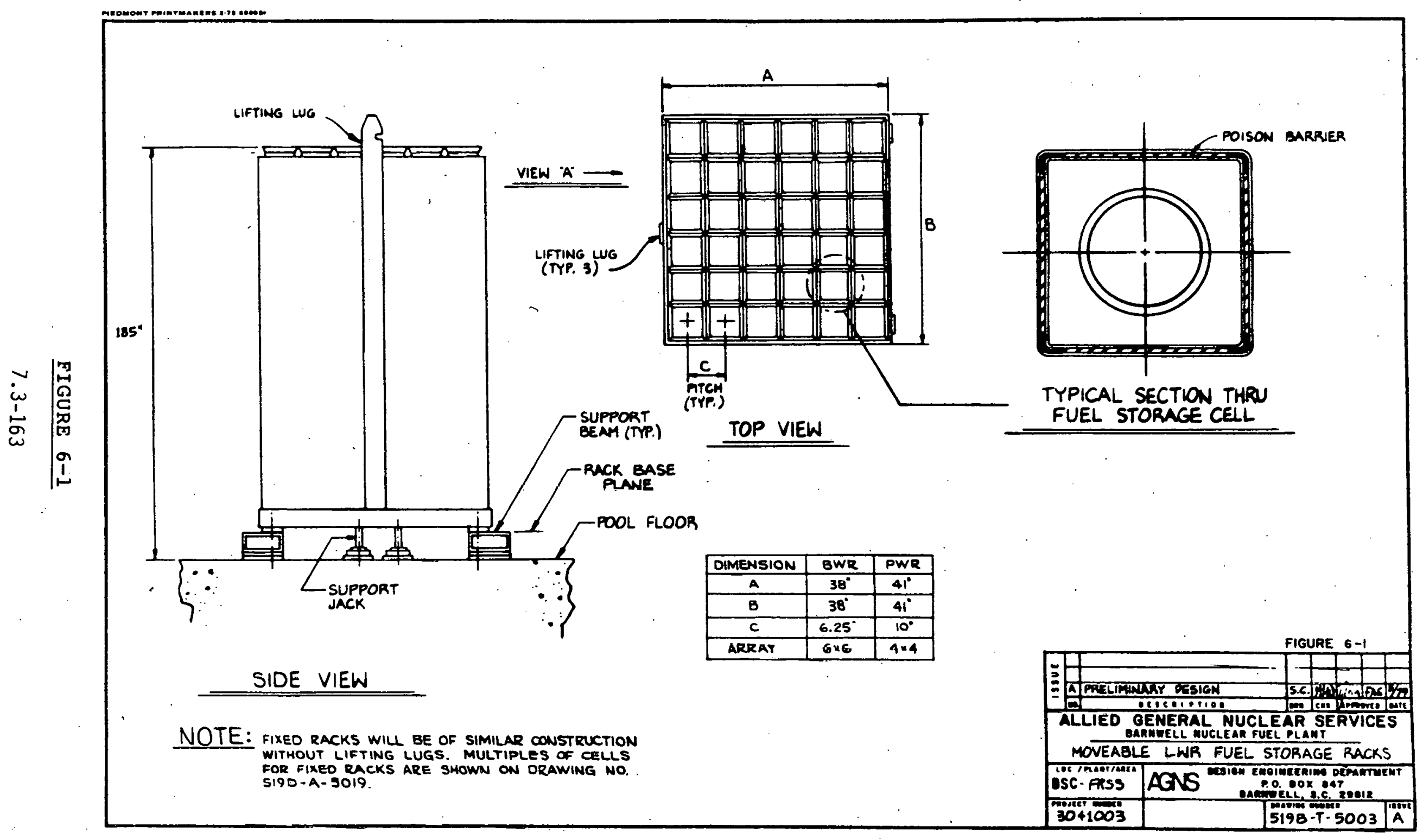


to a grid structure for seismic stability. A "movable" rack design can be employed for shallow pools (30-foot depth or less). This type of structure requires a floor grid structure for rack stability purposes.

Using current NRC-licensed technology, a center-to-center lattice spacing of about 12 inches can reasonably be obtained. High-density poison racks are approximately 308 more costly than "flux trap" nonpoisoned racks. They permit a 30 to $35 \%$ increase in compaction, which may be critical in this application. Most domestic spent fuel pools are now being reracked using poisons to obtain optimum pool loading. The estimated (1980) cost for high-density racks (non-leaker can assumed) is approximately $\$ 5000 / \mathrm{MTU}$.

From a pool space allocation standpoint, about 250 square feet of floor space would be needed. The aging and low burnup of the fuel reduces the source strength considerably. As a result, it is estimated that only approximately three to five feet of water is required over the fuel for shielding. Normally, a minimum of 9 to 11 feet is prescribed in a reactor pool. Figure $6-3$ shows the estimated radiation level in a pool for high burnup PWR fuel in a pool after five years of aging. Note that $4-1 / 2$ feet of water provides adequate coverage to reduce the dose levels to about $1 \mathrm{mR} /$ hour. The source strength of TMI-2 fuel after three years of aging (mid-1982) is approximately five to seven times lower than for this case. Hence, the minimum depth of water required in the pool is about 35 feet (for fixed racks). However, in this situation, there will be no capability of moving the fuel directly over the racks. In the minimum water depth case, a movable type of rack would have to be employed. In addition for off-site shipments, the fuel would have to be unloaded in a deeper area, typically at least 35 feet in depth. It is expected that the fuel would remain in the shipping cans utilized with the spent fuel cask.

The compatibility of the fuel can material with the pool water and the rack structure must be ascertained. The current state-of-the-art fuel racks are typically fabricated from stainless steel. It is expected that the fuel storage can will also be fabricated from a stainless steel alloy (see section 6.3). Conversely, fuel pool walls are normally lined with stainless steel. At PWR reactors, the water is typically borated even though many other plants are filled with demineralized water. If the TMI fuel is positioned in a sealed can, the only concern related to the interaction between the fuel and the pool water is the long-term possibility of in-leakage through the seal. In the event of in-leakage, there would be a potential for fuel pool contamination. Thermal concerns should be minimal due to the 


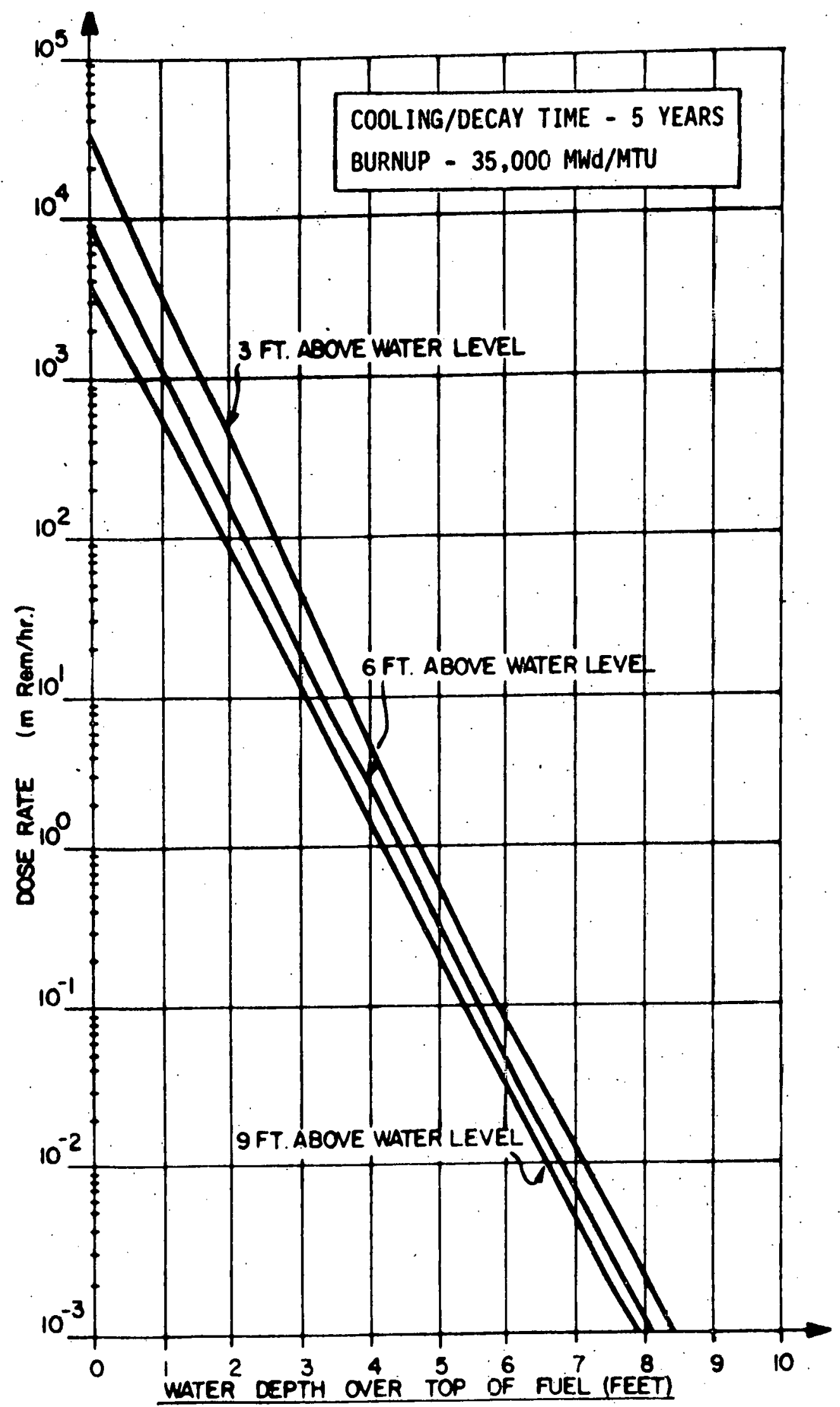

RADIATION LEVELS FOR PWR FUEL IN POOL

FIGURE $6-3$

(FROM REFERENCE 6.4)

$7.3-165$ 
extremely low decay heat levels. The interaction of the pool water and the materials used for the rack structure and the fuel can must be examined for possible corrosion mechanisms. It is believed that Type 304 stainless steel alloys should be adequate; however, more costly corrosion resistant metals for the can could be utilized to preclude any possible problems if long-term storage is envisioned.. Current day reactor fuel pools and expected AFR locations are serviced by demineralizers to maintain pool activity levels below set limits. Evaluation of potential wet storage areas will have to consider the adequacy of the capacity of the installed demineralizer system if one is available. The stability of the poison material must be considered during the fuel rack design portions. The poison position in most fuel storage racks is vented to the pool water.

The technology associated with high-density spent fuel racks has been refined for a reactor application over the last five years, as shown in Figure 6-2. The poison material for a spent fuel rack, normally composed of a boron carbide composite in a matrix, may be mounted on either two or four sides within the fuel slot hole. The poison axially extends to match the active fuel length of the assembly. The poison selected must withstand the radiation exposure (gamma) for the projected fuel lifetime. The NRC requires that a means of verifying poison efficacy be provided. Hence, either inspection ports or removable poisons may be supplied. The former is the more common situation. Preliminary examinations indicate that TMI-2 fuel should be a lesser problem than in reactor pool instaliations which cannot assume the prior aging of the fuel (as with TMI). Hence, any of several poison composites would be acceptable. The fuel slot within the rack must be designed to accommodate the cross-sectional dimension of the fuel can. For "normal" PWR fuel, this slot would be from 8.75 to 9.0 inches square. For canned fuel, as in this situation, the slot dimension would have to be opened to ascommodate the can dimensions. Normally, from 0.125- to 0.18-inch clearance is required on each side. However, due to the lack of present. knowledge of the fuel size, or the size of the shipping can, it may not be possible to design the racks until these areas of information have been defined. In section 5.4, it was noted that the fuel may be shipped in a cylindrical can. Obviously, racks could be fabricated to store this type of container; however, they would require a larger storage area and are not recommended.

There are certain technical areas which are historically evaluated for any racking of a spent fuel pool. The specific NRC guidelines are noted in Reference 6-3. In essence, they address nuclear criticality, thermal or fuel decay heat 


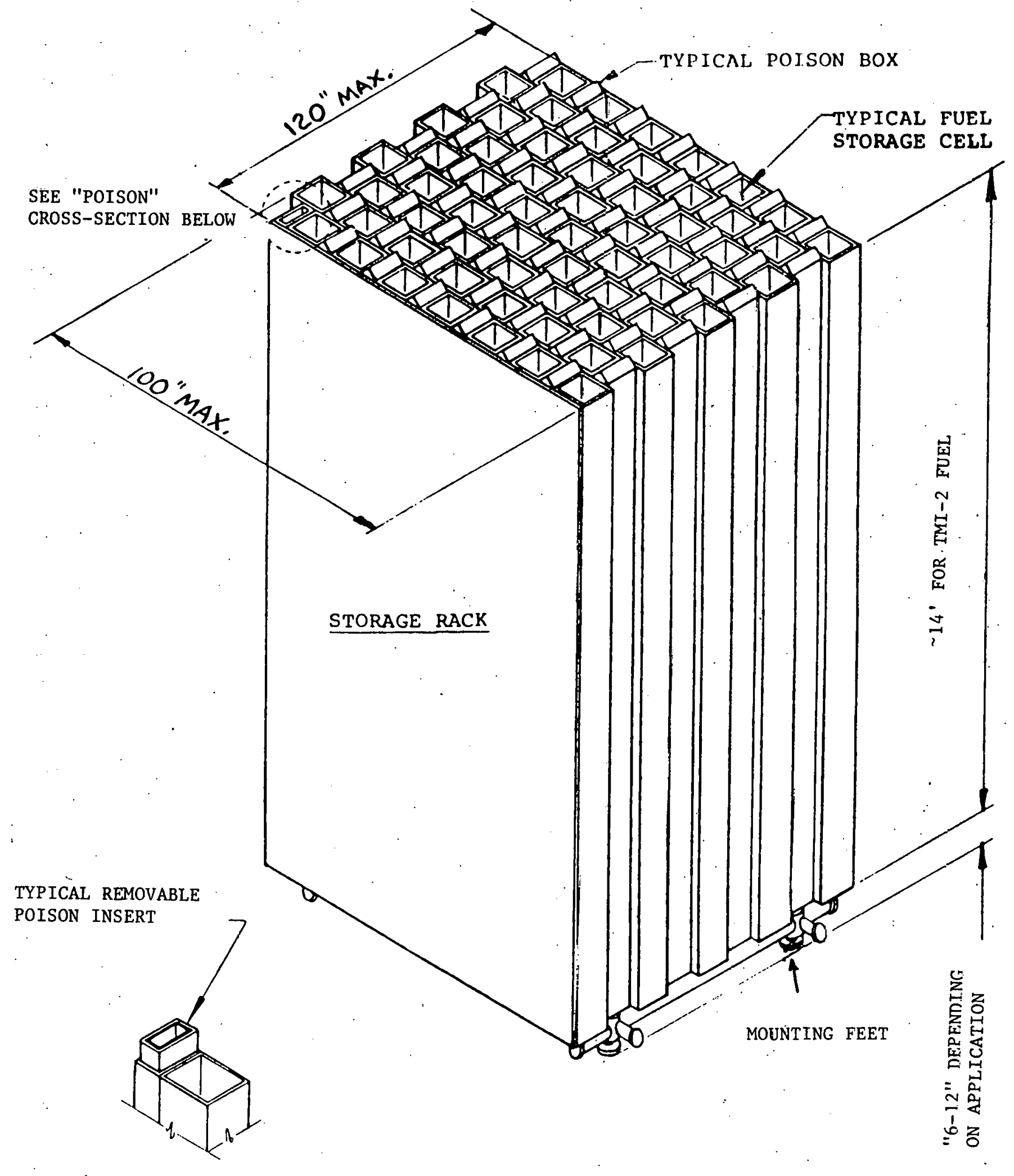

TYPICAL PWR SPENT FUEL STORAGE RACK POISON-FIXED DESIGN (7 $\times$.8)

FIGURE 6-2

7.3-167 
loads, seismic-structural aspects of the rack/can structure, and the pool and handling accident scenarios. These will have to be examined on a case basis considering the specific design characteristics expected. The design situation for this aged low burnup fuel should not present any major technical problems.

If the fuel is shipped to a wet pool storage site, there must be a means at the site of handling the fuel casks and unloading the fuel. As noted in section 5.4, site interface problems are minimized for truck casks which are lighter, smaller, and do not require special rail lines directly into the facility. The facility handling equipment (cranes, unloading areas, yokes, etc.) must have the capability of handling the cask and assuring that certain hypothetical handling accidents cannot occur. These accidents include crane failure, cask drop, etc. The mere existence of a pool of suitable size does not assure that the fuel could either be brought into or out of the facility.

Table 6-3 presents a list of items to be evaluated for an off-site wet pool installation preparatory to a decision being made to receive fuel. As further information becomes available on the availability of various sites, a portion of the feasibility study should include evaluating these factors. Reference 6-5 presents the results of a survey of the design and interface characteristics of a number of possible pool sites.

\subsubsection{Institutional problems}

Wet storage of spent fuel will always be viewed as an interim solution. Arguments have been made that movement to a pool off site involves the possible hazard of off-site shipping. Based on facts, this is more of an emotional argument with little technical basis.

It is inevitable that a certain quantity of fuel will be stored, if only for a period of a few months, at the TMI facility pending disposition. However, utilization of any off-site facility is dependent upon availability of adequate space, licensing, and operating constraints, along with the situation in the time frame needed.

\section{3 .3 .1 TMI Fuel Pool}

There is a need to store the bulk of the fuel for a time period extending probably into the early 1990 's when a program for ultimate disposition of fuel is hopefully in place. Storage of the remaining fuel for a 5- to lo-year period (1980 to $1990 / 1992)$ may be found within the TMI facility itself. Certainly, the most economic application 
TABLE 6-3

SPENT FUEL POOL INTERFACE CRITERIA (SUMMARY)

I. SHIPPING CASK INTERFACE

- Pool Water Depth

- Floor Structural Loading

- Crane Limits (structural and Lifting Load, Redundancy)

- Adequately Spaced Unloading Area

- Means of Moving Cask to Pool

- Roads (Truck), Rail Spur (Rail)

- Possible Accident Scenarios (Cask Drop, Etc.)

II. POOL INTERFACE

- Pool Lining

- Demineralizer/Filtration Systems for Water Control

- Pool Cooling System Capacity

- Floor Space, Water Depth

- Ventilation Systems, Pool Leak Detection System

III. SPENT FUEL RACK INTERFACE

- Pool/Rack structural and Seismic Adequacy

- Means of Mounting Rack

- Rack Loading Crane/Handling Tools

IV. NRC-LICENSING CRITERIA

- Regulatory Guide 3.44

- 10 CFR 50, 10 CFr 72

- Fuel Quantities

- Storage Period, NRC No. OTSB Position Guide. 
of pool storage would be at any of the pool areas currently constructed and on the TMI site itself. Figure 5-5 illustrates the available areas and the size and depth data. However, there is also a conflicting need for pool space for storage of equipment prior to decontamination. In addition, there may be public pressure to move all of the failed fuel off-site. Storage on-site would involve reracking selected areas of the pools and is expected to be the simplest alternative since of $\mathrm{f}-\mathrm{site}$ shipment is not required in the near-term.

\section{3 .3 .2 Reactor Pools}

Another possibility is transshipment to another reactor pool. In all likelihood, another reactor, particularly one within several hundred miles of the TMI site, would offer various handling and logistic advantages. It is expected that the reactor sites would be technically capable of handling the shipping casks and unloading the fuel as well as being designed and licensed to accommodate LWR type fuel. The primary disadvantage at this point in time is the erpected nonavailability of storage space. Reference.6.6, a report by a DOE official to Congress, indicates a potential shortfall in reactor on-site storage space in the United States by 1983 of 380 MTU. Even if a mutual sharing arrangement could be made with another utility or reactor plant with adequate storage space, and suitable technical and licensing questions resolved, there could be consideratie pressure brought to prevent or block such types of spent fuel transshipment. This problem has occurred previsusly with Duke Power. Their program includes movement of fuel from their Oconee to their McGuire plants. This is a relatively short shipping distance (less than 100 miles). However, this movement has been delayed for over two years and is not resolved at present (mid-1980). Currently, the Carolina Power and Light Company transships fuel between their Robinson and Brunswick plants. Commonwealth Edison has proposed similar fuel movements from their Dresden plants. Arguments used to prevent shipment have included the concern with possible shipping dangers.

\subsubsection{AFR Storage}

In recent policy addresses by the Department of Energy (DOE), Reference 6.6, the government has made known its intent to store spent nuclear fuel at an away-from-reactor facility (AFR). The rationale being that there will be insifficient at-reactor storage space available beyond the 1983 time frame, and that the resolution of a means of ultimate spent fuel disposition will not be addressed until 1985. This lack of storage space could lead to plant shutdown. The expected near-term sites involve utilization of 
one or several nonoperational reprocessing plant pools (i.e., Morris, NFS, West Valley,. AGNS-Barnwell). These storage facilities would be obtained via governmental acquisition and are proposed in current congressional legislation (H.R. 3000). It is also felt that these options will result in a lower cost to the electrical power industry than the utilization of colocated storage areas at large multireactor plant sites (Reference 6.6). However, current delay experienced in AFR site selection indicates that unless action is taken for purchase of these facilities, that the facilities would not be operational when needed (1983 to 1984 ).

\subsubsection{Government Facility}

A final alternative would be utilization of suitable spaces at governmental nuclear installations. Studies noted in. Reference 6.5 indicate that there are places which potentially have available space. Experience has been obtained with pool storage of spent research fuel or foreign fuel at a number of these installations. Since fuel will be shipped to many of these locations for $R \& D$ evaluation, there is the possibility that the total quantity of fuel could also be subdivided and stored at more than one of these facilities. The total quantity of fuel is not large, and it could be divided into amounts which could be manageably accommodated at several locations. Of major importance is the evaluation of the capability of these facilities to accommodate cask unloading and the physical dimensions and size of the individual packages. Prior evaluations have indicated that a number of potential facilities do not have the capability of handling current LWR spent fuel casks and full length current fuel. This is not the case if debris packages or smaller cans are developed which can be shipped in special casks. As discussed in succeeding paragraphs, the need for a government facility to obtain an NRC facility license for this receipt and storage is not clear. The utilization of this fuel for $R \& D$ may eliminate this requirement. A precedent is the movement of fuel from the Florida power and Light-Turkey Point plant to government installations at the Battelle Laboratories in Ohio, and the E-MAD facility near Las Vegas, Nevada.

In summary, for any off-site storage of TMI fuel, the capability of the facility to handle the fuel, the need for special racks to accommodate the canned fuel, and the space requirements must be evaluated. At present, it appears that no space is currently available for storage of the entire, core off-site. The possible intervention by antinuclear activist groups to prevent off-site shipments may be attempted no matter what option is selected. Hence, with the possible exception of a small portion of the fuel moved 
Iff-site for research interests, GPU should consider on-site pool storage of canned fuel for most of the core.

6.3.4 Facility Licensing

$h$ major area to be evaluated is the NRC licensing requirements for any facility used for storage.

- At TMI - A modification to the 10 CFR 50 license would be required to accommodate the procedures and equipment (canisters, racks, etc.) utilized.

- Transshipment to Another Reactor - The facility would also require an amendment to the 10 CFR 50 operating license to accommodate the TMI-2 fuel. (Precedent has been established at such places as the Carolina Power and Light-Brunswick installation.)

- AFR - A license for the facility would be required in accordance with 10 CFR 72, The facility license would specifically discuss the storage of failed (canned) fuels.

- Government Facility - Unknown.

6.3.5 Implementation Considerations

Implementation of each of these storage schemes can be esitimated. The current length of time for the NRC to approve a licensing amendment for reracking of a reactor facility is about 2 to $2-1 / 2$ years. The time to design, construct, and fabricate (and possibly test) the pool storage racks and cans is also about 2 to $2-1 / 2$ years. However, these two tasis can be performed in parallel if necessary. Hence, a minimum of $2-1 / 2$ years would be required to implement use of the TMI pool. The same time would be required for use of another LWR power reactor site. Licensing time could be extended for transshipment and for some necessary facility modifications. Hence, our estimate is a 3- to 3-1/2-year lead time until initial storage is available (mid-1983). AFR's are needed nationally by 1983. However, based on the current status of the government effort to purchase at least one of the inoperative reprocessing plant pools, the date of earliest availability is projected to be 1984 or beyond. Similarly, the utilization of government facilities (based on Reference 6.5) could take from two to six years depending on the facility, modifications required, etc. As can be seen, the lead time between initiation of specific action 'for storage and the time of initial receipt can extend from two to eight years. We suggest that tentative selection of several sites be identified as early as possible to complete more detailed studies of the places considered. 


\subsubsection{Advanced Wet Storage Techniques}

Spent fuel disassembly is a technique that now appears possible for "normal" intact fuel. This operation is utilized to compact the wet storage of spent fuel. The fuel assembly is dismantled into individual rods and canistered. The amount that can be stored within a given area is increased by $1-1 / 2$ to 2 times. The basic operational steps are:

- Removal of end fitting

- Rod pulling

- Canning of the fuel rods

- Compaction and disposal of the fuel skeleton.

This technique involves several handing steps which would normally pose no problems with fiel cladding structural integrity. However, in the case of the badly failed TMI-2 fuel, it is prudent to minimize fuel handling. It would not be recommended to purposely attempt to disassemble TMI-2 fuel for compacted storage.

Conceivably, the operational steps used for handling the research and examination of selected TMI-2 fuel assemblies will be similar to those used in disassembly. Fuel disassembly to some degree is required to make individual research evaluations of the interior rods within the fuel matrix. Hence, techniques for dismantling the fuel for hot cell examinations will probably parallel these disassembly steps. Eventually, the individual rods will require placement into a separate can for further disposition. Developmental studies with canning of individual rods and fuel components have been performed at AGNS in the 1978-1980 time frame. Figure 6-4 illustrates the storage of fuel rods from two complete assemblies within a rectangular container.

6.4 Dry Storage Concepts for TMI-2 Reactor Fuel

6.4.1 General

There are at least four possible means for dry storage of the fuel from the TMI-2 reactor: (1) storage at existing facilities (large hot cells) within the United States, (2) conceptualized vault storage, (3) conceptualized caisson storage, and (4) Federal repository storage (References 6.7 and 6.18$)$. Repository storage wculd entail placing TMI-2 fuel directly into geologic burial for permanent disposal (although a 10- to 50-year retrieval duration would probably be engineered into the repository design). The repository could be located in underground formations of salt, basalt, granite, shale, or some other stable geologic media. This repository is envisaged to be owned and regulated by the 


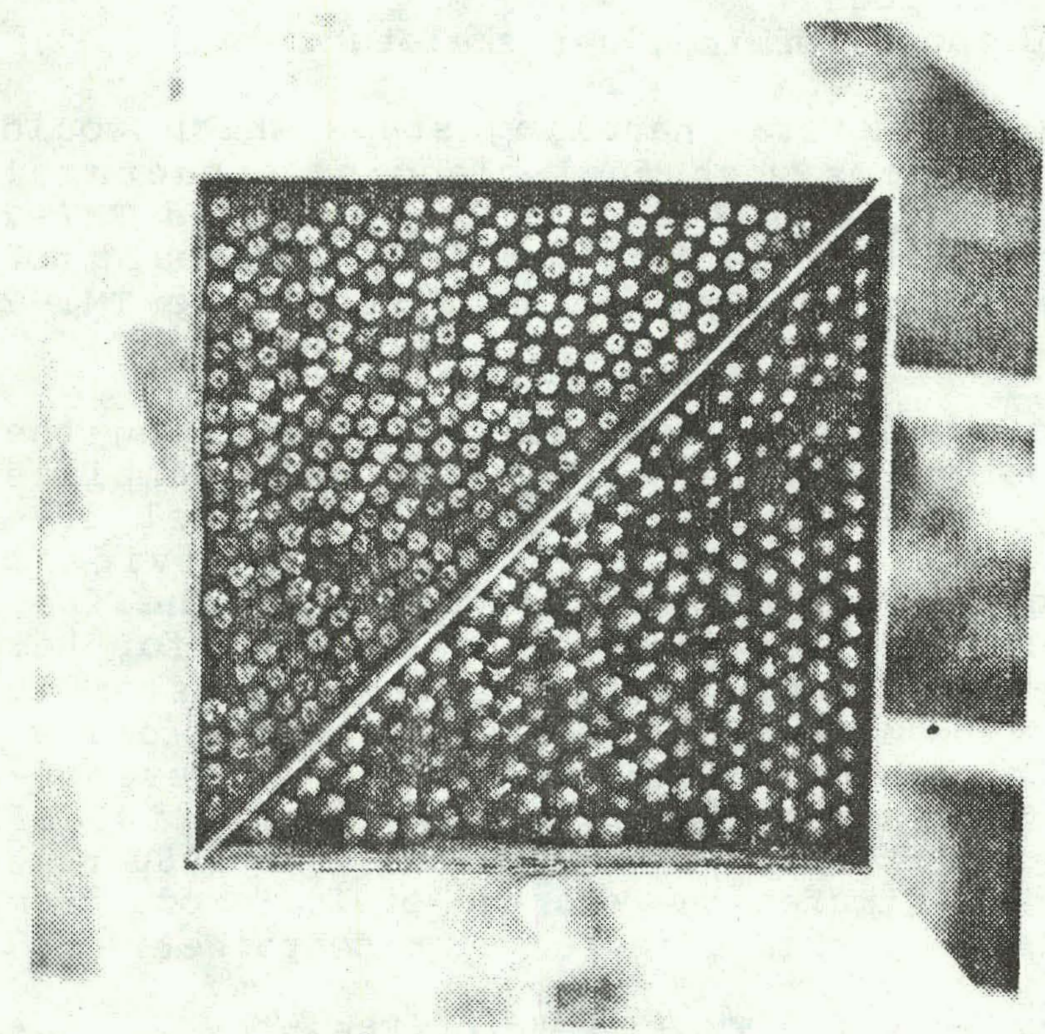

SQUARE (NINE-INCH) CAN LOADED WITH FUEL PINS FROM TWO COMPLETE PWR FUEL ASSEMBLY DUMMIES

F IGURE 6-4

$7 \cdot 3-174$ 
Federal Government and would be similar to those proposed for New Mexico (WIPP-salt media), Washington (BWIP-basalt media), and Nevada (NNWSI-tuff, granite, and shale media). It is estimated that the first government repository will not be on-line until at least 1990. In February of 1980, President Carter established a comprehensive radioactive waste management program that set the goals of a repository site selection by about 1985 and an operating facility by about 1995. Since a resolution of the disposition of TMI fuel needs to be made prior to 1990 , near-term storage in a Federal repository is not a viable solution. Repository storage will not be discussed any further in this report.

One possible means considered for storage of TMI-2 fuel is utilization of hot cells or vaults at nuclear facilities and laboratories operated by the Federal Government. Review of existing facilities is valuable from a timing standpoint, i.e., they may be modified to accept the TMI-2 fuel faster than building new dedicated facilities. However, technical descriptions of the existing facilities are covered in the discussion on vaults and caissons, particularly vaults. This report deals with these various vault and caisson concepts for the dry storage of TMI-2 fuel. Each concept is presented separately; and in the case of the caisson concepts, it is assumed that each caisson contains the equivalent of one fuel assembly. Some of the concepts for heat removal could be combined. For instance, cooling fins and forced convection cooling could be combined in a vault, or heat pipe cooling and natural convection could be combined in caisson storage. There are many such combinations; but to simplify the discussion, only the basic concepts are discussed individually. Caissons could probably store more than one to three fuel assemblies but this has not been addressed.

\subsubsection{Thermal Considerations}

To a large degree, the means of storing the TMI-2 spent fuel will be based on thermal considerations. Due to the very low burnup of the fuel, the fuel is relatively "cold" compared to most commercial power reactor fuels. As far as heat load is considered, the TMI-2 fuel heat load after three years is approximately equal to typical fuel discharged from an LWR and cooled for about 10 years. The heat load from TMI-2 fuel after three years of cooling is about 300 to 400 watts/MTU or 190 (maximum) watts/assembly. In the entire core, there is a total of 82.7 MTU ( $w$ ith a heat load of 30 kilowatts) contained in all of the $177 \mathrm{fuel}$ assemblies. After five years of cooling (mid-1984), the heat load drops to about 100 watts/assembly or less than 20 $\mathrm{kW}$ for the entire core. To place this in perspective, the design heat load for dry truck shipping casks (one assembly) 
is about $10 \mathrm{kWt}$ and for dry rail casks (NLI-10/24) is 70 kWt. Hence, the TMI-2 fuel is relatively cold, thermally, and would not be expected to present heat load problems to any of the designs, with the possible exception of the "no cooling" vault. Since this study is conceptual, a detailed thermal analysis was not performed. However, prior studies of dry-canistered fuel (Reference 6.9) indicate that the thermal gradient across the fuel bundle for this "aged" fuel is extremely small $\left(<50^{\circ} \mathrm{F}\right)$. In addition, the likelihood is that all of the free fission gases have been released from the fuel. Hence, since the canister will perform the containment function and the thermal load is very low, the dry storage does not appear to present a critical technical problem.

\subsubsection{Vault Storage Concepts}

The vault concepts considered for spent fuel storage consist of four possible designs. A vault is defined as a dedicated dry storage building. These four-vault concepts include: (1) vault with forced air cooling, (2) vault using heat pipes for cooling, (3) vault cooled by a natural draft, open to the outside environment, and (4) vault with no cooling, sealed to the outside environment. There are numerous variations of these four concepts, but for simplicity, only the four basic concepts are discussed. All the vault concepts have many features in common, i.e., they store many unit quantities of packaged reactor fuel and protect these packages from accidents. They differ mainly in the method used to remove radiolytic decay heat.

\subsubsection{Forced Air Cooling}

The vault concept with forced air cooling is shown in Figure 6-5. This is typical of the design of most existing vaults and hot cells. It utilizes well-understood existing technology and many existing facilities, particularly large hot cells, could be modified to store canned TMI-2 spent fuel in this manner. Nearly all nuclear facilities employ forced air ventilation followed by High Efficiency Particulate Air (HEPA) filtration to remove radioactive particles from the air prior to its exhaust to the environment. If any existing facilities were to be used for fuel storage, they would probably employ forced air cooling. As an example, Idaho National Engineering Laboratory (Idaho Falls, Idaho) has designed, built, and is operating two types of dry spent fuel storage facilities (Reference 6.10). The two installations, the Peach Bottom Storage Vaults and the Irradiated Fuels Storage Facility (IFSF), are both forced convection cooled storage vaults. 


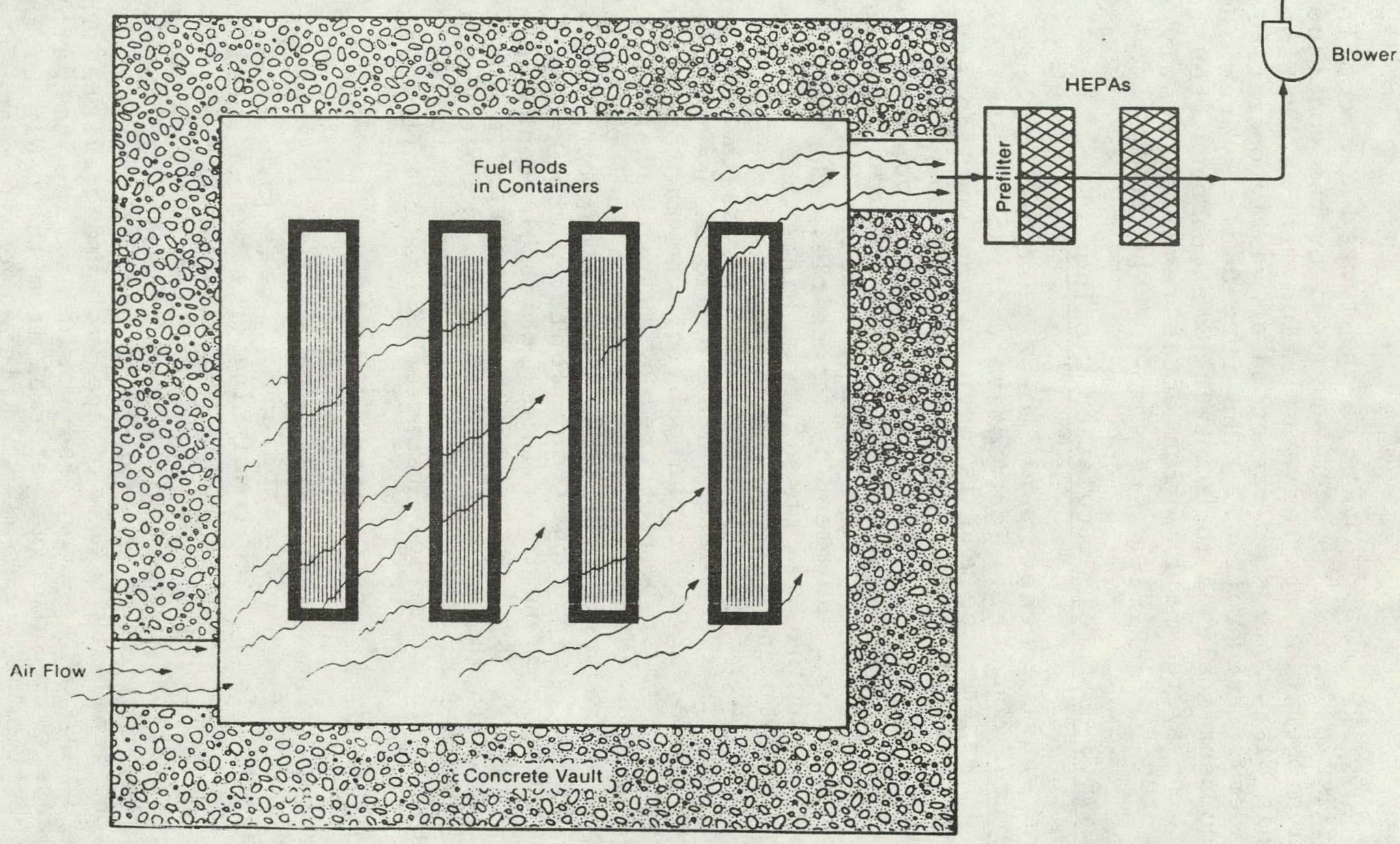

VAULT WITH FORCED AIR COOLING

FIGURE $\quad 6-5$ 
The obvious disadvantage of the forced air cooling is that it is an "active" system and requires the continuous effective operation of the ventilation and filtration systems. This disadvantage is reflected in yearly operating costs and safety considerations. The forced air system requires an accident analyses to determine what happens when components of the system fail, which often requires costly secondary backup ("fail-safe") systems or mechanisms.

\subsubsection{Natural Circulation Cooling}

The vault concept shown in Figure 6-6 relies upon the natural convection (or passive cooling of air) to cool the fuel assemblies and is open to the outside environment. Nukem GmbH of Germany (Reference 6.11) and Ontario Hydro of Canada (Reference 6.12) both have proposed a dry storage concept for spent fuel that utilizes a concrete vault cooled by natural convection. Loss of cooling due to the failure of mechanical equipment is impossible. The major disadvantage is in the area of safety. Since the air currents do not develop enough force to overcome the resistance of HEPA filters (due to the pressure drop across the filters), it is unlikely that absolute filters could be used. Therefore, to ensure the safety of the public, the radioactivity in the spent fuel must be protected by multiple barriers. A case car be made that the cladding of some fuel elements (in this case the majority of the TMI-2 core) cannot be classified as a barrier, since they may contain cracks, holes, etc. Therefore, to ensure a minimum of double containment of the spent fuel, the fuel rods (or entire assemblies) would be canned. In the case of TMI-2, even a double canning might be considered. Note that for proposed geological (repository) burial, multiple canisters have been proposed.

\subsubsection{Heat Pipe Cooling}

The vault concept shown in Figure $6-7$ relies on heat pipes to cool the fuel assemblies. A heat pipe is a "passive" heat-transfer device consisting of a sealed metal tube with an inner lining of wicklike capillary material and a small amount of fluid in a vacuum. Heat is absorbed at one end by vaporization of the fluid and is released at the other end by ccndensation of the vapor. Fluid circulation is enhanced by the capillary action of the wick and gravity. The tubes are completely sealed and would provide no pathway for release of radioactive material from the vault to the environment. The tubes may be finned to increase their heat transfer ability. The system is passive and does not require ventilation on a routine basis. A backup low capacity (low flow rate) ventilation system may be desirable to take air samples, allow major repairs/modifications to the heat pipes, provide clean airflow control for nonroutine 


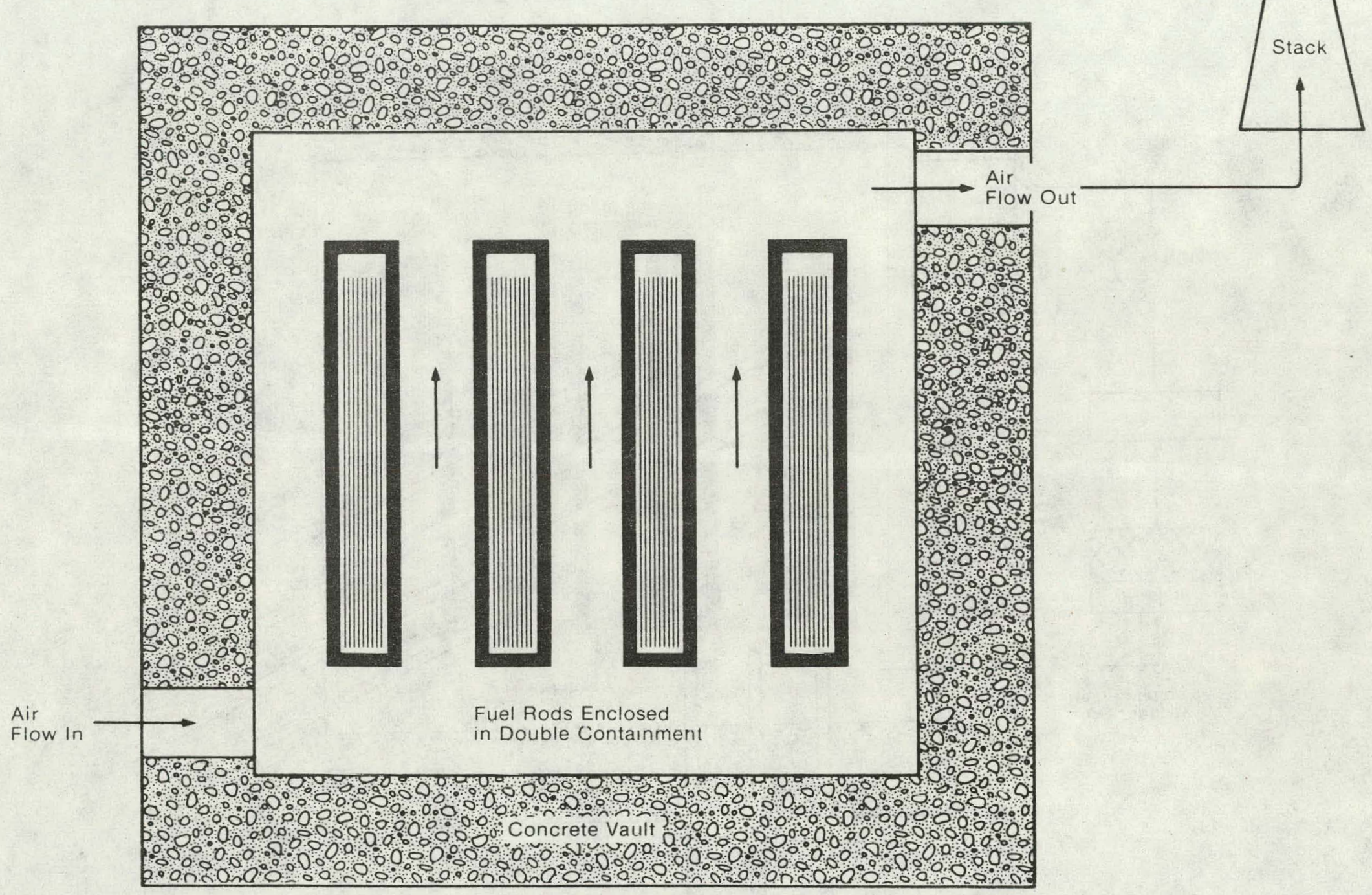

VAULT WITH NATURAL CONVECTION COOLING

FIGURE $\quad G-6$ 
Heat Released by Natural

or Forced Convection

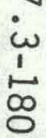

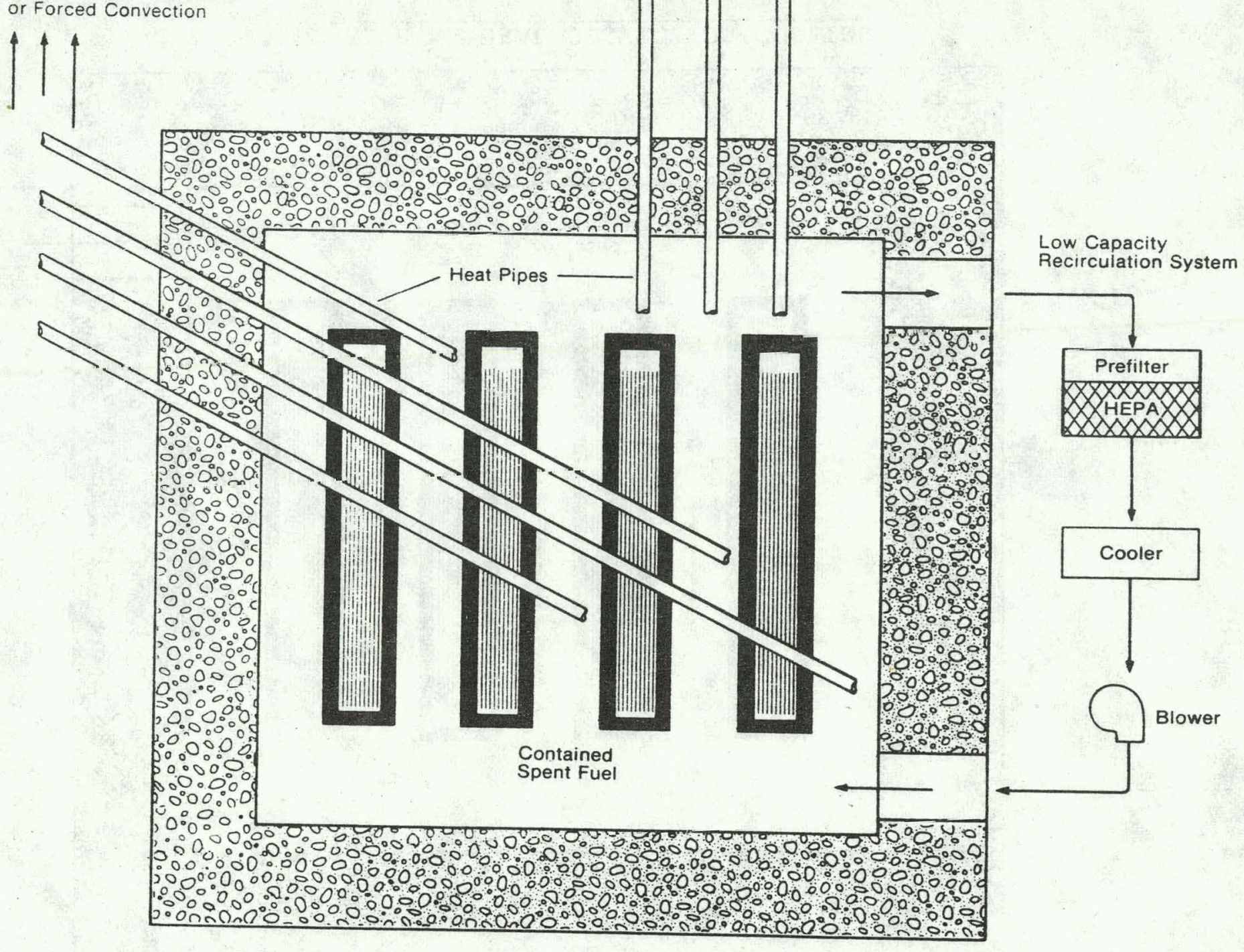

VAULT WITH HEAT PIPES

FIGURE $\quad 6-7$ 
manned entry, and allow a degree of safety by providing the required differential air pressure for normal operations. This system would probably not contain its own backup systems, such as redundant secondary blowers and HEPA filters, and could also be a recirculating system. This ventilation system would provide perhaps 0.5 to 2 entire vault air changes per hour versus about ten times that amount when a ventilation system is used on a routine basis for airflow and cooling.

\subsubsection{Fin Cooling}

A very similar concept to utilizing heat pipes is shown in Figure 6-8 in which metal fins are used to transfer heat, by conduction, out of the vault storage area. This cooling system is passive inside the vault. Forced (or perhaps natural) convection is used to sweep the heat from the fins outside the vault storage area. The air from this sweep would be noncontaminated and HتPA filtration may be optional. In this design, the entire ceiling of the vault could be metal which would assist transferring the heat out of the vault. This vault concept could also be provided an air recirculation system that would cool and filter relatively small volumes of air, i.e., about 0.5 to 2 vault air changes per hour. This system would provide a negative air pressure inside the vault, perhaps -1.0 ir.ch of water.

\section{4 .3 .5 No Cooling Devices}

The vault design in Figure 6-9 assumes a "closed" vault with no cooling devices. The vault would only be open during placement or removal of spent fuel. Ventilation would be similar to that discussed in the heat pipe or metal fin storage concepts, i.e., a recirculating, low capacity ventilation system employed mainly to retain a negative pressure and not used for cooling. The ability to use a vault design with no specific cooling devices (other than the heat sink provided by the vault's concrete structure) is only possible with spent fuel that has been cooled many years after reactor discharge. Fuel that is removed from the reactor with very low burnup, such as the TMI-2 fuel, with a very low heat load may be accommodatec in this manner.

\subsubsection{Caisson (Silo) Storage Concepts}

The attractiveness of the caisson concept is due to the fact that a vessel can be fabricated on demand to handle one or perhaps several fuel assemblies. These assemblies would be canned prior to being placed within the caisson for handing purposes and to provide multibarrier containment. The primary advantage of the caisson is that it is "siteindependent." The caisson could conceivably be located at 


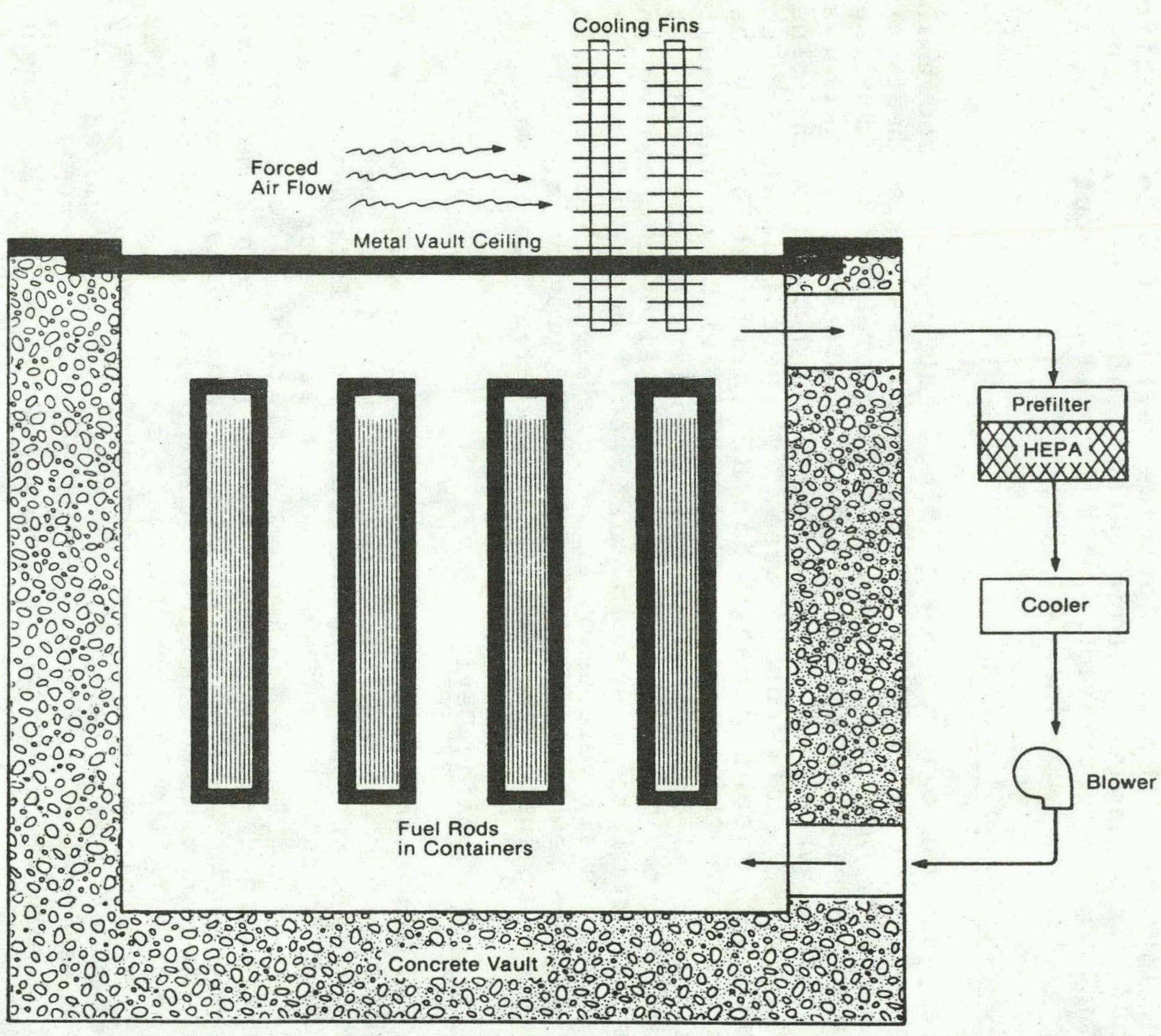

VAULT WITH COOLING FINS

FIGURE 6-8 


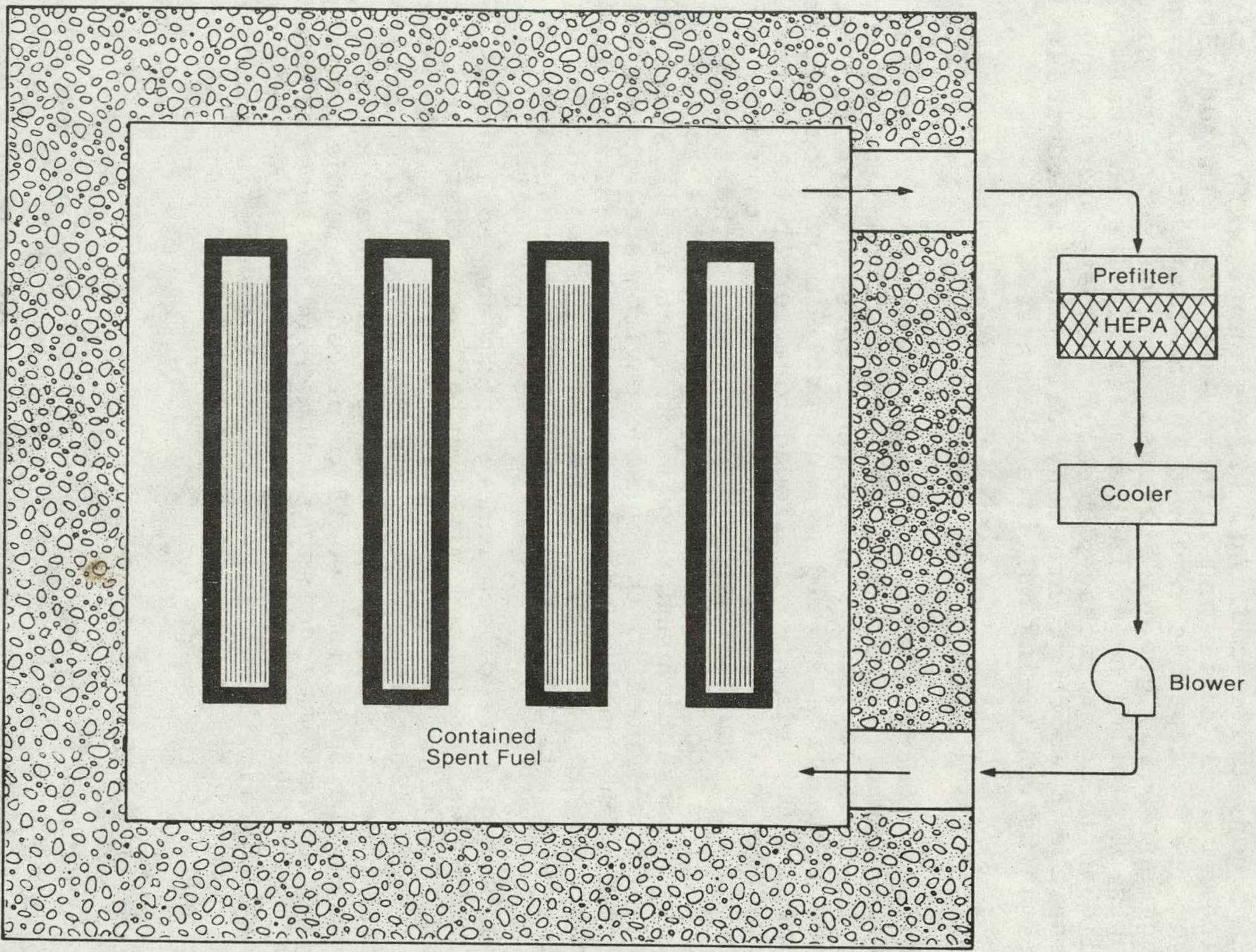

VAULT WITH NO COOLING DEVICES

FIGURE 6-9 
the TMI-2 site or a remote site depending upon the needs of the particular situation.

The caisson concepts reviewed include: (1) caissons with natural convection cooling, (2) caissons with heat pipe cooling, and (3) caissons with no cooling. In addition, each of the concepts is reviewed on the basis of aboveground storage and below ground storage. The caisson, or silo, concepts of storing spent fuel are all similar in that only small quantities of packaged reactor fuel are stored in each caisson and that there are many caissons, in contrast to large vaults containing multiple elements.

\subsubsection{Natural Circulation Caisson}

The caisson concepts with natural convection are shown in Figure 6-10 and Figure 6-11. The aboveground storage concept, Figure 6-10, uses a large concrete structure for each caisson to provide shielding and protection from natural phenomena. Outside air is allowed to enter at the bottom of the concrete caisson, and ratural convection currents move the air upward and out openings provided at the top of the structure. A concrete plug is provided at the top of the caisson for insertion and removal of spent fuel. Since the heated air is vented to the outside environment without HEPA filtration, the spent fuel must be confined within at least two containment barriers. These barriers would probably consist of a metal container with a metal overpack.

If the caissons are placed underground, the soil would act as a radiation shield and missile barrier; therefore, the need for a massive concrete structure is eliminated. Figure 6-1l illustrates a possible design utilizing natural convection cooling for spent fuel stored underground. In this case, the spent fuel would be placed in double containment and placed into caissons within an engineered berm. Vents are provided on the top and bottom of the storage caisson and pass through the soil to the outside environment. No filtration is provided because of the secure containment of the spent fuel.

\subsubsection{Heat Pipe Caisson}

Caisson storage using heat pipes for cooling, both above and below ground, are shown in Figures $6-12$ and $6-13$, respectively. The concept of aboveyround storage, Figure 6-12, is very similar to the natural convection aboveground storage showr in Figure 6-10. The relative size of the concrete structure and normal operations would be the same for both. A conceptual design combining natural convection and heat pipes for cooling stored spent fuel assemblies has been 
Access Plug

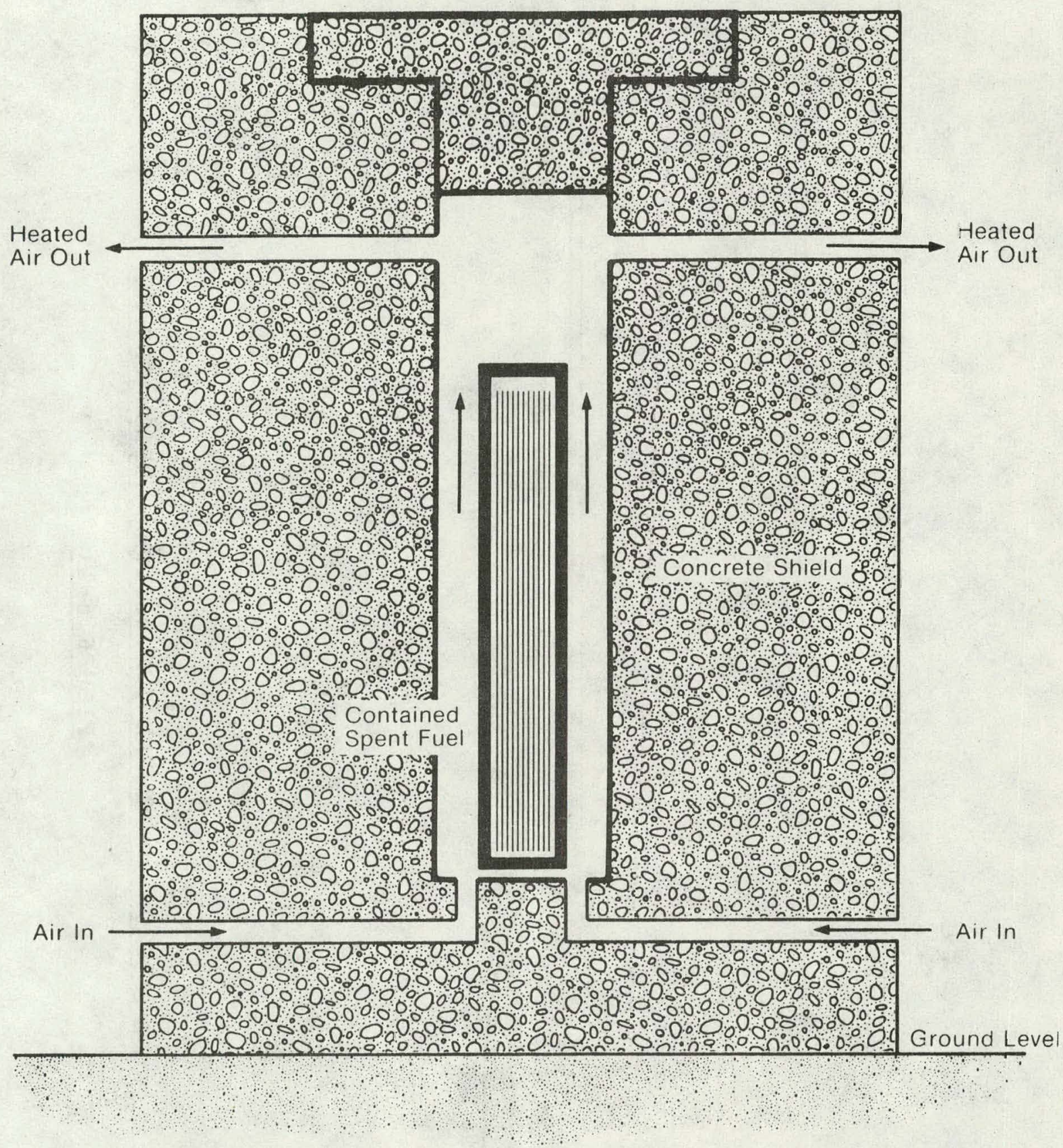

CAISSON - NATURAL CONVECTION - ABOVEGROUND

FIGURE $\quad 6-10$ 


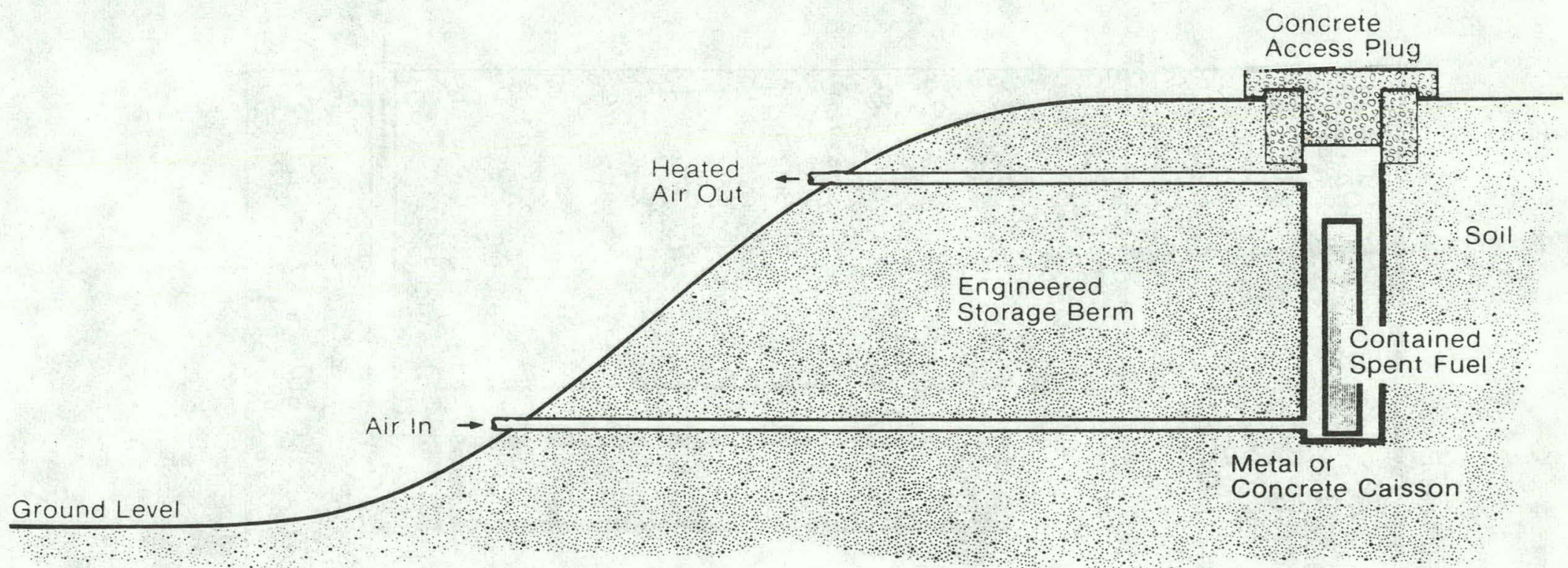

CAISSON - NATURAL CONVECTION - BELOW GROUND

FIGURE 6-11 
proposed by Electrowatt Engineering Services, Ltd. (Reference 6.13). Each concrete silo in the Electrowatt conceptual design would have the ability to store seven PWR fuel assemblies and could handle a maximum of 40-kilowatt heat load per silo. This is well in excess of that required in the aged TMI-2 fuel (by approximately a factor of 30 ). Heat pipes could also be introduced through the sides of the concrete shield. In either case, it is necessary to place the heat pipes in orientations that would minimize the amount of radiation "shining" through the heat pipe access openings. The heat pipes would transfer heat from the stored spent fuel to the outside air where it would be dispersed by natural convection.

The use of heat pipes in underground storage of spent fuel is shown in Figure 6-13. This concept may or may not use an engineered berm. The concept is shown not using a berm, which is probably less expensive. It is assumed that storage of spent fuel underground can be operated safely without employing an engineered berm. A detailed engineering safety analysis would have to be conducted to support this assumption. The heat pipes are inserted into the concrete shielding plug and draw heat from the spent fuel to be dispersed by natural convection.

\subsubsection{No Cooling Caisson}

The final caisson storage concept reviewed is above or below ground storage with no cooling. These concepts are shown in Figures 6-14 and 6-15, respectively. These concepts are the most simple and least costly designs. The aboveground caisson concept, shown in Figure 6-14, provides no cooling other than the heat sink effect of the large concrete structure. Nukem of Germany (Reference 6.11), Ontario Hydro of Canada (Reference 6.12), and the Nevada Test Site (References $6.14,6.15$ ) have proposed dry storage concepts for storing spent fuel that utilizes the aboveground caisson concept with no auxiliary cooling. The outside wall of the concrete will become heated slightly once equilibrium is reached. A slight degree of cooling will take place on the o uter concrete wall d ue to natural convection. This caisson concept could work either outside or inside a building.

The concept shown in Figure 6-15, underground spent fuel storage without auxiliary cooling, is similar to the conceptual caisson storage designed by Allied-General Nuclear Services (AGNS) for their proposed storage of hulls and general process trash (References 7,8 , and 9); again, this is waste material with small heat loads. The Nevada Test Site and the Atlantic Richfield Company (Reference 6, 16) have both proposed dry storage concepts for storing spent 


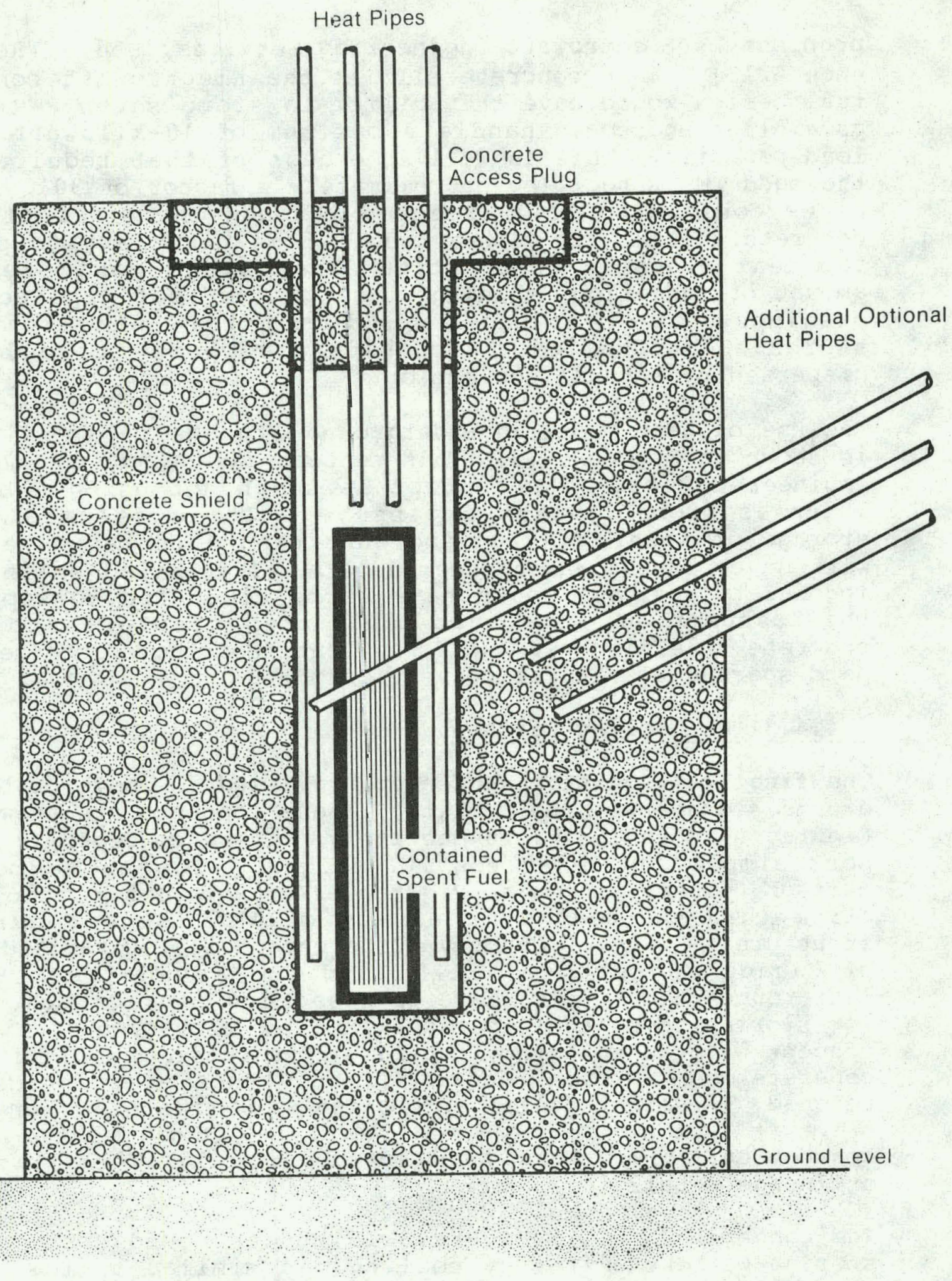

CAISSON ABOVEGROUND WITH HEAT PIPES

FIGURE 6-12 


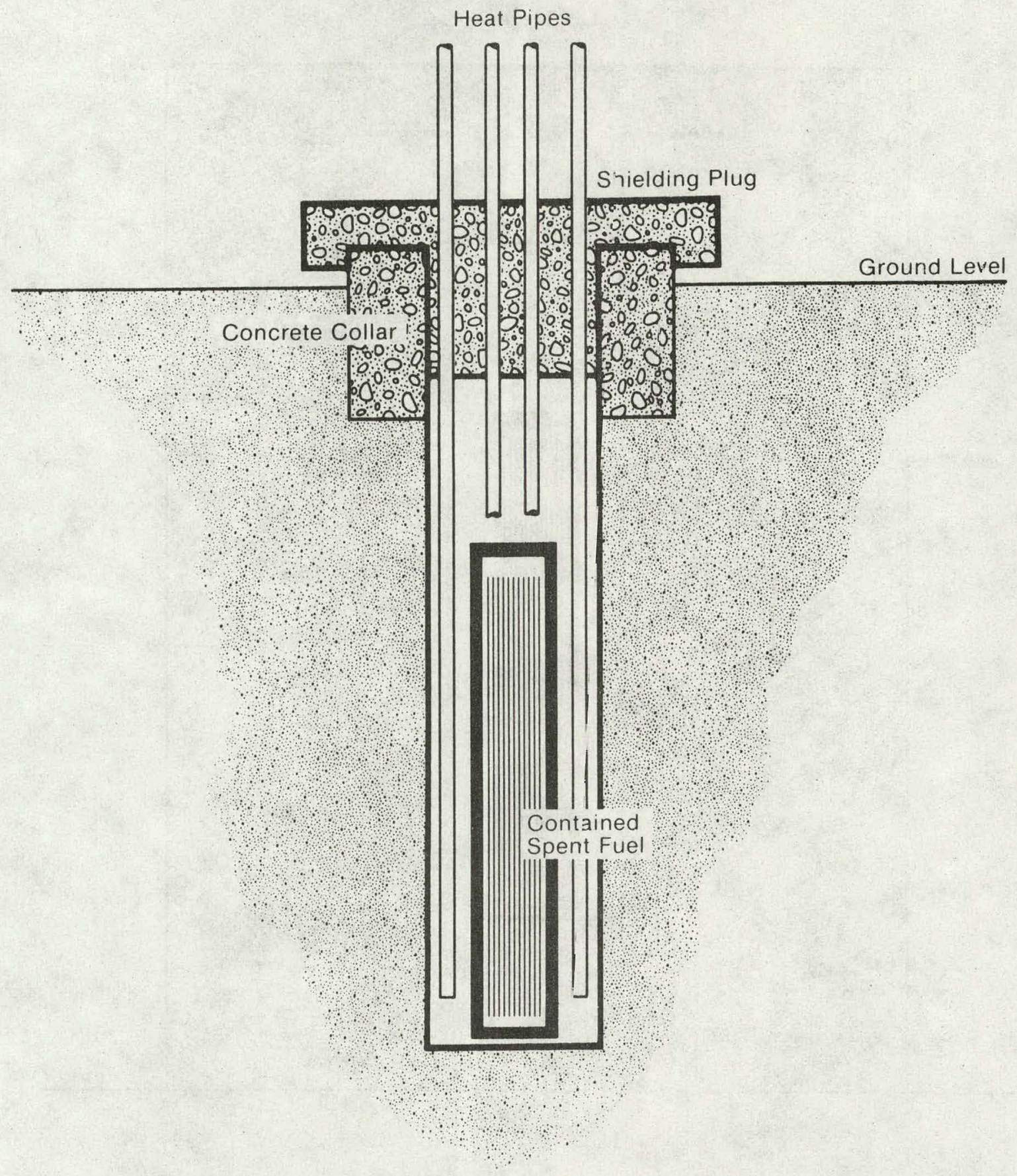

CAISSON BELOW GROUND WITH HEAT PIPES

FIGURE $\quad 6-13$ 
Concrete

Access Plug

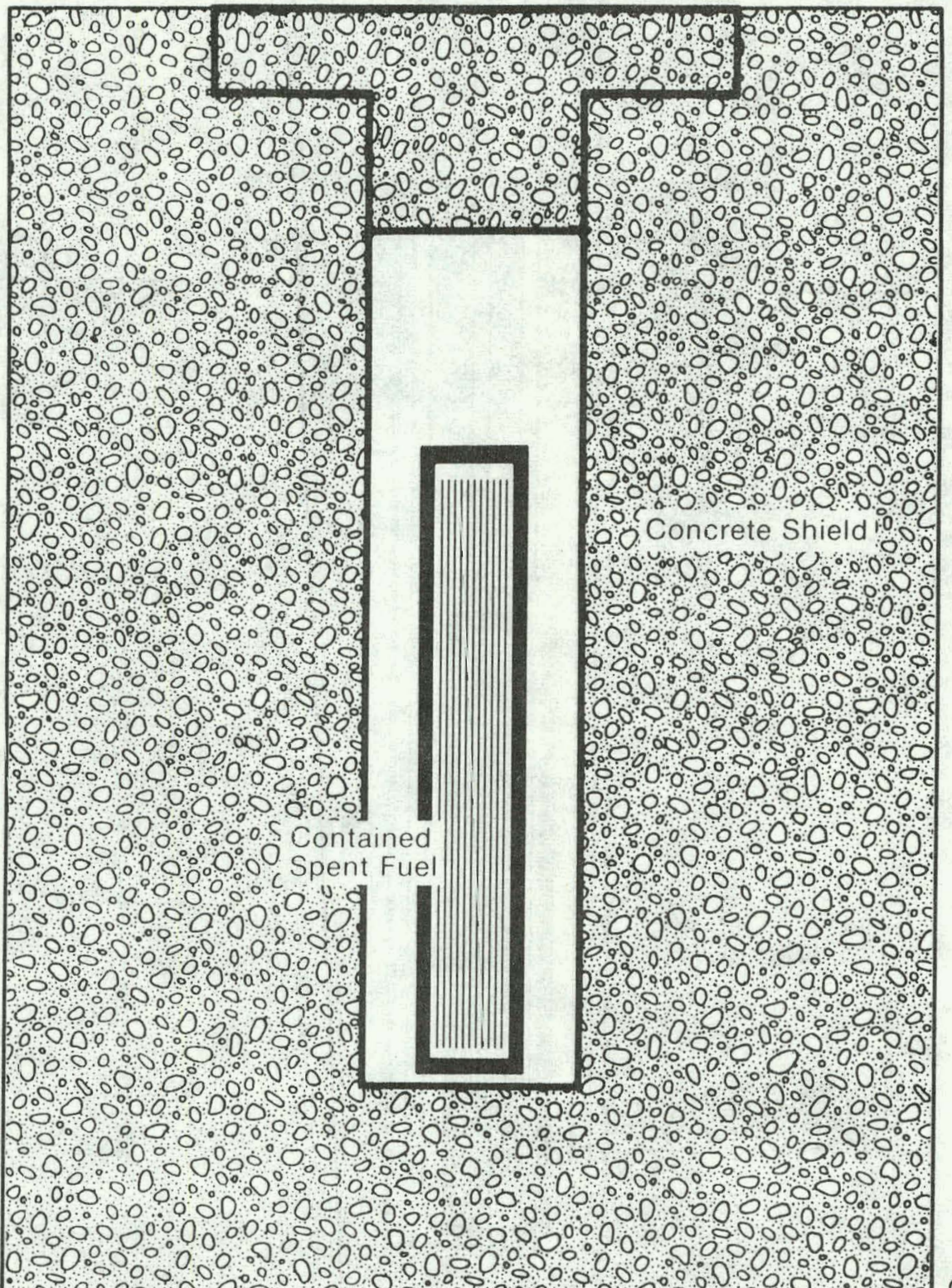

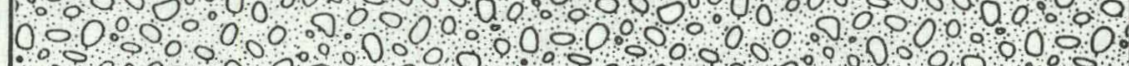

Ground Level

CAISSON ABOVEGROUND WITH NO COOLING

FIGURE 6-14 


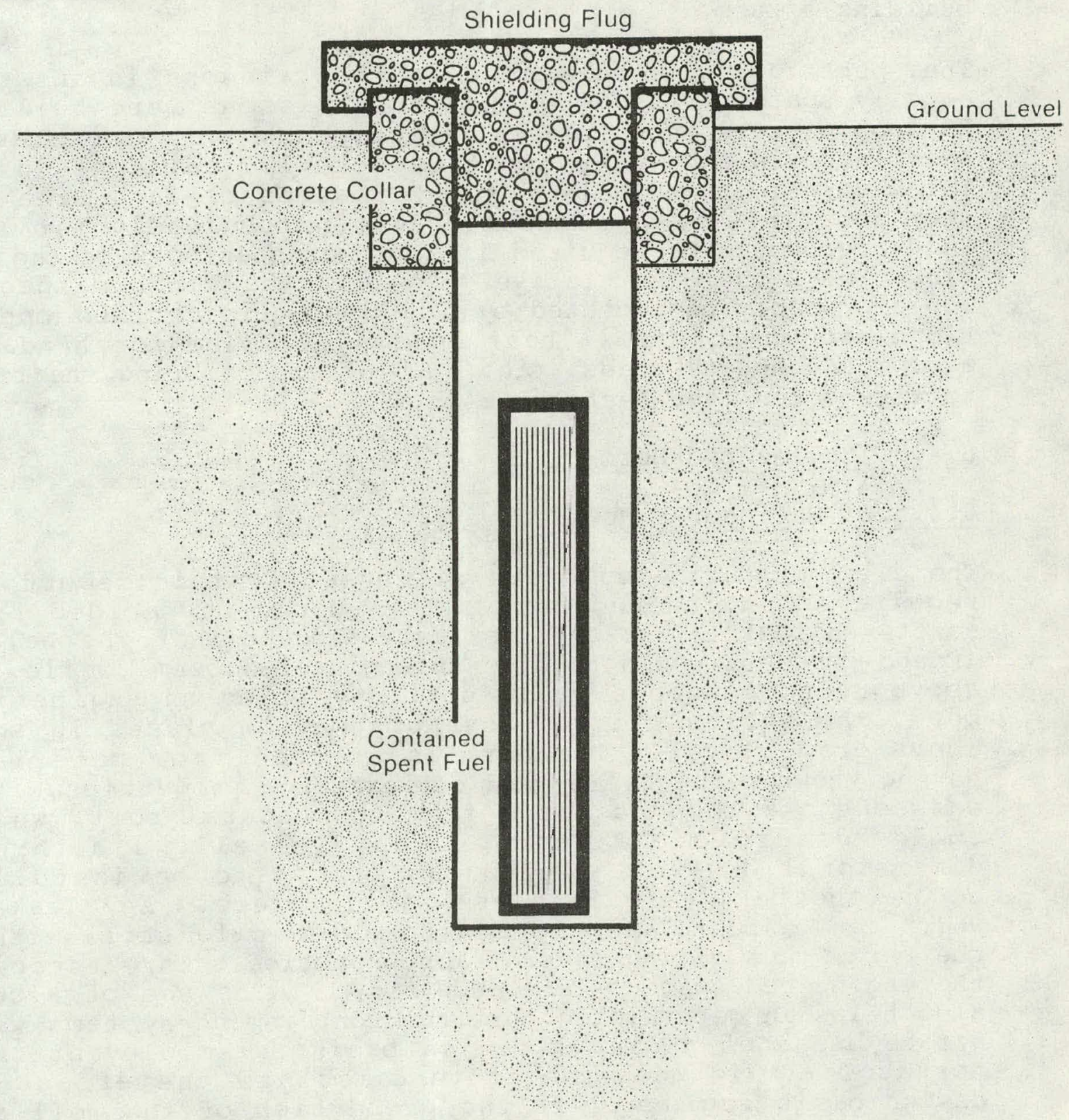

CAISSON BELOW GROUND WITH NO COOLING

FIGURE 6-15 
fuel that employ caissons, or silos, below ground with no auxiliary cooling. It was demonstrated that the use of an engineered berm would alleviate some of the design problems concerning earthquakes, floods, and heat removal. The details of such a berm are discussed in section 6.4. It would be possible at some locations to fabricate caissons directly in the ground without a berm. If this were possible, it could eliminate the expense of designing and building a berm.

The spent fuel will be placed in the caissons in an array equally spaced. Assuming that the caissons were built on 25-foot centers (conservative spacing) and only one assembly was placed in each caisson (there are 177 caissons in the TMI-2 core), it would take only 2.5 acres of land area for storage. This is an extreme case and obviously far less land area would be required if a closer spacing were chosen. Since TMI-2 fuel is relatively "cold," the minimum spacing would probably be dictated by handling equipment and operations rather than soil heat loading. Space for handling equipment, access roads, etc., would perhaps bring the total to five acres (see Section 6.4.5.2).

6.4.5 Engineered Berm

6.4.5.1 Engineered Berms

The caisson storage concepts discussed in this report may require an engineered berm. This would be decided by a safety analysis. The cost estimate assumed that the belowground concepts would require an engineered berm, while the aboveground concepts would not. By using an engineered berm, the entire caisson assembly may be placed in soil above grade level. The berm is erected from materials having known permeability and ion exchange properties. The caissons are thus isolated from the natural soil, which could be acidic. This approach also allows a threedimensional dry-well monitoring system to be installed befcre the berm materials are set in place. Any leakage which can be postulated is monitorable while still within the berm, thus giving several months notice before escaping the engineered system (Reference 6.17). The use of a berm also helps in determining that the containment systems will not be breached during a design basis seismic event or a tornado. It is much easier to calculate the effect of design basis accidents if the properties of the soil are knowr, as in the case of an engineered berm.

An example of an engineered berm is shown in Figure 6-16. A clay pad is laid at the existing grade level and is covered with a layer of relatively large aggregate. The purpose of 
the clay and gravel layers is to break the capillary communication between the original soil and the berm. The aggregate is then covered with another clay layer to provide additional capillary contrast. The remainder of the berm is constructed of a homogeneous fill having known ion exchange properties and a pore structure which is more open and free draining than the clay pads. In the fill, located above the clay pads, is laid a horizontal network of monitoring pipes. The berm material is added and capped with arother relatively impermeable clay layer. The toe of the berm is open, in the manner of an earth fill dam, to provide an escape route for water which may pass through any breach in the clay cap. A monitorable surface drainage systen is provided for surface run-off from the berm.

In the case of underground caisson storage concepts, holes are excavated in the berm and corrugated caissons (or concrete "silos") are set in place. Large aggregate is placed around the metallic caisson. The purpose of the aggregate in this case is to: (1) break the capillary communication between the caisson itself and the soil, and (2) insulate the metal from any acidic soil. The caisson may be equipped with a dip leg for monitoring purposes which is, in turn, equipped with a valve and a pressure gauge. Ar accumulation of water or change in activity level inside the caisson can be detected via this route. Beside each caisson is a dry well which, coupled with the underlying pipe network, yields a three-dimensional "fix" on any leaked radioactivity. The cover blocks are designed to seal the top of the caisson. Since caisson breathing will be ninor and since tornado criteria ( 3 psi) is not sufficient to break a properly designed cover-block seal, there is no reason to provide for continual airflow. If, however, the need arises, a small HEPA filter could be mounted on the dip tube and the valve left open.

\subsubsection{Siting Factors}

Depending upon the degree of compaction and spacing (one to three assemblies or more per container), the caisson storage concepts might take from $1 / 2$ to $2-1 / 2$ acres of 1 and, and the vault concepts would require less than $1 / 2$ an acre for the actual storage area. The "support" facilities, however, could require five acres or more (for an independent facility). These support facilities would include utility distribution, storage areas, operating areas, worker's change rooms, rest rooms, offices, receiving and shipping facilities, parking area, access roads, cleared security areas near perimeter fences, etc. Therefore, the space required for either vault or caisson storace will probably require a minimum of 5 to 10 acres for a stand-alone facility. If the storage of TMI-2 fuel were to be at an existing facility, 


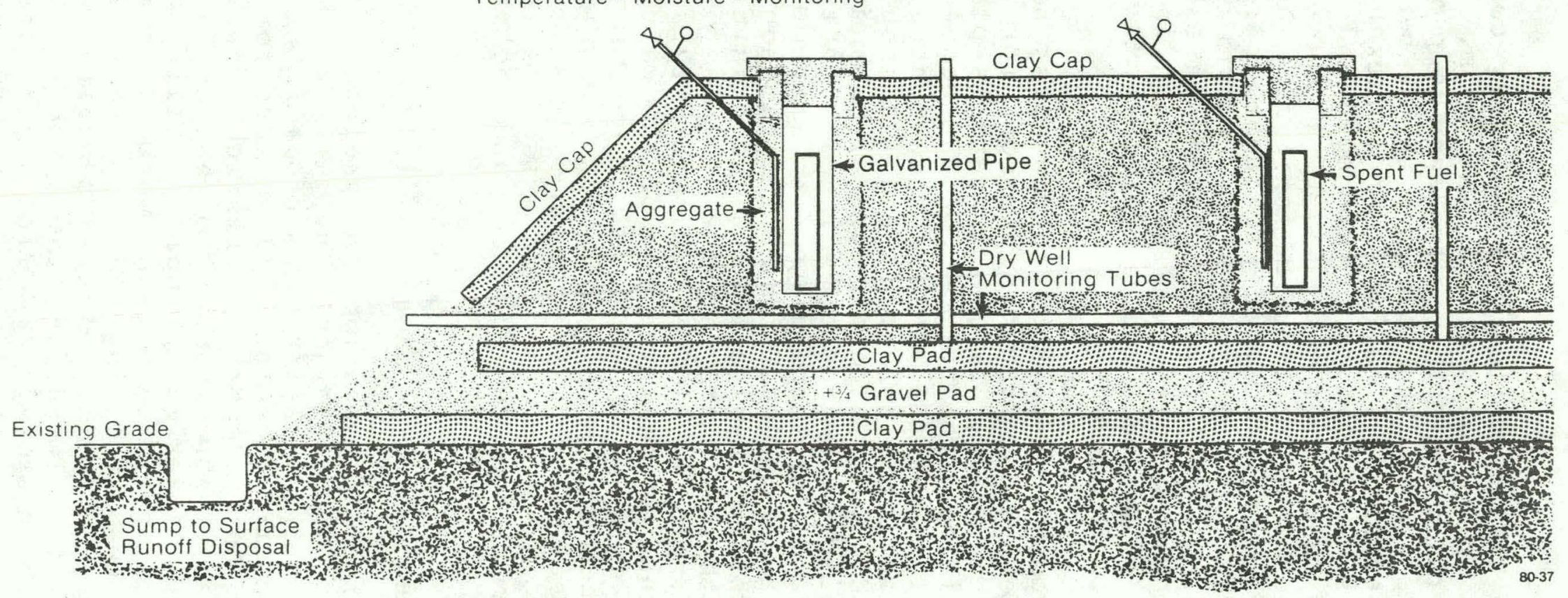

ENGINEERED BERM CONCEPT CAISSON STORAGE

FIGURE 6-16 
such as a national laboratory, most of the support facilities (roads, parking, etc.) would be available, and the land area required specifically for the TMI-2 dry fuel storage would be reduced.

The possible decision to store TMI-2 spent fuel on-site (in a caisson or possibly a spent fuel cask, at or near the TMI reactors site) depends upon trade-offs of technical feasibility, political factors, economics, and comparative risks. The feasibility of the concepts may be measured by the ability to store the fuel safely for an extended period of time, to meet all government laws/regulations/codes, and to be approved by appropriate authorities. A dry spent fuel storage facility would require the preparation and approval of a safety analysis that would confirm the facility's ability to operate safely even during design basis accidents (e.g., tornadoes or earthquakes). This has to be contrasted with the political realities associated with such things as local public acceptance, political pressures, and GPU company policy.

The last general area of concern, economics and schedules, is postulated below. It is expected that a new facility would take five to eight years to bring to an operational mode, unless special consideration was received from the Federal government. A storage facility built as an extension of an existing nuclear facility could, perhaps, be operational in three to five years. If political realities did not preclude on-site aboveground (TMI) caisson storage of spent fuel, the actual construction of the caissons could be performed to coincide with the canning of the fuel. Hence, considerable schedule time could be saved.

\subsubsection{Cost Estimates}

Initial capital costs and annual operating costs were estimated for the dry storage concepts under consideration. All of the storage concepts were assumed to accommodate disposition of the entire core. The costs would be le:is if more than one TMI-2 fuel assembly were placed into each container or caisson. This is particularly true for the caisson concepts. All of the concepts are based upon aistual construction taking between 2 to 4 years and being opelated for 20 years. It was assumed that the below ground caisson concepts would require an engineered berm while the akroveground concepts would not.

It is also important to note that the capacity of all. the storage concepts is enough to handle a single TMI-2 core, but not large enough to obtain cost savings from "economy of scale." For example, if one were to double the capacity of the vault concepts, the costs (capital and operating) would 
not double. They would increase by about 30 to 508 . If the caisson storage capacity were to be doubled, the costs would also not double, but in this case, they would increase by perhaps 60 to $80 \%$. It wolld appear that for a relatively small capacity storage system, the caisson concepts have an economic advantage, but for larger capacity storage centers (say 500 to 1000 assemblies), the vault concepts may be more economic.

\section{4 .7 Conclusions}

The storage of TMI-2 spent fuel in a dry environment appears to be feasible. For small quantities of spent fuel (e.g., the core of. TMI-2), it is nore economic to store the fuel in caissons rather than in large vaults. The most timely and least costly approach appears to be storing the TMI-2 fuel at an existing nuclear facility. For instance, previous studies (Reference 6.24) have surveyed existing hot cell facilities in the United States to determine their ability to receive, handle, disassemble, and reconstitute fulllength light water reactor spent fuel assemblies. Several of the hot cells examined would be adaptable to the storage of TMI-2 fuel. 


\section{REFERENCES}

6.1 Cumulative AFR Storage Requirements, Planning Base Total (US and Foreign), Spent Fuel Storage Requirement, DOE/NE 0002 (January 1980).

6.2 Carter, T. J., Status of Spent Fuel Pool Applications, 1979 Annual ANS Meeting (June 1979).

6.3 U. S. Nuclear Regulatory Commission, Staff Position Guide on Spent Fuel Storage and Handling Applications (April 1978).

6.4 Final Generic Environmental Impact Statement on Handling and storage of Spent light water Power Reactor Fuel, Document NUREG-0575, Vol. l, U. S. Nuclear Regulatory Commission (August 1979).

6.5 Spent Fuel Program Preliminary Technical Assessment of Existing Facilities for AFR Storage Capability, Document DOE/SRI0007, Rev. 1, Department of Energy (September 1979).

6.6. Myers, S. (DOE), Policy statement on DOE Remedial action for Spent Fuel Nuclear Programs to Committee on Interior and Insular Affairs, Subcommittee on Energy and the Environment (Februari 25, 1980).

6.7 Henry, K. H., and Turner, D. A., Storage of spent Unreprocessed Fuel (SURF), Rockwell International, Rockwell Hanford operation, Richland, Washington, RHO-SA-40 (March .1978).

6.8 Blackburn, L. D., et al, Maximum Allowable Temperature for storage of spent Nuclear Reactor Fuel, Hanford Eng ineering Development Laboratory, Richland, 'Washington, HEDL-TME 78-37, UC-71, an interim report !May 1978 ).

6.9 Ramspott, L. D., et al, Technical Concept for a Test of Geologic storage of spent Reactor Fuel in the Climax Granite, Nevada Test site, Lawrence Livermore Laboratory, UCRL-52796 (June 15, 1979).

6.10 Painter, M. J., and Meyer, H. S., Design and Operation of a Dry spent Fuel storage Installation, Final Summary for the 1979 annual ANS meeting (Atlanta, Georgia), CONF790602-25, Allied Chemical Corporation, Idaho National Engineering Laboratory, Idaho Falls, Idaho (1979). 
6.11 Klein, D. , Dry Storage of Spent Fuel from LightWater-Reactors with Inherently safe Air Cooling, Nukem GmbH (August 1979).

6.12 Barnes, R. W., and Drolet, T. S., Utilities Experience with the storage of Irradiated Fuel, Ontario Hydro, Toronto, Ontario, M5G 1X6, paper presented at workshop on the Management of spent Fuel and Radioactive Wastes, Washington, D. C. (September 16-19, 1979).

6.13 Doroszlai, P. G.; Ferroni, F. G.; and Watkins, G. Y., Transport and Dry storage of spent Fuel Elements, paper presented at the European Nuclear Conference $7 \overline{9}$, Hamburg, Electrowatt Engineering Services, Ltd.., Zurich, Switzerland (May 1979).

6.14 Staff, Fuel Temperature Test Interim Report, Nevada Test Site, Westinghouse Electric Corporation. WI : JBW : 79-367 (October 1979).

6.15 Staff, Drywell Spent Fuel Test Interim Thermal Report, Nevada Test Site, Westinghouse Electric Corporation, WI : JBW: 79-388 (November 1979).

6.16 Richards, L. M., and Szulinski, M. J., Subsur Storage of Commercial spent Nuclear Fuel, Nuclear Technology, Volume 43 (mid-April 1979).

6.17 Anderson, K. J., et al, Final Report: Engineering Evaluations of Wastes Handling and Storage Facilities, Allied-General Nuclear Services, AGNS-35900-4.2-26 (November 1979).

6.18 Menon, M. N., Spent Fuel Handling and Packaging Program - - A Survey of Hot Cell Facilities, Hanford Engineering Development Laboratory, HEDL-TME 78-53, UC-70 (July 1978). 


\subsection{ECONOMIC AND SCHEDULE EVALUATION}

\subsection{General}

Scoping evaluations were performed related to the economic comparisons of a number of TMI-2 core disposition options. These evaluations were based on a combination of published data, detailed cost estimates, and best judgment. Three specific interim alternatives for disposition, as noted in Section 6.0, were examined on a parametric basis to obtain the range of possible costs. Specifically, the interim options - chemical reprocessing, pool storage, dry storage -- were addressed, utilizing a number of possible scenarios related to off-site shipment date, storage period, and the initial availability of a waste repository for high-level nuclear material. Table 7-l presents the cost centers addressed for each specific case. Costs associated with on-site canning and storage were not addressed. It must be noted that such factors as inflation, time-value of money, and accountability for $O \& M$ labor were developed in a simplified manner due to the unique nature of this problem. These studies should be utilized solely to scope the magnitude of the extra costs involved with the core disposition. Circumstances and the accounting procedures used may cause these values to change considerably, however the general conclusions derived from these results will still be valid.

\subsection{Economic Study Results}

\subsubsection{Chemical Reprocessing}

Three cases were developed, each of which are summarized in Table 7-2. The cases represent the following scenarios:

Case 1 -- Mature (Domestic) Reprocessing -- Reprocessing starts in 1990 with recovered uranium and plutonium.

Case 2 - Mature (Domestic) Reprocessing -- Reprocessing starts in 1990 with no value for plutonium.

Case 3 - Foreign Reprocessing with equivalent (to United states) values for recovered uranium and plutonium.

Cases 1 through 3 assume on-site pool storage prior to reprocessing. Case 2 (no plutonium recovered) could describe either a coprocessing option or direct disposal of the plutonium with the high-level waste. In either event, the relative value of recovered plutonium does not greatly affect the results of these studies. In other words, the 
SABLE $7-1$

TMI-2 FUEL DISPOSITION COST CENTERS

1.0 Shipping Costs

1.1 Shipping to Storage Site

1.2 Shipping to Repository

1.2.1 Spent Fuel to Repository

1.2.2 Reprocessing Waste to Repository

2.0 Interim Disposition Costs (Off-site)

2.1 Chemical Reprocessing

2.1.1 AFR Storage

2.1.2 Reprocessing

2.1.3 Recovered Products (Benefit)

2.2 Pool storage

2.3 Dry Storage

2.3.1 Vault

2.3.2 Caisson

3.0 Repository Waste Disposal Cost

3.1 Reprocessing Waste

3.2 Spent Fuel 


\section{TABLE $7-2$}

\section{REPROCESSING SCENARIOS}

\section{ASSUMPTIONS}

Initial fuel off-site shipment - 1983

Reprocessing start date

- 1985 - Case 3

- 1990 - Cases 1 and 2

Geological repository open $\quad 2000$

CASE NUMBER:

1

2

3

$+$

DESCRIPTION

1.1 Shipping

2.1.2 Reprocessing

3.1 Repository Disposal

3.1.3 Recovered Product (Benefit) *

TOTAL COST

DOLLARS/KgU

*(Benefit)
IDEAL REPROCESS ING

$1,590,000$

$21,920,000$

$7,280,000$

$(53,820,000)$

$(23,030,000)$

$(280.80)$
$(19,160,000)$

(233.65)
FOREIGN

REPROCESSING

$6,620,000$

$62,050,000$

$7,280,000$

$(49,950,000)$

$(53,820,000)$

$14,850,000$

181.20 
disposition of plutonium does not represent a major economic variable for TMI-2 fuel.

The reprocessing cases do illustrate that the recovery value of the uranium for the TMI fuel is large. If there is a viable, reprocessing option available, the TMI fuel (more so than full burnup fuel) wou-d represent a prime economic candidate for such reprocessing. Reprocessing overseas. While seeming less attractive than the "idealized" domestic cases, appears attractive when the cost of storage prior to "throwaway" of the fuel in a Federal Repository is added. In summation, reprocessing to recover uranium has the potential for the greatest economic impact on this study if it is allowable with this fuel.

\subsubsection{Pool storage}

Three different pool storage scenarios (all related to a throwaway fuel cycle) were examined including on-site pool storage and early or late opening of the federal repository. These cases are summarized in Table 7-3. The following scenarios are presented:

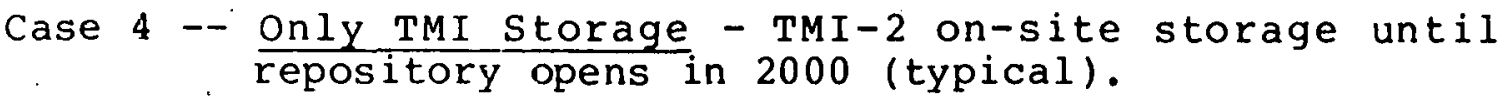

Case 5 -- Both TMI and AFR S=orage - TMI-2 on-site storage ( 8 years average) plus ten years AFR storage Repository opens in year 2000 (typical).

Case 6 -- Long-Term AFR Storage - Twenty years of AFR storage until repository opens in year 2005 (late).

The costs for these three cases range from $\$ 12$ to $\$ 31$ million. Obviously, the major factors in these cases are the shipping cost and the duration of off-site AFR storage.

On-site storage obviously is the least costly alternative, particularly since this evaluation does not factor in any unusual costs for this. This conclusion is probably reasonable so long as there is an operating crew at the TMI site. If it is necessary to either cecommission the plant or move the fuel off-site, cases 5 and 6 then become more realistic.

\subsubsection{Dry Storage}

Three different dry storage scenarios were examined including vault and caisson storage and the effect of a dedicated (small capacity) versus a nondedicated (large capacity) 


\section{TABLE $7-3$}

POOL STORAGE SCENARIOS

\section{CASE SUMMARY:}

CASE

DESCRIPTION

Initial Shipment from TMI

Initial Shipment From AFR

Repository Opens

1.1 Shipment to AFR

1.2 Shipment to Repository

2. 2 AFR Storage

3. 2 Repository Disposal

TOTAL COST

DOLLARS/KgU
4

$$
\text { NO AFR }
$$

STORAGE

2000

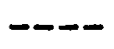

2000

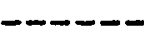

$5,030,000$

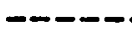

$7,280,000$

$\$ 12,310,000$

148.49
5

TMI \& AFR STORAGE

1990

2000

$20 \mathrm{C}: 0$

$1,590,000$

$4,260,000$

$10,750,000$

$7,280,000$

$\$ 23,880,000$

288.06
6.

LONG-TERM AFR STORAGE

1985

2005

2005

$1,590,000$

$4,260,000$

$18,200,000$

$7,280,000$

$\$ 31,330,000$

377.93 
facility. Table 7-4 summarizes the cases studied. The following scenarios are presented:

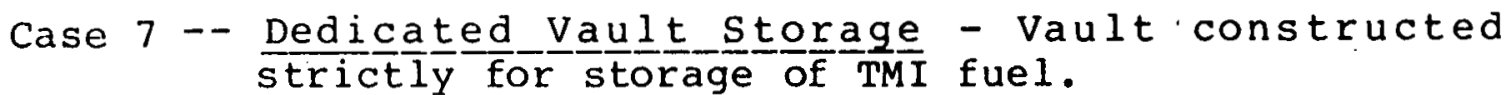

Case $8-$ Nondedicated Vault Storage - Hot cell with applicability for dry storage of other fuel.

Case 9 -- Caisson Storage.

The total costs range from $\$ 25$ to $\$ 45 \mathrm{million}$. This appears to be a less attractive option than pool storage. However, if an AFR or other pool site is not available, this represents a possible contingency or "back-up" option. It may be desirable to use on-site caisson storage for small quantities of severely damaged fuel.

\subsection{Cost Assumptions}

This section documents the means utilized to develop the costs presented in preceding sections in accordance with the cost centers presented in Table 7-1. "Annualization" of the fixed costs associated with capital expenditures for the TMI-2 recovery is a simplifying technique to permit comparison. Typically for a commercial or public utility this would include:

- depreciation of equipment

- return on capital investment (ROI).

Another unique area is the operation and maintenance (O\&M) costs associated with on-site canning and storage, off-site storage, etc. Normally, this work force is already inplace. Hence, the operational labor at a facility is prorated based on total facility operation costs. To simplify the problems associated with uncertainty in annualizing capital and prorating $O \& M$ cost, the following two guidelines were used:

- Specialized Capital - The installed "cost" is denoted the annualized "fixed charges" merely includes a linearized depreciation for the duration of the storage period.

- $\quad 0 \& M$ - An estimate of the number of direct man-years required is presented. An estimate of $\$ 50,000$ per manyear including both labor and materials was utilized.

In other questionable areas, there are published estimates of total charges and also estimates of approximate annualized costs (e.g., AFR costs). In this situation, both 


\section{TABLE $7-4$}

DRY STORAGE SCENARIOS

\section{ASSUMPTIONS}

Initial TMI-2 Fuel off-Site Shipment

- Vault

1980

- Caisson

1985

Shipping Distance

- TMI to Storage

- Storage to Repository

1000 miles

Initial Date of Repository Operation 2000

Case

\section{DESCRI PTION}

1.1 Shipment to Storage

1.2 Shipment to Repository

2.3 Dry Storage

3.2 Repository Disposal

TOTAL COST

DOLLARS/KgU
7 DEDICATED VAULT

$3,500,000$

$1,980,000$

$32,500,000$

$7,280,000$

$\$ 45,260,000$

551.95
8

NON-DEDICATED

VAULT

$3,500,000$

$1,980,000$

$12,500,000$

$7,280,000$

$20,900,000$

- $7,280,000$

$\$ 33,660,000$.

410.49 
values are presented and the published charge used as a basis.

\section{7. .1 Shipping Costs}

The cost assumptions were delineated in Section 5.5 of this; report and are as follows:

\begin{tabular}{crr} 
Distance (Miles) & $\$ / \mathrm{KgU}$ & \$/Entire Core \\
\cline { 2 - 3 } 750 & 19.23 & $\$ 1.59$ Million \\
1000 & 23.94 & 1.98 Million \\
2000 & 42.32 & 3.50 Million \\
3000 & 60.82 & 5.03 Million
\end{tabular}

Overseas Shipment - $80 \$ / \mathrm{KgU}$

\subsubsection{Interim Disposition Costs}

7.3.2.1 Chemical Reprocessing

The costs estimated with domestic reprocessing as a mature industry are presented in Reference 7.1 .

- Domestic Reprocessing

- Foreign Reprocessing (Rəference 7.2)

- Recovered Uranium (benefit) $\$ 604 / \mathrm{KgU}$ or $\$ 49.95$ Million

- Recovered Plutonium (benefit) $\$ 47 / \mathrm{KgU}$ or $\$ 3.87$ Million

\subsubsection{Pool storage}

The costs associated with wet storage at an external reactor (AFR or any other facility not dedicated solely to TMI-2 fuel) are as follows:

- AFR Pool Storage (Reference 7.3)

Independent estimates of temporary costs are $\$ 15-20 / \mathrm{KgU} /$ year during the initial years of receiving fuel and $\$ 8-12 / \mathrm{KgU} /$ year afterwards; hence, the preceding charge was utilized for up to 10 years storage. The graduated scale assumed was :

- up to 10 years storage a $\$ 130 / \mathrm{Kgu}$ or $\$ 10.75 \mathrm{million}$

- 20 years storage a $\$ 220 / \mathrm{KgU}$ or $\$ 18.20 \mathrm{million}$

- 30 years storage a $\$ 300 / \mathrm{KgU}$ or $\$ 24.81 \mathrm{million}$. 


\subsubsection{Dry Storage}

Vault storage

Two possibilities are evident concerning the case of a vault for dry storage. The first (noted in section 6.3) is dedicated solely to TMI-2 fuel. The second possibility is the use of an existing hot cell or a portion of a large storage vault. In the latter case where either new construction was not involved or a portion of the facility was prorated for use of TMI-2 fuel, it is expected that. lower capital or operating costs would result.

The following costs were estimated based on the data in Section 6.3:

- Dedicated Vault Storage

- Nondedicated Vault Storage
$\$ 25$ Million plus 15 manyears per year for $O \& M$

$\$ 10$ Million plus 5 manyears per year for O\&M.

\section{Caisson storage}

Estimates of the capital and operational costs associated with dedicated caissons were made. There is a possibility of using nondedicated equipment for storage caisson usage (e.g., utilization of spent fuel casks or temporary usage of similar equipment). In this case, the increased years of service life would lower the annual costs. The estimates are presented below:

- Dedicated Caisson Storage

- Nondedicated Caisson Storage
$\$ 13.4$ Million plus 10 manyears per year* O\&M

$\$ 8.0$ Million plus 5 manyears per year $O \& M$.

In order to obtain comparable costs for vauit and caisson storage, the capital was depreciated during the storage lifetime and added to the o\&M costs.

\subsubsection{Repository Costs}

These costs were obtained from Reference 7.2 which summarizes previous work performed by consultants to DOE related to repository use charges. The follcwing costs were utilized:

* Storage year. 
- Spent fuel (throw away) disposal-\$88/Kgu or $\$ 7.28$ Million

- Reprocessing Waste (including $-\$ 77 / \mathrm{KgU}$ or $\$ 6.34$ Million transportation). 


\section{REFERENCES}

7.1 Spent LWR Fuel Storage Costs - Reracking, AR Easins, and AFR Basins, Document DOz/SR-SF-2002, Rev. 1 , Department of Energy (January 1980).

7.2 AGNS Internal Document, Nuclear Fuel Cyclee_c log Alternatives: A Reassessment (May 1979).

7.3 AGNS Internal Document, A Procedure for Calculating the Salvage: Value of Spent Nuclear Fuel (August 1979: 
AGNS/DOE CONTRACT NO. DE-ACO9-78ET 35900

UC-85

Report No.: AGNS-35900-1.5-79

Standard AGNS Internal Distribution:

K. J. Bambas (1)

J. A. Buckham (1)

R. J. Cholister (1)

P. F. H1ghberger (1)

W. Knox (1)

R. C. Ravasz (1)

C. T. Stribling (1)

W. B. Sumner (1)

A. K. Williams (1)

Library (1)

Records Management (40)

Additional AGNS Internal Distribution:

K. J. Anderson (1)

R. T. Anders on (1)

W. H. Carr (1)

W. L. Godfrey (1)

J. H. Gray (1)

P. N. McCreery (1)

G. A. Townes (1)

V. R. Waltz (1)

Other Distribution:

Mr. W11lis Bixby (12)

Manager, DOE Site Office

P. 0. Box 88

Middletown, Pennsylvania 17057

Mr. Herbert Feinroth (12)

Nuclear Power Development Division

Department of Energy

Mail Stop. B107

Washington, D. C. 20545

[DOE Distribution:

Mr. J. W. Geiger, Project Englneer (2)

Waste and Fuel Cycle Technology office

DDE Savannah River Operations office

Post Office Box A

Aiken, South Carolina 29801

Dr. J. Spencer (2)

Savannah River Laboratory

E. I. du Pont de Nemours and Company

Aiken, South Carolina 29801
Date Issued: May 28, 1980

Will1am Burch (3)

Oak Ridge National Laboratory

Post Office Box X

Oak Ridge, Tennessee 37830

Mr. S. W. O'Rear, TIC (1)

Savannah River Laboratory

E. I. du Pont de Nemours and Company

Alken, South Carolina 29801

Mr. D. C. Drennon (1)

Contracting officer

DOE Savannah River Operations office

Post Office Box A

Aiken, South Carolina 29801

W. W. Ballard (3)

Chief

Nuclear Fuel Cycle Program Branch

DOE Germantown, Maryland 20767

Mr. M. J. Lawrence (3)

Director

Fuel Storage and Transfer

DOE Germantown, Maryland 20767

Mr. L. H. Meyer (3)

Savannah River Plant

E. I. du Pont de Nemours and Company

Alken, South Carolina 29801

TIC Distribution:

*DOE Technical Information Center (126)

Post Office Box 62

Oak Ridge, Tennessee 37830

*Per attached list. 
TIC STANDARD DISTRIBUTION (Total 119)

UC-85. Distribution List Away-From-Reactor Program

DOE Operations office

\author{
Albuquerque \\ Chicago \\ Savannah River, Manager \\ Savannah River, Contracting officer \\ Idaho \\ Oak Ridge \\ Richland \\ San Francisco \\ Nevada \\ Savannah River, AFRPO \\ Savannah River, Classification Officer \\ Columbus (Ohio) Area Office
}

\title{
DOE Prime Contractors
}

$\begin{array}{ll}1 & \text { Mound Lab, DAO } \\ 1 & \text { Rockwell International, RFP } \\ 1 & \text { Argonne National Lab, CH } \\ 1 & \text { Battelle Pactfic Northwest Lab, RL } \\ 2 & \text { Savannah River Lab, SR } \\ 1 & \text { Hanford. Engineering Development Lab, RL. } \\ 2 & \text { Los Alamos Scientific Lab, AL } \\ 2 & \text { Oak Ridge National Lab, OR } \\ 1 & \text { Sandia Lab, AL } \\ 1 & \text { Lawrence Livermore Lab, SAN } \\ 1 & \text { Rockwell Hanford Operations, RL } \\ 1 & \text { Oak Ridge Gaseous Diffusion Plant, OR } \\ 1 & \text { EG\&G Idaho Inc., ID } \\ 1 & \text { Brookhaven National Lab, Upton, NY } \\ 1 & \text { Exxon Nuclear Corporation, ID } \\ 1 & \text { Savannah River Ecology Lab., Alken, SC } \\ 2 & \text { Battelle Columbus OWI, Columbus, OH } \\ & \quad \text { Government, Other }\end{array}$

3 DOE Division of Spent Fuel Storage and Transfer, Director, HQ

1 Library of Congress

25 DOE TIC

25 National Technical Information Service (COMM)

1 DOE office of Safeguards and Security, HQ

2 DOE Office Waste Management Division, HQ

1 Nuclear Regulatory Commission, Washington, D.C.

1 Nuclear Regulatory Commission, Atlanta, GA

1. DOE Nuclear Power Division, HQ

2 DOE Division of Energy Research, HQ

1 DOE Office of Environmental and Safety, HQ 
TIC STANDARD DISTRIBUTION (CONTINUED)

UC-85 Distribution List Away-From-Reactor Program

\section{Industry}

1 Management Analysis Corporation, San Diego, CA

1 Babcock \& Wilcox Co., Lynchburg Research Facility, Lynchburg, VA

1 General Electric Uranlum Management Corporation, San Jose, CA

1 Battelle Columbus Lab., Columbus, $\mathrm{OH}$

1 Boeing Engineering and Construction, Seattle, WA

1 Kaman Sctence Corp., Colorado Springs, Co

1 Nuclear Assurance Corp., Atlanta, GA

1 General Atomic, San Diego, CA

1 All led-General Nuclear Services, Barnwell, SC

1 . S. M. Stoller Corp., New York, NY

1 Nuclear Services Corp., Campbell, CA

1. Westinghouse Corporation, Albuquerque, MM

\section{Institutes}

1. EPRI, Palo Al to, CA

\section{Universities}

1. University of South Carolina, Columbia, SC 
Frank Arsenault, Director

Division of Safeguards, Fuel Cycle, and Environmental Research

Office of Nuclear Regulatory Research U. S. Nuclear Regulatory Commission Washington, D. C. 20555

Dr. R. E. Brooksbank

Building 3019

Oak Ridge National Laboratory

Post Office Box X

Oak Ridge, Tennessee 37830

William H. Chambers

Deputy Associate Director for

Safeguards \& Security

Ma1l Stop 550

Post Office Box 1663

Los Alamos Scientific Laboratory

Los Alamos, New Mexico 87545

Dr. L. D. Chapman, Supervisor

Systems Analysis Division I

Sandia Laboratories

Albuquerque, New Mexico 87115

R. J. Jones, Chief

Materials Protection Standards Branch

Division of Siting, Health, and Safeguards Standards

Office of Standards Development

U. S. Nuclear Regulatory Commission

Washington, D. C. 20555

Dr. G. Robert Keepin

Associate Division Leader

Nuclear Safeguards Program Director

Mail Stop 550

Los Alamos Scientific Laboratory

Los Alamos, New Mexico 87545

George Weisz, Director

office of Safeguards and Security

U. S. Department of Energy

Washington, D. C. 20545

H. T. Kerr, Safeguards Task Leader

Consolidated Fuel Reprocessing Programs

Engineering Technology Division

Oak Ridge National Laboratory-Nuclear Div.

P. 0. Box Y

Oak Ridge, Tennessee 37830
R. G. Page; Special Assistant to the Director for Licensing

Division of Safeguards

Office of Nuclear Material Safety and Safeguards

U. S. Nuclear Regulatory Commission

Washington, D. C. 20555

Dr. P. J. Persiani

Argonne National Laboratory

9700 South Cass Avenue

Argonne, Illinois 60439

T. A. Sellers, Supervisor

Advanced Facilities Protection Division

Division 17.61.

Sandia Laboratories

Albuquerque, New Mexico 87115

Sylvester Suda

Technical Support Organization

Building 197

Brookhaven National Laboratory

Upton, Long Island, New York 11973

Don Crandall

Bechtel, Inc.

Post Office Box 3965

San Francisco, California 94105

R. W. Peterson

Battelle Project Management Division

Office of Nuclear Waste Isolation

505 King Avenue

Col umbus, Ohio 43201

F. A. O'Hara

Battelle Columbus Laboratories

505 King Avenue

Columbus, Ohio 43201

G. L. Stukenbroeker

Nuclear Transportation

NL Industries, Inc.

1130 Central Avenue

Albany, New York 12205 
J. V. Cupo

Westinghouse Electric Corporation

Power Systems

Nuclear Center

Post office Box 355

Pittsburgh, Pennsylvanta 15230

General E. C. Hardin

U. S. Department of Energy

Mail Number B-107

Washington, D. C. 20545

R. M. Jefferson, Manager

Nuclear Fuel Cycle Technology Development

Jepartment 5430

Sandia Laboratories

Albuquerque, New Mexico 87115

3. K. Rhode, Vice President

Niagara Mohawk

300 Erie Boulevard

Syracuse, New York 13202

K. B. Chitwood, Assistant Director

Transportation Branch

U. S. Department of Energy

Mail No. B-107

Washington, D. C. 20545

Charles E. MacDonald, Chief

Transportation Branch

Office of Nuclear Material Safecy

and Safeguards

U. S. Nuclear Regulatory Commission

Washington, D. C. 20555

R. R. Raw1

Macerials Transportation Bureau

U. S. Department of Transportation

Washington, D. C. 20591

Tom Carter.

Depaty Director

Division of Fuel Cycle and

Material Safety

U. S. Nuclear Regulatory Commission

Washingtor, D. C. 20555 

TMI-2 FUEL AND CORE COMPONENTS. EXAMINATIONS:

FINAL REPORT OF TMI-2 PLANNING GROUP 7.4

Compiled and Edited

by

J. E. Hanson

D. E. Owen

D. W. Croucher

EG\&G Idaho, Irc.

July 1980

$7.4-1$ 


\section{ABSTRACT}

This report summarizes the sequence of events which occurred during. the TMI-2 accident as these events -elate to fuel and core component behavior. It also identifies the highly unique, fully typical data which can be provided by an examination of the TMI core. These data can be used for advancing analytical method development, licensing criteria, safety, accident consequence mitigation, ano the design, operation, and maintenance of 1 ight water reactors. In addition, the report outlines potential fuel and core component examinations, depending on the condition of the core, and presents guidelines for transporting the fuel and the core components and for selecting examination and disposal facilities. Finally, the report considers the Task 7.2 recommendations on in-place TMI-2 core damage examination; and makes recommendatiors for TMI-2 fuel experiments, examination, and coordination with other tasks. 


\section{EXECUTIVE SUMMARY}

At the initial meeting of the Technical Working Group, held on September 11-13, 1979, 17 specific tasks in seven general areas were identified. The group agreed that most of the $1 ?$ tasks required a Technical Planning Group to develop a detailed definition of each task and to clarify its goals and methods of implementation. Among the tasks requiring detailed definition was one designated as Task 7.4, Fuel Experiments and Examination. The goal of Planning Group 7.4 was to develop a program plan for the detailed technical examination of the TMI-2 fuel and core components. To prepare the plan, members of Planning Group 7.4 met November 20, 1979 and February 7-8, 1980; and exchanged much correspondence. This report is a compilation of the efforts of Planning Group 7.4, and represents the final program plan for Task 7.4.

The report begins with a summary of the sequence of events which transpired during the TMI-2 accident. The estimated core candition is discussed and serves as a basis for examination recommendations which follow.

The next four report sections present rationale and justificaticn for performing the TMI-2 fuel and core component examinations. These sections indicate how examination data could be used to make a broad range of improvements to a number of generic LWR technologies.

One of the technologies to be advanced by TMI-2 is the development and assessment of computer models used to describe the course and.consequences of severe damage accidents. The development of such models would improve risk assessment and accident consequence analysis, and could be used to evaluate reactor design change recommendations.

The TMI-2 experience could also supply critical data in the area of future licensing criteria, since it appears that rulemaking hearings may be held to evaluate new reactor siting criteria and severe core damage accident analysis requirements. 
Data for accident consequence mitigation is a third area to which the TMI-2 examination would contribute. The TMI-2 examinations will reveal if inherent mitigation of savere accidents is possible in existing reactor designs; if any features of present designs exacerbate accident conditions; and will provide guidance for the development of inherent accident mitigators.

The fourth technology to which the TMI-2 examination would contribute is reactor operation and maintenance. These include such items as improved indications of reactor status, improved equipment testing procedures, better decontamination procedures, and development of techniques for accicent recovery.

Another section of the report is devoted to core examination recomendations. This section presents recommendations for a broad range of in:act assembly and fuel debris examinations, and for examinations of intermal core components and techniques for core hydraulic measurements.

(Ither. sections of the report address support questions such as removal, packaging, and shipping of core samples; examination facility criteria; fuel disposal; and coordination with Task 7.2 on core damage assessment.

The last section of the report presents the final recommendations of Planning Group 7.4. These recommendations will now be considered by the Technical Working Group and the Joint Coordinating Committee. Implementation of the tasks approved by these bodies will be at the direction of DOE.

Applicable postirradiation examination techniques are detailed in tabular form in Appendix A. 


\section{ACKNOWLEDGMENTS}

The following individuals are gratefully recognized for their contributions to Planning Group 7.4. The efforts of E. L. Ccurtright, J. S. Tulenko, and R. A. Copeland were particularly significant and represent a major portion of this report.

NAME

AFFILIATION

Andrew C. Millunzi--Planning Group Chairman

Harold M. Burton

Denn is $E$. Owen

Edward L. Courtright

John E. Hanson

Phil ip E. MacDonald

Dougl as. W. Croucher

Larry E. Hochre iter

Denn is L. Burman

George A. Last

Ernest L. Long

- David 0. Hobson

Len M. Mak i

Franklin D. Coffman

Raiph 0. Meyer

M. L. Picklesimer

Tom E. Morris

Howard Ocken

Art B. Carson

Richard 0ehlberg

Robert N. Whitesel

Jon A. Reuscher

George A. Sofer

Gary L. Sozzi

Tony Stathoplos
U. S. Department of Energy

EE\&G Idaho, Inc., TMI-2

Technical Integration office

Battelle Pacific Northwest Laboratories

EG\&G Idaho, Inc.

Westinghouse Electric Corporation

Westinghouse Hanford Co.

Oak Ridge National Laboratory

Rockwell International

U. S. Nuclear Regulatory

Commission

Bechtel Power Corporation

Electric Power Research

Institute

Sandia National Laboratory

Exxon Nuclear Company

General Electric Company

Combustion Engineering, Inc. 
Neil E. Todreas

James S. Tulenko George E. Kulynych

R. A. Copel and
Massachusetts Institute of Technology

Babcock \& Wilcox Company 


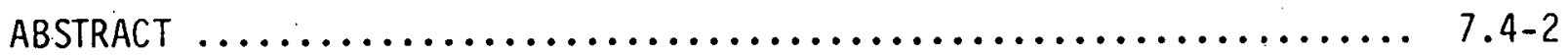

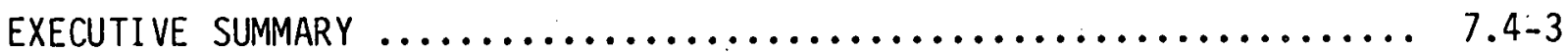

ACKNOWLEDGMENTS $\ldots \ldots \ldots \ldots \ldots \ldots \ldots \ldots \ldots \ldots \ldots \ldots \ldots \ldots \ldots \ldots \ldots \ldots \ldots \ldots, 7.4-5$

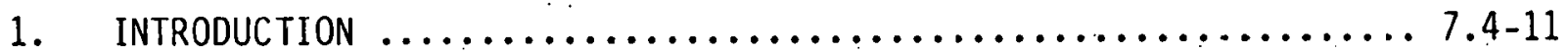

2. ESTIMATED TMI-2 CORE CONDITION $\ldots \ldots \ldots \ldots \ldots \ldots \ldots \ldots \ldots \ldots \ldots, 7.4-12$

2.1 Sequence of Events $\ldots \ldots \ldots \ldots \ldots \ldots \ldots \ldots \ldots \ldots \ldots \ldots, 7.4-12$

2.2 Condition of Core Components $\ldots \ldots \ldots \ldots \ldots \ldots \ldots \ldots \ldots \ldots .4 .4 .17$

2.2.1 Fuel Rods $\left(\mathrm{UO}_{2}\right.$ Fuel, Zircaloy-4 Cladding) ....... 7.4-17

2.2.2 Control Rods and Axial Power Shaping Rods

(80\% Ag-15\% In-5\% Cd Absorjer, 304SS Cladding) ... 7.4-17

2.2.3 Burnable Poison Rods ( $\mathrm{B}_{4} \mathrm{C}-\mathrm{AL}_{2} \mathrm{O}_{3}$

Absorber, Zry-4 Cladding) .................. 7.4-17

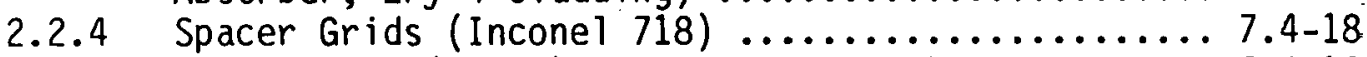

2.2.5 Guide Tubes $($ Zry-4) $\ldots \ldots \ldots \ldots \ldots \ldots \ldots \ldots \ldots \ldots \ldots \ldots .4 .4 .18$

2.2.6 Central Instrument Thimble

(Inconel 718 oversheath) .................... 7.4-18

2.3 Core Examination Objectives $\ldots \ldots \ldots \ldots \ldots \ldots \ldots \ldots \ldots \ldots \ldots \ldots \ldots \ldots .4 .18$.

3. DATA FOR ANALYTICAL TOOL DEVELOPMENT ANJ ASSESSMENT $\ldots \ldots \ldots \ldots 7.4-20$

3.1. Subtask Objectives $\ldots \ldots \ldots \ldots \ldots \ldots \ldots \ldots \ldots \ldots \ldots \ldots \ldots, 7.4-20$

3.2 Recommended Program $. \ldots \ldots \ldots \ldots \ldots \ldots \ldots \ldots \ldots \ldots \ldots . ., 7.4-c 0$

4. DATA FOR FUTURE LICENSING CRITERIA $\ldots \ldots \ldots \ldots \ldots \ldots \ldots \ldots \ldots .7 .4-23$

4.1 Subtask 0bjectives $\ldots \ldots \ldots \ldots \ldots \ldots \ldots \ldots \ldots \ldots \ldots \ldots \ldots .7 .4-23$

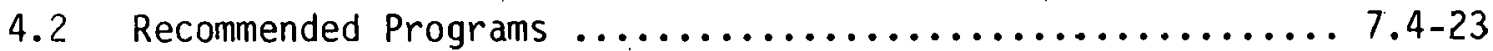

5. DATA FOR SAFETY AND ACCIDENT CONSEQUENCE MITIGATION $\ldots \ldots \ldots \ldots .7 .4-26$

5.1 Subtask Objectives $\ldots \ldots \ldots \ldots \ldots \ldots \ldots \ldots \ldots \ldots \ldots \ldots \ldots, 7.4-26$

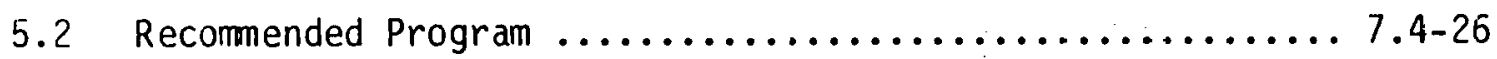

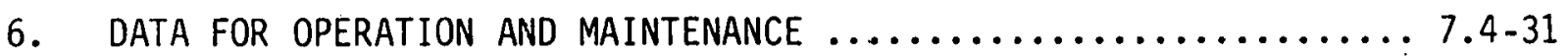

6.1 Subtask 0bjective $\ldots \ldots \ldots \ldots \ldots \ldots \ldots \ldots \ldots \ldots \ldots \ldots .7 .4-31$

6.2 Recommended Program $\ldots \ldots \ldots \ldots \ldots \ldots \ldots \ldots \ldots \ldots \ldots \ldots . .4 .4-31$ 
7. REMOVAL, PACKAGING, AND TRANSPORTATION OF TMI-2 FUEL

AND . CORE COMPONENT SAMPLES $\ldots \ldots \ldots \ldots \ldots \ldots \ldots \ldots \ldots \ldots \ldots \ldots . ., 7.4-33$

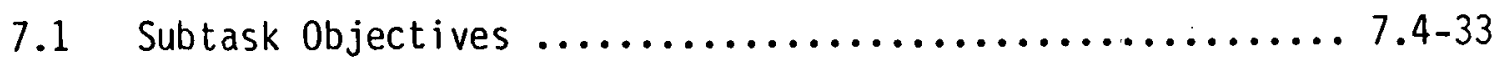

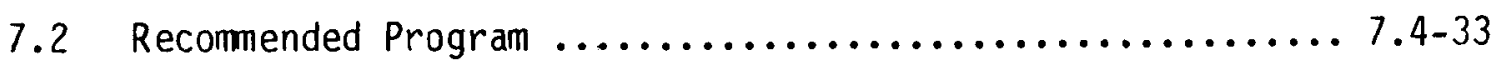

7.2.1 Removal .................................. 7.4-33

7.2 .2 Packaging $. . \ldots \ldots \ldots \ldots \ldots \ldots \ldots \ldots \ldots \ldots \ldots \ldots \ldots \ldots, 7.4-33$

7.2.3 Transportation $. \ldots \ldots \ldots \ldots \ldots \ldots \ldots \ldots \ldots \ldots \ldots \ldots \ldots .7 .4-33$

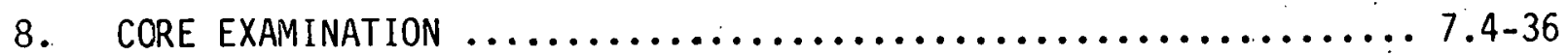

8.1 Subtask 0bjectives $\ldots \ldots \ldots \ldots \ldots \ldots \ldots \ldots \ldots \ldots \ldots \ldots \ldots \ldots .4 .4 .36$

8.2 Recommended Program $\ldots \ldots \ldots \ldots \ldots \ldots \ldots \ldots \ldots \ldots \ldots \ldots . . .4 .4-36$

8.2.1 Fuel Assembly Examination .................. 7.4-37

8.2.2 Examination of Other Core Components ............ 7.4-41

8.2.3 Core Hydraulic ests ..................... 7.4-45

9. CRITERIA FOR TMI-2 EXAMINATION FACILITIES $\ldots \ldots \ldots \ldots \ldots \ldots \ldots, 7.4-50$

9.1 Subtask 0bjectives $\ldots \ldots \ldots \ldots \ldots \ldots \ldots \ldots \ldots \ldots \ldots \ldots \ldots .4 .4 .50$

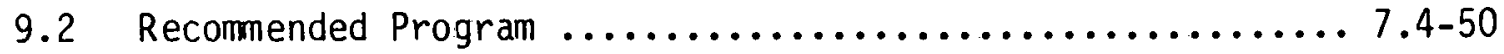

9.2.1 General Criteria ......................... 7.4-50

9.2.2 Specific Criteri.a...................... 7.4-53

9.2.3 Potential TMI-2 Examination Facilities .......... 7.4-53

10. FUEL DISPOSAL $\ldots \ldots \ldots \ldots \ldots \ldots \ldots \ldots \ldots \ldots \ldots \ldots \ldots \ldots \ldots \ldots \ldots \ldots \ldots, 7.58$

10.1 Subtask 0bjectịves $\ldots \ldots \ldots \ldots \ldots \ldots \ldots \ldots \ldots \ldots \ldots \ldots \ldots, 7.4-58$

10.2 Recommended Programs $\ldots \ldots \ldots \ldots \ldots \ldots \ldots \ldots \ldots \ldots \ldots \ldots \ldots .4 .4 .58$

11. REQUIREMENTS FOR DATA FROM TASK 7.2, CORE DAMAGE

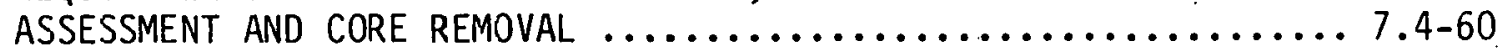

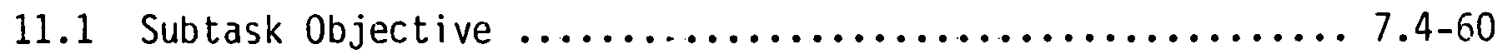

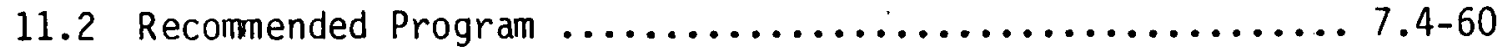

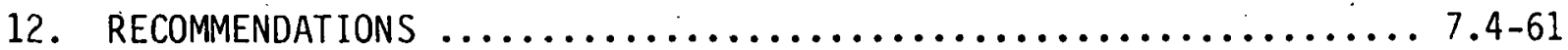

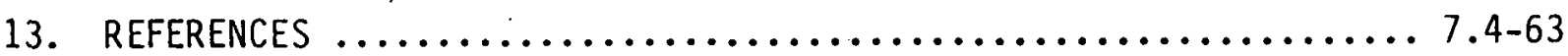

APPENDIX A--ANALYTICAL METHODS FOR EXAMINING TMI-2 FUEL AND

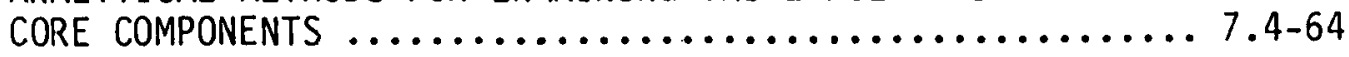




\section{FIGURES}

1. Calculated mixture level during uncovering of the TMI-2 core ....................................... 7.4-13

2. Fuel assembly design $\ldots \ldots \ldots \ldots \ldots \ldots \ldots \ldots \ldots \ldots \ldots \ldots \ldots \ldots, 7.4-38$

3. Axial power shaping rod assembly $\ldots \ldots \ldots \ldots \ldots \ldots \ldots \ldots \ldots \ldots, 7.4-40$

4. Burnable poison rod assembly ......................... 7.4-42

5. In-core instrumentation string $\ldots \ldots \ldots \ldots \ldots \ldots \ldots \ldots \ldots \ldots \ldots . . \ldots \ldots, 4.43$

6. Control rod drive mechanism $\ldots \ldots \ldots \ldots \ldots \ldots \ldots \ldots \ldots \ldots \ldots, 7.4-44$

7a. Side view of reactor vessel and internals showing

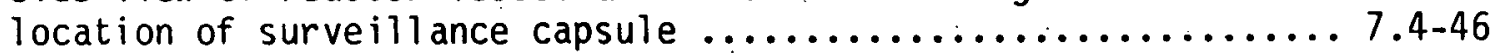

7b. Cross section of reactor vessel and internals showing

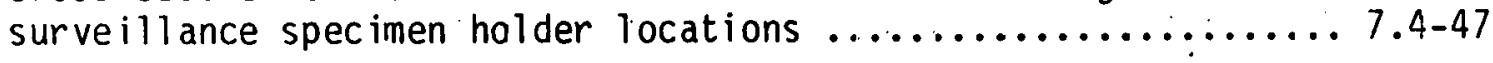

\section{TABLES}

1. Required and Obtainable Data for Develaping and Assessing Models for Severe Core Damage .................. 7.4-21

2. TMI Data Needs for Safety and Accident Mitigation $\ldots \ldots \ldots \ldots .7 .4-28$

3. Potential Domestic Facilities for TMI-2 Fuel and Core Component Examination ........................... 7.4-57

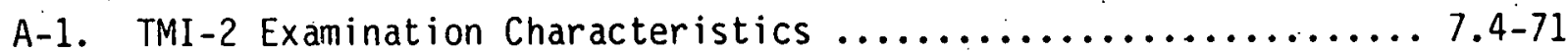

A-2. Component Melting ............................... 7.4

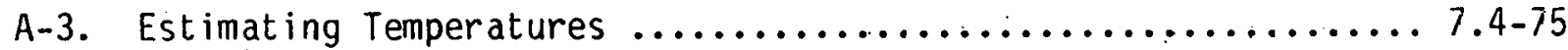

A-4. 0xide Thickness $\ldots \ldots \ldots \ldots \ldots \ldots \ldots \ldots \ldots \ldots \ldots \ldots \ldots \ldots \ldots \ldots, 7.4-81$

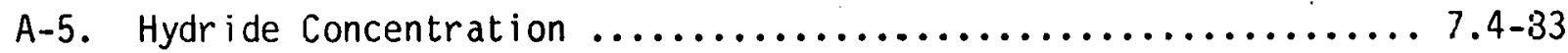

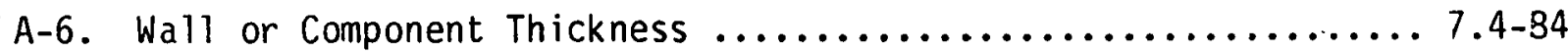

A-7. Dimensional and Functional Integrity $\ldots \ldots \ldots \ldots \ldots \ldots \ldots \ldots .4 .4 .85$

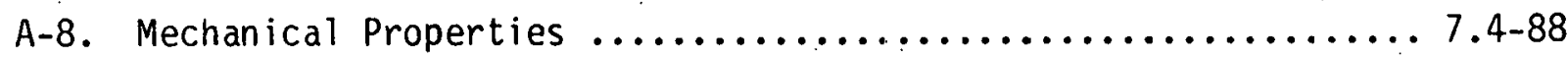

A-9. Retained Fission Products ........................... 7.4-90 


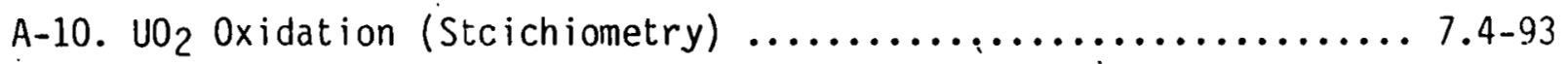

A-11. Debris and Rubble Bed Analysis $\ldots \ldots \ldots \ldots \ldots \ldots \ldots \ldots \ldots \ldots \ldots .4 .45$ 


\section{INTRODUCTION}

This report presents the recommendations of the Technical Planning Group for Fuel Experiments and Examination, Task 7.4. The purpose of this task has been to develop a plan for the detailed technical examination of the TMI-2 fuel and core components. The recommended program and its foundations are discussed herein.

Section 2 summarizes the sequence of events during the TMI-2 accident and indicates the estimated condition of the reactor core. Sections 3 through 6 present objectives and recommended programs to obtain data for development of analytic methods, future licensing criteria, safety and accident consequence mitigation, and design, operation, and maintenance of future light water reactors. Section 7 discusses major considerations in removal, packaging, and transportation of TMI-2 fuel and core component samples, and provides recommendations for areas of further planning and study. Section 8 discusses the core examination that should be performed to provide the data identified in Sections 3 through 6 . Sections 9 and 10 discuss the criteria for selection of TMI-2 examination and archiving facilities and the guidelines for disposal of the fuel and core component samples, respectively. Requirements for data from Task 7.2, Core Damage Assessment and Core Removal, are identified in Section 11. Recommendations for near- and long-term tasks are presented in Section 12.

Appendix A reviews specific examination techniques that may be applicable to examination of the TMI-2 core. 


\section{ESTIMATED TMI-2 CORE CONDITION}

The purpose of this section 's to briefly summarize the sequence of events at TMI- 2 as they relate to the behavior of the core materials. Thus, the stage will be set for tre following discussions on why and how the TMI-2 core should be examined. For core examination planning purposes only, this surmary is based primarily on the Rogovin Report ${ }^{1}$, and thus represents a worst-case core damage scenario. It is recognized that other evaluations completed to date predict less damage. However the planning group recommends that the worst case scenario be used to fully identify potential resource requirements.

\subsection{Sequence of Events}

The sequence of events described below occurred after the reactor was shut down. Core damage occurred due to a loss of adequate decay heat removal capability. The core experienced no damage during the first 100 minutes into the accident. During this time, the core was fully covered by water, even though the pilat operated relief valve (PORV) remained open, causing a decrease in pressure and a loss of coolant inventory from the primary coolant system.

At 73 minutes into the accident, the primary coolant pumps in the $B$ loop were stopped and at 100 minutes, the A loop pumps were stopped. As a result of continued loss of coolant through the PORV and the accompanying loss of primary coolant flow, the core began to uncover. The two-phase steam ard water mixture which remained homogeneous while the primary pumps were operating now separated, and steam collected in the high regions of the primary system. The calculated steam-water mixture level in the core during this period is shown in Figure $1 .^{2}$ Figure 1 was derived from calculated heat and mass balances and a variety of data taken from such instruments as the ex-core source range detectors and in-core self-powered neutron detectors (SPND) (see Reference 2). Below the mixture level, the coolant was near or at its saturation temperature and adequate heat transfer from the fuel rods to the coalant prevented fuel rod damage. Above the mixture level, relatively inefficient heat transfer occurred, and fuel rod temperatures increased dramatically. 


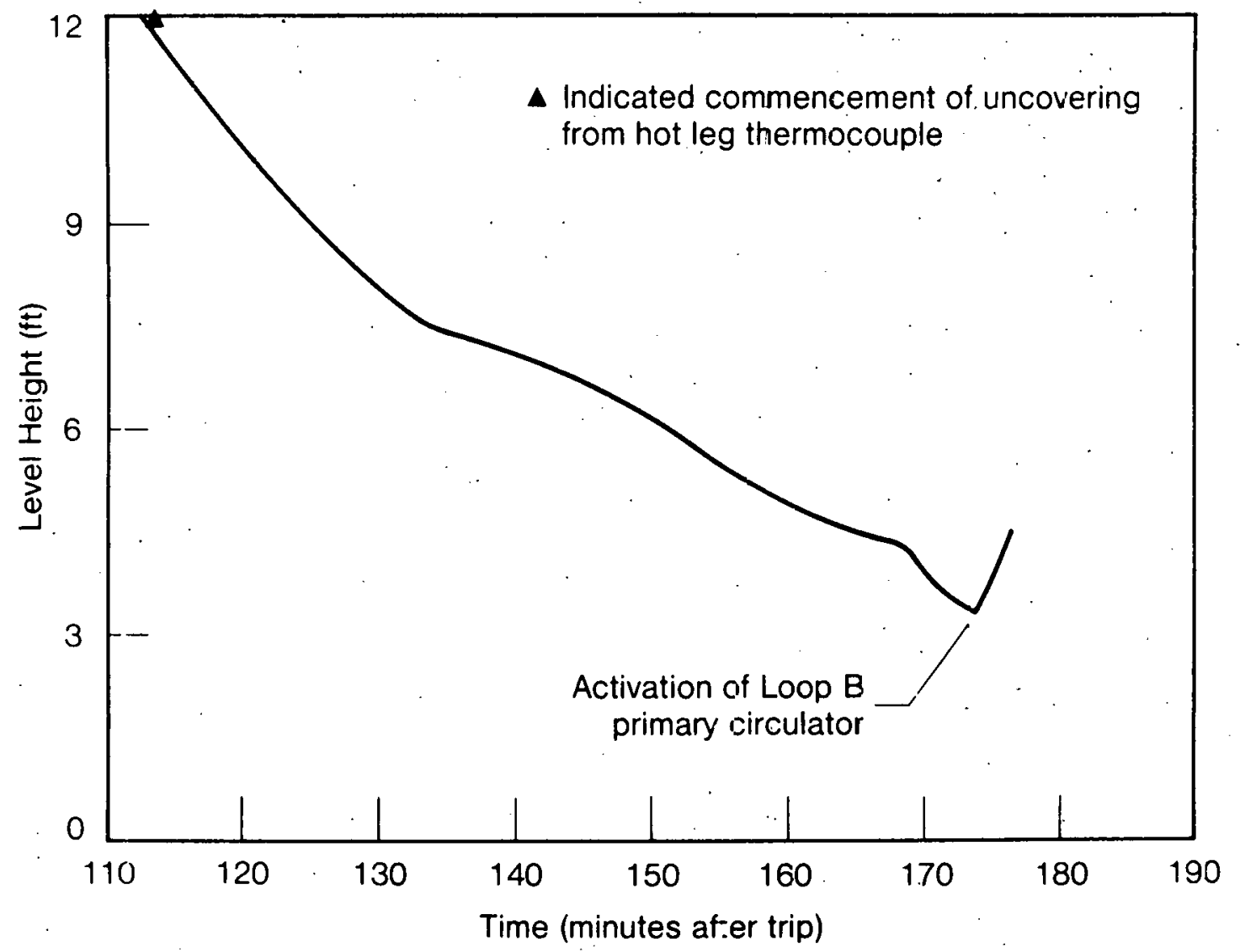

Figure 1. Calculated mixture level durirg uncovering of the TMI-2. core, 
Between 102 and 112 minutes, the core was no longer completely covered by water (see Reference 1, pp. 511,516). Based on core damage est imates and heat transfer calculations of the boildown of the water, the coolant mixture dropped to $1.2 \pm 0.15 \mathrm{~m}(4 \pm 1 / 2 \mathrm{feet})$ above the bottom of the core (see Reference 1, p. 511). A steady state coolant mixture level may have been achieved after about 120 minutes (see Reference 1, p. 511), although Figure 1 indicates a gradual decrease up to 174 minutes (see Reference 2). At 130 minutes, the primary system pressure began to rise, and at about 140 minutes, the PORV block valve was closed, stopping the loss of primary coolant (see Reference 1, p. 512). The primary system pressure continued to inzrease and the coolant mixture level in the core began to rise.

During this period of core uncovery, from about 102 to 174 minutes after reactor shutdown, the fuel rod temperatures rose. Cladding temperatures reached 1150 to $1650 \mathrm{~K}\left(1600\right.$ to $\left.2500^{\circ} \mathrm{F}\right)$ within 30 to 40 mirutes after the core began to uncover, causing all of the fuel rods tc burst with in 50 minutes of uncovering--that is, by about 150 minutes into the accident. The rate of cladding oxidation and hydrogen generation and release increased as the fuel rods continued to heat. The Rogovin report states that between 32 and $39 \%$ of the zircaloy in the fueled region, and between 26 to $31 \%$ of the total zircaloy in the core was probably converted to zirconium dioxide at 174 minutes into the accident. During this period, temperatures rose to $2500 \mathrm{~K}$ and higher, but did not exceed the melting point of $\mathrm{UO}_{2}(3100 \mathrm{~K})$. Approximately $50 \%$ of the fuel rod cladding was embrittled by oxidation. The cladding may have formed a eutectic liquid with the $\mathrm{ZrO}_{2}$ and flowed into the fuel-cladding gap of the fuel rods when it reached temperatures of $2175 \mathrm{~K}\left(3455^{\circ} \mathrm{F}\right)$. The eutectic then reacted with the $\mathrm{UO}_{2}$ fuel to form a liquid phase of $\mathrm{Zr}-U-0$. Estimates of the amount of "liquefied fuel" produced varies from a small amount in the upper portion of the highest power central fuel assembly to $10 \%$ of the fuel in the upper half of the core. Other core components were also damaged:

... Inconel grid spacers had been melted to at least 4 feet from the top; ... and the control rod guide tubes and ins:rumentation tubes remained in place and intact, although oxidized to a greater or lesser extent... (see Reference 1, p. 512) 
Instrumentation tubes and control rod guide tubes survived longer than the neighboring fuel rods because they were not significant heat sources and because they served as "percolator tubes" during depressurization, in which steam bubbles, formed in the annuli, caused liquid water to percolate above the average level in the core to reach higher temperature regions before evaporating. The net effect was to produce a much higher mass flow of steam, as well as velocity of steam flow, through the annuli between the guide tubes and the control rods (and in the double annuli of the instrumentation tubes) than occurred in the subchannels between neighboring fuel rods. Thus, the guide tubes, control rods, and instrumentation tubes stayed much cooler than otherwise expected during depressurization and, consequently, lagged significantly in temperature $r$ ise compared with their surroundings. Their heatup started later, and the heat absorbed by them was transferred by radiation from neighboring fuel rods and by conduction-convection interaction with the steam in the fuel subchannels (see Reference 1, p. 512).

At 174 minutes, with the coolant mixture level at about $1.5 \mathrm{~m}$ ( 5 feet) above the bottom of the core, one primary coolant pump in the B loop was turned on for 19 minutes. This produced a sudden influx of water to the core from the once-through steam generator B. Since the cladding was embrittled due to oxidation, the entering water would have produced a thermal shock to the cladding, causing fragmentation of the $\mathrm{ZrO}_{2}$ and $\mathrm{UO}_{2}$. This would have either formed a debris bed above the axial midplane of the core or increased the size of one already present. Although substantial quenching of the rods occurred, the debris bed itself remained hot and in steam.

The next major change in core condition occurred between 222 and 226 minutes into the accident. The source range monitors showed a sharp increase in activity, the primary system pressure increased even though the block valve was open, and the cold leg temperatures of both steam generators increased. Temperature estimates from thermocouples and SPNDS indicate that temperatures of $800 \mathrm{~K}\left(1000^{\circ} \mathrm{F}\right)$ were reached at locations 0.25 to $0.75 \mathrm{~m}$ (10 to 30 inches) above the bottom of the fuel rods. This behavior is discussed in the Rogovin report:

From the above evidence, we believe that the debris bed and shattered core produced in the prior period were further consolidated by additional formation of liquefied fuel to form a crust in the bed, which spread over much of the core. This 
crust effectively sealed the debris bed off from cooling by steam percolation, and a steam bubble was formed below the debris bed. This allowed additional oxidation of the fuel rod stubs by dryout, producing damage to a greater depth in the core. The debris bed and crust were penetrated by the increasing steam pressure below, and much of the bed and crust suddenly slumped to lower levels in the core, part of it dropping or dripping into the water in the lower part of the core. The sudden local pressure generation may have forced a radial displacement of part of the core material into the region between the core and the downcomer. Also, in some assemblies, liquefied fuel flowed down the small channels surrounding the instrumentation tube to reach levels as low as 10 inches from the bottom of the fuel in the rods, or lower, producing the activation of levels 1 and 2 SPNDs observed and forming a hot casing around it below the average water level in the core. This then would have formed a steam jet through the annuli of the instrumentation tube to keep the upper part of the tubes :ooled enough to survive until the overall system cooled below about $2600^{\circ} \mathrm{F}$... (see Reference 1, p. 519).

As one result of the additional oxidation, core slumping, and formation of liquefied fuel, more hydrogen was formed by reaction between zircaloy and steam. Additional sources of hydrogen would be oxidation of the stainless steel upper end fittings on each of the fuel assemblies and oxidation of some of the $\mathrm{UO}_{2}$ to a higher oxidation state... (see Reference 1, p. 519$)$.

The condition of the core was summarized as follows:

We believe the condition of the core at this time to. be raughly as follows: the debris bed plus crust has been lowered in the core so that its lower boundary may be as low as $4-1 / 2$ to 5 feet from the bottom of the fuel in the fuel rods, and its upfer boundary may be as low as 3 feet from the top of the fuel stack in the original fuel rods; its density has been increased toward $90 \%$ of full density; it rests on fuel rod stubs that may be no more than 5 to 6 feet long; and many assembly sections contain drips of frozen liquefied fuel reaching as far down as 10 inches from the bottom of the fuel. This would indicate that at least $50 \%$ and perhaps somewhat more of the Zircaloy in the core has reacted or been embrittled (see Reference 1, p. 519).

Based on available instrumentation, no apparent change in the condition of the core occurred after the event between 222 and 226 minutes. 


\subsection{Condition of Core Components}

This subsection summarizes the condition of the core components based on the preceding sequence of events. General damage to the core is expected to be in the shape of an inverted bell. Embrittled, fragmented cladding exists near the top periphery of the core and extends downward and toward the core centerline (a bell-shaped volume). At the center of the core, the damage may extend down to 4 or 5 feet above the battom of the core.

\subsubsection{Fuel Rods (U0 2 Fuel, Zircaloy-4 Cladding).}

All of the rods are expected to have ballooned, ruptured, and released fission products. Some flow blockage may have occurred. Fods at the periphery will be partially intact. Rods toward the center were embrittled due to oxidation and fragmented during the cosrse of the accident. A $U-Z r-0$ eutectic formed and probably resulted in several large areas of fused core components. Stubs of rods, perhaps 5 to 6 feet long, remain. The debris bed rests on these and at locations of grid spacers.

\subsubsection{Control Rods and Axial Power Shaping Rods ( $80 \% \mathrm{Ag}-15 \% \mathrm{In}-5 \% \mathrm{Cd}$ Absorber, 304SS Cladding)}

Since the melting points of the Ag-In-Cd alloy and the 304SS are about 1075 and $1675 \mathrm{~K}$, respectively, these rods melted over much of the same volume of the core in which the fuel rods were oxidized. The $\mathrm{Ag}-\mathrm{In}-\mathrm{Cd}$ alloy probably remained in place since it is an insoluble metal. Both materials contributed to formation of the debris bed and fusing of portions of the rubble.

\subsubsection{Burilable Poison Rods ( $\mathrm{B}_{4} \mathrm{C}-\mathrm{AL}_{2} \underline{0}_{3}$ Absorber, Zry-4 Cladding).}

The zircaloy cladding oxidized over the same region as the cladding on the fuel rods, so these rods are in the same fragmented condition. The rods are probably in place, but the boron absorber is known to leach out in 
the presence of water in a radiation environment. Gadolinia burnable poison rods $\left(\mathrm{UO}_{2}-\mathrm{Gd}_{2} \mathrm{O}_{3}\right)$ in two test assemblies are also oxidized and fragmented.

\subsection{Spacer Grids (Inconel 718)}

Since the melting point of Inconel 718 is about $1550 \mathrm{~K}$, the grids would have melted over most of region of the core in which the fuel rods were sxidized.

\subsubsection{Guide Tubes (Zry-4)}

The zircaloy guide tubes oxidized over the same region as the fuel rod cladding, contributing to the material in the debris bed.

\subsubsection{Central Instrument Thimble (Inconel 718 Oversheath)}

Since some of the thermocouples and self-powered neutron detectors were not affected, some of the instrument thimbles are expected to be partially intact. The Inconel oversheath is probably badly damaged, but the several layers of materials present in the thimbles may have contribated to the instrument's survival.

\subsection{Core Examination Objectives}

The foregoing is a summary of one postulated sequence of events and represents the expected worst-case condition of the TMI-2 core. It is a possible scenario, but not the only one. Therefore, two of the key. objectives in examining the TMI-2 core are: (a) to obtain data which will elucidate the accident scenario, and (b) to evaluate the adequacy of existing accident analysis and damage prediction tools.

Attaining these objectives may require that maximum temperatures for some or all core components be estimated from evidence of component melting, eutectic formation, oxidation, and changes in microstructure, mechanical properties, or dimensional integrity. Zircaloy cladding should 
be examined for bowing, ballooning deformation, and rupture. The stoichiometry of the fuel pellets and the debris bed should be determined to assess $\mathrm{UO}_{2}$ oxidation. The radial and axial extent of damage, and the mechanical, chemical, and thermal-hydraulic characteristics of rubble beds should be determined to evaluate the extent of core blockages and areas of reduced coolability. Fission products retained in the fuel and deposited on cold surfaces. should be measured to evaluate fission product release, transport, and deposition during the accident. 


\section{DATA FOR ANALYTICAL TOOL DEVELOPMENT \\ AND ASSESSMENT}

The TMI-2 accident graphically illustrates the need for development and issessment of analytic models to describe the course and consequences of severe damage accidents in light water reactors. The TMI-2 core represents a wealth of data from which the adequacy of present analytic techniques can be confirmed, guidance for the need for further development of analytic methods can be derived, and the need for new models identified.

Additionally, examination of TMI-2 will provide unique data. Specifically, TMI-2 will provide data on actual commercial nuclear fuel rods and assemblies in a severely damaged reactor that includes other core components. Areas of interest in which TMI-2 may provide unique data include the magnitude and extent of coplanar flow blockage due to rod ballooning, debris bed formation and coolability, $\mathrm{UO}_{2}$ dissolution by molten-zircaloy, and relocation and freezing of the liquid solution.

\subsection{Subtask Objectives}

The objective of this subtask is to identify the data obtainable from TMI-2 relevant to core and materials behavior phenomena which may be applicable to the formulation or confirmation of analytic models for assessirg the course and consequences of accidents in light water reactors. Such analytic models could be used for improved risk and consequence analyses and for the design of features to prevent severe damage azcidents.

\subsection{Recommended Programs}

The data required for the development and assessment of new or extended models and the data obtainable from TMI-2 are listed in Table 1. The $p 1$ an for acquisition of the data is presented in Section 8, Core Examination. 
Another important effort in this subtask is an evaluation of those present under-development analytic methods used in light water reactor accident analysis to confirm that key data needs have been identified and that plans have been made to satisfy them.

TABLE 1. Examples of Required and Obtainable Data for Developing and Assessing Models for Severe Core Damage

Data Needed

1. Coplanar flow blockage

2. $\mathrm{UO}_{2}$ dissolution by molten zircaloy

3. Liquid zircaloy relocation and freezing and slag

formation

4. Debris bed formation and coolability

5. High temperature oxidation kinetics of solid and liquid zircaloy, and $\mathrm{UO}_{2}$

6. Rod fragmentation upon quenching

7. Peak temperatures in the core

8. Fission product release, transport, and deposition

9. Extent and kinetics of inner surface cladding oxidation

10. Cladding deformation and rupture

11. High temperature metallurgical interaction between core materials, (e.g., eutectics)
Data Obtainable

1. Radial and axial extent of core blockages and areas of reduced cool ability

2. Extent of $\mathrm{UO}_{2}$ dissolution by molten zircaloy

3. Radial and axial extent of liquid zircaloy relocation and freezing, and slag formation

4. Radial and axial extent of formation and physical, chemical, and hydraulic characteristics of debris

5. Extent of oxidation of zircaloy and $\mathrm{UO}_{2}$

6. Thermal shock fragmentation of embrittled fuel rods and other core components during reflood

7. Peak core component temperatures

8. Fission product retention in fuel and deposition on core components and within the primary system piping

9. Extent and thicknesses of inner surface cladding oxidation

10. Bow, ballooning, and rupture of zircaloy cladding

11. Interactions between core materials 
TABLE 1. (cont inued)

Data Needed

12. Molten fuel and cladding interaction with coolant and associated energetics

13. Neutron absorber material behavior during severe core damage accidents

14. Hydrogen evolution

15. Behavior of molten fuel
Data Obtainable

12. Evidence, if any, of rapid deformations and failures of components from pressure pulses, and the formation and dispersal of liquid fuel droplets

13. Extent of damage to absorber materials and interaction with other core components

14. Hydrogen evolution can be assessed by measuring the extent of $\mathrm{UO}_{2}$ and zircaloy oxidation

15. Molten fuel is not expected in TMI-2, but any evidence of it will be investigated 


\section{DATA FOR FUTURE LICENSING CRITERIA}

As a result of NUREG-0660, ${ }^{3}$ core degradation and melting beyond the design basis may be considered in future safety reviews. This could involve new criteria, new analysis requirements, and new design approaches for all classes of. accidents. Rulemaking hearings for siting criteria and for severe core damage accident consequence mitigation are flanned for 1980 and 1981. The technical bases for the rulemaking may have to be developed after the fact since the time for conducting the requisite R\&D programs extends beyond 1981. The TMI-2 core examination program will provide a source of technical data for developing the basis for future licensing criteria.

\subsection{Subtask Objectives}

The overall objective of this subtask is to identify those data from the TMI-2 core examination program upon which future licensing criteria could be based.

\subsection{Recommended Programs}

The assessment and development of potential licensing criteria require information in the areas of the coolability and condition of severely damaged reactor cores. This information includes:

1. The thermal-hydraulic response of core rubble up to extended periods of dryout, both in the reactor vessel and in the reactor cavity

2. The thermal, chemical, mechanical, and structural interaction of melted fuel with concrete, steel, refractories, soil, etc.

3. The modeling of fuel behavior above $1480 \mathrm{~K}\left(2200^{\circ} \mathrm{F}\right)$ including rubble formation, flow blockage, and the extent of fuel liquefaction and relocation. 
In addition, this task of assessment and development need not focus only on expanding the licensing requirements for Class 9 accidents. The TMI-2 core damage may be $f a r$ in excess of what has been considered in most of the present licensing and safety analyses for nuclear plants; yet despite this damage, the TMI-2 core has remained coolable and the off-site radiological release for this acciJent has been at very low levels. Thus, it is expected that the. TMI-2 fuel experiments and examination will provide detailed data and information that can be the basis for an assessment (and. possibly some relaxation) of current licensing criteria and regulatory requirements for postulated acciderts. Specific areas which might be addressed include:

1. Greatly improved definition of what constitutes a coolable geometry

2. Assessment of 10 CFR 50.46 criteria for peak cladding temperature and maximum local metal reaction

3. Assessment of the 10 CFR 50.46 criterion for total hydrogen generation

4. Modifications to calculations of the radiological consequences of an accident.

Because the TMI-2 core remained coolable, it is important to measure the hydraulics (flow distribution characteristics) of the core. These measurements in conjunction with the characterization of the damage (e.g., rod ballooning, fragmentation, cladding melting and fuel liquefaction, and slag and rubble formation) will permit an improved definition of what constitutes a coolable geometry.

Determination via microstructure of cladding peak temperatures and measurement of the extent of cladding oxidation and fuel rod fragmentation in the TMI-2 core will allow assessment of the 10 CFR 50.46 criteria for peak cladding temperature and maximum local metal reaction. 
Initial analysis indicates that the 10 CFR 50.46 hydrogen generation criterion is conservative with respect to vessel or containment damage, but may not be with respect to damage to support sy'stems within the containment. The estimation of hydrogen generation from fuel and cladding oxidation in the TMI-2 core and the assessment of damage within the containment could be used to assess the hydrogen criterion.

Measurement of the fission products retained in the fual, those deposited on surfaces within the core and within the primary system, and those dissolved in the primary water will permit improved requirements with respect to calculations of the radiological consequences of an accident. 


\section{DATA FOR SAFETY AND ACCIDENT CONSEQUENCE MITIGATION}

As a result of the President's Commission report and other related studies on the TMI-2 accident, future reactor safety reviews may require detailed evaluations of $\mathrm{plant}$ desịn features which mitigate the consequences of beyond design basis accidents. Data from examination of the TMI-2 core which will be useful for evaluating these design considerations are identified in this section. These data will provide information to:

1. Evaluate inherent mitigators which may have acted during the accident in minimizing core damage or fission product releases.

2. Gain insights into potential additional mitigators, either inherent or specifically engineered, which may have prevented further core damage or releases.

3. Evaluate core component design features which enhance or degrade core coolability during an accident.

4. Identify material incompatibilities under accident conditions.

\subsection{Subtask Objectives}

The overall objective of this subtask is to identify those data from TMI-2 which can be used to improve rector safety and reduce the consequences of an accident. This will include identifying what TMI-2 data are needed, justifying the data needs, specifying how the data will be utilized, and specifying when the data should be obtained.

\subsection{Recommended Program}

The careful disassembly of the TMi-2 core will reveal its final condition. Various examinations of the core components will provide a description of the conditions and environment experienced as a result of 
the accident. This information, coupled with the B\&W design information, will. provide insight into potential design-related modifications which will enhance reactor safety. The program will investigate reactor systems, subsystems, and components, and their performance during three stages of the accident listed below:

1. Up to initiation of severe core damage

2. During the period of severe core damage

3. During the period of radioactivity release from the containment.

There are presently no mechanistically-based analytic models for safety evaluation of the complete course of a core melt accident. Bounding analyses have typically been employed, but these are generally inadequate for evaluating various detailed core designs or design-related accident consequence mitigators. Therefore, the proposed program includes some data requirements for the reactor vessel internals, in addition to the core design itself, which will be used to more thoroughly identify those design features which tend to mitigate or aggravate the severity of core damage or core melt.

Specific core and internal components data needs are 1 isted in Table 2. This table also indicates why the data are needed and how they will be used. These data will generally be obtained during core removal after the vessel head has been removed. However, some data can be obtained now and periodically up to the time of head removal; these are also indicated in Table 2.

The physical, mechanical, and metallurgical properties and characteristics of the core and components listed are needed to evaluate the overall integrity of the components and design features. Some of the existing design features in TMI-2 will be evaluated to determine their effect on the course and consequences of the accident and whether they acted to mitigate or aggravate the event. These features specifically include the following: 
TABLE 2. Data Needs for Safety and Accident Mitigation

\begin{tabular}{llll}
\hline \multicolumn{1}{c}{ Data Source and Type } & Phenomena ur Feature Evaluated & When/Where \\
\hline $\begin{array}{l}\text { Physical, mechanical metallurgical } \\
\text { properties of }\end{array}$ & Effects on coolant blockage & In situ and removal \\
- Core & $\begin{array}{l}\text { Integrity of components and } \\
\text { - Cladding }\end{array}$ & In situ and during removal, hot \\
- Fuel pellets & $\begin{array}{l}\text { Accessibility and removability of } \\
\text { - Fuel end fittings. }\end{array}$ & In situ and during removal \\
- Spacer grids & Materials interactions & Hot cell \\
- Control rods & & \\
- Guide tubes & & \\
- Core support structures & & \\
- Pressure vessel (include & & \\
- seals, gaskets, "0" rings, & &
\end{tabular}

$\begin{array}{lll}\begin{array}{l}\text { Chemical analys is of fission } \\ \text { products }\end{array} & \begin{array}{l}\text { Interaction potential of Zr, } \\ \text { Inconel, SS, and other materials }\end{array} & \text { Hot cell } \\ & \text { Retention in fuel/cladding } & \text { Hot cell } \\ & \text { Retention in containment } & \text { Hot cell } \\ & \text { Retention in control materials } & \text { Hot cell } \\ & \text { (e.g., formation of AgI) } & \end{array}$


TABLE 2. (continued)

\begin{tabular}{|c|c|c|}
\hline Data Source and Type & Phenomena or Feature Evaluated & When/Where \\
\hline Debris bed & Geometry, coolability/flow paths & $\frac{\text { In situ }}{\text { cell }}$ and during removal, hot \\
\hline Control rod system & $\begin{array}{l}\text { System operability of component } \\
\text { degradation }\end{array}$ & $\begin{array}{l}\text { Now, and during removal, hot } \\
\text { cell }\end{array}$ \\
\hline $\begin{array}{l}\text { - Components } \\
\text { - Electrical (wires, etc.) } \\
\text { - Hydraulic systems } \\
\text { - Mechanical systems }\end{array}$ & . & $\cdot$ \\
\hline
\end{tabular}


1. Core support and upper plenum structure

2. Bypass flow configuration

3. Fuel assembly spacer grids

4. Core formers

5. Material incompatibilities and eutectic formations at high temperatures.

From the examinations, it will be possible to provide more detailed evaluations of future reactor safety improvements and design features. 


\section{DATA FOR OPERATION AND MAINTENANCE}

Examination of the TMI-2 core will provide information that can be used to evaluate the operation and maintenance of light water reactor power plants and potential revisions to operation and maintenance procedures.

\subsection{Subtask Objective}

The objective of this subtask is to gather data from t'e examination of the TMI-2 core components which would be applicable to the operation and maintenance of light water reactor power plants.

\subsection{Recommended Program}

The programs to improve reactor operation and maintenance should begin with a review of operation and maintenance procedures prior to and following the accident. Recommendations could then be formulated which would lead to the collection of operation and maintenance related data during the course of the fuel and core component examinations. Examples of the types of improvements to reactor operation and maintenance during both normal and accident situations include:

1. Improved safety instrumentation inclúding ability to survive accidents.

2. Techniques for removal and replacement of damaged core components.

3. Techniques for assessment of core component structural integrity.

4. Techniques for debris removal with minimum reactor contamination.

5. Improved techniques for core component decontamination. 
6. Improved training of maintenance personnel.

7. Modifications to controi rooms.

8. Improved techniques for minimizing radiation exposure during fuel handling operations. 


\section{REMOVAL, PACKAGING, AND TRANSPORTATION OF TMI-2 FUEL AND CORE COMPONENT SAMPLES}

The ireatment of TMI-2 fuel and core component samples from the time of their removal to their arrival at an examination facility must be carefully controlled to prevent sample damage or alteration. The purpose of this section is to address the major aspects of this problem and to make recommendations for areas of further planning and study. The subtasks of selecting, handling, and shipping of TMI-2 samples for scientific examination will have a broad range of generic applicability to LWR technology. This section relies heavily on the Allied-General Nuclear Services study ${ }^{4}$ of the TMI-2 core disposition.

\subsection{Subtask Objectives}

The objectives of this subtask are to develop a representative TMI-2 core sampling $\mathrm{plan}$ and to develop fuel debris handling, packaging, and transportation techniques which preserve TMI-2 scientific data and have generic applicability to LWR accident recovery technology.

\subsection{Recommended Program}

This section is divided into discussions of sample removal, packaging, and transportation.

\subsubsection{Removal}

For planning purposes it should be assumed that the TMI-2 core contains both relatively intact fuel elements as well as fuel debris in a variety of configurations. Removal of these samples must be preceded by the establishment of a core sampling $\mathrm{plan}$. This $\mathrm{plan}$ will attempt to ensure that the specimens selected for analysis are representative of the entire spectrum of core damage. Formulation of a detailed plan must await the results of the early core damage assessment activities; however, the general features of the sampling $\mathrm{plan}$ are clear. The plan must consider the full range of core damage from integral fuel assemblies (undamaged, 
distorted, failed) to debris (large, small, fused, loose). The damage must be sampled as a function of radial position and height within the core. Any small loose debris, present in the vessel plenum structure or below the lower core grid plate, must be sampled to determine the extent of debris migration. If it can be shown that the damage is radially symmetrical, then a smaller sampling $\mathrm{plan}$ from a certain core segment may be acceptable. In addition, all of the in-core structures must be sampled and analyzed. These include the plenum assembly, core barrel, control rod guide tubes, core support shield, vessel thermal shield, flow distributor, and the instrument guide tubes. The extent of sampling of these structures will be highly dependent on their apparent damage.

The actual removal of the bulk of the core is not expected to be significantly different from the removal of the examination specimens. Very general guidelines for this process are discussed in Reference 4. Because of core distortion, it is likely that the main and auxiliary fuel handling bridges will require modification. Special grapples and tongs will be required because of the reduced structural integrity of the fuel assemblies. Underwater cutting capabilities will be required because of the likelihood of fused debris. Too ing will be developed for core debris and exaaination sample removal. Special removal procedures may be necessary, including such things as the addition of a load cell to lifting equipment to measure extraction forc $\in S$, or specifying very smal1 acceleration values to prevent relocation of very loose debris. An underwater vacuuming system to collect fine debris will also be required.

\subsubsection{Fackaging}

A variety of buckets, containers, and shipping cans will be required for examination samples. Conceptual designs have been developed for such devices which are also required for general core removal (see Reference 4). Once early core damage assessment has been performed, a better idea of the size range of the examination specimens will be available. Fabrication of the shipping containers should then begin. The design of the containers should follow, as closely as possible, the designs selected by GPU for bulk core removal. 
The integrity of the examination samples during handling and shipping is very important to the success of the examination. Therefore, it is recommended that a techique be developed to encapsulate highly embrittled fuel examination samples for shipping. The encapsulation technique would be used on critical samples which, if damaged, could compromise the value of their subsequent study and analysis. Ideally, the encapsulation material would be applied in situ prior to renoval of the sample from the core; the material would not degrade in the radiation and thermal environment, and would be removable so that hydraulic flow tests could be conducted on some debris samples. Some orgarics (such as resins, waxes, gels, and silicone formulations) or low melting metal alloys may meet some or all of these requirements. It is recommended that early encapsulation studies be initiated, and that candidate materials be screened by laboratory experiments, so that an encapsulation technology will be available prior to the start of core removal.

\subsubsection{Transportation}

During removal of a damaged core, the use of large spent fuel casks to reduce the number and cost of shipments should be emphasized. Many of the debris samples selected for examination will, however, be fairly small. Therefore, a study must be initiated to identify suitable radioactive material shipping casks for transportation of these smaller debris samples.

A transportation option which should be evaluated is that in which none of the examination samples would be shipped until all (or most) had been removed from the core, packaged, and stored at an interim TMI location in one of the spent fuel pools. Then, a large cask would be used to transport all of the samples to a central remote handling facility in a single (or very few) shipment(s). At the central facility, the samples could be photographed, catalogued, decontaminated, and repackaged for shipment to analytical. laboratories. The central facility could house a TMI archive facility where samples remained in controlled storage while specific examination plans were formulated. This procedure could also reduce the number of shipments originating from TMI, and thereby reduce the impact on the overall TMI-2 recovery effort. 


\section{CORE EXAMINATION}

The TMI-2 core postirradiation examinations will be extremely important due to the nature of the accident and the intense amount of public and scientific interest that it generated. Questions relating to core behavior during the course of the accident extend beyond previous fue 1 behavior studies or analysis of design basis accidents. The information obtained from these examinations will be vital to understanding what actually happened, and determining the risk of other accidents which might occur. Many of the analytical techniques that will be required to evaluate both fuel and core components will involve advanced methods that depend heavily upon the expertise and knowledge of the analyst. State-of-the-art postirradiation examination techniques will be used to supplement the special analytical methods and to provide a complete and thorough evaluation.

\subsection{Subtask Objectives}

The basic objective of the examinations is to provide the data identified in Sections 3 through 6 . These data will be obtained by performing those examinations and analyses required to determine the core thermal history and the chemical, ceramographic, metallurgical, and physical changes that occurred in the fuel and core components during the course of the accident.

\subsection{Recommended Program}

This section is divided into three subsections. Subsection 8.2.1 discusses the examination of the fuel assemblies, including structural components and control elements. Subsection 8.2.2 discusses the examination of other core components such as instrumentation, experimental assemblies, and reactor vessel internals. Hydraulic tests to characterize coolant channeling and flow blockages are discussed in Subsection 8.2.3. In addition, Appendix $A$ contains a sumary of the analytical techniques available for obtaining specific types of data. Appendix $A$ can be used to determine what specific analyses could be used to perform the recommended examinat: ons. 


\subsubsection{Fuel Assembly Examination}

The design of the TMI-2 fuel assembly is shown in Figure 2. Its overall fuel rod length is $12.75 \mathrm{ft}$. In addition to extensive fuel assembly debris, it is likely that part-length and perhaps even some full-length fuel assemblies will be present in the TMI-2 cor?. The fuel rods of the part-length assembly provide one of the most important data sources. The deformation and integrity of the cladding is important for coolability assessment. The mechanical properties and damaçe structures are needed to evaluate fuel rod performance during the incident. The oxidation (both inner and outer surface) should be measured to assess the temperature history as we 11 as to provide data on hydrogen generation. The presence of eutectic reactions (with grids or other components) should be ascertained. Cladding microstructures should be evaluated to determine the axial temperature profiles. The inspection of the part-length fuel assemblies should also include fuel assembly geometry measurements, e.g. : bow, twist, water channel spacing, flow blockage determinations. These examinations will determine if there was an axial or radia pattern to the damage.

Relatively intact full-length fuel assemblies are most likely located near the core periphery. As with the part-length assembly, the fuel assembly configuration (e.g., bow, twist, water channel sfacing, deformation) and resultant coolability should be determined. The upper ent fitting configuration, and the hold-down spring relaxation properties are needed before fuel disassembly to provide temperature data. After disassembly, metallurgical examinations of the upper end fittings and hold-down springs are needed for oxide and secondary phase measurements. The grids should be inspected to determine any axial gradient in the microstructure, possible eutectic formation with the fuel rods, and extent of oxidation. The fuel rod characterizations should include dimensional and mechanical integrity tests.

$\mathrm{Cl}$ adding mechanical properties and metallurgical analys is snould yield data on axial temperature gradients, oxidation, and hydrogen absorption. Fuel analysis for retained fission products, structure $\left(\mathrm{UO}_{2}\right.$ or $\left.\mathrm{U}_{3} \mathrm{O}_{8}\right)$, 


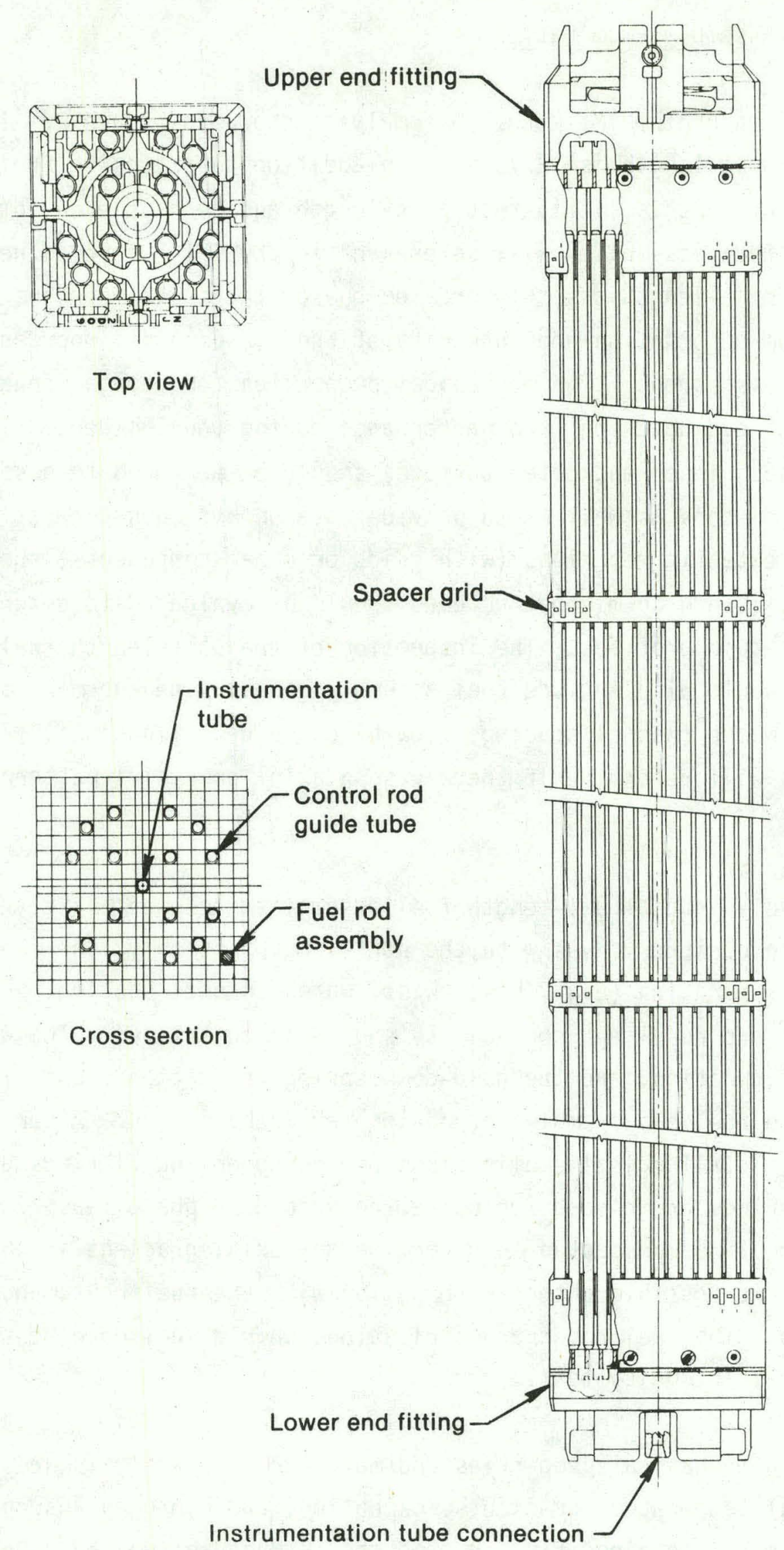

Figure 2. Fuel assembly design. 
extent of oxidation, swelling, and melting should be performed. Gamma scans and fuel rod neutron radiographs should indicate fuel relocation within the rods.

Metallurgical and mechanical tests of the upper and lower fuel rod springs should give data on the temperature conditions at the ends of the rods. Mechanical and metallurgical inspections of the control rod guide tubes are necessary since the guide tubes are the structural members of a fuel assembly and provide paths for control rod insertion. Metallurgical examinations of the lower end fittings should yield temperature data on the part of the core that did not become uncovered during the accident. Since the upper and lower fittings are of the same naterial, variations in performance based on environmental differences should be evident.

The control elements, consisting of the control rods, the axial power shaping rods (APSR), and the burnable poison rods, must be thoroughly examined. The short axial power shaping rods are a silver-indium-cadmium alloy clad in 304 stainless steel attached to a CF3M (a cast stainless steel) spider (as shown in Figure 3 ). These rods were parked in an operating mode in the lower four feet of the core; therefore, metallurgical and mechanical analys is of the ASPRs can provide indications of the conditions in the region above the core. The control rods (also silver-indium-cadmium alloy clad in 304 stainless) were inserted into the core at the time of reactor scram. Rod deformations in the poison area, (i.e., creep-collapse) may provide time-temperature information when coupled with the metallurgical data. Eddy current integrity checks may provide specific failure locations for further analysis. The silver-indium-cadmium poison in the rods has a low melting point $(1075 \mathrm{~K})$. Examination of the poison zones should provide insight into shutdown responses during accident conditions. By examining melting or remelting profiles, information about the time-temperature history may be obtained. Metallurgical determinations of poison-cladding compatibility, secondary phase segregation, etc., should also be conducted. If there was a large cladding breach, erosion patterns in the poison may give information about flow patterns in the core during the the accident. 


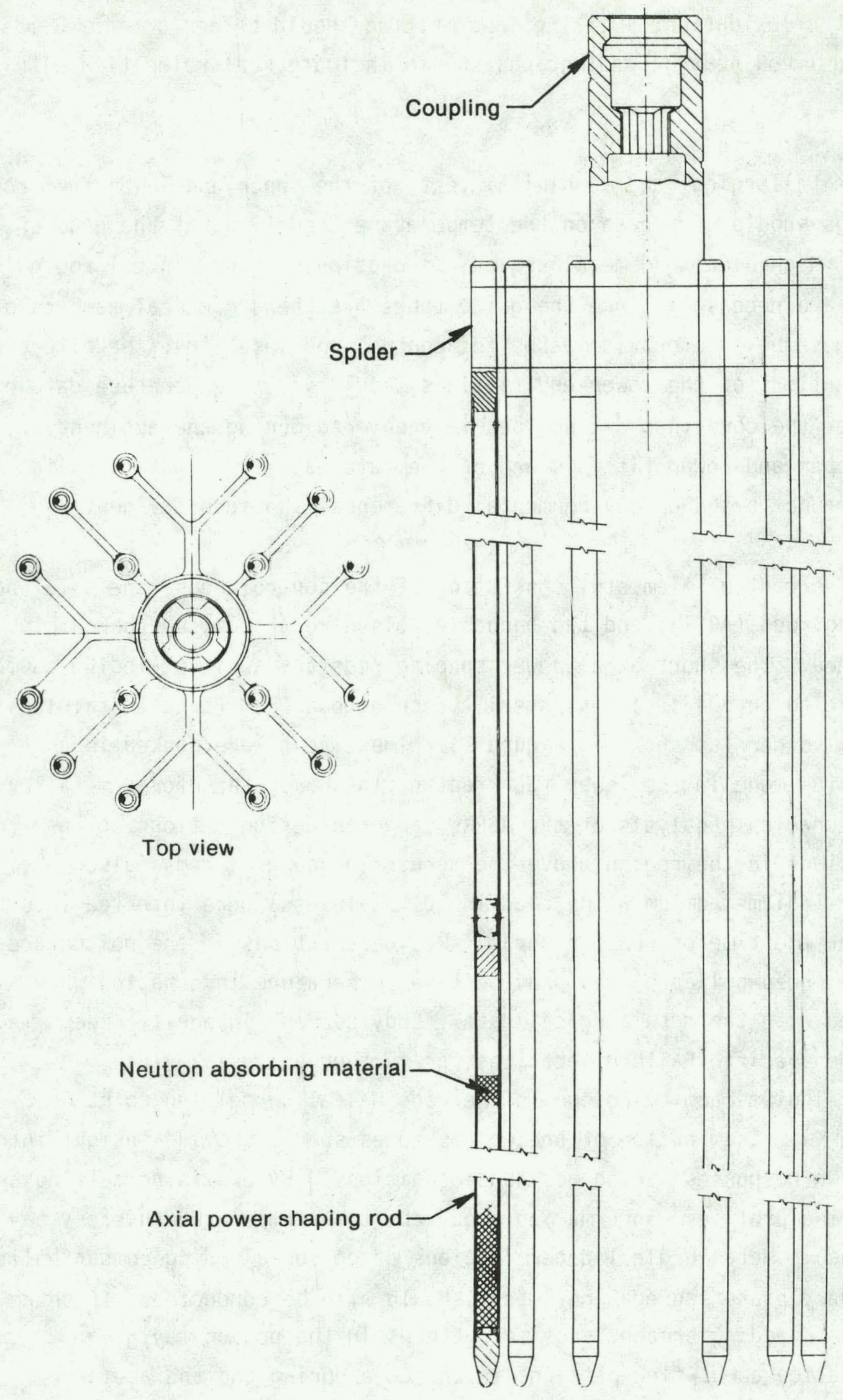

Figure 3. Axial power shaping rod assembly. 
The burnable poison rods (Figure 4 ) are pelletized alumina-boron carbide $\left(\mathrm{Al}_{2} \mathrm{O}_{3}-\mathrm{B} 4 \mathrm{C}\right) \mathrm{clad}$ with zircaloy-4, the same cladding material as the fuel rods. The rod geometry (ballooning or creepdown), integrity, and types of defects, can be compared to the fuel rods. Excessive swelling resulting from poison volume changes upon reaction with the coolant may have occurred. Metallurgical determinations (for oxides and secondary phases) can yield information about the thermal history. The burnable poison rod assembly spiders are made of CF3M and 17-4 PH (a stainless steel). They should be thoroughly examined and compared to other examples of the same materials in the core. Examination of the $\mathrm{Al}_{2} \mathrm{O}_{3}-\mathrm{B}_{4} \mathrm{C}$ pellets could yield data about system mechanical and thermal shocks. Since the pellets are normally very strong, if the pellets are not intact, transient severity estimates can be made, and the stresses and the corresponding temperatures can be approximated.

The burnable poison rod assembly retainers are other components which should be examined. The retainers are manufactured from 304 stainless steel and Inconel X-750 material. Metallurgical and hardness examinations should provide temperature history information of the top of the fuel assembly.

\subsubsection{Examination of Other Core Components}

Fifty-two fuel assemblies in TMI-2 are instrumented with in-core detectors consisting of a string of self-powered neutron detectors and a thermocouple, as shown in Figure 5. If intact, this instrumentation can be removed prior to core access. Damaged instruments can be retrieved during core removal. Metallurgical investigations of instruments may provide tire temperature histories. Selected instruments should be recalibrated to help understand some of the anomalies in the online measurements made during tre course of the accident. Detailed planning for examination of the ir-core instrumentation is being addressed by Planning Group 1.0, Instrumentation and Electrical Equipment Survivability.

The condition of the control rod drive mechanism lead screws shown in Figure 6 will reflect the temperatures reached in the upper part of the 


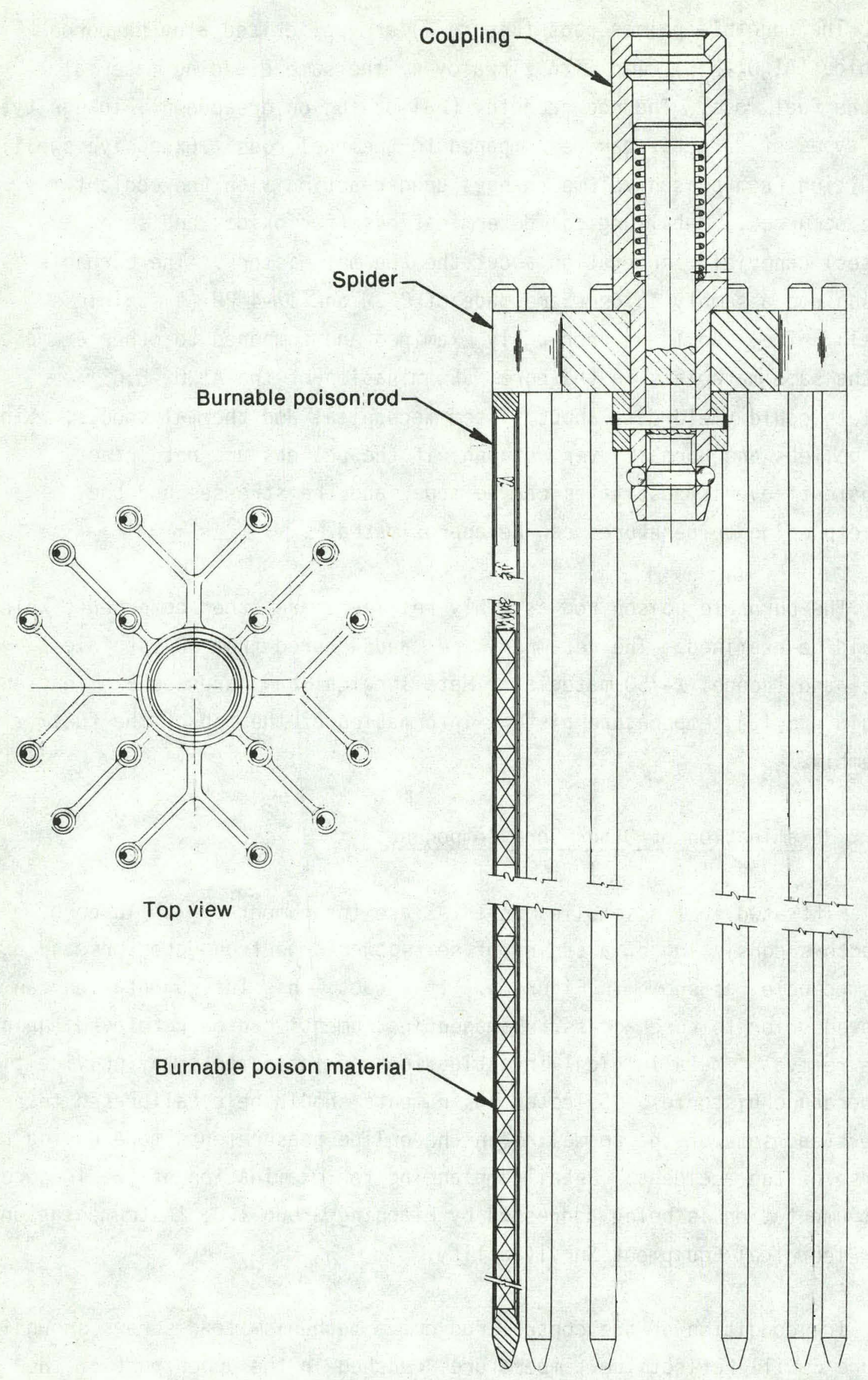

Figure 4. Burnable poison rod assembly. 


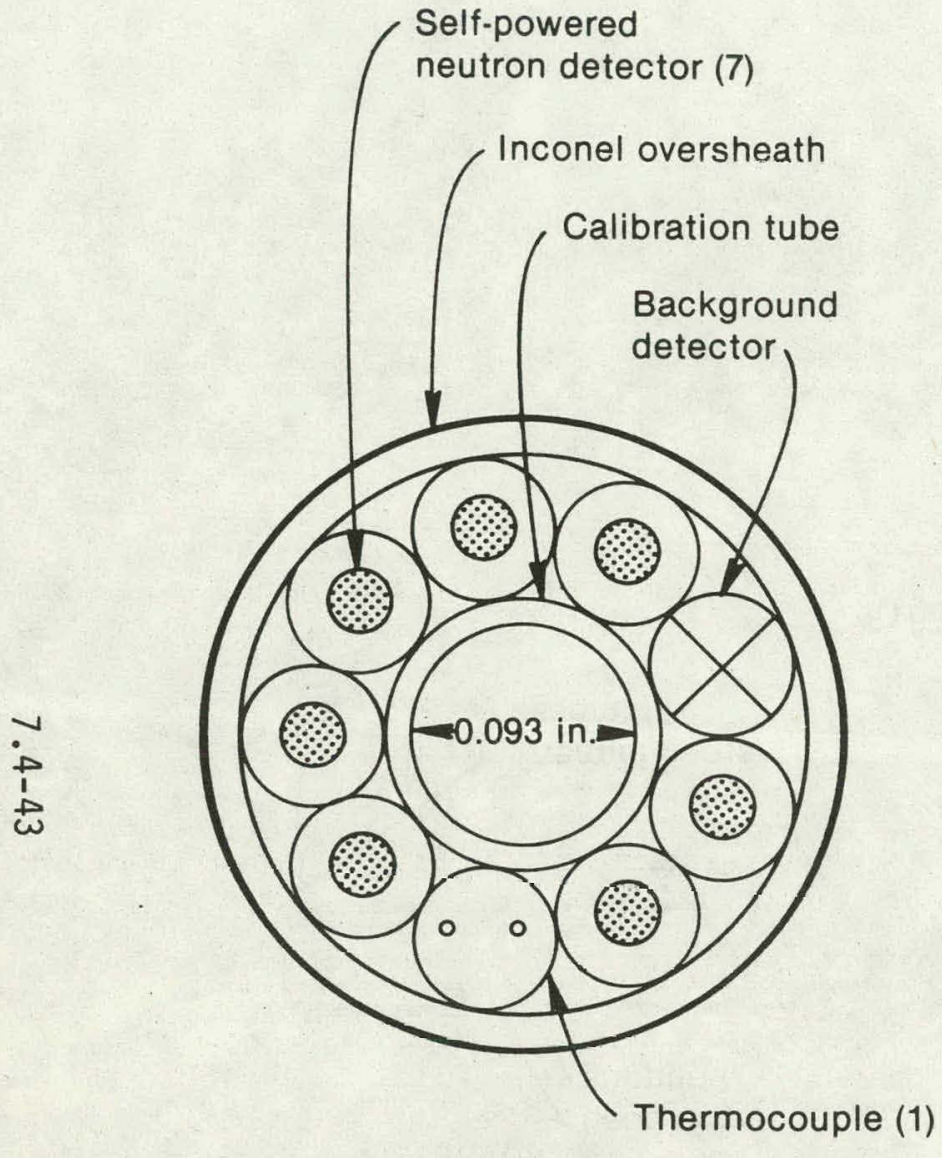

a. Fixed SPND assembly cross section

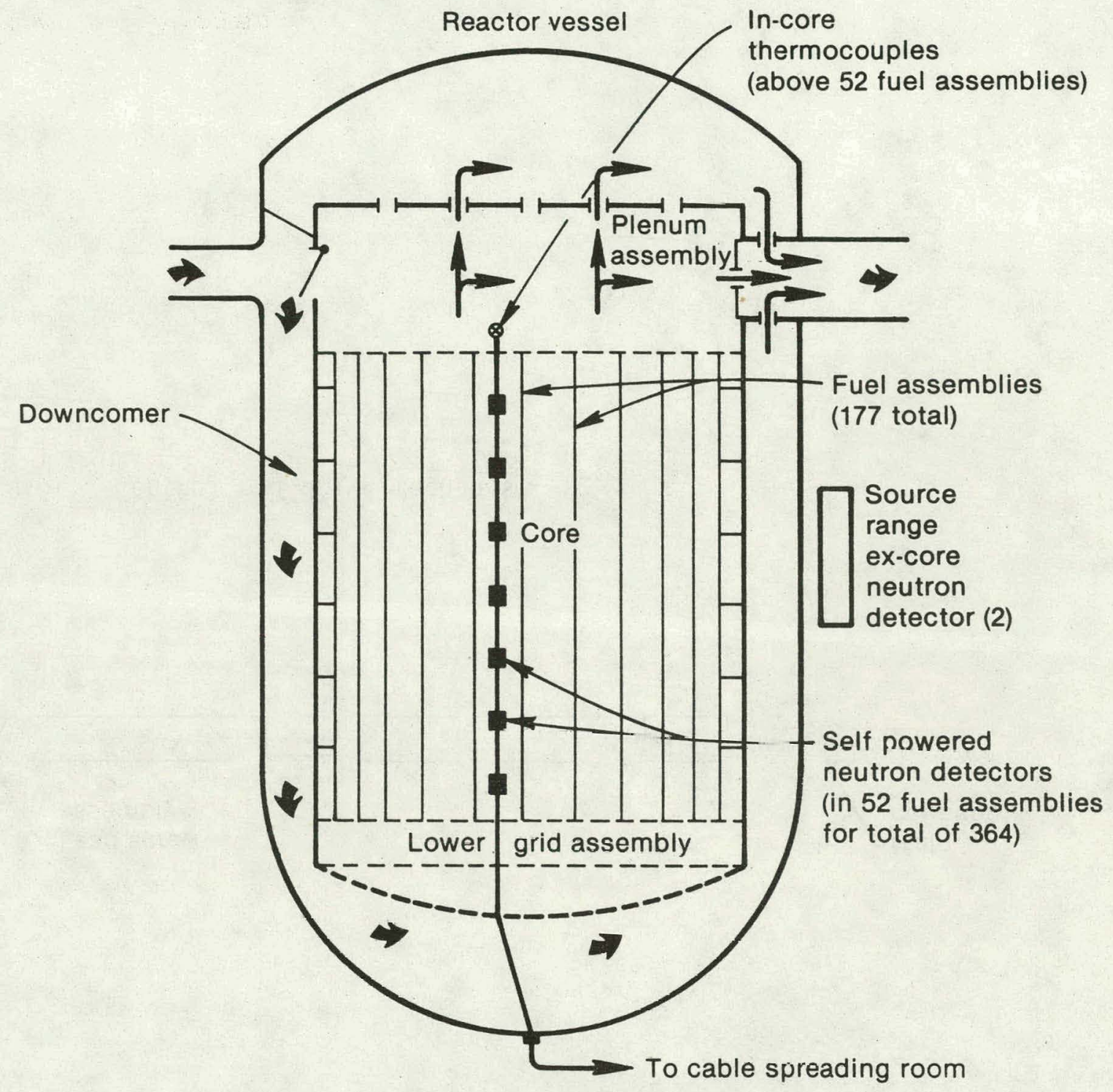

b. Instrument locations

INEL-A-16 285

Figure 5. In-Core instrumentation string. 


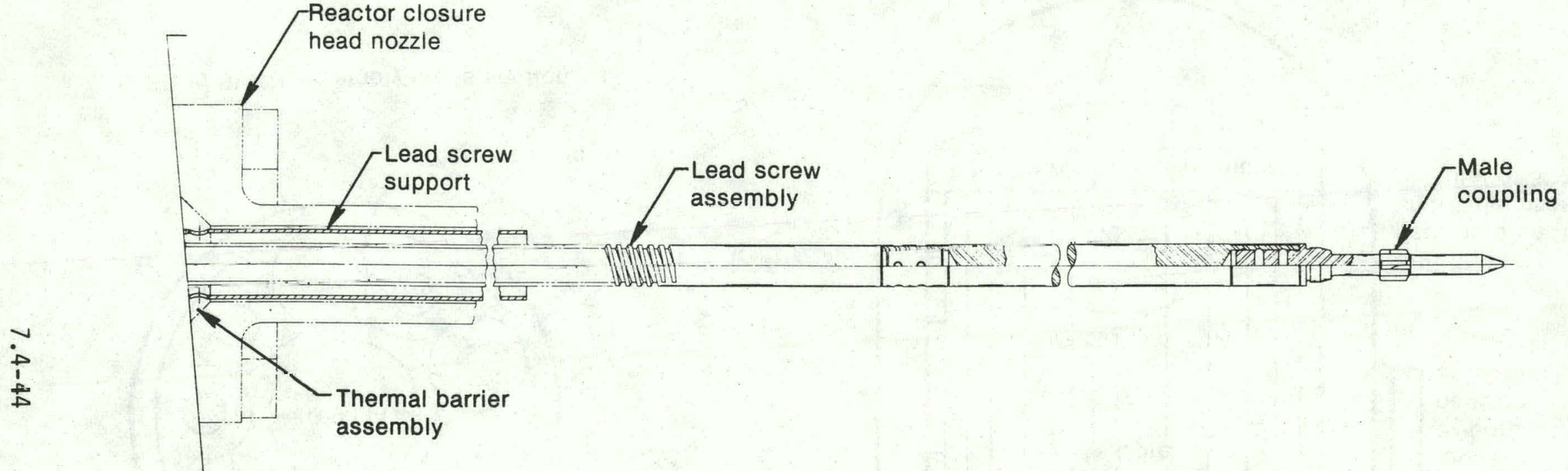

Figure 6. Control rod drive mechanism. 
core. Analysis of oxide layers, metallurgical structure, hardness, and melting will provide a good preliminary estimate of the temperature conditions across the upper core.

The pressure vessel surveillance capsule shown in Figures $7 \mathrm{a}$ and $7 \mathrm{~b}$ can provide an estimate of the environment in the reactor vesse?. There are six such capsules in TMI-2. The capsules contain vessel samples, welding samples, dosimetry, and alloy temperature monitor specimens. An examination of one of these capsules could provide boundary: temperat ure estimates. The initial specimen inspection should include mechanical testing and metallurgical determinations. Also, the specinen holder should be inspected for oxidation and microstructural changes. Since the frimary value of the surveillance capsule specimens will be to aid the pressure vessel requalification, it is important that these examination recommendations be integrated into the requalification pragram.

TMI-2 contained several experimental assemblies. These include $\mathrm{Gd}_{2} \mathrm{O}_{3}-\mathrm{UO}_{2}$ fuel rods and some borated graphite burnable poison rods. Detailed inspection of the gadolinia rods should provide data on the effect of $\mathrm{Gd}_{2} \mathrm{O}_{3}$ on $\mathrm{UO}_{2}$ fuel during accident conditions. Melting, gas release or gas retention, phase changes, and microstructure changes can be measured as a function of axial position to assess behavior over a range of conditions. Investigation of the borated graphite behavior should provide an estimate of the upper limit of the core temperatures. The borated graphite is very stable at high temperatures; thus, the major changes would be caused by water corrosion. Possible graphite-claddirg reactions may be present and may help provide temperature history data.

\subsubsection{Core Hydraulic Tests}

The loss of ability to cool the TMI- 2 core during and after the accident due to flow restrictions, flow blockages, and degradation of heat transfer was a major concern. The fact that the core remained coolable despite apparent major damage means that nydraulic tes:s on portions of the core will greatly improve understanding of reactor accident heat removal. Full- and partial-length bundles, representative of moderately and severely 


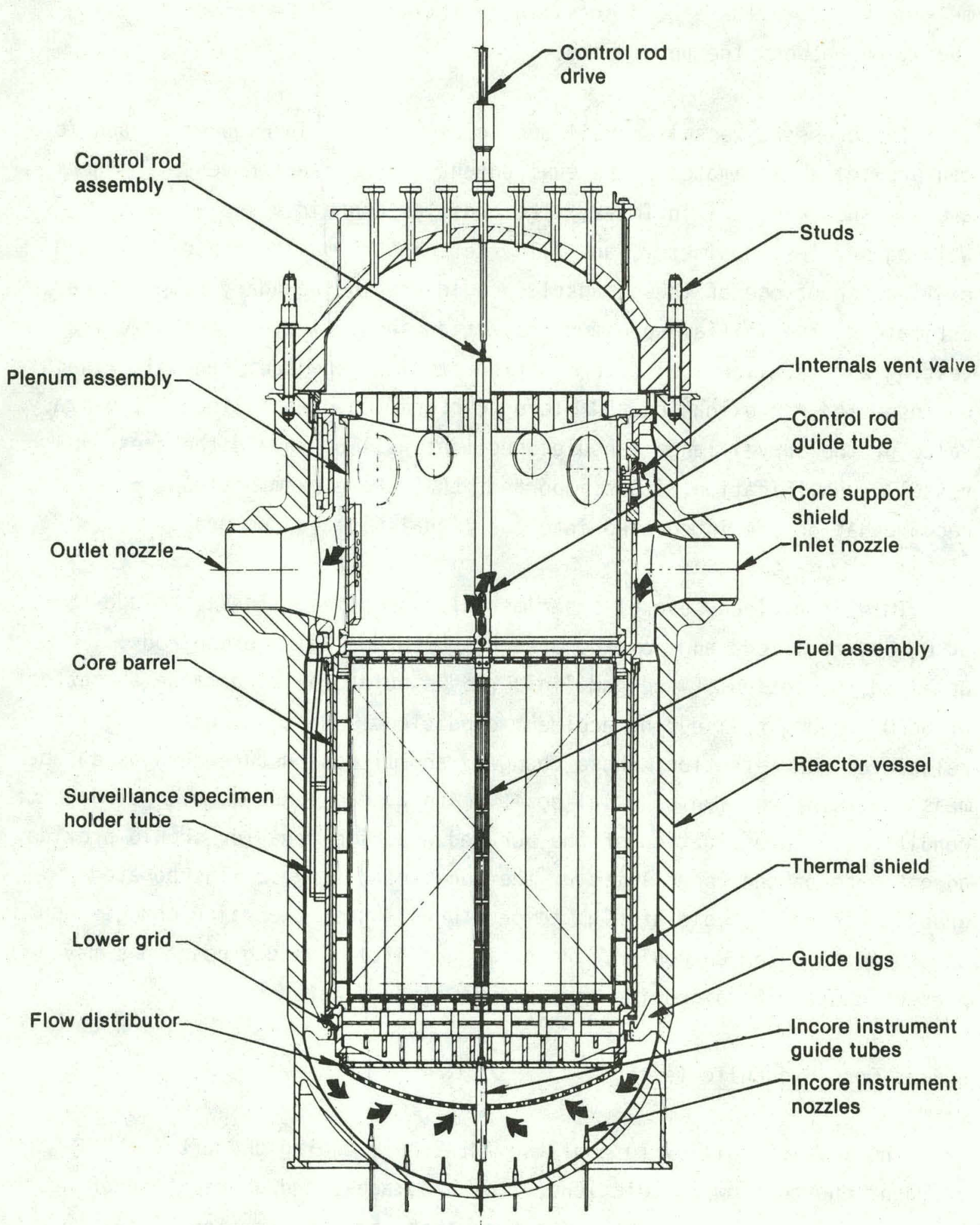

Figure 7a. Side view of reactor vessel and internals showing location of surveillance capsule. 


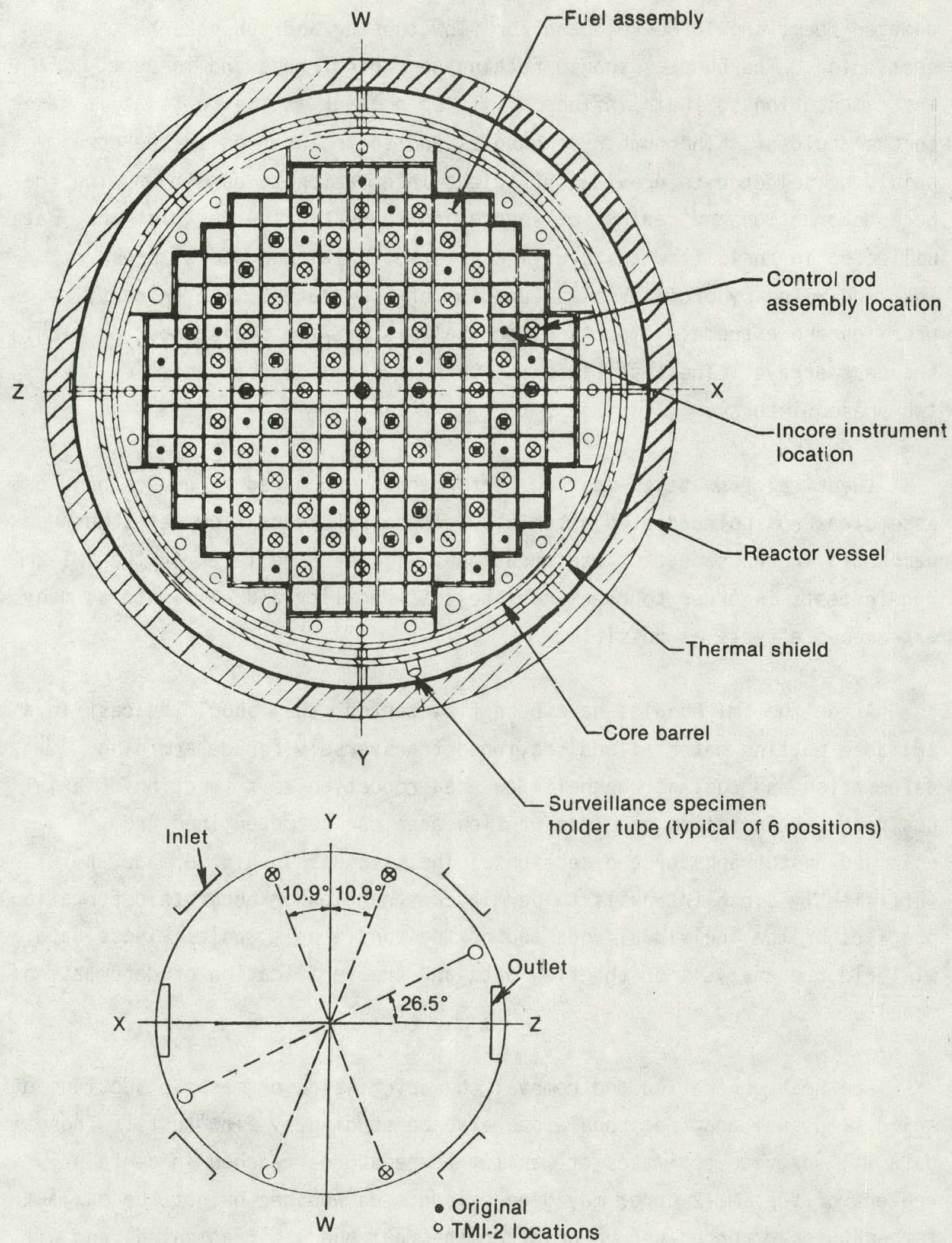

Surveillance specimen holder tube azimuthal location

Figure $7 \mathrm{~b}$. Cross section of reactor vessel and internals showirg surveillance specimen holder locations. 
damaged fuel, should be selected for flow testing and subsequent sectioning. The bundles should retain the control rods and in-core instrumentation so their influence will be present in the tests as it was in the accident. The number of bundles and their location in the core should be selected to provide sufficient information to permit mapping the core deformation and regions of severe and moderate flow restriction. Dat collected in these flow tests will consist of axial and radial pressure loss and velocity profiles as a function of flow rates. The velocity profiles are extremely important for analysis of flow redistribution within the test arrays. The test fluid may be suitable liquids, gases, or two-phase mixtures.

Identical flow tests must be performed on undamaged reference bundles assem jled from unirradiated materials. These reference flow tests must be performed in the same test equipment that will be used later in the TMI-2 bundle tests in order to check out the test facility and eliminate as many extraieous effects as possible.

After the TMI bundles have been flow tested, they should be cast in a suitable potting material and sectioned transversely for determining deforration and coolant channel flow area reduction as a function of axial position. Deformation and loss of flow area can be determined from enlarged photographs of the sections. The sections should be made at sufficiently close intervals to permit construction of accurate deformation profiles of the individual rods and of the bundle as a whole. These data will a d the analysis of the flow data and the verification of deformation models.

The heat generation and removal characteristics of massive sections of solidified fuel and fuel rubble can also be studied by flow tests. These data will provide estimates of maximum temperatures reached in certain regions of the TMI-2 core, may give evidence on whether or not the maximum temperatures occurred before or after the fuel bundles fragmented, and will provide important data for modeling coolable geometries of severely damaged cores. To obtain the necessary data, massive sections of debris representative of known regions of the core should be placed into large 
flow housings. Flow tests should be conducted using suitable liquids., gases, or two-phase mixtures. Hot test fluids, in combination with suitably located thermocouples, will provide data on effective thermal parameters, temperature distributions, etc.

After the flow test, the conglomerates must be characterized for properties such as volume fraction of the constituents, porosity, density, heat capacity, thermal conductivity, etc. These data will be needed for analys is of the flow tests, will provide input for estimating heat generation, cooling, and temperature levels Juring the accident, and will become important for constructing predictive models for saverely damaged cores. 


\section{CRITERIA FOR TMI-2 EXAMINATION FACILITIES}

This section presents recommendations for general criteria for facilities and organizations responsible for examination, analysis, and temporary storage of fuel materials and samples taken as part of the TMI-2 Information and Examination Program. The development of these general criteria will enable early identification of potentially acceptable facilities and organizations and allow for accurate cost estimating and planning for all of the tesks necessary to ensure removal of materials from TMI-2. shipping to examination locations, relocation to archival storage facilities, and ultimate disposal. More specific requirements will have to be prepared for each examination, analysis, and storage task.

The following subsections discuss general and specific examination facility criteria. Potential United States examination facilities are 1 isted.

\subsection{Subtask Objectives.}

There is broad interest in participating in TMI-2 core examinations by domestic remote handling failities. The objectives of this subtask are to develof examination facility criteria, identify unique examination capabilities, and encourage broad participation in the postirradiation examination of TMI-2.

\subsection{Recommended Program}

\subsubsection{General Criteria}

9.2.1.1 Applicable Experienze. The facility processing contaminated componerits and samples should have experience in related tasks. Desirable experience includes:

1. Hot cell operations, material archiving, and retrieval systems with thorough material records 
2. Irradiated and contaminated materials receiving, handling, decontamination, and storage

3. Postirradiation examination (PIE) data interpretation and analys is

4. Equipment, component, and materials requalification or certification

5. Use of accurate equipment for radijactive materials analys is

6. Nondestructive and destructive exanination of radioactive material

7. Familiarity and experience with dccumentation and $Q A$ requirements for government radioactive material licenses

8. Coordination of work with other related facilities.

9.2.1.2 Quality Assurance. The examination, processing, or storage participant will be required to have an organizational and facility QA plan including, but not limited to, the following:

1. Inspection and documentation of raterial as received

2. Traceability records for work performed

3. Handling and storage procedures and criteria to prevent degradation of materials prior to analys is

4...Calibration and/or statistical records for processes or equipment used in analysis

5. Organizational $Q A$ independence. 
9.2.1.3 Safety and Security. The performing organization shall have a safety program that ensures operations are conducted in a manner that protects the health and safety of employees and the general public and that minimizes danger from all hazards to life and property. The safety program shall comply with applicable federal, state, and local health, safety, and fire sratection regulations and requirements. In particular, the safety program shall conform to applicable DOE (DOE Order 5483.1) and OSHA (29 CFR 1910 and 29 CFR 1926) safety regulctions.

The facility in which work is performed shall have appropriate approved safety assessments under which authorization to perform the work is defined.

The performing organization shall have a security program which in combination with administrative con that materials are secure from theft, loss, or sabotage. If handling of special nuclear materials is involved, the program shall conform to the provis:ons of the DOE Order 5632 Series, as applicable.

\subsubsection{Licensing Requirements. Any organization that receives,} possesses, uses, or transfers radioactive material shall be licens ed in accordance with the applicable regulations in Title 10, Parts 20, 30 through 35, 40, 50, or 70 of the Code of Federal Regulations (Private Facilities) or be operated under the Atomic Energy Act of 1954, as amended, the Enemgy Reorganization Act of 1974, and the Department of Energy' Organization Act of 1977 (Government Facilities).

\subsubsection{Documentation and Configuration Control. The performing} organization shall have a documentation and configuration control system which ersures the latest approved procedures and processes are used in handling or examination of materials or samples. Details, procedures, and special processes for accomplishing examinations shall be prepared, approved as required, and utilized in performing work tasks. The configuration control system shall ensure positive identification of material; and their location, and pertinent data, such as special handling requirements and fissile material content. 
9.2.1.6 Performance Management System. The performing organization shall use the TMI-2 standard reporting system presently being developed oy the Technical Integration office. The reporting system must include schedule and cost analysis reports. The performing organization should have a proven record of maintaining established schedule and cost agreements. All schedule and cost estimates shall be requirec in sufficient detail to allow proper evaluation.

9.2.1.7 Institutional. The performing organization and facility location shall satisfy a number of institutional guidelines. The following is a partial list of items to be considered:

1. Compliance with EEO, minority business, and other federal and state requirements

2. Disclosure of any current or pending litigations

3. Community acceptability

4. Public affairs and image

5. Possession of required state, regional, local, and federal permits.

\subsubsection{Specific Criteria}

9.2.2.1 Facility Requirements. Facilities are required for processing TMI-2 fuel components and samples that will enable the nuclear community and general public to gain the maximum information possible from the events that occurred. The processing of components will require facilities to perform the following services:

1. Receiving of high level radioactive material packaged in standard licensed shipping casks and containers 
2. Removal of material from casks for general inspection including dimensional, photography, radiography, weight, visual, etc.

3. Disassembly and assembly of fuel and core components and samples in controlled environments

c. Decontamination of shipping containers

5. Ensuring interim storage in a controlled environment for fuel and core components material prior to disposition

6. Packaging and shipping of fuel and core components material for further analysis or disposal.

Specific equipment which may be required includes:

1. Ventilation equipment adequate to meet federal radiological standards for the following:

a. Stack gas monitors (particulate and gas)

b. Air monitoring in facility containment (particulate and gas)

2. Fuel handling equipment (strong backs, pallets, manipulators, etc.) compatible with requirements (e.g., acceleration, deceleration, velocity, handling forces) for disassembling and handling highly damaged, brittle fuel assemblies, and capable of disassembling fuel bundles with brittle, ballooned, previously molten, or fractured fuel. rods

3. Biological shielding adequate to meet federal guidelines for exposure to radioactive components and samples 
4. Chemical and mechanical decontamination equipment to meet federal contamination control guidelines for handling and shipping classifications

5. Equipment to provide remote handling, disassemblj, receiving, inspection, and analys is (e.g., manipulators, turntables, fixtures, etc.) of radioactive hardware within allowable personnel exposure guidelines without Jamage or degradation to the fuel component or sample under investigation

6. Receiving facilities, including cranes and manifulators for unloading components and samples from shipping containers and casks

7. Examination/analysis equipment necassary to periorm stateof-the-art nondestructive and destructive examination of fuel and other radioactive material

8. Personnel with capabilities for interpreting arid analyzing (e.g., recognized technical expertise in PIE, metallurgy, chemistry, materials science, etc.)

9. Facilities for obtaining, interpreting, and storing photographs, radiographs, microfilm, etc., of fuel, components, and samples as required

10. A readily accessible facility location that can be easily entered by 1 icensed commercial carriers.

9.2.2.2 Examination, Analys is and Testing. The facilities and organizations responsible for performing specific examinations, analyses, and testing of fuel materials and samples from TMI-2 should have the capabilities for performing state-of-the-art nondestructive and destructive examinations. In order to minimize health hazards, the potential for loss or damage, and reduce program cost, it is desirable that the facilities 
identified for the examinations have sufficient in-house analys is capability to minimize the need for sample relocation for examinations. Furteer, it is desirable that the facility have the capability for temporary archive storage of the fuel materials and samples.

9.2.2.3 Disposal or Retention. Following examination and processing, all material will be identified for disposal or retention. Items will be packaged in accordance with DOT standards for shipment, if required. Packaging will ensure contamination-free handling during disposal or for the retention time identified. All storage will be in a protected environment to ensure contamination-free retrievability and all disposal will be made in an officially designated disposal area in conformance with requirements at that facility. Shielding requirements for radiation levels at package surfaces will conform to established regulations.

\subsubsection{Decontamination. Shipping casks will undergo initial} decontamination at the TMI-2 site to the level required for transportation to the examination facility. Cask decontamination at the examination facility may require a deionized water flush, a detergent flush, or steam cleaning. Sample material decontamination may require all of the above plus chemical treatment. The examination facility is responsible for handling and disposal of the decontamination solutions.

\subsubsection{Segregation. Selected TMI-2 samples may require segregated} storage to prevent physical damage or cross-contamination with other samples. Damaged fuel will require secondary, water and gas tight encapsulation tubes. Some small solid samples (cladding, etc.) may require dry storage.

\subsubsection{Potential TMI-2 Examination Facilities}

Tat.le 3 contains a listing of potential domestic facilities for examination of TMI-2 fuel and core components. The 1 ist was formulated by contacting the members of Planning Group 7.4 and, in some cases, the laboratories directly. The list is believed to be complete; however, addition; to the 1 ist are welcome. Determination of whether these 
facilities meet all of the general and specific criteria outlined above has not yet been made, but the nature of their ongoing work indicates that they are qualified to participate in the TMI-2 fuel and core component examinations. It is recomended that the Technical Integration office continue to develop specific examination criteria and match these with established administrative and technical capabilities of these laboratories. This will permit competitive bidding except in those cases where a laboratory has unique capabilites.

TABLE 3. Potential Domestic Facilities for TMI-2 Fuel and Core Component Examinationa

Industrial Laboratories

Babcock and Wilcox Company

Battelle Columbus Laboratories

Combustion Engineering, Inc.

Exxon Nuclear Company

General Atomic Company

General Electric Nuclear Center

Rockwell International
Government Laboratories

Argonne National Laboratory-East

Argonne National. Laboratory-West

Battelle Pacific Northwest Laboratories

Brookhaven National Laboratory

Hanford Engineering Development Laboratory

Idaho National Engineering Laboratory

Los Alamos Scientific Laboratory

Oak Ridge National Laboratory

Sandia Mational Laboratories

a. In addition to the facilities listed, several universities with remote-handling facilities may be qualified to participate in the TMI-2 examinations. 
Advance consideration must be given to the disposition of the TMI-2 fuel and core component samples once their examinations have been completed. Inter im and ultimate disposition of the TMI-2 core has been considered (see Reference 4). This study, which considered chemical reprocessing, pool storage, and dry storage, concluded that definitive recommendations are preseitly impossible becalse of political, institutional, and economic uncertainties. However, a likely disposition sequence would almost certainly begin with a period of temporary pool storage at TMI or elsewhere. Dry storage in special caissons or in existing vautts (such as those located at governmen: hot cell facilities) was found to have some economic benefits because of the extremely low TMI-2 core decay heat. Chemical reprocessing with disposal of high-level waste was recognized as the best technical solutior, but one which would probably not be available until the mid- to late-199Cs.

\section{-0.1 Subtask Objectives}

The objectives of this subtask are to recommend general guidel ines for the disposal of fuel examined under the TMI-2 Information and Examination Program, and to ensure that disposal options are in-place and adequately funded when the TMI-2 fuel Examination begins.

\subsection{Recommended Programs}

Sirze the fuel selected for examination will be only a small. fraction of the core, Planning Group ?.4 does not recommend pursuing disposal options ndependent of those being developed by the utility. We suggest that the facilities selected to perform the technical examinations be responsicle for storing the fuel for' a short interim period of $<1$ year, while the examination results are reviewed and analyzed. Thereafter the fuel samples should be properly contained and shipped to the facility holding the largest part of the core, and from that point on be handled by the utili=y or its agent as part of the general TMI-2 core debris. 
Spec if ically we recommend:

1. Monitoring of TMI-2 core disposition plans and prepare a program to integrate the disposal $0^{-}$fuel examination samples into these plans.

2. Storage of the fuel for an inter im period followed by shipment to the designated TMI-2 core disposition site.

3. Ensuring that fuel and core component specimens prepared for examination can be disposed of when the ceramograph or metallographic examinations are completed.

4. Providing for archival storage of specimens for future examination and experiments as identified by advances in technology. 


\section{REQUIREMENTS FOR DATA FROM TASK 7.2, CORE DAMAGE ASSESSMENT AND CORE REMOVAL}

The principal objective of $\mathrm{Planning}$ Group 7.2 is to determine the examinations needed to establish the undisturbed condition of the core prior to its removal. It is imperative that the results of the examinations be used at the earliest possible time to further refine the planning presented in this report since, as stated earlier, the Task 7.4 planning is based solely on analytic estimates of the extent of core damage.

\subsection{Subtask Objective}

The objective of this subtask is to coordinate, to the maximum extent possible, the core damage assessment and fuel examination planning.

\subsection{Recommended Program}

It is particularly important to Task $7.4 \mathrm{planning}$ that information on the following items be obtained at the earliest possible time:

1. Radial and axial extent of fuel assembly damage

2. Presence and extent of a rubble bed

3. Symmetry of damage

4. Apparent structurai integrity of core components

5. Extent of damage to control rods

Information of this type is needed to finalize plans for the selection of samples for archiving and for detailed examinations. It is recommended that a very close liaison between the groups responsible for implementation of the Task 7.2 and $7.4 \mathrm{plans}$ be mainiained so that final Task $7.4 \mathrm{planning}$ reflects the best assessment of actuai core damage. 


\section{RECOMMENDATIONS}

The recommendations of Planning Group 7.4 are listed below.

1. The fuel and core component examination plan will be highly dependent on the results from the Task 7.2 (core damage assessment) activities. Accordingly, a technical evaluation group should be maintained to revise the $\mathrm{plan}$ presented herein as the Task 7.2 activity progresses.

2. It is evident that the data obtained from implementation of the Task 7.2 and $7.4 \mathrm{plans}$ may differ in value and may be applied in a different fashion for each of the areas described in Sections 3, 4, 5, and 6 of this report. Thus, it is recommended that a small number of experts from each of these areas be retained to evaluate and catalog the data for application in their areas of interest and expertise and to provide an overview of examination procedures.

3. Further develop specific criteria for the detailed examination of selected TMI-2 fuei and core components. These should include the following:

a. Intact fuel assemblies

b. Debris of various anticipated forms

c. Pressure vessel surveillance capsules

d. Experimental assemblies

e. Debris flow tests.

4. Assess candidate examination laboratories against the criteria presented in Section 9 and identify in detail the technical capabilities of each facility. 
5. Integrate disposal plar.s of examined fuel and core component samples with the plans for ultimate disposal of the TMI-2 core.

6. Assess present regulatony guides to identify inconsistencies and instances where the TMI-2 accident reveals either excessively conservative or nonconservative criteria. Use the results of this study to further define the examination and analyses programs.

7. Evaluate reactor accident analysis methods to more precisely define the state-of-the-art and ascertain that key data needs have been identified and that $p l$ ans have been made to satisfy them.

8. Review accident consequence mitigation design studies (such as those of Zion and Indian Point, the Floating Nuclear Power Plant and the current EG\&G Idaho study) to ascertain that key data needs have been identified and that $p l a n s$ have been made to satisfy them.

G. Establish a TMI-2 fuel and core component archive facility so that samples of scient $f i c$ interest may be preserved for future examinations.

10. Develop schedules and cost estimates for implementation of the examination program.

11. Evaluate environmental effects on fuel. 


\section{REFERENCES}

1. M. Rogovin (Director, NRC Special Inquiry Group), Three Mile Isl and--A Report to the Commissioners and to the Public, U. S. Nuclear Regulatory Commission Special Inquiry Group, NUREG/CR-1250, Vol. II, Part 2, January 1980.

2. Electric Power Research Institute, Analysis of the Three Mile Island Unit No. 2 Accident, NSAC-80-1, March 1930.

3. NRC Action Plan Developed as a Result of the TMI-2 Accident, NUREG-0660, Vols. I, II, May 1980.

4. R. T. Anderson, Scoping Studies of the Alternate Options for Defueling, Packaging, Shipping, and Disposing of the TMI-2 Spent Fuel Core, AGNS-35900-1.5-79, May 1980. 


\section{APPENDIX A}

ANALYT:CAL METHODS FOR EXAMINING TMI-2

FUEL AND CORE COMPONENTS 
ANALYTICAL METHODS FOR EXAMINING TMI-2

FUEL AND CORE COMPONENTS

Compiled by

Edward L. Courtright Jr. Materials Department

Pacific Northwest Labcratories

Contributing Organizations:

Argonne National Laboratory

J. P. Bacca - Argonne-West

H. M. Chung

S. M. Gehl

Babcox \& Wilcox

G. M. Bain

D. L. Baty

M. A. Rigdon

Battelle Columbus Laboratories

J. S. Perrin

Battelle Pacific Northwest

L. R. Bunne 11

J. L. Daniel

P. E. Hart

R. Wang
EG\&G Idaho, Inc.

D. E. Owen

General Electric Company

R. B. Adamson

E. L. Esch

S. Y. Ogawa

Hanford Engineering Development Laboratory

G. J. Alkire

Nuclear Regulatory Commission

F. D. Coffman

U. L. Pickles mer

Oak Ridge Nationæl Laboratory

D. 0. Hobson

E. L. Long 


\section{APPENDIX A}

\section{ANALYTICAL METHODS FOR EXAMINING TMI-2 \\ FUEL AND CORE COMPONENTS}

The principal characteristics needed in the TMI-2 fuel and component examinations have been selected from criteria recommended by Planning Group 7.4 members. These data characteristics are divided into ten major categories 1 isted in Table A-1 with the major components to be examined, and principal regions or lacations from which samples will most likely be taken.

The analytical techniques that will be needed to provide basic information for each of the ten categories are presented in Tables A-2 through $A-11$. The objectives in preparing this information were to:

1. Identify techniques for obtaining microstructural and macrostructural information

2. Identify the chemical, microchemical, or other analytical techniques that would be needed to analyze the range of potentiat materials or comoosites

3. Ident ify core component metrology requirements

4. Identify NDT techniques thit can be used to support destructive analysis

5. Identify the analysis techniques that will require expert interpretation prior to the final reporting or transmitting of data

6. Obtain thermal-hydraulic information for fuel bundles or. bundle segments

7. Discover where archive samples will be needed. 
In each of these tables, applicable measurement techniques are indicated, along with the specific parameter or information to be obtained. Practical degrees of accuracy and precision for each technique are also included, $i=$ they are available, although equally precise methods of sample selection and removal are considered to be equally important to a successful evaluation. However, limited or poor understanding of sample location or history could have a greater impact on data analysis than experimental uncertainties.

For illustrative and comparative purposes, comments on each of the analytical techniques are also included in the tables. In addition, ways to use and apply the data are suggested. The listing order of each measurement technique is purely arbitrary--no attempt is made to rank the techniques with respect to accuracy, applicability, or usefulness. In many instances, the techniques complement one another, so the use of more than one method may be necessary to verify examination results.

It will be apparent that the scope of the presented material is great enough to cover all possible examination subjects, from an intact fuel rod to a pile of rubble. The most appropriate selection of an examination technique, or an experimental method, cannot be made, however, without consideration of the specific sample to be analyzed. As an example, elemental and isotopic distribution can have quite different meanings, depending on whether one is analyzing a sample of an intact rod or a piece of debris from the rubble bed. Many of the techniques will also be highly dependent upon the knowledge and expertise of the individual performing the analysis. For these techniques, the skill of the interpmeter will be as important to proper evaluation of the infornation as the analytical technique itself. In addition, many of the newer techniques may require some development or calibration before they can be applied to the myriad of special analysis problems that are likely to be encountered.

The basic objectives of the examinations will be to determine the core thermal history along with the chemical and physical changes that occurred to the fuel and core components during the course of the accident. The bulk of the important information will be obtained from microstructural examinations or chemical analysis. 
The microstructural evaluations would include the extent of cladding oxidation and oxygen uptake, the type and extent of fuel-zircaloy interactions, the occurrence of fuel oxidation and/or reduction, evidence of grain growth, liquation or melting of stainless steel components, and characterization of the debris bej. In most cases, these studies will directly or indirectly answer temperature related questions (e.g., microstructural evidence of grain boundary liquation in stainless steel or the formation of a $\mathrm{UO}_{2}-\mathrm{Zr}$ eutectic are clear signs that the solidus and eutectic temperatures, respectively, were exceeded). Similarly, a study of $\mathrm{ZrO}_{2}$ and oxygen-stabilized alpha layer thicknesses can be used to infer the envelope of time-temperature conditions that could have produced the observed microstructures. The amount and distribution of cladding hydrides and the morphology of the alpha phase will provide information about the mechanism of hydrogen uptake and the cooling history of the cladding.

The most useful tools for microstructural investigations will probably be optical or scanning electron microscopy. For phase identification and other.crystallography studies (i.e., $U_{2} 0_{2} 1$ attice parameter measurements to determine $0 / M), X$-ray diffraction and electron microprobe analys is should prove useful. Electron channeling patterns obtained from SEM analys is may also have some limited applicability in evaluating the complex material structures that may be discovered in the course of this examination.

An atlas of microstructures will probably have to be prepared to assist in proper interpretation of the resultant microstructures. The PBF tests of LWR fuel behavior, as well as the TREAT and SLSF tests, should provide micrographs for a variety of well-characterized conditions.

Chemical characterization of fuel materials, cladding segments, and debris rubble (particularly for retained fission products) will also be an integral and important part of the evaluation. By measuring the amount and type of fission products remaining in the fuel, a temperature-time history can be estimated. Such measurements will necessarily be complicated by the low concentration of fission products; however, careful radiochemical 
techniques involving wet chemical element separation should provide adequate sensitivity for most isotopes. For stable isotopes, atomic absorption spectrometry or neutron activation analysis may prove useful.

Gradients in fission product concentration will probably be most advantageously studied with an ion microprobe which has better sensitivity than the electron microprobe by several orders of magnitude and is also capable of isotopic analysis. The utility of the electron microprobe in fission product characterizations will probably be limited to the study of more massive fission product concentrations (e.g., cesium liranate phases). If thin films of fission product are present (e.g., on grain boundaries), surface analysis tools, such as scanning Auger microscopy analysis. may have to be employed. The use of precision gamma spectroscopy will most likely be 1 imited to the interrogation of intact fuel elements for the longer lived isotopes, but could help in the construction of a qualitative core wide temperature map based on the temperature-dependent redistribution of the fission products.

Fission gas measurements of the plenum gas from unbreached rods, if any are left, will contribute to constructing the qualitative corewide temperature map. However, direct measuremen:s of the fission gas retai eed in recovered fuel, whether from breached or unbreached rods, will assist in establishing the amount of fission gas released during the accident. Some of the techniques to be employed will involve total dissolution or melting of samples followed by gas chromatography or mass spectrometry.

The hydrogen concentration of the cladding will alsc provide a valuable piece of chemical information. The axial profile of hydrogen concentration in the vicinity of a cladding rupture will help to determine temperature history during the accident. Vacuum fusion and other techniques $c$ an be used to determine hydrogen concentrations:

Two important considerations associated with sample selection are the problem of sampling the core, and the fact that all of the core material will have been washed in hot water for at least two years prior to the examination. The continued presence of water in contact with the core 
materials means that the exposed surfaces may be considerably changed from their condition during and just after the accident. Corrosion products, other deposits, and subsurface leaching will have obscured and perhaps destroyed parts of the evidence upon which to base the accident history. Much effort will be required to separate changes that occurred during the accident from those that have occurred since. As a result, special examination and specimen preparation techniques may have to be considered. All materials removed during the examinations must be documented in terms of their location and orientation within the core. 
TABLE A-1. TMI-2 EXAMINATION DATA CHARACTERISTICS

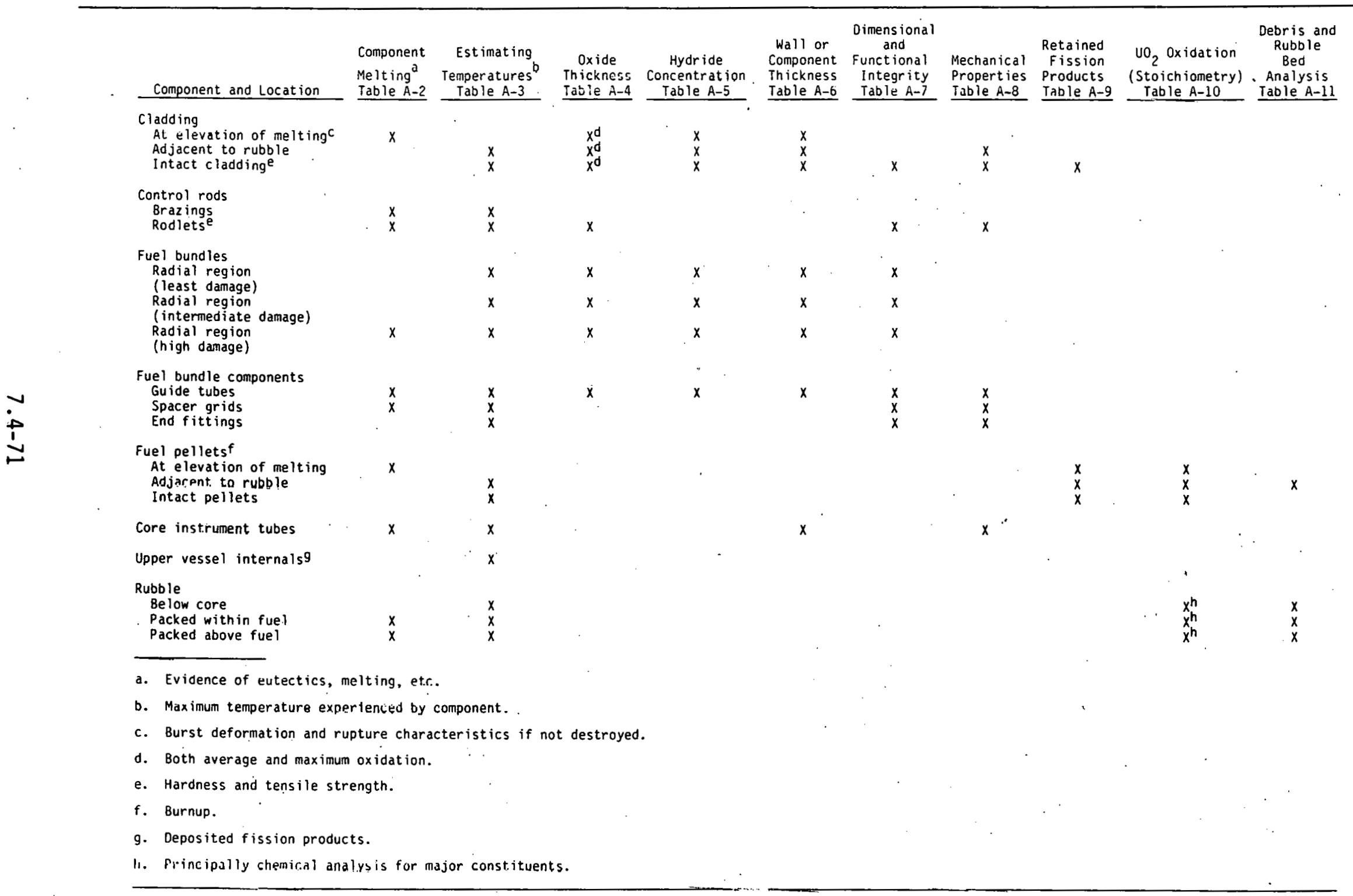


TABLE A-2. COMPONENT MELTING

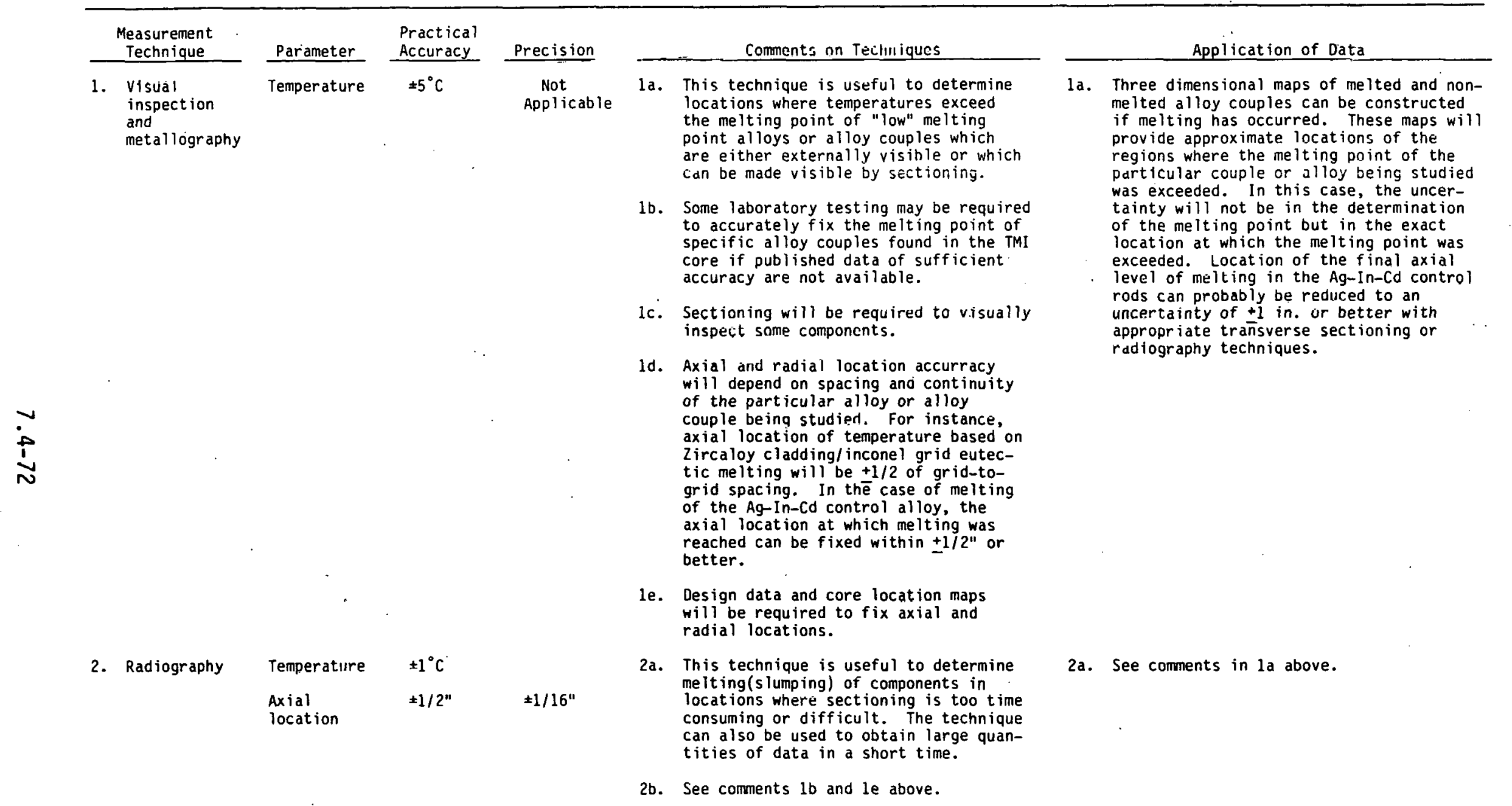


TABLE A-2. (cont inued)

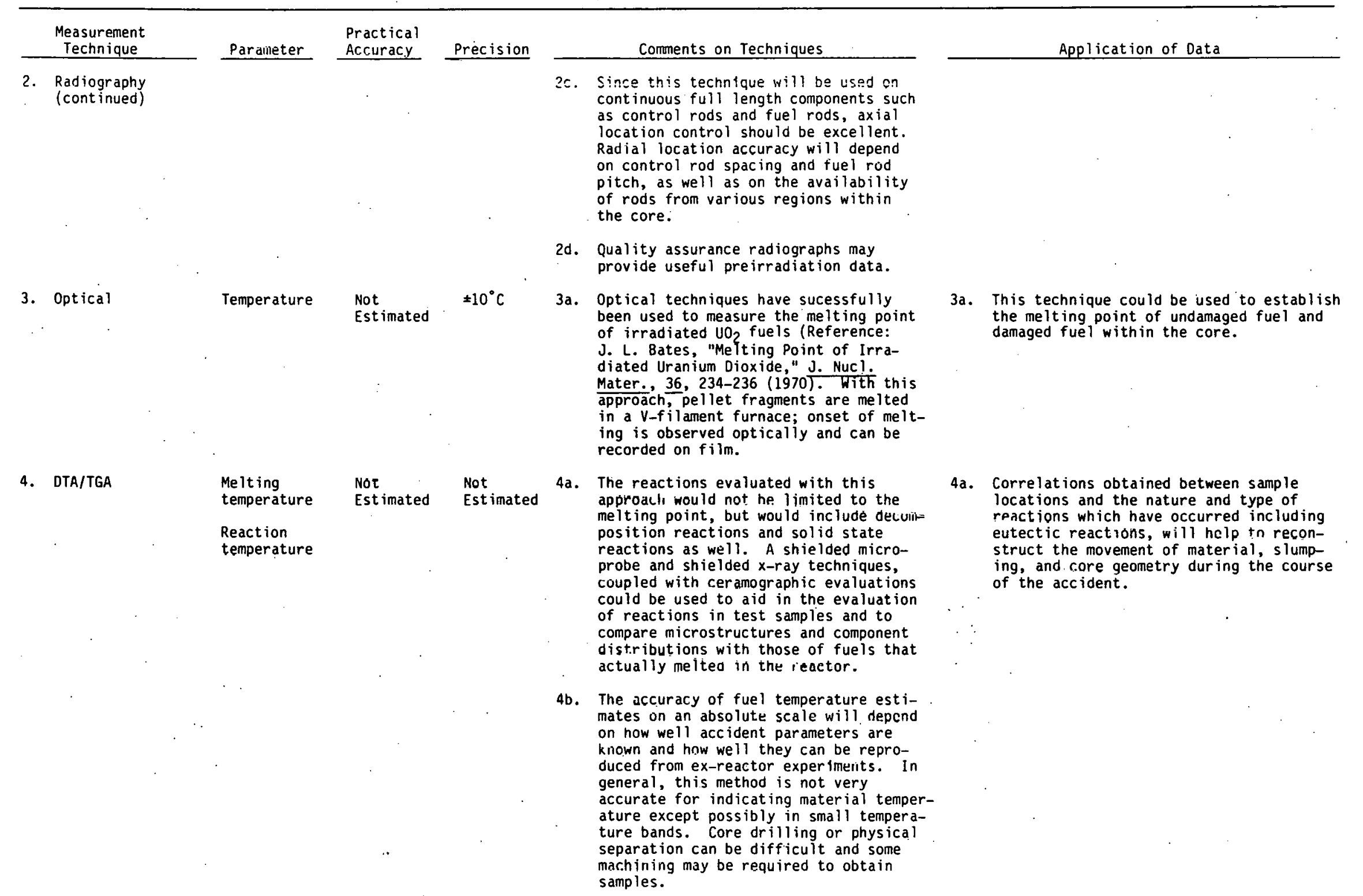


TABLE A-2. (continued)

Measurement
Technique

4. DTA/TGA

(cont inued)
Parameter Accuracy Precision

Precision

Comments on Techniques

Application of Data

c. The opt imum samples for use in this

evaluation would be irradiated samples

that had not melted but had the same

irradiation history as those portions

of fuel that exhibiteo melting. There

would be some value, however, in eval-

uating the behavior of non irradiated

materials containing appropriate levels

of dopents to establish experience

and a data base prior to conducting

extensive post-irradiation examina-

tions. This data base should be estab-

lished prior to TMI-2 samples being

lished prior to TMI-2 samples being

4d. The DTA-TGA approach would also be applicable to examining $\mathrm{UO}_{2}-\mathrm{ZrO}_{2}-\mathrm{Zr}$. reactions under nonequilibrium conditions. Reactions of this nature may be as important, if nnt more impor lane, in terms of forming liquid phase than melting, per se, under equilibrium conditions. 
TABLE A-3. ESTIMATING TEMPERATURES

\begin{tabular}{|c|c|c|c|c|c|c|}
\hline & $\begin{array}{c}\text { Measurement } \\
\text { Technique }\end{array}$ & Parameter & $\begin{array}{r}\text { Practical } \\
\text { Accuracy }\end{array}$ & Precision & & Comments on Techniques \\
\hline 1. & $\begin{array}{l}\text { Microstructural } \\
\text { evaluation }\end{array}$ & $\begin{array}{l}\text { Time/temperatıre } \\
\text { relationships }\end{array}$ & $\begin{array}{l}\text { Sop } \\
\text { Comments }\end{array}$ & & $1 a$ & $\begin{array}{l}\text { Microstructural changes whirh necur- } \\
\text { red in the cladding during the acci- } \\
\text { dent will basically be a function of } \\
3 \text { variables: time; temperature, and } \\
\text { processing history. Therefore, a } \\
\text { precise temperature fix will not be } \\
\text { possible, but a time-temperature } \\
\text { envelope might be deduced. (Chem- } \\
\text { ical composition, principally oxygen } \\
\text { may aiso be an additional variable } \\
\text { affect ing the a }+ \text { B/B phase tran- } \\
\text { sition. A microstructure atlas pre- } \\
\text { pared from available information on } \\
\text { fuel and core materials subjected to } \\
\text { extreme conditions where the temper- } \\
\text { ature is known should be employed. } \\
\text { The thermal-mechanical history will } \\
\text { have to be documented for each com- } \\
\text { ponent.) Accuracy will be limited } \\
\text { to the variables which contribute } \\
\text { to microstructural changes (residual } \\
\text { stress, radiation induced stresses, } \\
\text { prior temperature history, etc.) }\end{array}$ \\
\hline
\end{tabular}

1b. Extensive metallographic, x-ray diffraction, microprobe, and SEM cxaminations have been perfor Thus, it should be possible during postirradiation examination to est imate the maximum temperature experienced by the fuel.

1c. An atlas of microstructures can be prepared from laboratory specimens exposed to known thermal histories, cooling rates, and with known oxygen contents. Results from previous LOCA/PCM/RIA tests performed in LOFT and PBF may also be useful to determine or confirm the time and temperature combinations which would likely produce the observed microstructures. The time/-temperature envelopes for different materials will be compared to narrow the temperature range at locations where

\section{Application of. Data}

1a. In interpreting zircaloy cladding micrnstructures, some of the following physical phenomena can be employed: recovery occurs at about $480^{\circ} \mathrm{C}$, recrystallization begins around $540^{\circ} \mathrm{C}$ and is basicaliy com plete by $620^{\circ} \mathrm{C}$, pronounced grain growth will occur above $700^{\circ} \mathrm{C}$, the initial phase change from a to $\alpha+\beta$ will occur at

$825^{\circ} \mathrm{C}$ and the final transformation to all beta at about $960^{\circ} \mathrm{C}$, depending on oxygen content. Microstructural interpretation based on these phase regions can provide only a rough estimate of temperature since the transformations are also sensitive to cooling rate and oxygen content. However prior beta and oxyen stabilized However, prior beta and oxygen stabilized temperatures, if prior temperature history is known.

1b. If centerline melting $\left(2800^{\circ} \mathrm{C}+50^{\circ} \mathrm{C}\right)$ has occurred in the fuel, a radius of melting should be ident ifiable. In the region are for +100 , are formed ${ }^{\circ} \mathrm{Columin}$ grains form at about $1950{ }^{\circ} \mathrm{C}+150^{\circ} \mathrm{C}$. Between the colum nar grain region and the as-fabricated is region, a ring of equiaxed arain region is generally observed. The lower temper -

equiaxed region is about $1200^{\circ} \mathrm{C} \pm 100^{\circ} \mathrm{C}$.

These temperature regimes have been established for fuel exposed to steep temperature gradients as opposed to the more or less isothermal profiles experienced by the TWI fuel; however, they will provide valuable benchmarks and can be coupled with comparisons of isotherma treatments given to archive specimens. 
TABLE A-3. (continued)

\begin{tabular}{|c|c|c|c|c|c|c|c|c|c|}
\hline & $\begin{array}{c}\text { Measurement } \\
\text { Terhnique }\end{array}$ & fánguter & & $\begin{array}{r}\text { Practical } \\
\text { Aciuraly } \\
\end{array}$ & Precision & & Comments on Techniques & & Application of Data \\
\hline \multirow[t]{2}{*}{1.} & $\begin{array}{l}\text { Microstructural } \\
\text { evaluation } \\
\text { (cont inued) }\end{array}$ & & & & & & $\begin{array}{l}\text { more than one material is avail- } \\
\text { able. Time estimates from the acci- } \\
\text { dent history can be used to further } \\
\text { refine the temperature estimates. }\end{array}$ & & . \\
\hline & & & . & & & $1 d$. & $\begin{array}{l}\text { Archive materials should be used, } \\
\text { if possible, in the laboratory sim- } \\
\text { ulations. The samples should be } \\
\text { subjected to the estimated steam } \\
\text { conditions predicted during the } \\
\text { course of accident since the } \\
\text { effects of oxygen and hydrogen } \\
\text { uptake on the grain structural } \\
\text { changes must be considered when } \\
\text { making comparisons to the accident- } \\
\text { damaged materials. }\end{array}$ & & \\
\hline \multirow[t]{3}{*}{2.} & $\begin{array}{l}x \text {-ray } \\
\text { diffraction }\end{array}$ & $\begin{array}{l}\text { Phase } \\
\text { identification }\end{array}$ & & $\begin{array}{l}\text { See } \\
\text { Comments }\end{array}$ & & $2 a$. & $\begin{array}{l}\text { X-ray diffraction provides supple- } \\
\text { mentary data for use in microstrue- } \\
\text { tural evaluations (see comment la } \\
\text { above). For example, this technique } \\
\text { can be used to identify, conf irm, } \\
\text { and quantify the presence of high } \\
\text { temperature phases, precipitates, } \\
\text { etc. }\end{array}$ & $2 a$. & $\begin{array}{l}\text { X-ray diffraction would supplement the } \\
\text { microstructural interpretation discussed } \\
\text { in la and } 1 \mathrm{~b} \text { above. }\end{array}$ \\
\hline & & $\begin{array}{l}\text { Oxide } \\
\text { morphology }\end{array}$ & $\therefore$ & $\begin{array}{l}\text { See } \\
\text { Comments }\end{array}$ & . & $2 \mathrm{~b}$. & $\begin{array}{l}\text { Structures of transformed oxides } \\
\text { that have formed at high tempera- } \\
\text { tures can be useful in est imating } \\
\text { maximum cladding temperatures } \\
\text { reached ( } V \text {. F. Urbanic and } \\
T \text {. R. Heidrick, J. Nucl. Mater., } 75 \text {, } \\
251 \text { (1978). For T }>1577^{2} \mathrm{C} \text { the } \\
\text { volume fraction is } 10 \text { in contrast } \\
\text { to } 1-2 \text { for oxidation at } T<1577^{\circ} \mathrm{C} \text {. }\end{array}$ & & . \\
\hline & & . & & & & $2 c$. & $\begin{array}{l}\text { Metastable tetragonal } \mathrm{ZrO}_{2} \text { accom- } \\
\text { panies the monoclinic pliase in the } \\
\text { oxidation temperature range of } \\
\text { stable monoclinic } 2 \mathrm{rO} \mathrm{O}_{2}\left(\mathrm{~T} \leq 1150^{\circ} \mathrm{C}\right) \\
\text { if the oxidatiun Lakes plaçe in a } \\
\text { hydrogen containing atmosphere } \\
\text { (T. Nakayama and T. Koizumi, J. } \\
\text { Japan Inst. Metals, } 31,839 \text {, } 1967 \text {. } \\
\text { Accordingly, an observation of outer- } \\
\text { surface oxide layers containing both }\end{array}$ & & . \\
\hline
\end{tabular}


TABLE A-3. (continued)

\begin{tabular}{|c|c|c|c|c|}
\hline $\begin{array}{c}\text { Measurement } \\
\text { Technique }\end{array}$ & Parameter & $\begin{array}{l}\text { Practical } \\
\text { Accuracy }\end{array}$ & Precision & Comments on Techniques \\
\hline $\begin{array}{ll}\text { 2. } & \text { X-ray } \\
\text { diffidaction } \\
\text { (continued) }\end{array}$ & r & & - & $\begin{array}{l}\text { monoclinic and tetragonal modifica- } \\
\text { tions would indicate oxidation of } \\
\text { TMI-2 fuel cladding in a steam- } \\
\text { hydrogen mixture. Under such a sit- } \\
\text { uation, the relative intensity of } \\
\text { x-ray diffraction peaks correspon- } \\
\text { ding to monoclinic and tetragonal } \\
\mathrm{ZrO}_{2} \text { should be measured and com- } \\
\text { pared with out-of-reactor simulation } \\
\text { tests. }\end{array}$ \\
\hline
\end{tabular}

$\begin{array}{lll}\text { 3. Hardness Annealing See } & \\ & \text { Comments }\end{array}$

4. Microhardness

Localized

$\pm 5 \mathrm{KHN}$

5. Oxide

thickness
Temperature

1 um 3a. Accuracy and precision depend on equipment and hardness scale used for the test.

3b. Hardness tests on thermal simulations performed in the laboratory on archive or duplicate materials could be used to describe a timetemperature enve lope

3c. This technique can be used to process a large number of samples relatively quickly.

4a. Microhardness medsur einents can be used to define the oxygen enriched a-zircaloy boundary.

Not
Est imate

3a. Hardness data from core components can be compared with hardness data from laboratory simulations to assist in determining the time-temperature envelopes. Core radial and axial hardness scans will also serve to locate temperature transition points more accurately. For instance, there will be an abrupt change in hardness at the axial location on the zircaloy fuel cladding where recrystallization temperature was exceeded. (See comment la above.)

a. The thickness of the oxygen enriched zircaloy layer provides a 3rd variablic which can be used along with diffusion and time data from the accident history to help define a temperature profile.

5a. Computer codes such as ZORO, COBILD, and SIMTRAN can utilize the oxide and oxygen stabilized alpha thickness measurements to compare predicted reaction times and temperatures which would have provided those thicknesses. Verification of current "state-of-the-art" analytical models to predict oxidation under a accident conditions will be one of the major uses of the TMI fuel examinations. Most models currently employ parabolic rate constants and may not be entirely valid for the transient thermal exposure history experienced by the TMI fuel rods.

5b. Metallography on zircaloy tubes will provide measurements of oxide layer thicknesses, oxygen-stabilized,

5b. The appearance of such features as a 
TABLE A-3. (continued)

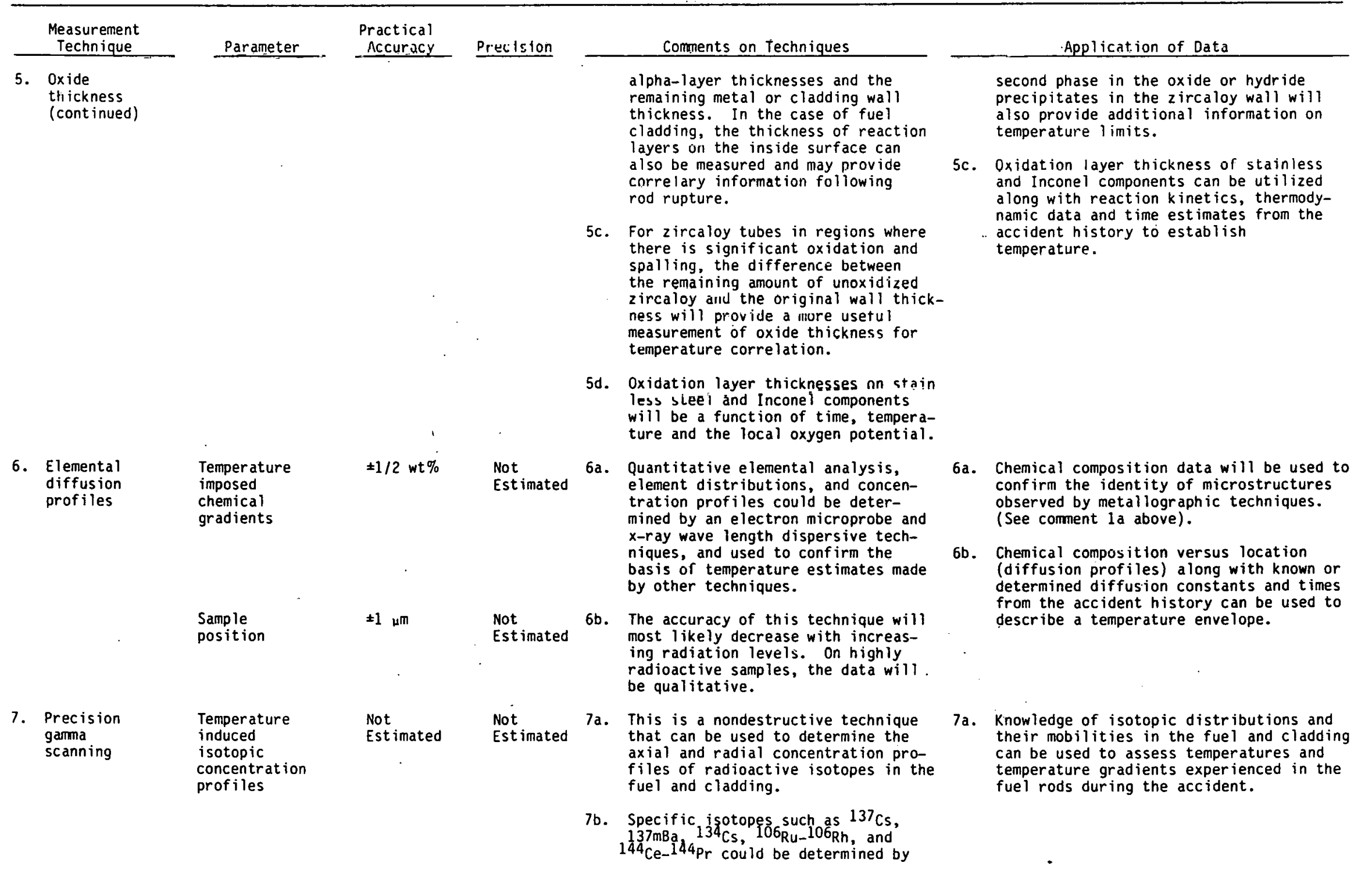


TABLE A-3. (continued)

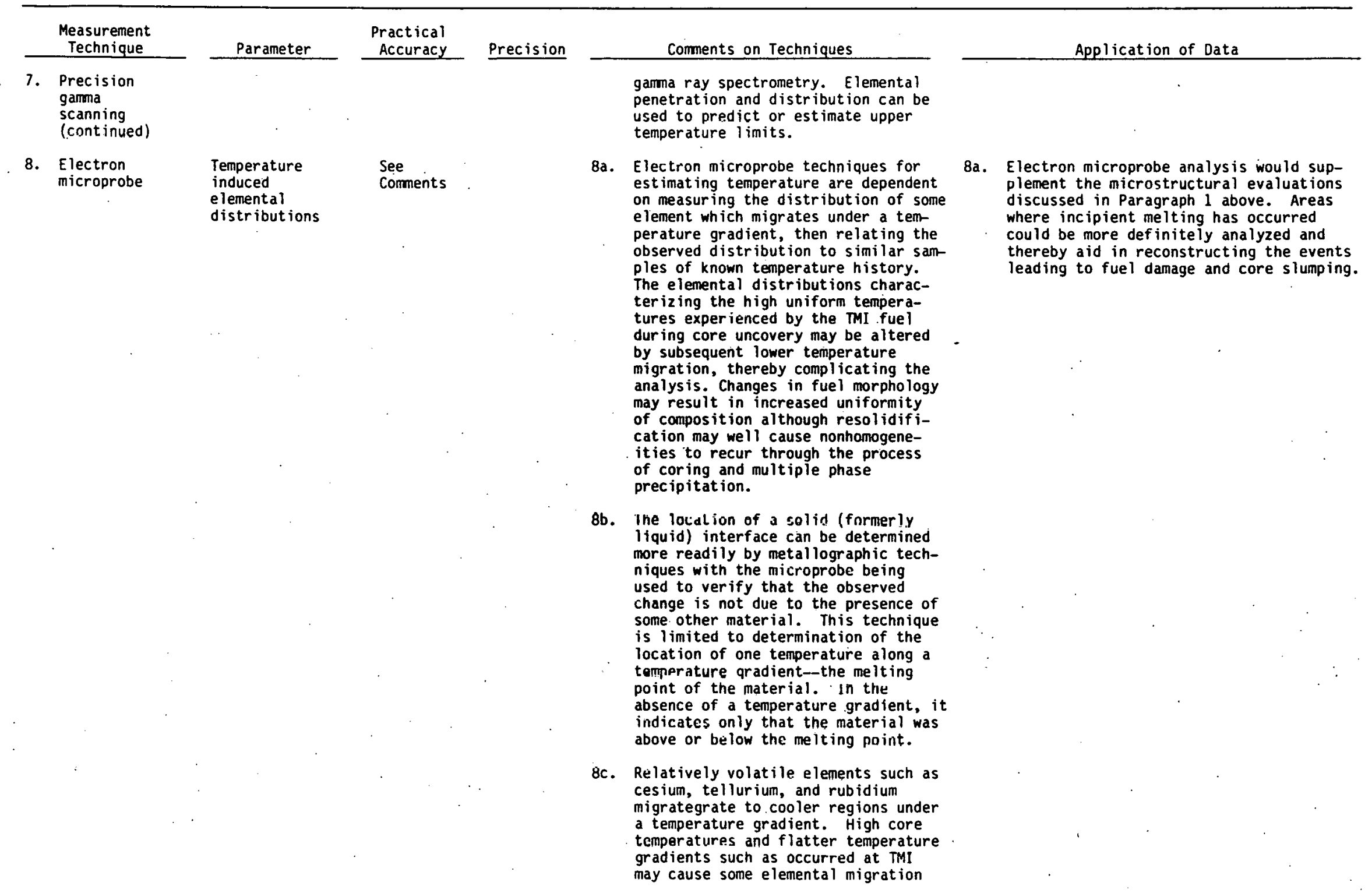


TABLE A-3. (continued)

\begin{tabular}{|c|c|c|c|c|c|c|c|c|}
\hline & $\begin{array}{c}\text { Measurement } \\
\text { Technique }\end{array}$ & Parameter & $\begin{array}{r}\text { Practical } \\
\text { Accuracy } \\
\end{array}$ & Precision & & Comments on Techniques & & Application of Data \\
\hline 8. & $\begin{array}{l}\text { Electron } \\
\text { microprobe } \\
\text { (continued) }\end{array}$ & . & & . & & $\begin{array}{l}\text { back toward the fuel center. The } \\
\text { experimental technique is to gather } \\
\text { a series of photomicrographs of } \\
\text { cesium distribution (for example) } \\
\text { and correlate the depth of penetra- } \\
\text { tion of cesium aloriy fuel grain } \\
\text { hnundariac, oto.3 with teiluelalures } \\
\text { from similar fuel at known tempera- } \\
\text { ture. Since cesium profiles can } \\
\text { only be measured by electron micro- } \\
\text { probe in fuels with burnups greater } \\
\text { than - } 2 \% \text {, this technique may not be } \\
\text { generally applicablp to the TMI fuel } \\
\text { examinat ions except in local regions } \\
\text { of high cesium concentration. Huw- } \\
\text { ever, an ion microprobe may be used } \\
\text { to determine qualitative concentra- } \\
\text { tion profiles at much lower cesium } \\
\text { levels. }\end{array}$ & & \\
\hline 9. & $\begin{array}{l}\text { Pustirraolation } \\
\text { heating of } \\
\text { cladding }\end{array}$ & Temperature & $\begin{array}{l}\text { Not } \\
\text { Estimated }\end{array}$ & \pm 5 & 9a. & $\begin{array}{l}\text { This technique is based on the mea- } \\
\text { surement of the rate of release of } \\
\text { Kr-85 during controlled heating of } \\
\text { irradiated fuel cladding samples. } \\
\text { (Swanson, et al. J. Nucl. Mater.. } \\
3: 302-310 \text { (1960). The precision of } \\
\text { this technique is + } 3 \% \text { where minimal } \\
\text { clad attack has occurred; the pre- } \\
\text { cision degenerates to +8\% where } \\
\text { severe attack is evident (Buzzelli, } \\
\text { et al., GA-A14459 (April 1978). }\end{array}$ & $9 a$. & $\begin{array}{l}\text { The } \mathrm{Kr}-85 \text { annealing technique has been } \\
\text { used as means of determining in-reactor } \\
\text { fuel cladding temperatures. This method } \\
\text { is based on the measurement of the rate } \\
\text { of release of } \mathrm{kr}-85 \text { during controlled } \\
\text { heating of irradiated fuel cladding sam- } \\
\text { ples. An increased rate of release of } \\
\mathrm{Kr}-85 \text {, which had impregnated the inner } \\
\text { surface of the cladding from fission } \\
\text { recoil, will occur when the maximum oper- } \\
\text { ating temperature is exceeded during } \\
\text { annealing. }\end{array}$ \\
\hline
\end{tabular}


TABLE A-4. OXIDE THICKNESS

\begin{tabular}{|c|c|c|c|c|c|}
\hline $\begin{array}{c}\text { Measurement } \\
\text { Technique }\end{array}$ & Parameter & $\begin{array}{l}\text { Practical } \\
\text { Accuracy } \\
\end{array}$ & Precision & Comments on Techniques & Application of Data \\
\hline 1. Metallography & $\begin{array}{l}\text { Oxide } \\
\text { thickness/ } \\
\text { alpha } \\
\text { stabilized } \\
\text { layer }\end{array}$ & $\pm 3 \quad(10)$ & $\begin{array}{l}\text { Not } \\
\text { Estimated }\end{array}$ & $\begin{array}{l}\text { 1a. Samples of material to be examined } \\
\text { would be mounted in standard metal- } \\
\text { lographic mounts suitable for oxide } \\
\text { thickness measurements at magn if i- } \\
\text { cations up to } 500 x \text {. For thicknes- } \\
\text { ses less than } 10 \text { microns, scanning } \\
\text { electron microscopy could be used at } \\
\text { magnifications up to } 3,000 x \text {. Micro- }\end{array}$ & $\begin{array}{l}\text { 1a. The oxide thickness data will be userui } \\
\text { in postaccident heat transfer, calcula- } \\
\text { tions. Microstructural features such as } \\
\text { morphology, grain size and shape, and } \\
\text { fracture appearances will provide evi- } \\
\text { dence of fuel rod damage that occurred } \\
\text { during the accident. }\end{array}$ \\
\hline
\end{tabular}

$\begin{array}{lll}\text { 2. NDT-Eddy } & \begin{array}{l}\text { Oxide } \\ \text { current }\end{array} & \begin{array}{l}\text { Alpha } \\ \text { thickness }\end{array} \\ & & \begin{array}{l}\text { labilized } \\ \text { (possibly) }\end{array} \\ +\infty & & \end{array}$

$\pm 1 \mu \mathrm{m}$

Not
Estimated
structurations up to 3,000X. Micro structural features, such as morphology, grain size and shape, and fracture appe

2a. 0xide layer thicknesses measured by eddy current techniques have been perfected for zirconium oxide films on irradiated fuel rods. A specially designed probe is used as the sensor. Measurements can be made underwater as
well as in-cell. In addition, measurements can be made at discrete points or as cont inuous traces a long a rod length. Accuracy is best on thicker oxide layers ( $>2 \mathrm{mils}$ ) which should be the case for most of the exposed cladding material.

2b. Comparisons have been made between thickness measurements obtained using the eddy current technique and the same measurements' obtained by metallography. Results compare ver favorably. At film thicknesses less than $5 \mu \mathrm{m}$, the eddy current results tend to be less accurate; hnwever. they should be sat isfactory. over film thicknesses that range from five to several hundred microns.

2c. Practical accuracy will be limited by (a) ability to accurately measure oxide thicknesses on standards and (b) the extent of oxide removal caused by sliding contact between the probe and any nonadherent oxide 2a. The data can be compared with calculations from computer codes such as ZORO, COBILD, or SIMTRAN which predict both oxide and oxy given reaction time and nesses from given reacti

2b. Oxidation layer thicknesses on stainless and Inconel components can be utilized along with reaction $k$ inetics, thermodynamic data, and time estimales from: the accident history to estimate temperature. 
TABLE A-4. (continued)

\begin{tabular}{|c|c|c|c|c|c|c|c|}
\hline $\begin{array}{c}\text { Measurement } \\
\text { Techniquc }\end{array}$ & Pardineter & $\begin{array}{l}\text { Practical } \\
\text { Accuracy }\end{array}$ & Precision & & Comments on Techniques & & Application of Data \\
\hline $\begin{array}{l}\text { 2. NDT-Eddy } \\
\text { current }\end{array}$ & & & & $2 d$. & $\begin{array}{l}\text { Oxide thicknesses measurements can } \\
\text { be made quickly on a larger number } \\
\text { of rods or other components. }\end{array}$ & & \\
\hline \multirow[t]{3}{*}{$\begin{array}{l}\text { 3. Color } \\
\text { standards }\end{array}$} & $\begin{array}{l}\text { 0xide } \\
\text { thickness }\end{array}$ & $\begin{array}{l}\text { Not } \\
\text { Applicable } \\
\text { (material } \\
\text { and } \\
\text { operator } \\
\text { dependent) }\end{array}$ & $\begin{array}{l}\text { Not } \\
\text { Applicable }\end{array}$ & $3 a$. & $\begin{array}{l}\text { Because the color of zirconium oxide } \\
\text { film varies with thickness, a qual- } \\
\text { itative indication of film thickness } \\
\text { can be obtained from observing film } \\
\text { color. A series of color standards } \\
\text { have been defined which correlates } \\
\text { with zirconium oxide film thickness. } \\
\text { The standards are applicable over a } \\
\text { thickness range of a few to several } \\
\text { hundred microns. However, because } \\
\text { of its qualitalive nature, the } \\
\text { method is not appropriate for preci- } \\
\text { sion measurements. In addition, the } \\
\text { oxide thicknesses on the zircaloy } \\
\text { fuel cladding at TMI will likg? bo } \\
\text { beyond the color sensitive range. }\end{array}$ & $3 a$. & $\begin{array}{l}\text { The principal use of color standards } \\
\text { would likely be to provide a quick } 3 \text { - } \\
\text { dimensional perspective of oxidation and } \\
\text { temperature distribution in the peri- } \\
\text { pheral core region and, would therefore, } \\
\text { aid in the selection of samples for more } \\
\text { detailed examinations by one of the } \\
\text { other techniques listed above. }\end{array}$ \\
\hline & & & & $3 b$. & $\begin{array}{l}\text { This technique may be more useful } \\
\text { for estimating oxide thicknesses on } \\
\text { Inconel and stainless steel compon- } \\
\text { ents in areas where the temperatures } \\
\text { were not extremely high and the oxi- } \\
\text { dation effects were not severe. }\end{array}$ & & \\
\hline & & & & $3 c$. & $\begin{array}{l}\text { This technique is fast, nondestruc- } \\
\text { tive, and can be used to process a } \\
\text { large number of samples or to view a } \\
\text { cross-section of components to ob- } \\
\text { tain a 3-dimensional perspective of } \\
\text { oxidation outside heavily damaged } \\
\text { regions. }\end{array}$ & & . \\
\hline
\end{tabular}


TABLE A-5. HYDRIDE CONCENTRATION

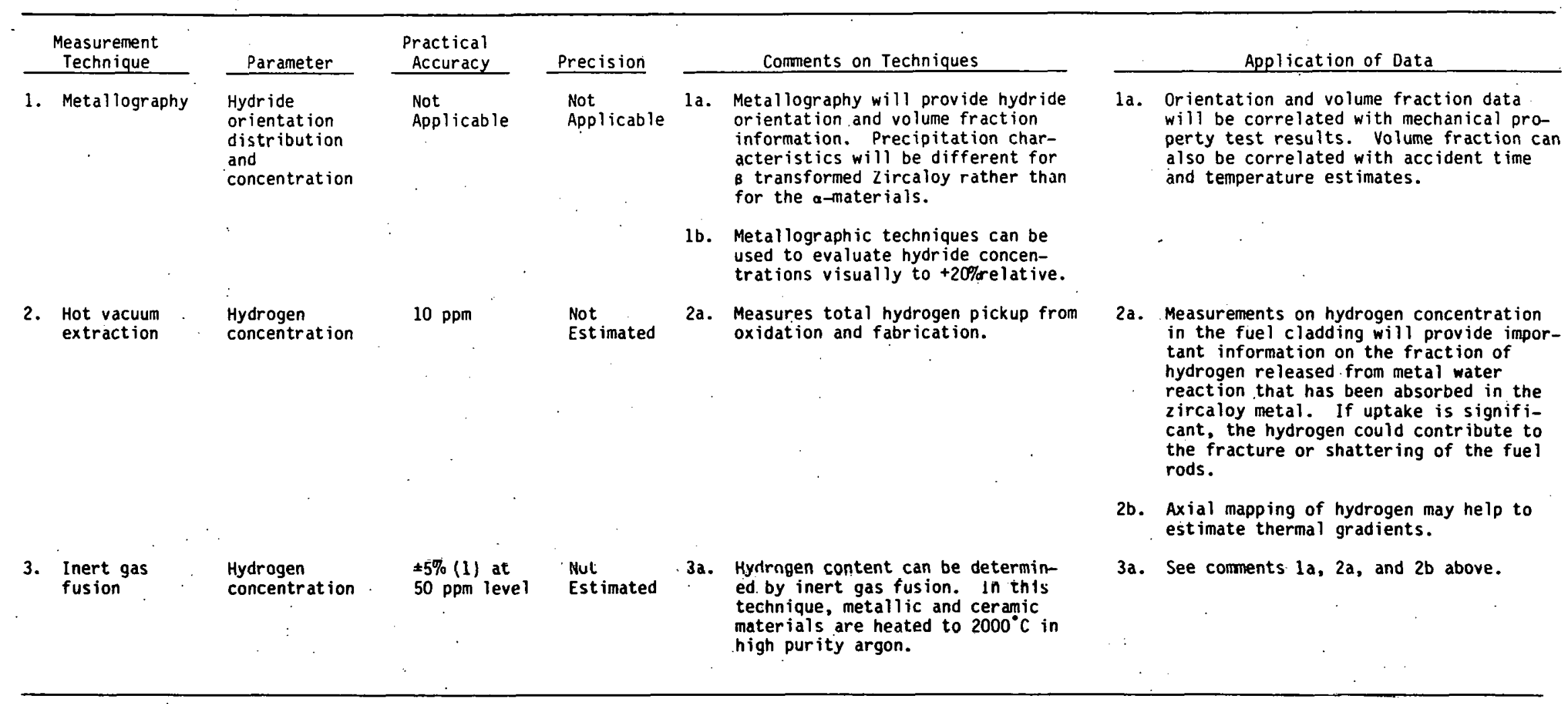


TABLE A-6. WALL OR COMPONENT THICKNESS

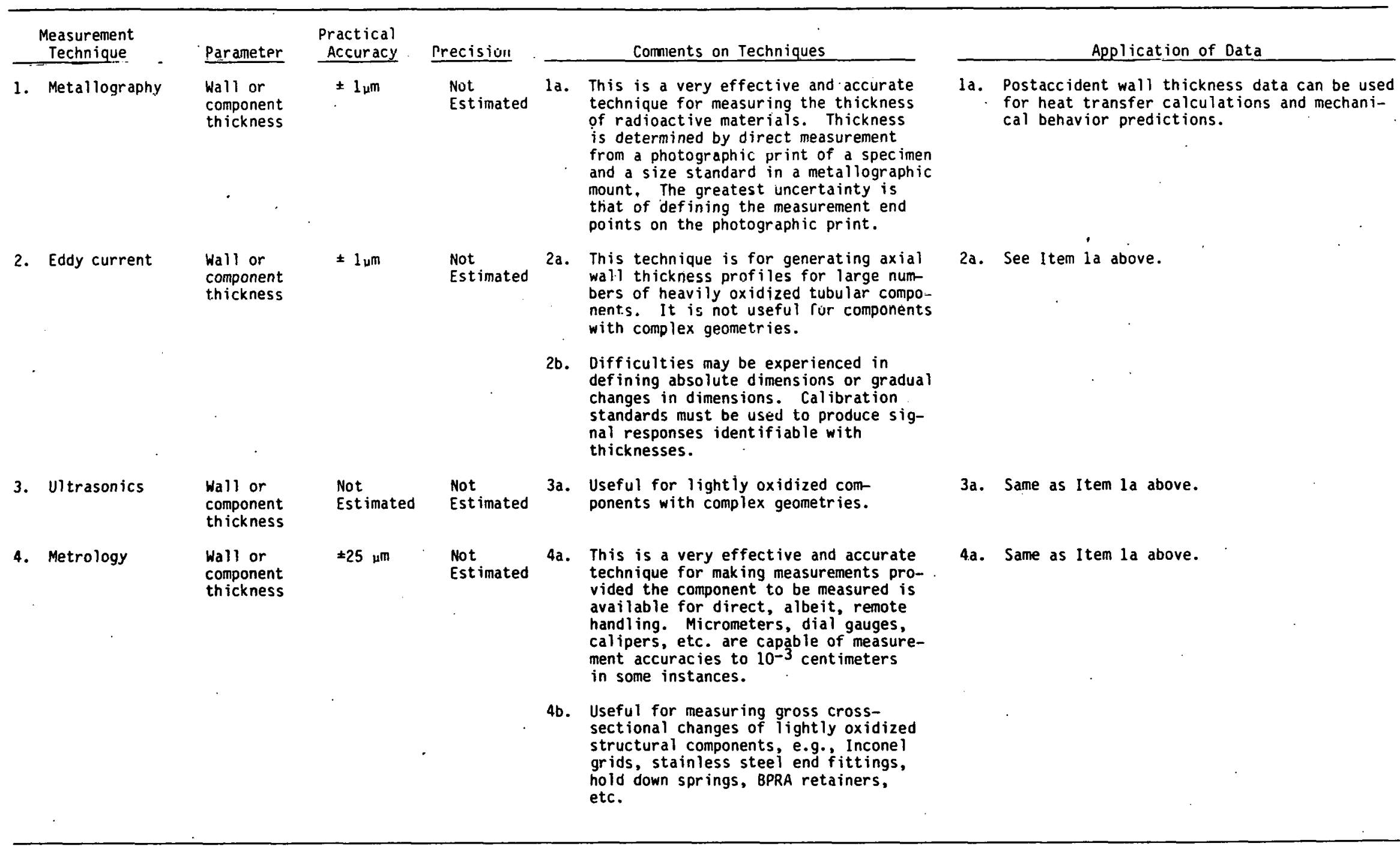


TABLE *A-7. DIMENSTIONAL AND FUNCTIONAL INTEGRITY

\begin{tabular}{|c|c|c|c|c|c|c|}
\hline & $\begin{array}{c}\text { Measurement } \\
\text { Technique }\end{array}$ & Parameter & $\begin{array}{l}\text { Practical } \\
\text { Accuracy }\end{array}$ & Precision & & Comments on Techniques \\
\hline 1. & Sulo probe & $\begin{array}{l}\text { Water } \\
\text { channel } \\
\text { spacing }\end{array}$ & $\pm 25 \mu \mathrm{m}$ & \pm 25 นm & la. & $\begin{array}{l}\text { This measurement will provide rod-to-rod } \\
\text { spacing on the intact portions of fuel } \\
\text { assemblies and will also provide informa- } \\
\text { tion in situ fuel rod bow. }\end{array}$ \\
\hline 2. & LVDT & $\begin{array}{l}\text { Assemb ly } \\
\text { bow }\end{array}$ & $250 \mu \mathrm{m}$ & $250 \mu \mathrm{m}$ & $2 \mathrm{a}$ & $\begin{array}{l}\text { This technique is more useful for measur- } \\
\text { ing fuel assembly envelope dimensions to } \\
\text { determine overall bow. }\end{array}$ \\
\hline 3. & $\begin{array}{l}\text { Stretch wire } \\
\text { techn ique }\end{array}$ & $\begin{array}{l}\text { Bow and } \\
\text { twist }\end{array}$ & $\pm 50 \mathrm{mils}$ & $\begin{array}{l}\text { Not } \\
\text { Est imated }\end{array}$ & $3 a$. & $\begin{array}{l}\text { Both bow and twist measurements of the } \\
\text { entire fuel assembly are possible using a } \\
\text { stretch wire technique. By using the top } \\
\text { and bottom nozzle plates as reference } \\
\text { points, it is possible to measure bow in } \\
\text { the assembly to within } \pm 50 \text { mils although } \\
\text { only a qualitative measure of the amount } \\
\text { of twist in the assembly is possible. } \\
\text { The position and distortion of the indi- } \\
\text { vidual components within an assembiy can } \\
\text { be determined with a Sulo type probe. } \\
\text { Channel closure, bow, and general distor- } \\
\text { tion of the fuel rods and control rod } \\
\text { cluster tubes could be measured to accu- } \\
\text { racies of } 100 \text { mils with this approach. }\end{array}$ \\
\hline
\end{tabular}

3b. Individual core components can also be measured for distortion onle they have been removed from the assemblies. The stretch wire technique can be employed to measure the bowing of components such as fuel rods, control rods, and neutron monitors to accuracies of \pm 20 mils.

$\begin{array}{llll}\text { 4. Laser } & \text { Fuel rod } & \pm 2.5-\mu m & \text { Not } \\ \text { profilometry } & \text { diameter }\end{array}$

5. X-radiography
Flaw

detection
Not

Estimated $\quad$ Not 4a. This technique will provide the overall fuel rod envelope and its noncontacting nature is particularly suited for measuring fragile samples. It would only be applicable to individual rods or peripheral rods in the fuel assembly.

5a. X-radiography is not likely to be of much use in examining the irradiated fuel rod assemblies or their components. The heavy fogging of the radiographic film by the act ivated components. will result in little or no detail.

\section{Application of Data}

1a. These data can be used to predict thermal-hydraulic behavior in the postaccident condition if intact assemblies still exist.

2a. Same as la above.

3a. Same as la above.

4a. These data can be used to measure average and local diametral strains as. a function of rod axial position (e.g., the amount of ballooning and its location). They can also be used lo predict thermal hydraulic performance. 
TABLE A-7. (continued)

\begin{tabular}{|c|c|c|c|c|c|c|c|}
\hline $\begin{array}{c}\text { Measurement } \\
\text { Technique }\end{array}$ & Parameter & $\begin{array}{l}\text { Practical } \\
\text { Accuracy }\end{array}$ & Precision & & Comments on Techniques & & Application of Data \\
\hline $\begin{array}{l}\text { 6. Neutron } \\
\text { radiography }\end{array}$ & $\begin{array}{l}\text { Flaws, } \\
\text { defects } \\
\text { fissile } \\
\text { content } 1\end{array}$ & $0.5 \mathrm{mi} 7 \mathrm{~s}$ & $0.5 \mathrm{mils}$ & $6 a$ & $\begin{array}{l}\text { Neutron radiography is frequently used to } \\
\text { provide valuable postirradiation data. A } \\
\text { high flux }\left(-2 \text { to } 5 \times 10^{6} \mathrm{n} / \mathrm{cm}^{2} \mathrm{sec}\right) \\
\text { neutron (both thermal and epithermal) } \\
\text { source is reguired. The neutron beall must } \\
\text { be well collimated (with } L / 0 \text { ratios of at } \\
\text { least } 50 \text { to } 1 \text { ) and the sample handling mech- } \\
\text { anisms designed to minimize scattering in }\end{array}$ & $6 a$. & $\begin{array}{l}\text { This technique will locate cracks or } \\
\text { other surface flaws that would contri- } \\
\text { bute to a degradation in the structural } \\
\text { integrity of various core components. } \\
\text { It will provide evidence of damage and } \\
\text { an indication of whether or not some } \\
\text { components which have not. failed are } \\
\text { at or near the damage thresholds. }\end{array}$ \\
\hline
\end{tabular}

7. Eddy current

8. Dye penetrant
Flaw

detection

Not

Surface
flaws
Est imated

Not $\begin{array}{ll}\text { Not } & \text { Not } \\ \text { Estimated } & \text { Estimated }\end{array}$ order to provide adequate resolution. The capabilities of computer assisted axial tomography using neutron radiography is rapidly fuel istortion.

6b. Fuel redistribution in a large number of ruds or a block of solidified debris may be difficult to determine if the interior rod images are observed in the radiograph. It may be possible, however, to measure total and axial fissile content wilh dll accuracy of -1

6c. Neutron radiography would be an excellent technique for characterizing defects and structural aspects of the fuel assemblies and their individual components. By util-
izing the proper techniques, fuel rod condiizing the proper techniques, fuel rod cond
tions - such as centerline fuel melting, internal void formation, fuel pellet cracking and fragmentation, and relocation of any fuel fragments - could be easily resolved. Dimensional changes should be repeatable and accurate to $0.5 \mathrm{mils}$.

6d. Radiography will be extremely useful for detecting internal flaws in large section components (e.g., end fittings and spider-couplings).

7a. Multi-frequency eddy current will be useful for locating flaws in tubular components (e.g., fuel and control rod components).

7a. See 6a above.

8a. This technique would be useful for detecting surface flaws in regularly shaped components upper vessel internals. 8a. See 6a above. 
TABLE A-7. (continued)

\begin{tabular}{|c|c|c|c|c|c|}
\hline $\begin{array}{l}\text { Measurement } \\
\text { Technique }\end{array}$ & Parameter & $\begin{array}{l}\text { Practical } \\
\text { Accuracy }\end{array}$ & Precision. & Corments on Techniques & Application of Data \\
\hline $\begin{array}{l}\text { 8. Oye } \\
\text { penetrant } \\
\text { (cont inued) }\end{array}$ & & . & & $\begin{array}{l}\text { 8b. Dye penetrant would be the most effective } \\
\text { technique for the inspection of a large } \\
\text { number of samples. }\end{array}$ & \\
\hline
\end{tabular}


TABLE A-8. MECHANICAL PROPERTIES

\begin{tabular}{|c|c|c|c|}
\hline $\begin{array}{c}\text { Measurement } \\
\text { Technique }\end{array}$ & Parameter & $\begin{array}{l}\text { Practical } \\
\text { Acrilurary }\end{array}$ & Precisinil \\
\hline \multirow{2}{*}{$\begin{array}{l}\text { 1. Uniaxial } \\
\text { and } \\
\text { biaxial } \\
\text { tension }\end{array}$} & \multirow{2}{*}{$\begin{array}{l}\text { Tensile } \\
\text { strength, } \\
\text { yield } \\
\text { strength, } \\
\text { ductility }\end{array}$} & $\begin{array}{l} \pm 2 \mathrm{ksi} \\
\text { (strength) }\end{array}$ & $\begin{array}{l} \pm 2 \mathrm{ksi} \\
\text { (strength) }\end{array}$ \\
\hline & & $\begin{array}{l} \pm 0.1 \% \\
\text { (ductility) }\end{array}$ & $\begin{array}{l} \pm 0.1 \% \\
\text { (ductility) }\end{array}$ \\
\hline
\end{tabular}

Curments on Techniques

1a. Tests under various loading conditions should be conducted to evaluate mechan $i$ cal properties of intact cladding, control rod components, spacer grid material, and end fittings.

1b. Samples should be selected from core axial and radial locations which represent the full range of expected property rostructure, evidence of the low ductility $\alpha+\beta$ phase region, or a fully transformed prior a microstructure.

1c. Uniaxial tension tests at room temperature and elevated temperatures can be performed on all the structures listed above. For materials which were originally anisotropic rings tensile and burst tests w111 provide useful supplemental information.

1d. All samples should be tested in the asreceived condition if possible. No attempt should be made to remove oxide layers or other deposits from the samples.

\section{Tube burst Transverse} strength and circumferential ductility

\section{Not}

Estimated

Not

Estimated

a. The tube burst test is a good means of evaluating the performance of fuel rod cladding tested in a bi-axial axial stress state. This test will provide an indication of circum-
ferential strength and ductility.

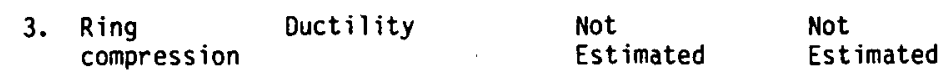

2a. The tube burst tests would provide information on the circumferential ductility of oxidized samples. The data, however, would not correlate with the balloon-rupture characteristics of the fuel cladding during the early stages of the transient.

3a. Compression tests, along with bend tests, will provide supplementary information to fully evaluate cladding ductility and mechanical behavior. 
TABLE A-8. (continued)

\begin{tabular}{|c|c|c|c|c|c|c|c|}
\hline $\begin{array}{c}\text { Measurement } \\
\text { Technique }\end{array}$ & Parameter & $\begin{array}{l}\text { Practical } \\
\text { Accuracy }\end{array}$ & Precision & & Comments on Techniques & & Application of Data \\
\hline 4. Bending & $\begin{array}{l}\text { Ductility and } \\
\text { embrittlement }\end{array}$ & $\begin{array}{l}\text { Not } \\
\text { Estimated } \\
\cdots\end{array}$ & $\begin{array}{l}\text { Not } \\
\text { Estimated }\end{array}$ & $4 a$. & $\begin{array}{l}\text { Experience with bend testing of irradi- } \\
\text { ated zircaloy fuel cladding at tempera- } \\
\text { tures to } 1300^{\circ} \mathrm{F}\left(700^{\circ} \mathrm{C}\right) \text { indicates that } \\
\text { this test does not provide a sensitive } \\
\text { measure of embrittlement as a result of } \\
\text { irradiation. The bend test should be a } \\
\text { more effective tool as an indication of } \\
\text { cladding embrittlement and ductility } \\
\text { for heavily oxidized specimens. }\end{array}$ & $4 a$. & Same as $3 a$ above. \\
\hline $\begin{array}{l}\text { 5. Load/ } \\
\text { def lection } \\
\text { tests }\end{array}$ & $\begin{array}{l}\text { Changes in } \\
\text { component } \\
\text { elasticity }\end{array}$ & $\begin{array}{l}\text { Not } \\
\text { Estimated }\end{array}$ & $\begin{array}{l}\text { Not } \\
\text { Estimated }\end{array}$ & $5 a$. & $\begin{array}{l}\text { Changes in spring constant and permanent } \\
\text { set should be measured for the hold down } \\
\text { springs and fuel rod internal springs } \\
\text { to evaluate changes in functional } \\
\text { integrity. } \\
\text { Changes in lift-off force and spring } \\
\text { rate can be measured on Incone? } \\
\text { spacer grids. }\end{array}$ & $5 a$. & $\begin{array}{l}\text { Load/deflection tests will provide } \\
\text { data to evaluate the functional in- } \\
\text { tegrity of the various spring and } \\
\text { spacer components within the core. } \\
\text { This information should prove useful } \\
\text { in assessing changes in core geome- } \\
\text { try during the course of the } \\
\text { accident. }\end{array}$ \\
\hline $\begin{array}{l}\text { 6. Expanding } \\
\text { mandrel }\end{array}$ & $\begin{array}{l}\text { Strength and } \\
\text { ductility }\end{array}$ & $\begin{array}{l}\text { Not } \\
\text { Estimated }\end{array}$ & $\begin{array}{l}\text { Not } \\
\text { Estimated. }\end{array}$ & $6 a$. & $\begin{array}{l}\text { Expanding mandrel tests have been used } \\
\text { successfully to duplicate pellet clad- } \\
\text { interaction resulting from fuel expan- } \\
\text { sion. However, the stress state in the } \\
\text { cladding may not be representative of a } \\
\text { LOCA unless the cladding has been col- } \\
\text { lapsed onto the fuel, which is not } \\
\text { thought to be the case for TMI-2. }\end{array}$ & & . \\
\hline 7. Impact & Embritt lement & $\begin{array}{l}\text { Not } \\
\text { Est imated }\end{array}$ & $\begin{array}{l}\text { Not } \\
\text { Estimated }\end{array}$ & $7 a$. & $\begin{array}{l}\text { Recent research efforts have been } \\
\text { directed towards determining the impact } \\
\text { propert ies of irradiated zircaloy } \\
\text { (H. M. Chung and T. F. Kassner, } \\
\text { ANL-79-48, NUREG/CR-1344, January 1980). } \\
\text { Due to the' highly strain-rate sensitive } \\
\text { nature of zircaloy, impact tests seem to } \\
\text { provide the most adaptable meains of } \\
\text { dcfining appropriate embrittlement } \\
\text { criteria. Impact tests will not } \\
\text { only be most useful in assessing the. } \\
\text { degree of embrittlement in heavily } \\
\text { oxidized sperimens, but can help to } \\
\text { verify the applicability of newly } \\
\text { developed embrittlement criteria. }\end{array}$ & $7 a$. & $\begin{array}{l}\text { Information obtained from impact } \\
\text { testing will provide data to evalu- } \\
\text { ate embrittlement failure criteria } \\
\text { under core-wide accident conditions } \\
\text { and can be used to compare with ex- } \\
\text { reactor tests. } \\
\end{array}$ \\
\hline
\end{tabular}


TABLE A-9. RETAINED FISSION PRODUCTS

\begin{tabular}{|c|c|c|c|c|c|c|c|}
\hline $\begin{array}{l}\text { Measurement } \\
\text { Technique }\end{array}$ & Parameter & $\begin{array}{l}\text { Practical } \\
\text { Accuracy }\end{array}$ & Precision & & Comments on Techniques & & Application of Data \\
\hline \multirow[t]{3}{*}{$\begin{array}{l}\text { Microprobe } \\
\text { analys is }\end{array}$} & $\begin{array}{l}\text { Quantitative } \\
\text { and gradient } \\
\text { analys is }\end{array}$ & $\begin{array}{l} \pm 10 \% \text { low } \\
\text { concentration } \\
\pm \text { high } \\
\text { concentration }\end{array}$ & $\begin{array}{l}\text { Not } \\
\text { Est imated }\end{array}$ & $1 \mathrm{a}$. & $\begin{array}{l}\text { By use of a shielded electron microprobe } \\
\text { (EMP), quantitative and gradient analysis } \\
\text { could be performed on materials to } 100 \mathrm{R} / \mathrm{hr} \\
\text { at } 1 \text { foot for elements Atomic No. } 11 \text { (Na) } \\
\text { through Atomic No. } 94(\mathrm{Pu}) \text {. }\end{array}$ & 1 a. & $\begin{array}{l}\text { These data can be used to deter- } \\
\text { mine the effects of transient } \\
\text { behavior on fission product dis- } \\
\text { tribution or redistribution } \\
\text { within the fuel. }\end{array}$ \\
\hline & & & & lh & $\begin{array}{l}\text { Ficcion producls lil the } 1 \text { range (total } \\
\text { elemental) could be analyzed to } \pm 10 \% \text {. } \\
\text { Three elements could be step-scañed simul- } \\
\text { taneously with a wave length dispersive } \\
\text { x-ray detector system for radia! or } \\
\text { cross-sectional gradients. }\end{array}$ & & ' \\
\hline & & . & . & $1 c$. & $\begin{array}{l}\text { The electron microprobe is useful for deter- } \\
\text { mining retained fission products in both the } \\
\text { display mode or as a quantitative determina- } \\
\text { tion of concentration with position along the } \\
\text { fuel radius. In the display mode, photo- } \\
\text { micrographs of the elemental distribution in } \\
\text { a ylven area are prepared. A separate photo- } \\
\text { micrograph is required for each element in } \\
\text { each area. Concentrations must be a few } \\
\text { percent or higher to produce a meaningful } \\
\text { signal. }\end{array}$ & & . \\
\hline \multirow[t]{2}{*}{$\begin{array}{l}\text { 2. Ion } \\
\text { microprobe }\end{array}$} & $\begin{array}{l}\text { Isotopic } \\
\text { analys is }\end{array}$ & $\begin{array}{l}\text { Accuracy and } \\
\text { precision are } \\
\text { typical of a } \\
\text { semi- } \\
\text { quantitative } \\
\text { technique }\end{array}$ & $\begin{array}{l}\text { Accuracy and } \\
\text { precision are } \\
\text { typical of a } \\
\text { semi- } \\
\text { quantitative } \\
\text { technique }\end{array}$ & $2 a$. & $\begin{array}{l}\text { Isotopic analysis of retained fission pro- } \\
\text { ducts can be accomplished using an ion micro- } \\
\text { probe mass analyzer or ion microscope. If } \\
\text { shielded versions of these instruments are } \\
\text { not available in current facilities, work must } \\
\text { be done using thin sample techniques and seg- } \\
\text { ments of fuel pin cross-sections. Displays } \\
\text { of isotope distribution, as well as quanti- } \\
\text { tative isotope ratios as a function of } \\
\text { position can be obtained. }\end{array}$ & $2 a$. & Same as la above. \\
\hline & . & & - & $2 b$. & $\begin{array}{l}\text { The ion microprobe is useful for determining. } \\
\text { relative concentrations profiles but not } \\
\text { absolute magnitudes. The sensitivity of the. } \\
\text { ion microprobe is in the parts per million } \\
\text { range or better for most elements. }\end{array}$ & $2 b$. & $\begin{array}{l}\text { The most likely application is } \\
\text { for determining I-129, a low- } \\
\text { activity isotope which will be } \\
\text { present in low concentrations. A } \\
\text { quant itative core-wide, I-129 } \\
\text { concentration will contribute to } \\
\text { the determination of a radiolog- } \\
\text { ical source time. Local varia- } \\
\text { tions in concentration can } \\
\text { contribute qualitatively to } \\
\text { determining the temperature } \\
\text { range. }\end{array}$ \\
\hline
\end{tabular}


TABLE A-9. (continued)

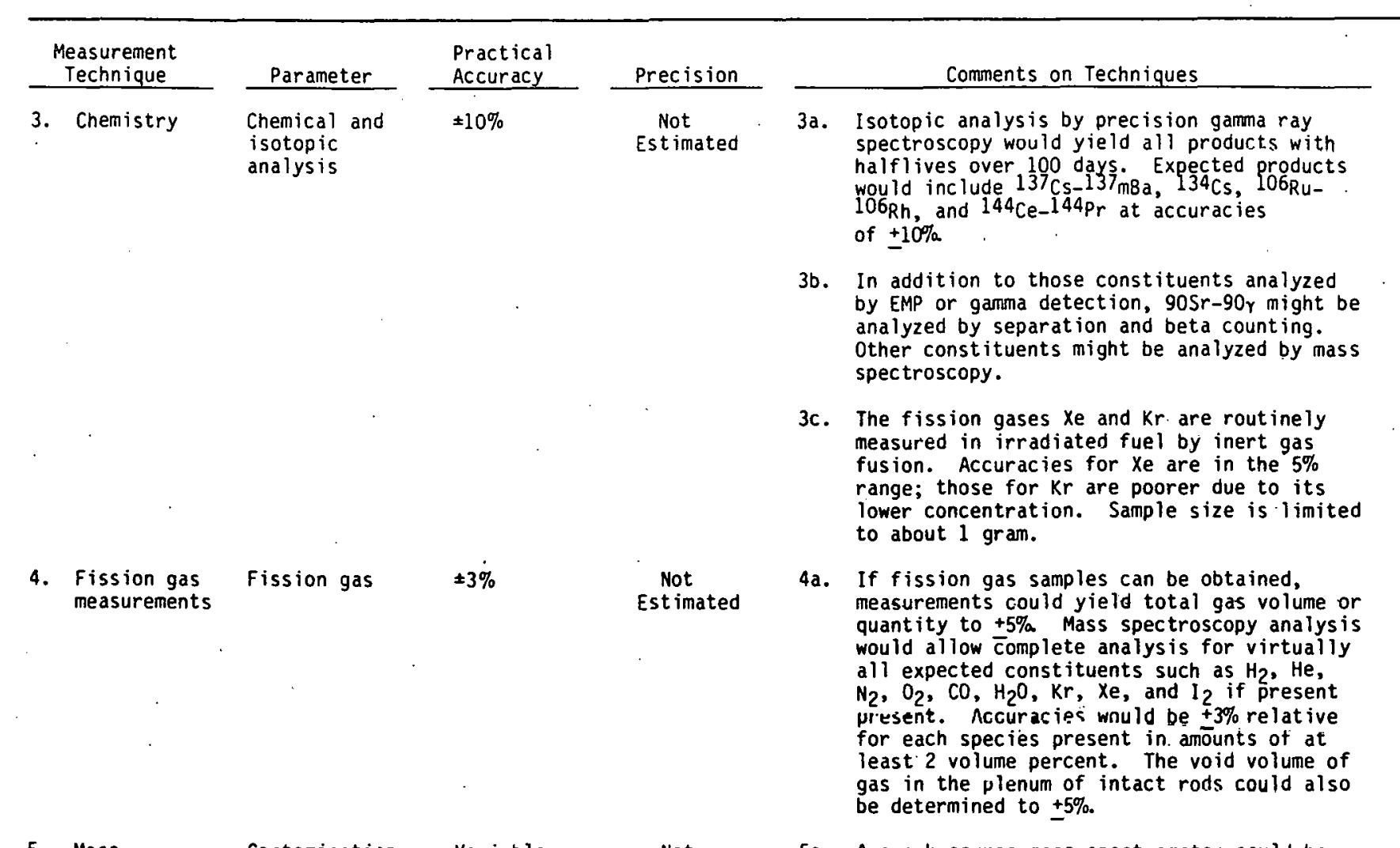
spectrometry and impurity Estimated used for trace impurity analysis of fuel segtration: at $100 \mathrm{ppm}$, it will be $0.1-0.5 \%$ reiative; at $10 \mathrm{ppm}, 10-50 \%$ relative; and at $1 \mathrm{ppm}$ it would be $50 \%$ relative.

6. Precision

Isotopic

distribution

2 to $5 \%$

Not

spectroscopy

Estimated

6a. Precision gamma spectrometry techniques

involve the use of multichannel analyzers

coupled to high resolution Ge-Li detectors.

nalys is usually requires hot cell sample dis

solution prior to radio chemical analysis.

For a few isotopes, chemical separation is

needed. Under favorable conditions, accura-

cies in the $2-5 \%$ range can be obtained.

4a. Same as la above.

5a. This analysis might provide some information on the mass transport of various elements during the course of the accident. 
TABLE A-9. (cont inued)

\begin{tabular}{|c|c|c|c|c|c|c|c|c|}
\hline \multicolumn{2}{|c|}{$\begin{array}{c}\text { Measurement } \\
\text { Technique }\end{array}$} & \multirow[t]{2}{*}{ Parameter } & \multirow[t]{2}{*}{$\begin{array}{l}\text { Practical } \\
\text { Acrouracy }\end{array}$} & \multirow[t]{2}{*}{ Precisiun } & \multicolumn{2}{|r|}{ Comments on Techniques } & \multicolumn{2}{|r|}{ Application of Data } \\
\hline 6. & $\begin{array}{l}\text { Precision } \\
\text { gamma } \\
\text { spectroscopy } \\
\text { (cont inued) }\end{array}$ & & & & $6 \mathrm{~b}$. & $\begin{array}{l}\text { Data from low burnup fuel which operated } \\
\text { under normal operating conditions can be } \\
\text { obtained and used for comparison. }\end{array}$ & & \\
\hline 7. & $\begin{array}{l}\text { Atomic } \\
\text { absorption } \\
\text { spectrometry }\end{array}$ & $\begin{array}{l}\text { Radiological } \\
\text { source term }\end{array}$ & $\begin{array}{l}\text { Not } \\
\text { Est imated }\end{array}$ & $\begin{array}{l}\text { Not } \\
\text { Estimated }\end{array}$ & $7 a$. & $\begin{array}{l}\text { The accuracy of this technique will depend } \\
\text { upon the concentration and the chemical } \\
\text { separation technique. }\end{array}$ & $7 a$. & $\begin{array}{l}\text { Could be used to help make quan- } \\
\text { titat ive determinations of fis- } \\
\text { sion products for radiological } \\
\text { source term, temperature, and } \\
\text { chemical interaction studies. }\end{array}$ \\
\hline
\end{tabular}


TABLE A-10. UO 2 OXIDATION (STOICHIOMETRY)

\begin{tabular}{|c|c|c|c|c|c|c|}
\hline & $\begin{array}{c}\text { Measurement } \\
\text { Technique }\end{array}$ & Parameter & $\begin{array}{l}\text { Practical } \\
\text { Accuracy }\end{array}$ & \multicolumn{2}{|l|}{ Precision } & Comments on Techniques \\
\hline 1. & Polarography & $0 / 0$ & $\begin{array}{l} \pm 10 \% \text { Not } \\
\text { Est imated }\end{array}$ & $\begin{array}{c}\text { Not } \\
\text { Est imated }\end{array}$ & $1 \mathrm{a}$. & $\begin{array}{l}\text { 0xygen-to-metal ratios by polarography } \\
\text { would be the most accurate means of } \\
\text { determining stoichiometry since compari- } \\
\text { sons can be made with known standards. } \\
\text { oxygen-to-metal ratios for } \mathrm{U}_{2} .0^{c} \text { an be } \\
\text { determined to } 10 \% \text { relative. }\end{array}$ \\
\hline 2. & Coulometry & $0 / U$ & $\pm 5 \quad(U)$ & $\begin{array}{l}\text { Not } \\
\text { Est imated }\end{array}$ & $2 a$. & $\begin{array}{l}\text { Another method for determination of } \mathrm{UO}_{2} \\
\text { oxidation is uranium analys is (also } \mathrm{Pu}^{2} \\
\text { if required) by coulometric techniques } \\
\text { for known } \mathrm{UO}_{2} \text { fuel weights. Accuracy } \\
\text { of elemental uranium could be quant if ied } \\
\text { to } \pm 5 \%\end{array}$ \\
\hline 3. & Thermogravimetric & $0 / U$ & $\begin{array}{c}\text { Not } \\
\text { Est imated }\end{array}$ & 0.0005 & $3 a$. & $\begin{array}{l}\text { Fuel pellets would first be exposed to a } \\
\text { He- } \mathrm{H}_{2}-\mathrm{H}_{2} \mathrm{O} \text { atmosphere at elevated } \\
\text { temperatures. The initial oxidation } \\
\text { rate data, acquired in a real-time mode } \\
\text { would be used to predict the oxidation } \\
\text { end point and to calculate the addi- } \\
\text { tional oxygen-to-metal ratio. Precision } \\
\text { is } 0.0005 \text {. }\end{array}$ \\
\hline & . & . & \pm 0.01 & $\begin{array}{l}\text { Not } \\
\text { Estimated }\end{array}$ & 3b. & 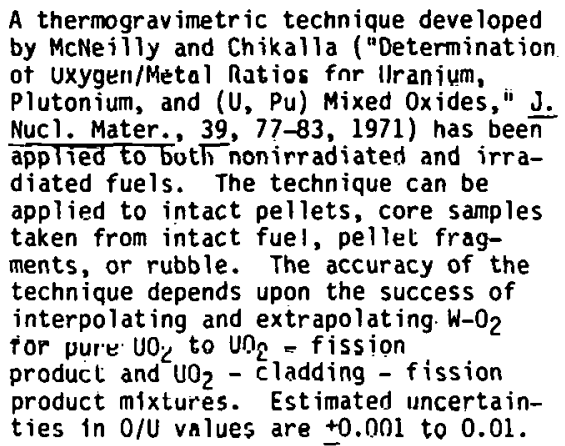 \\
\hline
\end{tabular}

$\begin{array}{lcc}\text { Lattice } & \text { Not } & \text { Not } \\ \text { distortion } & \text { Estimated } & \text { Estimated }\end{array}$

4a. Qualitative and general electron chanexamination of small samples. These patterns can be used to determine changes or distortion to the crystal lattice. Electron microprobe anaiys is might also be considered as a supplemental analys is for determining $\mathrm{UO}_{2}$ variations or gradients.

Data_ication of

1a. Analysis of selected fuel samples for $0 / 0$ will indicate the degree of oxidation of exposed fuel during the course of the accident. Comparisions with adjacent seg-
ments of cladding will indicate the relathe fue 1 and interior zircaloy surfaces.

2a. Same as la above.

3a. Thermogravimetric techniques should be useful to analyze liquified fuel rubble when dissolution of $\mathrm{UO}_{2}$ in oxygen saturated a $\mathrm{Zr}$ has taken place. The stoichio metry in the $(U, Z r) 0_{2-x}$ will provide measure of the maximum temperature
reached above that of the liquids.

4a. Crystallographic measurements will supplement the $0 / U$ measurements by providing information on the mechanics of the oxidation process. It will also be useful in analyzing liquef ied fuel samples where ternary phase interactions have taken place. 
TABLE A-10. U02 OXIDATION (STOICHIOMETRY)

\begin{tabular}{|c|c|c|c|c|c|c|c|c|}
\hline & $\begin{array}{c}\text { Measurement } \\
\text { Technique }\end{array}$ & Parameter & $\begin{array}{l}\text { Practical } \\
\text { Accuracy }\end{array}$ & Precision & & Comments on Techniques & & Application of Data \\
\hline 5. & $\begin{array}{l}\text { Electrolyte } \\
\text { probe }\end{array}$ & $0 / 4$ & \pm 0.1 & $\begin{array}{l}\text { Not } \\
\text { Est imated }\end{array}$ & $5 a$. & $\begin{array}{l}\text { An electrolyte probe technique developed } \\
\text { by Bones and Carney, ("A Galvanic Cell } \\
\text { Technique for the Rapid Measurement of } \\
\text { O/U Ratios In-0xide Fuel Pellets," } \\
\text { AERE-R-6301, 1969) has been applied to } \\
\text { non-irradiated and irradiated fuels. } \\
\text { This technique is most readily applied } \\
\text { to samples with flat surfaces due to the } \\
\text { need for electrical contact between } \\
\text { probe and sample. The accuracy of this } \\
\text { technique is similar to that described } \\
\text { in } 3 b \text { above. }\end{array}$ & 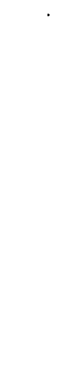 & . \\
\hline 6. & Electrochemical & $\begin{array}{l}\mathrm{UO}_{2} \\
\text { oxidation }\end{array}$ & $\begin{array}{c}\text { Not } \\
\text { Est imated }\end{array}$ & $\begin{array}{l}\text { Not } \\
\text { Estimated }\end{array}$ & $6 a$. & $\begin{array}{l}\text { Electrochemical dissolution of oxidized } \\
\text { fuel can be used to evaluate the charac- } \\
\text { teristics of pellet surfaces. The } \\
\text { results are compared to laboratory } \\
\text { samples which have been oxidized under } \\
\text { controlled conditions and the oxidation } \\
\text { films charactcrized by x-ray diffrac- } \\
\text { tion, SEM, or Auger analysis. The dis- } \\
\text { solution products can also be used to } \\
\text { provide information on the chemical dis- } \\
\text { tribution of radionuclides in the } \\
\text { sample. }\end{array}$ & $6 a$. & $\begin{array}{l}\text { This analysis technique may prove useful } \\
\text { in separating out the effects of low tem- } \\
\text { perature oxidation occurring over the long } \\
\text { time period since the accident, as the } \\
\text { exposed } \mathrm{UO}_{2} \text { reacts with oxygen dissolved } \\
\text { in the coolant. }\end{array}$ \\
\hline 7. & $\begin{array}{l}\text { x-ray } \\
\text { diffraction }\end{array}$ & $\begin{array}{l} \pm 0.010 / U \\
\text { unit }\end{array}$ & $\begin{array}{l} \pm 0.0050 / U \\
\text { unit }\end{array}$ & & $7 a$. & $\begin{array}{l}\text { This method relies on standardized tech- } \\
\text { niques. The specimen can be either a } \\
\text { polished metallographic mount or a } \\
\text { powdered compact. Other phases in the } \\
\text { U- } 0 \text { systems (e.g., U metal, } U_{3} 0_{8} \text { ) can } \\
\text { can be also identified by } x \text {-ray } \\
\text { diffraction. }\end{array}$ & $7 a$. & $\begin{array}{l}\text { This information should be useful in } \\
\text { estimating core thermal history, determin- } \\
\text { ation of gas atmosphere in contact with } \\
\text { fuel, mechanisms of fission-product } \\
\text { release, etc. }\end{array}$ \\
\hline
\end{tabular}


TABLE A-11. DEBRIS AND RUBBLE BEO ANALYSIS

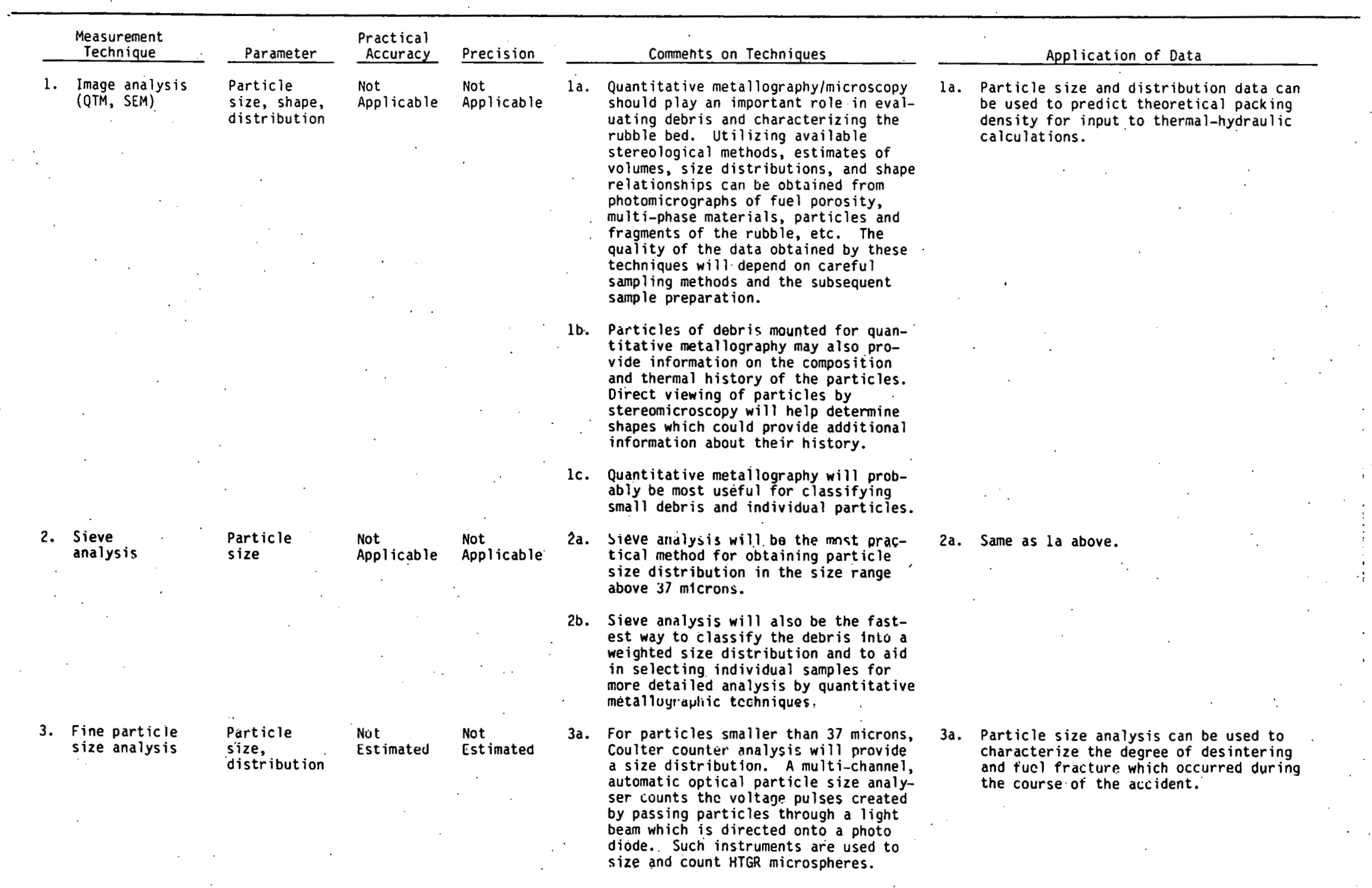


TABLE A-11. (continued)

\section{Measurement}

Technique

3. Fine particle

size analysis

lcontinued

4. Gamma and

emission

spectroscopy

Composition

Composition

Not

Estimated

Not

Est imated

-1
1
8

5. Chemical

analys is

Composition

\section{Not}

Estimated

6. Visual

examinations
General size, Not Not shape, and Applicable Applicable
Comments on Techniques

3b. Fischer subsieve analys is can be used to provide the average particle size for the fraction below 37 microns.

3c. Separation of particles for more detailed analysis may be desirable and could best be done by alluviation or using a microparticle separator such as a Bahco Unit.

4a. These techniques $c$ an be used to deter mine the composition and source of individual pleces of debris.

4b. The particles could be analyzed by gamma spectroscopy and possibly by alpha spectroscopy to determine the Seyeral transuranic materjals such as might be determined by alpha pulse height spectroscopy. Accuracy would depend on the nature of the sample. Filter samples may be imited to qualitative identification only. More definitive samples with purification may allow quantitative analysis.

5a. Chemical analyses of the debris could help determine the origin of the paricles. This information could be used to complement and conf irm metallograpnic and activity analysis.

6a. One appropriate visual method for debris size and shape analys is would be to use a television optic system supported by an image analys is computer system and a digital videosignal recording or video tape system.

6b. A Questar telescope coupled with an Omni-scope ( $1 x$ image) can also be used for detailed analysis of selected
Application of Data

4a. Data on debris composition indicating the isotopes present can be used to predict heat generation rates in the rubble pile for input into thermo-hydraulic calculations. 
TABLE A-11. (cont inued)

\begin{tabular}{|c|c|c|c|c|c|c|c|c|}
\hline & $\begin{array}{c}\text { Measurement } \\
\text { Technique }\end{array}$ & Parameter & $\begin{array}{l}\text { Practical } \\
\text { Accuracy } \\
\end{array}$ & Precision & & Comments on Techniques & & Application of Data \\
\hline 7. & $\begin{array}{l}\text { Impregnation } \\
\text { and neutron } \\
\text { radiography }\end{array}$ & $\begin{array}{l}\text { Packing } \\
\text { density }\end{array}$ & $\begin{array}{l}\text { Not } \\
\text { Applicable }\end{array}$ & $\begin{array}{l}\text { Not } \\
\text { Applicable }\end{array}$ & 7a. & $\begin{array}{l}\text { Impregnation of the rubble bed with } \\
\text { epoxy or some other suitable filler } \\
\text { medium followed by the sectioning } \\
\text { would permit quant itative metal- } \\
\text { lography that would determine density } \\
\text { and an est fimation of the amount of } \\
\text { interconnected porosity that would } \\
\text { permit water flow through the bed. } \\
\text { Radiography could also be used to } \\
\text { estimate the resistance of the bed to } \\
\text { water flow. }\end{array}$ & $7 a$. & $\begin{array}{l}\text { Analyses of the rubble bed packing den- } \\
\text { sity and interconnected flow paths which } \\
\text { would permit water flow through the bed } \\
\text { will provide information on the thermo- } \\
\text { hydraulic performance and coolability } \\
\text { of a badly perturbed core geometry. }\end{array}$ \\
\hline
\end{tabular}

$\stackrel{v}{i}$ 